\title{
Catalytic Asymmetric Tandem Reaction of o-Alkynylbenzaldehydes, Amines, and Diazo Compounds
}

Wei Wu, Na Liao, Qi Wei, Jiaying Huang, Qi Huang and Yungui Peng*

Key Laboratory of Applied Chemistry of Chongqing Municipality, School of Chemistry and Chemical Engineering, Southwest University, Chongqing 400715, China

*E-mail address: pengyungui@hotmail.com; pyg@swu.edu.cn

\section{Table of Contents}

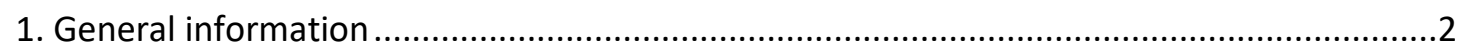

2. General procedure for the preparation of o-alkynylbenzaldehyde derivatives ${ }^{1} \ldots \ldots \ldots \ldots \ldots \ldots . . .2$

3. Synthesis of chiral phosphoric acid and imidodiphosphate ................................................

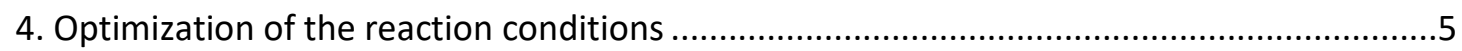

5. General procedure for the catalytic asymmetric three-component reaction .......................8

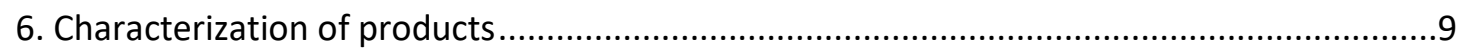

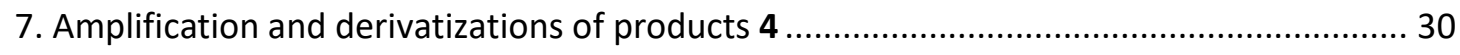

8. Determination of the absolute configuration of product 4 iaa and 7................................. 34

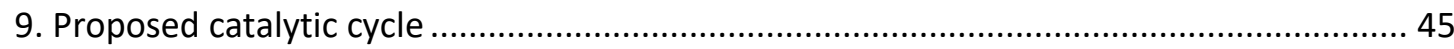

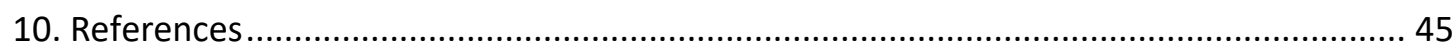

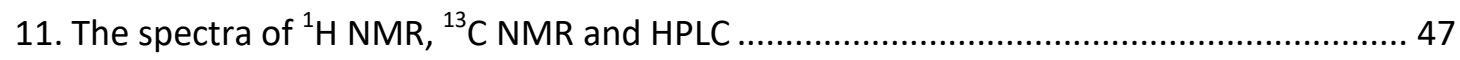




\section{General information}

Unless stated otherwise, all reactions were carried out under argon atmosphere. All the reactions that need to be heated, the oil bath is used as a heating source. All solvents were purified by standard procedures and distilled prior to use. Reagents obtained from commercial source were used without further purification. Petroleum ether and ethyl acetate for flash column chromatography was distilled before use. All reactions were monitored by TLC with silica gel coated plates. Flash column chromatography was performed on silica gel (300-400 mesh). NMR spectra were recorded on Bruker Avance $600 \mathrm{MHz}$ instruments. Chemical shifts $(\delta)$ are given in ppm relative to TMS, coupling constants $(J)$ in Hz. ${ }^{1} \mathrm{H}$ NMR chemical shifts are reported in ppm relative to tetramethylsilane (TMS) with the solvent signal as the internal standard $\left(\mathrm{CDCl}_{3}\right.$ at $\left.7.26 \mathrm{ppm}\right) .{ }^{13} \mathrm{C}$ NMR chemical shifts are reported in ppm from tetramethylsilane (TMS) with the solvent resonance as the internal standard $\left(\mathrm{CDCl}_{3}\right.$ at $\left.77.00 \mathrm{ppm}\right)$. Melting points were determined on an $\mathrm{X}-6$ digital melting-point apparatus and were uncorrected. Optical rotations were measured on a Perkin Elmer 341 Polarimeter at $\lambda=589 \mathrm{~nm}$. Analytical high performance liquid chromatography (HPLC) was carried out on WATERS 510 instrument (2487 Dual $\lambda$ Absorbance Detector and 515 HPLC Pump). ESI HRMS (Bio TOF Q) spectra were recorded on P-SIMS-Gly of Bruker Daltonics Inc. $3 \AA$ MS, $4 \AA$ MS and $5 \AA$ MS were purchased from J\&K Chemicals and used as received (500 mesh powder). The all starting compounds are known compounds.

\section{General procedure for the preparation of o-alkynylbenzaldehyde derivatives ${ }^{1}$}

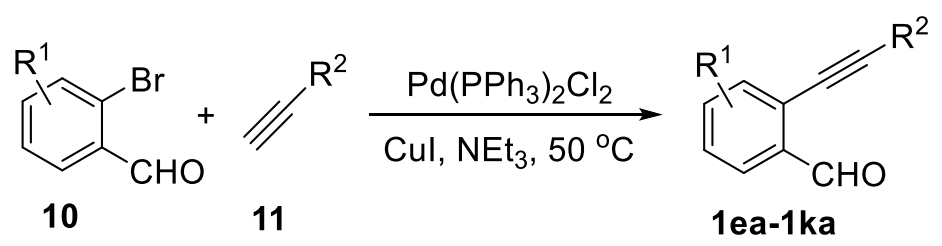

Under Ar atmosphere, the appropriate acetylene 11 (1.2 equiv) was added to the solution of the corresponding 2-bromobenzaldehyde 10 (1.0 equiv), $\mathrm{PdCl}_{2}\left(\mathrm{PPh}_{3}\right)_{2}$ (2 mol \%), and $\mathrm{CuI}(1 \mathrm{~mol} \%)$ in $\mathrm{NEt}_{3}(0.25 \mathrm{M})$. The resulted mixture was heated at $50{ }^{\circ} \mathrm{C}$ for $12 \mathrm{~h}$ under Ar atmosphere. After the reaction was completed, quenched with distilled water and extracted with $\mathrm{CH}_{2} \mathrm{Cl}_{2}$ (three times). Combined organic layer and washed with brine, dried over $\mathrm{Na}_{2} \mathrm{SO}_{4}$, concentrated under reduce pressure. The residue was purified by column chromatography on silica gel to afford the desired product 1ea-1ka. 


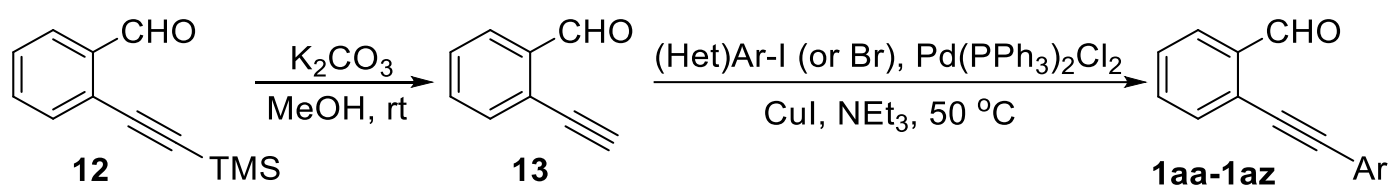

Compound $12(1.0 \mathrm{mmol})$ was dissolved in $\mathrm{MeOH}(14 \mathrm{~mL}, 0.07 \mathrm{M})$ and treated with $\mathrm{K}_{2} \mathrm{CO}_{3}$ (276.4 mg, 2 equiv), stirred at $\mathrm{rt}$ for $1 \mathrm{~h}$, the reaction mixture was diluted with water and extracted with $\mathrm{CH}_{2} \mathrm{Cl}_{2}$ (three times). Combined organic layer and dried over $\mathrm{Na}_{2} \mathrm{SO}_{4}$, concentrated under reduce pressure and the residue was purified by column chromatography on silica gel to afford the desired product $\mathbf{1 3}$.

Aryl halide (3.6 mmol, 1.2 equiv) was added to the solution of compound 13 (390 $\mathrm{mg}, 3.0 \mathrm{mmol}), \mathrm{PdCl}_{2}\left(\mathrm{PPh}_{3}\right)_{2}(42.0 \mathrm{mg}, 0.06 \mathrm{mmol}, 2 \mathrm{~mol} \%)$ and $\mathrm{CuI}(5.7 \mathrm{mg}, 0.03$ mmol, $1 \mathrm{~mol} \%)$ in $\mathrm{NEt}_{3}(12 \mathrm{ml}, 0.25 \mathrm{M})$, stirred at $50{ }^{\circ} \mathrm{C}$ for $8 \mathrm{~h}$ under Ar atmosphere, the reaction mixture was quenched with distilled water and extracted with $\mathrm{CH}_{2} \mathrm{Cl}_{2}$ (three times). Combined organic layer and washed with brine, dried over $\mathrm{Na}_{2} \mathrm{SO}_{4}$, concentrated under reduce pressure and the residue was purified by column chromatography on silica gel to afford the desired product 1aa-1az.

\section{Synthesis of chiral phosphoric acid and imidodiphosphate}

Ia-Ik were synthesized following the procedure described in the literature. ${ }^{2}$

Partial intermediates for the synthesis of chiral imidodiphosphate were prepared as the following procedure:

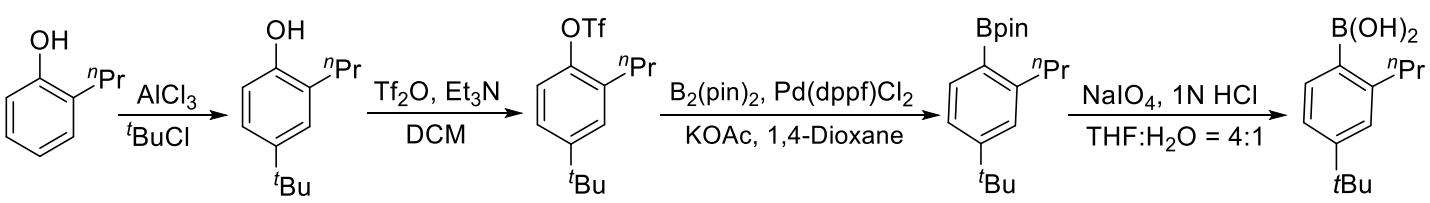

2-propylphenol (5.0 g, $36.74 \mathrm{mmol})$ was dissolved in $50 \mathrm{~mL}$ dry dichloromethane and $4.8 \mathrm{~mL}$ (44.10 mmol) 2-chloro-2-methylpropane was added. Aluminum chloride (0.367 g, $2.75 \mathrm{mmol})$ was slowly added and the mixture was stirred under nitrogen overnight, untill the starting material was disappeared. The reaction was quenched with water and the organic layer was separated, dried over with $\mathrm{Na}_{2} \mathrm{SO}_{4}$ and concentrated under reduce pressure. The residue was purified by flash chromatography on silica gel $(\mathrm{PE} / \mathrm{EA}=10 / 1)$ to give 4-tert-butyl-2-propylphenol with colorless liquid $\left(5.43 \mathrm{~g}, 77 \%\right.$ yield). ${ }^{3}$

$\mathrm{Tf}_{2} \mathrm{O} 5.3 \mathrm{~mL}(31.2 \mathrm{mmol})$ was added dropwise to the solution of 4-tert-butyl-2propylphenol $5.0 \mathrm{~g}(26.0 \mathrm{mmol})$ and $\mathrm{Et}_{3} \mathrm{~N} 5.4 \mathrm{~mL}(39.0 \mathrm{mmol})$ in $50 \mathrm{~mL}$ dry DCM at $0{ }^{\circ} \mathrm{C}$. The mixture was stirred until the start material was disappeared, after 
concentrated under reduce pressure. The residue was purified by flash chromatography on silica gel $(\mathrm{PE} / \mathrm{EA}=20 / 1)$ to give 4-tert-butyl-2-propylphenyl trifluoromethanesulfonate with colorless liquid $(5.90 \mathrm{~g}, 70 \%$ yield).

$\mathrm{Pd}(\mathrm{dppf}) \mathrm{Cl}_{2} 563 \mathrm{mg}(0.77 \mathrm{mmol})$ was added to the mixture of the above product $5.0 \mathrm{~g}(15.4 \mathrm{mmol}), \mathrm{B}(\mathrm{pin})_{2} 4.7 \mathrm{~g}(18.5 \mathrm{mmol})$ and KOAc $10.6 \mathrm{~g}(107.8 \mathrm{mmol})$ in degassing dioxane $50 \mathrm{~mL}$ under an Ar atmosphere in a round bottomed flask. The mixture was refluxed untill the start material was disappeared, the mixture was filtrated and washed with $\mathrm{Et}_{2} \mathrm{O}$, combined organic layer and washed with saturated $\mathrm{NaCl}$, dried over with $\mathrm{Na}_{2} \mathrm{SO}_{4}$ and concentrated under reduce pressure, and the boronate was achieved by flash chromatography on silica gel (2.85 g, $88 \%$ yield).

$\mathrm{NaIO}_{4} 10.36 \mathrm{~g}$ (48 mmol) was added to the solution of the above obtained boronate $2.8 \mathrm{~g}(16 \mathrm{mmol})$ in $\mathrm{THF} / \mathrm{H}_{2} \mathrm{O}(\mathrm{v}: \mathrm{v}=4: 1,50 \mathrm{~mL})$, the mixture was stirred for $30 \mathrm{~min}$ at $\mathrm{rt}$, then $1 \mathrm{~N} \mathrm{HCl}(20 \mathrm{~mL})$ was added. The mixture was stirred at room temperature untill the start material was disappeared, THF was removed, and the residue was extracted with $\mathrm{Et}_{2} \mathrm{O}$, combined the organic layers and dried over with $\mathrm{Na}_{2} \mathrm{SO}_{4}$, concentrated under reduce pressure and the residue was purified by flash chromatography on silica gel to achieve the 4-tert-butyl-2-propylphenylboronic acid. ${ }^{4}$

IIa-IIm were synthesized following the procedure described in the literature. ${ }^{5}$

\section{Imidodiphosphate IIh}

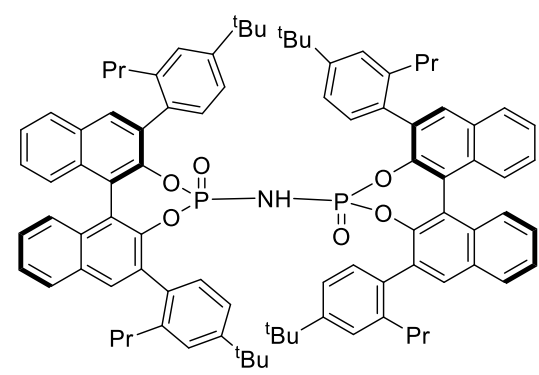

Colorless solid, mp: 142.7-143.9 ${ }^{\circ} \mathrm{C} ;{ }^{1} \mathrm{H}$ NMR (600 $\left.\mathrm{MHz}, \mathrm{CDCl}_{3}\right) \delta 8.01(\mathrm{~d}, J=8.2 \mathrm{~Hz}, 2 \mathrm{H}), 7.91(\mathrm{~d}, J=$ $8.2 \mathrm{~Hz}, 2 \mathrm{H}), 7.88-7.80(\mathrm{~m}, 4 \mathrm{H}), 7.64(\mathrm{~d}, J=8.5 \mathrm{~Hz}$, $2 \mathrm{H}), 7.60-7.53(\mathrm{~m}, 2 \mathrm{H}), 7.50-7.44(\mathrm{~m}, 4 \mathrm{H}), 7.38-$ $7.27(\mathrm{~m}, 4 \mathrm{H}), 7.23(\mathrm{~s}, 2 \mathrm{H}), 7.11(\mathrm{~s}, 2 \mathrm{H}), 6.96(\mathrm{~s}, 2 \mathrm{H})$, $6.39-5.62(\mathrm{~m}, 6 \mathrm{H}), 5.16(\mathrm{~s}, 1 \mathrm{H}), 2.56-2.14(\mathrm{~m}$, 9H), $1.35-1.27(\mathrm{~m}, 22 \mathrm{H}), 1.07-0.79(\mathrm{~m}, 23 \mathrm{H}), 0.65-0.40(\mathrm{~m}, 10 \mathrm{H}) \mathrm{ppm} .{ }^{13} \mathrm{C}$ NMR $\left(151 \mathrm{MHz}, \mathrm{CDCl}_{3}\right) \delta 150.3,149.7,145.5,140.9,140.0,134.7,133.0,132.3$, 132.2 , 131.9, 131.4, 131.3, 129.5, 128.7, 128.3, 127.4, 126.6, 126.2, 125.7, 125.4, 124.7, 122.6, 122.1, 121.6, 36.1, 34.5, 31.3, 23.8, 14.1 ppm. HRMS (ESI) m/z: $[\mathrm{M}+\mathrm{H}]^{+}$Calcd. for $\mathrm{C}_{92} \mathrm{H}_{98} \mathrm{NO}_{6} \mathrm{P}_{2} 1374.6869$, found 1374.6864 


\section{Optimization of the reaction conditions}

Table S1. Metal screening for the reaction
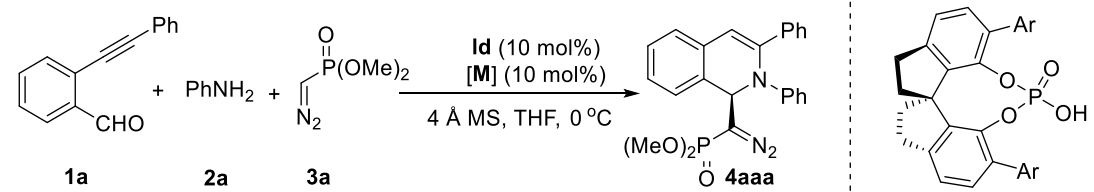

Id: $\mathrm{Ar}=3,5-(\mathrm{Me})_{2}-4-(4-$ $\left.\mathrm{MeOC}_{6} \mathrm{H}_{4}\right)-\mathrm{C}_{6} \mathrm{H}_{2}$

\begin{tabular}{ccccc}
\hline Entry $^{a}$ & {$[\mathbf{M}]$} & Ligand & Yield of 4aaa $^{b}$ & $e e(\%)^{c}$ \\
\hline 1 & & Id & $\mathrm{NR}$ & - \\
2 & $\mathrm{AgNO}_{3}$ & - & 70 & - \\
3 & $\mathrm{AgOTf}$ & - & 79 & 0 \\
4 & $\mathrm{AgOAc}$ & - & 68 & 56 \\
$5^{d}$ & $\mathrm{AgOAc}^{d, e}$ & Id & 51 & 60 \\
$6^{d, e}$ & $\mathrm{Ag}_{2} \mathrm{CO}_{3}$ & Id & 41 & - \\
$7^{d}$ & $\mathrm{Cu}(\mathrm{OAc})_{2}$ & Id & $\mathrm{NR}$ & - \\
$8^{d}$ & $\mathrm{Fe}(\mathrm{acac})_{3}$ & Id & $\mathrm{NR}$ & - \\
$9^{d}$ & $\mathrm{Pd}(\mathrm{OAc})_{2}$ & Id & $\mathrm{NR}$ & \\
\hline
\end{tabular}

${ }^{a}$ Unless otherwise specified, the reaction was performed with $\mathbf{1 a}(0.24 \mathrm{mmol}), \mathbf{2 a}(0.22 \mathrm{mmol}), \mathbf{3 a}(0.2 \mathrm{mmol}), 4 \AA$ MS (200 mg), ligand (10 mol \%), and [M] (10 mol \%) in $1.0 \mathrm{~mL}$ of THF at $\mathrm{rt}$ for $24 \mathrm{~h}$ under argon. ${ }^{b}$ Isolated yield.

${ }^{c}$ Determined by chiral HPLC. ${ }^{d}$ Reaction at $0{ }^{\circ} \mathrm{C}$ for $24 \mathrm{~h} .{ }^{e} \mathrm{Ag}_{2} \mathrm{CO}_{3}(5 \mathrm{~mol} \%)$.

Table S2. Catalyst screening for the reaction

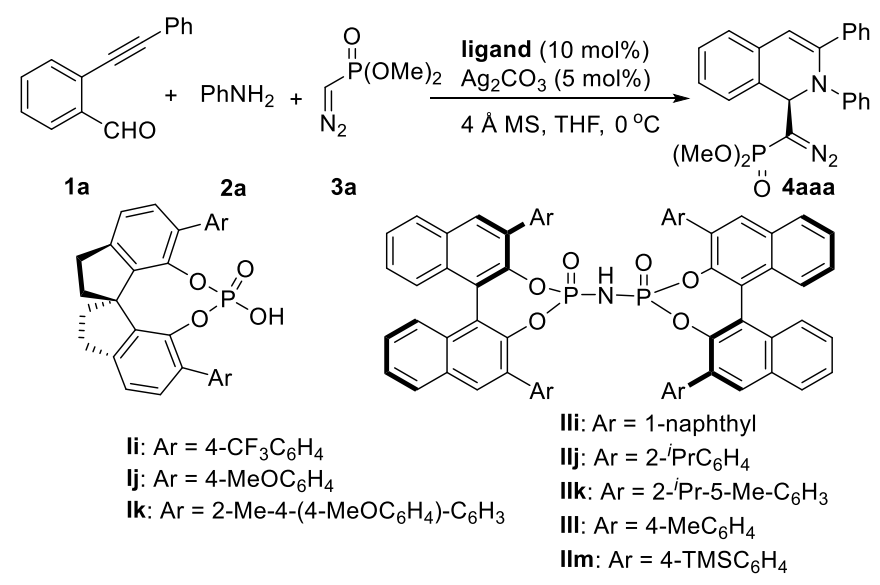

\begin{tabular}{cccc}
\hline Entry $^{a}$ & Ligand & Yield of 4aaa $(\%)^{b}$ & $e e(\%)^{c}$ \\
\hline 1 & Ii & 65 & 6 \\
2 & Ij & 67 & 19 \\
3 & Ik & 87 & 37 \\
& & 5 &
\end{tabular}




\begin{tabular}{cccc}
4 & IIi & 95 & 22 \\
5 & IIj & 38 & 11 \\
6 & IIk & 75 & 18 \\
7 & IIl & 88 & 29 \\
8 & IIm & 94 & 44 \\
\hline
\end{tabular}

${ }^{a}$ Unless otherwise specified, the reaction was performed with $\mathbf{1 a}(0.24 \mathrm{mmol}), \mathbf{2 a}(0.22 \mathrm{mmol}), \mathbf{3 a}(0.2 \mathrm{mmol}), 4 \AA$ MS (200 mg), ligand (10 mol \%), and $\mathrm{Ag}_{2} \mathrm{CO}_{3}(5 \mathrm{~mol} \%)$ in $1.0 \mathrm{~mL}$ of THF at $0{ }^{\circ} \mathrm{C}$ for $24 \mathrm{~h}$ under argon. ${ }^{b}$ Isolated yield. ${ }^{c}$ Determined by chiral HPLC.

Table S3. The effect of solvent and concentration on the reaction

\begin{tabular}{|c|c|c|c|}
\hline Entry $^{a}$ & Solvent & Yield $(\%)^{b}$ & $e e(\%)^{c}$ \\
\hline 1 & $\mathrm{PhMe}$ & 96 & 70 \\
\hline 2 & $\mathrm{PhF}$ & 96 & 82 \\
\hline 3 & DCM & 95 & 75 \\
\hline 4 & THF & 93 & 90 \\
\hline 5 & 2-Me-THF & 96 & 73 \\
\hline 6 & MTBE & 93 & 70 \\
\hline 7 & $\mathrm{Et}_{2} \mathrm{O}$ & 99 & 45 \\
\hline 8 & DME & 55 & 93 \\
\hline 9 & DEE & 98 & 80 \\
\hline 10 & $\mathrm{DME}^{d}$ & 89 & 93 \\
\hline 11 & $\mathrm{DME}^{e}$ & 41 & 93 \\
\hline
\end{tabular}

${ }^{\mathrm{a}}$ Unless otherwise specified, the reaction was performed with $\mathbf{1 a}(0.24 \mathrm{mmol}), \mathbf{2 a}(0.22 \mathrm{mmol}), \mathbf{3 a}(0.2 \mathrm{mmol}), 4 \AA$ MS (200 mg), IIh (10 mol \%), and $\mathrm{Ag}_{2} \mathrm{CO}_{3}(5 \mathrm{~mol} \%)$ in $1.0 \mathrm{~mL}$ of solvent at $0{ }^{\circ} \mathrm{C}$ for $24 \mathrm{~h}$ under argon. ${ }^{b}$ Isolated yield. ${ }^{c}$ Determined by chiral HPLC. ${ }^{d} 1.3 \mathrm{~mL}$ of DME. ${ }^{e} 2.0 \mathrm{~mL}$ of DME. 
Table S4. Optimization of the reaction temperature and time.
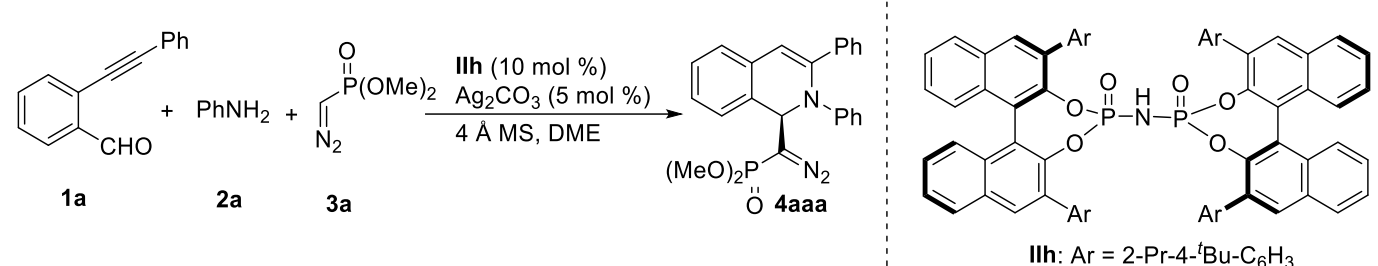

\begin{tabular}{ccccc}
\hline Entry $^{a}$ & $\mathrm{~T}\left({ }^{\circ} \mathrm{C}\right)$ & Time $(\mathrm{h})$ & ${\text { Yield }(\%)^{b}}^{b}$ & $e e(\%)^{c}$ \\
\hline 1 & 0 & 24 & 89 & 93 \\
2 & -10 & 36 & 90 & 94 \\
3 & -20 & 24 & 73 & 97 \\
4 & -20 & 36 & 81 & 97 \\
5 & -20 & 48 & 89 & 97 \\
\hline
\end{tabular}

${ }^{a}$ Unless otherwise specified, the reaction was performed with $1 \mathbf{a}(0.24 \mathrm{mmol}), \mathbf{2 a}(0.22 \mathrm{mmol}), \mathbf{3 a}(0.2 \mathrm{mmol}), 4 \AA$ MS (200 mg), IIh (10 mol \%), and $\mathrm{Ag}_{2} \mathrm{CO}_{3}(5 \mathrm{~mol} \%)$ in $1.3 \mathrm{~mL}$ of DME at the temperature under argon. ${ }^{b}$ Isolated yield. ${ }^{c}$ Determined by chiral HPLC.

Table S5. The effect of molecular sieve on the reaction.

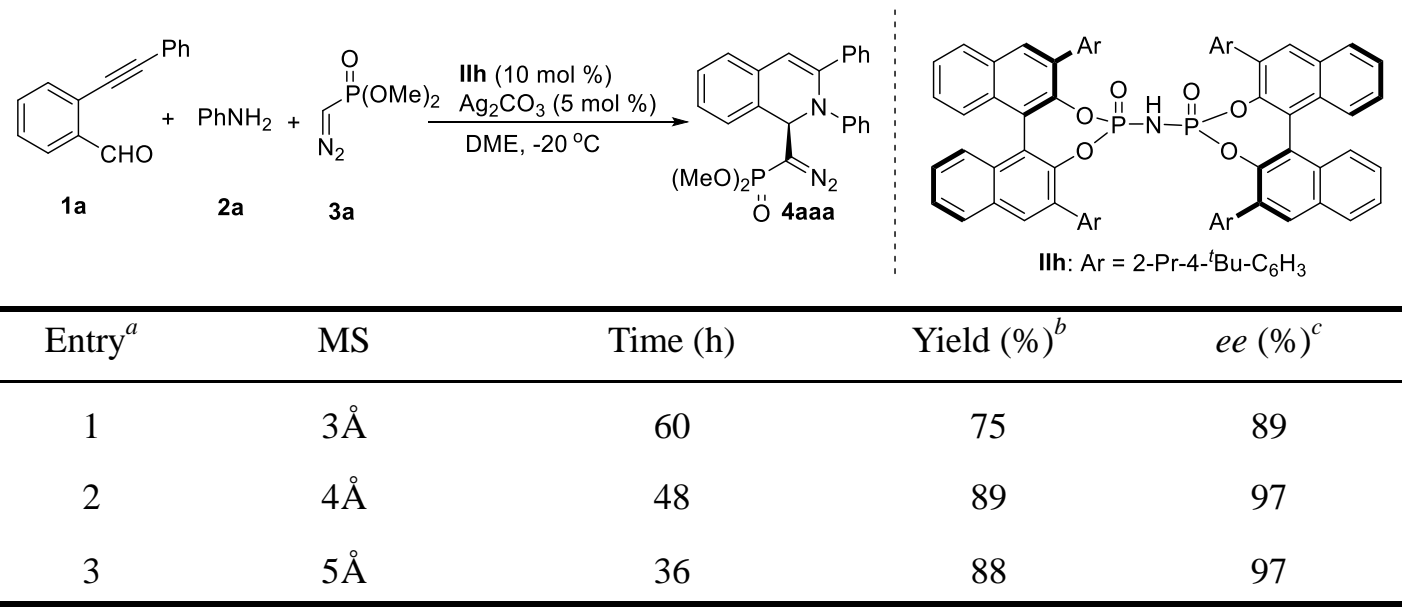

${ }^{a}$ Unless otherwise specified, the reaction was performed with $\mathbf{1 a}(0.24 \mathrm{mmol}), \mathbf{2 a}(0.22 \mathrm{mmol}), \mathbf{3 a}(0.2 \mathrm{mmol}), 200$ mg MS, IIh (10 mol \%), and $\mathrm{Ag}_{2} \mathrm{CO}_{3}(5 \mathrm{~mol} \%)$ in $1.3 \mathrm{~mL}$ of DME at $-20{ }^{\circ} \mathrm{C}$ under argon. ${ }^{b}$ Isolated yield. ${ }^{c}$ Determined by chiral HPLC. 
Table S6: The effect of counter ion on the reaction

\begin{tabular}{|c|c|c|c|c|}
\hline Entry $^{\mathrm{a}}$ & $\mathrm{X}$ & Time (h) & Yield $(\%)^{b}$ & $e e(\%)^{c}$ \\
\hline 1 & $\mathrm{CO}_{3}^{2-}$ & 48 & 89 & 97 \\
\hline 2 & $\mathrm{AcO}^{-}$ & 72 & 47 & 97 \\
\hline 3 & $4-\mathrm{CH}_{3} \mathrm{C}_{6} \mathrm{H}_{4} \mathrm{SO}_{3}^{-}$ & 72 & 67 & 96 \\
\hline 4 & $\mathrm{TfO}^{-}$ & 72 & 58 & 93 \\
\hline 5 & $\mathrm{NO}_{3}^{-}$ & 72 & 73 & 93 \\
\hline 6 & $\mathrm{BF}_{4}^{-}$ & 72 & 57 & 94 \\
\hline 7 & $\mathrm{CF}_{3} \mathrm{COO}^{-}$ & 72 & 74 & 83 \\
\hline
\end{tabular}

${ }^{a}$ Unless otherwise specified, the reaction was performed with $1 \mathbf{a}(0.24 \mathrm{mmol}), \mathbf{2 a}(0.22 \mathrm{mmol}), \mathbf{3 a}(0.2 \mathrm{mmol}), 4 \AA$ MS (200 mg), IIh (10 mol \%), and AgX (5 mol \%) in $1.3 \mathrm{~mL}$ of DME at $-20{ }^{\circ} \mathrm{C}$ under argon. ${ }^{b}$ Isolated yield. ${ }^{c}$ Determined by chiral HPLC.

\section{General procedure for the catalytic asymmetric three-component reaction}

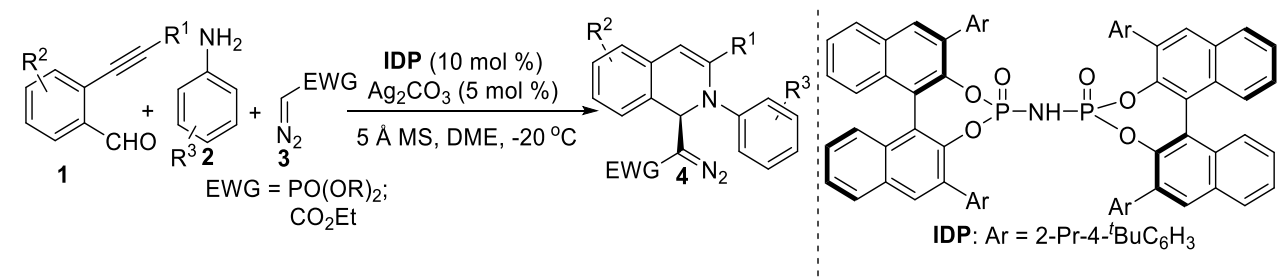

Chiral imidodiphosphate IIh (0.01mmol, 0.1 equiv.), $\mathrm{Ag}_{2} \mathrm{CO}_{3}$ (0.005 mmol, 0.05 equiv.) and anhydrous DME $(1.3 \mathrm{~mL})$ were added to a pre-dried tube under argon and stirred for $2 \mathrm{~h}$ at $30{ }^{\circ} \mathrm{C}$ and then the corresponding $o$-alkynylbenzaldehyde derivatives 1 (0.24 mmol, 1.2 equiv.), amine 2 (0.22 mmol, 1.1 equiv.) and $5 \AA$ MS (200 mg) powder were added, the mixture reacted at $30{ }^{\circ} \mathrm{C}$ for $0.5 \mathrm{~h}$. Then the reaction mixture was cooled down to $-20^{\circ} \mathrm{C}$, the diazo compounds 3 (0.2 mmol, 1.0 equiv.) was added and the mixture was stirred until $\mathbf{3}$ had been completely consumed. The reaction mixture was concentrated under reduce pressure then purified directly by cryogenic flash chromatography on silica gel to obtain the corresponding product 4 . 


\section{Characterization of products}

Dimethyl $\quad(R)-($ diazo(2,3-diphenyl-1,2-dihydroisoquinolin-1-yl) methyl) phosphonate (4aaa)<smiles>COP(OC)C(=N)C1c2ccccc2C=C(c2ccccc2)N1c1ccccc1</smiles>

Following the general procedure, reaction were performed with $o$-alkynylbenzaldehyde 1a $(0.24 \mathrm{mmol}), \mathbf{2 a}(0.22 \mathrm{mmol}), 3 \mathbf{3}(0.20$ mmol), $5 \AA$ MS (200 mg), IIh (10 mol \%), and $\mathrm{Ag}_{2} \mathrm{CO}_{3}(5 \mathrm{~mol} \%)$ in the $1.3 \mathrm{~mL}$ of $\mathrm{MTBE}$ at $-20^{\circ} \mathrm{C}$ for 3 days under argon, followed by column chromatography $(\mathrm{PE} / \mathrm{EA}=10: 1 \sim 1: 1)$ to afford 4aaa as yellow sticky oil, $76.7 \mathrm{mg}(89 \%$ yield, $97 \% e e) ;[\alpha]_{\mathrm{D}}^{25}=+496.1^{\circ}\left(c=0.77,{ }^{i} \operatorname{PrOH}\right)$; HPLC (IC, n-hexane $/{ }^{i} \mathrm{PrOH}=70 / 30$, flow rate $=0.5 \mathrm{~mL} / \mathrm{min}, \mathrm{I}=238 \mathrm{~nm}$ ): $t_{\mathrm{R}}=13.2$ min (major), $15.4 \mathrm{~min}$ (minor). ${ }^{1} \mathrm{H} \mathrm{NMR}\left(600 \mathrm{MHz}, \mathrm{CDCl}_{3}\right) \delta 7.64-7.56(\mathrm{~m}, 2 \mathrm{H})$, $7.36-7.28(\mathrm{~m}, 2 \mathrm{H}), 7.26-7.18(\mathrm{~m}, 4 \mathrm{H}), 7.13(\mathrm{~d}, J=7.5 \mathrm{~Hz}, 1 \mathrm{H}), 7.10$ (d, $J=4.3 \mathrm{~Hz}$, $4 \mathrm{H}), 6.89-6.81(\mathrm{~m}, 1 \mathrm{H}), 6.69(\mathrm{~s}, 1 \mathrm{H}), 5.89(\mathrm{~d}, J=5.4 \mathrm{~Hz}, 1 \mathrm{H}), 3.78(\mathrm{dd}, J=11.8$, $2.4 \mathrm{~Hz}, 6 \mathrm{H}) \mathrm{ppm} .{ }^{13} \mathrm{C} \mathrm{NMR}\left(151 \mathrm{MHz}, \mathrm{CDCl}_{3}\right) \delta 146.4,141.6,136.8,132.1,128.7$, $128.4,128.3,128.2,127.5,127.2,127.2,125.5,124.6,122.7,122.7,112.6,62.2$ (d, $J$ $=10.7 \mathrm{~Hz}), 53.0(\mathrm{~d}, J=6.1 \mathrm{~Hz}), 53.0(\mathrm{~d}, J=5.7 \mathrm{~Hz}), 46.0(\mathrm{~d}, J=225.3 \mathrm{~Hz}) \mathrm{ppm}$. HRMS (ESI) m/z: $[\mathrm{M}+\mathrm{H}]^{+}$Calcd. for $\mathrm{C}_{24} \mathrm{H}_{23} \mathrm{~N}_{3} \mathrm{O}_{3} \mathrm{P} 432.1472$; found 432.1473.

Dimethyl $\quad(R)$-(diazo(2-phenyl-3-(p-tolyl)-1,2-dihydroisoquinolin-1-yl)methyl) phosphonate (4baa)

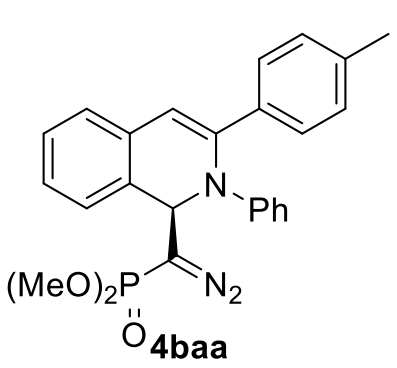

Following the general procedure, reaction were performed with $o$-alkynylbenzaldehyde $\mathbf{1 a b}(0.24 \mathrm{mmol})$, 2a $(0.22$

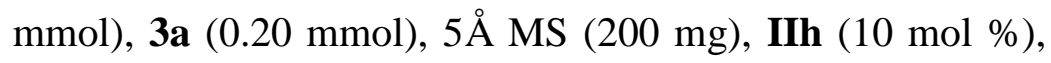
and $\mathrm{Ag}_{2} \mathrm{CO}_{3}(5 \mathrm{~mol} \%)$ in the $1.0 \mathrm{~mL}$ of THF/DME (1:1) at $-20{ }^{\circ} \mathrm{C}$ for 3 days under argon, followed by column chromatography $(\mathrm{PE} / \mathrm{EA}=10: 1 \sim 1: 1)$ to afford 4 baa as yellow sticky oil, $78.3 \mathrm{mg}(88 \%$ yield, $93 \% e e) ;[\alpha]_{\mathrm{D}}^{25}=+491.6^{\circ}\left(c=1.58,{ }^{i} \mathrm{PrOH}\right)$; HPLC $\left(\mathrm{IC}, \mathrm{n}\right.$-hexane $/{ }^{i} \mathrm{PrOH}=70 / 30$, flow rate $\left.=0.5 \mathrm{~mL} / \mathrm{min}, \mathrm{I}=238 \mathrm{~nm}\right): t_{\mathrm{R}}=12.9$ min (major), $14.8 \mathrm{~min}$ (minor). ${ }^{1} \mathrm{H}$ NMR $\left(600 \mathrm{MHz} \mathrm{CDCl}_{3}\right) \delta 7.51$ (d, $J=8.1 \mathrm{~Hz}$, 2H), $7.35-7.28(\mathrm{~m}, 2 \mathrm{H}), 7.24-7.21(\mathrm{~m}, 1 \mathrm{H}), 7.16-7.09(\mathrm{~m}, 5 \mathrm{H}), 7.07(\mathrm{~d}, J=8.0$ $\mathrm{Hz}, 2 \mathrm{H}), 6.92-6.83(\mathrm{~m}, 1 \mathrm{H}), 6.67(\mathrm{~s}, 1 \mathrm{H}), 5.89(\mathrm{~d}, J=5.4 \mathrm{~Hz}, 1 \mathrm{H}), 3.80(\mathrm{dd}, J=11.7$, $3.3 \mathrm{~Hz}, 6 \mathrm{H}), 2.30$ (s, 3H) ppm. ${ }^{13} \mathrm{C} \mathrm{NMR}\left(151 \mathrm{MHz}, \mathrm{CDCl}_{3}\right) \delta 146.6,141.7,138.1$, 134.0, 132.2, 129.1, 128.7, 128.3, 127.4, 127.2 (d, $J=6.1 \mathrm{~Hz}), 127.0,125.5,124.5$, 122.7, 122.6, 112.0, 62.2 (d, $J=10.8 \mathrm{~Hz}), 53.0$ (d, $J=6.2 \mathrm{~Hz}), 53.0(\mathrm{~d}, J=6.4 \mathrm{~Hz})$, $46.0(\mathrm{~d}, J=224.7 \mathrm{~Hz}) 21.1 \mathrm{ppm}$. HRMS (ESI) m/z: $[\mathrm{M}+\mathrm{H}]^{+}$Calcd for $\mathrm{C}_{25} \mathrm{H}_{25} \mathrm{~N}_{3} \mathrm{O}_{3} \mathrm{P}$ 
446.1628, found 446.1632 .

Dimethyl $\quad(R)$-(diazo(3-(4-ethylphenyl)-2-phenyl-1,2-dihydroisoquinolin-1-yl) methyl)phosphonate (4caa)

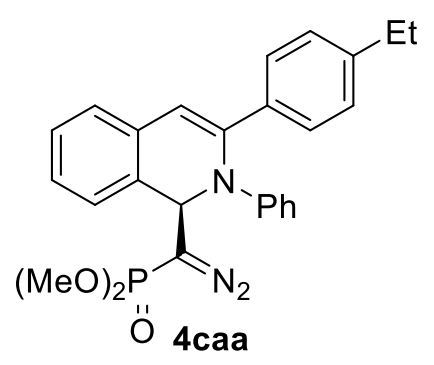

Following the general procedure, reaction were performed with $o$-alkynylbenzaldehyde 1 ac $(0.24 \mathrm{mmol}), \mathbf{2 a}(0.22$ mmol), 3a (0.20 mmol), $5 \AA$ MS (200 mg), IIh (10 mol \%), and $\mathrm{Ag}_{2} \mathrm{CO}_{3}(5 \mathrm{~mol} \%)$ in the $1.3 \mathrm{~mL}$ of MTBE at $-20{ }^{\circ} \mathrm{C}$ for 3 days under argon, followed by column chromatography $(\mathrm{PE} / \mathrm{EA}=10: 1 \sim 1: 1)$ to afford 4caa as yellow sticky oil, $75.3 \mathrm{mg}$ (88\% yield, $98 \% e e)$; $[\alpha]_{\mathrm{D}}^{25}=+453.4^{\circ}\left(c=0.76,{ }^{i} \operatorname{PrOH}\right)$; HPLC (IC, n-hexane $/{ }^{i} \mathrm{PrOH}=70 / 30$, flow rate $=0.5 \mathrm{~mL} / \mathrm{min}, \mathrm{I}=238 \mathrm{~nm}$ ): $t_{\mathrm{R}}=12.8$ min (major), $14.7 \mathrm{~min}$ (minor). ${ }^{1} \mathrm{H} \mathrm{NMR}\left(600 \mathrm{MHz}, \mathrm{CDCl}_{3}\right) \delta 7.54(\mathrm{~d}, J=8.1 \mathrm{~Hz}, 2 \mathrm{H})$, $7.38-7.28(\mathrm{~m}, 2 \mathrm{H}), 7.25-7.19(\mathrm{~m}, 1 \mathrm{H}), 7.18-7.06(\mathrm{~m}, 7 \mathrm{H}), 6.87(\mathrm{p}, J=4.2 \mathrm{~Hz}$, 1H), $6.68(\mathrm{~s}, 1 \mathrm{H}), 5.89(\mathrm{~d}, J=5.5 \mathrm{~Hz}, 1 \mathrm{H}), 3.80(\mathrm{dd}, J=11.7,5.2 \mathrm{~Hz}, 6 \mathrm{H}), 2.60(\mathrm{q}, J$ $=7.7 \mathrm{~Hz}, 2 \mathrm{H}), 1.21(\mathrm{t}, J=7.6 \mathrm{~Hz}, 3 \mathrm{H}) \mathrm{ppm} .{ }^{13} \mathrm{C} \mathrm{NMR}\left(151 \mathrm{MHz}, \mathrm{CDCl}_{3}\right) \delta 146.6$, 144.4, 141.8, 134.2, 132.3, 128.7, 128.3, 127.8, 127.5, 127.2 (d, $J=6.4 \mathrm{~Hz}$ ), 127.0, 125.5, 124.6, 122.7, 122.6, 112.1, 62.3 (d, $J=10.7 \mathrm{~Hz}), 53.0$ (d, $J=6.0 \mathrm{~Hz}), 53.0$ (d, $J=6.0 \mathrm{~Hz}), 46.0(\mathrm{~d}, J=225.4 \mathrm{~Hz}) .28 .5,15.1 \mathrm{ppm}$. HRMS (ESI) m/z: $[\mathrm{M}+\mathrm{H}]^{+} \mathrm{Calcd}$ for $\mathrm{C}_{26} \mathrm{H}_{27} \mathrm{~N}_{3} \mathrm{O}_{3} \mathrm{P} 460.1785$, found 460.1780 .

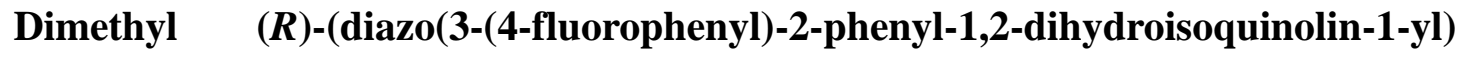
methyl)phosphonate (4daa)

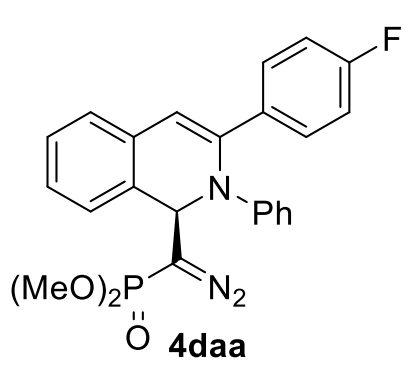

Following the general procedure, reaction were performed with $o$-alkynylbenzaldehyde1ad $(0.24 \mathrm{mmol}), 2 \mathbf{a}(0.22$ mmol), 3a (0.20 mmol), $5 \AA$ MS (200 mg), IIh (10 mol \%), and $\mathrm{Ag}_{2} \mathrm{CO}_{3}(5 \mathrm{~mol} \%)$ in the $1.3 \mathrm{~mL}$ of MTBE at $-20{ }^{\circ} \mathrm{C}$ for 3 days under argon, followed by column chromatography $(\mathrm{PE} / \mathrm{EA}=10: 1 \sim 1: 1)$ to afford 4daa as yellow sticky oil, $84.4 \mathrm{mg}$ (94\% yield, 95\% ee); $[\alpha]_{\mathrm{D}}^{25}=+433.3^{\circ}\left(c=1.69,{ }^{i} \operatorname{PrOH}\right)$; HPLC (IC, n-hexane $/{ }^{i} \mathrm{PrOH}=70 / 30$, flow rate $=0.5 \mathrm{~mL} / \mathrm{min}, \mathrm{I}=238 \mathrm{~nm}$ ): $t_{\mathrm{R}}=12.9$ $\min$ (major), $14.5 \mathrm{~min}$ (minor). ${ }^{1} \mathrm{H} \mathrm{NMR}\left(600 \mathrm{MHz}, \mathrm{CDCl}_{3}\right) \delta 7.64-7.56(\mathrm{~m}, 2 \mathrm{H})$, $7.36-7.29(\mathrm{~m}, 2 \mathrm{H}), 7.26-7.22(\mathrm{~m}, 1 \mathrm{H}), 7.16-7.08(\mathrm{~m}, 5 \mathrm{H}), 6.95(\mathrm{t}, J=8.6 \mathrm{~Hz}, 2 \mathrm{H})$, $6.92-6.85(\mathrm{~m}, 1 \mathrm{H}), 6.65(\mathrm{~s}, 1 \mathrm{H}), 5.89(\mathrm{~d}, J=5.2 \mathrm{~Hz}, 1 \mathrm{H}), 3.80(\mathrm{dd}, J=11.7,4.7 \mathrm{~Hz}$, $6 \mathrm{H}) \mathrm{ppm} .{ }^{13} \mathrm{C}$ NMR $\left(151 \mathrm{MHz}, \mathrm{CDCl}_{3}\right) \delta 162.6(\mathrm{~d}, J=248.4 \mathrm{~Hz}), 146.2,140.7,132.9$ $(\mathrm{d}, J=3.1 \mathrm{~Hz}), 132.0,129.2(\mathrm{~d}, J=8.0 \mathrm{~Hz}), 128.8,128.4,127.2,127.0(\mathrm{~d}, J=6.2 \mathrm{~Hz})$, 
125.6, 124.6, 122.9, 122.9, 115.4, 115.2, 112.3, 62.2 (d, $J=10.6 \mathrm{~Hz}), 53.0$ (d, $J=6.0$ $\mathrm{Hz}), 53.0(\mathrm{~d}, J=5.7 \mathrm{~Hz}), 46.0(\mathrm{~d}, J=225.6 \mathrm{~Hz}) \mathrm{ppm}$. HRMS (ESI) m/z: $[\mathrm{M}+\mathrm{H}]^{+}$ Calcd for $\mathrm{C}_{24} \mathrm{H}_{22} \mathrm{FN}_{3} \mathrm{O}_{3} \mathrm{P} 450.1377$ found 450.1388 .

Dimethyl (R)-((3-(4-chlorophenyl)-2-phenyl-1,2-dihydroisoquinolin-1-yl) (diazo)methyl)phosphonate (4eaa)

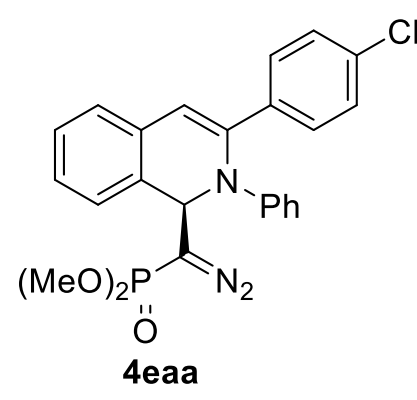

Following the general procedure, reaction were performed with $o$-alkynylbenzaldehyde $\mathbf{1 a e}(0.24 \mathrm{mmol}), \mathbf{2 a}(0.22 \mathrm{mmol})$, 3a (0.20 mmol), $5 \AA \mathrm{MS}(200 \mathrm{mg})$, IIh (10 mol \%), and $\mathrm{Ag}_{2} \mathrm{CO}_{3}(5 \mathrm{~mol} \%)$ in the $1.0 \mathrm{~mL}$ of THF/DME $(1: 1)$ at $-20{ }^{\circ} \mathrm{C}$ for 3 days under argon, followed by column chromatography $(\mathrm{PE} / \mathrm{EA}=10: 1 \sim 1: 1)$ to afford 4eaa as yellow sticky oil, 76.3 mg $(82 \%$ yield, $96 \% e e) ;[\alpha]_{\mathrm{D}}^{25}=+446.2^{\circ}\left(c=1.52,{ }^{i} \mathrm{PrOH}\right) ;$ HPLC (IC, n-hexane/ ${ }^{i} \mathrm{PrOH}=70 / 30$, flow rate $=0.5 \mathrm{~mL} / \mathrm{min}, \mathrm{I}=238 \mathrm{~nm}$ ): $t_{\mathrm{R}}=13.0 \mathrm{~min}$ (major), $14.5 \mathrm{~min}$ (minor). ${ }^{1} \mathrm{H}$ NMR (600 MHz, $\left.\mathrm{CDCl}_{3}\right) . \delta 7.58-7.52(\mathrm{~m}, 2 \mathrm{H}), 7.37-7.29(\mathrm{~m}, 2 \mathrm{H})$, $7.26-7.21(\mathrm{~m}, 3 \mathrm{H}), 7.16-7.06(\mathrm{~m}, 5 \mathrm{H}), 6.94-6.85(\mathrm{~m}, 1 \mathrm{H}), 6.69$ (s, 1H), 5.89 (d, $J$ $=5.2 \mathrm{~Hz}, 1 \mathrm{H}), 3.79(\mathrm{dd}, J=11.7,4.5 \mathrm{~Hz}, 6 \mathrm{H}) \mathrm{ppm} .{ }^{13} \mathrm{C} \mathrm{NMR}\left(151 \mathrm{MHz}, \mathrm{CDCl}_{3}\right) . \delta$ $146.2,140.6,135.3,134.0,131.9,128.9,128.8,128.7,128.5,128.5,127.4,127.2(\mathrm{~d}, J$ $=6.5 \mathrm{~Hz}), 125.6,124.8,123.0,122.8,112.8,62.2(\mathrm{~d}, J=10.8 \mathrm{~Hz}), 53.0(\mathrm{~d}, J=6.1$ $\mathrm{Hz}), 53.0(\mathrm{~d}, J=5.4 \mathrm{~Hz}), 46.0(\mathrm{~d}, J=225.4 \mathrm{~Hz}) \mathrm{ppm}$. HRMS (ESI) m/z: $[\mathrm{M}+\mathrm{H}]^{+}$ Calcd for $\mathrm{C}_{24} \mathrm{H}_{22} \mathrm{ClN}_{3} \mathrm{O}_{3} \mathrm{P} 466.1082$, found 466.1095.

Dimethyl $(R)-((3-(4-b r o m o p h e n y l)-2-p h e n y l-1,2-d i h y d r o i s o q u i n o l i n-1-y l)(d i a z o)$ methyl)phosphonate (4faa)<smiles>COP(=O)(OC)C(=N)C1C(c2ccc(Br)cc2)=Cc2ccccc21</smiles>

Following the general procedure, reaction were performed with $o$-alkynylbenzaldehyde $1 \mathrm{af}(0.24 \mathrm{mmol})$, 2a (0.22 mmol), 3a (0.20 mmol), $5 \AA$ MS (200 mg), IIh (10 mol \%), and $\mathrm{Ag}_{2} \mathrm{CO}_{3}(5 \mathrm{~mol} \%$ ) in the $1.0 \mathrm{~mL}$ of THF/DME (1:1) at $-20{ }^{\circ} \mathrm{C}$ for 3 days under argon, followed by column chromatography $(\mathrm{PE} / \mathrm{EA}=10: 1 \sim$

1:1) to afford 4faa as yellow sticky oil, $78.4 \mathrm{mg}(77 \%$ yield, $96 \% e e) ;[\alpha]_{\mathrm{D}}^{25}=+367.8^{\circ}$ $\left(c=1.56,{ }^{i} \mathrm{PrOH}\right) ; \mathrm{HPLC}\left(\mathrm{IC}, \mathrm{n}-\right.$ hexane $/{ }^{i} \mathrm{PrOH}=70 / 30$, flow rate $=0.5 \mathrm{~mL} / \mathrm{min}, \mathrm{I}=$ $238 \mathrm{~nm}$ ): $t_{\mathrm{R}}=13.3 \mathrm{~min}$ (major), $14.9 \mathrm{~min}$ (minor). ${ }^{1} \mathrm{H} \mathrm{NMR} \mathrm{(600} \mathrm{MHz}, \mathrm{CDCl}_{3}$ ). $\delta$ $7.51-7.47(\mathrm{~m}, 2 \mathrm{H}), 7.41-7.36(\mathrm{~m}, 2 \mathrm{H}), 7.35-7.29(\mathrm{~m}, 2 \mathrm{H}), 7.26-7.22(\mathrm{~m}, 1 \mathrm{H})$, $7.16-7.07(\mathrm{~m}, 5 \mathrm{H}), 6.93-6.85(\mathrm{~m}, 1 \mathrm{H}), 6.70(\mathrm{~s}, 1 \mathrm{H}), 5.88(\mathrm{~d}, J=5.2 \mathrm{~Hz}, 1 \mathrm{H}), 3.79$ $(\mathrm{dd}, J=11.7,4.2 \mathrm{~Hz}, 6 \mathrm{H}) \mathrm{ppm} .{ }^{13} \mathrm{C} \mathrm{NMR}\left(151 \mathrm{MHz}, \mathrm{CDCl}_{3}\right) . \delta 146.1,140.6,135.8$, 
131.8, 131.5, 129.0, 128.9, 128.5, 127.5, 127.2 (d, $J=6.1 \mathrm{~Hz}), 125.6,124.8,123.0$, 122.8, 122.2, 112.9, $62.2(\mathrm{~d}, J=10.6 \mathrm{~Hz}), 53.0(\mathrm{~d}, J=6.2 \mathrm{~Hz}), 53.0(\mathrm{~d}, J=3.9 \mathrm{~Hz})$, $46.0(\mathrm{~d}, J=225.2 \mathrm{~Hz})$ ppm HRMS (ESI) $\mathrm{m} / \mathrm{z}$ : $[\mathrm{M}+\mathrm{H}]^{+}$Calcd for $\mathrm{C}_{24} \mathrm{H}_{22} \mathrm{BrN}_{3} \mathrm{O}_{3} \mathrm{P}$ 510.0577 found 510.0572 .

Dimethyl $(R)-($ diazo(3-(4-methoxyphenyl)-2-phenyl-1,2-dihydroisoquinolin-1-yl) methyl)phosphonate (4gaa)

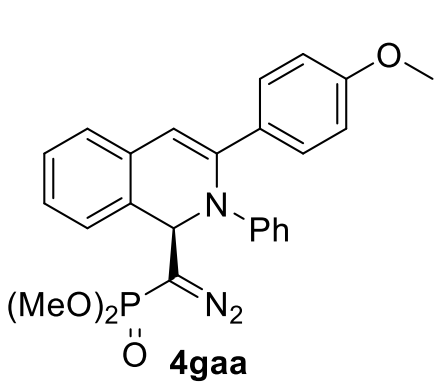

Following the general procedure, reaction were performed with $o$-alkynylbenzaldehyde $\mathbf{1 a g}(0.24 \mathrm{mmol}), \mathbf{2 a}(0.22$ mmol), 3a (0.20 mmol), $5 \AA$ MS (200 mg), IIh (10 mol \%), and $\mathrm{Ag}_{2} \mathrm{CO}_{3}(5 \mathrm{~mol} \%)$ in the $1.0 \mathrm{~mL}$ of THF/DME (1:1) at $-20{ }^{\circ} \mathrm{C}$ for 3 days under argon, followed by column chromatography $(\mathrm{PE} / \mathrm{EA}=10: 1 \sim 1: 1)$ to afford 4gaa as yellow sticky oil, $79.3 \mathrm{mg}$ (86\% yield, 94\% ee); $[\alpha]_{\mathrm{D}}^{25}=+449.4^{\circ}\left(c=1.59,{ }^{i} \operatorname{PrOH}\right)$; HPLC (IC, n-hexane $/{ }^{i} \mathrm{PrOH}=70 / 30$, flow rate $=0.5 \mathrm{~mL} / \mathrm{min}, \mathrm{I}=238 \mathrm{~nm}$ ): $t_{\mathrm{R}}=15.3$ min (major), $18.4 \mathrm{~min}$ (minor). ${ }^{1} \mathrm{H}$ NMR (600 MHz, $\left.\mathrm{CDCl}_{3}\right) \delta 7.54(\mathrm{~d}, J=8.5 \mathrm{~Hz}, 2 \mathrm{H})$, $7.33-7.28(\mathrm{~m}, 2 \mathrm{H}), 7.23-7.18(\mathrm{~m}, 1 \mathrm{H}), 7.15-7.06(\mathrm{~m}, 5 \mathrm{H}), 6.91-6.84(\mathrm{~m}, 1 \mathrm{H})$, $6.79(\mathrm{~d}, J=8.5 \mathrm{~Hz}, 2 \mathrm{H}), 6.62(\mathrm{~s}, 1 \mathrm{H}), 5.87(\mathrm{~d}, J=5.3 \mathrm{~Hz}, 1 \mathrm{H}), 3.87-3.68(\mathrm{~m}, 9 \mathrm{H})$ ppm. ${ }^{13} \mathrm{C} \mathrm{NMR}\left(151 \mathrm{MHz}, \mathrm{CDCl}_{3}\right) \delta 159.7,146.6,141.5,132.4,129.3,128.8,128.7$, 128.4, 127.1 (d, $J=6.4 \mathrm{~Hz}), 126.8,125.5,124.4,122.9,122.7,113.8,111.2,62.3$ (d, $J$ $=10.8 \mathrm{~Hz}), 55.2,53.0(\mathrm{~d}, J=6.1 \mathrm{~Hz}), 53.0(\mathrm{~d}, J=5.8 \mathrm{~Hz}), 45.9(\mathrm{~d}, J=225.0 \mathrm{~Hz})$ ppm. HRMS (ESI) m/z: [M+H] $]^{+}$Calcd for $\mathrm{C}_{25} \mathrm{H}_{25} \mathrm{~N}_{3} \mathrm{O}_{4} \mathrm{P} 462.1577$ found 462.1576 .

\section{Dimethyl (R)-(diazo(2-phenyl-3-(4-(trifluoromethoxy)phenyl)-1,2-dihydro isoquinolin-1-yl)methyl)phosphonate (4haa)}

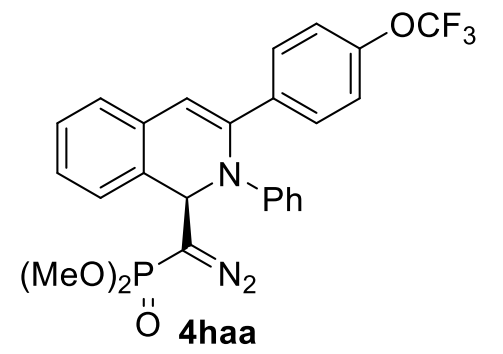

Following the general procedure, reaction were performed with $o$-alkynylbenzaldehyde 1ah $(0.24$ mmol), 2a (0.22 mmol), 3a (0.20 mmol), $5 \AA$ MS (200 $\mathrm{mg})$, IIh (10 mol \%), and $\mathrm{Ag}_{2} \mathrm{CO}_{3}(5 \mathrm{~mol} \%)$ in the 1.0 $\mathrm{mL}$ of THF/DME (1:1) at $-20{ }^{\circ} \mathrm{C}$ for 3 days under argon, followed by column chromatography $(\mathrm{PE} / \mathrm{EA}=10: 1 \sim$

1:1) to afford 4gaa as yellow sticky oil, $79.3 \mathrm{mg}(86 \%$ yield, $94 \% e e) ;[\alpha]_{\mathrm{D}}^{25}=+407.5^{\circ}$ $\left(c=1.94,{ }^{i} \mathrm{PrOH}\right) ; \mathrm{HPLC}\left(\mathrm{IC}, \mathrm{n}-\mathrm{hexane} /{ }^{i} \mathrm{PrOH}=70 / 30\right.$, flow rate $=0.5 \mathrm{~mL} / \mathrm{min}, \mathrm{I}=$ $238 \mathrm{~nm}$ ): $t_{\mathrm{R}}=10.6 \mathrm{~min}$ (major), $11.5 \mathrm{~min}$ (minor). ${ }^{1} \mathrm{H} \mathrm{NMR}\left(600 \mathrm{MHz}, \mathrm{CDCl}_{3}\right) \delta 7.67$ - $7.62(\mathrm{~m}, 2 \mathrm{H}), 7.37-7.29(\mathrm{~m}, 2 \mathrm{H}), 7.27-7.24(\mathrm{~m}, 1 \mathrm{H}), 7.18-7.05(\mathrm{~m}, 7 \mathrm{H}), 6.93-$ $6.87(\mathrm{~m}, 1 \mathrm{H}), 6.69(\mathrm{~s}, 1 \mathrm{H}), 5.89(\mathrm{~d}, J=5.3 \mathrm{~Hz}, 1 \mathrm{H}), 3.80(\mathrm{dd}, J=11.7,3.7 \mathrm{~Hz}, 6 \mathrm{H})$ 
ppm. ${ }^{13} \mathrm{C}$ NMR (151 MHz, $\left.\mathrm{CDCl}_{3}\right) \delta 149.0,146.1,140.4,135.5,131.8,128.9,128.8$, 128.5, 127.5, $127.2(\mathrm{~d}, J=6.4 \mathrm{~Hz}), 125.6,124.8,123.1,122.8,120.6,120.4$ (d, $J=$ $257.5 \mathrm{~Hz}), 113.2,62.3(\mathrm{~d}, J=10.6 \mathrm{~Hz}), 53.0(\mathrm{~d}, J=5.5 \mathrm{~Hz}), 46.1(\mathrm{~d}, J=226.2 \mathrm{~Hz})$ ppm. HRMS (ESI) m/z: $[\mathrm{M}+\mathrm{H}]^{+}$Calcd. for $\mathrm{C}_{25} \mathrm{H}_{22} \mathrm{~F}_{3} \mathrm{~N}_{3} \mathrm{O}_{4} \mathrm{P} 516.1295$ found 516.1298.

Dimethyl $(R)-((3-(4-c y a n o p h e n y l)-2-p h e n y l-1,2-d i h y d r o i s o q u i n o l i n-1-y l)(d i a z o)$ methyl)phosphonate (4iaa)

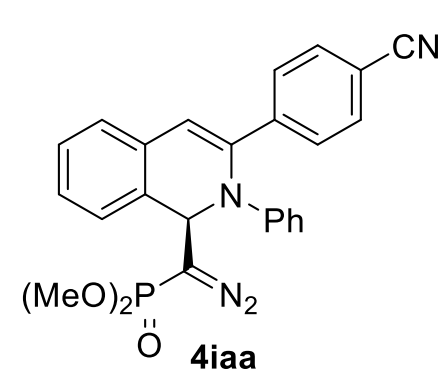

Following the general procedure, reaction were performed with $o$-alkynylbenzaldehyde 1ai $(0.24 \mathrm{mmol})$, 2a $(0.22 \mathrm{mmol}), 3 \mathbf{3}(0.20 \mathrm{mmol}), 5 \AA \mathrm{MS}(200 \mathrm{mg}), \mathbf{I I h}$ (10 mol \%), and $\mathrm{Ag}_{2} \mathrm{CO}_{3}(5 \mathrm{~mol} \%)$ in the $1.0 \mathrm{~mL}$ of THF/DME (1:1) at $-20{ }^{\circ} \mathrm{C}$ for 3 days under argon, followed by column chromatography $(\mathrm{PE} / \mathrm{EA}=10: 1 \sim$ 1:1) to afford 4iaa as yellow solid, $81.2 \mathrm{mg}\left(89 \%\right.$ yield, $91 \%$ ee), mp: $166-167.9{ }^{\circ} \mathrm{C}$; $[\alpha]_{\mathrm{D}}^{25}=+477.2^{\circ}\left(c=0.82,{ }^{i} \mathrm{PrOH}\right) ; \mathrm{HPLC}\left(\mathrm{ADH}, \mathrm{n}\right.$-hexane $/{ }^{i} \mathrm{PrOH}=50 / 50$, flow rate $=0.5 \mathrm{~mL} / \mathrm{min}, \mathrm{I}=238 \mathrm{~nm}$ ): $t_{\mathrm{R}}=12.7 \mathrm{~min}$ (major), $22.4 \mathrm{~min}$ (minor). ${ }^{1} \mathrm{H}$ NMR $(600$ $\left.\mathrm{MHz}, \mathrm{CDCl}_{3}\right) \delta 7.71(\mathrm{~d}, J=8.4 \mathrm{~Hz}, 2 \mathrm{H}), 7.60-7.47(\mathrm{~m}, 2 \mathrm{H}), 7.39-7.32(\mathrm{~m}, 2 \mathrm{H})$, $7.31-7.27(\mathrm{~m}, 1 \mathrm{H}), 7.17-7.10(\mathrm{~m}, 3 \mathrm{H}), 7.09-7.06(\mathrm{~m}, 2 \mathrm{H}), 6.95-6.87(\mathrm{~m}, 1 \mathrm{H})$, $6.80(\mathrm{~s}, 1 \mathrm{H}), 5.90(\mathrm{~d}, J=5.2 \mathrm{~Hz}, 1 \mathrm{H}), 3.79(\mathrm{dd}, J=11.3 \mathrm{~Hz}, 6 \mathrm{H}) \mathrm{ppm} .{ }^{13} \mathrm{C}$ NMR $(151$ $\left.\mathrm{MHz}, \mathrm{CDCl}_{3}\right) \delta 145.9,141.4,139.8,132.1,131.3,129.1,128.6,128.2,127.8,127.3$ (d, $J=6.5 \mathrm{~Hz}), 125.7,125.2,123.4,122.7,118.6,114.9,111.5,62.2(\mathrm{~d}, J=10.5 \mathrm{~Hz})$, $53.1(\mathrm{~d}, J=5.6 \mathrm{~Hz}), 53.1(\mathrm{~d}, J=5.8 \mathrm{~Hz}), 46.2(\mathrm{~d}, J=225.9 \mathrm{~Hz}) \mathrm{ppm}$. HRMS (ESI) $\mathrm{m} / \mathrm{z}: \quad[\mathrm{M}+\mathrm{H}]^{+}$Calcd. for $\mathrm{C}_{25} \mathrm{H}_{22} \mathrm{~N}_{4} \mathrm{O}_{3} \mathrm{P} \quad 457.1424$ found 457.1421. Ethyl (R)-4-(1-(diazo(dimethoxyphosphoryl)methyl)-2-phenyl-1,2-dihydro

\section{isoquinolin-3-yl)benzoate (4jaa)}

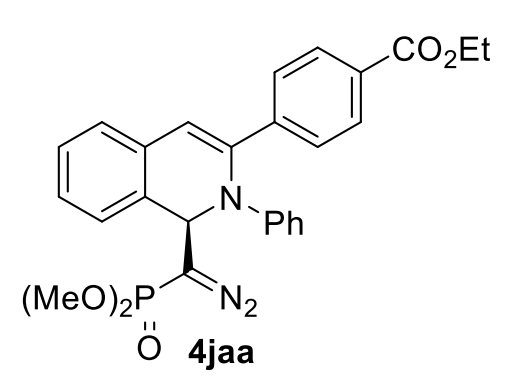

Following the general procedure, reaction were performed with $o$-alkynylbenzaldehyde 1aj $(0.24$ mmol), 2a (0.22 mmol), 3a (0.20 mmol), $5 \AA$ MS (200 $\mathrm{mg})$, IIh (10 mol \%), and $\mathrm{Ag}_{2} \mathrm{CO}_{3}(5 \mathrm{~mol} \%)$ in the 1.0 $\mathrm{mL}$ of THF/DME $(1: 1)$ at $-20{ }^{\circ} \mathrm{C}$ for 3 days under argon, followed by column chromatography $(\mathrm{PE} / \mathrm{EA}=$ 10:1 1:1) to afford 4 jaa as yellow sticky oil, $90.5 \mathrm{mg}(90 \%$ yield, $93 \%$ ee $) ;[\alpha]_{\mathrm{D}}^{25}=$ $+407.5^{\circ}\left(c=1.91,{ }^{i} \mathrm{PrOH}\right)$; HPLC $\left(\mathrm{IC}, \mathrm{n}\right.$-hexane $/{ }^{i} \mathrm{PrOH}=70 / 30$, flow rate $=0.5$ $\mathrm{mL} / \mathrm{min}, \mathrm{I}=238 \mathrm{~nm}$ ): $t_{\mathrm{R}}=20.8 \mathrm{~min}$ (major), $30.9 \min$ (minor). ${ }^{1} \mathrm{H} \mathrm{NMR}(600 \mathrm{MHz}$, $\left.\mathrm{CDCl}_{3}\right) \delta 8.01-7.88(\mathrm{~m}, 2 \mathrm{H}), 7.74-7.61(\mathrm{~m}, 2 \mathrm{H}), 7.39-7.35(\mathrm{~m}, 1 \mathrm{H}), 7.35-7.30$ 
(m, 1H), $7.29-7.24(\mathrm{~m}, 1 \mathrm{H}), 7.16(\mathrm{~d}, J=7.5 \mathrm{~Hz}, 1 \mathrm{H}), 7.13-7.06(\mathrm{~m}, 4 \mathrm{H}), 6.90-$ $6.85(\mathrm{~m}, 1 \mathrm{H}), 6.80(\mathrm{~s}, 1 \mathrm{H}), 5.91(\mathrm{~d}, J=5.3 \mathrm{~Hz}, 1 \mathrm{H}), 4.33(\mathrm{q}, J=7.1 \mathrm{~Hz}, 2 \mathrm{H}), 3.80(\mathrm{dd}$, $J=11.7,2.7 \mathrm{~Hz}, 6 \mathrm{H}), 1.35(\mathrm{t}, J=7.1 \mathrm{~Hz}, 3 \mathrm{H}) \mathrm{ppm} .{ }^{13} \mathrm{C} \mathrm{NMR}\left(151 \mathrm{MHz}, \mathrm{CDCl}_{3}\right) \delta$ $166.1,146.1,141.2,140.6,131.7,129.9$, 129.6, 128.9, 128.5, 127.7, 127.3, 127.3, 125.6, 125.0, 123.0, 122.7, 114.1, 62.2 (d, $J=10.6 \mathrm{~Hz}), 60.9,53.0(\mathrm{~d}, J=5.8 \mathrm{~Hz})$, $46.1(\mathrm{~d}, J=225.9 \mathrm{~Hz}), 14.2 \mathrm{ppm}$. HRMS (ESI) m/z: $[\mathrm{M}+\mathrm{H}]^{+}$Calcd. for $\mathrm{C}_{27} \mathrm{H}_{27} \mathrm{~N}_{3} \mathrm{O}_{5} \mathrm{P}$ 504.1683 found 504.1691 .

Dimethyl

(R)-(diazo(2-phenyl-3-(4-(trifluoromethyl)phenyl)-1,2-dihydro isoquinolin-1-yl)methyl)phosphonate (4kaa)

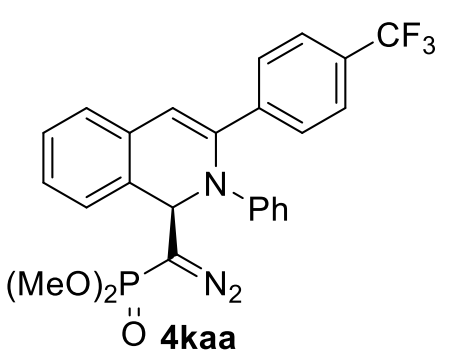

Following the general procedure, reaction were performed with $o$-alkynylbenzaldehyde 1ak $(0.24 \mathrm{mmol})$, 2a $(0.22 \mathrm{mmol}), 3 \mathbf{a}(0.20 \mathrm{mmol}), 5 \AA \mathrm{MS}(200 \mathrm{mg})$, IIh (10 mol \%), and $\mathrm{Ag}_{2} \mathrm{CO}_{3}(5 \mathrm{~mol} \%)$ in the $1.0 \mathrm{~mL}$ of THF/DME (1:1) at $-20{ }^{\circ} \mathrm{C}$ for 3 days under argon, followed by column chromatography $(\mathrm{PE} / \mathrm{EA}=10: 1 \sim$

1:1) to afford 4kaa as yellow sticky oil, $89.8 \mathrm{mg}(90 \%$ yield, $84 \% e e)$; $[\alpha]_{\mathrm{D}}^{25}=+405.8^{\circ}$ $\left(c=1.85,{ }^{i} \mathrm{PrOH}\right) ; \mathrm{HPLC}\left(\mathrm{IC}, \mathrm{n}-\right.$ hexane $/{ }^{i} \mathrm{PrOH}=70 / 30$, flow rate $=0.5 \mathrm{~mL} / \mathrm{min}, \mathrm{I}=$ $238 \mathrm{~nm}): t_{\mathrm{R}}=11.1 \mathrm{~min}$ (major), $11.8 \mathrm{~min}$ (minor). ${ }^{1} \mathrm{H} \mathrm{NMR}\left(600 \mathrm{MHz}, \mathrm{CDCl}_{3}\right) \delta 7.74$ $(\mathrm{d}, J=8.1 \mathrm{~Hz}, 2 \mathrm{H}), 7.52(\mathrm{~d}, J=8.2 \mathrm{~Hz}, 2 \mathrm{H}), 7.41-7.36(\mathrm{~m}, 1 \mathrm{H}), 7.36-7.31(\mathrm{~m}, 1 \mathrm{H})$, $7.30-7.26$ (m, 1H), $7.20-7.05$ (m, 5H), $6.94-6.84$ (m, 1H), 6.78 (s, 1H), 5.91 (d, J $=5.3 \mathrm{~Hz}, 1 \mathrm{H}), 3.81(\mathrm{dd}, J=11.7,2.8 \mathrm{~Hz}, 6 \mathrm{H}) \mathrm{ppm} .{ }^{13} \mathrm{C} \mathrm{NMR}\left(151 \mathrm{MHz}, \mathrm{CDCl}_{3}\right) \delta$ 146.0, 140.4 (d, $J=23.7 \mathrm{~Hz}), 131.6,130.0$ (q, $J=32.0 \mathrm{~Hz}), 129.0,128.6,127.9$, $127.6,127.3(\mathrm{~d}, J=6.5 \mathrm{~Hz}), 125.7,125.3(\mathrm{q}, J=4.0 \mathrm{~Hz}), 125.0,124.0(\mathrm{q}, J=272.3$ Hz)., 123.2, 122.7, $114.2,62.3(\mathrm{~d}, J=10.9 \mathrm{~Hz}), 53.1(\mathrm{~d}, J=5.8 \mathrm{~Hz}), 46.1(\mathrm{~d}, J=$ $225.9 \mathrm{~Hz}$ ) ppm. HRMS (ESI) m/z: $[\mathrm{M}+\mathrm{H}]^{+}$Calcd. for $\mathrm{C}_{25} \mathrm{H}_{22} \mathrm{~F}_{3} \mathrm{~N}_{3} \mathrm{O}_{3} \mathrm{P} 500.1345$ found 500.1342 .

Dimethyl (R)-(diazo(3-(3-fluorophenyl)-2-phenyl-1,2-dihydroisoquinolin-1-yl) methyl)phosphonate (4laa)

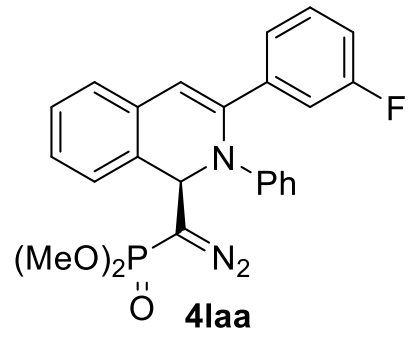

Following the general procedure, reaction were performed with $o$-alkynylbenzaldehyde 1al $(0.24 \mathrm{mmol}), \mathbf{2 a}(0.22$ mmol), 3a (0.20 mmol), $5 \AA$ MS (200 mg), IIh (10 mol \%), and $\mathrm{Ag}_{2} \mathrm{CO}_{3}(5 \mathrm{~mol} \%)$ in the $1.0 \mathrm{~mL}$ of THF/DME (1:1) at $-20{ }^{\circ} \mathrm{C}$ for 3 days under argon, followed by column chromatography $(\mathrm{PE} / \mathrm{EA}=10: 1 \sim 1: 1)$ to afford 4laa as 
yellow sticky oil, $78.1 \mathrm{mg}$ (87\% yield, 98\% ee); $[\alpha]_{\mathrm{D}}^{25}=+522.7^{\circ}\left(c=1.56,{ }^{i} \operatorname{PrOH}\right)$; HPLC (IC, n-hexane $/{ }^{i} \mathrm{PrOH}=70 / 30$, flow rate $\left.=0.5 \mathrm{~mL} / \mathrm{min}, \mathrm{I}=238 \mathrm{~nm}\right): t_{\mathrm{R}}=12.2$ min (major), $14.2 \mathrm{~min}$ (minor). ${ }^{1} \mathrm{H} \mathrm{NMR}\left(600 \mathrm{MHz}, \mathrm{CDCl}_{3}\right) \delta 7.50-7.46(\mathrm{~m}, 1 \mathrm{H})$, $7.42-7.35(\mathrm{~m}, 3 \mathrm{H}), 7.34-7.26(\mathrm{~m}, 2 \mathrm{H}), 7.23-7.14(\mathrm{~m}, 5 \mathrm{H}), 7.00-6.91(\mathrm{~m}, 2 \mathrm{H})$, $6.77(\mathrm{~s}, 1 \mathrm{H}), 5.95(\mathrm{~d}, J=5.3 \mathrm{~Hz}, 1 \mathrm{H}), 3.87(\mathrm{dd}, J=11.7,10.5 \mathrm{~Hz}, 6 \mathrm{H}) \mathrm{ppm} .{ }^{13} \mathrm{C} \mathrm{NMR}$ $\left(151 \mathrm{MHz}, \mathrm{CDCl}_{3}\right) \delta 162.9(\mathrm{~d}, J=245.3 \mathrm{~Hz}), 146.2,140.6,139.4(\mathrm{~d}, J=7.6 \mathrm{~Hz})$, 131.7, $129.7(\mathrm{~d}, J=8.3 \mathrm{~Hz}), 128.9,128.5,127.6,127.3(\mathrm{~d}, J=6.6 \mathrm{~Hz}), 125.6,124.9$, 123.1, $123.0(\mathrm{~d}, J=2.8 \mathrm{~Hz}), 122.6,115.0(\mathrm{~d}, J=21.3 \mathrm{~Hz}), 114.4(\mathrm{~d}, J=22.9 \mathrm{~Hz})$, $113.5,62.3(\mathrm{~d}, J=10.5 \mathrm{~Hz}), 53.0(\mathrm{~d}, J=5.7 \mathrm{~Hz}), 46.1(\mathrm{~d}, J=225.9 \mathrm{~Hz}) \mathrm{ppm}$. HRMS (ESI) m/z: $[\mathrm{M}+\mathrm{H}]^{+}$Calcd. for $\mathrm{C}_{24} \mathrm{H}_{22} \mathrm{FN}_{3} \mathrm{O}_{3} \mathrm{P} 450.1377$ found 450.1369 .

\section{Dimethyl (R)-((3-(3-chlorophenyl)-2-phenyl-1,2-dihydroisoquinolin-1-yl)(diazo) methyl)phosphonate (4maa)}

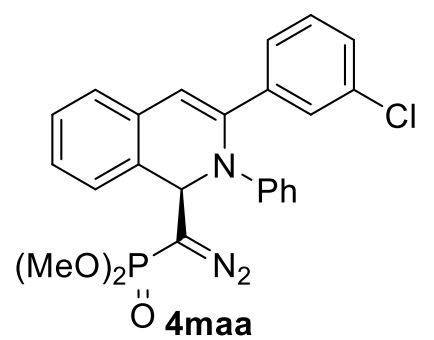

Following the general procedure, reaction were performed with $o$-alkynylbenzaldehyde $\mathbf{1 a m}(0.24 \mathrm{mmol}), \mathbf{2 a}(0.22$ mmol), 3a (0.20 mmol), 5^ MS (200 mg), IIh (10 mol \%), and $\mathrm{Ag}_{2} \mathrm{CO}_{3}(5 \mathrm{~mol} \%)$ in the $1.0 \mathrm{~mL}$ of THF/DME (1:1) at $-20{ }^{\circ} \mathrm{C}$ for 3 days under argon, followed by column chromatography $(\mathrm{PE} / \mathrm{EA}=10: 1 \sim 1: 1)$ to afford 4 maa as yellow sticky oil, $90.2 \mathrm{mg}(97 \%$ yield, $94 \% e e) ;[\alpha]_{\mathrm{D}}^{25}=+391.8^{\circ}\left(c=1.81,{ }^{i} \mathrm{PrOH}\right)$; HPLC (IC, n-hexane $/{ }^{i} \mathrm{PrOH}=70 / 30$, flow rate $=0.5 \mathrm{~mL} / \mathrm{min}, \mathrm{I}=238 \mathrm{~nm}$ ): $t_{\mathrm{R}}=12.2$ min (major), $14.9 \mathrm{~min}$ (minor). ${ }^{1} \mathrm{H}$ NMR (600 MHz, $\left.\mathrm{CDCl}_{3}\right) \delta 7.67-7.59(\mathrm{~m}, 1 \mathrm{H})$, $7.53-7.46(\mathrm{~m}, 1 \mathrm{H}), 7.37-7.30(\mathrm{~m}, 2 \mathrm{H}), 7.28-7.24(\mathrm{~m}, 1 \mathrm{H}), 7.21-7.16(\mathrm{~m}, 2 \mathrm{H})$, $7.16-7.08(\mathrm{~m}, 5 \mathrm{H}), 6.93-6.85(\mathrm{~m}, 1 \mathrm{H}), 6.71(\mathrm{~s}, 1 \mathrm{H}), 5.88(\mathrm{~d}, J=5.2 \mathrm{~Hz}, 1 \mathrm{H}), 3.83$ $(\mathrm{t}, J=11.9 \mathrm{~Hz}, 6 \mathrm{H}) \mathrm{ppm} .{ }^{13} \mathrm{C} \mathrm{NMR}\left(151 \mathrm{MHz}, \mathrm{CDCl}_{3}\right) \delta 146.1,140.4,139.0,134.3$, $131.7,129.5,128.9,128.5,128.2,127.6,127.5,127.2(\mathrm{~d}, J=6.4 \mathrm{~Hz}), 125.6,125.6$, $124.9,123.0,122.7,113.7,62.3(\mathrm{~d}, J=10.4 \mathrm{~Hz}), 53.1(\mathrm{~d}, J=5.2 \mathrm{~Hz}), 46.1(\mathrm{~d}, J=$ $226.5 \mathrm{~Hz}$ ) ppm. HRMS (ESI) m/z: $[\mathrm{M}+\mathrm{H}]^{+}$Calcd. for $\mathrm{C}_{24} \mathrm{H}_{22} \mathrm{ClN}_{3} \mathrm{O}_{3} \mathrm{P} 466.1082$, found 466.1084 . 


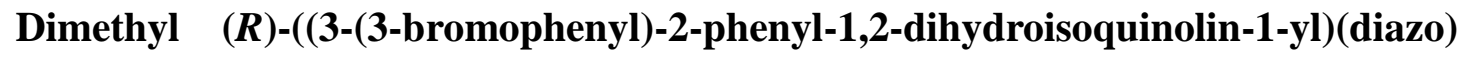
methyl)phosphonate (4naa)<smiles>COP(=O)(OC)C(=N)C1c2ccccc2C=C(c2cccc(Br)c2)N1c1ccccc1</smiles>

Following the general procedure, reaction were performed with $o$-alkynylbenzaldehyde $\mathbf{1 a n}(0.24 \mathrm{mmol})$, 2a $(0.22$ mmol), 3a (0.20 mmol), $5 \AA$ MS (200 mg), IIh (10 mol \%), and $\mathrm{Ag}_{2} \mathrm{CO}_{3}(5 \mathrm{~mol} \%)$ in the $1.0 \mathrm{~mL}$ of THF/DME (1:1) at $-20{ }^{\circ} \mathrm{C}$ for 3 days under argon, followed by column chromatography $(\mathrm{PE} / \mathrm{EA}=10: 1 \sim 1: 1)$ to afford 4naa as yellow sticky oil, $84.5 \mathrm{mg}$ (83\% yield, $86 \% e e) ;[\alpha]_{\mathrm{D}}^{25}=+335.7^{\circ}\left(c=1.67,{ }^{i} \mathrm{PrOH}\right)$; HPLC (IC, n-hexane $/{ }^{i} \mathrm{PrOH}=70 / 30$, flow rate $=0.5 \mathrm{~mL} / \mathrm{min}, \mathrm{I}=238 \mathrm{~nm}$ ): $t_{\mathrm{R}}=12.7$ min (major), $15.5 \mathrm{~min}$ (minor). ${ }^{1} \mathrm{H} \mathrm{NMR}\left(600 \mathrm{MHz}, \mathrm{CDCl}_{3}\right) \delta 7.85-7.74(\mathrm{~m}, 1 \mathrm{H})$, $7.57-7.50(\mathrm{~m}, 1 \mathrm{H}), 7.38-7.30(\mathrm{~m}, 3 \mathrm{H}), 7.28-7.24(\mathrm{~m}, 1 \mathrm{H}), 7.17-7.07(\mathrm{~m}, 6 \mathrm{H})$, $6.94-6.84(\mathrm{~m}, 1 \mathrm{H}), 6.70(\mathrm{~s}, 1 \mathrm{H}), 5.88(\mathrm{~d}, J=5.2 \mathrm{~Hz}, 1 \mathrm{H}), 3.83(\mathrm{dd}, J=12.7,11.7 \mathrm{~Hz}$, $6 \mathrm{H}) \mathrm{ppm} .{ }^{13} \mathrm{C} \mathrm{NMR}\left(151 \mathrm{MHz}, \mathrm{CDCl}_{3}\right) \delta 146.0,140.3,139.2,131.7,131.1,130.4$, 129.8, 128.9, 128.5, 127.7, $127.2(\mathrm{~d}, J=6.8 \mathrm{~Hz}), 126.1,125.6,124.9,123.1,122.7$, 122.5, 113.7, 62.3 (d, $J=10.4 \mathrm{~Hz}), 53.1$ (d, $J=5.7 \mathrm{~Hz}), 46.1$ (d, $J=226.0 \mathrm{~Hz}) \mathrm{ppm}$. HRMS (ESI) m/z: [M+H] ${ }^{+}$Calcd. for $\mathrm{C}_{24} \mathrm{H}_{22} \mathrm{BrN}_{3} \mathrm{O}_{3} \mathrm{P} 510.0577$, found 510.0580.

Dimethyl (R)-(diazo(2-phenyl-3-(m-tolyl)-1,2-dihydroisoquinolin-1-yl)methyl) phosphonate (4oaa)<smiles>COP(=O)(OC)OC(=N)C1c2ccccc2C=C(c2cccc(C)c2)N1c1ccccc1</smiles>

Following the general procedure, reaction were performed with $o$-alkynylbenzaldehyde 1 ao $(0.24 \mathrm{mmol}), \mathbf{2 a}(0.22$ mmol), 3a (0.20 mmol), $5 \AA$ MS (200 mg), IIh (10 mol \%), and $\mathrm{Ag}_{2} \mathrm{CO}_{3}(5 \mathrm{~mol} \%)$ in the $1.0 \mathrm{~mL}$ of THF/DME (1:1) at $-20{ }^{\circ} \mathrm{C}$ for 3 days under argon, followed by column chromatography $(\mathrm{PE} / \mathrm{EA}=10: 1 \sim 1: 1)$ to afford 4oaa as yellow sticky oil, $85.4 \mathrm{mg}(96 \%$ yield, $95 \% e e) ;[\alpha]_{\mathrm{D}}^{25}=+435.5^{\circ}\left(c=1.70,{ }^{i} \mathrm{PrOH}\right)$; HPLC (IC, n-hexane $/{ }^{i} \mathrm{PrOH}=70 / 30$, flow rate $=0.5 \mathrm{~mL} / \mathrm{min}, \mathrm{I}=238 \mathrm{~nm}$ ): $t_{\mathrm{R}}=12.5$ $\min$ (major), $15.4 \mathrm{~min}$ (minor). ${ }^{1} \mathrm{H} \mathrm{NMR}\left(600 \mathrm{MHz}, \mathrm{CDCl}_{3}\right) \delta 7.47(\mathrm{~d}, J=1.9 \mathrm{~Hz}, 1 \mathrm{H})$, $7.39(\mathrm{~d}, J=7.8 \mathrm{~Hz}, 1 \mathrm{H}), 7.36-7.28(\mathrm{~m}, 2 \mathrm{H}), 7.25-7.20(\mathrm{~m}, 1 \mathrm{H}), 7.17-7.08(\mathrm{~m}$, $6 \mathrm{H}), 7.04(\mathrm{~d}, J=7.4 \mathrm{~Hz}, 1 \mathrm{H}), 6.92-6.79(\mathrm{~m}, 1 \mathrm{H}), 6.68(\mathrm{~s}, 1 \mathrm{H}), 5.89(\mathrm{~d}, J=5.5 \mathrm{~Hz}$, $1 \mathrm{H}), 3.81(\mathrm{dd}, J=11.7,5.7 \mathrm{~Hz}, 6 \mathrm{H}), 2.31(\mathrm{~s}, 3 \mathrm{H}) \mathrm{ppm} .{ }^{13} \mathrm{C} \mathrm{NMR}\left(151 \mathrm{MHz}, \mathrm{CDCl}_{3}\right) \delta$ $146.5,141.8,137.8,136.8,132.2,129.0,128.7,128.3,128.1,128.1,127.2$ (d, $J=6.4$ $\mathrm{Hz}), 127.1,125.5,124.8,124.6,122.6,122.6,112.6,62.2$ (d, $J=10.6 \mathrm{~Hz}), 53.0$ (d, $J$ $=5.4 \mathrm{~Hz}), 46.0(\mathrm{~d}, J=225.7 \mathrm{~Hz}), 21.4 \mathrm{ppm}$. HRMS $(\mathrm{ESI}) \mathrm{m} / \mathrm{z}:[\mathrm{M}+\mathrm{H}]^{+}$Calcd. for 
$\mathrm{C}_{25} \mathrm{H}_{25} \mathrm{~N}_{3} \mathrm{O}_{3} \mathrm{P} 446.1628$, found 446.1629

Dimethyl $(R)-(\operatorname{diazo}$ (3-(3-methoxyphenyl)-2-phenyl-1,2-dihydroisoquinolin-1-yl) methyl)phosphonate (4paa)

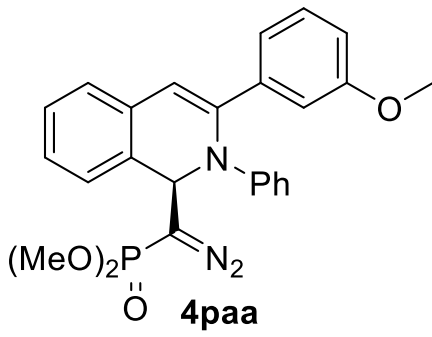

Following the general procedure, reaction were performed with $o$-alkynylbenzaldehyde 1ap $(0.24 \mathrm{mmol})$, 2a $(0.22$ mmol), 3a (0.20 mmol), $5 \AA ̊$ MS (200 mg), IIh (10 mol \%), and $\mathrm{Ag}_{2} \mathrm{CO}_{3}(5 \mathrm{~mol} \%)$ in the $1.0 \mathrm{~mL}$ of THF/DME (1:1) at $-20{ }^{\circ} \mathrm{C}$ for 3 days under argon, followed by column chromatography $(\mathrm{PE} / \mathrm{EA}=10: 1 \sim 1: 1)$ to afford 4paa as yellow sticky oil, $86.6 \mathrm{mg}$ (94\% yield, $95 \% e e) ;[\alpha]_{\mathrm{D}}^{25}=+496.7^{\circ}\left(c=1.74,{ }^{i} \mathrm{PrOH}\right)$; HPLC (IC, $\mathrm{n}$-hexane $/{ }^{i} \operatorname{PrOH}=70 / 30$, flow rate $\left.=0.5 \mathrm{~mL} / \mathrm{min}, \mathrm{I}=238 \mathrm{~nm}\right): t_{\mathrm{R}}=13.5$ $\min$ (major), $16.9 \mathrm{~min}$ (minor). ${ }^{1} \mathrm{H} \mathrm{NMR}\left(600 \mathrm{MHz}, \mathrm{CDCl}_{3}\right) \delta 7.36-7.29$ (m, 2H), $7.26-7.21(\mathrm{~m}, 2 \mathrm{H}), 7.20-7.16(\mathrm{~m}, 2 \mathrm{H}), 7.16-7.10(\mathrm{~m}, 5 \mathrm{H}), 6.92-6.84(\mathrm{~m}, 1 \mathrm{H})$, $6.80-6.74(\mathrm{~m}, 1 \mathrm{H}), 6.71(\mathrm{~s}, 1 \mathrm{H}), 5.89(\mathrm{~d}, J=5.4 \mathrm{~Hz}, 1 \mathrm{H}), 3.80(\mathrm{dd}, J=11.6,0.8 \mathrm{~Hz}$, $6 \mathrm{H}), 3.72(\mathrm{~s}, 3 \mathrm{H}) \mathrm{ppm} .{ }^{13} \mathrm{C} \mathrm{NMR}\left(151 \mathrm{MHz}, \mathrm{CDCl}_{3}\right) \delta 159.6,146.5,141.5,138.3$, 132.0, 129.2, 128.8, 128.4, 127.2, 127.2, 125.5, 124.7, 122.8, 122.6, 120.0, 113.9, 113.2, 112.7, $62.2(\mathrm{~d}, J=10.8 \mathrm{~Hz}), 55.1,53.0(\mathrm{~d}, J=9.3 \mathrm{~Hz}), 53.0(\mathrm{~d}, J=8.7 \mathrm{~Hz})$, $46.0\left(\mathrm{~d}, J=225.3 \mathrm{~Hz}\right.$ ) ppm. HRMS (ESI) m/z: $[\mathrm{M}+\mathrm{H}]^{+}$Calcd. for $\mathrm{C}_{25} \mathrm{H}_{25} \mathrm{~N}_{3} \mathrm{O}_{4} \mathrm{P}$ 462.1577 , found 462.1578 .

Dimethyl $\quad(R)$-(diazo(2-phenyl-3-(o-tolyl)-1,2-dihydroisoquinolin-1-yl)methyl) phosphonate (4qaa)

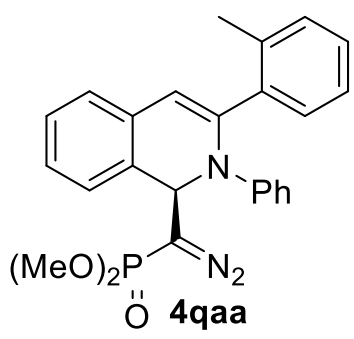

Following the general procedure, reaction were performed with $o$-alkynylbenzaldehyde $\mathbf{1 a q}(0.24 \mathrm{mmol}), \mathbf{2 a}(0.22$ mmol), 3a (0.20 mmol), $5 \AA$ MS (200 mg), IIh (10 mol \%), and $\mathrm{Ag}_{2} \mathrm{CO}_{3}(5 \mathrm{~mol} \%)$ in the $1.0 \mathrm{~mL}$ of THF/DME (1:1) at $-20{ }^{\circ} \mathrm{C}$ for 3 days under argon, followed by column chromatography $(\mathrm{PE} / \mathrm{EA}=10: 1 \sim 1: 1)$ to afford 4qaa as yellow sticky oil, $81.9 \mathrm{mg}$ (92\% yield, $84 \% e e)$; $[\alpha]_{\mathrm{D}}^{25}=+94.3^{\circ}\left(c=1.33,{ }^{i} \mathrm{PrOH}\right)$; HPLC (ADH, n-hexane $/{ }^{i} \mathrm{PrOH}=50 / 50$, flow rate $\left.=0.5 \mathrm{~mL} / \mathrm{min}, \mathrm{I}=238 \mathrm{~nm}\right): t_{\mathrm{R}}=9.4$ min (major), $17.1 \mathrm{~min}$ (minor). ${ }^{1} \mathrm{H} \mathrm{NMR}\left(600 \mathrm{MHz}, \mathrm{CDCl}_{3}\right) \delta 7.51-7.47(\mathrm{~m}, 1 \mathrm{H})$, $7.33-7.27(\mathrm{~m}, 2 \mathrm{H}), 7.26-7.22(\mathrm{~m}, 1 \mathrm{H}), 7.17(\mathrm{~d}, J=7.5 \mathrm{~Hz}, 1 \mathrm{H}), 7.15-7.05(\mathrm{~m}$, $7 \mathrm{H}), 6.90-6.81(\mathrm{~m}, 1 \mathrm{H}), 6.13(\mathrm{~s}, 1 \mathrm{H}), 5.77(\mathrm{~d}, J=7.0 \mathrm{~Hz}, 1 \mathrm{H}), 3.81(\mathrm{dd}, J=39.3$, $11.6 \mathrm{~Hz}, 6 \mathrm{H}), 2.52$ (s, 3H) ppm. ${ }^{13} \mathrm{C} \mathrm{NMR}\left(151 \mathrm{MHz}, \mathrm{CDCl}_{3}\right) \delta 145.9,141.9,136.7$, 136.6, 131.9, 130.8, 130.5, 128.5, 128.1, 128.0, 127.0, 126.8 (d, $J=5.0 \mathrm{~Hz}), 125.7$, 
125.1, 124.2, 122.9, 122.9, 113.2, 61.8 (d, $J=12.1 \mathrm{~Hz}), 53.2(\mathrm{~d}, J=6.0 \mathrm{~Hz}), 53.0$ (d, $J=5.6 \mathrm{~Hz}), 47.1(\mathrm{~d}, J=226.7 \mathrm{~Hz}), 20.5 \mathrm{ppm}$. HRMS (ESI) m/z: $[\mathrm{M}+\mathrm{H}]^{+}$Calcd. for $\mathrm{C}_{25} \mathrm{H}_{25} \mathrm{~N}_{3} \mathrm{O}_{3} \mathrm{P} 446.1628$ found 446.1627 .

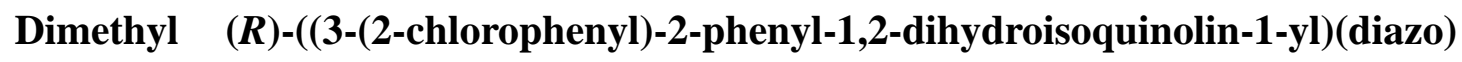
methyl)phosphonate (4raa)

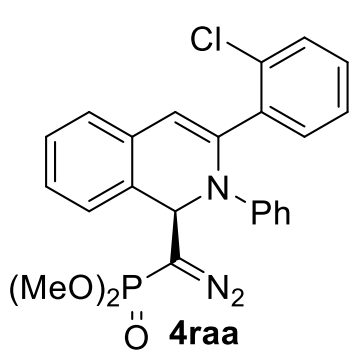

Following the general procedure, reaction were performed with $o$-alkynylbenzaldehyde 1ar $(0.24 \mathrm{mmol})$, 2a $(0.22$ mmol), 3a (0.20 mmol), $5 \AA$ MS (200 mg), IIh (10 mol \%), and $\mathrm{Ag}_{2} \mathrm{CO}_{3}(5 \mathrm{~mol} \%)$ in the $1.0 \mathrm{~mL}$ of THF/DME (1:1) at $-20{ }^{\circ} \mathrm{C}$ for 3 days under argon, followed by column chromatography $(\mathrm{PE} / \mathrm{EA}=10: 1 \sim 1: 1)$ to afford 4raa as yellow sticky oil,29.7 mg (32\% yield, 80\% ee); $[\alpha]_{\mathrm{D}}^{25}=+152.6^{\circ}\left(c=0.46,{ }^{i} \mathrm{PrOH}\right)$; HPLC (IC, n-hexane $/{ }^{i} \mathrm{PrOH}=70 / 30$, flow rate $=0.5 \mathrm{~mL} / \mathrm{min}, \mathrm{I}=238 \mathrm{~nm}$ ): $t_{\mathrm{R}}=25.9$ min (major), $27.8 \mathrm{~min}$ (minor). ${ }^{1} \mathrm{H} \mathrm{NMR}\left(600 \mathrm{MHz}, \mathrm{CDCl}_{3}\right) \delta 7.61-7.54(\mathrm{~m}, 1 \mathrm{H})$, $7.32(\mathrm{t}, J=5.3 \mathrm{~Hz}, 3 \mathrm{H}), 7.30-7.26(\mathrm{~m}, 1 \mathrm{H}), 7.19(\mathrm{~d}, J=7.5 \mathrm{~Hz}, 1 \mathrm{H}), 7.17-7.10(\mathrm{~m}$, $6 \mathrm{H}), 6.93-6.83(\mathrm{~m}, 1 \mathrm{H}), 6.41(\mathrm{~s}, 1 \mathrm{H}), 5.80(\mathrm{~d}, J=6.6 \mathrm{~Hz}, 1 \mathrm{H}), 3.83(\mathrm{dd}, J=36.4$, $11.7 \mathrm{~Hz}, 6 \mathrm{H}) \mathrm{ppm} .{ }^{13} \mathrm{C} \mathrm{NMR}\left(151 \mathrm{MHz}, \mathrm{CDCl}_{3}\right) \delta 145.7,138.5,135.8,133.3,131.7$, 131.3, 130.4, 129.0, 128.6, 128.2, 127.5, 127.0 (d, $J=5.4$ Hz), 126.6, 125.3, 124.6, 123.0, 122.6, 115.1, $61.6(\mathrm{~d}, J=11.9 \mathrm{~Hz}), 53.2(\mathrm{~d}, J=6.0 \mathrm{~Hz}), 53.0(\mathrm{~d}, J=5.8 \mathrm{~Hz})$, $47.0(\mathrm{~d}, J=227.2 \mathrm{~Hz}) \mathrm{ppm}$. HRMS (ESI) m/z: $[\mathrm{M}+\mathrm{H}]^{+}$Calcd. for $\mathrm{C}_{24} \mathrm{H}_{22} \mathrm{ClN}_{3} \mathrm{O}_{3} \mathrm{P}$ 466.1082, found 466.1076 .

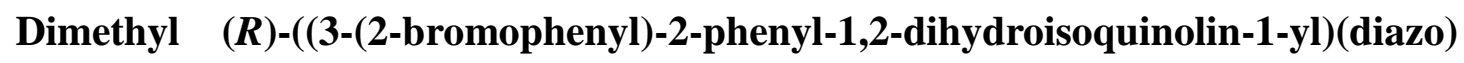
methyl)phosphonate (4saa)<smiles>COP(=O)(OC)C(=N)C1c2ccccc2C=C(c2ccccc2Br)N1c1ccccc1</smiles>

Following the general procedure, reaction were performed with $o$-alkynylbenzaldehyde $1 \mathbf{a s}(0.24 \mathrm{mmol}), \mathbf{2 a}(0.22 \mathrm{mmol})$, 3a (0.20 mmol), $5 \AA$ MS (200 mg), IIh (10 mol \%), and $\mathrm{Ag}_{2} \mathrm{CO}_{3}(5 \mathrm{~mol} \%)$ in the $1.0 \mathrm{~mL}$ of THF/DME (1:1) at $-20{ }^{\circ} \mathrm{C}$ for 3 days under argon, followed by column chromatography $(\mathrm{PE} / \mathrm{EA}=10: 1 \sim 1: 1)$ to afford 4saa as yellow sticky oil, 35.6 mg $(35 \%$ yield, $76 \% e e) ;[\alpha]_{\mathrm{D}}^{25}=+237.3^{\circ}\left(c=0.22,{ }^{i} \mathrm{PrOH}\right)$; HPLC (IC, n-hexane/ ${ }^{i} \mathrm{PrOH}=70 / 30$, flow rate $=0.5 \mathrm{~mL} / \mathrm{min}, \mathrm{I}=238 \mathrm{~nm}$ ): $t_{\mathrm{R}}=12.2 \mathrm{~min}$ (major), $12.8 \mathrm{~min}$ (minor). ${ }^{1} \mathrm{H}$ NMR (600 MHz, $\left.\mathrm{CDCl}_{3}\right) \delta 7.58-7.51(\mathrm{~m}, 2 \mathrm{H}), 7.36-7.31(\mathrm{~m}, 2 \mathrm{H}), 7.30$ - 7.27 (m, 1H), $7.22-7.09$ (m, 6H), $7.08-7.02$ (m, 1H), $6.91-6.86$ (m, 1H), 6.39 (s, $1 \mathrm{H}), 5.80(\mathrm{~d}, J=6.5 \mathrm{~Hz}, 1 \mathrm{H}), 3.82(\mathrm{dd}, J=33.3,11.7 \mathrm{~Hz}, 6 \mathrm{H}) \mathrm{ppm} .{ }^{13} \mathrm{C}$ NMR $(151$ 
$\left.\mathrm{MHz}, \mathrm{CDCl}_{3}\right) \delta 145.7,139.8,137.4,133.8,131.9,131.3,129.2,128.6,128.2,127.4$, $127.1,126.8$ (d, $J=5.2 \mathrm{~Hz}), 125.2,124.5,123.1,123.0,122.8,115.1,61.7$ (d, $J=11.7$ $\mathrm{Hz}), 53.2(\mathrm{~d}, J=6.1 \mathrm{~Hz}), 53.0(\mathrm{~d}, J=6.0 \mathrm{~Hz}), 47.1(\mathrm{~d}, J=228.0 \mathrm{~Hz}) \mathrm{ppm}$. HRMS (ESI) $\mathrm{m} / \mathrm{z}$ : $[\mathrm{M}+\mathrm{H}]^{+}$Calcd. for $\mathrm{C}_{24} \mathrm{H}_{22} \mathrm{BrN}_{3} \mathrm{O}_{3} \mathrm{P} 510.0577$ found 510.0573 .

Dimethyl (R)-(diazo(3-(3,5-dimethylphenyl)-2-phenyl-1,2-dihydroisoquinolin-1-yl) methyl)phosphonate (4taa)

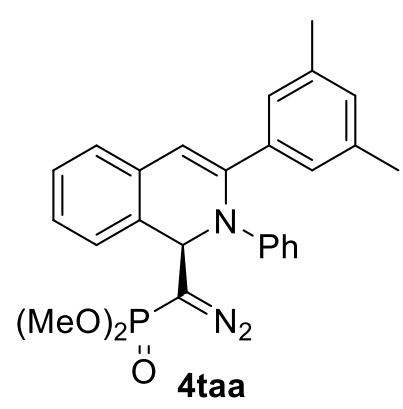

Following the general procedure, reaction were performed with $o$-alkynylbenzaldehyde 1at $(0.24 \mathrm{mmol}), 2 \mathrm{a}(0.22$ mmol), 3a (0.20 mmol), $5 \AA$ MS (200 mg), IIh (10 mol \%), and $\mathrm{Ag}_{2} \mathrm{CO}_{3}(5 \mathrm{~mol} \%)$ in the $1.0 \mathrm{~mL}$ of THF/DME (1:1) at $-20{ }^{\circ} \mathrm{C}$ for 3 days under argon, followed by column chromatography $(\mathrm{PE} / \mathrm{EA}=10: 1 \sim 1: 1)$ to afford 4taa as yellow sticky oil, $80.7 \mathrm{mg}(88 \%$ yield, $89 \% e e) ;[\alpha]_{\mathrm{D}}^{25}=$ $+413.4^{\circ}\left(c=1.62,{ }^{i} \mathrm{PrOH}\right)$; HPLC $\left(\mathrm{IC}, \mathrm{n}\right.$-hexane $/{ }^{i} \mathrm{PrOH}=70 / 30$, flow rate $=0.5$ $\mathrm{mL} / \mathrm{min}, \mathrm{I}=238 \mathrm{~nm}): t_{\mathrm{R}}=11.6 \mathrm{~min}$ (major), $15.0 \mathrm{~min}$ (minor). ${ }^{1} \mathrm{H} \mathrm{NMR}(600 \mathrm{MHz}$, $\left.\mathrm{CDCl}_{3}\right) \delta 7.35-7.29(\mathrm{~m}, 2 \mathrm{H}), 7.26-7.25(\mathrm{~m}, 2 \mathrm{H}), 7.25-7.21(\mathrm{~m}, 1 \mathrm{H}), 7.16-7.10$ $(\mathrm{m}, 5 \mathrm{H}), 6.93-6.83(\mathrm{~m}, 2 \mathrm{H}), 6.67(\mathrm{~s}, 1 \mathrm{H}), 5.88(\mathrm{~d}, J=5.5 \mathrm{~Hz}, 1 \mathrm{H}), 3.83(\mathrm{dd}, J=11.7$, $8.1 \mathrm{~Hz}, 6 \mathrm{H}), 2.26(\mathrm{~s}, 6 \mathrm{H}) \mathrm{ppm} .{ }^{13} \mathrm{C} \mathrm{NMR}\left(151 \mathrm{MHz}, \mathrm{CDCl}_{3}\right) \delta 146.6,142.0,137.6$, 136.9, 132.2, 130.0, 128.7, 128.3, 127.2 (d, $J=6.5 \mathrm{~Hz}), 127.0,125.5,125.4$, 124.6, 122.6, 122.6, 112.6, $62.2(\mathrm{~d}, J=10.7 \mathrm{~Hz}), 53.0(\mathrm{~d}, J=6.0 \mathrm{~Hz}), 52.9(\mathrm{~d}, J=5.5 \mathrm{~Hz})$. $46.0(\mathrm{~d}, J=225.8 \mathrm{~Hz}), 21.3 \mathrm{ppm}$. HRMS (ESI) m/z: $[\mathrm{M}+\mathrm{H}]^{+}$Calcd. for $\mathrm{C}_{26} \mathrm{H}_{27} \mathrm{~N}_{3} \mathrm{O}_{3} \mathrm{P}$ 460.1785 , found 460.1786 .

Dimethyl (R)-(diazo(3-(3,5-dichlorophenyl)-2-phenyl-1,2-dihydroisoquinolin-1-yl) methyl)phosphonate (4uaa)

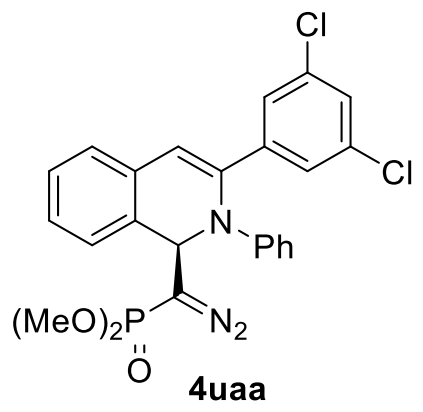

Following the general procedure, reaction were performed with $o$-alkynylbenzaldehyde 1au $(0.24 \mathrm{mmol}), 2 \mathbf{a}(0.22$ mmol), 3a (0.20 mmol), $5 \AA$ MS (200 mg), IIh (10 mol \%), and $\mathrm{Ag}_{2} \mathrm{CO}_{3}(5 \mathrm{~mol} \%)$ in the $1.0 \mathrm{~mL}$ of THF/DME (1:1) at $-20{ }^{\circ} \mathrm{C}$ for 3 days under argon, followed by column chromatography $(\mathrm{PE} / \mathrm{EA}=10: 1 \sim 1: 1)$ to afford 4uaa as yellow sticky oil, $71.8 \mathrm{mg}(72 \%$ yield, $82 \% e e) ;[\alpha]_{\mathrm{D}}^{25}=$ $+352.6^{\circ}\left(c=1.44,{ }^{i} \mathrm{PrOH}\right)$; HPLC $\left(\mathrm{IC}, \mathrm{n}\right.$-hexane $/{ }^{i} \mathrm{PrOH}=70 / 30$, flow rate $=0.5$ $\mathrm{mL} / \mathrm{min}, \mathrm{I}=238 \mathrm{~nm}): t_{\mathrm{R}}=11.4 \mathrm{~min}$ (major), $13.5 \min$ (minor). ${ }^{1} \mathrm{H} \mathrm{NMR}(600 \mathrm{MHz}$, $\left.\mathrm{CDCl}_{3}\right) \delta 7.52(\mathrm{~d}, J=1.9 \mathrm{~Hz}, 2 \mathrm{H}), 7.37-7.31(\mathrm{~m}, 2 \mathrm{H}), 7.30-7.26(\mathrm{~m}, 1 \mathrm{H}), 7.22-$ 
$7.19(\mathrm{~m}, 1 \mathrm{H}), 7.18-7.12(\mathrm{~m}, 3 \mathrm{H}), 7.11-7.06(\mathrm{~m}, 2 \mathrm{H}), 6.96-6.87(\mathrm{~m}, 1 \mathrm{H}), 6.71(\mathrm{~s}$, $1 \mathrm{H}), 5.87(\mathrm{~d}, J=5.0 \mathrm{~Hz}, 1 \mathrm{H}), 3.86(\mathrm{dd}, J=22.7,11.7 \mathrm{~Hz}, 6 \mathrm{H}) \mathrm{ppm} .{ }^{13} \mathrm{C}$ NMR $(151$ $\left.\mathrm{MHz}, \mathrm{CDCl}_{3}\right) \delta 145.7,140.4,139.3,135.0,131.4,129.1,128.6,128.1,128.0,127.2(\mathrm{~d}$, $J=7.2 \mathrm{~Hz}),, 125.9,125.7,125.1,123.4,122.6,114.7,, 62.4(\mathrm{~d}, J=10.0 \mathrm{~Hz}), 53.2(\mathrm{~d}$, $J=5.7 \mathrm{~Hz}), 53.1(\mathrm{~d}, J=6.1 \mathrm{~Hz}), 46.2(\mathrm{~d}, J=227.0 \mathrm{~Hz}) \mathrm{ppm}$. HRMS (ESI) m/z: $[\mathrm{M}+\mathrm{H}]^{+}$Calcd. for $\mathrm{C}_{24} \mathrm{H}_{21} \mathrm{Cl}_{2} \mathrm{~N}_{3} \mathrm{O}_{3} \mathrm{P} 500.0692$, found 500.0696 .

Dimethyl (R)-(diazo(3-(naphthalen-1-yl)-2-phenyl-1,2-dihydroisoquinolin-1-yl) methyl)phosphonate (4vaa)<smiles>COP(=O)(O)C(=N)C1c2ccccc2C=C(c2cccc3ccccc23)N1c1ccccc1</smiles>

Following the general procedure, reaction were performed with $o$-alkynylbenzaldehyde $\mathbf{1 a v}(0.24 \mathrm{mmol}), \mathbf{2 a}(0.22$ mmol), 3a (0.20 mmol), $5 \AA$ MS (200 mg), IIh (10 mol \%), and $\mathrm{Ag}_{2} \mathrm{CO}_{3}(5 \mathrm{~mol} \%)$ in the $1.0 \mathrm{~mL}$ of THF/DME (1:1) at $-20{ }^{\circ} \mathrm{C}$ for 3 days under argon, followed by column chromatography $(\mathrm{PE} / \mathrm{EA}=10: 1 \sim 1: 1)$ to afford 4vaa as yellow sticky oil, $32.7 \mathrm{mg}(34 \%$ yield, $72 \% e e) ;[\alpha]_{\mathrm{D}}^{25}=+63.3^{\circ}\left(c=0.12,{ }^{i} \operatorname{PrOH}\right)$; HPLC (IC, n-hexane $/{ }^{i} \mathrm{PrOH}=70 / 30$, flow rate $=0.5 \mathrm{~mL} / \mathrm{min}, \mathrm{I}=238 \mathrm{~nm}$ ): $t_{\mathrm{R}}=13.2$ $\min$ (major), $14.7 \mathrm{~min}$ (minor). ${ }^{1} \mathrm{H}$ NMR (600 MHz, $\left.\mathrm{CDCl}_{3}\right) \delta 8.74$ (d, $J=8.5 \mathrm{~Hz}, 1 \mathrm{H}$ ), $7.82(\mathrm{~d}, J=8.1 \mathrm{~Hz}, 1 \mathrm{H}), 7.76-7.68(\mathrm{~m}, 2 \mathrm{H}), 7.59-7.53(\mathrm{~m}, 1 \mathrm{H}), 7.51-7.45(\mathrm{~m}$, $1 \mathrm{H}), 7.41-7.35(\mathrm{~m}, 3 \mathrm{H}), 7.33-7.28(\mathrm{~m}, 1 \mathrm{H}), 7.25(\mathrm{~d}, J=7.4 \mathrm{~Hz}, 1 \mathrm{H}), 7.17(\mathrm{~d}, J=$ $8.0 \mathrm{~Hz}, 2 \mathrm{H}), 7.05-6.96(\mathrm{~m}, 2 \mathrm{H}), 6.84-6.74(\mathrm{~m}, 1 \mathrm{H}), 6.42(\mathrm{~s}, 1 \mathrm{H}), 5.93(\mathrm{~d}, J=6.6$ $\mathrm{Hz}, 1 \mathrm{H}), 3.88(\mathrm{dd}, J=43.5,11.7 \mathrm{~Hz}, 6 \mathrm{H}) \mathrm{ppm} .{ }^{13} \mathrm{C} \mathrm{NMR}\left(151 \mathrm{MHz}, \mathrm{CDCl}_{3}\right) \delta 146.1$, $140.2,134.7,133.9,131.9,131.8,128.6,128.5,128.5,128.2,127.9,127.2,126.8$ (d, $J$ $=5.2 \mathrm{~Hz}), 126.3,125.6,125.3,125.2,125.1,124.3,122.8,122.5,114.8,61.7(\mathrm{~d}, J=$ $11.8 \mathrm{~Hz}), 53.2(\mathrm{~d}, J=5.9 \mathrm{~Hz}), 53.1(\mathrm{~d}, J=5.7 \mathrm{~Hz}), 46.9(\mathrm{~d}, J=226.5 \mathrm{~Hz}) \mathrm{ppm}$. HRMS (ESI) m/z: $[\mathrm{M}+\mathrm{H}]^{+}$Calcd. for $\mathrm{C}_{28} \mathrm{H}_{25} \mathrm{~N}_{3} \mathrm{O}_{3} \mathrm{P} 482.1634$, found 482.1637 .

Dimethyl $\quad(R)$-(diazo(2-phenyl-3-(pyridin-2-yl)-1,2-dihydroisoquinolin-1-yl) methyl)phosphonate (4waa)

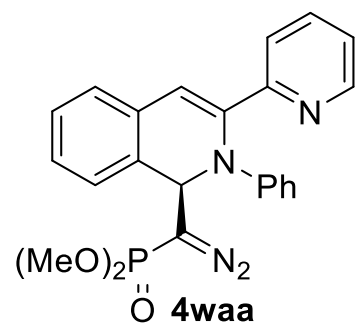

Following the general procedure, reaction were performed with $o$-alkynylbenzaldehyde 1 aw $(0.24 \mathrm{mmol}), \mathbf{2 a}(0.22 \mathrm{mmol}), 3 \mathbf{a}$ (0.20 mmol), $5 \AA$ MS (200 mg), IIh (10 mol \%), and $\mathrm{Ag}_{2} \mathrm{CO}_{3}(5$ mol \%) in the $1.0 \mathrm{~mL}$ of THF/DME $(1: 1)$ at $-20{ }^{\circ} \mathrm{C}$ for 3 days under argon, followed by column chromatography $(\mathrm{PE} / \mathrm{EA}=$ 10:1 1:1) to afford 4waa as yellow sticky oil, $33.7 \mathrm{mg}(39 \%$ yield, $17 \% e e) ;[\alpha]_{\mathrm{D}}^{25}=+72.2^{\circ}\left(c=0.68,{ }^{i} \mathrm{PrOH}\right), \mathrm{HPLC}\left(\mathrm{IC}, \mathrm{n}\right.$-hexane $/{ }^{i} \mathrm{PrOH}=70 / 30$, 
flow rate $=0.5 \mathrm{~mL} / \mathrm{min}, \mathrm{I}=238 \mathrm{~nm}$ ): $t_{\mathrm{R}}=14.9 \min$ (major), $15.8 \mathrm{~min}$ (minor). ${ }^{1} \mathrm{H}$ NMR (600 MHz, $\left.\mathrm{CDCl}_{3}\right) \delta 8.67-8.58(\mathrm{~m}, 1 \mathrm{H}), 7.50-7.41(\mathrm{~m}, 3 \mathrm{H}), 7.38-7.34(\mathrm{~m}$, $1 \mathrm{H}), 7.33-7.29(\mathrm{~m}, 1 \mathrm{H}), 7.28-7.24(\mathrm{~m}, 1 \mathrm{H}), 7.17-7.08(\mathrm{~m}, 6 \mathrm{H}), 6.94-6.85(\mathrm{~m}$, 1H), $5.91(\mathrm{~d}, J=5.2 \mathrm{~Hz}, 1 \mathrm{H}), 3.77(\mathrm{dd}, J=20.6,11.7 \mathrm{~Hz}, 6 \mathrm{H}) \mathrm{ppm} .{ }^{13} \mathrm{C}$ NMR $(151$ $\left.\mathrm{MHz} \mathrm{CDCl}_{3}\right) \delta 154.2,149.3,146.6,140.0,136.2,131.6,129.0,128.6,127.9,127.6$ (d, $J=6.6 \mathrm{~Hz}), 125.8,125.6,122.9,122.6,122.3,115.7,62.4(\mathrm{~d}, J=10.3 \mathrm{~Hz}), 53.0(\mathrm{~d}, J$ $=4.4 \mathrm{~Hz}), 53.0(\mathrm{~d}, J=5.1 \mathrm{~Hz}) .45 .8(\mathrm{~d}, J=225.4 \mathrm{~Hz}) \mathrm{ppm}$. HRMS (ESI) m/z: $[\mathrm{M}+\mathrm{H}]^{+}$Calcd. for $\mathrm{C}_{23} \mathrm{H}_{22} \mathrm{~N}_{4} \mathrm{O}_{3} \mathrm{P} 433.1424$, found 433.1422.

Dimethyl (R)-(diazo(3-(furan-2-yl)-2-phenyl-1,2-dihydroisoquinolin-1-yl)methyl) phosphonate (4xaa)

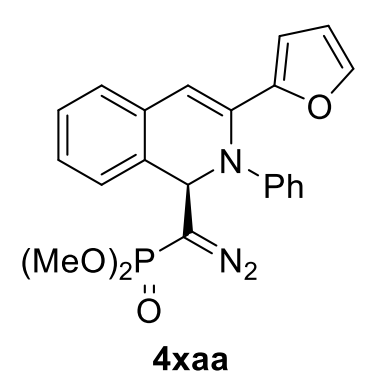

Following the general procedure, reaction were performed with $o$-alkynylbenzaldehyde $\mathbf{1 a x}(0.24 \mathrm{mmol}), \mathbf{2 a}(0.22 \mathrm{mmol})$, 3a $(0.20 \mathrm{mmol}), 5 \AA \mathrm{MS}(200 \mathrm{mg})$, IIh (10 mol \%), and $\mathrm{Ag}_{2} \mathrm{CO}_{3}(5 \mathrm{~mol} \%)$ in the $1.0 \mathrm{~mL}$ of THF/DME $(1: 1)$ at $-20{ }^{\circ} \mathrm{C}$ for 3 days under argon, followed by column chromatography $(\mathrm{PE} / \mathrm{EA}=10: 1 \sim 1: 1)$ to afford 4xaa as yellow sticky oil, 37.0 mg (44\% yield, 69\% ee); $[\alpha]_{\mathrm{D}}^{25}=+289.5^{\circ}\left(c=0.67,{ }^{i} \mathrm{PrOH}\right) ; \mathrm{HPLC}$ (ADH, n-hexane/ ${ }^{i} \mathrm{PrOH}=50 / 50$, flow rate $=0.5 \mathrm{~mL} / \mathrm{min}, \mathrm{I}=238 \mathrm{~nm}$ ): $t_{\mathrm{R}}=10.8 \mathrm{~min}$ (major), $12.3 \mathrm{~min}$ (minor). ${ }^{1} \mathrm{H}$ NMR (600 MHz, $\left.\mathrm{CDCl}_{3}\right) \delta 7.35(\mathrm{~d}, J=1.7 \mathrm{~Hz}, 1 \mathrm{H}), 7.34-7.28(\mathrm{~m}, 2 \mathrm{H})$, $7.24-7.20(\mathrm{~m}, 1 \mathrm{H}), 7.20-7.13(\mathrm{~m}, 4 \mathrm{H}), 7.10(\mathrm{~d}, J=7.5 \mathrm{~Hz}, 1 \mathrm{H}), 7.00-6.92(\mathrm{~m}$, $1 \mathrm{H}), 6.89(\mathrm{~s}, 1 \mathrm{H}), 6.31-6.25(\mathrm{~m}, 1 \mathrm{H}), 6.12(\mathrm{~d}, J=3.4 \mathrm{~Hz}, 1 \mathrm{H}), 5.75(\mathrm{~d}, J=5.2 \mathrm{~Hz}$, $1 \mathrm{H}), 3.77(\mathrm{dd}, J=11.8,4.2 \mathrm{~Hz}, 6 \mathrm{H}) \mathrm{ppm} .{ }^{13} \mathrm{C} \mathrm{NMR}\left(151 \mathrm{MHz}, \mathrm{CDCl}_{3}\right) \delta 150.9,147.0$, 142.2, 132.5, 131.5, 128.9, 128.5, 127.4, 127.0 (d, $J=6.3 \mathrm{~Hz}), 125.8,125.0,123.3$, 122.4, 111.5, 111.1, 109.8, 62.3 (d, $J=10.4 \mathrm{~Hz}), 52.9$ (d, $J=5.0 \mathrm{~Hz}), 52.9$ (d, $J=5.2$ $\mathrm{Hz}$ ), 45.7 (d, $J=223.8 \mathrm{~Hz}$ ) ppm. HRMS (ESI) m/z: $[\mathrm{M}+\mathrm{H}]^{+}$Calcd. for $\mathrm{C}_{22} \mathrm{H}_{21} \mathrm{~N}_{3} \mathrm{O}_{4} \mathrm{P}$ 422.1270 found 422.1270 .

Dimethyl $\quad(R)-($ diazo(2-phenyl-3-(thiophen-2-yl)-1,2-dihydroisoquinolin-1-yl) methyl)phosphonate (4yaa)<smiles>COC(=O)C1c2ccccc2C=C(c2cccs2)N1c1ccccc1</smiles>

Following the general procedure, reaction were performed with $o$-alkynylbenzaldehyde 1ay $(0.24 \mathrm{mmol}), \mathbf{2 a}(0.22 \mathrm{mmol})$, 3a (0.20 mmol), $5 \AA \mathrm{MS}$ (200 mg), IIh (10 mol \%), and $\mathrm{Ag}_{2} \mathrm{CO}_{3}(5 \mathrm{~mol} \%)$ in the $1.0 \mathrm{~mL}$ of THF/DME (1:1) at $-20{ }^{\circ} \mathrm{C}$ for 3 days under argon, followed by column chromatography $(\mathrm{PE} / \mathrm{EA}=10: 1 \sim 1: 1)$ to afford 4yaa as yellow sticky oil, 59.4 
mg (68\% yield, $60 \% e e) ;[\alpha]_{\mathrm{D}}^{25}=+277.4^{\circ}\left(c=1.19,{ }^{i} \mathrm{PrOH}\right)$; HPLC (IC, n-hexane/ ${ }^{i} \mathrm{PrOH}=70 / 30$, flow rate $=0.5 \mathrm{~mL} / \mathrm{min}, \mathrm{I}=238 \mathrm{~nm}$ ): $t_{\mathrm{R}}=14.1 \mathrm{~min}$ (major), $16.3 \mathrm{~min}$ (minor). ${ }^{1} \mathrm{H}$ NMR (600 MHz, $\left.\mathrm{CDCl}_{3}\right) \delta 7.34-7.28(\mathrm{~m}, 2 \mathrm{H}), 7.25-7.20(\mathrm{~m}, 1 \mathrm{H}), 7.20$ $-7.09(\mathrm{~m}, 7 \mathrm{H}), 6.97-6.91(\mathrm{~m}, 1 \mathrm{H}), 6.91-6.86(\mathrm{~m}, 1 \mathrm{H}), 6.75(\mathrm{~s}, 1 \mathrm{H}), 5.80(\mathrm{~d}, J=$ $5.5 \mathrm{~Hz}, 1 \mathrm{H}), 3.80(\mathrm{dd}, J=11.8,7.9 \mathrm{~Hz}, 6 \mathrm{H}) \mathrm{ppm} .{ }^{13} \mathrm{C} \mathrm{NMR}\left(151 \mathrm{MHz}, \mathrm{CDCl}_{3}\right) \delta$ 146.7, 141.4, 136.0, 131.7, 128.8, 128.4, 127.3, 127.0 (d, $J=6.2 \mathrm{~Hz}), 126.3,125.7$, 125.6, 124.6, 123.2, 122.8, 111.9, 62.5 (d, $J=10.8 \mathrm{~Hz}), 53.0$ (d, $J=5.5 \mathrm{~Hz}), 52.9$ (d, $J=5.0 \mathrm{~Hz}), 45.8(\mathrm{~d}, J=224.9 \mathrm{~Hz}) \mathrm{ppm}$. HRMS (ESI) m/z: $[\mathrm{M}+\mathrm{H}]^{+}$Calcd. for $\mathrm{C}_{22} \mathrm{H}_{21} \mathrm{~N}_{3} \mathrm{O}_{3} \mathrm{PS}$ 438.1036, found 438.1038.

Dimethyl $\quad(R, E)$-(diazo(2-phenyl-3-styryl-1,2-dihydroisoquinolin-1-yl)methyl) phosphonate (4zaa)

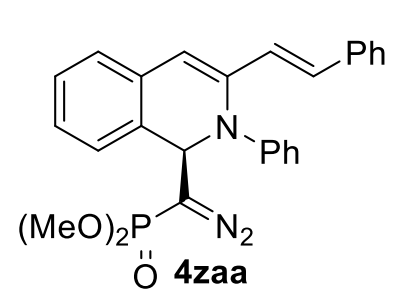

Following the general procedure, reaction were performed with $o$-alkynylbenzaldehyde $\mathbf{1 a z}(0.24 \mathrm{mmol}), \mathbf{2 a}(0.22 \mathrm{mmol}), \mathbf{3 a}$ (0.20 mmol), $5 \AA$ MS (200 mg), IIh (10 mol \%), and $\mathrm{Ag}_{2} \mathrm{CO}_{3}(5$ $\mathrm{mol} \%)$ in the $1.0 \mathrm{~mL}$ of THF/DME (1:1) at $-20{ }^{\circ} \mathrm{C}$ for 3 days under argon, followed by column chromatography $(\mathrm{PE} / \mathrm{EA}=$ $10: 1 \sim 1: 1)$ to afford 4zaa as yellow sticky oil, $74.0 \mathrm{mg}(81 \%$ yield, $48 \% e e) ;[\alpha]_{\mathrm{D}}^{25}=$ $+103.4^{\circ}\left(c=1.48,{ }^{i} \mathrm{PrOH}\right) ; \mathrm{HPLC}\left(\mathrm{ADH}, \mathrm{n}\right.$-hexane $/{ }^{i} \mathrm{PrOH}=50 / 50$, flow rate $=0.5$ $\mathrm{mL} / \mathrm{min}, \mathrm{I}=238 \mathrm{~nm}$ ): $t_{\mathrm{R}}=10.6 \min$ (major), $17.5 \mathrm{~min}$ (minor). ${ }^{1} \mathrm{H} \mathrm{NMR}(600 \mathrm{MHz}$, $\left.\mathrm{CDCl}_{3}\right) \delta 7.28(\mathrm{~d}, J=7.7 \mathrm{~Hz}, 6 \mathrm{H}), 7.25-7.18(\mathrm{~m}, 6 \mathrm{H}), 7.11(\mathrm{~d}, J=7.5 \mathrm{~Hz}, 1 \mathrm{H}), 6.99$ $-6.93(\mathrm{~m}, 1 \mathrm{H}), 6.70(\mathrm{~s}, 2 \mathrm{H}), 6.64(\mathrm{~s}, 1 \mathrm{H}), 5.77(\mathrm{~d}, J=5.1 \mathrm{~Hz}, 1 \mathrm{H}), 3.78(\mathrm{t}, J=12.0$ $\mathrm{Hz}, 6 \mathrm{H}) \mathrm{ppm} .{ }^{13} \mathrm{C} \mathrm{NMR}\left(151 \mathrm{MHz}, \mathrm{CDCl}_{3}\right) \delta 146.8,140.0,136.8,132.4,131.9,129.0$, $128.6,128.5,127.8,127.5,127.4,126.5,125.8,125.3,124.8,123.0,122.4,115.8$, $62.3(\mathrm{~d}, J=10.4 \mathrm{~Hz}), 52.9(\mathrm{~d}, J=5.3 \mathrm{~Hz}), 45.7(\mathrm{~d}, J=224.1 \mathrm{~Hz}) \mathrm{ppm}$. HRMS (ESI) $\mathrm{m} / \mathrm{z}:[\mathrm{M}+\mathrm{H}]^{+}$Calcd. for $\mathrm{C}_{26} \mathrm{H}_{25} \mathrm{~N}_{3} \mathrm{O}_{3} \mathrm{P} 458.1634$ found 458.1619 .

\section{Dimethyl $(\boldsymbol{R})$-(diazo(6-fluoro-2,3-diphenyl-1,2-dihydroisoquinolin-1-yl)methyl) phosphonate (4aea)}

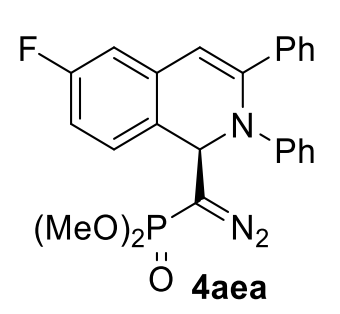

Following the general procedure, reaction were performed with $o$-alkynylbenzaldehyde $\mathbf{1 e a}(0.24 \mathrm{mmol}), \mathbf{2 a}(0.22 \mathrm{mmol})$, 3a (0.20 mmol), $5 \AA \mathrm{MS}$ (200 mg), IIh (10 mol \%), and $\mathrm{Ag}_{2} \mathrm{CO}_{3}(5 \mathrm{~mol} \%)$ in the $1.0 \mathrm{~mL}$ of THF/DME (1:1) at $-20{ }^{\circ} \mathrm{C}$ for 3 days under argon, followed by column chromatography $(\mathrm{PE} / \mathrm{EA}=10: 1 \sim 1: 1)$ to afford 4aea as yellow sticky oil, $86.2 \mathrm{mg}$ (96\% yield, 94\% $e e) ;[\alpha]_{\mathrm{D}}^{25}=+327.1^{\circ}\left(c=1.72,{ }^{i} \mathrm{PrOH}\right)$; HPLC (IC, n-hexane $/{ }^{i} \mathrm{PrOH}=70 / 30$, flow rate 
$=0.5 \mathrm{~mL} / \mathrm{min}, \mathrm{I}=238 \mathrm{~nm}$ ): $t_{\mathrm{R}}=12.1 \mathrm{~min}$ (major), $13.0 \mathrm{~min}$ (minor). ${ }^{1} \mathrm{H} \mathrm{NMR}(600$ $\left.\mathrm{MHz}, \mathrm{CDCl}_{3}\right) \delta 7.63-7.53(\mathrm{~m}, 2 \mathrm{H}), 7.26-7.19(\mathrm{~m}, 3 \mathrm{H}), 7.10(\mathrm{~d}, J=6.5 \mathrm{~Hz}, 5 \mathrm{H})$, $7.03-6.99(\mathrm{~m}, 1 \mathrm{H}), 6.93-6.85(\mathrm{~m}, 2 \mathrm{H}), 6.60(\mathrm{~s}, 1 \mathrm{H}), 5.87(\mathrm{~d}, J=5.2 \mathrm{~Hz}, 1 \mathrm{H}), 3.78$ (dd, $J=11.8,3.3 \mathrm{~Hz}, 6 \mathrm{H}) \mathrm{ppm} .{ }^{13} \mathrm{C} \mathrm{NMR}\left(151 \mathrm{MHz}, \mathrm{CDCl}_{3}\right) . \delta 162.6(\mathrm{~d}, J=245.6$ $\mathrm{Hz}), 146.2,143.0,136.5,134.1(\mathrm{~d}, J=8.8 \mathrm{~Hz}), 128.8,128.5,128.4,127.7,127.2(\mathrm{~d}, J$ $=8.8 \mathrm{~Hz}), 123.1,122.9,122.9(\mathrm{~d}, J=2.8 \mathrm{~Hz}), 122.8(\mathrm{~d}, J=2.7 \mathrm{~Hz}), 113.9(\mathrm{~d}, J=22.5$ $\mathrm{Hz}), 111.5(\mathrm{~d}, J=2.6 \mathrm{~Hz}), 110.8(\mathrm{~d}, J=22.3 \mathrm{~Hz}), 61.8$ (d, $J=11.0 \mathrm{~Hz}), 53.1$ (d, $J=$ $6.2 \mathrm{~Hz}), 46.2(\mathrm{~d}, J=225.3 \mathrm{~Hz}) \mathrm{ppm}$. HRMS (ESI) m/z: $[\mathrm{M}+\mathrm{H}]^{+}$Calcd. for $\mathrm{C}_{24} \mathrm{H}_{22} \mathrm{FN}_{3} \mathrm{O}_{3} \mathrm{P} 450.1377$, found 450.1375 .

Dimethyl (R)-((6-chloro-2,3-diphenyl-1,2-dihydroisoquinolin-1-yl)(diazo)methyl) phosphonate (4afa)<smiles>COP(=O)(OC)C(=N)C1c2ccc(Cl)cc2C=C(c2ccccc2)N1c1ccccc1</smiles>

Following the general procedure, reaction were performed with $o$-alkynylbenzaldehyde $\mathbf{1 f a}(0.24 \mathrm{mmol}), \mathbf{2 a}(0.22 \mathrm{mmol}), 3 \mathbf{3 a}$ (0.20 mmol), $5 \AA$ MS (200 mg), IIh (10 mol \%), and $\mathrm{Ag}_{2} \mathrm{CO}_{3}(5$ $\mathrm{mol} \%)$ in the $1.0 \mathrm{~mL}$ of THF/DME (1:1) at $-20{ }^{\circ} \mathrm{C}$ for 3 days under argon, followed by column chromatography $(\mathrm{PE} / \mathrm{EA}=$ 10:1 1:1) to afford 4afa as yellow sticky oil, $86.5 \mathrm{mg}(93 \%$ yield, $91 \% e e) ;[\alpha]_{\mathrm{D}}^{25}=$ $+316.5^{\circ}\left(c=0.72,{ }^{i} \mathrm{PrOH}\right)$; HPLC $\left(\mathrm{IC}, \mathrm{n}\right.$-hexane $/{ }^{i} \mathrm{PrOH}=70 / 30$, flow rate $=0.5$ $\mathrm{mL} / \mathrm{min}, \mathrm{I}=238 \mathrm{~nm}$ ): $t_{\mathrm{R}}=12.2 \mathrm{~min}$ (major), $13.4 \mathrm{~min}$ (minor). ${ }^{1} \mathrm{H} \mathrm{NMR}(600 \mathrm{MHz}$, $\left.\mathrm{CDCl}_{3}\right) \delta 7.62-7.53(\mathrm{~m}, 2 \mathrm{H}), 7.31(\mathrm{~d}, J=2.1 \mathrm{~Hz}, 1 \mathrm{H}), 7.26-7.20(\mathrm{~m}, 3 \mathrm{H}), 7.19-$ $7.16(\mathrm{~m}, 1 \mathrm{H}), 7.13-7.04(\mathrm{~m}, 5 \mathrm{H}), 6.91-6.83(\mathrm{~m}, 1 \mathrm{H}), 6.57(\mathrm{~s}, 1 \mathrm{H}), 5.84(\mathrm{~d}, J=5.4$ $\mathrm{Hz}, 1 \mathrm{H}), 3.78(\mathrm{dd}, J=11.7,4.1 \mathrm{~Hz}, 6 \mathrm{H}) \mathrm{ppm} .{ }^{13} \mathrm{C} \mathrm{NMR}\left(151 \mathrm{MHz}, \mathrm{CDCl}_{3}\right) \delta 146.1$, $143.1,136.4,134.0,133.7,128.9,128.6,128.4,127.7,127.0,126.9,125.3$ (d, $J=6.2$ $\mathrm{Hz}), 124.3,123.1,122.9,111.1,61.7(\mathrm{~d}, J=11.2 \mathrm{~Hz}), 53.1(\mathrm{~d}, J=6.1 \mathrm{~Hz}), 53.1(\mathrm{~d}, J$ $=5.5 \mathrm{~Hz}), 46.0(\mathrm{~d}, J=225.0 \mathrm{~Hz}) \mathrm{ppm}$. HRMS (ESI) m/z: $[\mathrm{M}+\mathrm{H}]^{+}$Calcd. for $\mathrm{C}_{24} \mathrm{H}_{22} \mathrm{ClN}_{3} \mathrm{O}_{3} \mathrm{P} 466.1087$ found 466.1082 .

Dimethyl $(R)$-(diazo(6-methyl-2,3-diphenyl-1,2-dihydroisoquinolin-1-yl)methyl) phosphonate (4aga)<smiles>COP(=O)(OC)C(=N)C1c2ccc(C)cc2C=C(c2ccccc2)N1c1ccccc1</smiles>

Following the general procedure, reaction were performed with $o$-alkynylbenzaldehyde 1 ga $(0.24 \mathrm{mmol}), 2 \mathbf{2 a}(0.22 \mathrm{mmol}), 3 \mathbf{a}$ (0.20 mmol), $5 \AA$ MS (200 mg), IIh (10 mol \%), and $\mathrm{Ag}_{2} \mathrm{CO}_{3}(5$ $\mathrm{mol} \%)$ in the $1.0 \mathrm{~mL}$ of THF/DME/DMF $(5: 4: 1)$ at $-20{ }^{\circ} \mathrm{C}$ for 3 days under argon, followed by column chromatography $(\mathrm{PE} / \mathrm{EA}=$ $10: 1 \sim 1: 1)$ to afford 4aga as yellow sticky oil, $70.3 \mathrm{mg}(79 \%$ yield, 92\% ee $) ;[\alpha]_{\mathrm{D}}^{25}=$ 
$+81.6^{\circ}\left(c=1.40,{ }^{i} \mathrm{PrOH}\right) ; \mathrm{HPLC}\left(\mathrm{IC}, \mathrm{n}\right.$-hexane $/{ }^{i} \mathrm{PrOH}=70 / 30$, flow rate $=0.5$ $\mathrm{mL} / \mathrm{min}, \mathrm{I}=238 \mathrm{~nm}$ ): $t_{\mathrm{R}}=14.6 \mathrm{~min}$ (major), $16.9 \mathrm{~min}$ (minor). ${ }^{1} \mathrm{H}$ NMR $(600 \mathrm{MHz}$, $\left.\mathrm{CDCl}_{3}\right) \delta 7.66-7.48(\mathrm{~m}, 2 \mathrm{H}), 7.24(\mathrm{~d}, J=7.5 \mathrm{~Hz}, 2 \mathrm{H}), 7.23-7.18(\mathrm{~m}, 1 \mathrm{H}), 7.14(\mathrm{~s}$, 1H), $7.12-7.06(\mathrm{~m}, 4 \mathrm{H}), 7.06-7.00(\mathrm{~m}, 2 \mathrm{H}), 6.89-6.80(\mathrm{~m}, 1 \mathrm{H}), 6.65(\mathrm{~s}, 1 \mathrm{H}), 5.85$ $(\mathrm{d}, J=5.4 \mathrm{~Hz}, 1 \mathrm{H}), 3.79(\mathrm{dd}, J=11.7,2.2 \mathrm{~Hz}, 6 \mathrm{H}), 2.36(\mathrm{~s}, 3 \mathrm{H}) \mathrm{ppm} .{ }^{13} \mathrm{C}$ NMR $(151$ $\left.\mathrm{MHz}, \mathrm{CDCl}_{3}\right) \delta 146.5,141.6,138.0,136.9,132.0,128.7,128.3,128.1,128.1,127.5$, 125.4, 125.2, 124.6 (d, $J=6.2 \mathrm{~Hz}), 122.7,122.6,112.8,62.1(\mathrm{~d}, J=10.7 \mathrm{~Hz}), 53.0$ (d, $J=5.7 \mathrm{~Hz}), 53.0(\mathrm{~d}, J=5.7 \mathrm{~Hz}), 46.1(\mathrm{~d}, J=225.2 \mathrm{~Hz}) \mathrm{ppm}$. HRMS (ESI) m/z: $[\mathrm{M}+\mathrm{H}]^{+}$Calcd. for $\mathrm{C}_{25} \mathrm{H}_{25} \mathrm{~N}_{3} \mathrm{O}_{3} \mathrm{P} 446.1628$, found 446.1630.

Dimethyl (R)-(diazo(6-methoxy-2,3-diphenyl-1,2-dihydroisoquinolin-1-yl)methyl) phosphonate (4aha)<smiles>COCCCCCCCC(=N)[C@@H]1c2ccc(OC)cc2C=C(c2ccccc2)N1c1ccccc1</smiles>

Following the general procedure, reaction were performed with $o$-alkynylbenzaldehyde $\mathbf{1 h a}(0.24 \mathrm{mmol}), \mathbf{2 a}(0.22 \mathrm{mmol})$, 3a (0.20 mmol), $5 \AA \mathrm{MS}(200 \mathrm{mg})$, IIh (10 $\mathrm{mol} \%)$, and $\mathrm{Ag}_{2} \mathrm{CO}_{3}(5 \mathrm{~mol} \%)$ in the $1.0 \mathrm{~mL}$ of THF/DME/DMF (5:4:1) at $-20{ }^{\circ} \mathrm{C}$ for 3 days under argon, followed by column chromatography $(\mathrm{PE} / \mathrm{EA}=10: 1 \sim 1: 1)$ to afford 4aha as yellow sticky oil, $38.7 \mathrm{mg}$ ( $42 \%$ yield, $74 \%$ ee $)$; $[\alpha]_{\mathrm{D}}^{25}=+199.0^{\circ}\left(c=0.77,{ }^{i} \mathrm{PrOH}\right)$; HPLC (ADH, n-hexane/ ${ }^{i} \mathrm{PrOH}=50 / 50$, flow rate $=0.5 \mathrm{~mL} / \mathrm{min}, \mathrm{I}=238 \mathrm{~nm}$ ): $t_{\mathrm{R}}=15.1 \mathrm{~min}$ (major), $20.3 \mathrm{~min}$ (minor). ${ }^{1} \mathrm{H}$ NMR (600 MHz, $\left.\mathrm{CDCl}_{3}\right) \delta 7.60(\mathrm{dt}, J=6.4,1.4 \mathrm{~Hz}, 2 \mathrm{H}), 7.26-7.23(\mathrm{~m}$, $2 \mathrm{H}), 7.23-7.19(\mathrm{~m}, 1 \mathrm{H}), 7.12-7.07(\mathrm{~m}, 4 \mathrm{H}), 7.05(\mathrm{~d}, J=8.3 \mathrm{~Hz}, 1 \mathrm{H}), 6.88-6.82$ $(\mathrm{m}, 2 \mathrm{H}), 6.81-6.73(\mathrm{~m}, 1 \mathrm{H}), 6.64(\mathrm{~s}, 1 \mathrm{H}), 5.84(\mathrm{~d}, J=5.1 \mathrm{~Hz}, 1 \mathrm{H}), 3.83(\mathrm{~s}, 3 \mathrm{H}), 3.78$ $(\mathrm{d}, J=11.7 \mathrm{~Hz}, 6 \mathrm{H}) \mathrm{ppm} .{ }^{13} \mathrm{C} \mathrm{NMR}\left(151 \mathrm{MHz}, \mathrm{CDCl}_{3}\right) \delta 159.6,146.5,142.1,136.8$, 133.4, 128.8, 128.3, 128.3, 127.6, 126.7, 122.8, 122.7, 120.0 (d, $J=6.5 \mathrm{~Hz}), 113.4$, 112.6, 109.1, $62.0(\mathrm{~d}, J=10.8 \mathrm{~Hz}), 55.3,53.0(\mathrm{~d}, J=5.7 \mathrm{~Hz}), 53.0(\mathrm{~d}, J=5.5 \mathrm{~Hz})$, $46.3(\mathrm{~d}, J=224.7 \mathrm{~Hz})$ ppm. HRMS (ESI) $\mathrm{m} / \mathrm{z}$ : $[\mathrm{M}+\mathrm{H}]^{+}$Calcd. for $\mathrm{C}_{25} \mathrm{H}_{25} \mathrm{~N}_{3} \mathrm{O}_{4} \mathrm{P}$ 462.1583 found 462.1584 .

Dimethyl (R)-((7-chloro-2,3-diphenyl-1,2-dihydroisoquinolin-1-yl)(diazo)methyl) phosphonate (4aia)<smiles>COP(=O)(OC)C(=N)C1c2cc(Cl)ccc2C=C(c2ccccc2)N1c1ccccc1</smiles>

Following the general procedure, reaction were performed with $o$-alkynylbenzaldehyde 1ia $(0.24 \mathrm{mmol}), \mathbf{2 a}(0.22 \mathrm{mmol}), 3 \mathbf{a}$ (0.20 mmol), $5 \AA$ MS (200 mg), IIh (10 mol \%), and $\mathrm{Ag}_{2} \mathrm{CO}_{3}(5$ $\mathrm{mol} \%)$ in the $1.0 \mathrm{~mL}$ of THF/DME (1:1) at $-20{ }^{\circ} \mathrm{C}$ for 3 days under argon, followed by column chromatography $(\mathrm{PE} / \mathrm{EA}=$ 
$10: 1 \sim 1: 1)$ to afford 4aia as yellow sticky oil, $75.3 \mathrm{mg}(81 \%$ yield, $35 \% e e) ;[\alpha]_{\mathrm{D}}^{25}=$ $+201.5^{\circ}\left(c=0.54{ }^{i} \mathrm{PrOH}\right)$; HPLC (IC, n-hexane/ ${ }^{i} \mathrm{PrOH}=90 / 10$, flow rate $=0.5$ $\mathrm{mL} / \mathrm{min}, \mathrm{I}=238 \mathrm{~nm}$ ): $t_{\mathrm{R}}=23.7 \mathrm{~min}$ (major), $22.0 \mathrm{~min}$ (minor). ${ }^{1} \mathrm{H}$ NMR $(600 \mathrm{MHz}$, $\left.\mathrm{CDCl}_{3}\right) \delta 7.71-7.58(\mathrm{~m}, 2 \mathrm{H}), 7.33-7.29(\mathrm{~m}, 4 \mathrm{H}), 7.28(\mathrm{~d}, J=7.0 \mathrm{~Hz}, 1 \mathrm{H}), 7.19$ (s, $1 \mathrm{H}), 7.18-7.11(\mathrm{~m}, 4 \mathrm{H}), 6.97-6.89(\mathrm{~m}, 1 \mathrm{H}), 6.70(\mathrm{~s}, 1 \mathrm{H}), 5.88(\mathrm{~d}, J=5.5 \mathrm{~Hz}, 1 \mathrm{H})$, $3.86(\mathrm{dd}, J=11.8,10.0 \mathrm{~Hz}, 6 \mathrm{H}) \mathrm{ppm} .{ }^{13} \mathrm{C} \mathrm{NMR}\left(151 \mathrm{MHz}, \mathrm{CDCl}_{3}\right) . \delta 146.1,142.2$, 136.5, 132.3, 130.6, 128.9, 128.5, 128.4, 128.4, 128.4, 127.5, 125.8, 125.6, 123.1, 122.8, 111.5, $61.8(\mathrm{~d}, J=11.1 \mathrm{~Hz}), 53.1(\mathrm{~d}, J=5.7 \mathrm{~Hz}), 46.0(\mathrm{~d}, J=226.1 \mathrm{~Hz}) \mathrm{ppm}$. HRMS (ESI) m/z: [M+H] $]^{+}$Calcd. for $\mathrm{C}_{24} \mathrm{H}_{22} \mathrm{ClN}_{3} \mathrm{O}_{3} \mathrm{P} 466.1087$ found 466.1078 .

Dimethyl $(R)$-(diazo(7-methyl-2,3-diphenyl-1,2-dihydroisoquinolin-1-yl)methyl) phosphonate (4aja)<smiles>COP(=O)(OC)C(=N)C1c2cc(C)ccc2C=C(c2ccccc2)N1c1ccccc1</smiles>

Following the general procedure, reaction were performed with $o$-alkynylbenzaldehyde $\mathbf{1 j a}(0.24 \mathrm{mmol}), \mathbf{2 a}(0.22 \mathrm{mmol}), \mathbf{3 a}$ (0.20 mmol), $5 \AA$ MS (200 mg), IIh (10 mol \%), and $\mathrm{Ag}_{2} \mathrm{CO}_{3}(5$ $\mathrm{mol} \%)$ in the $1.0 \mathrm{~mL}$ of THF/DME/DMF $(5: 4: 1)$ at $-20^{\circ} \mathrm{C}$ for 3 days under argon, followed by column chromatography (PE/EA $=10: 1 \sim 1: 1)$ to afford 4aja as yellow sticky oil, $81.0 \mathrm{mg}(91 \%$ yield, $49 \% e e) ;[\alpha]_{\mathrm{D}}^{25}$ $=+271.0^{\circ}\left(c=0.52,{ }^{i} \mathrm{PrOH}\right) ; \mathrm{HPLC}\left(\mathrm{ADH}, \mathrm{n}\right.$-hexane $/{ }^{i} \mathrm{PrOH}=50 / 50$, flow rate $=0.5$ $\mathrm{mL} / \mathrm{min}, \mathrm{I}=238 \mathrm{~nm}$ ): $t_{\mathrm{R}}=10.4 \mathrm{~min}$ (major), $40.4 \mathrm{~min}$ (minor). ${ }^{1} \mathrm{H} \mathrm{NMR}(600 \mathrm{MHz}$, $\left.\mathrm{CDCl}_{3}\right) \delta 7.65-7.59(\mathrm{~m}, 2 \mathrm{H}), 7.29-7.23(\mathrm{~m}, 3 \mathrm{H}), 7.23-7.19(\mathrm{~m}, 1 \mathrm{H}), 7.15-7.06$ $(\mathrm{m}, 5 \mathrm{H}), 6.96(\mathrm{~s}, 1 \mathrm{H}), 6.89-6.83(\mathrm{~m}, 1 \mathrm{H}), 6.70(\mathrm{~s}, 1 \mathrm{H}), 5.84(\mathrm{~d}, J=5.5 \mathrm{~Hz}, 1 \mathrm{H}), 3.81$ $(\mathrm{dd}, J=11.7,2.0 \mathrm{~Hz}, 6 \mathrm{H}), 2.34(\mathrm{~s}, 3 \mathrm{H}) \mathrm{ppm} .{ }^{13} \mathrm{C} \mathrm{NMR}\left(151 \mathrm{MHz}, \mathrm{CDCl}_{3}\right) \delta 146.6$, $140.8,137.3,136.9,129.5,129.2,128.8,128.3,128.0,127.4,127.4,126.1,124.6$, 122.6, 122.5, 112.9, $62.3(\mathrm{~d}, J=10.6 \mathrm{~Hz}), 53.0(\mathrm{~d}, J=5.9 \mathrm{~Hz}), 53.0(\mathrm{~d}, J=5.6 \mathrm{~Hz})$, $46.0\left(\mathrm{~d}, J=225.3 \mathrm{~Hz}\right.$ ), $21.3 \mathrm{ppm}$. HRMS (ESI) m/z: $[\mathrm{M}+\mathrm{H}]^{+}$Calcd. for $\mathrm{C}_{25} \mathrm{H}_{25} \mathrm{~N}_{3} \mathrm{O}_{3} \mathrm{P}$ 446.1628 , found 446.1631 .

Dimethyl (R)-(diazo(7-methoxy-2,3-diphenyl-1,2-dihydroisoquinolin-1-yl)methyl) phosphonate (4aka)<smiles>COC(=O)C1c2cc(OC)ccc2C=C(c2ccccc2)N1c1ccccc1</smiles>

Following the general procedure, reaction were performed with $o$-alkynylbenzaldehyde $1 \mathbf{k a}(0.24 \mathrm{mmol}), \mathbf{2 a}(0.22 \mathrm{mmol})$, 3a (0.20 mmol), $5 \AA \mathrm{MS}$ (200 mg), IIh (10 mol \%), and $\mathrm{Ag}_{2} \mathrm{CO}_{3}(5 \mathrm{~mol} \%)$ in the $1.0 \mathrm{~mL}$ of THF/DME $(1: 1)$ at $-20^{\circ} \mathrm{C}$ for 3 days under argon, followed by column chromatography $(\mathrm{PE} / \mathrm{EA}=10: 1 \sim 1: 1)$ to afford 4aka as yellow sticky oil, $69.1 \mathrm{mg}$ (75\% yield, $47 \%$ 
$e e) ;[\alpha]_{\mathrm{D}}^{25}=+126.4^{\circ}\left(c=1.38,{ }^{i} \mathrm{PrOH}\right) ; \mathrm{HPLC}\left(\mathrm{IC}, \mathrm{n}-\right.$ hexane $/{ }^{i} \mathrm{PrOH}=70 / 30$, flow rate $=0.5 \mathrm{~mL} / \mathrm{min}, \mathrm{I}=238 \mathrm{~nm}$ ): $t_{\mathrm{R}}=17.7 \mathrm{~min}$ (major), $13.2 \mathrm{~min}$ (minor). ${ }^{1} \mathrm{H} \mathrm{NMR}(600$ $\left.\mathrm{MHz}, \mathrm{CDCl}_{3}\right) \delta 7.63-7.58(\mathrm{~m}, 2 \mathrm{H}), 7.29-7.23(\mathrm{~m}, 3 \mathrm{H}), 7.22-7.18(\mathrm{~m}, 1 \mathrm{H}), 7.11(\mathrm{~d}$, $J=6.5 \mathrm{~Hz}, 4 \mathrm{H}), 6.92-6.83(\mathrm{~m}, 2 \mathrm{H}), 6.72-6.65(\mathrm{~m}, 2 \mathrm{H}), 5.84(\mathrm{~d}, J=5.6 \mathrm{~Hz}, 1 \mathrm{H})$, $3.81(\mathrm{dd}, J=11.7,3.8 \mathrm{~Hz}, 6 \mathrm{H}), 3.79(\mathrm{~s}, 3 \mathrm{H}) \mathrm{ppm} .{ }^{13} \mathrm{C} \mathrm{NMR}\left(151 \mathrm{MHz}, \mathrm{CDCl}_{3}\right) \delta$ 159.3, 146.6, 139.5, 136.9, 128.8 (d, $J=6.5 \mathrm{~Hz}), 128.8,128.3,127.9,127.2$, 126.1, $125.3,122.5,122.4,114.6,112.7,110.6,62.3$ (d, $J=10.9 \mathrm{~Hz}), 55.4,53.1$ (d, $J=6.3$ $\mathrm{Hz}), 53.0(\mathrm{~d}, J=6.6 \mathrm{~Hz}), 45.8(\mathrm{~d}, J=225.4 \mathrm{~Hz}) \mathrm{ppm}$. HRMS (ESI) m/z: $[\mathrm{M}+\mathrm{H}]^{+}$ Calcd. for $\mathrm{C}_{25} \mathrm{H}_{25} \mathrm{~N}_{3} \mathrm{O}_{4} \mathrm{P} 462.1583$ found 462.1573 .

Dimethyl (R)-(diazo(3-phenyl-2-(m-tolyl)-1,2-dihydroisoquinolin-1-yl)methyl) phosphonate (4aab)<smiles>COP(=O)(OC)OC(=N)C1c2ccccc2C=C(c2ccccc2)N1c1cccc(C)c1</smiles>

Following the general procedure, reaction were performed with $o$-alkynylbenzaldehyde $\mathbf{1 a}(0.24 \mathrm{mmol}), \mathbf{2 b}(0.22$ mmol), 3a (0.20 mmol), $5 \AA$ MS (200 mg), IIh (10 mol \%), and $\mathrm{Ag}_{2} \mathrm{CO}_{3}(5 \mathrm{~mol} \%)$ in the $1.0 \mathrm{~mL}$ of THF/DME (1:1) at $-20{ }^{\circ} \mathrm{C}$ for 3 days under argon, followed by column chromatography $(\mathrm{PE} / \mathrm{EA}=10: 1 \sim 1: 1)$ to afford 4aab as yellow sticky oil, $83.6 \mathrm{mg}$ $(94 \%$ yield, $93 \% e e) ;[\alpha]_{\mathrm{D}}^{25}=+420.7^{\circ}\left(c=0.84,{ }^{i} \mathrm{PrOH}\right)$; HPLC (IC, n-hexane $/{ }^{i} \mathrm{PrOH}$ $=70 / 30$, flow rate $=0.5 \mathrm{~mL} / \mathrm{min}, \mathrm{I}=238 \mathrm{~nm}$ ): $t_{\mathrm{R}}=12.2 \mathrm{~min}$ (major), $14.9 \mathrm{~min}$ (minor). ${ }^{1} \mathrm{H}$ NMR (600 MHz, $\left.\mathrm{CDCl}_{3}\right) \delta 7.62-7.57$ (m, 2H), $7.34-7.27$ (m, 2H), 7.27 $-7.23(\mathrm{~m}, 2 \mathrm{H}), 7.23-7.18(\mathrm{~m}, 2 \mathrm{H}), 7.13(\mathrm{~d}, J=7.5 \mathrm{~Hz}, 1 \mathrm{H}), 7.00-6.93(\mathrm{~m}, 2 \mathrm{H})$, $6.87-6.82(\mathrm{~m}, 1 \mathrm{H}), 6.70-6.63(\mathrm{~m}, 2 \mathrm{H}), 5.86(\mathrm{~d}, J=5.4 \mathrm{~Hz}, 1 \mathrm{H}), 3.78(\mathrm{dd}, J=11.7$, $3.0 \mathrm{~Hz}, 6 \mathrm{H}), 2.17$ (s, 3H) ppm. ${ }^{13} \mathrm{C} \mathrm{NMR}\left(151 \mathrm{MHz}, \mathrm{CDCl}_{3}\right) \delta 146.4,141.8,138.5$, 137.0, 132.1, 128.5, 128.3, 128.3, 128.1, 127.5, 127.2 (d, $J=6.1 \mathrm{~Hz}), 127.1,125.6$, 124.6, 123.6, 123.4, 120.0, 112.4, 62.1 (d, $J=10.7 \mathrm{~Hz}), 53.0$ (d, $J=5.3 \mathrm{~Hz}), 53.0$ (d, $J=5.1 \mathrm{~Hz}), 46.1(\mathrm{~d}, J=226.0 \mathrm{~Hz}), 21.4 \mathrm{ppm}$. HRMS (ESI) m/z: $[\mathrm{M}+\mathrm{H}]^{+}$Calcd. for $\mathrm{C}_{25} \mathrm{H}_{25} \mathrm{~N}_{3} \mathrm{O}_{3} \mathrm{P} 446.1628$, found 446.1623 .

Dimethyl (R)-(diazo(2-(3-methoxyphenyl)-3-phenyl-1,2-dihydroisoquinolin-1-yl) methyl)phosphonate (4aac)<smiles>COc1cccc(N2C(c3ccccc3)=Cc3ccccc3C2C(=N)P(=O)(O)OC(C)(C)C)c1</smiles>

Following the general procedure, reaction were performed with $o$-alkynylbenzaldehyde 1 a $(0.24 \mathrm{mmol}), 2 \mathrm{c}(0.22$ mmol), 3a (0.20 mmol), $5 \AA$ MS (200 mg), IIh (10 mol \%), and $\mathrm{Ag}_{2} \mathrm{CO}_{3}(5 \mathrm{~mol} \%)$ in the $1.0 \mathrm{~mL}$ of THF/DME (1:1) at $-20{ }^{\circ} \mathrm{C}$ for 3 days under argon, followed by column 
chromatography $(\mathrm{PE} / \mathrm{EA}=10: 1 \sim 1: 1)$ to afford 4aac as yellow sticky oil, $48.8 \mathrm{mg}$ (53\% yield, $80 \% e e) ;[\alpha]_{\mathrm{D}}^{25}=+361.9^{\circ}\left(c=0.98,{ }^{i} \mathrm{PrOH}\right)$; HPLC (IC, n-hexane $/{ }^{i} \mathrm{PrOH}$ $=70 / 30$, flow rate $=0.5 \mathrm{~mL} / \mathrm{min}, \mathrm{I}=238 \mathrm{~nm}$ ): $t_{\mathrm{R}}=15.1 \mathrm{~min}$ (major), $16.7 \mathrm{~min}$ (minor). ${ }^{1} \mathrm{H}$ NMR (600 MHz, $\left.\mathrm{CDCl}_{3}\right) \delta 7.66-7.58(\mathrm{~m}, 2 \mathrm{H}), 7.35-7.30(\mathrm{~m}, 2 \mathrm{H}), 7.29$ $-7.21(\mathrm{~m}, 4 \mathrm{H}), 7.16(\mathrm{~d}, J=7.5 \mathrm{~Hz}, 1 \mathrm{H}), 6.98(\mathrm{t}, J=8.1 \mathrm{~Hz}, 1 \mathrm{H}), 6.74(\mathrm{t}, J=2.3 \mathrm{~Hz}$, $1 \mathrm{H}), 6.69-6.59(\mathrm{~m}, 2 \mathrm{H}), 6.46-6.38(\mathrm{~m}, 1 \mathrm{H}), 5.90(\mathrm{~d}, J=5.6 \mathrm{~Hz}, 1 \mathrm{H}), 3.80(\mathrm{dd}, J=$ 11.7, $6.0 \mathrm{~Hz}, 6 \mathrm{H}), 3.66$ (s, 3H) ppm. ${ }^{13} \mathrm{C} \mathrm{NMR}\left(151 \mathrm{MHz}, \mathrm{CDCl}_{3}\right) \delta 160.1,147.7$, 141.6, 137.0, 132.1, 129.3, 128.4, 128.4, 128.2, 127.4, 127.3, 125.6, 124.7, 115.1, $112.7,108.8,108.3,62.1(\mathrm{~d}, J=10.7 \mathrm{~Hz}), 55.2,53.1(\mathrm{~d}, J=5.9 \mathrm{~Hz}), 53.0(\mathrm{~d}, J=5.5$ $\mathrm{Hz}$ ), 46.1 (d, $J=225.5 \mathrm{~Hz}$ ) ppm. HRMS (ESI) m/z: $[\mathrm{M}+\mathrm{H}]^{+}$Calcd. for $\mathrm{C}_{25} \mathrm{H}_{25} \mathrm{~N}_{3} \mathrm{O}_{4} \mathrm{P}$ 462.1583 found 462.1580 .

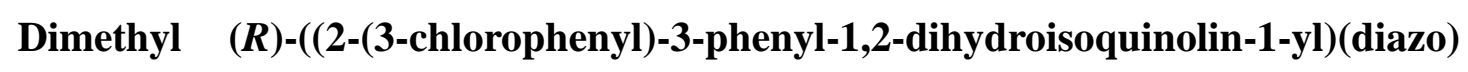
methyl)phosphonate (4aad)<smiles>COP(=O)(OC)C(=N)C1c2ccccc2C=C(c2ccccc2)N1c1cccc(Cl)c1</smiles>

Following the general procedure, reaction were performed with $o$-alkynylbenzaldehyde $\mathbf{1 a}(0.24 \mathrm{mmol}), \mathbf{2 d}(0.22$

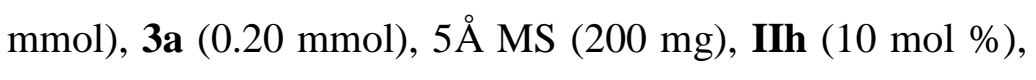
and $\mathrm{Ag}_{2} \mathrm{CO}_{3}(5 \mathrm{~mol} \%)$ in the $1.0 \mathrm{~mL}$ of THF/DME (1:1) at $-20{ }^{\circ} \mathrm{C}$ for 3 days under argon, followed by column chromatography $(\mathrm{PE} / \mathrm{EA}=10: 1 \sim 1: 1)$ to afford 4aad as yellow sticky oil, $30.7 \mathrm{mg}$ (33\% yield, $40 \% e e)$; $[\alpha]_{\mathrm{D}}^{25}=+180.9^{\circ}\left(c=0.61,{ }^{i} \mathrm{PrOH}\right)$; HPLC (IC, n-hexane $/{ }^{i} \mathrm{PrOH}$ $=70 / 30$, flow rate $=0.5 \mathrm{~mL} / \mathrm{min}, \mathrm{I}=238 \mathrm{~nm}$ ): $t_{\mathrm{R}}=12.5 \mathrm{~min}$ (major), $14.4 \mathrm{~min}$ (minor). ${ }^{1} \mathrm{H}$ NMR (600 MHz, $\left.\mathrm{CDCl}_{3}\right) \delta 7.66-7.55(\mathrm{~m}, 2 \mathrm{H}), 7.37-7.31(\mathrm{~m}, 2 \mathrm{H}), 7.31$ $-7.23(\mathrm{~m}, 4 \mathrm{H}), 7.19-7.14(\mathrm{~m}, 2 \mathrm{H}), 7.04-6.92(\mathrm{~m}, 2 \mathrm{H}), 6.89-6.80(\mathrm{~m}, 1 \mathrm{H}), 6.72$ (s, $1 \mathrm{H}), 5.85(\mathrm{~d}, J=5.4 \mathrm{~Hz}, 1 \mathrm{H}), 3.82(\mathrm{~d}, J=11.7 \mathrm{~Hz}, 6 \mathrm{H}) \mathrm{ppm} .{ }^{13} \mathrm{C} \mathrm{NMR}(151 \mathrm{MHz}$, $\left.\mathrm{CDCl}_{3}\right) \delta 147.6,141.0,136.4,134.3,131.8,129.7,128.6,128.5,128.4,127.5,127.4$, $127.3(\mathrm{~d}, J=6.5 \mathrm{~Hz}), 125.5,124.9,122.7,122.6,120.8,113.4,62.1(\mathrm{~d}, J=10.6 \mathrm{~Hz})$, $53.1(\mathrm{~d}, J=2.8 \mathrm{~Hz}), 53.1(\mathrm{~d}, J=3.0 \mathrm{~Hz}), 46.0(\mathrm{~d}, J=226.1 \mathrm{~Hz}) \mathrm{ppm}$. HRMS (ESI) $\mathrm{m} / \mathrm{z}:[\mathrm{M}+\mathrm{H}]^{+}$Calcd. for $\mathrm{C}_{24} \mathrm{H}_{22} \mathrm{ClN}_{3} \mathrm{O}_{3} \mathrm{P} 466.1087$ found 466.1074 .

\section{Dimethyl (R)-(diazo(2-(4-fluorophenyl)-3-phenyl-1,2-dihydroisoquinolin-1-yl) methyl)phosphonate (4aae)}

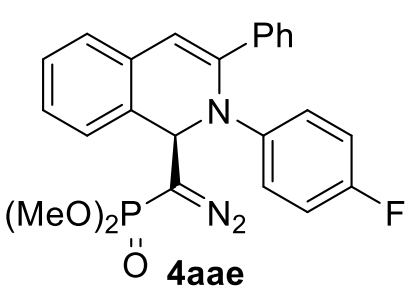

Following the general procedure, reaction were performed with $o$-alkynylbenzaldehyde $\mathbf{1 a}(0.24 \mathrm{mmol}), \mathbf{2 e}(0.22$ mmol), 3a (0.20 mmol), $5 \AA$ MS (200 mg), IIh (10 mol \%), and $\mathrm{Ag}_{2} \mathrm{CO}_{3}(5 \mathrm{~mol} \%)$ in the $1.0 \mathrm{~mL}$ of THF/DME (1:1) at 
$-20{ }^{\circ} \mathrm{C}$ for 3 days under argon, followed by column chromatography (PE/EA = 10:1 $1: 1)$ to afford 4aae as yellow sticky oil, $72.7 \mathrm{mg}(81 \%$ yield, $54 \% e e)$; $[\alpha]_{\mathrm{D}}^{25}=+236.7^{\circ}$ $\left(c=0.73,{ }^{i} \mathrm{PrOH}\right)$; HPLC (IC, n-hexane $/{ }^{i} \mathrm{PrOH}=70 / 30$, flow rate $=0.5 \mathrm{~mL} / \mathrm{min}, \mathrm{I}=$ $238 \mathrm{~nm}): t_{\mathrm{R}}=12.2 \mathrm{~min}$ (major), $14.7 \mathrm{~min}$ (minor). ${ }^{1} \mathrm{H} \mathrm{NMR}\left(600 \mathrm{MHz}, \mathrm{CDCl}_{3}\right) \delta 7.61$ $-7.55(\mathrm{~m}, 2 \mathrm{H}), 7.34-7.28(\mathrm{~m}, 2 \mathrm{H}), 7.26-7.19(\mathrm{~m}, 4 \mathrm{H}), 7.12(\mathrm{~d}, J=7.5 \mathrm{~Hz}, 1 \mathrm{H})$, $7.10-7.05(\mathrm{~m}, 2 \mathrm{H}), 6.80-6.74(\mathrm{~m}, 2 \mathrm{H}), 6.67(\mathrm{~s}, 1 \mathrm{H}), 5.79(\mathrm{~d}, J=5.2 \mathrm{~Hz}, 1 \mathrm{H}), 3.77$ $(\mathrm{dd}, J=11.7,8.5 \mathrm{~Hz}, 6 \mathrm{H}) \mathrm{ppm} .{ }^{13} \mathrm{C} \mathrm{NMR}\left(151 \mathrm{MHz}, \mathrm{CDCl}_{3}\right) \delta 158.8(\mathrm{~d}, J=242.7$ $\mathrm{Hz}), 142.6$ (d, $J=2.7 \mathrm{~Hz}), 141.8,136.6,132.1,128.5,128.4,127.6,127.3,126.7$ (d, $J$ $=6.8 \mathrm{~Hz}), 125.6,124.7,124.5(\mathrm{~d}, J=8.1 \mathrm{~Hz}), 115.5(\mathrm{~d}, J=22.5 \mathrm{~Hz}), 112.3,62.8(\mathrm{~d}, J$ $=10.8 \mathrm{~Hz}), 53.1(\mathrm{~d}, J=6.0 \mathrm{~Hz}), 53.0(\mathrm{~d}, J=5.6 \mathrm{~Hz}), 45.9(\mathrm{~d}, J=224.7 \mathrm{~Hz}) \mathrm{ppm}$. HRMS (ESI) m/z: [M+H] $]^{+}$Calcd. for $\mathrm{C}_{24} \mathrm{H}_{22} \mathrm{FN}_{3} \mathrm{O}_{3} \mathrm{P} 450.1377$, found 450.1370.

Dimethyl (R)-(diazo(2-(4-methoxyphenyl)-3-phenyl-1,2-dihydroisoquinolin-1-yl) methyl)phosphonate (4aaf)<smiles>COc1ccc(N2C(c3ccccc3)=Cc3ccccc3C2C(=N)P(=O)(O)OC)cc1</smiles>

Following the general procedure, reaction were performed with $o$-alkynylbenzaldehyde $1 \mathrm{a}(0.24 \mathrm{mmol})$, 2f $(0.22 \mathrm{mmol}), 3 \mathbf{a}(0.20 \mathrm{mmol}), 5 \AA \mathrm{MS}(200 \mathrm{mg})$, IIh (10 mol \%), and $\mathrm{Ag}_{2} \mathrm{CO}_{3}(5 \mathrm{~mol} \%)$ in the $1.0 \mathrm{~mL}$ of THF/DME (1:1) at $-20{ }^{\circ} \mathrm{C}$ for 3 days under argon, followed by column chromatography $(\mathrm{PE} / \mathrm{EA}=10: 1 \sim 1: 1)$ to afford 4aaf as yellow sticky oil, $75.6 \mathrm{mg}(82 \%$ yield, $42 \% e e) ;[\alpha]_{\mathrm{D}}^{25}=+184.1^{\circ}\left(c=1.51,{ }^{i} \mathrm{PrOH}\right)$; HPLC $\left(\mathrm{IC}, \mathrm{n}\right.$-hexane $/{ }^{i} \mathrm{PrOH}=70 / 30$, flow rate $\left.=0.5 \mathrm{~mL} / \mathrm{min}, \mathrm{I}=238 \mathrm{~nm}\right): t_{\mathrm{R}}=17.4 \mathrm{~min}$ (major), $20.6 \mathrm{~min}$ (minor). ${ }^{1} \mathrm{H} \mathrm{NMR}\left(600 \mathrm{MHz}, \mathrm{CDCl}_{3}\right) \delta 7.69-7.53(\mathrm{~m}, 2 \mathrm{H}), 7.34-$ $7.28(\mathrm{~m}, 2 \mathrm{H}), 7.26-7.18(\mathrm{~m}, 4 \mathrm{H}), 7.11(\mathrm{~d}, J=7.5 \mathrm{~Hz}, 1 \mathrm{H}), 7.06-6.97(\mathrm{~m}, 2 \mathrm{H}), 6.68$ $-6.56(\mathrm{~m}, 3 \mathrm{H}), 5.77(\mathrm{~d}, J=5.2 \mathrm{~Hz}, 1 \mathrm{H}), 3.78(\mathrm{dd}, J=11.7,4.5 \mathrm{~Hz}, 6 \mathrm{H}), 3.65(\mathrm{~s}, 3 \mathrm{H})$ ppm. ${ }^{13} \mathrm{C} \mathrm{NMR}\left(151 \mathrm{MHz}, \mathrm{CDCl}_{3}\right) \delta 155.7,142.2,140.0,136.9,132.3,128.4,128.2$, $128.2,127.7,127.0,126.5(\mathrm{~d}, J=6.5 \mathrm{~Hz}), 125.7,124.6,124.5,114.1,111.5,62.9(\mathrm{~d}, J$ $=10.6 \mathrm{~Hz}), 55.3,53.0(\mathrm{~d}, J=6.3 \mathrm{~Hz}), 53.0(\mathrm{~d}, J=6.1 \mathrm{~Hz}), 46.1(\mathrm{~d}, J=224.7 \mathrm{~Hz})$ ppm. HRMS (ESI) m/z: $[\mathrm{M}+\mathrm{H}]^{+}$Calcd. for $\mathrm{C}_{25} \mathrm{H}_{25} \mathrm{~N}_{3} \mathrm{O}_{4} \mathrm{P} 462.1583$ found 462.1572 . 
Diethyl $(R)$-(diazo(2,3-diphenyl-1,2-dihydroisoquinolin-1-yl)methyl)phosphonate (4aag)<smiles>CCOC(=N)C(c1ccccc1)P(=O)(OCC)OC(F)(F)F</smiles>
Following the general procedure, reaction were performed with $o$-alkynylbenzaldehyde $\mathbf{1 a}(0.24 \mathrm{mmol}), \mathbf{2 a}(0.22 \mathrm{mmol}), \mathbf{3 b}(0.20$ mmol), $5 \AA$ MS (200 mg), IIh (10 mol \%), and $\mathrm{Ag}_{2} \mathrm{CO}_{3}(5 \mathrm{~mol} \%)$ in the $1.0 \mathrm{~mL}$ of THF/DME $(1: 1)$ at $-20{ }^{\circ} \mathrm{C}$ for 3 days under argon, followed by column chromatography $(\mathrm{PE} / \mathrm{EA}=10: 1 \sim 1: 1)$ to afford 4aag as yellow sticky oil, $51.4 \mathrm{mg}(56 \%$ yield, 97\% ee $) ;[\alpha]_{\mathrm{D}}^{25}=+370.2^{\circ}(c=$ $\left.1.04,{ }^{i} \mathrm{PrOH}\right) ; \mathrm{HPLC}\left(\mathrm{IC}, \mathrm{n}-\right.$ hexane $/{ }^{i} \mathrm{PrOH}=70 / 30$, flow rate $=0.5 \mathrm{~mL} / \mathrm{min}, \mathrm{I}=238$ $\mathrm{nm}): t_{\mathrm{R}}=11.3 \min$ (major), $13.5 \min$ (minor). ${ }^{1} \mathrm{H} \mathrm{NMR}\left(600 \mathrm{MHz}, \mathrm{CDCl}_{3}\right) \delta 7.67-$ $7.58(\mathrm{~m}, 2 \mathrm{H}), 7.35-7.27(\mathrm{~m}, 2 \mathrm{H}), 7.26-7.19(\mathrm{~m}, 4 \mathrm{H}), 7.16-7.07$ (m, 5H), $6.89-$ $6.80(\mathrm{~m}, 1 \mathrm{H}), 6.66(\mathrm{~s}, 1 \mathrm{H}), 5.90(\mathrm{~d}, J=5.5 \mathrm{~Hz}, 1 \mathrm{H}), 4.28-4.03(\mathrm{~m}, 4 \mathrm{H}), 1.36(\mathrm{t}, J=$ $7.1 \mathrm{~Hz}, 6 \mathrm{H}) \mathrm{ppm} .{ }^{13} \mathrm{C} \mathrm{NMR}\left(151 \mathrm{MHz}, \mathrm{CDCl}_{3}\right) \delta 146.5,141.7,137.0,132.1,128.7$, 128.3, 128.2, 128.1, 127.6, 127.5 (d, $J=6.3 \mathrm{~Hz}), 127.2$, 125.5, 124.6, 122.7, 122.5, 112.7, 62.7 (d, $J=4.9 \mathrm{~Hz}), 62.6(\mathrm{~d}, J=5.4 \mathrm{~Hz}), 62.1(\mathrm{~d}, J=10.8 \mathrm{~Hz}), 46.9(\mathrm{~d}, J=$ $224.0 \mathrm{~Hz}), 16.2(\mathrm{~d}, J=2.8 \mathrm{~Hz}), 16.1(\mathrm{~d}, J=2.6 \mathrm{~Hz}) \mathrm{ppm}$. HRMS (ESI) m/z: $[\mathrm{M}+\mathrm{H}]^{+}$ Calcd. for $\mathrm{C}_{26} \mathrm{H}_{27} \mathrm{~N}_{3} \mathrm{O}_{3} \mathrm{P} 460.1785$, found 460.1783 .

\section{Di-tert-butyl} (R)-(diazo(2,3-diphenyl-1,2-dihydroisoquinolin-1-yl)methyl) phosphonate (4aah)<smiles>CC(C)(C)OP(=O)(OC(C)(C)C)C1c2ccccc2C=C(c2ccccc2)N1c1ccccc1</smiles>

Following the general procedure, reaction were performed with $o$-alkynylbenzaldehyde $\mathbf{1 a}(0.24 \mathrm{mmol}), \mathbf{2 a}(0.22 \mathrm{mmol}), \mathbf{3 c}(0.20$ mmol), $5 \AA$ MS (200 mg), IIh (10 mol \%), and $\mathrm{Ag}_{2} \mathrm{CO}_{3}(5 \mathrm{~mol} \%)$ in the $1.0 \mathrm{~mL}$ of $\mathrm{THF} / \mathrm{DME}(1: 1)$ at $-20{ }^{\circ} \mathrm{C}$ for 3 days under argon, followed by column chromatography (PE/EA $=10: 1 \sim 3: 1)$ to afford 4aah as yellow sticky oil, $52.5 \mathrm{mg}(51 \%$ yield, $84 \% e e) ;[\alpha]_{\mathrm{D}}^{25}=+325.7^{\circ}(c=$ 1.06, $\left.{ }^{i} \mathrm{PrOH}\right)$; HPLC (IC, n-hexane $/{ }^{i} \mathrm{PrOH}=70 / 30$, flow rate $=0.5 \mathrm{~mL} / \mathrm{min}, \mathrm{I}=238$ $\mathrm{nm}): t_{\mathrm{R}}=9.9 \min$ (major), $10.8 \min$ (minor). ${ }^{1} \mathrm{H} \mathrm{NMR}\left(600 \mathrm{MHz}, \mathrm{CDCl}_{3}\right) \delta 7.65-$ $7.60(\mathrm{~m}, 2 \mathrm{H}), 7.32-7.20(\mathrm{~m}, 6 \mathrm{H}), 7.18(\mathrm{~d}, J=8.0 \mathrm{~Hz}, 2 \mathrm{H}), 7.13(\mathrm{~d}, J=7.5 \mathrm{~Hz}, 1 \mathrm{H})$, $7.11-7.06(\mathrm{~m}, 2 \mathrm{H}), 6.83(\mathrm{t}, J=7.3 \mathrm{~Hz}, 1 \mathrm{H}), 6.56(\mathrm{~s}, 1 \mathrm{H}), 5.89(\mathrm{~d}, J=6.1 \mathrm{~Hz}, 1 \mathrm{H})$, $1.56(\mathrm{~d}, J=1.7 \mathrm{~Hz}, 18 \mathrm{H}) \mathrm{ppm} .{ }^{13} \mathrm{C} \mathrm{NMR}\left(151 \mathrm{MHz}, \mathrm{CDCl}_{3}\right) \delta 146.6,141.9,137.5$, $132.1,128.5,128.2,128.2,128.0(\mathrm{~d}, J=4.3 \mathrm{~Hz}), 127.8,127.0,125.4,124.5,122.5$, 122.1, 113.0, $83.4(\mathrm{~d}, J=2.6 \mathrm{~Hz}), 83.4(\mathrm{~d}, J=2.1 \mathrm{~Hz}), 61.8(\mathrm{~d}, J=11.1 \mathrm{~Hz}), 50.6(\mathrm{~d}$, $J=229.3 \mathrm{~Hz}), 30.3(\mathrm{~d}, J=4.1 \mathrm{~Hz}), 30.1(\mathrm{~d}, J=4.0 \mathrm{~Hz}) \mathrm{ppm}$. HRMS (ESI) m/z: $[\mathrm{M}+\mathrm{H}]^{+}$Calcd. for $\mathrm{C}_{30} \mathrm{H}_{35} \mathrm{~N}_{3} \mathrm{O}_{3} \mathrm{P}$ 516.2411, found 516.2414. 
<smiles>CCOC(=O)C(C#N)C1c2ccccc2C=C(c2ccccc2)N1c1ccccc1</smiles>

Following the general procedure, reaction were performed with $o$-alkynylbenzaldehyde $1 \mathbf{a}(0.24 \mathrm{mmol}), \mathbf{2 a}(0.22 \mathrm{mmol}), \mathbf{3 e}(0.20$ mmol), $5 \AA$ MS (200 mg), IIh (10 mol \%), and $\mathrm{Ag}_{2} \mathrm{CO}_{3}$ (5 mol \%) in the $1.0 \mathrm{~mL}$ of $\mathrm{THF} / \mathrm{DME}(1: 1)$ at $-20{ }^{\circ} \mathrm{C}$ for 3 days under argon, followed by column chromatography $(\mathrm{PE} / \mathrm{EA}=10: 1 \sim 1: 1)$ to afford 4aai as yellow sticky oil, $42.7 \mathrm{mg}(54 \%$ yield, $92 \% e e) ;[\alpha]_{\mathrm{D}}^{25}=+581.7^{\circ}(c=$ 0.06, ${ }^{i} \mathrm{PrOH}$ ); HPLC (ADH, n-hexane $/{ }^{i} \mathrm{PrOH}=90 / 10$, flow rate $=0.5 \mathrm{~mL} / \mathrm{min}, \mathrm{I}=$ $238 \mathrm{~nm}$ ): $t_{\mathrm{R}}=12.1 \mathrm{~min}$ (major), $15.0 \mathrm{~min}$ (minor). ${ }^{1} \mathrm{H} \mathrm{NMR}\left(600 \mathrm{MHz}, \mathrm{CDCl}_{3}\right) \delta 7.58$ - $7.50(\mathrm{~m}, 2 \mathrm{H}), 7.34-7.27(\mathrm{~m}, 2 \mathrm{H}), 7.27-7.17(\mathrm{~m}, 6 \mathrm{H}), 7.15(\mathrm{~d}, J=7.5 \mathrm{~Hz}, 1 \mathrm{H})$, $7.10(\mathrm{t}, J=8.0 \mathrm{~Hz}, 2 \mathrm{H}), 6.85(\mathrm{t}, J=7.3 \mathrm{~Hz}, 1 \mathrm{H}), 6.61(\mathrm{~s}, 1 \mathrm{H}), 6.14(\mathrm{~s}, 1 \mathrm{H}), 4.34(\mathrm{q}, J$ $=6.9,6.1 \mathrm{~Hz}, 2 \mathrm{H}), 1.32(\mathrm{t}, J=7.2 \mathrm{~Hz}, 3 \mathrm{H}) \mathrm{ppm} .{ }^{13} \mathrm{C} \mathrm{NMR}\left(151 \mathrm{MHz}, \mathrm{CDCl}_{3}\right) \delta 166.2$, 146.4 , 142.0, 137.2, 132.1, 128.6, 128.4, 128.3, 128.2, 127.5, 127.4, 127.2, 125.7, 124.7, 122.6, 122.4, 112.6, 77.0, 61.0, 60.4, 14.5 ppm. HRMS (ESI) m/z: $[\mathrm{M}+\mathrm{H}]^{+}$ Calcd. for $\mathrm{C}_{25} \mathrm{H}_{22} \mathrm{~N}_{3} \mathrm{O}_{2} 396.1712$, found 396.1710.

\section{Amplification and derivatizations of products 4}

\subsection{Amplification reaction}

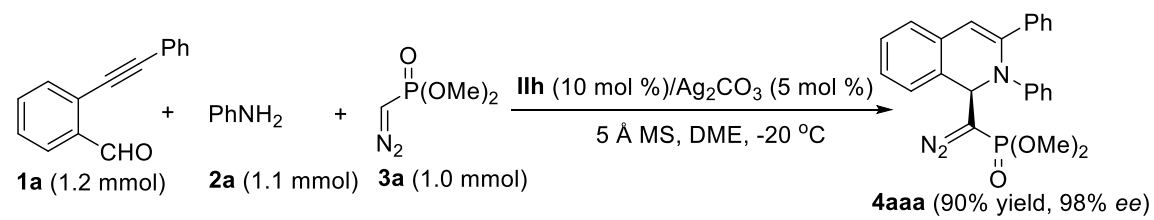

Chiral imidodiphosphate IIh (68.7 mg, $0.05 \mathrm{mmol}, 0.1$ equiv.), $\mathrm{Ag}_{2} \mathrm{CO}_{3}$ (6.9 mg, $0.025 \mathrm{mmol}, 0.05$ equiv.) and anhydrous DME $(6.5 \mathrm{~mL})$ were added to a pre-dried tube under argon and stirred for $2 \mathrm{~h}$ at $30{ }^{\circ} \mathrm{C}$ and then the corresponding $o$-alkynylbenzaldehyde 1 a $(0.247 \mathrm{~g}, 1.2 \mathrm{mmol}, 1.2$ equiv.), amine $2 \mathbf{a}(0.1 \mathrm{~mL}, 1.1$ mmol, 1.1 equiv.) and $5 \AA$ MS (1.0 g) powder were added, the mixture reacted at $30{ }^{\circ} \mathrm{C}$ for $0.5 \mathrm{~h}$. Then the reaction mixture was cooled down to $-20{ }^{\circ} \mathrm{C}$, the diazo compounds 3a ( $0.150 \mathrm{~g}, 1.0 \mathrm{mmol}, 1.0$ equiv.) was added and the mixture was stirred until 3a had been completely consumed. The reaction mixture was then purified directly by flash chromatography on silica gel to obtain the corresponding product 4aaa $(0.390 \mathrm{~g}, 90 \%$ yield, $98 \%$ ee $)$. 


\subsection{Derivatizations of products}

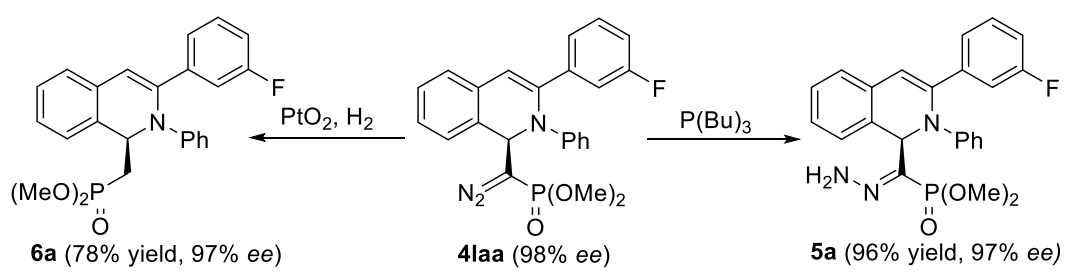

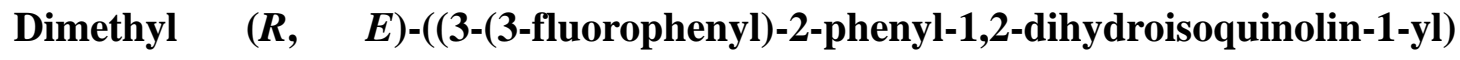
(hydrazono)methyl)phosphonate (5a)<smiles>COP(=O)(OC)/C(=N/N)C1c2ccccc2C=C(c2cccc(F)c2)N1c1ccccc1</smiles>
corresponding product 5a $(43.3 \mathrm{mg}, 96 \%$ yield, $97 \%$ ee $)$. Yellow sticky oil, $[\alpha]_{\mathrm{D}}^{25}=$ $+353.0^{\circ}(c=0.04, \mathrm{EA})$; HPLC (IC, n-hexane $/{ }^{i} \mathrm{PrOH}=70 / 30$, flow rate $=0.5 \mathrm{~mL} / \mathrm{min}$, $\mathrm{I}=238 \mathrm{~nm}$ ): $t_{\mathrm{R}}=15.6 \mathrm{~min}$ (major), $13.7 \mathrm{~min}$ (minor). ${ }^{1} \mathrm{H} \mathrm{NMR}\left(600 \mathrm{MHz}, \mathrm{CDCl}_{3}\right) \delta$ $7.63-7.48(\mathrm{~m}, 4 \mathrm{H}), 7.25(\mathrm{~d}, J=4.2 \mathrm{~Hz}, 2 \mathrm{H}), 7.18-7.13(\mathrm{~m}, 1 \mathrm{H}), 7.12-7.06(\mathrm{~m}$, $3 \mathrm{H}), 7.02(\mathrm{~d}, J=8.1 \mathrm{~Hz}, 2 \mathrm{H}), 6.92(\mathrm{t}, J=8.5 \mathrm{~Hz}, 2 \mathrm{H}), 6.86(\mathrm{t}, J=7.3 \mathrm{~Hz}, 1 \mathrm{H}), 6.57(\mathrm{~s}$, $1 \mathrm{H}), 5.83(\mathrm{~d}, J=5.7 \mathrm{~Hz}, 1 \mathrm{H}), 3.67-3.58(\mathrm{~m}, 6 \mathrm{H}) \mathrm{ppm} .{ }^{13} \mathrm{C} \mathrm{NMR}\left(151 \mathrm{MHz}, \mathrm{CDCl}_{3}\right)$ $\delta 162.2(\mathrm{~d}, J=247.4 \mathrm{~Hz}), 147.4,140.4,133.5,132.9,129.9,129.1(\mathrm{~d}, J=7.9 \mathrm{~Hz})$, $129.0(\mathrm{~d}, J=3.9 \mathrm{~Hz}), 128.4,127.4,126.6,126.0,124.0,122.4,122.1,114.9,114.8$, 111.7, $67.5(\mathrm{~d}, J=29.0 \mathrm{~Hz}), 52.2(\mathrm{~d}, J=5.1 \mathrm{~Hz}), 51.9(\mathrm{~d}, J=5.5 \mathrm{~Hz})$ ppm. HRMS (ESI) m/z: $[\mathrm{M}+\mathrm{H}]^{+}$Calcd. for $\mathrm{C}_{24} \mathrm{H}_{24} \mathrm{FN}_{3} \mathrm{O}_{3} \mathrm{P} 452.1539$, found 452.1534 .

\section{Dimethyl methyl)phosphonate $(6 a)$}

$\mathrm{PtO}_{2}(2.3 \mathrm{mg}, 10 \mathrm{~mol} \%)$ was added to the solution of 4 laa $(44.9 \mathrm{mg}, 0.1 \mathrm{mmol})$ in $\mathrm{EA}: \mathrm{AcOH}=10: 1(2.0 \mathrm{~mL})$. The reaction vessel was purged with $\mathrm{H}_{2}$ (3 times), the mixture then stirred under 1 atm of $\mathrm{H}_{2}$ (balloon). After the reaction completed, filtrated through a plug of celite, then the filtrate was concentrated under vacuum, the residue was purified by flash chromatography on silica gel to give the compound $\mathbf{6 a}$ with $78 \%$ yield $(33.0 \mathrm{mg}, 97 \% e e)$.Yellow sticky oil, $[\alpha]_{\mathrm{D}}^{25}=+514.7^{\circ}(c=0.05$, EA); HPLC (IC, n-hexane $/{ }^{i} \mathrm{PrOH}=70 / 30$, flow rate $=0.5 \mathrm{~mL} / \mathrm{min}, \mathrm{I}=238 \mathrm{~nm}$ ): $t_{\mathrm{R}}=12.2$ $\min$ (major), $13.8 \mathrm{~min}$ (minor). ${ }^{1} \mathrm{H} \mathrm{NMR}\left(600 \mathrm{MHz} \mathrm{CDCl}_{3}\right) \delta 7.43(\mathrm{~d}, J=7.8 \mathrm{~Hz}, 1 \mathrm{H}$ ), $7.36-7.29(\mathrm{~m}, 1 \mathrm{H}), 7.27-7.22(\mathrm{~m}, 2 \mathrm{H}), 7.22-7.19(\mathrm{~m}, 1 \mathrm{H}), 7.18-7.14(\mathrm{~m}, 1 \mathrm{H})$, 
<smiles>COP(=O)(O)C[C@H]1c2ccccc2C=C(c2cccc(F)c2)N1c1ccccc1</smiles>

$7.08(\mathrm{~d}, J=6.7 \mathrm{~Hz}, 5 \mathrm{H}), 6.91-6.81(\mathrm{~m}, 2 \mathrm{H}), 6.75(\mathrm{~s}, 1 \mathrm{H})$, $5.50-5.43(\mathrm{~m}, 1 \mathrm{H}), 3.74(\mathrm{~d}, J=11.0 \mathrm{~Hz}, 3 \mathrm{H}), 3.67(\mathrm{~d}, J=$ $10.9 \mathrm{~Hz}, 3 \mathrm{H}), 2.60-2.48(\mathrm{~m}, 1 \mathrm{H}), 2.01-1.91(\mathrm{~m}, 1 \mathrm{H})$ ppm. ${ }^{13} \mathrm{C}$ NMR $\left(151 \mathrm{MHz}, \mathrm{CDCl}_{3}\right) \delta 162.9(\mathrm{~d}, J=244.9$ $\mathrm{Hz}), 146.6,139.8$ (d, $J=7.7 \mathrm{~Hz}), 139.3,132.0(\mathrm{~d}, J=13.1$ $\mathrm{Hz}), 130.9,129.6(\mathrm{~d}, J=8.2 \mathrm{~Hz}), 128.8,127.7,127.2$, 125.3, 124.6, $123.0(\mathrm{~d}, J=2.7 \mathrm{~Hz}), 122.6,122.6,114.9(\mathrm{~d}, J=21.4 \mathrm{~Hz}), 114.5$ (d, $J=$ $22.8 \mathrm{~Hz}), 112.8,60.4(\mathrm{~d}, J=2.5 \mathrm{~Hz}), 52.4(\mathrm{~d}, J=6.5 \mathrm{~Hz}), 52.1(\mathrm{~d}, J=6.7 \mathrm{~Hz}), 30.0(\mathrm{~d}$, $J=136.7 \mathrm{~Hz}$ ) ppm. HRMS (ESI) m/z: $[\mathrm{M}+\mathrm{H}]^{+}$Calcd. for $\mathrm{C}_{24} \mathrm{H}_{24} \mathrm{FNO}_{3} \mathrm{P} 424.1478$, found 424.1472 .

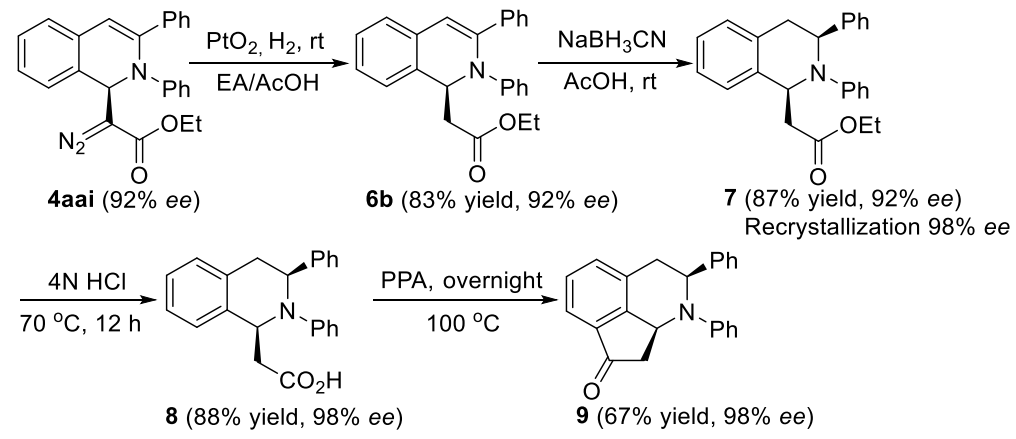

Ethyl (S)-2-(2, 3-diphenyl-1,2-dihydroisoquinolin-1-yl)acetate (6b)<smiles>CCOC(=O)CC1c2ccccc2C=C(c2ccccc2)N1c1ccccc1</smiles>

$\mathrm{PtO}_{2}(6.8 \mathrm{mg}, 10 \mathrm{~mol} \%)$ was added to the solution of 4aai (118.5 $\mathrm{mg}, 0.3 \mathrm{mmol})$ in $\mathrm{EA}: \mathrm{AcOH}=10: 1(3.0 \mathrm{~mL})$. The reaction vessel was purged with $\mathrm{H}_{2}$ (3 times), the mixture then stirred under $1 \mathrm{~atm}$ of $\mathrm{H}_{2}$ (balloon). After the reaction completed, filtrated through a plug of celite, then the filtrate was concentrated under vacuum, the residue was purified by flash chromatography on silica gel to give the title compound $\mathbf{6 b}$ in $83 \%$ yield $(91.9 \mathrm{mg}, 92 \%$ ee $)$. Yellow sticky oil, $[\alpha]_{\mathrm{D}}^{25}=$ $+847.7^{\circ}\left(c=0.10,{ }^{i} \mathrm{PrOH}\right) ; \mathrm{HPLC}\left(\mathrm{ADH}, \mathrm{n}\right.$-hexane $/{ }^{i} \mathrm{PrOH}=90 / 10$, flow rate $=0.5$ $\mathrm{mL} / \mathrm{min}, \mathrm{I}=238 \mathrm{~nm}$ ): $t_{\mathrm{R}}=22.1 \mathrm{~min}$ (major), $11.7 \min$ (minor). ${ }^{1} \mathrm{H}$ NMR $(600 \mathrm{MHz}$, $\left.\mathrm{CDCl}_{3}\right) \delta 7.55-7.50(\mathrm{~m}, 2 \mathrm{H}), 7.27-7.20(\mathrm{~m}, 4 \mathrm{H}), 7.18(\mathrm{t}, J=7.1 \mathrm{~Hz}, 1 \mathrm{H}), 7.16-$ $7.10(\mathrm{~m}, 1 \mathrm{H}), 7.05(\mathrm{dd}, J=7.7,3.6 \mathrm{~Hz}, 3 \mathrm{H}), 7.00(\mathrm{~d}, J=8.0 \mathrm{~Hz}, 2 \mathrm{H}), 6.80(\mathrm{t}, J=7.2$ $\mathrm{Hz}, 1 \mathrm{H}), 6.73$ (s, 1H), $5.51-5.46(\mathrm{~m}, 1 \mathrm{H}), 4.37-4.18$ (m, 2H), 3.00 (dd, $J=14.9$, $10.0 \mathrm{~Hz}, 1 \mathrm{H}), 2.48(\mathrm{dd}, J=14.9,4.8 \mathrm{~Hz}, 1 \mathrm{H}), 1.27(\mathrm{t}, J=7.2 \mathrm{~Hz}, 3 \mathrm{H}) \mathrm{ppm} .{ }^{13} \mathrm{C} \mathrm{NMR}$ $\left(151 \mathrm{MHz}, \mathrm{CDCl}_{3}\right) \delta 171.7,146.9,140.5,137.5,131.8,131.7,128.6,128.3,128.0$, 127.6, 127.4, 126.8, 125.2, 124.6, 122.3, 122.1, 112.5, 77.0, 62.2, 60.7, 39.6, 14.3 
ppm. HRMS (ESI) m/z: [M+H] ${ }^{+}$Calcd. for $\mathrm{C}_{25} \mathrm{H}_{24} \mathrm{NO}_{2} 370.1807$, found 370.1802 .

Ethyl 2-((1S)-2, 3-diphenyl-1,2,3,4-tetrahydroisoquinolin-1-yl)acetate (7)<smiles>CCOC(=O)C[C@@H]1c2ccccc2C[C@H](c2ccccc2)N1c1ccccc1</smiles>

with EA (three times), combined organic layers and washed with brine, dried over $\mathrm{Na}_{2} \mathrm{SO}_{4}$, and concentrated under reduce pressure and the residue was purified by column chromatography on silica gel to give 7 with $87 \%$ yield $(77.5 \mathrm{mg}, 92 \% e e, d r>$ 20:1). The enantioselectivities was up to $98 \%$ by recrystallization of EA/PE. Yellow solid, mp: $60.1-61.5^{\circ} \mathrm{C} ;[\alpha]_{\mathrm{D}}^{25}=+10.0^{\circ}\left(c=0.02,{ }^{i} \mathrm{PrOH}\right)$; HPLC (ADH, n-hexane/ ${ }^{i} \mathrm{PrOH}=90 / 10$, flow rate $=0.5 \mathrm{~mL} / \mathrm{min}, \mathrm{I}=254 \mathrm{~nm}$ ): $t_{\mathrm{R}}=12.5 \mathrm{~min}$ (major), $18.1 \mathrm{~min}$ (minor). ${ }^{1} \mathrm{H}$ NMR $\left(600 \mathrm{MHz},\left(\mathrm{CD}_{3}\right)_{2} \mathrm{CO}\right) \delta 7.51(\mathrm{~d}, J=7.6 \mathrm{~Hz}, 2 \mathrm{H}), 7.35-7.29(\mathrm{~m}$, $3 \mathrm{H}), 7.29-7.23(\mathrm{~m}, 2 \mathrm{H}), 7.23-7.18(\mathrm{~m}, 2 \mathrm{H}), 7.10-7.01(\mathrm{~m}, 2 \mathrm{H}), 6.89(\mathrm{~d}, J=8.3$ $\mathrm{Hz}, 2 \mathrm{H}), 6.61(\mathrm{t}, J=7.2 \mathrm{~Hz}, 1 \mathrm{H}), 5.45(\mathrm{dd}, J=9.6,5.0 \mathrm{~Hz}, 1 \mathrm{H}), 4.72(\mathrm{dd}, J=12.1$, $4.9 \mathrm{~Hz}, 1 \mathrm{H}), 4.24-4.07(\mathrm{~m}, 2 \mathrm{H}), 3.32(\mathrm{dd}, J=14.8,4.9 \mathrm{~Hz}, 1 \mathrm{H}), 3.22(\mathrm{dd}, J=15.4$, $4.9 \mathrm{~Hz}, 1 \mathrm{H}), 3.16-3.07(\mathrm{~m}, 1 \mathrm{H}), 2.91-2.83(\mathrm{~m}, 1 \mathrm{H}), 1.21(\mathrm{t}, J=7.1 \mathrm{~Hz}, 3 \mathrm{H}) \mathrm{ppm}$. ${ }^{13} \mathrm{C}$ NMR $\left(151 \mathrm{MHz},\left(\mathrm{CD}_{3}\right)_{2} \mathrm{CO}\right) \delta 171.5,150.2,146.1,139.4,135.9,129.4,129.4$, 128.4, 128.2, 127.6, 127.4, 127.2, 127.2, 118.6, 116.3, 61.1, 61.1, 60.8, 44.7, 39.6, 14.6 ppm. HRMS (ESI) m/z: $[\mathrm{M}+\mathrm{H}]^{+}$Calcd. for $\mathrm{C}_{25} \mathrm{H}_{26} \mathrm{NO}_{2}$ 372.1964, found 372.1957.

\section{2-((1S)-2,3-Diphenyl-1,2,3,4-tetrahydroisoquinolin-1-yl)acetic acid (8)}<smiles>O=C(O)C[C@H]1c2ccccc2CC(c2ccccc2)N1c1ccccc1</smiles>

A $10.0 \mathrm{~mL}$ of oven-dried round-bottom flask equipped with a magnetic stir bar was charged with $7(55.6 \mathrm{mg}, 0.15 \mathrm{mmol})$ and $\mathrm{HCl}$ (4.0 $\mathrm{M}$ in dioxane, $2.0 \mathrm{~mL}$ ). The reaction was stirred at $70{ }^{\circ} \mathrm{C}$ for 12 $\mathrm{h}$ under argon atmosphere. The solution was allowed to cool down to room temperature. and extracted with EA (three times), combined organic layers and washed with brine, dried over $\mathrm{Na}_{2} \mathrm{SO}_{4}$, and concentrated under reduce pressure and the residue was purified by column chromatography on silica gel to give 8 with $88 \%$ yield $(45.3 \mathrm{mg}, 98 \%$ ee $)$. White solid, mp: $163.5-164.7{ }^{\circ} \mathrm{C} ;[\alpha]_{\mathrm{D}}^{25}=$ $+75.6^{\circ}\left(c=0.25\right.$, EA); HPLC $\left(\right.$ ASH, n-hexane $/{ }^{i} \mathrm{PrOH}=90 / 10$, flow rate $=0.5$ $\mathrm{mL} / \mathrm{min}, \mathrm{I}=254 \mathrm{~nm}$ ): $t_{\mathrm{R}}=17.3 \mathrm{~min}$ (major), $23.6 \mathrm{~min}$ (minor). ${ }^{1} \mathrm{H}$ NMR $(600 \mathrm{MHz}$, 
$\left.\left(\mathrm{CD}_{3}\right)_{2} \mathrm{CO}\right) \delta 11.02(\mathrm{~s}, 1 \mathrm{H}), 7.51(\mathrm{~d}, J=7.6 \mathrm{~Hz}, 2 \mathrm{H}), 7.36-7.18(\mathrm{~m}, 7 \mathrm{H}), 7.12-7.02$ $(\mathrm{m}, 2 \mathrm{H}), 6.91(\mathrm{~d}, J=8.3 \mathrm{~Hz}, 2 \mathrm{H}), 6.71-6.57(\mathrm{~m}, 1 \mathrm{H}), 5.54-5.43(\mathrm{~m}, 1 \mathrm{H}), 4.73$ (dd, $J=12.0,4.8 \mathrm{~Hz}, 1 \mathrm{H}), 3.34(\mathrm{dd}, J=15.3,4.3 \mathrm{~Hz}, 1 \mathrm{H}), 3.22(\mathrm{dd}, J=15.2,4.6 \mathrm{~Hz}, 1 \mathrm{H})$, $3.19-3.08(\mathrm{~m}, 1 \mathrm{H}), 2.98-2.84(\mathrm{~m}, 1 \mathrm{H}) \mathrm{ppm} .{ }^{13} \mathrm{C} \mathrm{NMR}\left(151 \mathrm{MHz},\left(\mathrm{CD}_{3}\right)_{2} \mathrm{CO}\right) \delta$ $171.5,149.2$, 145.1, 138.6, 135.0, 128.5, 127.4, 127.2, 126.7, 126.5, 126.4, 126.4, 126.4, 117.8, 115.6, 60.2, 59.8, 43.3, 38.7 ppm. HRMS (ESI) m/z: $[\mathrm{M}+\mathrm{H}]^{+}$Calcd. for $\mathrm{C}_{23} \mathrm{H}_{22} \mathrm{NO}_{2} 344.1651$, found 344.1645.

(2S,8aS)-1,2-Diphenyl-2,3,8,8a-tetrahydrocyclopenta[ij]isoquinolin-7(1H)-one (9)

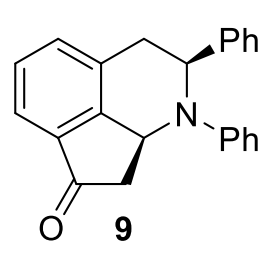

Polyphosphoric acid (1.0 g) was added to a suspension of $\mathbf{8}(34.3$ $\mathrm{mg}, 0.1 \mathrm{mmol}$ ) and the mixture was allowed to react at $100^{\circ} \mathrm{C}$ for overnight. The crude was poured to a mixture of ice/water to destroy the PPA, and extracted with EA (three times). The

combined organic layers were dried with $\mathrm{Na}_{2} \mathrm{SO}_{4}$, evaporated in vacuo and purified by silicagel chromatography to afford $9(21.8 \mathrm{mg}, 67 \%$ yield, $98 \%$ ee $)$. Yellow solid, mp: $195.1-196.7{ }^{\circ} \mathrm{C} ;[\alpha]_{\mathrm{D}}^{25}=+40.0^{\circ}\left(c=0.07\right.$, EA); HPLC (ASH, n-hexane $/{ }^{i} \mathrm{PrOH}=$ 90/10, flow rate $=0.5 \mathrm{~mL} / \mathrm{min}, \mathrm{I}=254 \mathrm{~nm}$ ): $t_{\mathrm{R}}=36.9 \mathrm{~min}$ (major), $33.8 \mathrm{~min}$ (minor). ${ }^{1} \mathrm{H}$ NMR (600 MHz, $\left.\mathrm{CDCl}_{3}\right) \delta 7.60(\mathrm{~d}, J=7.7 \mathrm{~Hz}, 1 \mathrm{H}), 7.30(\mathrm{t}, J=8.2 \mathrm{~Hz}, 1 \mathrm{H}), 7.25$ $-7.17(\mathrm{~m}, 3 \mathrm{H}), 7.16-7.06(\mathrm{~m}, 3 \mathrm{H}), 6.96(\mathrm{~d}, J=7.3 \mathrm{~Hz}, 2 \mathrm{H}), 6.89-6.71(\mathrm{~m}, 3 \mathrm{H})$, $5.20-5.08(\mathrm{~m}, 2 \mathrm{H}), 3.70-3.55(\mathrm{~m}, 2 \mathrm{H}), 3.21(\mathrm{~d}, J=14.9 \mathrm{~Hz}, 1 \mathrm{H}), 2.76(\mathrm{~d}, J=16.9$ $\mathrm{Hz}, 1 \mathrm{H}) \mathrm{ppm} .{ }^{13} \mathrm{C} \mathrm{NMR}\left(151 \mathrm{MHz}, \mathrm{CDCl}_{3}\right) \delta 202.4,152.7,149.4,143.9,134.5,133.5$, $132.4,129.1,128.4,126.8,125.8,121.1,118.7,115.3,77.0,63.7,51.3,48.8,36.7$ ppm. HRMS (ESI) m/z: [M+H] ${ }^{+}$Calcd. for $\mathrm{C}_{23} \mathrm{H}_{20} \mathrm{NO} 326.1545$, found 326.1539 .

\section{Determination of the absolute configuration of product 4iaa and 7}

Single-crystal X-ray diffraction measurements were carried out on an Agilent SuperNova EosS2 diffractometer using graphite monochromated $\mathrm{Cu} K \alpha$ radiation $(\lambda=$ $1.54184 \AA$ A). The crystals were kept at 150 (10) $\mathrm{K}$ during data collection. The structures were solved by the Superflip3 or ShelXT4 structure solution program in Olex25 and refined using Full-matrix Least Squares based on F2 with program SHELXL-20186 within Olex2. Disorder was modelled using standard crystallographic methods including constraints and restraints where necessary. Crystal data and experimental data for 4iaa and 7 are summarized in Table S6 and S7.

The single crystals of $\mathrm{C}_{25} \mathrm{H}_{21} \mathrm{~N}_{4} \mathrm{O}_{3} \mathrm{P}$ [4iaa] was prepared from a acetone/n-hexane solution at $-20{ }^{\circ} \mathrm{C}$. The single crystals of $\mathrm{C}_{25} \mathrm{H}_{25} \mathrm{NO}_{2}$ [7] was prepared from a 
n-hexane solution at $-20{ }^{\circ} \mathrm{C}$. Both molecular structure of 4iaa and 7, ellipsoids shown at $30 \%$ probability.

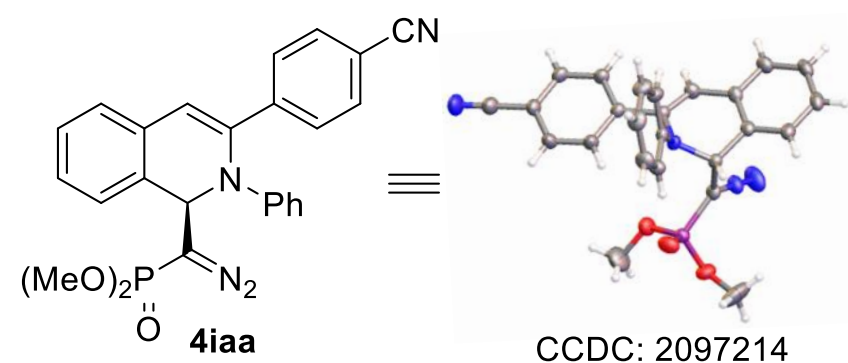

\begin{tabular}{|c|c|}
\hline \multicolumn{2}{|c|}{ Table S7. Crystal data and structure refinement for 4 iaa } \\
\hline Empirical formula & $\mathrm{C}_{25} \mathrm{H}_{21} \mathrm{~N}_{4} \mathrm{O}_{3} \mathrm{P}$ \\
\hline Formula weight & 456.43 \\
\hline Temperature/K & $179.4(9)$ \\
\hline Crystal system & orthorhombic \\
\hline Space group & P212121 \\
\hline $\mathrm{a} / \AA$ & $11.61271(12)$ \\
\hline $\mathrm{b} / \AA ̊ \AA$ & $13.57269(13)$ \\
\hline$c / \AA$ & $14.65006(16)$ \\
\hline$\alpha /^{\circ}$ & 90 \\
\hline$\beta /{ }^{\circ}$ & 90 \\
\hline$\gamma /{ }^{\circ}$ & 90 \\
\hline Volume $/ \AA^{3}$ & 2309.08(4) \\
\hline $\mathrm{Z}$ & 4 \\
\hline$\rho_{\text {calc }} \mathrm{g} / \mathrm{cm}^{3}$ & 1.313 \\
\hline$\mu / \mathrm{mm}^{-1}$ & 1.342 \\
\hline $\mathrm{F}(000)$ & 952 \\
\hline Crystal size $/ \mathrm{mm}^{3}$ & $0.2 \times 0.1 \times 0.1$ \\
\hline Radiation & $\mathrm{CuK} \alpha(\lambda=1.54184)$ \\
\hline $2 \Theta$ range for data collection ${ }^{\circ}$ & 8.882 to 146.048 \\
\hline Index ranges & $-14 \leq \mathrm{h} \leq 13,-16 \leq \mathrm{k} \leq 10,-17 \leq 1 \leq 17$ \\
\hline Reflections collected & 12268 \\
\hline Independent reflections & $4271[$ Rint $=0.0254$, Rsigma $=0.0274]$ \\
\hline
\end{tabular}




\begin{tabular}{|c|c|}
\hline Data/restraints/parameters & $4271 / 0 / 300$ \\
\hline Goodness-of-fit on $\mathrm{F}^{2}$ & 1.039 \\
\hline Final R indexes $[\mathrm{I}>=2 \sigma(\mathrm{I})]$ & $\mathrm{R} 1=0.0324, \mathrm{wR} 2=0.0898$ \\
\hline Final R indexes [all data] & $\mathrm{R} 1=0.0333, \mathrm{wR} 2=0.0909$ \\
\hline Largest diff. peak/hole / e $\AA^{-3}$ & $0.16 /-0.28$ \\
\hline
\end{tabular}

Table S8 Fractional Atomic Coordinates $\left(\times 10^{4}\right)$ and Equivalent Isotropic Displacement Parameters $\left(\AA^{2} \times 1^{3}\right)$ for 4 iaa. $U_{\text {eq }}$ is defined as 1/3 of of the trace of the orthogonalised $U_{I J}$ tensor.

$\begin{array}{ccccc}\text { Atom } & \boldsymbol{x} & \boldsymbol{y} & \boldsymbol{z} & \mathbf{U}(\mathbf{e q}) \\ \text { P1 } & -7287.8(5) & -6455.9(4) & -3193.4(4) & 25.38(15) \\ \text { O1 } & -6845.7(15) & -7463.5(13) & -3207.8(14) & 39.0(4) \\ \text { O2 } & -7491.1(16) & -5985.1(14) & -2233.9(12) & 38.1(4) \\ \text { O3 } & -8513.4(14) & -6317.9(14) & -3641.5(13) & 37.8(4) \\ \text { C3 } & -6328.3(19) & -5656.2(16) & -3741.7(15) & 26.4(5) \\ \text { N1 } & -6698.3(18) & -4775.9(16) & -3972.6(14) & 33.5(5) \\ \text { N3 } & -4472.9(15) & -6114.3(13) & -3063.9(12) & 23.2(4) \\ \text { C14 } & -3986.3(19) & -7858.1(16) & -3213.9(17) & 29.3(5) \\ \text { C12 } & -4210(2) & -5252.6(17) & -2559.4(15) & 25.3(4) \\ \text { C19 } & -4202.4(19) & -5362.6(16) & -1552.4(15) & 24.3(4) \\ \text { C5 } & -4473.1(18) & -5149.4(17) & -4484.9(16) & 25.6(5) \\ \text { C4 } & -5074.7(18) & -5930.7(16) & -3932.5(15) & 23.8(4) \\ \text { C18 } & -2478(2) & -6690.1(18) & -2906.6(16) & 30.7(5) \\ \text { C10 } & -4013.4(19) & -4355.5(17) & -3990.1(16) & 26.7(5) \\ \text { C22 } & -4291(2) & -5535.1(19) & 348.8(16) & 31.0(5) \\ \text { C17 } & -1685(2) & -7459(2) & -2878.5(19) & 38.7(6) \\ \text { C13 } & -3636.7(18) & -6889.0(15) & -3061.6(14) & 23.1(4) \\ \text { C20 } & -4742(2) & -6168.2(18) & -1141.2(17) & 31.9(5) \\ \text { C6 } & -4410.7(19) & -5160.6(19) & -5429.6(16) & 30.0(5) \\ \text { C11 } & -4032.4(19) & -4393.9(17) & -2996.1(16) & 27.3(5) \\ \text { C25 } & -3693(2) & -4651.0(16) & -992.2(16) & 27.1(5) \\ \text { C21 } & -4788(2) & -6258.2(19) & -201.1(16) & 34.6(5) \\ \text { C24 } & -3743(2) & -4729.7(18) & -49.7(17) & 30.1(5) \\ \text { N4 } & -4370(3) & -5720(2) & 2103.4(16) & 58.0(7) \\ \text { N2 } & -7029(2) & -4030.3(19) & -4184(2) & 55.3(7) \\ \text { C9 } & -3524(2) & -3567.2(18) & -4468.8(17) & 32.5(5) \\ \text { C8 } & -3475(2) & -3583(2) & -5415.4(17) & 35.3(5) \\ \text { C7 } & -3908(2) & -4376(2) & -5891.6(17) & 34.7(6) \\ \text { C15 } & -3192(2) & -8616.0(17) & -3193.7(18) & 36.3(5)\end{array}$




$\begin{array}{ccccc}\text { C23 } & -4333(2) & -5636(2) & 1328.9(18) & 39.3(6) \\ \text { C16 } & -2037(2) & -8422(2) & -3018.3(19) & 40.7(6) \\ \text { C1 } & -8389(3) & -6368(3) & -1653(2) & 67.3(10) \\ \text { C2 } & -8755(4) & -6836(4) & -4473(3) & 87.0(15)\end{array}$

Table S9 Anisotropic Displacement Parameters $\left(\AA^{2} \times 10^{3}\right)$ for 4iaa. The Anisotropic displacement factor exponent takes the form:

$$
-2 \pi^{2}\left[h^{2} a^{* 2} U_{11}+2 h k a * b * U_{12}+\ldots .\right] \text {. }
$$

$\begin{array}{ccccccc}\text { Atom } & \mathbf{U}_{\mathbf{1 1}} & \mathbf{U}_{\mathbf{2 2}} & \mathbf{U}_{\mathbf{3 3}} & \mathbf{U}_{\mathbf{2 3}} & \mathbf{U}_{\mathbf{1 3}} & \mathbf{U}_{\mathbf{1 2}} \\ \mathrm{P} 1 & 23.6(3) & 23.3(3) & 29.2(3) & -1.9(2) & 0.4(2) & 0.5(2) \\ \mathrm{O} 1 & 34.0(8) & 25.9(8) & 57.2(11) & 1.7(8) & 4.8(8) & 1.6(7) \\ \mathrm{O} 2 & 40.6(10) & 44.7(10) & 29.0(8) & -5.3(7) & 6.1(7) & -2.4(8) \\ \text { O3 } & 25.8(8) & 43.7(10) & 43.9(10) & -6.1(8) & -4.2(7) & 1.2(7) \\ \text { C3 } & 27.8(11) & 24.4(10) & 26.9(11) & 2.6(9) & -1.2(9) & 4.9(9) \\ \text { N1 } & 32.2(11) & 33.7(10) & 34.5(10) & 9.5(9) & 5.0(8) & 6.7(8) \\ \text { N3 } & 26.0(9) & 21.9(8) & 21.8(9) & -1.2(7) & -3.1(7) & 2.4(7) \\ \text { C14 } & 27.2(10) & 26.8(10) & 33.8(12) & 0.8(10) & 0.3(9) & 0.5(9) \\ \text { C12 } & 26.5(10) & 24.7(10) & 24.7(11) & -2.2(9) & -0.5(8) & 1.4(9) \\ \text { C19 } & 24.4(10) & 25.2(10) & 23.2(11) & -2.4(8) & -1.7(8) & 2.6(8) \\ \text { C5 } & 23.1(10) & 28.2(11) & 25.5(11) & 2.9(9) & 0.2(8) & 4.6(9) \\ \text { C4 } & 25.2(10) & 23.2(9) & 22.9(10) & -1.0(8) & -1.3(8) & 2.3(8) \\ \text { C18 } & 28.0(11) & 34.4(12) & 29.8(11) & -4.7(9) & -3.6(9) & -1.4(9) \\ \text { C10 } & 27.7(10) & 25(1) & 27.3(11) & 1.4(9) & 3.2(9) & 4.9(9) \\ \text { C22 } & 32.2(12) & 38.4(12) & 22.3(11) & -2.1(10) & 0.8(9) & -1.5(10) \\ \text { C17 } & 25.3(11) & 47.3(14) & 43.3(14) & -5.9(12) & -6.2(10) & 6.3(11) \\ \text { C13 } & 25.9(10) & 25(1) & 18.3(9) & 1.4(8) & 0.0(8) & 2.3(8) \\ \text { C20 } & 37.6(12) & 32.0(11) & 26.1(11) & -1(1) & -1.9(10) & -9.2(10) \\ \text { C6 } & 27.1(11) & 38.1(12) & 24.8(11) & 0.5(10) & -0.1(9) & 3.3(10) \\ \text { C11 } & 30.9(11) & 23.4(10) & 27.5(11) & -2.8(9) & 2.7(9) & 0.5(9) \\ \text { C25 } & 29.6(11) & 23.2(10) & 28.6(12) & -1.8(9) & -1.5(9) & -0.3(9) \\ \text { C21 } & 40.3(13) & 36.8(13) & 26.8(11) & 1.5(10) & 1.5(10) & -12.1(11) \\ \text { C24 } & 31.9(12) & 31.3(11) & 27.1(11) & -6.5(10) & -3.8(9) & 0.0(9) \\ \text { N4 } & 68.3(17) & 81(2) & 25.0(12) & 0.7(13) & -0.2(11) & -19.7(16) \\ \text { N2 } & 54.8(15) & 44.8(14) & 66.4(17) & 24.0(13) & 14.4(13) & 21.2(12) \\ \text { C9 } & 34.4(11) & 27.3(10) & 35.7(12) & 4.1(10) & 3.2(10) & 0.9(10) \\ \text { C8 } & 32.5(12) & 36.5(12) & 36.9(13) & 14.0(11) & 7.3(10) & 2.3(11) \\ \text { C7 } & 30.1(11) & 48.0(14) & 26.0(12) & 9.5(11) & 4.2(9) & 6.6(11) \\ \text { C15 } & 41.0(13) & 24.0(11) & 43.8(13) & 1.2(11) & 0.5(11) & 5(1) \\ \text { C23 } & 41.8(14) & 46.6(15) & 29.5(14) & -4.8(12) & 0.9(10) & -8.2(12) \\ \text { C16 } & 38.2(13) & 39.1(13) & 44.7(14) & 1.9(12) & -2.1(11) & 16.2(11) \\ \text { C1 } & 61(2) & 92(3) & 48.8(18) & 0.3(19) & 26.7(16) & -5(2) \\ & & & 37 & & & \end{array}$


$\begin{array}{lllllll}\mathrm{C} 2 & 62(2) & 123(4) & 76(3) & -49(3) & -34(2) & 8(2)\end{array}$

Table S10 Bond Lengths for 4iaa.

$\begin{array}{cccccc}\text { Atom } & \text { Atom } & \text { Length/A } & \text { Atom } & \text { Atom } & \text { Length/A } \\ \text { P1 } & \text { O1 } & 1.4608(17) & \text { C5 } & \text { C4 } & 1.506(3) \\ \text { P1 } & \text { O2 } & 1.5621(17) & \text { C5 } & \text { C10 } & 1.404(3) \\ \text { P1 } & \text { O3 } & 1.5785(17) & \text { C5 } & \text { C6 } & 1.386(3) \\ \text { P1 } & \text { C3 } & 1.751(2) & \text { C18 } & \text { C17 } & 1.393(3) \\ \text { O2 } & \text { C1 } & 1.443(4) & \text { C18 } & \text { C13 } & 1.391(3) \\ \text { O3 } & \text { C2 } & 1.435(4) & \text { C10 } & \text { C11 } & 1.457(3) \\ \text { C3 } & \text { N1 } & 1.314(3) & \text { C10 } & \text { C9 } & 1.400(3) \\ \text { C3 } & \text { C4 } & 1.528(3) & \text { C22 } & \text { C21 } & 1.394(3) \\ \text { N1 } & \text { N2 } & 1.126(3) & \text { C22 } & \text { C24 } & 1.393(3) \\ \text { N3 } & \text { C12 } & 1.417(3) & \text { C22 } & \text { C23 } & 1.443(3) \\ \text { N3 } & \text { C4 } & 1.473(3) & \text { C17 } & \text { C16 } & 1.385(4) \\ \text { N3 } & \text { C13 } & 1.431(3) & \text { C20 } & \text { C21 } & 1.384(3) \\ \text { C14 } & \text { C13 } & 1.395(3) & \text { C6 } & \text { C7 } & 1.390(3) \\ \text { C14 } & \text { C15 } & 1.382(3) & \text { C25 } & \text { C24 } & 1.386(3) \\ \text { C12 } & \text { C19 } & 1.483(3) & \text { N4 } & \text { C23 } & 1.141(4) \\ \text { C12 } & \text { C11 } & 1.346(3) & \text { C9 } & \text { C8 } & 1.388(3) \\ \text { C19 } & \text { C20 } & 1.397(3) & \text { C8 } & \text { C7 } & 1.377(4) \\ \text { C19 } & \text { C25 } & 1.399(3) & \text { C15 } & \text { C16 } & 1.390(4)\end{array}$

Table S11 Bond Angles for 4iaa.

$\begin{array}{cccccccc}\text { Atom } & \text { Atom } & \text { Atom } & \text { Angle }^{\circ} & \text { Atom } & \text { Atom } & \text { Atom } & \text { Angle }{ }^{\circ} \\ \text { O1 } & \text { P1 } & \text { O2 } & 116.68(11) & \text { N3 } & \text { C4 } & \text { C5 } & 111.30(18) \\ \text { O1 } & \text { P1 } & \text { O3 } & 114.96(11) & \text { C5 } & \text { C4 } & \text { C3 } & 111.62(18) \\ \text { O1 } & \text { P1 } & \text { C3 } & 110.49(10) & \text { C13 } & \text { C18 } & \text { C17 } & 120.0(2) \\ \text { O2 } & \text { P1 } & \text { O3 } & 100.92(10) & \text { C5 } & \text { C10 } & \text { C11 } & 118.9(2) \\ \text { O2 } & \text { P1 } & \text { C3 } & 104.80(10) & \text { C9 } & \text { C10 } & \text { C5 } & 118.8(2) \\ \text { O3 } & \text { P1 } & \text { C3 } & 108.03(10) & \text { C9 } & \text { C10 } & \text { C11 } & 122.3(2) \\ \text { C1 } & \text { O2 } & \text { P1 } & 119.5(2) & \text { C21 } & \text { C22 } & \text { C23 } & 119.6(2) \\ \text { C2 } & \text { O3 } & \text { P1 } & 118.1(2) & \text { C24 } & \text { C22 } & \text { C21 } & 119.9(2) \\ \text { N1 } & \text { C3 } & \text { P1 } & 118.28(17) & \text { C24 } & \text { C22 } & \text { C23 } & 120.5(2) \\ \text { N1 } & \text { C3 } & \text { C4 } & 119.1(2) & \text { C16 } & \text { C17 } & \text { C18 } & 120.5(2) \\ \text { C4 } & \text { C3 } & \text { P1 } & 122.62(16) & \text { C14 } & \text { C13 } & \text { N3 } & 119.68(19) \\ \text { N2 } & \text { N1 } & \text { C3 } & 178.6(3) & \text { C18 } & \text { C13 } & \text { N3 } & 120.93(19) \\ \text { C12 } & \text { N3 } & \text { C4 } & 114.41(17) & \text { C18 } & \text { C13 } & \text { C14 } & 119.4(2) \\ \text { C12 } & \text { N3 } & \text { C13 } & 117.33(17) & \text { C21 } & \text { C20 } & \text { C19 } & 121.0(2) \\ \text { C13 } & \text { N3 } & \text { C4 } & 116.62(17) & \text { C5 } & \text { C6 } & \text { C7 } & 120.0(2)\end{array}$




$\begin{array}{cccccccc}\text { C15 } & \text { C14 } & \text { C13 } & 120.3(2) & \text { C12 } & \text { C11 } & \text { C10 } & 120.6(2) \\ \text { N3 } & \text { C12 } & \text { C19 } & 115.92(19) & \text { C24 } & \text { C25 } & \text { C19 } & 120.9(2) \\ \text { C11 } & \text { C12 } & \text { N3 } & 120.0(2) & \text { C20 } & \text { C21 } & \text { C22 } & 119.8(2) \\ \text { C11 } & \text { C12 } & \text { C19 } & 124.0(2) & \text { C25 } & \text { C24 } & \text { C22 } & 119.8(2) \\ \text { C20 } & \text { C19 } & \text { C12 } & 120.4(2) & \text { C8 } & \text { C9 } & \text { C10 } & 120.3(2) \\ \text { C20 } & \text { C19 } & \text { C25 } & 118.5(2) & \text { C7 } & \text { C8 } & \text { C9 } & 120.2(2) \\ \text { C25 } & \text { C19 } & \text { C12 } & 121.1(2) & \text { C8 } & \text { C7 } & \text { C6 } & 120.3(2) \\ \text { C10 } & \text { C5 } & \text { C4 } & 116.1(2) & \text { C14 } & \text { C15 } & \text { C16 } & 120.5(2) \\ \text { C6 } & \text { C5 } & \text { C4 } & 123.6(2) & \text { N4 } & \text { C23 } & \text { C22 } & 179.6(4) \\ \text { C6 } & \text { C5 } & \text { C10 } & 120.3(2) & \text { C17 } & \text { C16 } & \text { C15 } & 119.4(2) \\ \text { N3 } & \text { C4 } & \text { C3 } & 109.58(18) & & & & \end{array}$

Table S12 Hydrogen Atom Coordinates $\left(\AA \times 10^{4}\right)$ and Isotropic Displacement Parameters $\left(\AA^{2} \times 10^{3}\right)$ for 4iaa.

$\begin{array}{ccccc}\text { Atom } & \boldsymbol{x} & \boldsymbol{y} & \boldsymbol{z} & \mathbf{U}(\mathbf{e q}) \\ \text { H14 } & -4757.34 & -7995.08 & -3329.42 & 35 \\ \text { H4 } & -5068.85 & -6543.45 & -4286.3 & 29 \\ \text { H18 } & -2233.48 & -6044.14 & -2821.75 & 37 \\ \text { H17 } & -912.31 & -7324.94 & -2765.06 & 46 \\ \text { H20 } & -5074.97 & -6651.89 & -1505.02 & 38 \\ \text { H6 } & -4705.06 & -5692.77 & -5754.34 & 36 \\ \text { H11 } & -3920.8 & -3819.94 & -2660.75 & 33 \\ \text { H25 } & -3315.3 & -4117.86 & -1255.68 & 33 \\ \text { H21 } & -5148.48 & -6799.31 & 63.17 & 42 \\ \text { H24 } & -3411.45 & -4246.38 & 315.54 & 36 \\ \text { H9 } & -3229.78 & -3029.95 & -4151.08 & 39 \\ \text { H8 } & -3149.2 & -3057.18 & -5729.16 & 42 \\ \text { H7 } & -3863.73 & -4386.59 & -6525.32 & 42 \\ \text { H15 } & -3431.02 & -9260.06 & -3298.27 & 44 \\ \text { H16 } & -1506.63 & -8934.74 & -2995.02 & 49 \\ \text { H1A } & -8482.52 & -7060.22 & -1765.42 & 101 \\ \text { H1B } & -9097.99 & -6032.37 & -1781.24 & 101 \\ \text { H1C } & -8182.62 & -6266.52 & -1025.25 & 101 \\ \text { H2A } & -8293.47 & -6568.81 & -4957.5 & 130 \\ \text { H2B } & -9555.23 & -6764.32 & -4622.94 & 130 \\ \text { H2C } & -8577.07 & -7521.8 & -4396.24 & 130\end{array}$




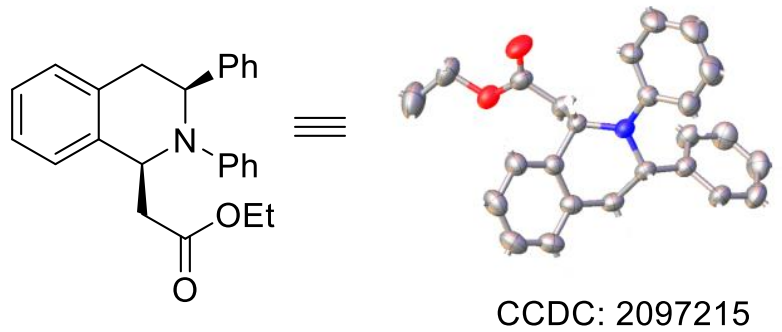

\begin{tabular}{|c|c|}
\hline \multicolumn{2}{|c|}{ Table S13. Crystal data and structure refinement for 7} \\
\hline Empirical formula & $\mathrm{C}_{25} \mathrm{H}_{25} \mathrm{NO}_{2}$ \\
\hline Formula weight & 371.46 \\
\hline Temperature/K & $290.07(11)$ \\
\hline Crystal system & monoclinic \\
\hline Space group & $\mathrm{P} 21$ \\
\hline $\mathrm{a} / \AA ̊$ & $11.00550(10)$ \\
\hline $\mathrm{b} / \AA ̊ \AA$ & $7.38740(10)$ \\
\hline $\mathrm{c} / \AA$ & $12.8816(2)$ \\
\hline$\alpha /^{\circ}$ & 90 \\
\hline$\beta /{ }^{\circ}$ & $98.2430(10)$ \\
\hline$\gamma /{ }^{\circ}$ & 90 \\
\hline Volume $/ \AA^{3}$ & $1036.48(2)$ \\
\hline $\mathrm{Z}$ & 2 \\
\hline$\rho_{\text {calc }} \mathrm{g} / \mathrm{cm}^{3}$ & 1.19 \\
\hline$\mu / \mathrm{mm}^{-1}$ & 0.588 \\
\hline $\mathrm{F}(000)$ & 396 \\
\hline Crystal size $/ \mathrm{mm}^{3}$ & $0.2 \times 0.2 \times 0.2$ \\
\hline Radiation & $\mathrm{CuK} \alpha(\lambda=1.54184)$ \\
\hline $2 \Theta$ range for data collection $/^{\circ}$ & 6.934 to 143.59 \\
\hline Index ranges & $-13 \leq \mathrm{h} \leq 13,-9 \leq \mathrm{k} \leq 6,-15 \leq 1 \leq 15$ \\
\hline Reflections collected & 10618 \\
\hline Independent reflections & $3420[$ Rint $=0.0188$, Rsigma $=0.0178]$ \\
\hline Data/restraints/parameters & $3420 / 1 / 254$ \\
\hline Goodness-of-fit on $\mathrm{F}^{2}$ & 1.043 \\
\hline Final $\mathrm{R}$ indexes $[\mathrm{I}>=2 \sigma(\mathrm{I})]$ & $\mathrm{R} 1=0.0349, \mathrm{wR} 2=0.0905$ \\
\hline Final $\mathrm{R}$ indexes [all data] & $\mathrm{R} 1=0.0358, \mathrm{wR} 2=0.0921$ \\
\hline Largest diff. peak/hole / e $\AA^{-3}$ & $0.10 /-0.18$ \\
\hline
\end{tabular}


Table S14 Fractional Atomic Coordinates $\left(\times 10^{4}\right)$ and Equivalent Isotropic Displacement Parameters $\left(\AA^{2} \times 10^{3}\right)$ for $7 . U_{\text {eq }}$ is defined as $1 / 3$ of of the trace of the orthogonalised $U_{\text {IJ }}$ tensor.

$\begin{array}{ccccc}\text { Atom } & \boldsymbol{x} & \boldsymbol{y} & \boldsymbol{z} & \mathbf{U}(\mathbf{e q}) \\ \mathrm{O} 2 & 3184.5(12) & 4799(3) & 6578.9(10) & 59.0(4) \\ \mathrm{N} 1 & 3151.2(12) & 4578(2) & 2890.1(10) & 44.5(4) \\ \mathrm{O} 1 & 4923.2(13) & 4190(3) & 5953.9(12) & 81.0(6) \\ \mathrm{C} 6 & 2050.0(15) & 2637(3) & 3996.6(14) & 44.0(4) \\ \mathrm{C} 11 & 925.8(15) & 3140(3) & 3432.6(15) & 46.2(4) \\ \mathrm{C} 14 & 2001.0(16) & 6775(3) & 1660.7(14) & 45.8(4) \\ \mathrm{C} 5 & 3144.0(15) & 3861(3) & 3952.2(12) & 41.0(4) \\ \mathrm{C} 3 & 3886.2(16) & 4726(3) & 5828.9(13) & 50.2(5) \\ \mathrm{C} 20 & 4248.4(15) & 4481(3) & 2466.8(14) & 45.5(4) \\ \mathrm{C} 13 & 1997.6(15) & 4954(3) & 2203.1(13) & 43.1(4) \\ \mathrm{C} 7 & 2150.6(19) & 1037(3) & 4561.3(16) & 56.1(5) \\ \mathrm{C} 15 & 1333.6(18) & 6973(4) & 664.0(15) & 55.0(5) \\ \mathrm{C} 12 & 913.7(15) & 4875(3) & 2832.2(14) & 48.4(4) \\ \mathrm{C} 4 & 3243.2(17) & 5382(3) & 4786.9(14) & 48.6(4) \\ \mathrm{C} 19 & 2594(2) & 8273(3) & 2134.3(15) & 56.3(5) \\ \mathrm{C} 21 & 5378.6(16) & 4379(3) & 3120.8(16) & 54.8(5) \\ \mathrm{C} 10 & -80.0(17) & 2004(3) & 3434.3(18) & 58.1(5) \\ \mathrm{C} 25 & 4267.2(19) & 4501(4) & 1381.0(16) & 60.1(5) \\ \mathrm{C} 16 & 1249(2) & 8631(4) & 169.8(17) & 66.2(6) \\ \mathrm{C} 9 & 21(2) & 421(4) & 4006(2) & 67.2(6) \\ \mathrm{C} 22 & 6465(2) & 4258(4) & 2708(2) & 72.6(7) \\ \text { C8 } & 1129(2) & -59(4) & 4574(2) & 66.8(6) \\ \mathrm{C} 18 & 2518(2) & 9927(3) & 1631(2) & 67.8(6) \\ \mathrm{C} 17 & 1843(2) & 10111(4) & 651(2) & 69.0(6) \\ \mathrm{C} 2 & 3762(2) & 4249(5) & 7624.7(15) & 74.2(7) \\ \mathrm{C} 23 & 6469(2) & 4238(5) & 1640(2) & 85.5(9) \\ \mathrm{C} 24 & 4370(5) & 988.7(19) & 79.3(7) \\ \mathrm{C} 1 & 4130(6) & 8290(2) & 106.0(12) \\ & 5374(2) & & & \end{array}$

Table S15 Anisotropic Displacement Parameters $\left(\AA^{2} \times 10^{3}\right)$ for 7. The Anisotropic displacement factor exponent takes the form: $-2 \pi^{2}\left[h^{2} a^{* 2} U_{11}+2 h k a * b * U_{12}+\ldots\right]$.

$\begin{array}{ccccccc}\text { Atom } & \mathbf{U}_{\mathbf{1 1}} & \mathbf{U}_{\mathbf{2 2}} & \mathbf{U}_{\mathbf{3 3}} & \mathbf{U}_{\mathbf{2 3}} & \mathbf{U}_{\mathbf{1 3}} & \mathbf{U}_{\mathbf{1 2}} \\ \mathrm{O} 2 & 57.7(7) & 74.9(11) & 43.1(6) & 0.4(7) & 2.1(5) & 7.6(7) \\ \mathrm{N} 1 & 36.8(6) & 54.9(10) & 40.4(7) & 5.0(7) & 1.2(5) & 2.4(7) \\ \mathrm{O} 1 & 51.2(8) & 130.5(19) & 58.1(8) & 8.4(10) & -3.4(6) & 22.1(10) \\ \mathrm{C} 6 & 41.7(8) & 46.4(11) & 44.4(8) & -1.3(8) & 8.0(7) & 0.4(8) \\ \mathrm{C} 11 & 39.1(8) & 49.3(12) & 50.1(8) & -5.7(9) & 6.0(7) & 0.7(8)\end{array}$




$\begin{array}{ccccccc}\text { C14 } & 42.5(8) & 48.8(11) & 44.6(9) & 0.6(8) & 0.9(7) & 4.7(8) \\ \text { C5 } & 37.1(7) & 45.0(11) & 39.7(7) & 2.7(7) & 2.1(6) & 3.0(7) \\ \text { C3 } & 45.4(9) & 57.5(13) & 45.4(8) & -3.8(9) & -1.7(7) & -0.8(9) \\ \text { C20 } & 45.0(8) & 42.7(11) & 49.7(9) & 0.5(8) & 9.5(7) & 2.8(8) \\ \text { C13 } & 40.2(8) & 45.3(11) & 41.5(7) & -3.0(8) & -2.3(6) & 1.2(7) \\ \text { C7 } & 52.3(10) & 56.3(13) & 60.5(11) & 7.9(10) & 10.6(8) & 0.9(9) \\ \text { C15 } & 52.1(10) & 63.4(14) & 46.4(9) & 2.9(9) & -3.4(8) & -0.2(10) \\ \text { C12 } & 37.0(7) & 54.1(12) & 52.0(9) & -0.2(9) & -1.2(7) & 3.9(8) \\ \text { C4 } & 48.2(8) & 48.6(12) & 47.1(9) & -0.1(8) & -0.3(7) & 0.9(8) \\ \text { C19 } & 65.7(11) & 47.5(13) & 52.2(10) & -2.9(10) & -3.8(9) & 2.6(10) \\ \text { C21 } & 41.9(8) & 62.7(14) & 60(1) & 5.1(10) & 8.1(7) & 2.1(9) \\ \text { C10 } & 40.5(9) & 64.5(15) & 69.7(12) & -7.5(11) & 9.7(8) & -3.9(9) \\ \text { C25 } & 61.3(11) & 69.7(15) & 50.7(9) & 0.4(11) & 13.1(8) & 6.7(11) \\ \text { C16 } & 64.8(12) & 79.2(18) & 52.7(10) & 16.4(12) & 1.9(9) & 13.6(12) \\ \text { C9 } & 55.2(11) & 62.5(16) & 88.0(15) & -6.8(13) & 24.2(11) & -13.8(11) \\ \text { C22 } & 46.4(10) & 88(2) & 85.8(15) & 11.7(15) & 16.7(10) & 10.1(12) \\ \text { C8 } & 68.7(12) & 55.7(14) & 80.3(14) & 9.6(12) & 25.4(11) & -4.7(12) \\ \text { C18 } & 84.1(15) & 44.8(13) & 73.9(13) & -3.7(11) & 9.4(11) & 0.4(12) \\ \text { C17 } & 85.2(15) & 51.6(14) & 72.4(13) & 15.9(12) & 18.6(11) & 14.8(12) \\ \text { C2 } & 88.3(15) & 88(2) & 42.8(9) & 1.8(11) & -2.3(10) & 14.1(15) \\ \text { C23 } & 62.9(13) & 106(2) & 95.9(18) & 8.5(18) & 38.1(13) & 15.1(15) \\ \text { C24 } & 83.2(15) & 96(2) & 65.3(12) & 1.9(15) & 34.7(12) & 8.9(16) \\ \text { C1 } & 130(3) & 134(3) & 58.7(13) & 17.9(17) & 30.2(15) & 32(2)\end{array}$

Table S16 Bond Lengths for 7.

\begin{tabular}{|c|c|c|c|c|c|}
\hline Atom & Atom & Length/Å & Atom & Atom & Length/Å \\
\hline $\mathrm{O} 2$ & $\mathrm{C} 3$ & $1.322(2)$ & $\mathrm{C} 20$ & $\mathrm{C} 21$ & $1.401(3)$ \\
\hline $\mathrm{O} 2$ & $\mathrm{C} 2$ & $1.462(2)$ & $\mathrm{C} 20$ & $\mathrm{C} 25$ & $1.402(3)$ \\
\hline $\mathrm{N} 1$ & C5 & $1.468(2)$ & C13 & $\mathrm{C} 12$ & $1.536(2)$ \\
\hline $\mathrm{N} 1$ & $\mathrm{C} 20$ & $1.396(2)$ & $\mathrm{C} 7$ & $\mathrm{C} 8$ & $1.387(3)$ \\
\hline $\mathrm{N} 1$ & $\mathrm{C} 13$ & $1.4663(19)$ & $\mathrm{C} 15$ & $\mathrm{C} 16$ & $1.378(3)$ \\
\hline O1 & $\mathrm{C} 3$ & $1.197(2)$ & C19 & $\mathrm{C} 18$ & $1.380(3)$ \\
\hline C6 & $\mathrm{C} 11$ & $1.392(2)$ & $\mathrm{C} 21$ & $\mathrm{C} 22$ & $1.380(3)$ \\
\hline C6 & $\mathrm{C} 5$ & $1.513(2)$ & $\mathrm{C} 10$ & C9 & $1.378(4)$ \\
\hline C6 & C7 & $1.384(3)$ & $\mathrm{C} 25$ & $\mathrm{C} 24$ & $1.387(3)$ \\
\hline $\mathrm{C} 11$ & $\mathrm{C} 12$ & $1.497(3)$ & C16 & $\mathrm{C} 17$ & $1.375(4)$ \\
\hline $\mathrm{C} 11$ & $\mathrm{C} 10$ & $1.389(3)$ & C9 & $\mathrm{C} 8$ & $1.376(3)$ \\
\hline $\mathrm{C} 14$ & $\mathrm{C} 13$ & $1.516(3)$ & $\mathrm{C} 22$ & $\mathrm{C} 23$ & $1.376(4)$ \\
\hline $\mathrm{C} 14$ & $\mathrm{C} 15$ & $1.392(2)$ & C18 & $\mathrm{C} 17$ & $1.376(4)$ \\
\hline $\mathrm{C} 14$ & $\mathrm{C} 19$ & $1.382(3)$ & $\mathrm{C} 2$ & $\mathrm{C} 1$ & $1.496(4)$ \\
\hline
\end{tabular}




$\begin{array}{llllll}\mathrm{C} 5 & \mathrm{C} 4 & 1.548(3) & \mathrm{C} 23 & \mathrm{C} 24 & 1.369(4) \\ \mathrm{C} 3 & \mathrm{C} 4 & 1.505(2) & & & \end{array}$

Table S17 Bond Angles for 7.

\begin{tabular}{|c|c|c|c|c|c|c|c|}
\hline Atom & Atom & Atom & Angle $/^{\circ}$ & Atom & Atom & Atom & Angle ${ }^{\circ}$ \\
\hline $\mathrm{C} 3$ & $\mathrm{O} 2$ & $\mathrm{C} 2$ & $116.09(16)$ & $\mathrm{C} 21$ & $\mathrm{C} 20$ & $\mathrm{C} 25$ & $117.50(17)$ \\
\hline $\mathrm{C} 20$ & $\mathrm{~N} 1$ & $\mathrm{C} 5$ & $117.77(14)$ & $\mathrm{N} 1$ & $\mathrm{C} 13$ & $\mathrm{C} 14$ & $112.78(15)$ \\
\hline $\mathrm{C} 20$ & $\mathrm{~N} 1$ & $\mathrm{C} 13$ & $119.77(13)$ & N1 & $\mathrm{C} 13$ & $\mathrm{C} 12$ & $110.18(13)$ \\
\hline $\mathrm{C} 13$ & $\mathrm{~N} 1$ & $\mathrm{C} 5$ & $120.72(13)$ & $\mathrm{C} 14$ & $\mathrm{C} 13$ & $\mathrm{C} 12$ & $109.26(15)$ \\
\hline $\mathrm{C} 11$ & C6 & $\mathrm{C} 5$ & $118.25(17)$ & C6 & $\mathrm{C} 7$ & $\mathrm{C} 8$ & $120.06(19)$ \\
\hline $\mathrm{C} 7$ & C6 & C11 & $120.00(18)$ & $\mathrm{C} 16$ & $\mathrm{C} 15$ & $\mathrm{C} 14$ & $120.7(2)$ \\
\hline $\mathrm{C} 7$ & C6 & $\mathrm{C} 5$ & $121.73(16)$ & $\mathrm{C} 11$ & $\mathrm{C} 12$ & $\mathrm{C} 13$ & $110.79(15)$ \\
\hline C6 & $\mathrm{C} 11$ & $\mathrm{C} 12$ & $116.06(16)$ & $\mathrm{C} 3$ & $\mathrm{C} 4$ & $\mathrm{C} 5$ & $111.29(17)$ \\
\hline $\mathrm{C} 10$ & $\mathrm{C} 11$ & C6 & 119.1(2) & $\mathrm{C} 18$ & C19 & $\mathrm{C} 14$ & $120.55(18)$ \\
\hline $\mathrm{C} 10$ & $\mathrm{C} 11$ & $\mathrm{C} 12$ & $124.76(17)$ & $\mathrm{C} 22$ & $\mathrm{C} 21$ & $\mathrm{C} 20$ & $121.0(2)$ \\
\hline $\mathrm{C} 15$ & $\mathrm{C} 14$ & $\mathrm{C} 13$ & $118.82(18)$ & C9 & $\mathrm{C} 10$ & $\mathrm{C} 11$ & $120.7(2)$ \\
\hline $\mathrm{C} 19$ & $\mathrm{C} 14$ & $\mathrm{C} 13$ & $122.65(16)$ & $\mathrm{C} 24$ & $\mathrm{C} 25$ & $\mathrm{C} 20$ & $120.1(2)$ \\
\hline $\mathrm{C} 19$ & $\mathrm{C} 14$ & C15 & $118.46(19)$ & $\mathrm{C} 17$ & $\mathrm{C} 16$ & $\mathrm{C} 15$ & $120.17(19)$ \\
\hline N1 & $\mathrm{C} 5$ & C6 & $111.18(14)$ & $\mathrm{C} 8$ & C9 & $\mathrm{C} 10$ & $120.0(2)$ \\
\hline N1 & $\mathrm{C} 5$ & $\mathrm{C} 4$ & $112.11(16)$ & $\mathrm{C} 23$ & $\mathrm{C} 22$ & $\mathrm{C} 21$ & $120.9(2)$ \\
\hline C6 & $\mathrm{C} 5$ & $\mathrm{C} 4$ & $112.62(14)$ & C9 & $\mathrm{C} 8$ & $\mathrm{C} 7$ & $120.1(2)$ \\
\hline $\mathrm{O} 2$ & $\mathrm{C} 3$ & $\mathrm{C} 4$ & $112.93(15)$ & $\mathrm{C} 17$ & $\mathrm{C} 18$ & C19 & $120.4(2)$ \\
\hline $\mathrm{O} 1$ & $\mathrm{C} 3$ & $\mathrm{O} 2$ & $124.11(17)$ & $\mathrm{C} 16$ & $\mathrm{C} 17$ & $\mathrm{C} 18$ & $119.6(2)$ \\
\hline $\mathrm{O} 1$ & $\mathrm{C} 3$ & $\mathrm{C} 4$ & $122.96(17)$ & $\mathrm{O} 2$ & $\mathrm{C} 2$ & $\mathrm{C} 1$ & $106.5(2)$ \\
\hline $\mathrm{N} 1$ & $\mathrm{C} 20$ & $\mathrm{C} 21$ & $120.73(16)$ & $\mathrm{C} 24$ & $\mathrm{C} 23$ & $\mathrm{C} 22$ & $118.9(2)$ \\
\hline N1 & $\mathrm{C} 20$ & $\mathrm{C} 25$ & $121.76(16)$ & $\mathrm{C} 23$ & $\mathrm{C} 24$ & $\mathrm{C} 25$ & $121.6(2)$ \\
\hline
\end{tabular}

Table S18 Torsion Angles for 7.

$\begin{array}{cccccccccc}\mathbf{A} & \mathbf{B} & \mathbf{C} & \mathbf{D} & \text { Angle }^{\circ} & \mathbf{A} & \mathbf{B} & \mathbf{C} & \mathbf{D} & \text { Angle }^{\circ} \\ \mathrm{O} 2 & \mathrm{C} 3 & \mathrm{C} 4 & \mathrm{C} 5 & -118.18(19) & \mathrm{C} 20 & \mathrm{C} 21 & \mathrm{C} 22 & \mathrm{C} 23 & 0.1(4) \\ \mathrm{N} 1 & \mathrm{C} 5 & \mathrm{C} 4 & \mathrm{C} 3 & -149.78(15) & \mathrm{C} 20 & \mathrm{C} 25 & \mathrm{C} 24 & \mathrm{C} 23 & -0.7(5) \\ \mathrm{N} 1 & \mathrm{C} 20 & \mathrm{C} 21 & \mathrm{C} 22 & 179.1(2) & \mathrm{C} 13 & \mathrm{~N} 1 & \mathrm{C} 5 & \mathrm{C} 6 & 32.9(2) \\ \mathrm{N} 1 & \mathrm{C} 20 & \mathrm{C} 25 & \mathrm{C} 24 & -178.9(2) & \mathrm{C} 13 & \mathrm{~N} 1 & \mathrm{C} 5 & \mathrm{C} 4 & -94.2(2) \\ \mathrm{N} 1 & \mathrm{C} 13 & \mathrm{C} 12 & \mathrm{C} 11 & -52.1(2) & \mathrm{C} 13 & \mathrm{~N} 1 & \mathrm{C} 20 & \mathrm{C} 21 & 170.9(2) \\ \mathrm{O} 1 & \mathrm{C} 3 & \mathrm{C} 4 & \mathrm{C} 5 & 61.5(3) & \mathrm{C} 13 & \mathrm{~N} 1 & \mathrm{C} 20 & \mathrm{C} 25 & -8.3(3) \\ \mathrm{C} 6 & \mathrm{C} 11 & \mathrm{C} 12 & \mathrm{C} 13 & 47.3(2) & \mathrm{C} 13 & \mathrm{C} 14 & \mathrm{C} 15 & \mathrm{C} 16 & 175.94(19) \\ \mathrm{C} 6 & \mathrm{C} 11 & \mathrm{C} 10 & \mathrm{C} 9 & 1.6(3) & \mathrm{C} 13 & \mathrm{C} 14 & \mathrm{C} 19 & \mathrm{C} 18 & -176.6(2) \\ \mathrm{C} 6 & \mathrm{C} 5 & \mathrm{C} 4 & \mathrm{C} 3 & 83.94(19) & \mathrm{C} 7 & \mathrm{C} 6 & \mathrm{C} 11 & \mathrm{C} 12 & -178.98(17) \\ \mathrm{C} 6 & \mathrm{C} 7 & \mathrm{C} 8 & \mathrm{C} 9 & 1.5(4) & \mathrm{C} 7 & \mathrm{C} 6 & \mathrm{C} 11 & \mathrm{C} 10 & -0.9(3) \\ \mathrm{C} 11 & \mathrm{C} 6 & \mathrm{C} 5 & \mathrm{~N} 1 & -39.9(2) & \mathrm{C} 7 & \mathrm{C} 6 & \mathrm{C} 5 & \mathrm{~N} 1 & 138.84(18)\end{array}$




$\begin{array}{cccccccccc}\mathrm{C} 11 & \mathrm{C} 6 & \mathrm{C} 5 & \mathrm{C} 4 & 86.9(2) & \mathrm{C} 7 & \mathrm{C} 6 & \mathrm{C} 5 & \mathrm{C} 4 & -94.4(2) \\ \mathrm{C} 11 & \mathrm{C} 6 & \mathrm{C} 7 & \mathrm{C} 8 & -0.6(3) & \mathrm{C} 15 & \mathrm{C} 14 & \mathrm{C} 13 & \mathrm{~N} 1 & 148.18(17) \\ \mathrm{C} 11 & \mathrm{C} 10 & \mathrm{C} 9 & \mathrm{C} 8 & -0.7(4) & \mathrm{C} 15 & \mathrm{C} 14 & \mathrm{C} 13 & \mathrm{C} 12 & -88.93(19) \\ \mathrm{C} 14 & \mathrm{C} 13 & \mathrm{C} 12 & \mathrm{C} 11 & -176.50(14) & \mathrm{C} 15 & \mathrm{C} 14 & \mathrm{C} 19 & \mathrm{C} 18 & 0.4(3) \\ \mathrm{C} 14 & \mathrm{C} 15 & \mathrm{C} 16 & \mathrm{C} 17 & 1.1(3) & \mathrm{C} 15 & \mathrm{C} 16 & \mathrm{C} 17 & \mathrm{C} 18 & -0.2(4) \\ \mathrm{C} 14 & \mathrm{C} 19 & \mathrm{C} 18 & \mathrm{C} 17 & 0.4(4) & \mathrm{C} 12 & \mathrm{C} 11 & \mathrm{C} 10 & \mathrm{C} 9 & 179.4(2) \\ \mathrm{C} 5 & \mathrm{~N} 1 & \mathrm{C} 20 & \mathrm{C} 21 & -24.0(3) & \mathrm{C} 19 & \mathrm{C} 14 & \mathrm{C} 13 & \mathrm{~N} 1 & -34.9(2) \\ \mathrm{C} 5 & \mathrm{~N} 1 & \mathrm{C} 20 & \mathrm{C} 25 & 156.8(2) & \mathrm{C} 19 & \mathrm{C} 14 & \mathrm{C} 13 & \mathrm{C} 12 & 88.0(2) \\ \mathrm{C} 5 & \mathrm{~N} 1 & \mathrm{C} 13 & \mathrm{C} 14 & 134.17(17) & \mathrm{C} 19 & \mathrm{C} 14 & \mathrm{C} 15 & \mathrm{C} 16 & -1.2(3) \\ \mathrm{C} 5 & \mathrm{~N} 1 & \mathrm{C} 13 & \mathrm{C} 12 & 11.8(3) & \mathrm{C} 19 & \mathrm{C} 18 & \mathrm{C} 17 & \mathrm{C} 16 & -0.5(4) \\ \mathrm{C} 5 & \mathrm{C} 6 & \mathrm{C} 11 & \mathrm{C} 12 & -0.2(2) & \mathrm{C} 21 & \mathrm{C} 20 & \mathrm{C} 25 & \mathrm{C} 24 & 1.8(4) \\ \mathrm{C} 5 & \mathrm{C} 6 & \mathrm{C} 11 & \mathrm{C} 10 & 177.84(17) & \mathrm{C} 21 & \mathrm{C} 22 & \mathrm{C} 23 & \mathrm{C} 24 & 1.1(5) \\ \mathrm{C} 5 & \mathrm{C} 6 & \mathrm{C} 7 & \mathrm{C} 8 & -179.3(2) & \mathrm{C} 10 & \mathrm{C} 11 & \mathrm{C} 12 & \mathrm{C} 13 & -130.66(19) \\ \mathrm{C} 3 & \mathrm{O} 2 & \mathrm{C} 2 & \mathrm{C} 1 & -170.4(3) & \mathrm{C} 10 & \mathrm{C} 9 & \mathrm{C} 8 & \mathrm{C} 7 & -0.9(4) \\ \mathrm{C} 20 & \mathrm{~N} 1 & \mathrm{C} 5 & \mathrm{C} 6 & -132.09(18) & \mathrm{C} 25 & \mathrm{C} 20 & \mathrm{C} 21 & \mathrm{C} 22 & -1.6(4) \\ \mathrm{C} 20 & \mathrm{~N} 1 & \mathrm{C} 5 & \mathrm{C} 4 & 100.86(19) & \mathrm{C} 22 & \mathrm{C} 23 & \mathrm{C} 24 & \mathrm{C} 25 & -0.8(5) \\ \mathrm{C} 20 & \mathrm{~N} 1 & \mathrm{C} 13 & \mathrm{C} 14 & -61.2(2) & \mathrm{C} 2 & \mathrm{O} 2 & \mathrm{C} 3 & \mathrm{O} 1 & 2.7(3) \\ \mathrm{C} 20 & \mathrm{~N} 1 & \mathrm{C} 13 & \mathrm{C} 12 & 176.45(17) & \mathrm{C} 2 & \mathrm{O} 2 & \mathrm{C} 3 & \mathrm{C} 4 & -177.7(2)\end{array}$

Table S19 Hydrogen Atom Coordinates $\left(\AA \times 10^{4}\right)$ and Isotropic Displacement Parameters $\left(\AA^{2} \times 10^{3}\right)$ for 7.

$\begin{array}{ccccc}\text { Atom } & \boldsymbol{x} & \boldsymbol{y} & \boldsymbol{z} & \mathbf{U}(\mathbf{e q}) \\ \text { H5 } & 3879.48 & 3107.63 & 4115.06 & 49 \\ \text { H13 } & 1876.94 & 4011.73 & 1662.46 & 52 \\ \text { H7 } & 2903.51 & 696.48 & 4932.45 & 67 \\ \text { H15 } & 940.65 & 5973.86 & 328.21 & 66 \\ \text { H12A } & 962.91 & 5888.27 & 3315.69 & 58 \\ \text { H12B } & 148.81 & 4975.06 & 2357.14 & 58 \\ \text { H4A } & 3692.71 & 6396.05 & 4551.5 & 58 \\ \text { H4B } & 2426.58 & 5800.89 & 4867.43 & 58 \\ \text { H19 } & 3049.23 & 8166.52 & 2797.98 & 68 \\ \text { H21 } & 5396.87 & 4393.05 & 3844.8 & 66 \\ \text { H10 } & -829.1 & 2315.89 & 3045.84 & 70 \\ \text { H25 } & 3536.35 & 4602.37 & 922.74 & 72 \\ \text { H16 } & 788.93 & 8749.59 & -490.96 & 79 \\ \text { H9 } & -660.37 & -323.53 & 4007.12 & 81 \\ \text { H22 } & 7204.94 & 4189.54 & 3156.7 & 87 \\ \text { H8 } & 1193.9 & -1119.04 & 4967.54 & 80 \\ \text { H18 } & 2925.54 & 10924.53 & 1955.29 & 81 \\ \text { H17 } & 1787.95 & 11229.54 & 316.44 & 83\end{array}$




$\begin{array}{lcccc}\text { H2A } & 4370.99 & 5133.06 & 7911.24 & 89 \\ \text { H2B } & 4162.82 & 3084.55 & 7593.98 & 89 \\ \text { H23 } & 7202.56 & 4136.34 & 1364.97 & 103 \\ \text { H24 } & 5372.13 & 4372.84 & 266.52 & 95 \\ \text { H1A } & 2157.47 & 3261.06 & 7992.42 & 159 \\ \text { H1B } & 2374.7 & 5292.94 & 8318.21 & 159 \\ \text { H1C } & 3094.45 & 3758.04 & 8985.92 & 159\end{array}$

\section{Proposed catalytic cycle}

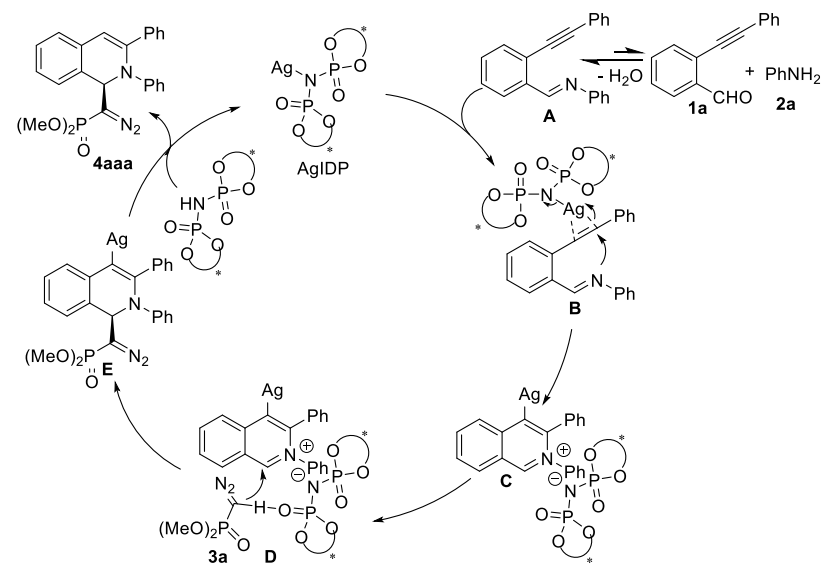

First, the $o$-alkynylbenzaldehyde and aniline dehydrated to form imine $\mathbf{A}$ (We have also employed the preformed imine of $1 \mathbf{a}$ and $\mathbf{2 a}$ in the reaction, the same good results were obtained), and the $\pi$ acidity of the silver salt (AgIDP) allows it to activate the triple bond by coordination. This is followed by an intramolecular imine-yne 6-endo-dig cyclization to give the zwitterionic intermediate $\mathbf{C}$ with silver attached at the 4-position. Then, the (diazomethyl)phosphonate nucleophile attacks the C1-position of the isoquinolinium ion from the Si-face while the oxygen of the phosphine oxide double bond in the chiral imidodiphosphate interacts with the $\alpha$-hydrogen of the (diazomethyl)phosphonate at the same time to promote the process and abstracting the $\alpha$-hydrogen to give intermediate E. Finally, protonation of intermediate $\mathbf{E}$ affords the target product $4 \mathbf{a a a}$ and regenerates the catalyst AgIDP.

\section{References}

(1) (a) Jong, H. P.; Sachin, V. B.; So, W. Y. Org. Lett. 2011, 13, 2228. (b) Zou, L.; Huang, J.; Liao, N.; Liu, Y.; Guo, Q.; Peng, Y. Org. Lett. 2020, 22, 6932.

(2) (a) Wu, W.; Wang, Y.; Guo, J.; Cai, L.; Chen, Y.; Huang, Y.; Peng, Y. Chem. Commun. 2020, 56, 11235. (b) Chen, J.; Wen, X. J.; Wang, Y.; Du, F.; Cai, L.; Peng, 
Y. Org. Lett. 2016, 18, 4336. (c) Xu, B.; Zhu, S. F.; Xie, X. L.; Shen, J. J.; Zhou, Q. L. Angew. Chem. Int. Ed. 2011, 50, 11483.

(3) Rosevear, J.; Wilshire, F. K. Aust. J. Chem. 1985, 38, 1163.

(4) Zheng, B.; Chen, H. H.; Zhu, L.; Hou, X. Q.; Wang, Y.; Lan, Y.; Peng, Y. Org. Lett. 2019, 21, 593.

(5) (a) Coric, I.; List, B. Nature 2012, 483, 315. (b) Liu, L.; Leutzsch, M.; Zheng, Y.; Alachraf, M. W.; Thiel, W.; List, B. J. Am. Chem. Soc. 2015, 137, 13268. (c) Tsui, G. C.; Liu, L.; List, B. Angew. Chem. Int. Ed. 2015, 54, 7703. (d) Xie, Y.; Cheng, G. J.; Lee, S.; Kaib, P. S.; Thiel, W.; List, B. J. Am. Chem. Soc. 2016, 138, 14538. 


\section{The spectra of ${ }^{1} \mathrm{H}$ NMR, ${ }^{13} \mathrm{C}$ NMR and HPLC}

$600 \mathrm{MHz}, \mathrm{CDCl}_{3},{ }^{1} \mathrm{H} \mathrm{NMR}$

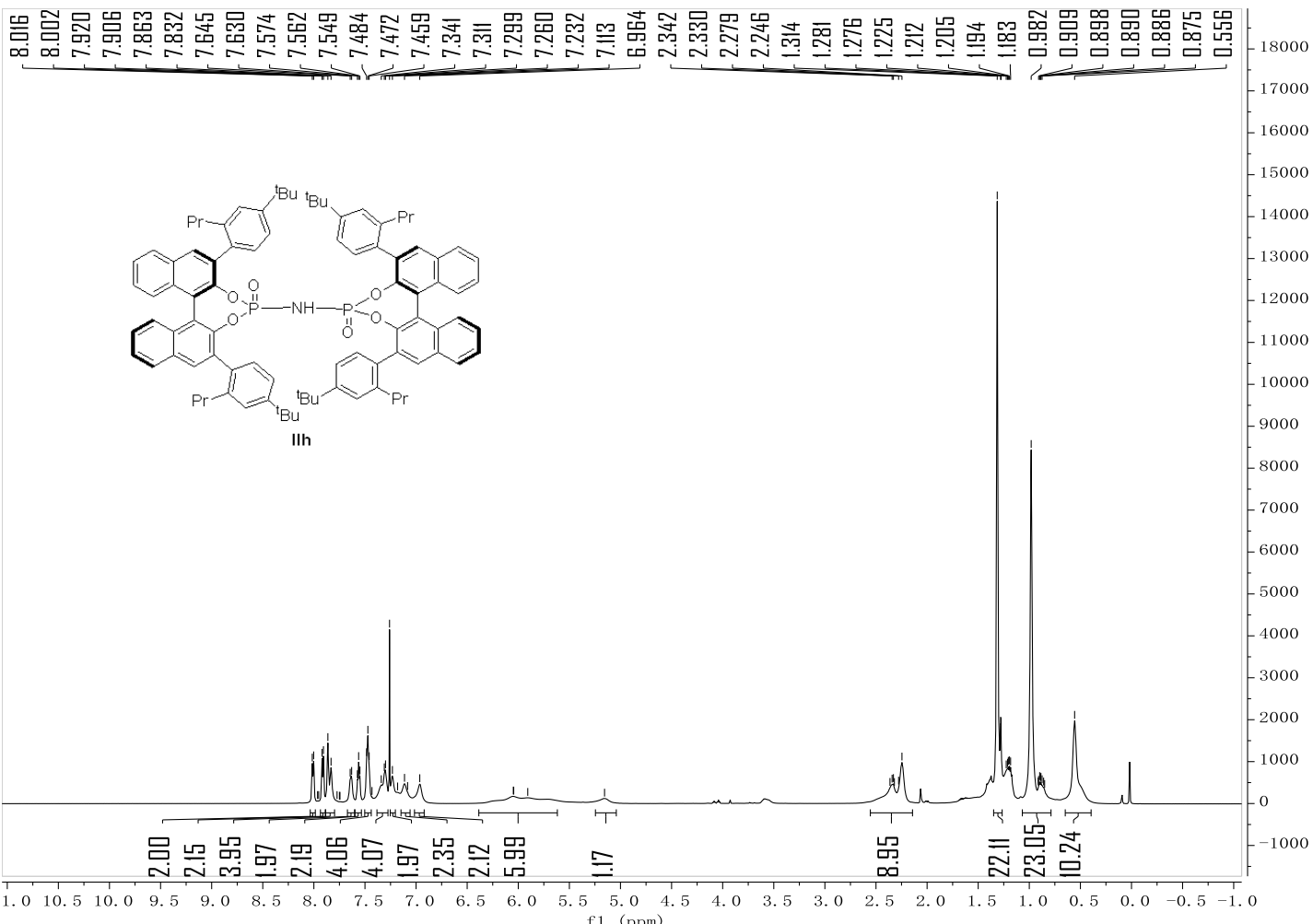

$151 \mathrm{MHz}, \mathrm{CDCl}_{3},{ }^{13} \mathrm{C} \mathrm{NMR}$

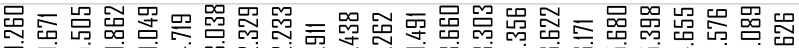

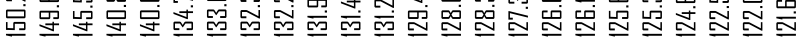
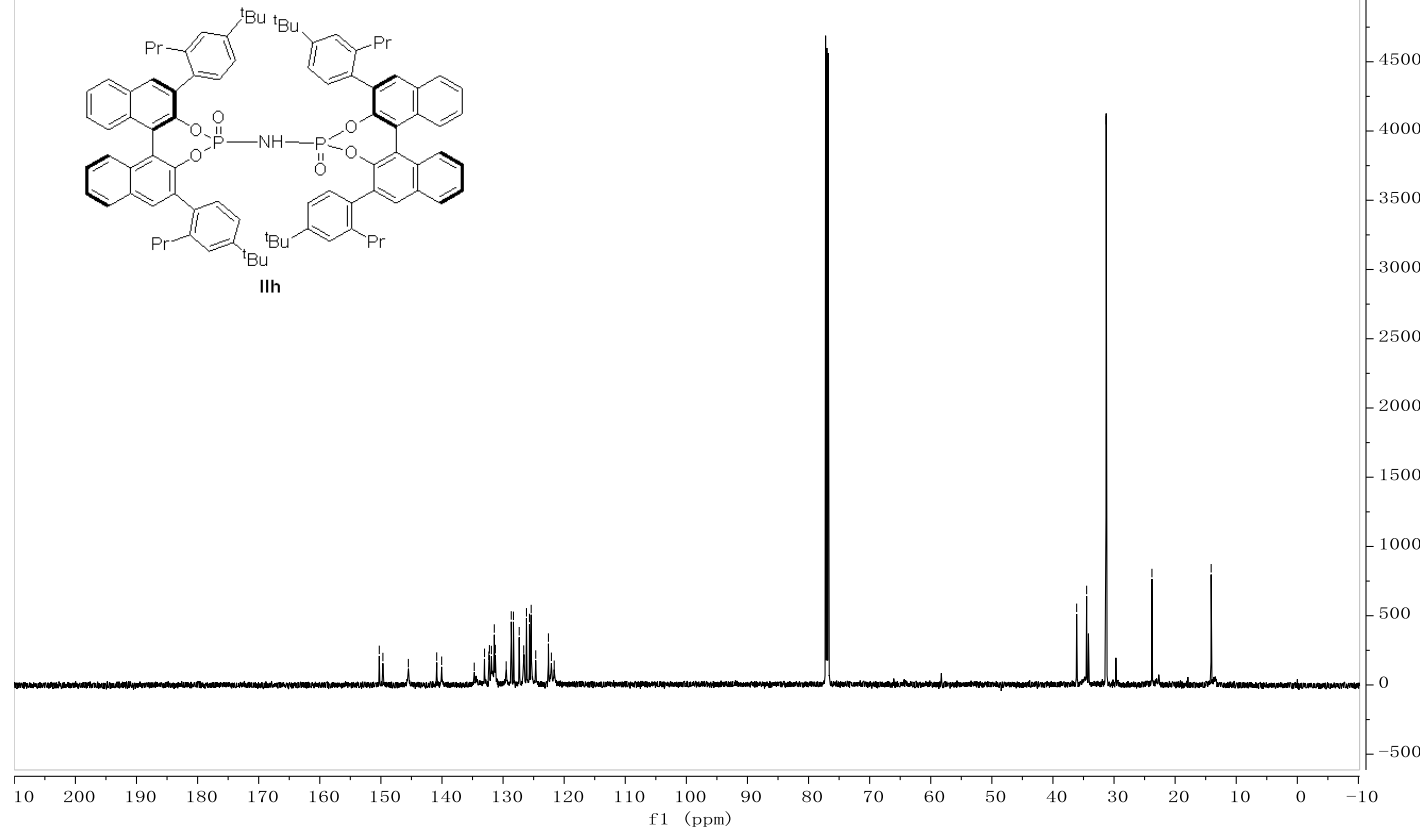

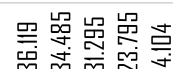

m的式

6000 
$600 \mathrm{MHz}, \mathrm{CDCl}_{3},{ }^{1} \mathrm{H} \mathrm{NMR}$

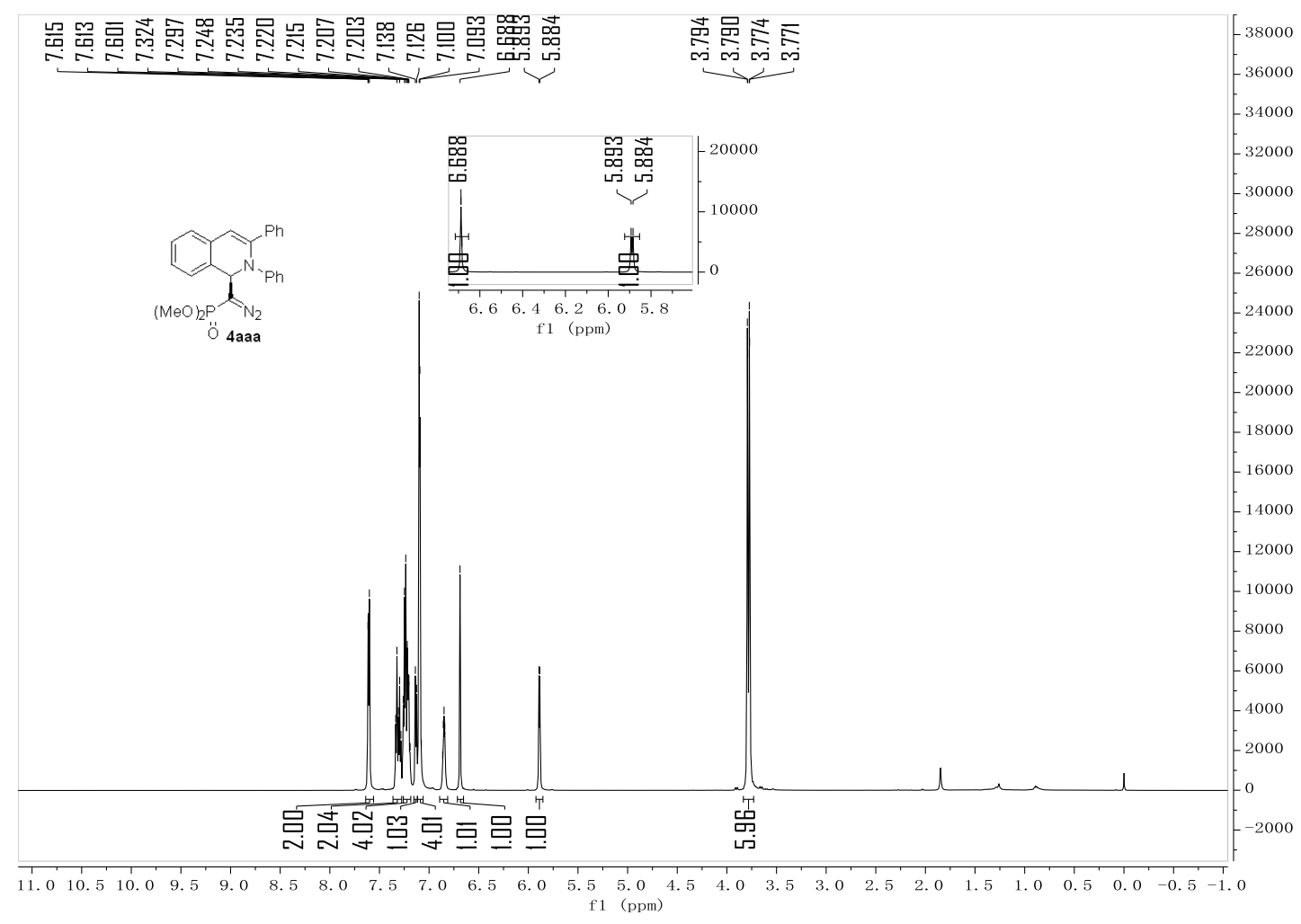

$151 \mathrm{MHz}, \mathrm{CDCl}_{3},{ }^{13} \mathrm{C} \mathrm{NMR}$

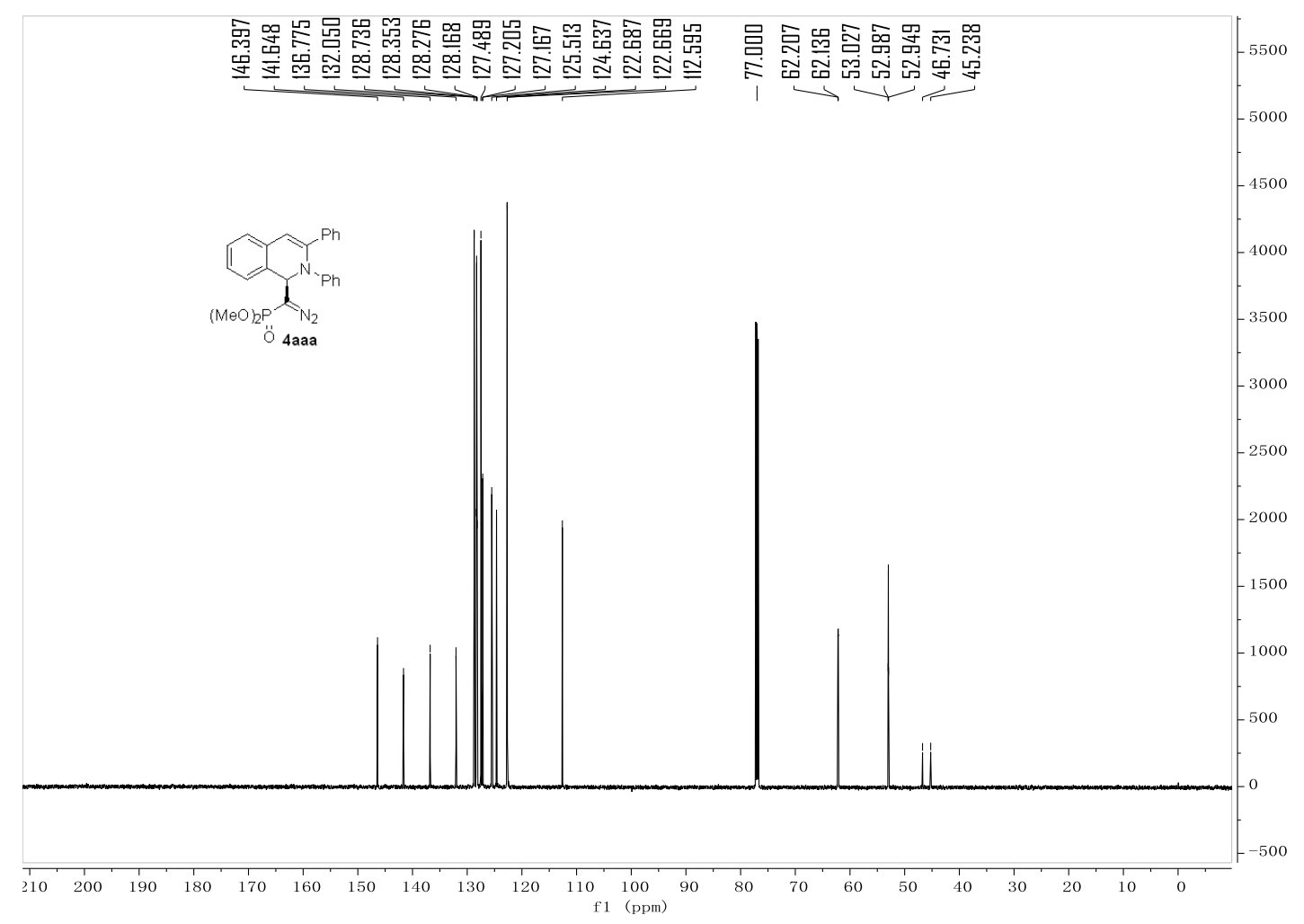




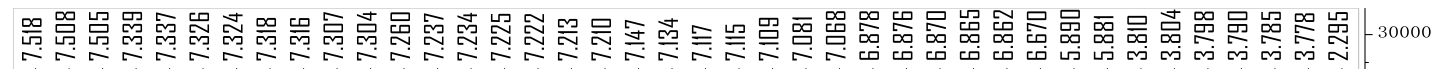

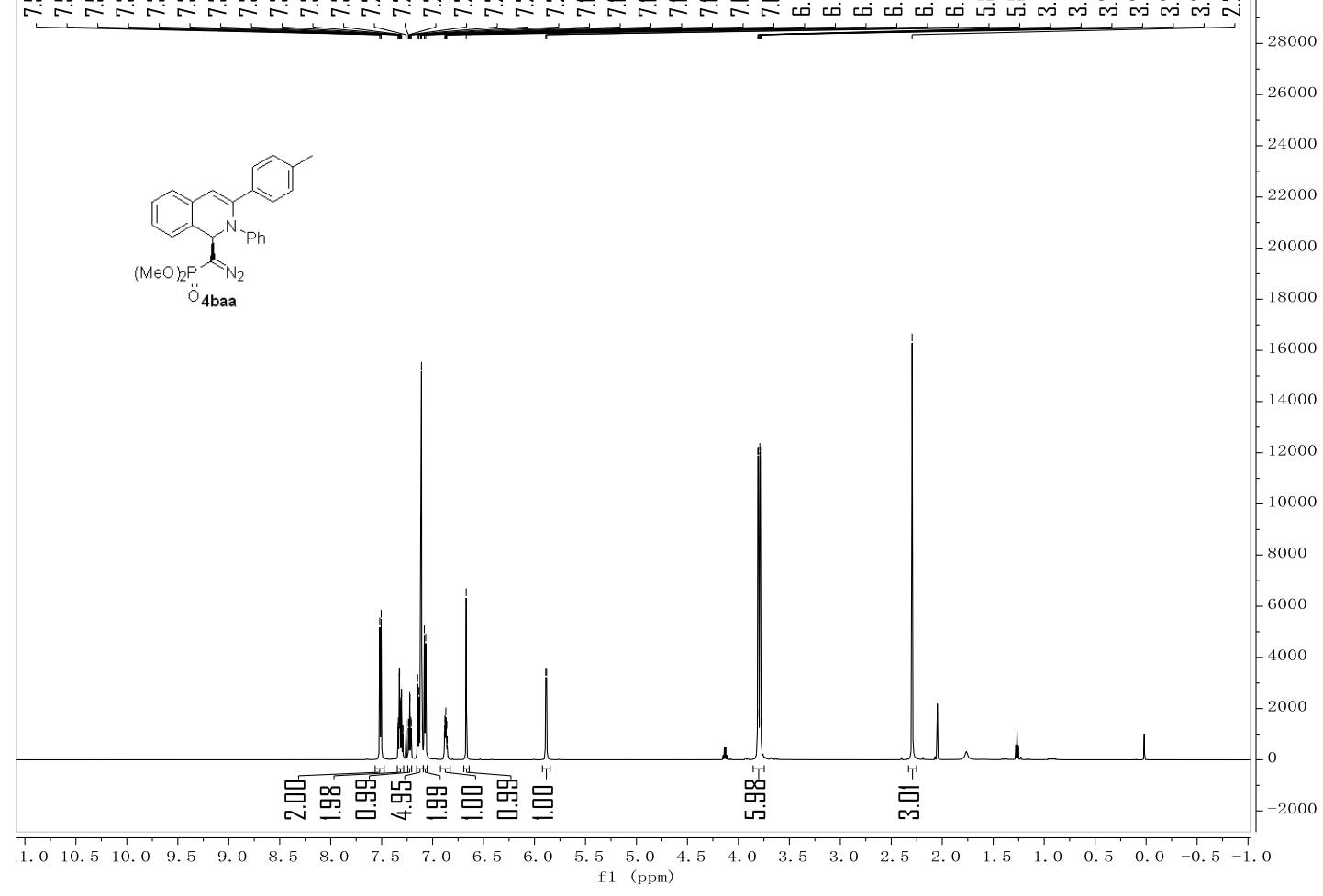

$151 \mathrm{MHz}, \mathrm{CDCl}_{3},{ }^{13} \mathrm{C} \mathrm{NMR}$

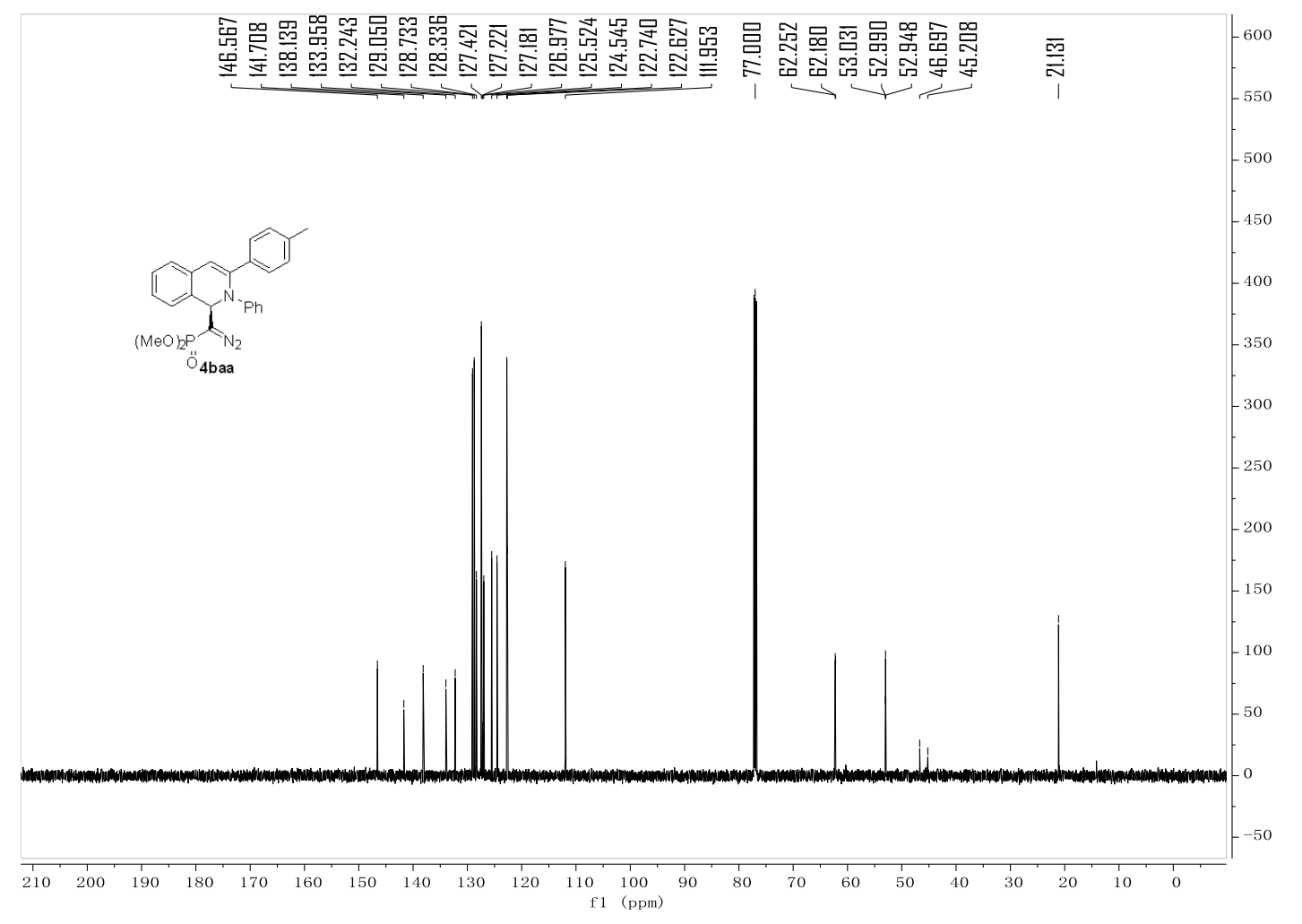




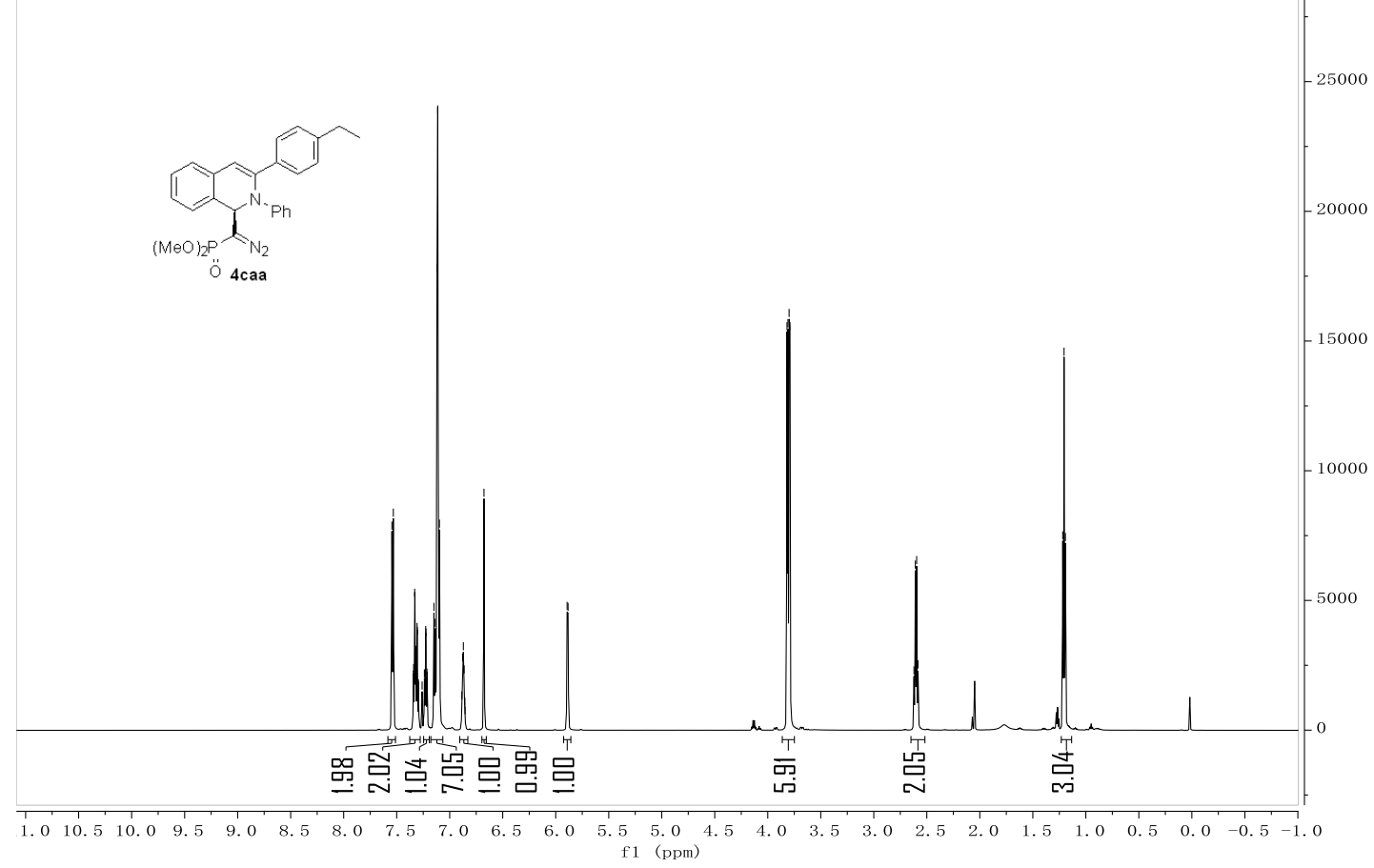

$151 \mathrm{MHz}, \mathrm{CDCl}_{3},{ }^{13} \mathrm{C} \mathrm{NMR}$

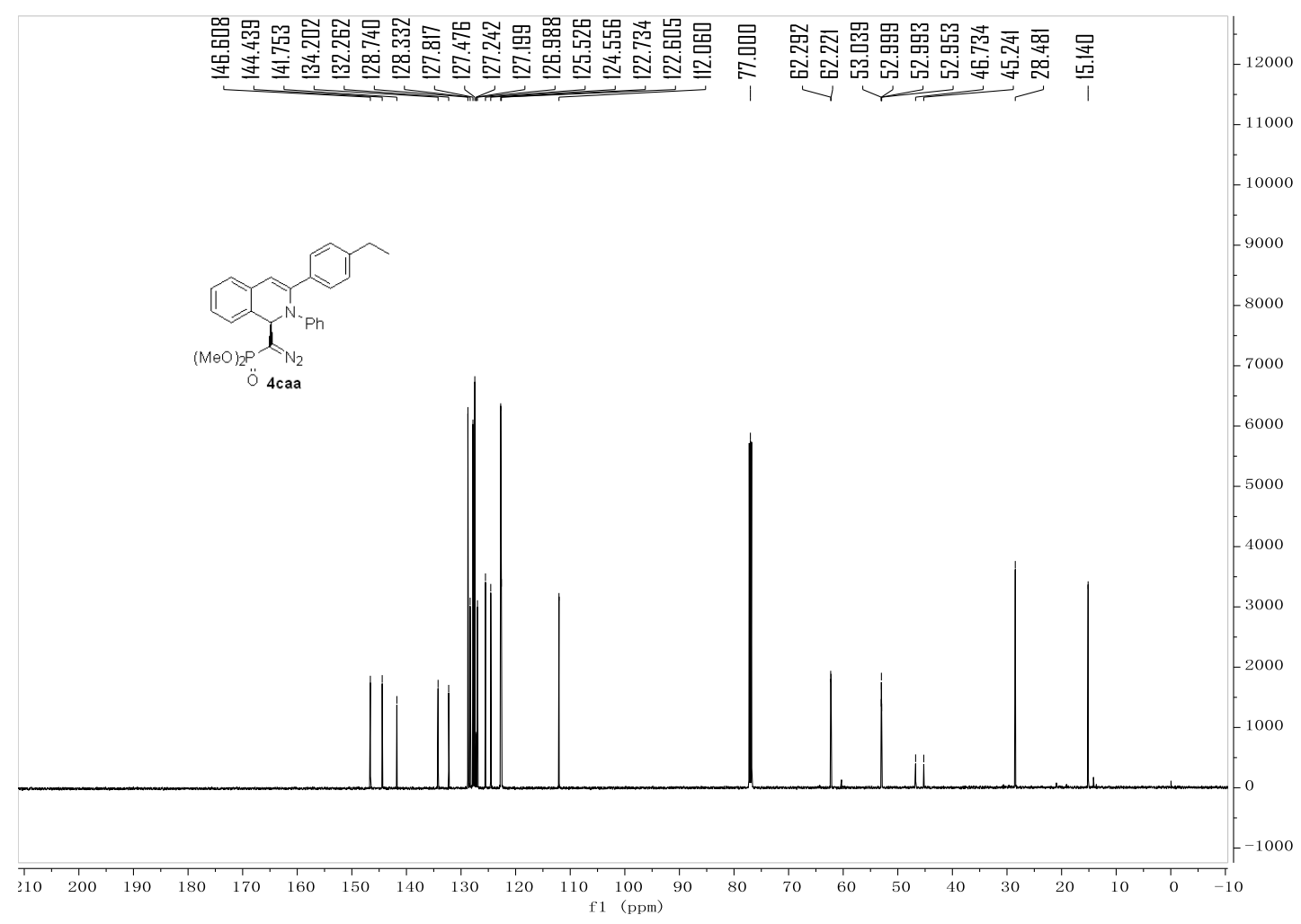




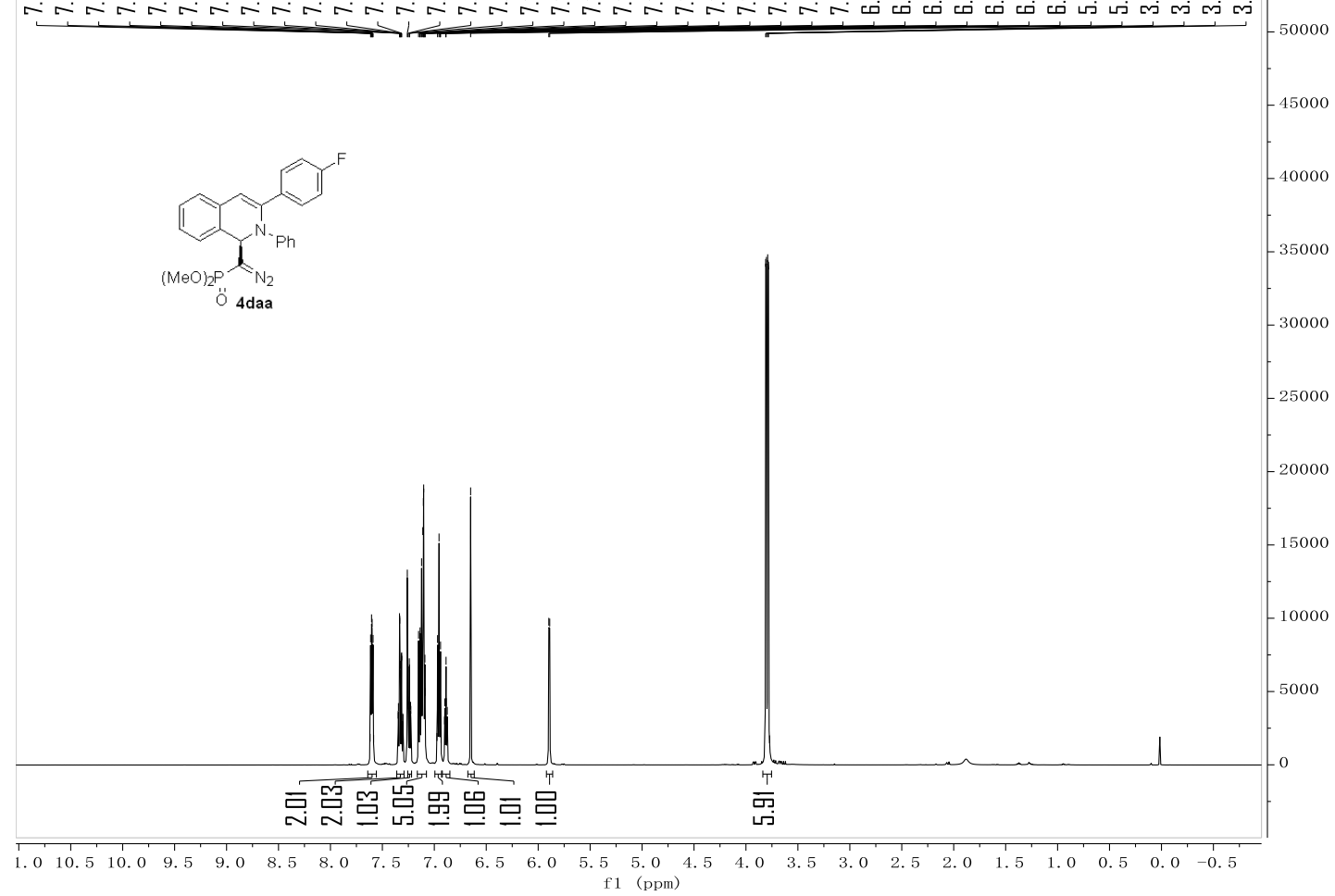

$151 \mathrm{MHz}, \mathrm{CDCl}_{3},{ }^{13} \mathrm{C} \mathrm{NMR}$

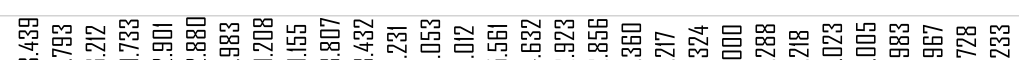

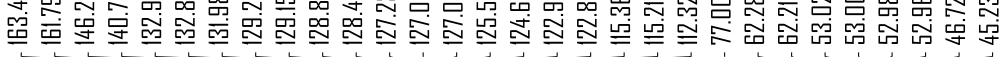

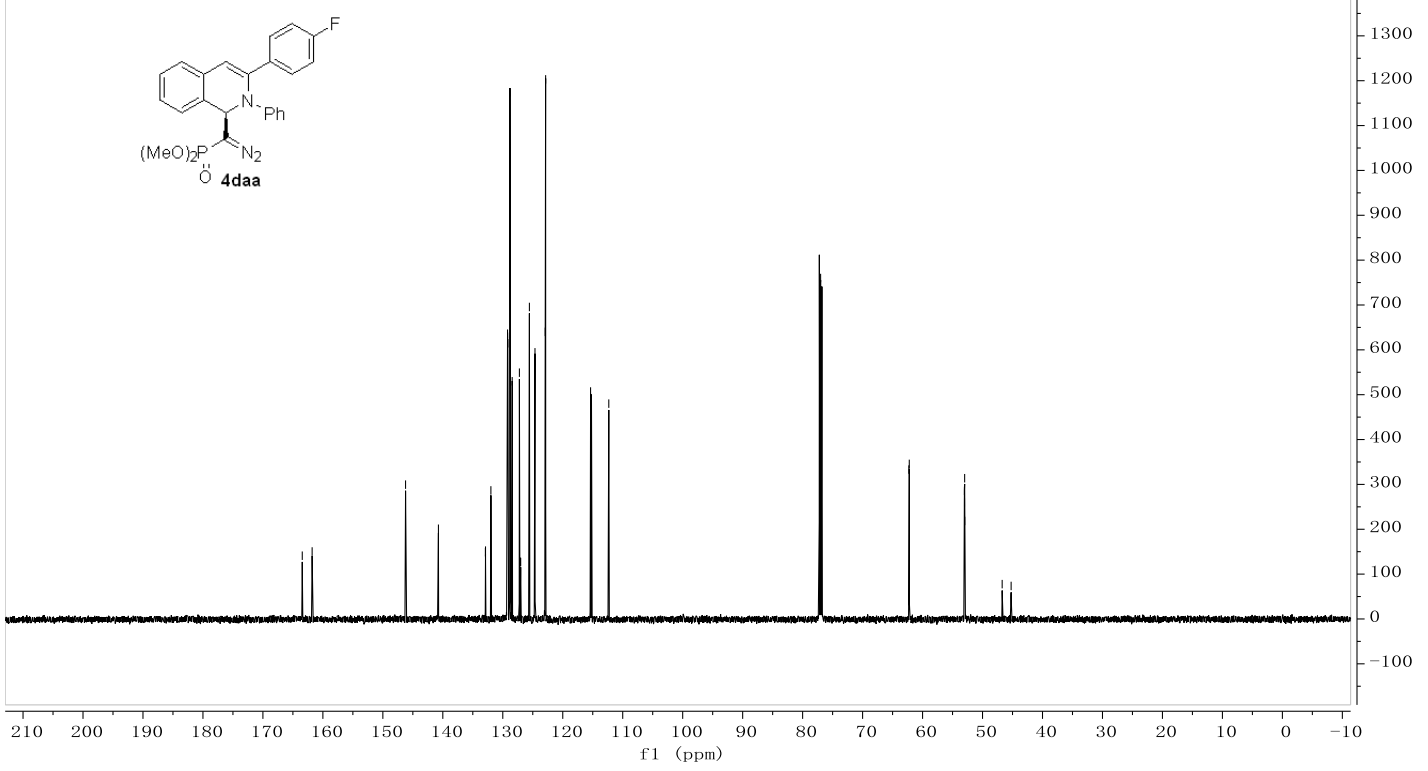




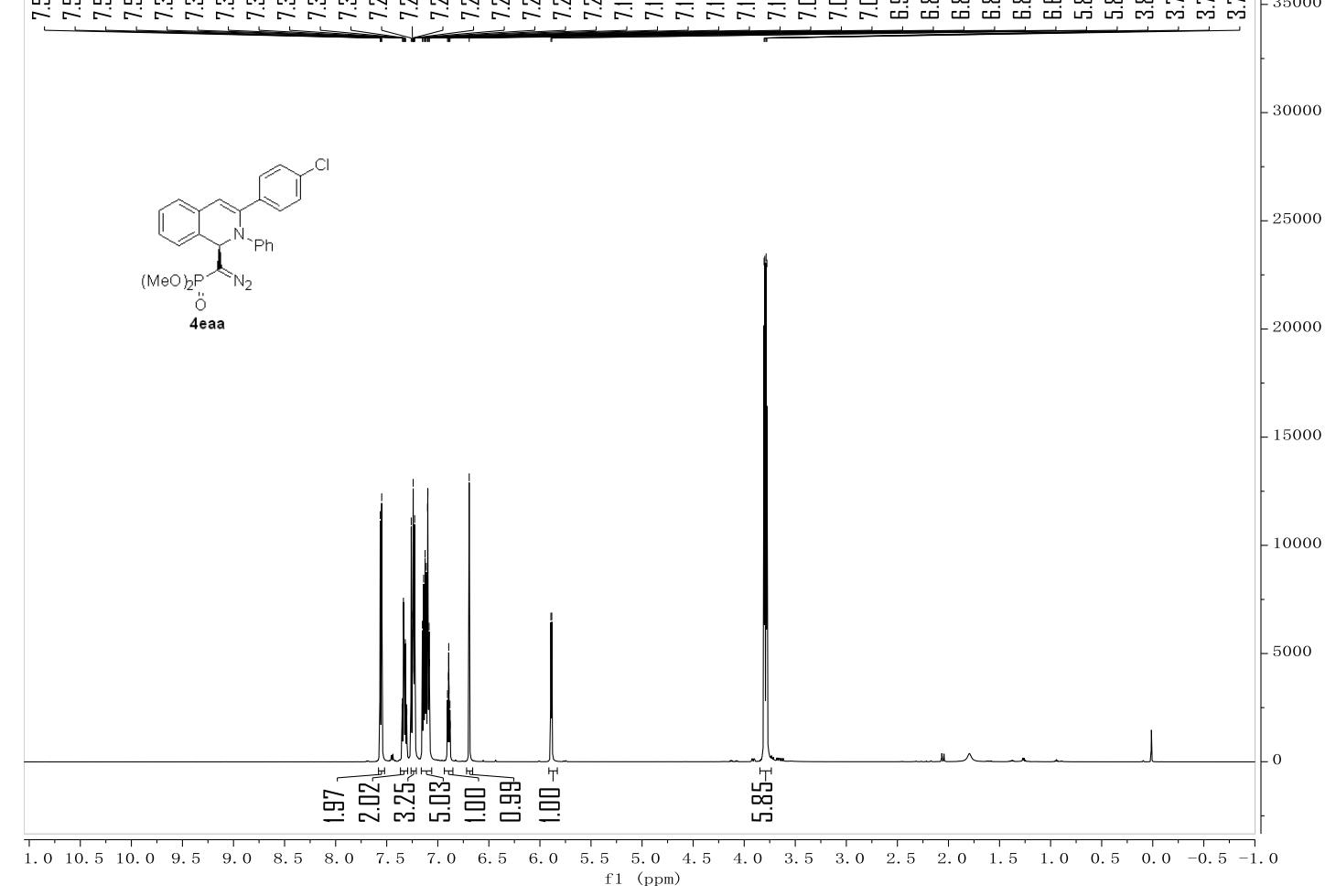

$151 \mathrm{MHz}, \mathrm{CDCl}_{3},{ }^{13} \mathrm{C} \mathrm{NMR}$

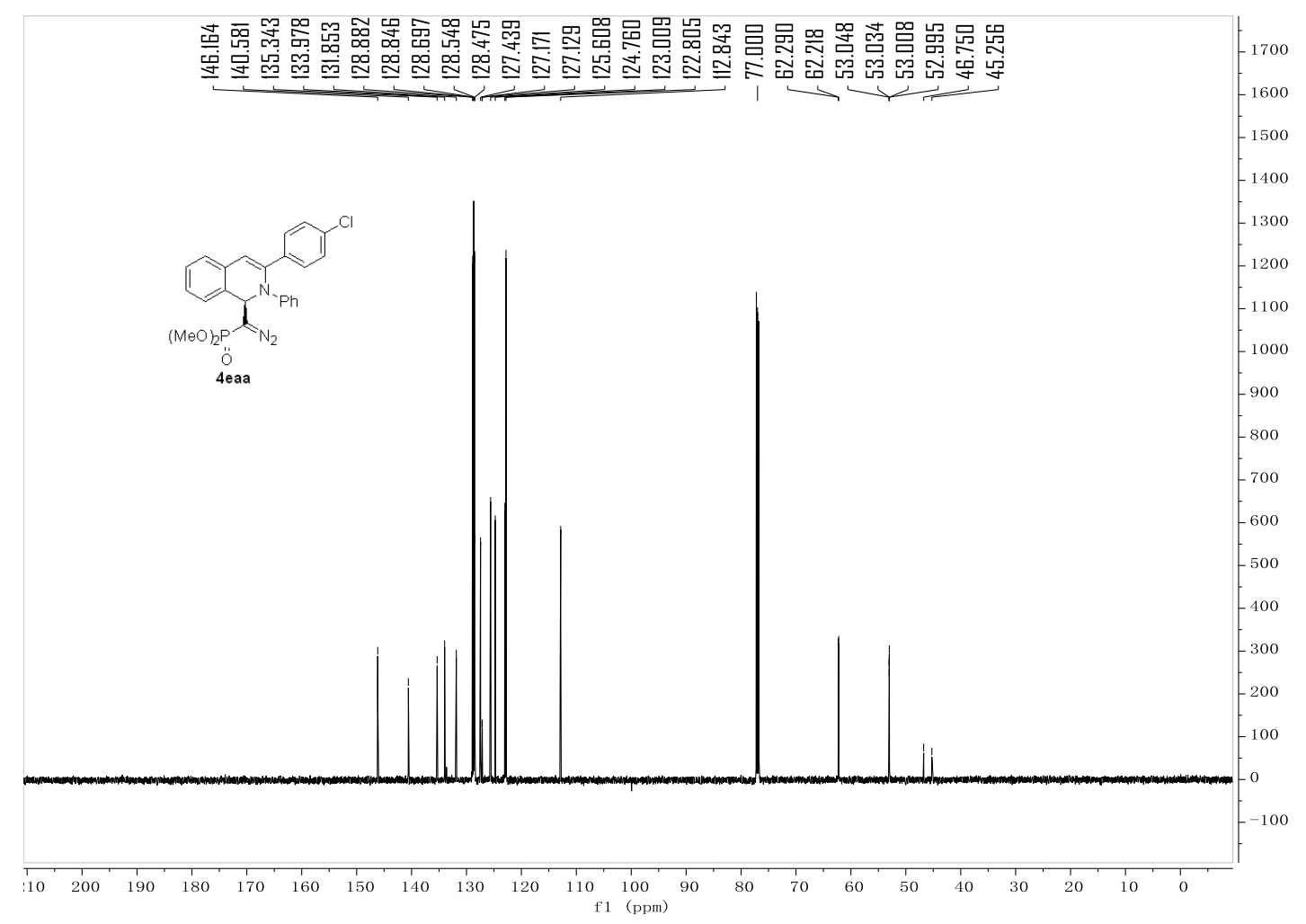




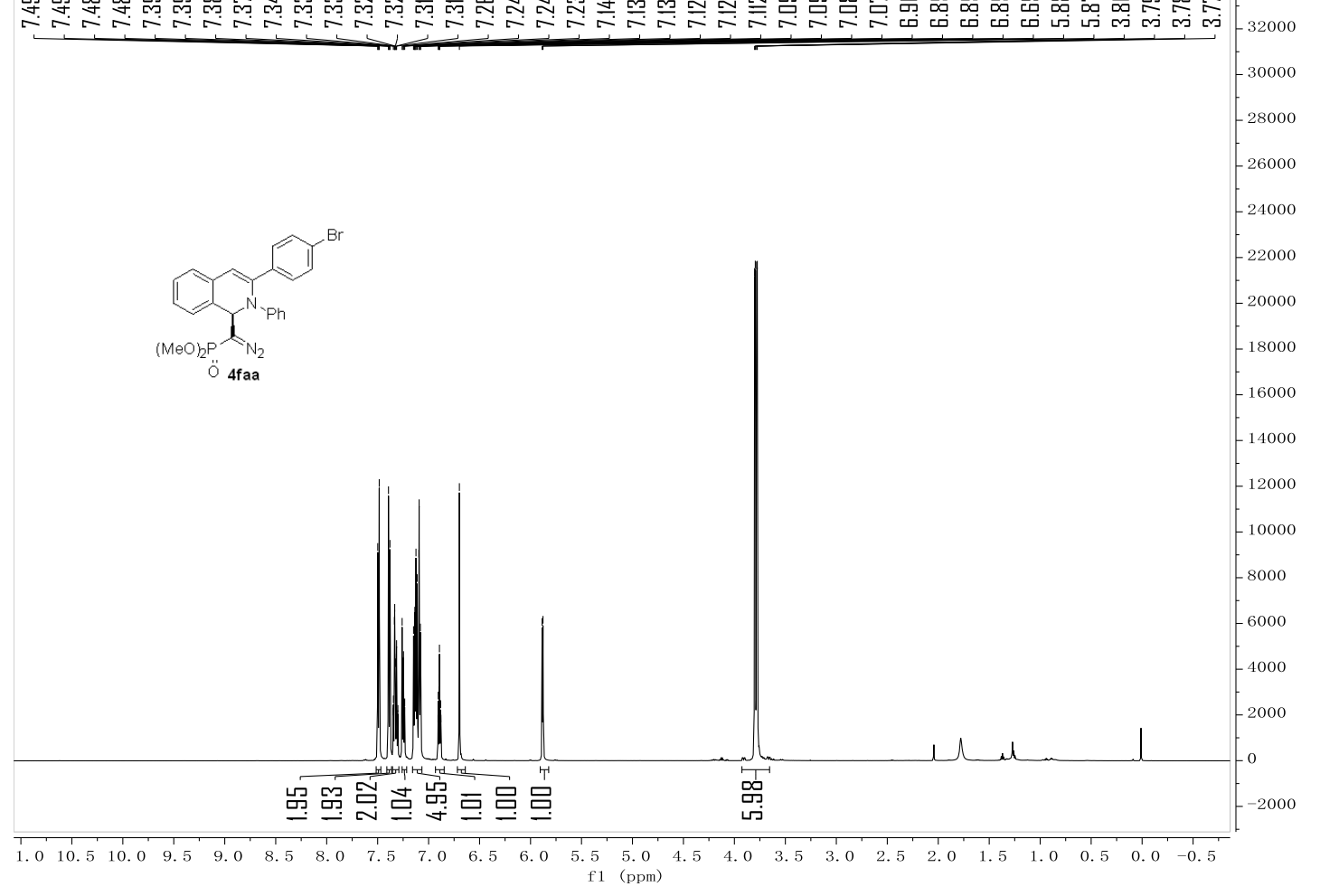

$151 \mathrm{MHz}, \mathrm{CDCl}_{3},{ }^{13} \mathrm{C}$ NMR

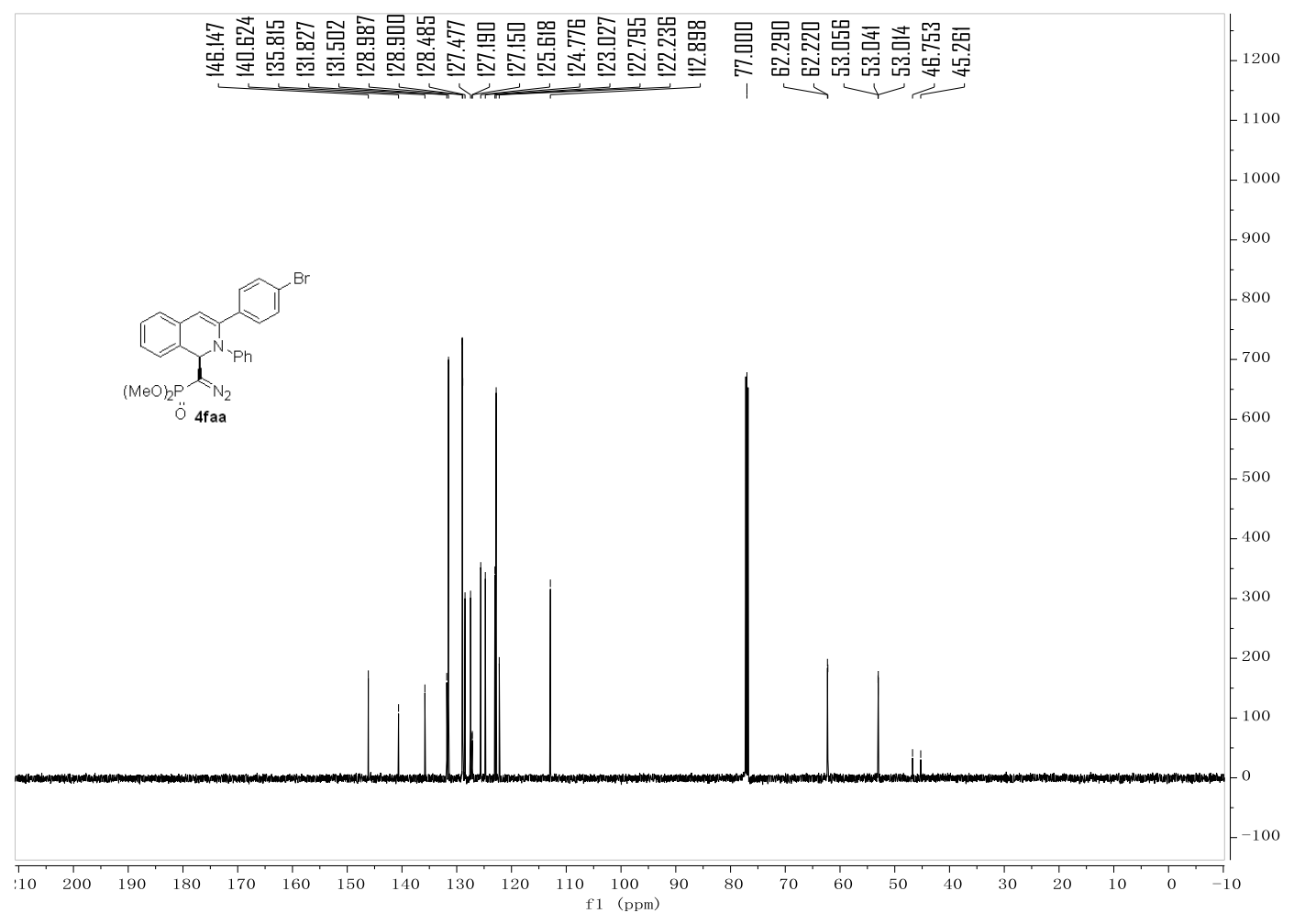




\section{$600 \mathrm{MHz}, \mathrm{CDCl}_{3},{ }^{1} \mathrm{H} \mathrm{NMR}$}

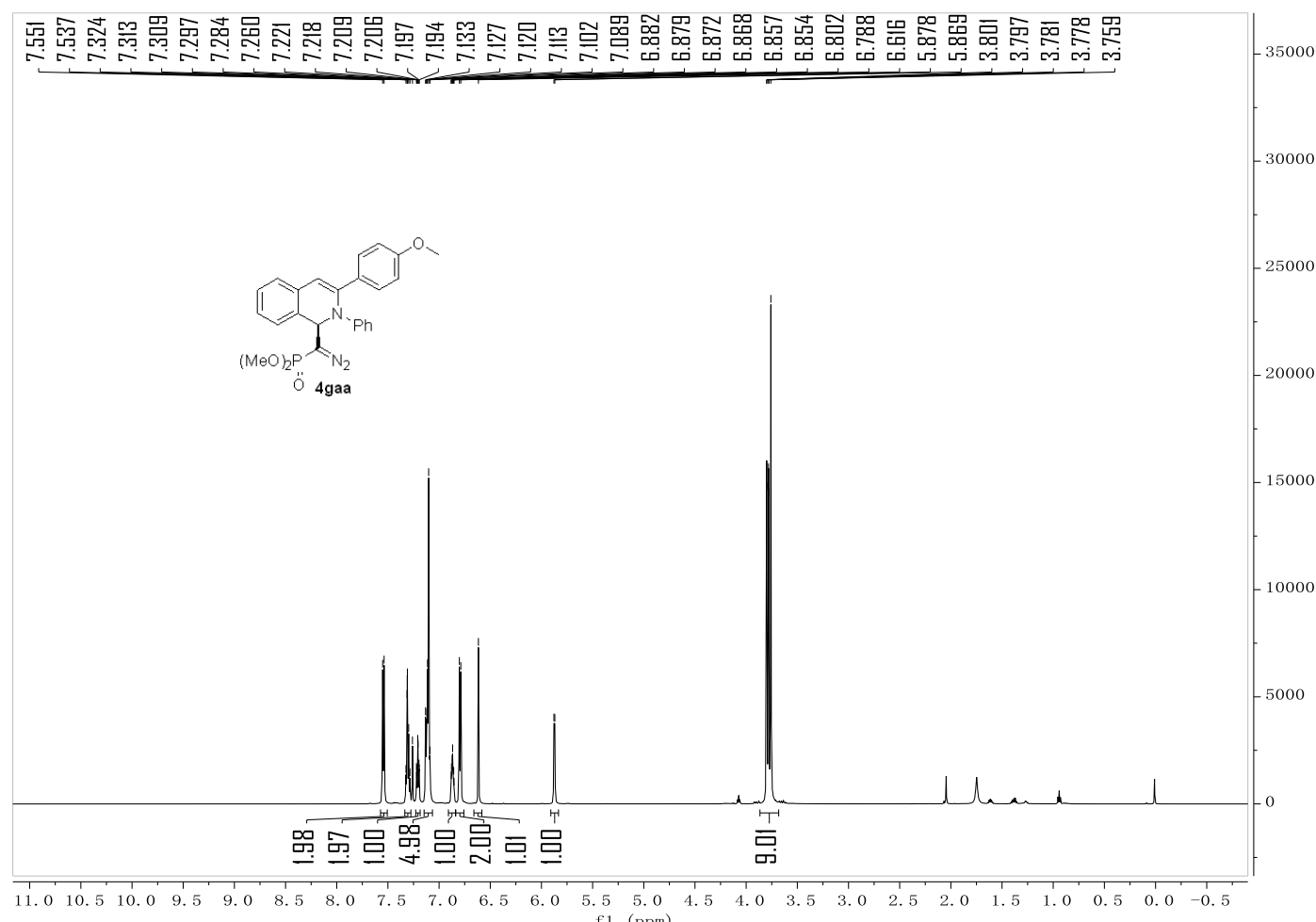

$151 \mathrm{MHz}, \mathrm{CDCl}_{3},{ }^{13} \mathrm{C} \mathrm{NMR}$

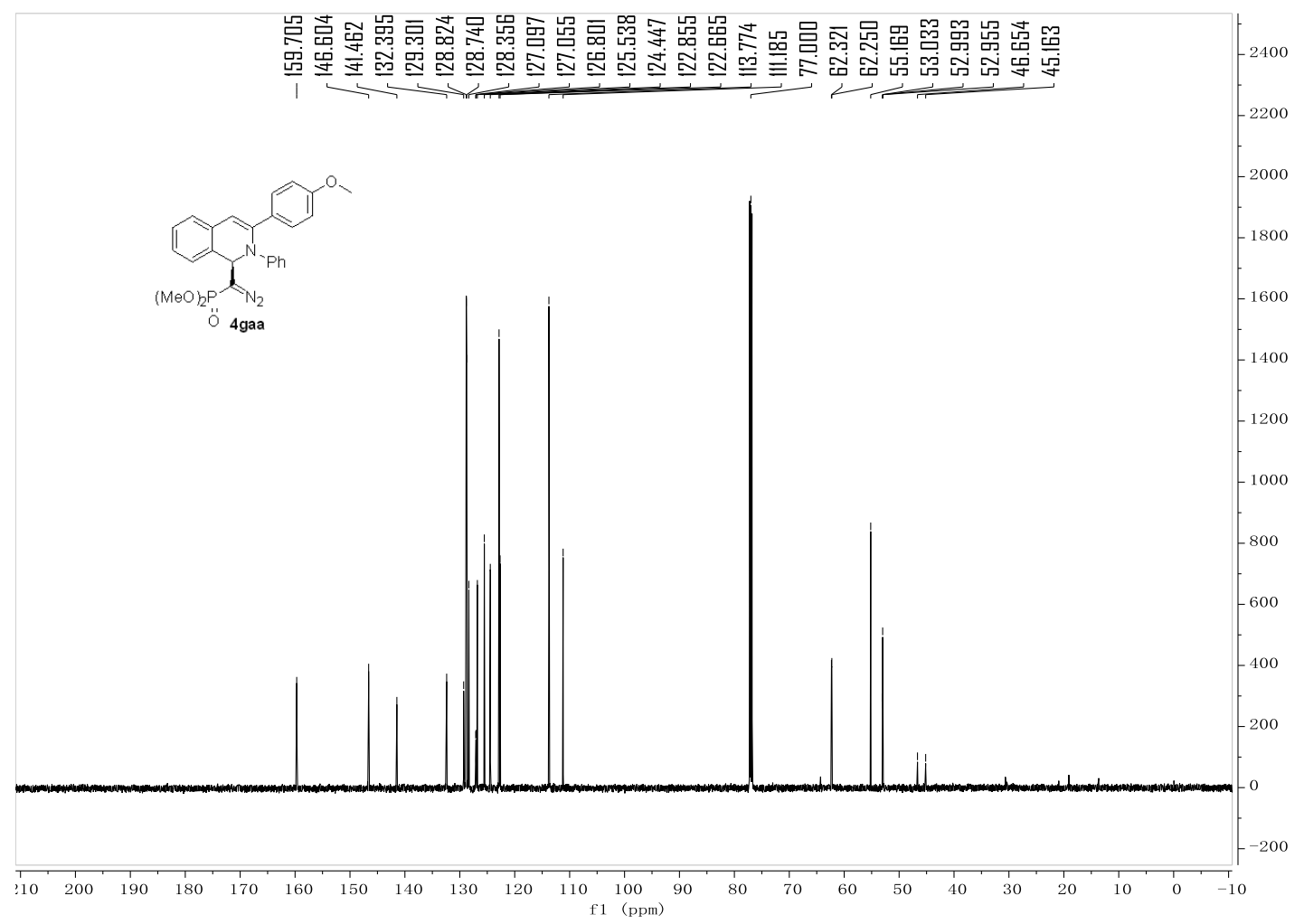




\section{$600 \mathrm{MHz}, \mathrm{CDCl}_{3},{ }^{1} \mathrm{H} \mathrm{NMR}$}

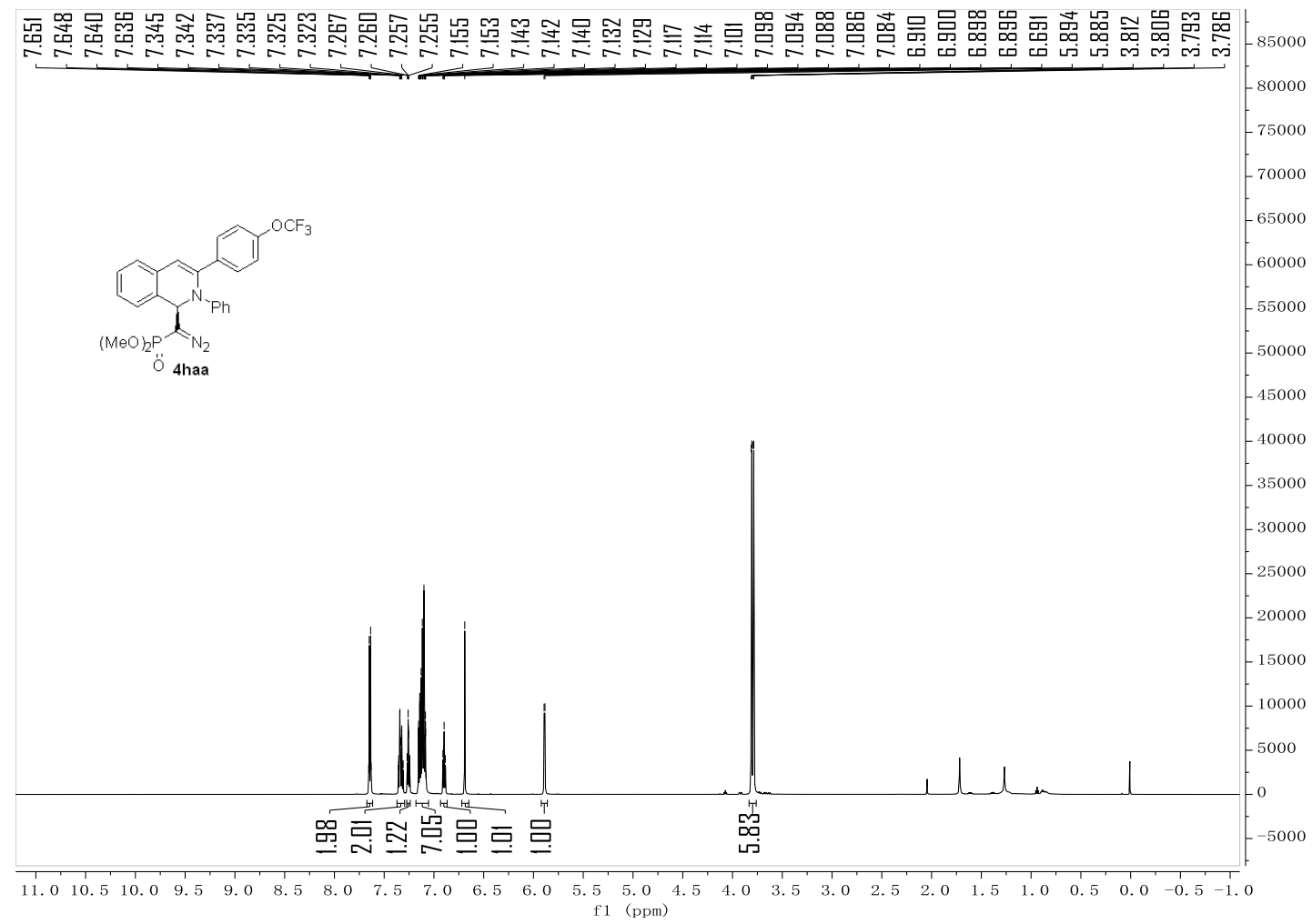

$151 \mathrm{MHz}, \mathrm{CDCl}_{3},{ }^{13} \mathrm{C} \mathrm{NMR}$

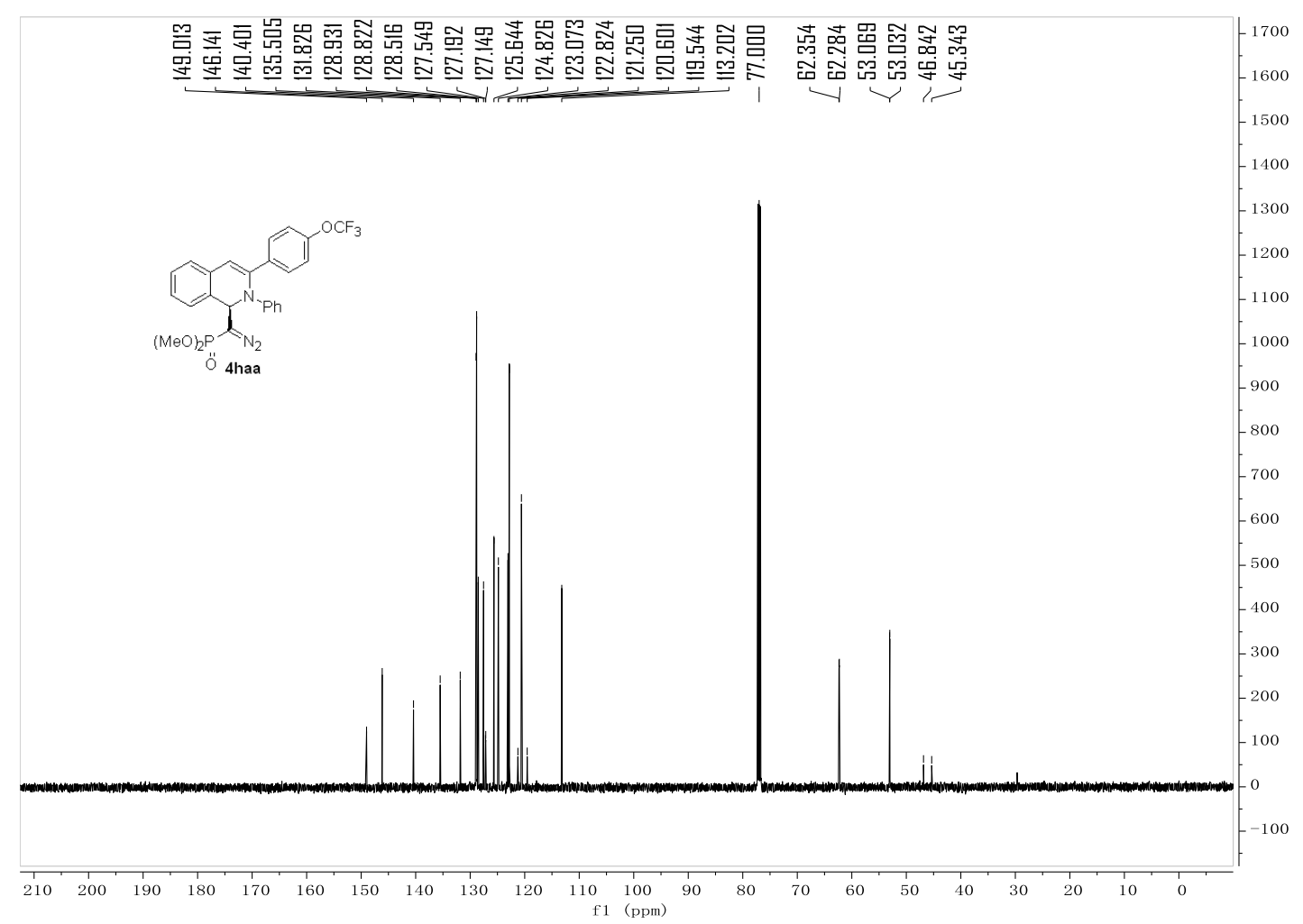


$600 \mathrm{MHz}, \mathrm{CDCl}_{3},{ }^{1} \mathrm{H} \mathrm{NMR}$

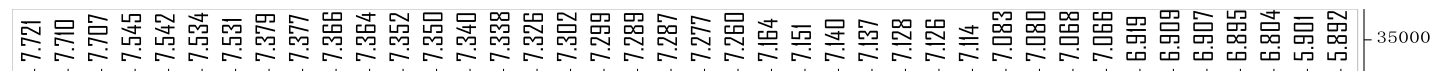

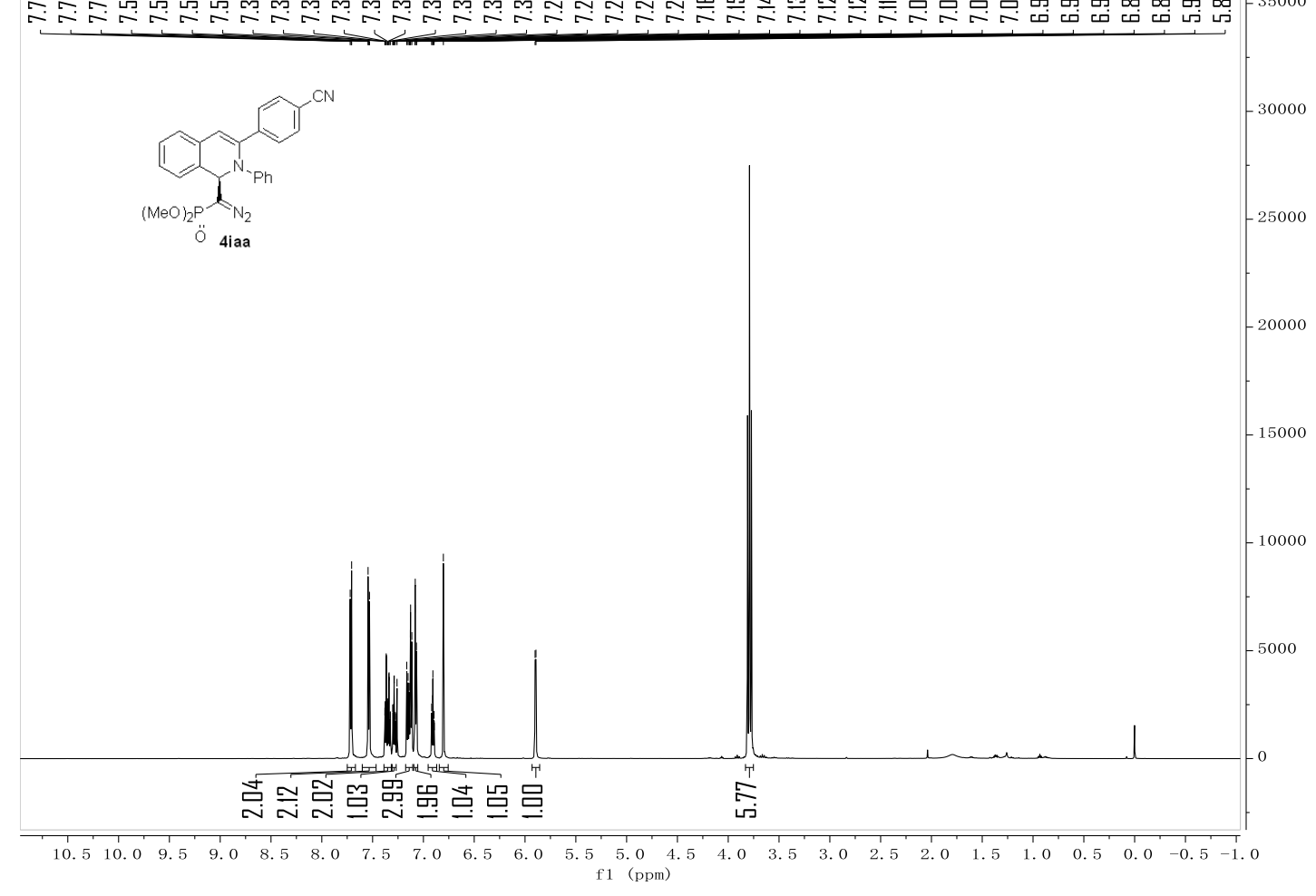

$151 \mathrm{MHz}, \mathrm{CDCl}_{3},{ }^{13} \mathrm{C} \mathrm{NMR}$

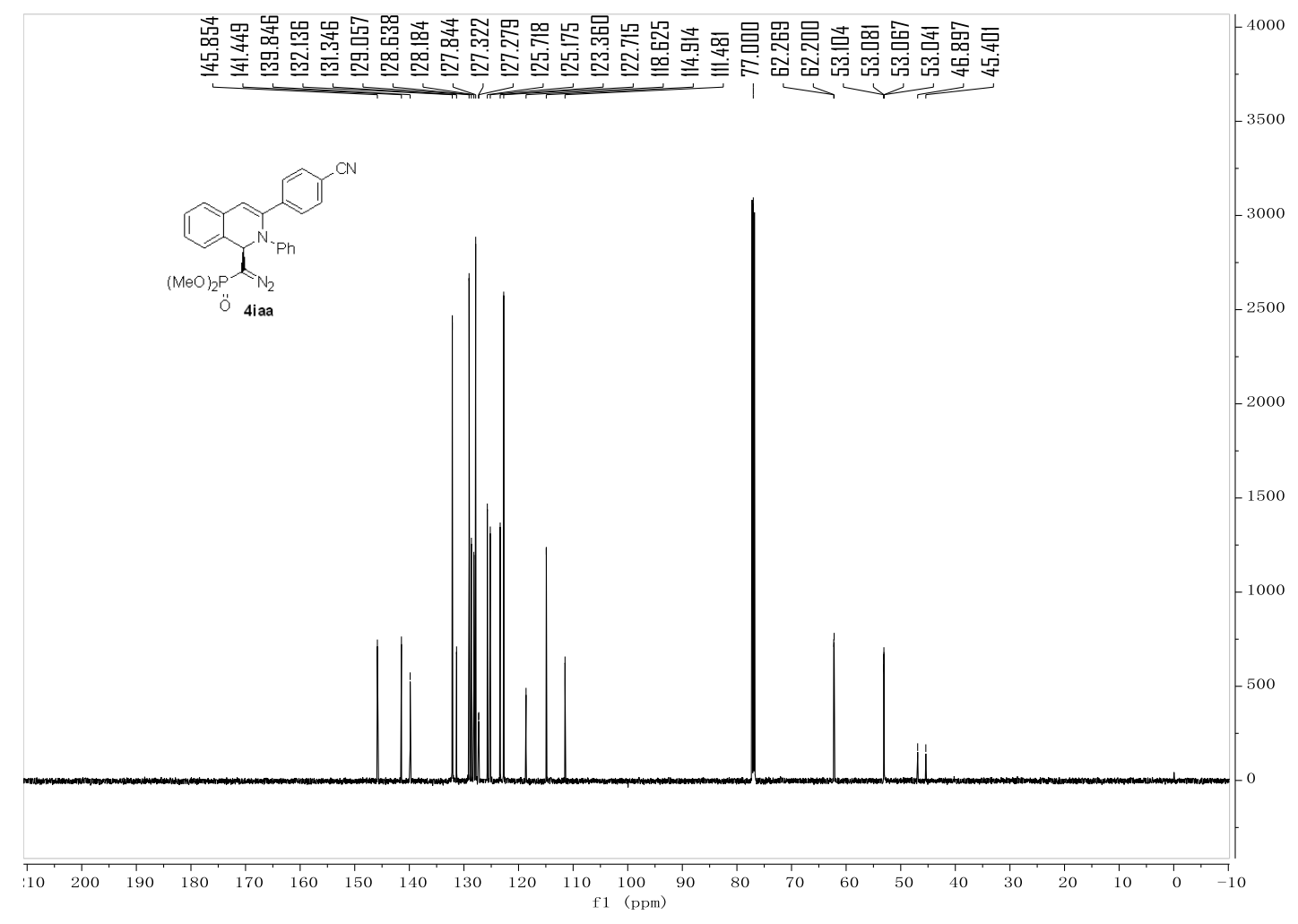


$600 \mathrm{MHz}, \mathrm{CDCl}_{3},{ }^{1} \mathrm{H} \mathrm{NMR}$

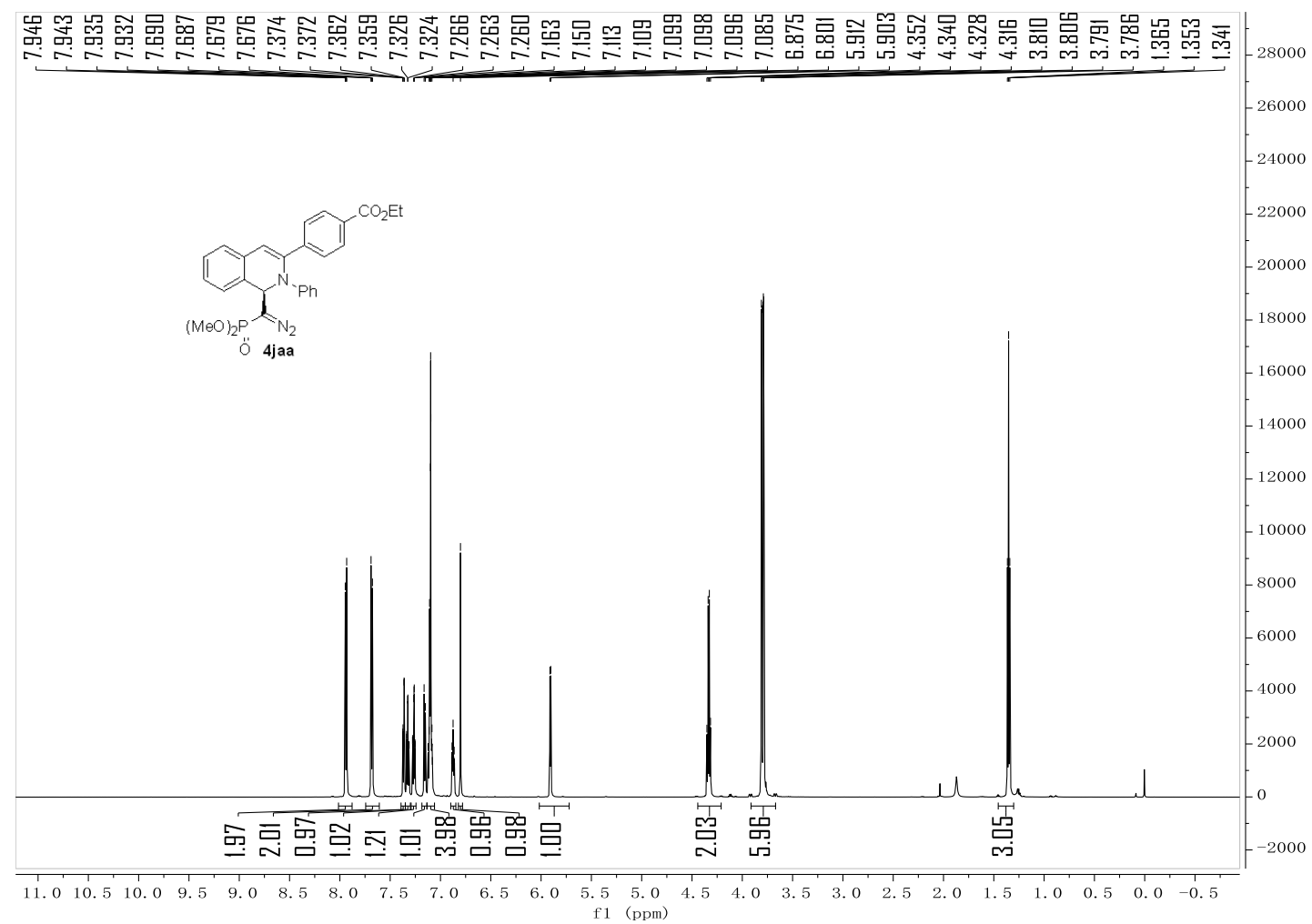

$151 \mathrm{MHz}, \mathrm{CDCl}_{3},{ }^{13} \mathrm{C} \mathrm{NMR}$

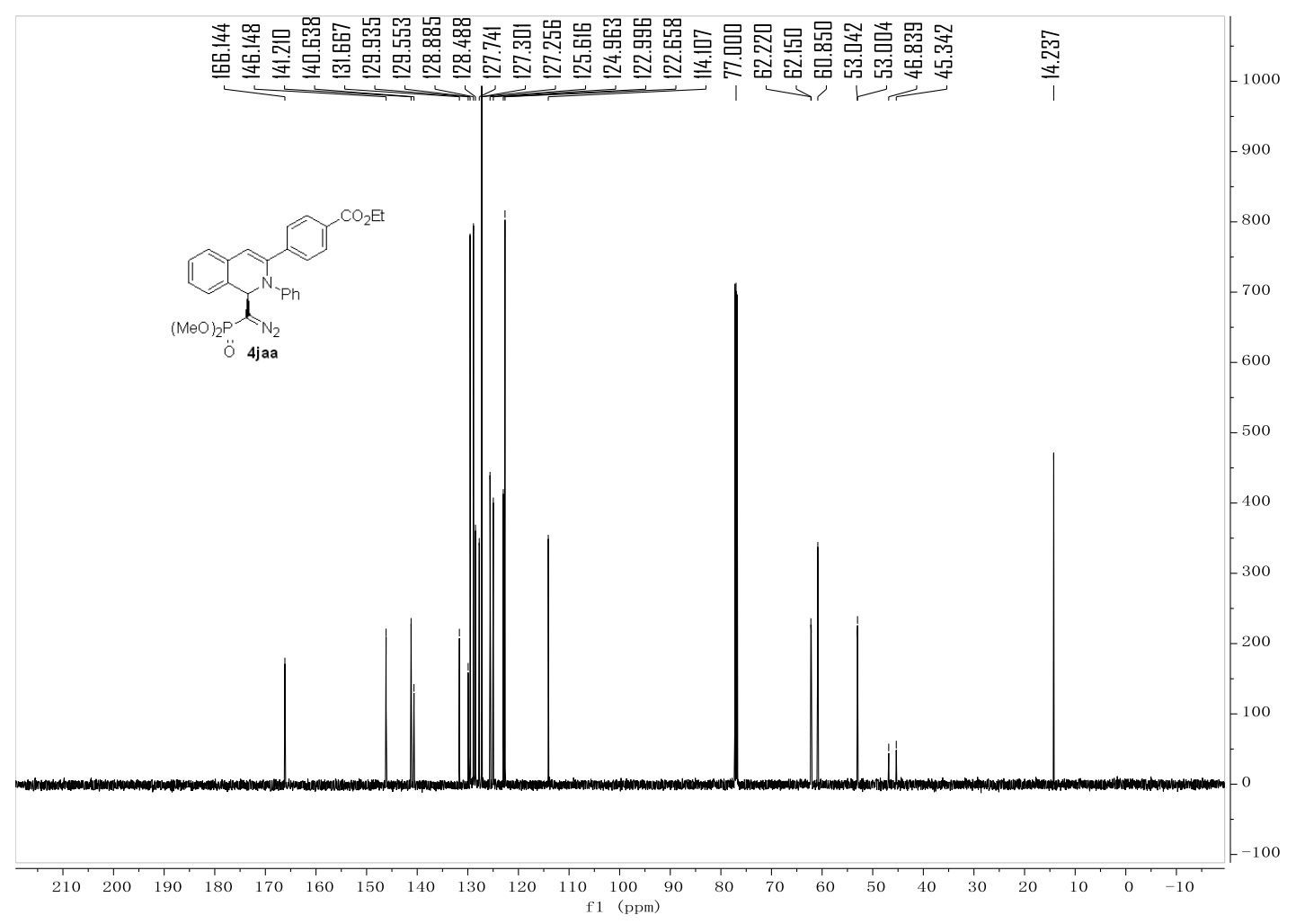




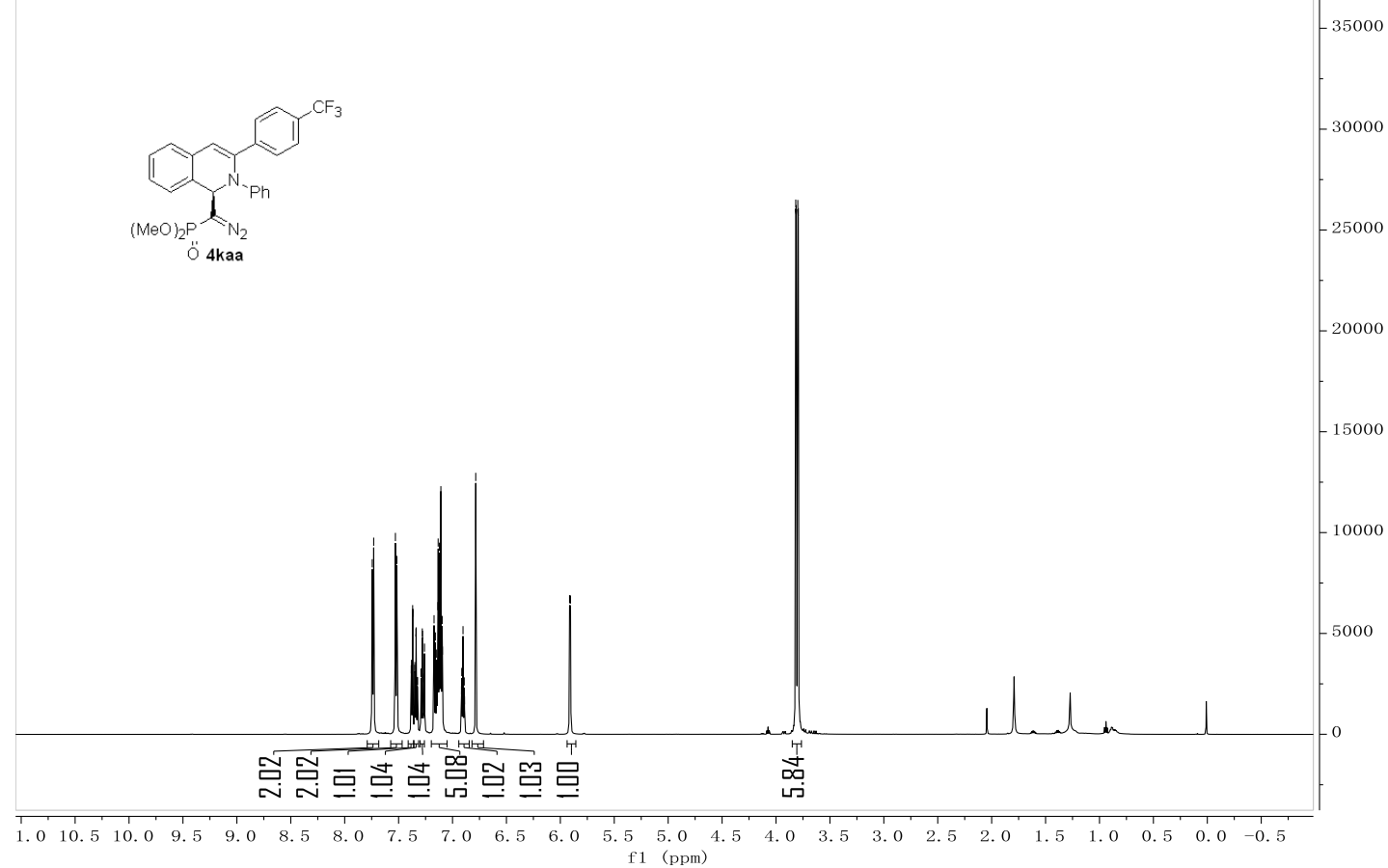

$151 \mathrm{MHz}, \mathrm{CDCl}_{3},{ }^{13} \mathrm{C} \mathrm{NMR}$

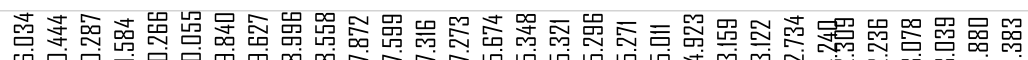

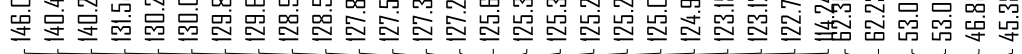

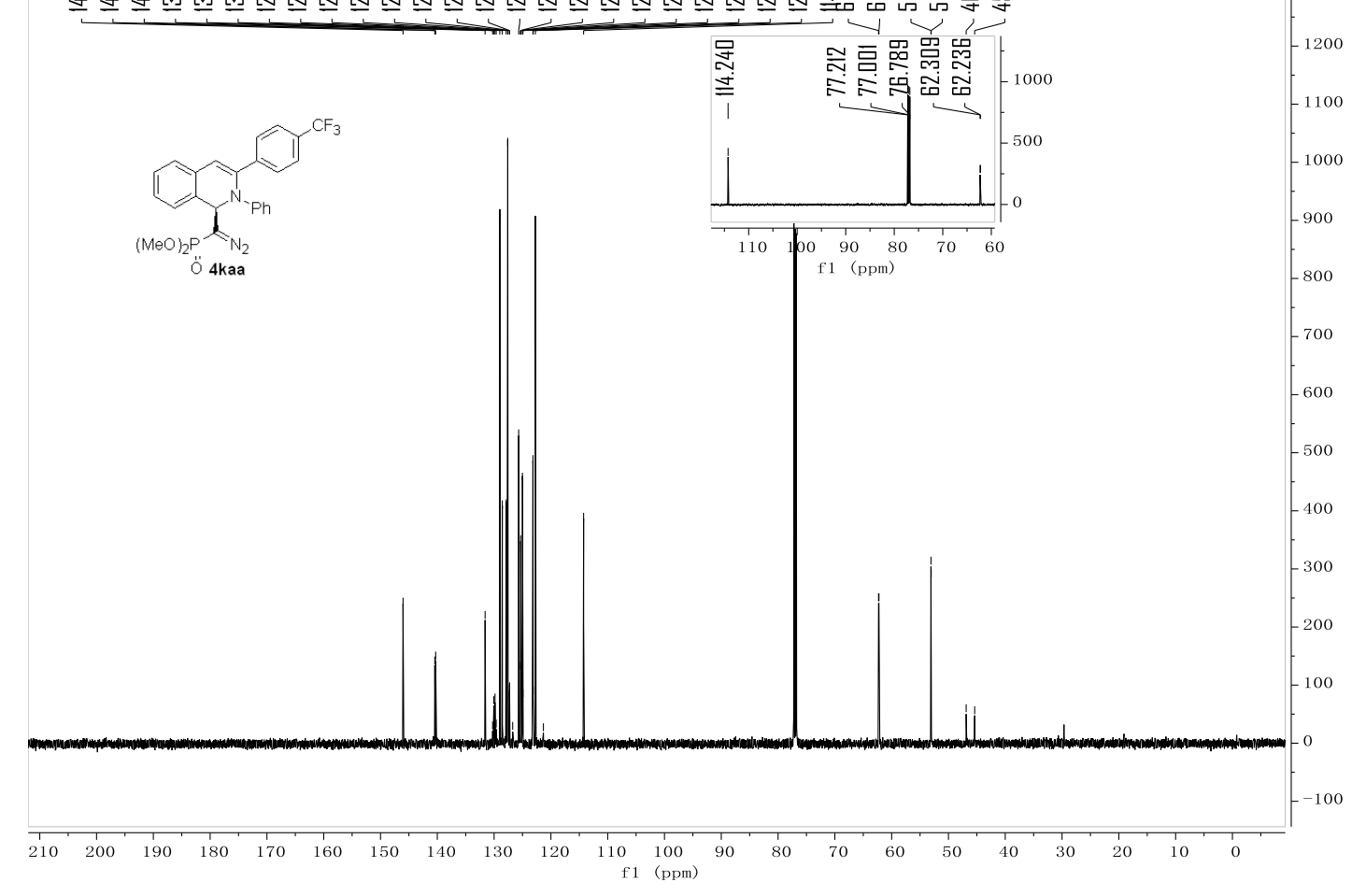




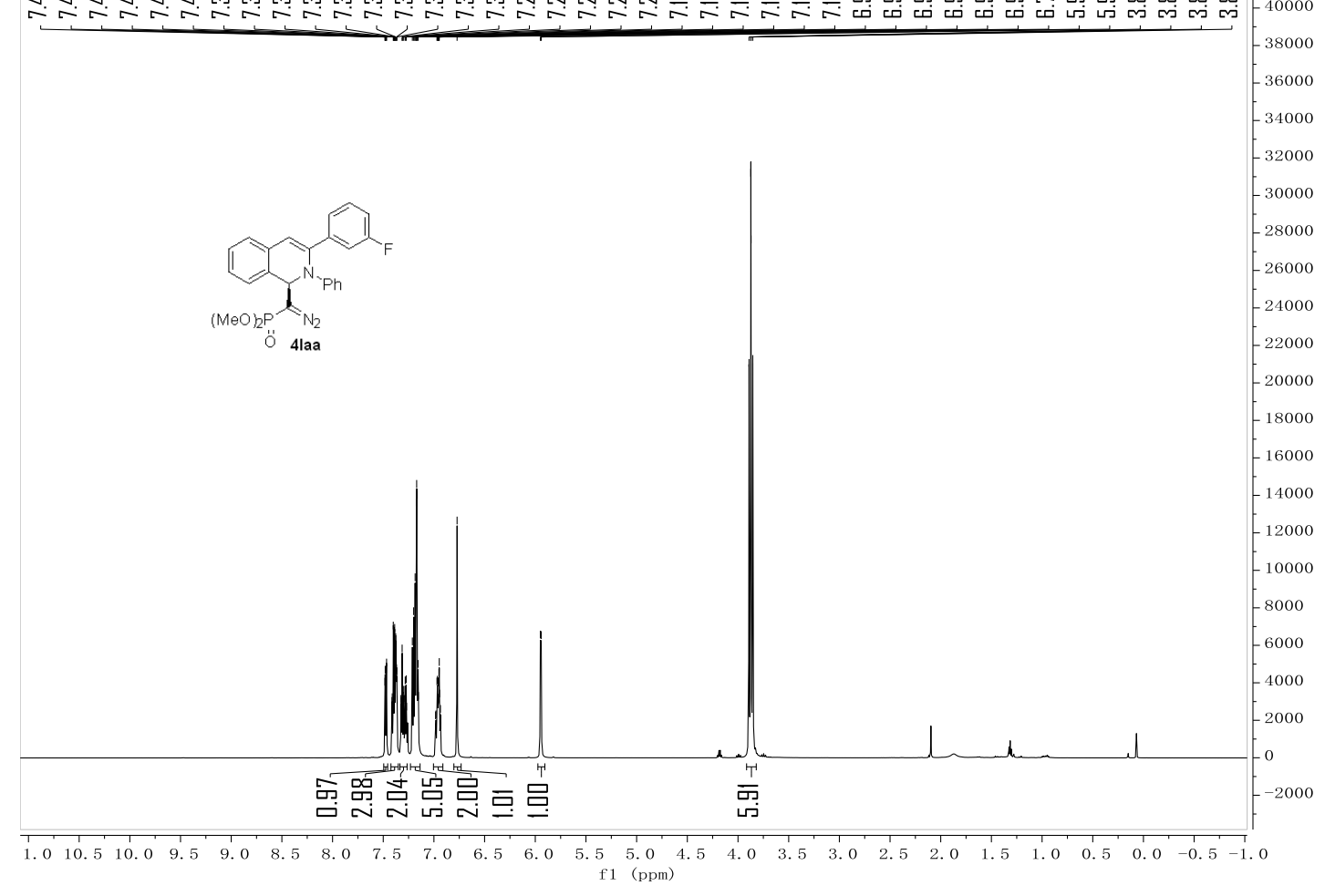

$151 \mathrm{MHz}, \mathrm{CDCl}_{3},{ }^{13} \mathrm{C} \mathrm{NMR}$

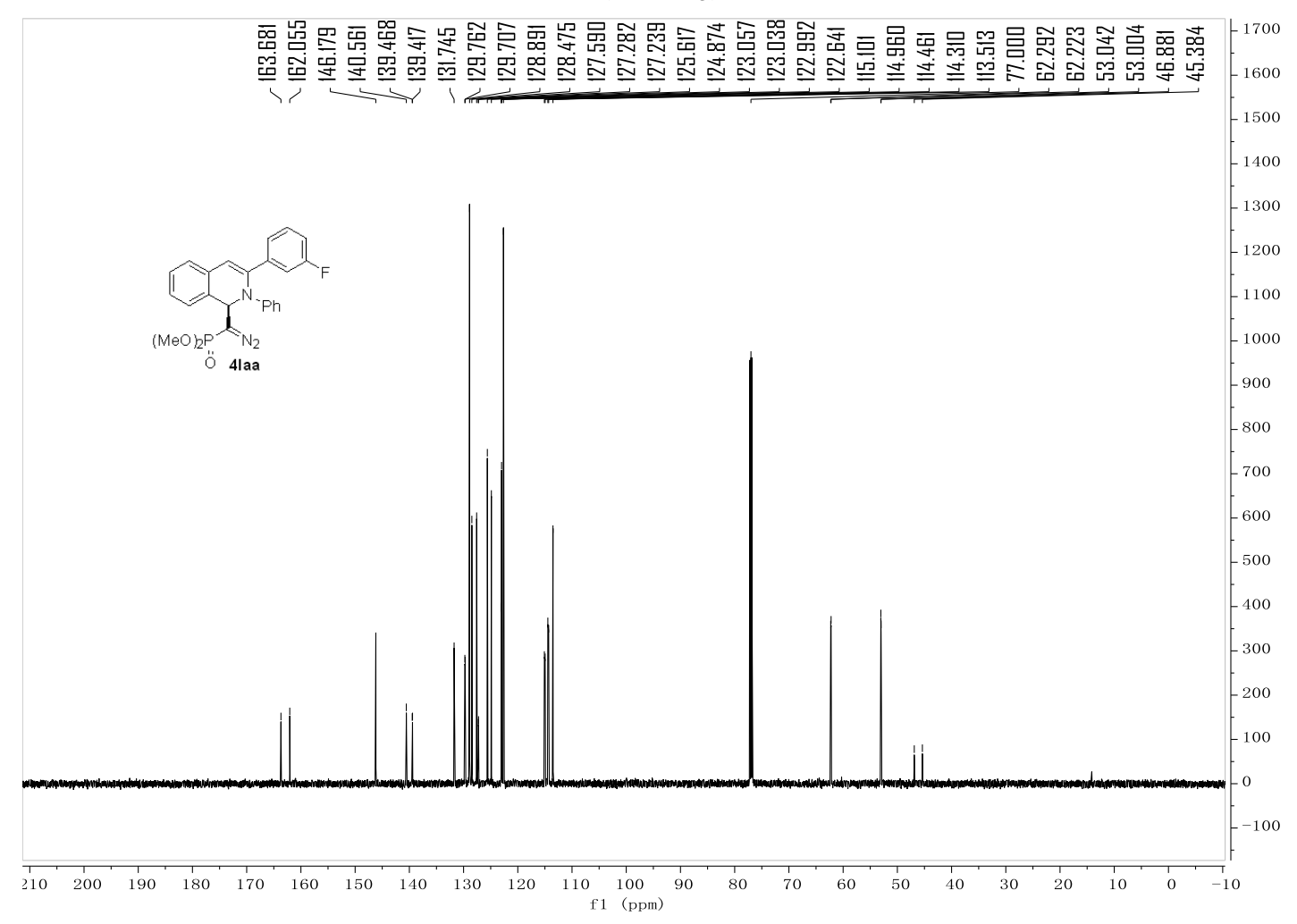




\section{$600 \mathrm{MHz}, \mathrm{CDCl}_{3},{ }^{1} \mathrm{H} \mathrm{NMR}$}

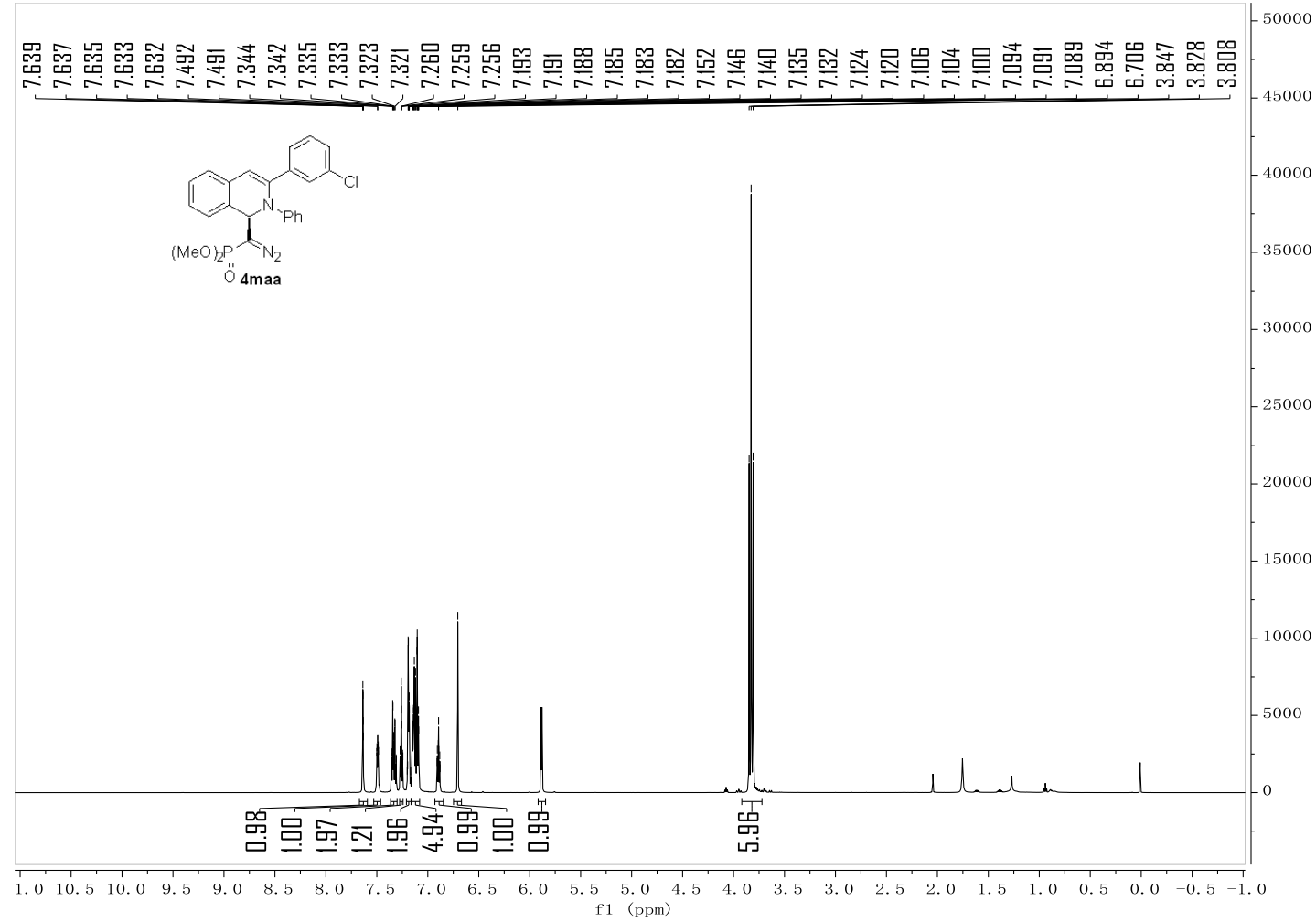

$151 \mathrm{MHz}, \mathrm{CDCl}_{3},{ }^{13} \mathrm{C} \mathrm{NMR}$

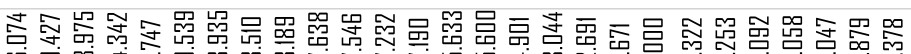

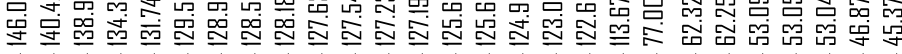

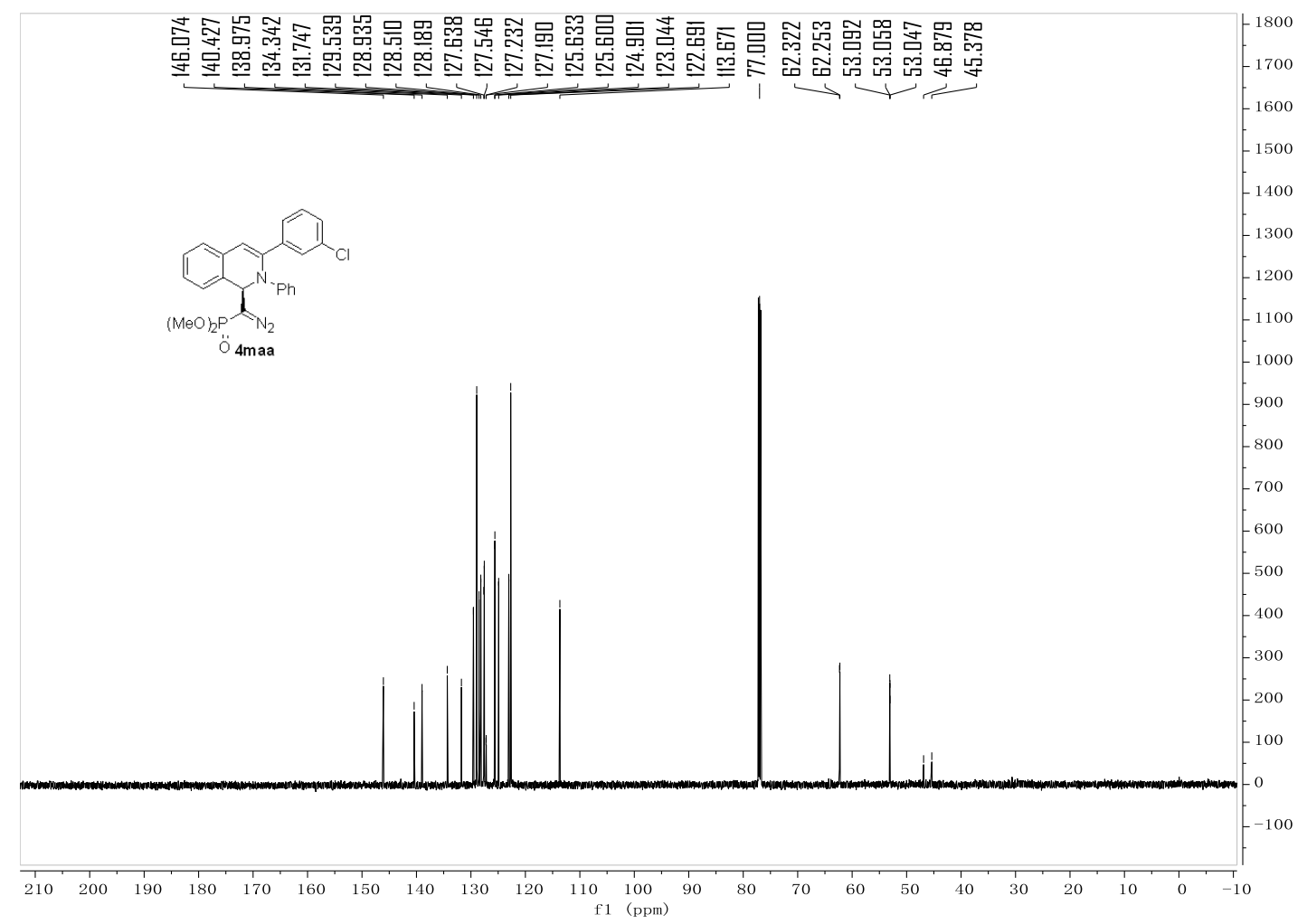


$600 \mathrm{MHz}, \mathrm{CDCl}_{3},{ }^{1} \mathrm{H} \mathrm{NMR}$

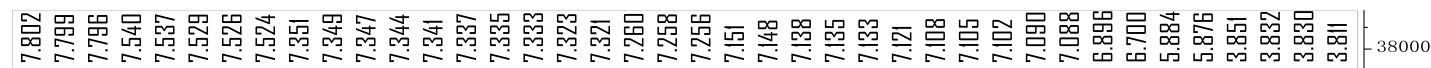

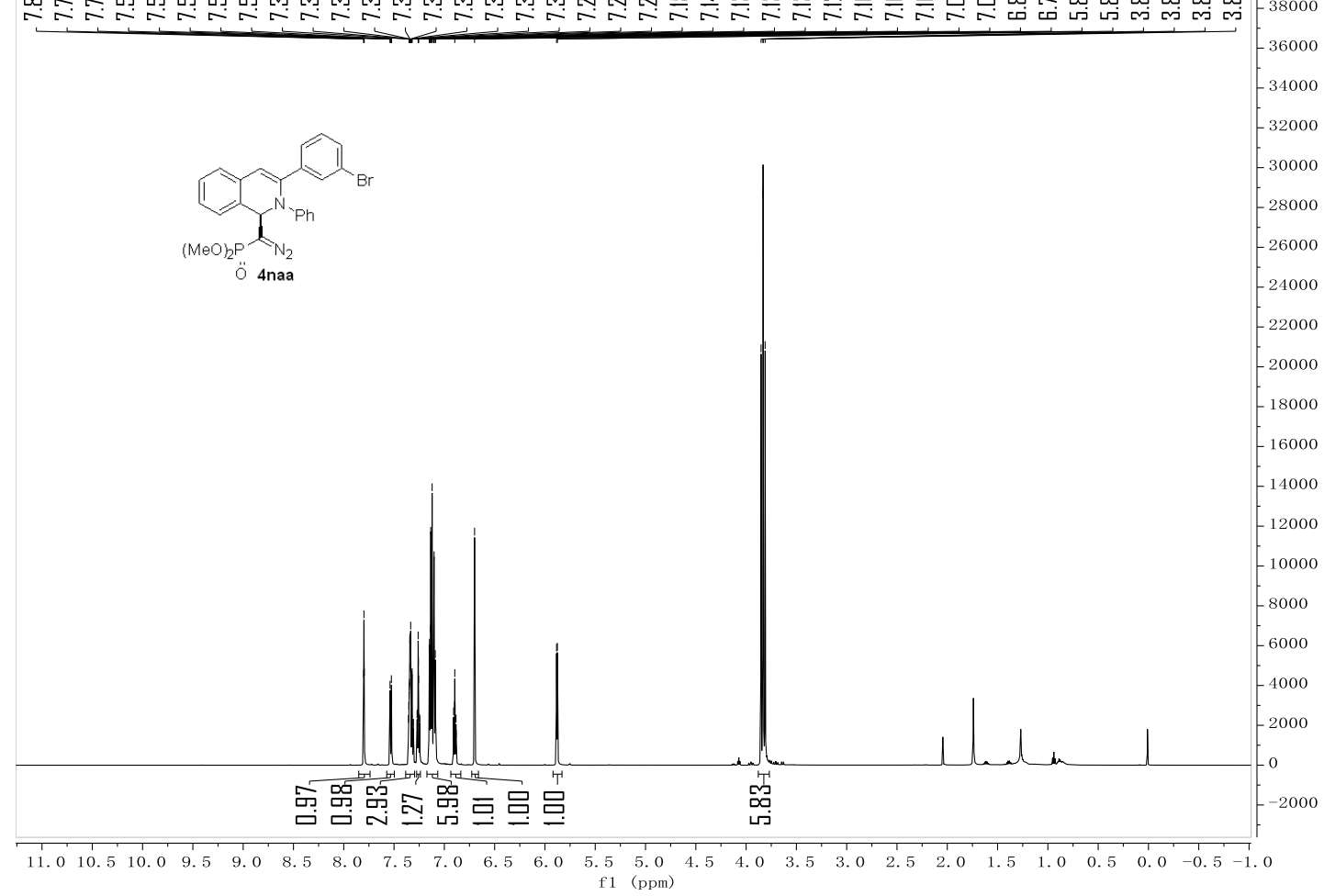

$151 \mathrm{MHz}, \mathrm{CDCl}_{3},{ }^{13} \mathrm{C} \mathrm{NMR}$

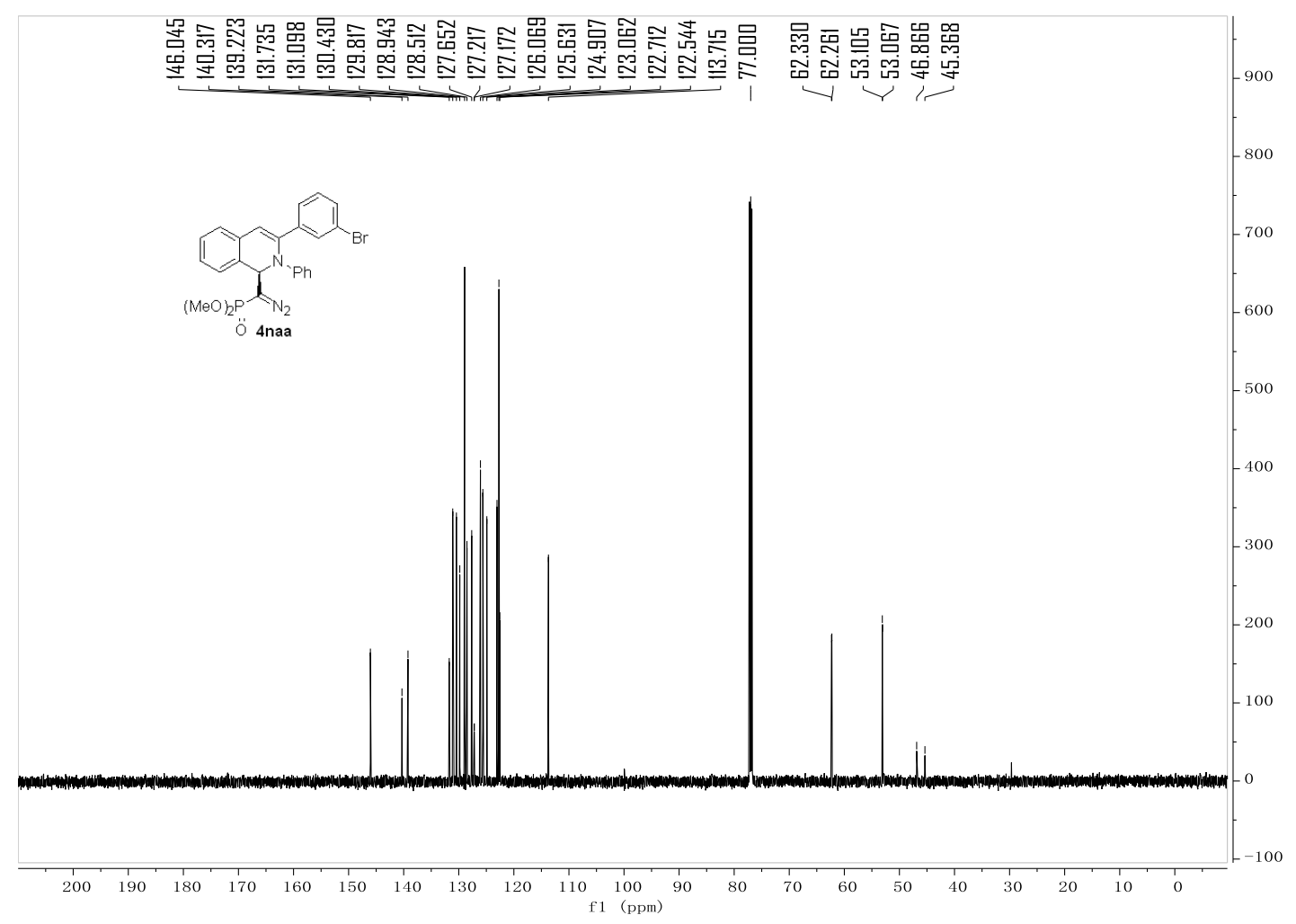




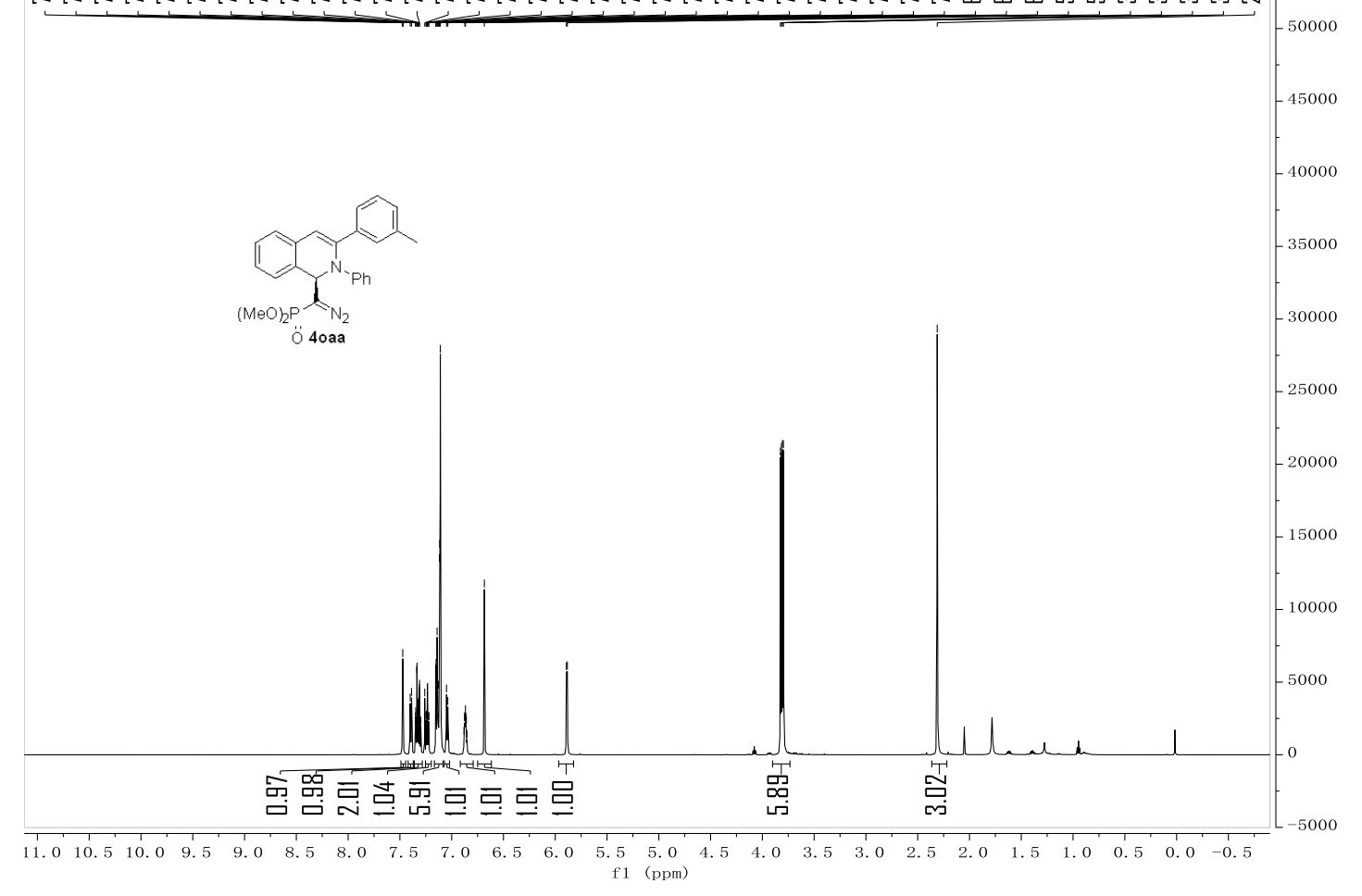

$151 \mathrm{MHz}, \mathrm{CDCl}_{3},{ }^{13} \mathrm{C} \mathrm{NMR}$

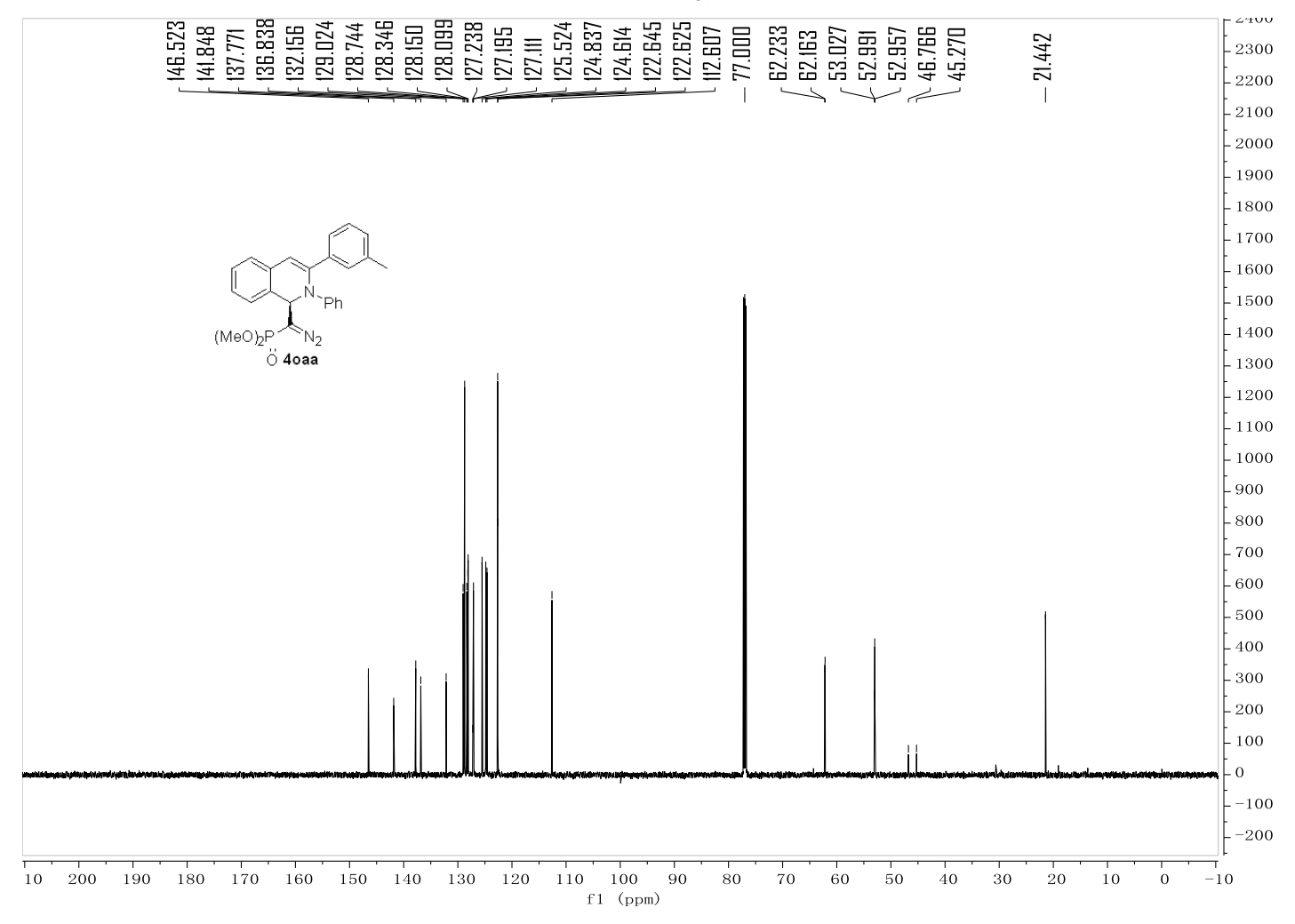




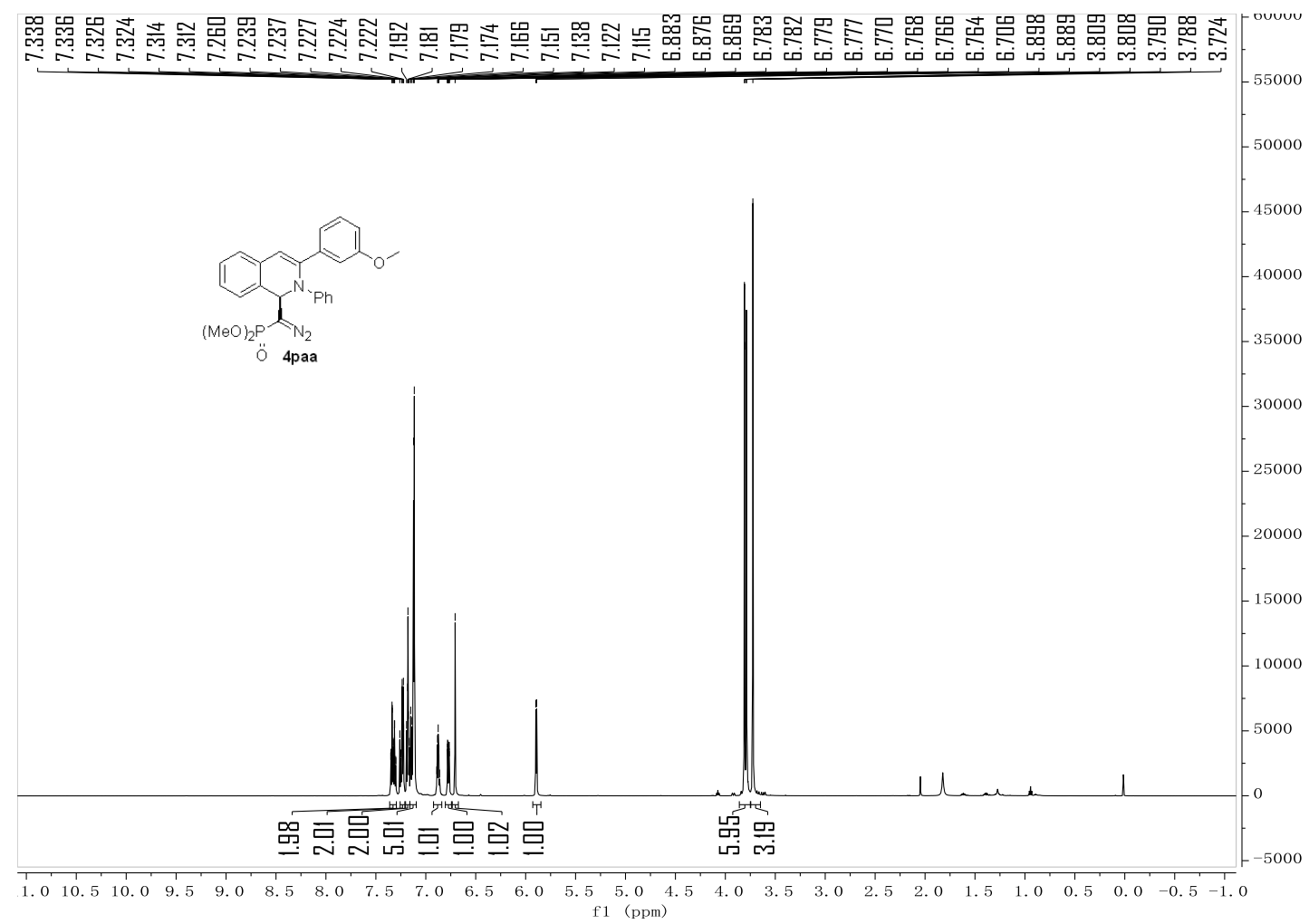

$151 \mathrm{MHz}, \mathrm{CDCl}_{3},{ }^{13} \mathrm{C} \mathrm{NMR}$

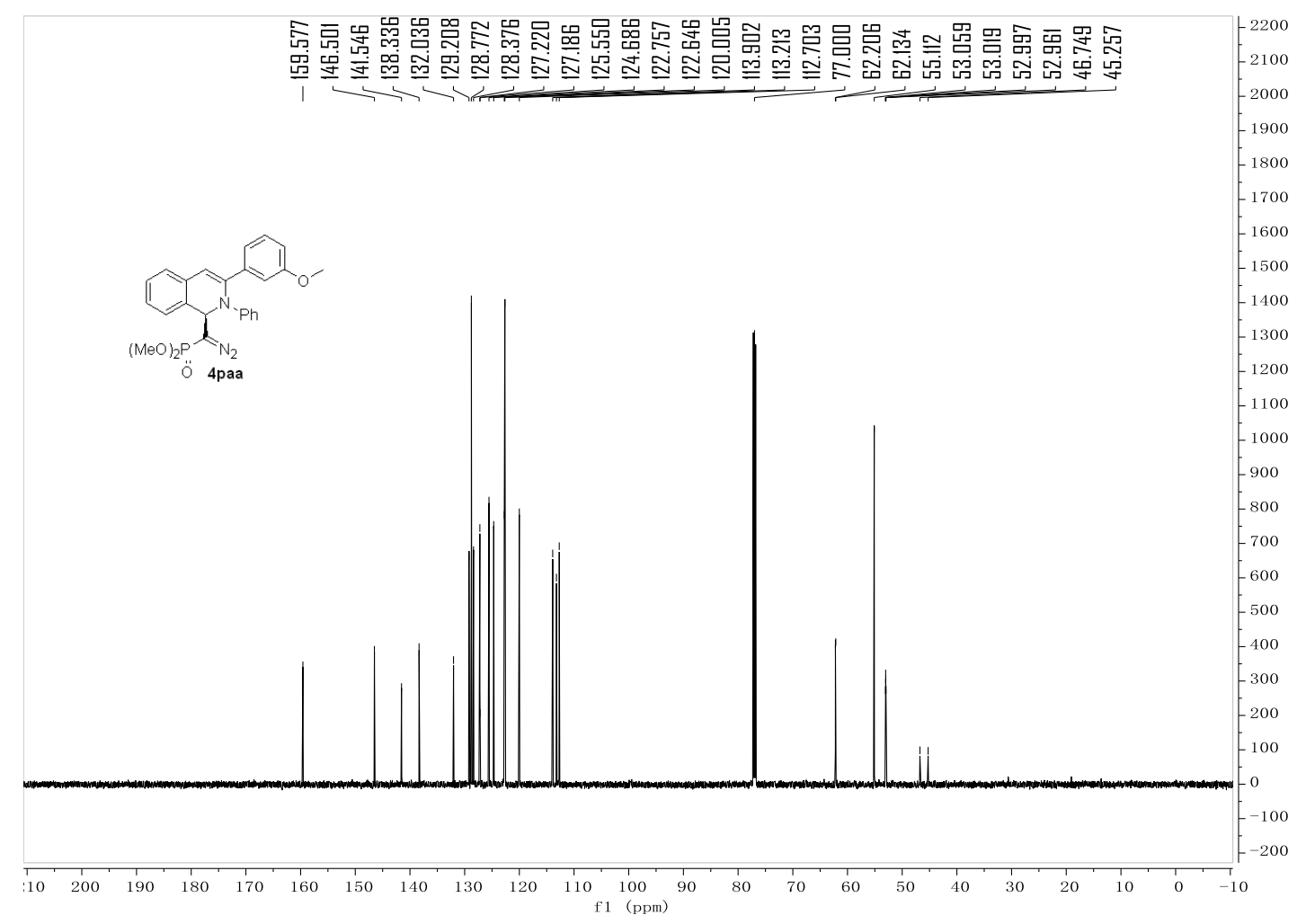




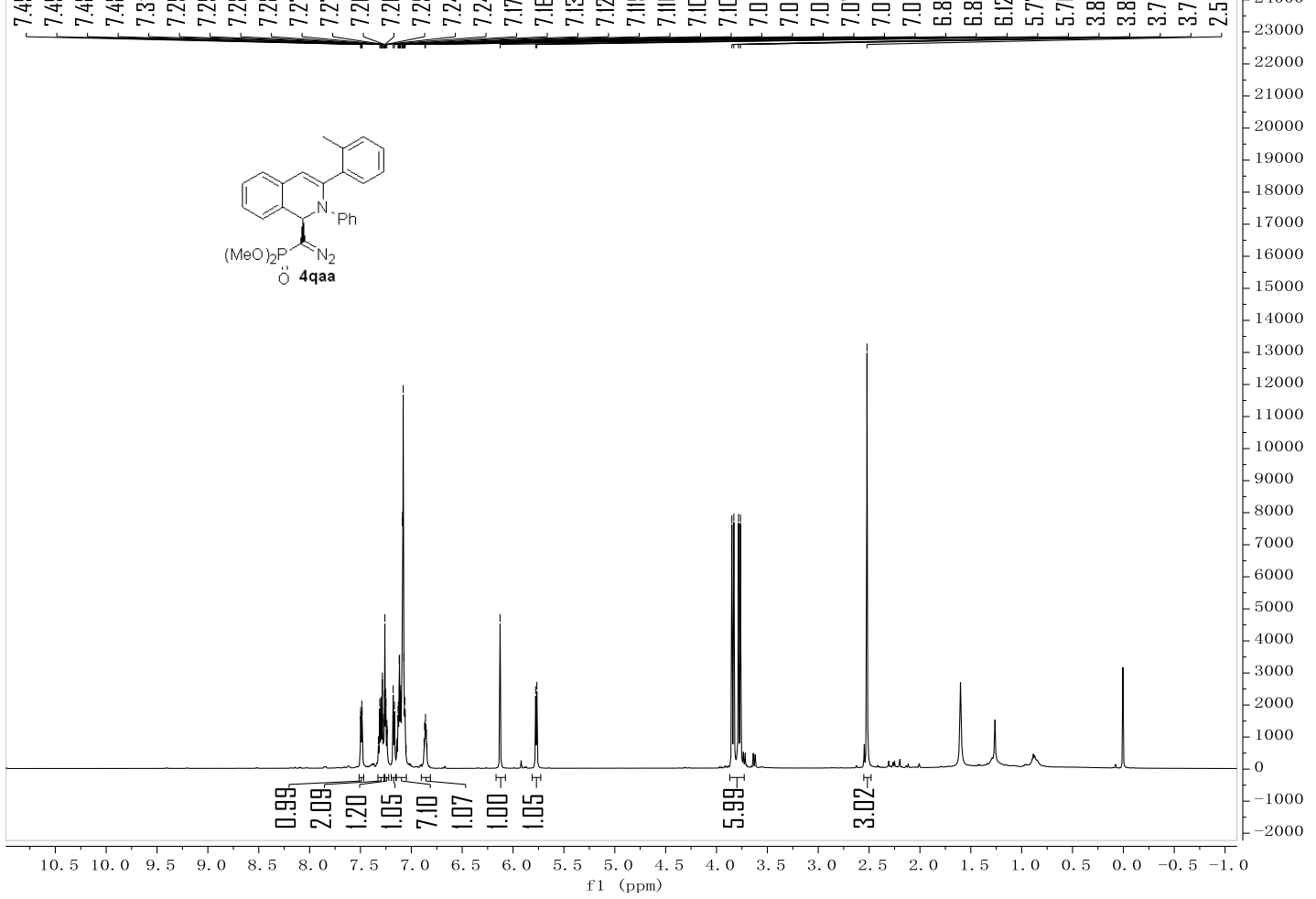

$151 \mathrm{MHz}, \mathrm{CDCl}_{3},{ }^{13} \mathrm{C} \mathrm{NMR}$

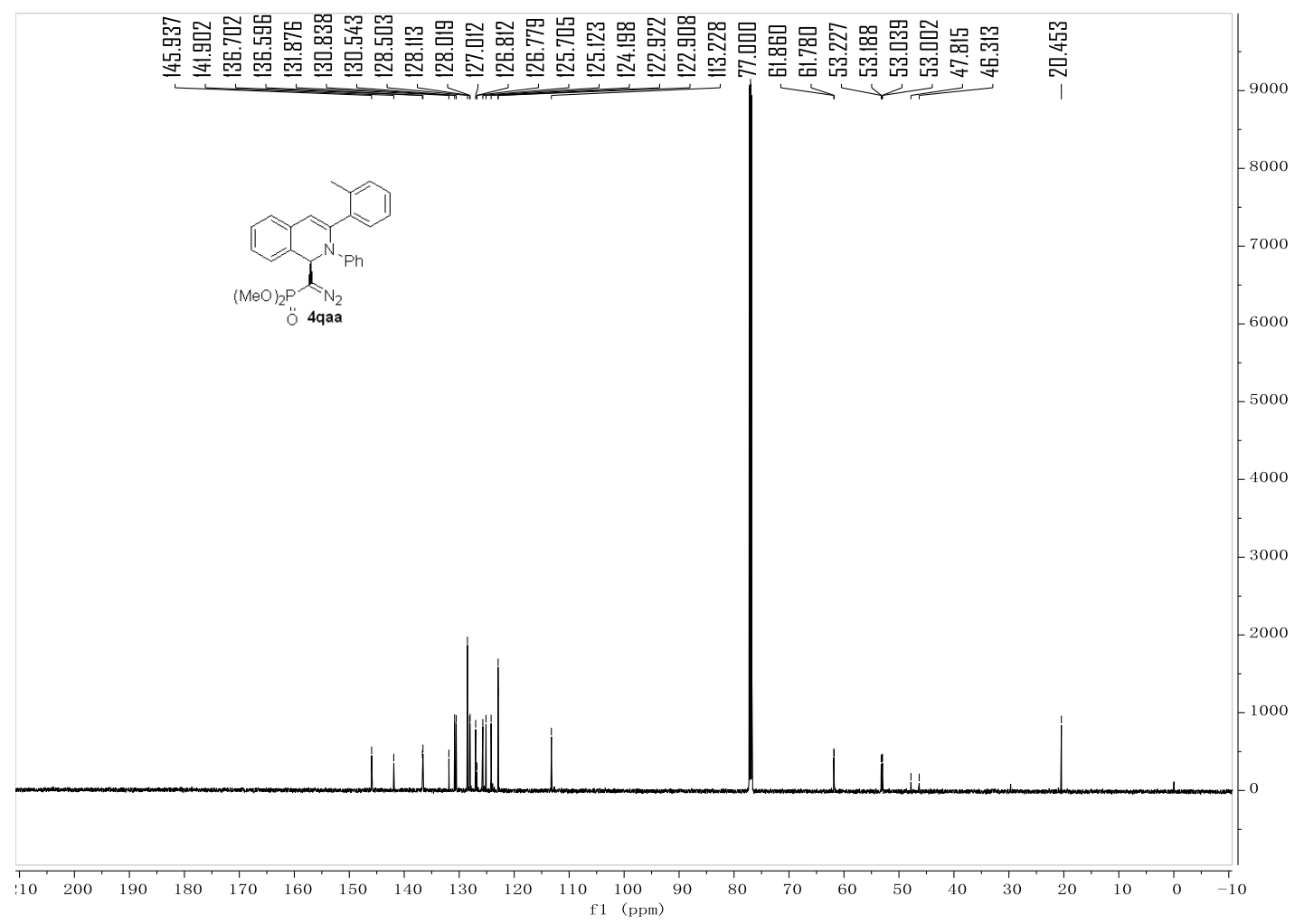


$600 \mathrm{MHz}, \mathrm{CDCl}_{3},{ }^{1} \mathrm{H} \mathrm{NMR}$

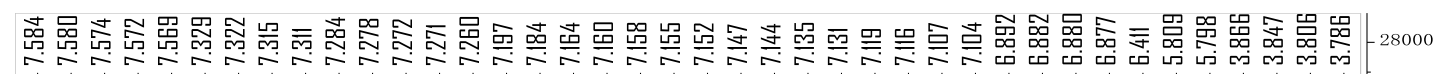

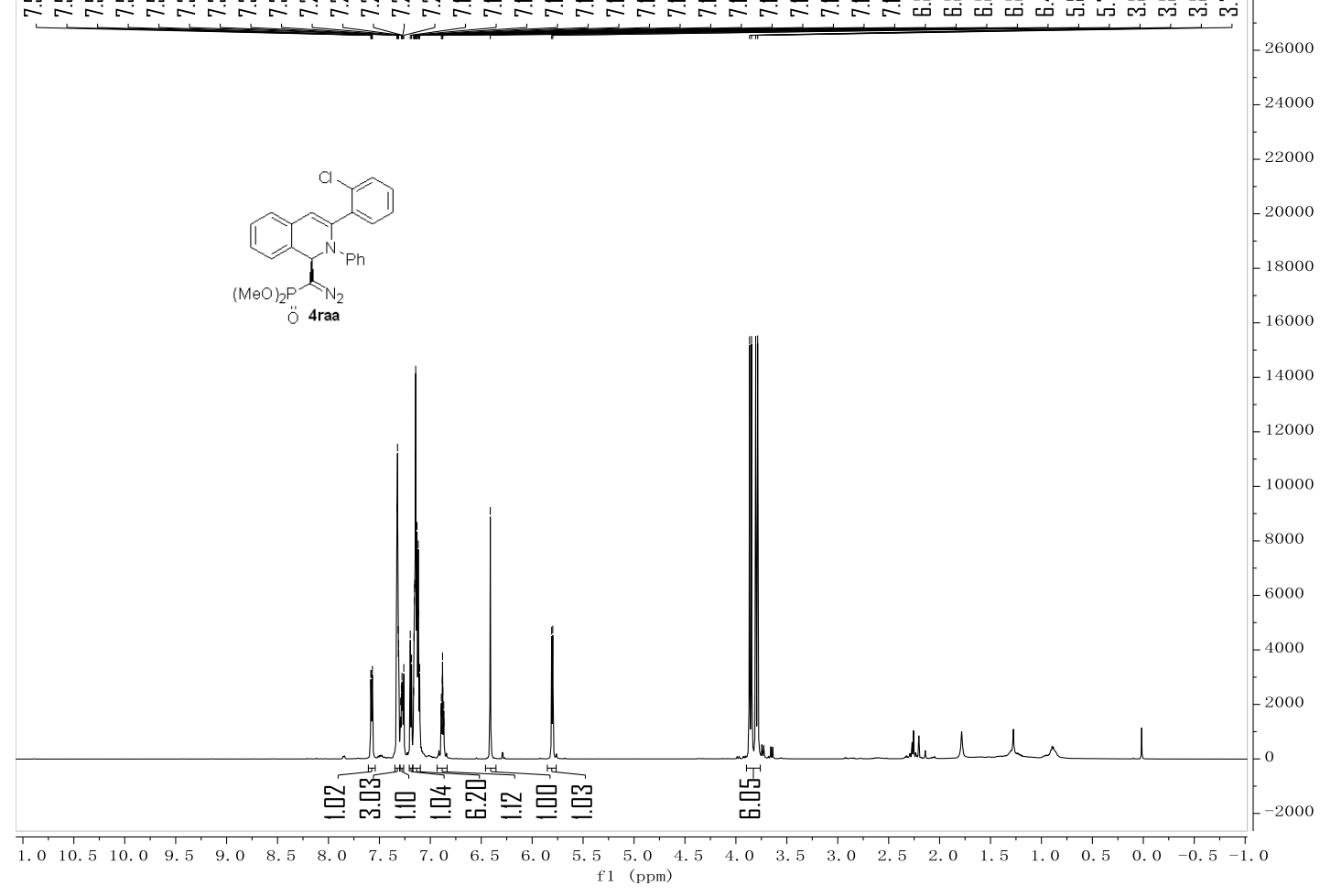

$151 \mathrm{MHz}, \mathrm{CDCl}_{3},{ }^{13} \mathrm{C} \mathrm{NMR}$

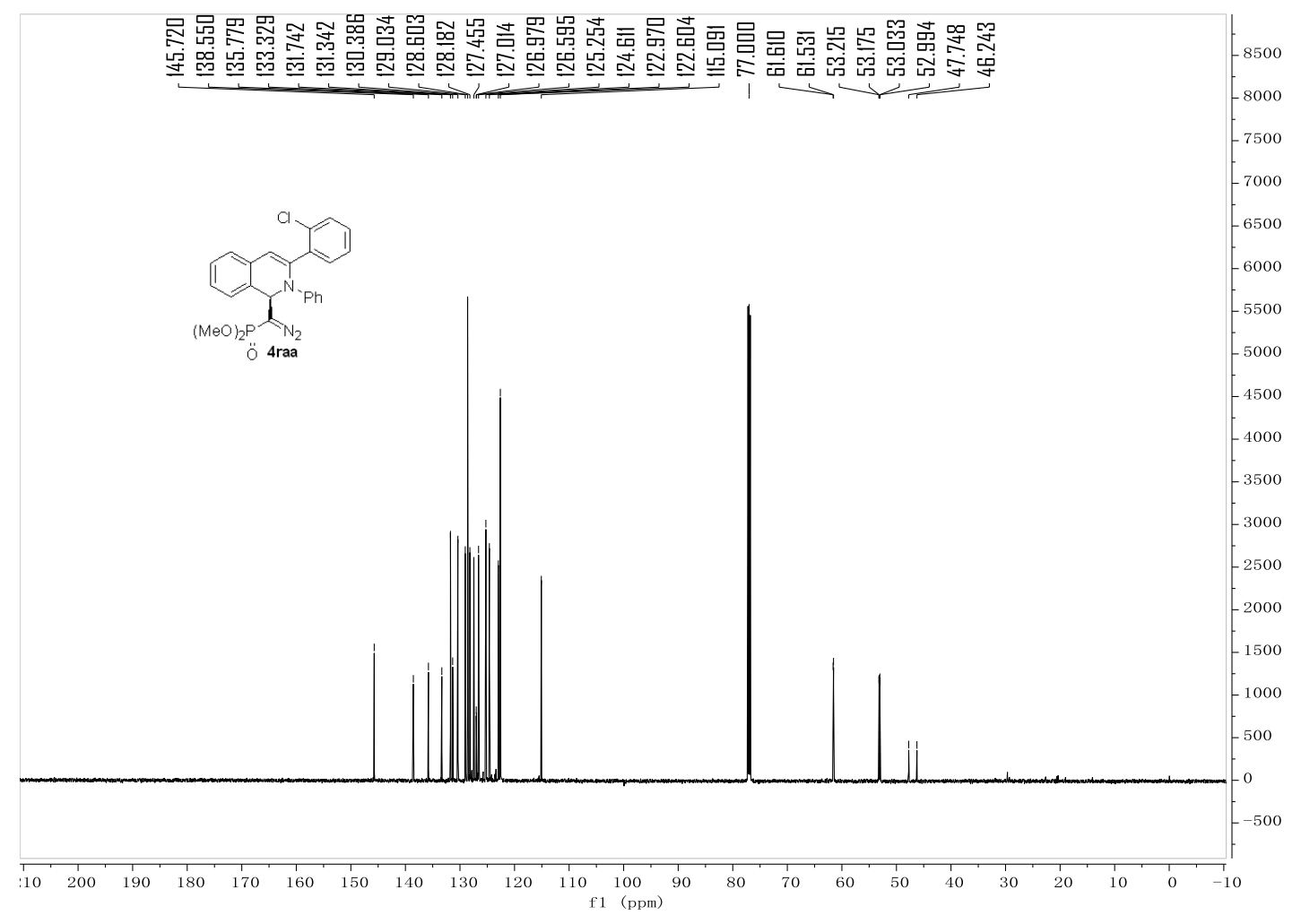




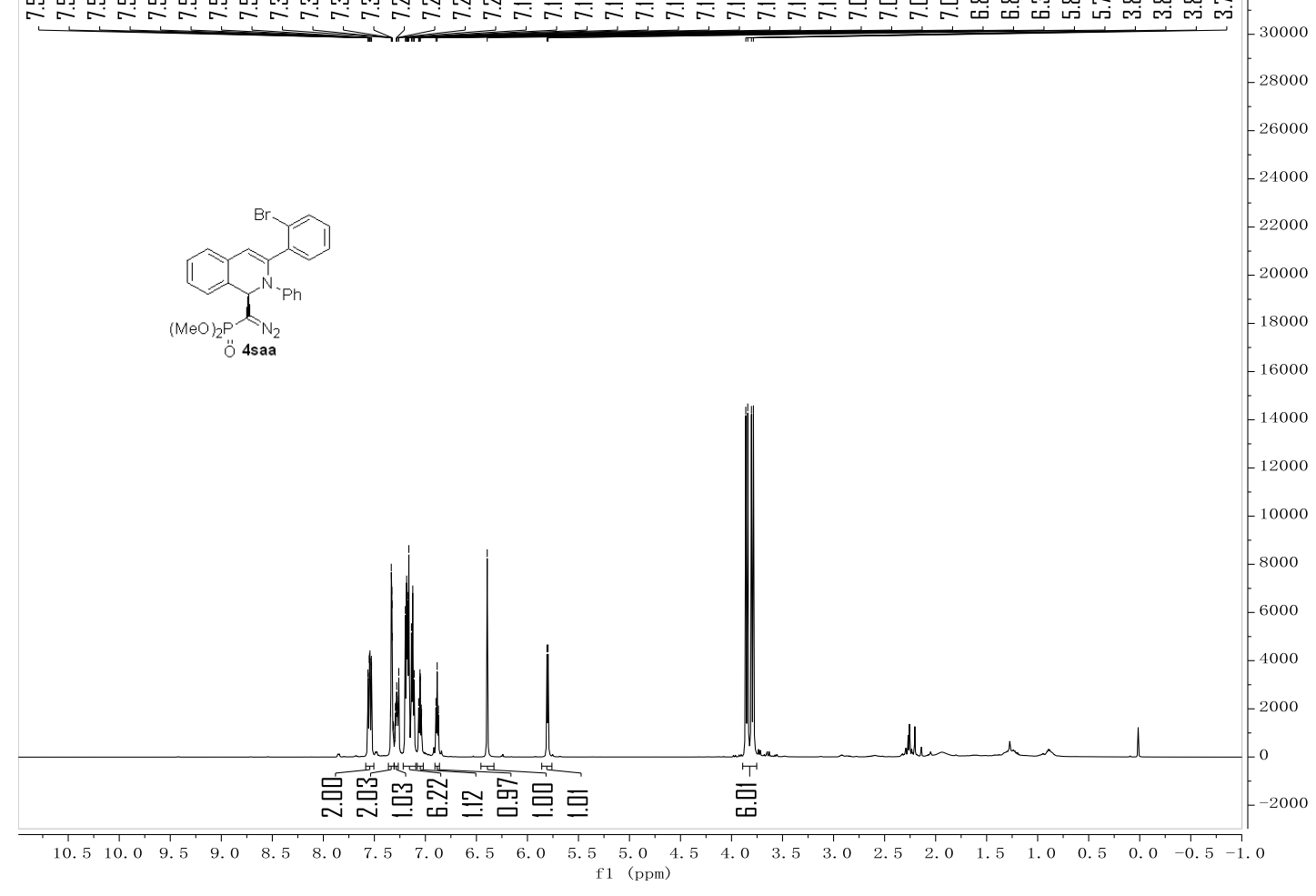

$151 \mathrm{MHz}, \mathrm{CDCl}_{3},{ }^{13} \mathrm{C} \mathrm{NMR}$

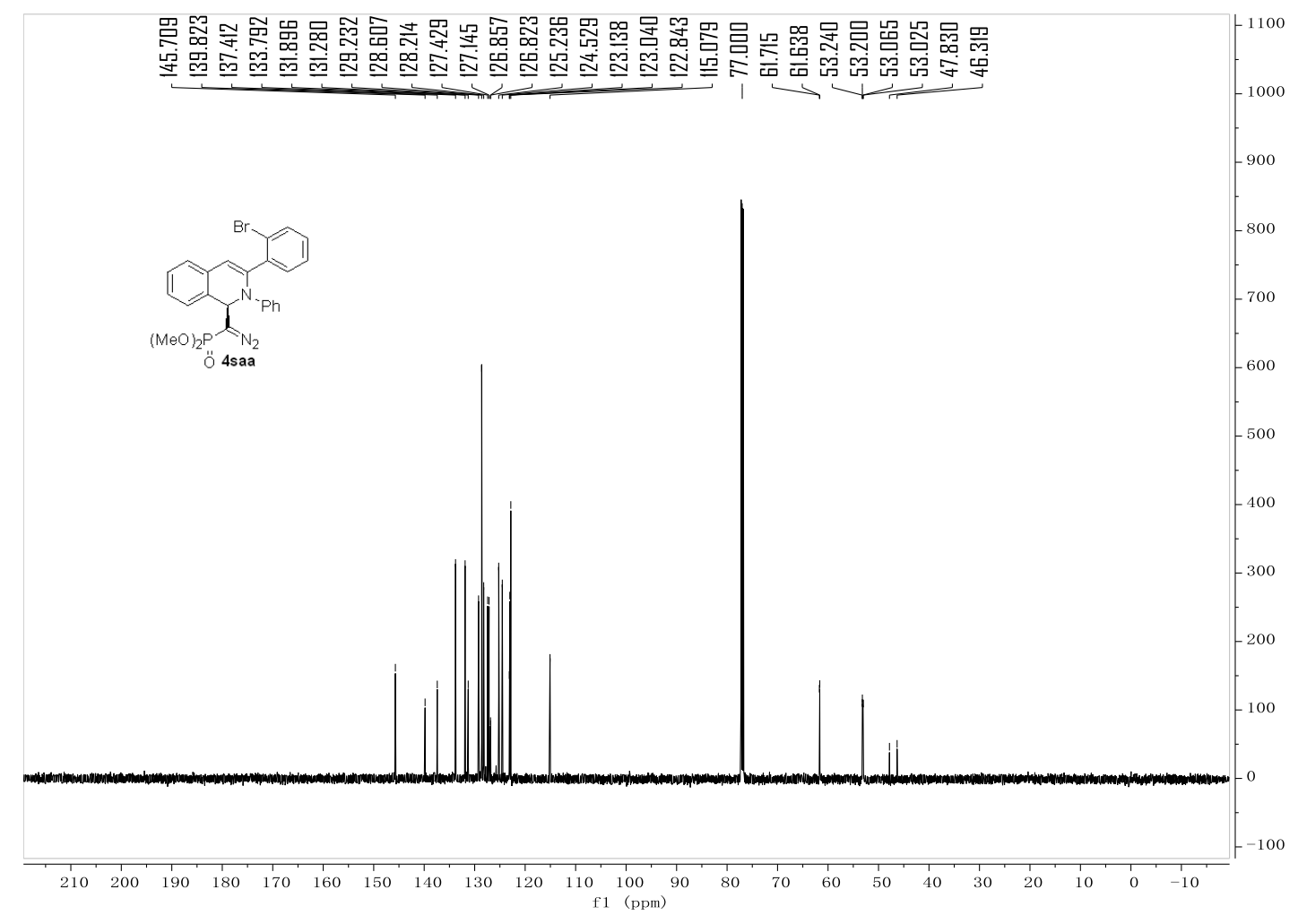




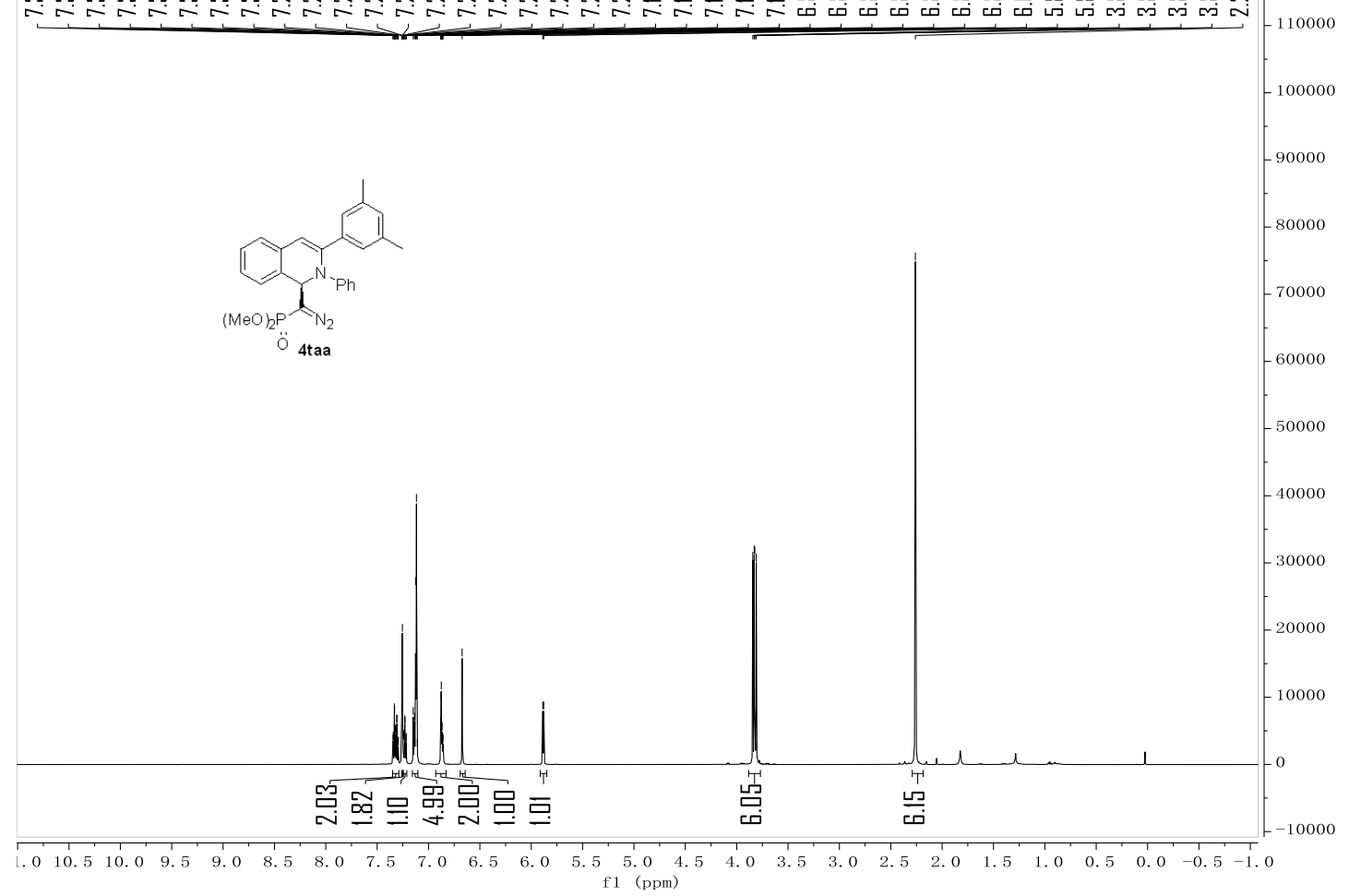

$151 \mathrm{MHz}, \mathrm{CDCl}_{3},{ }^{13} \mathrm{C} \mathrm{NMR}$

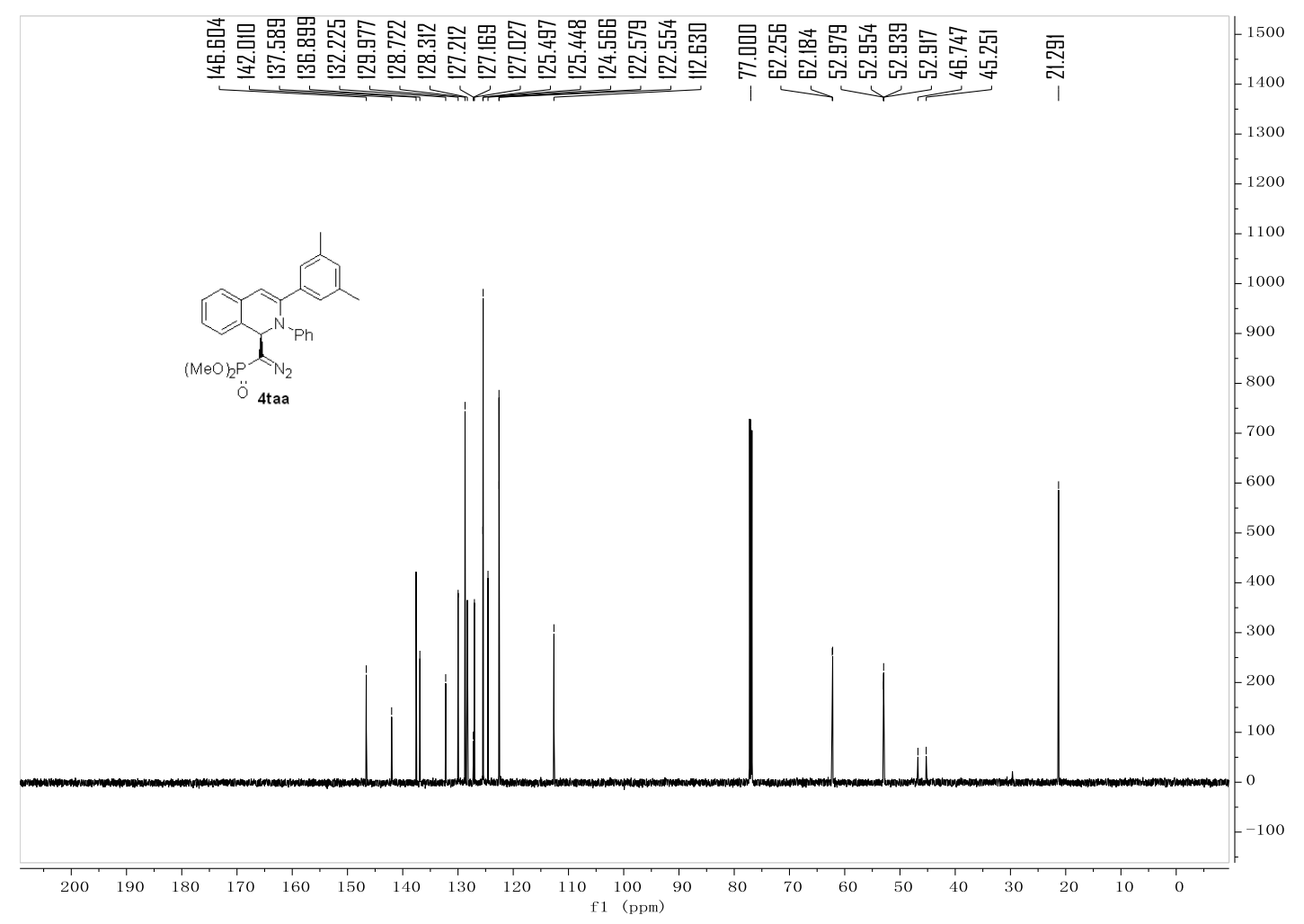



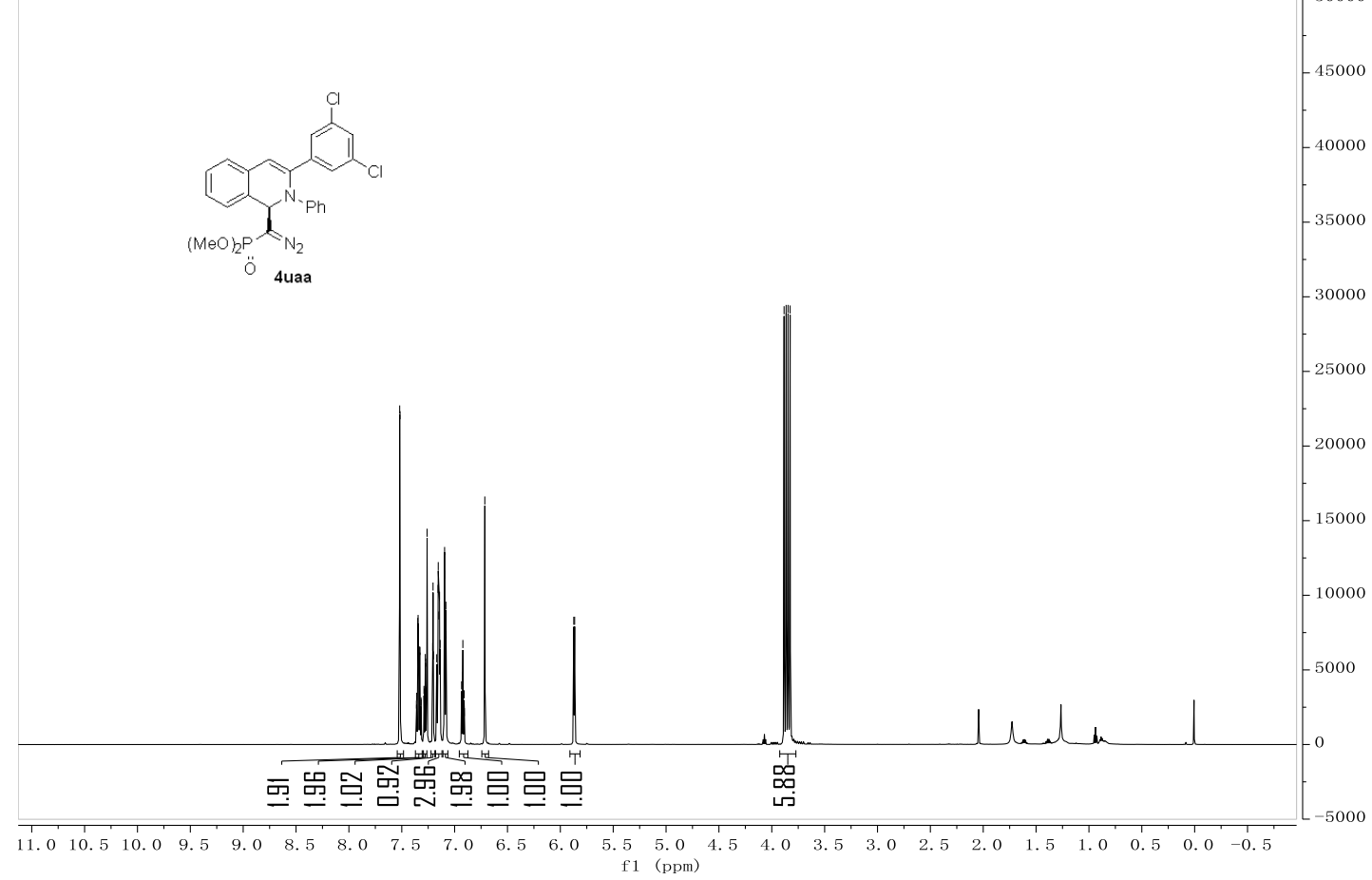

$151 \mathrm{MHz}, \mathrm{CDCl}_{3},{ }^{13} \mathrm{C} \mathrm{NMR}$

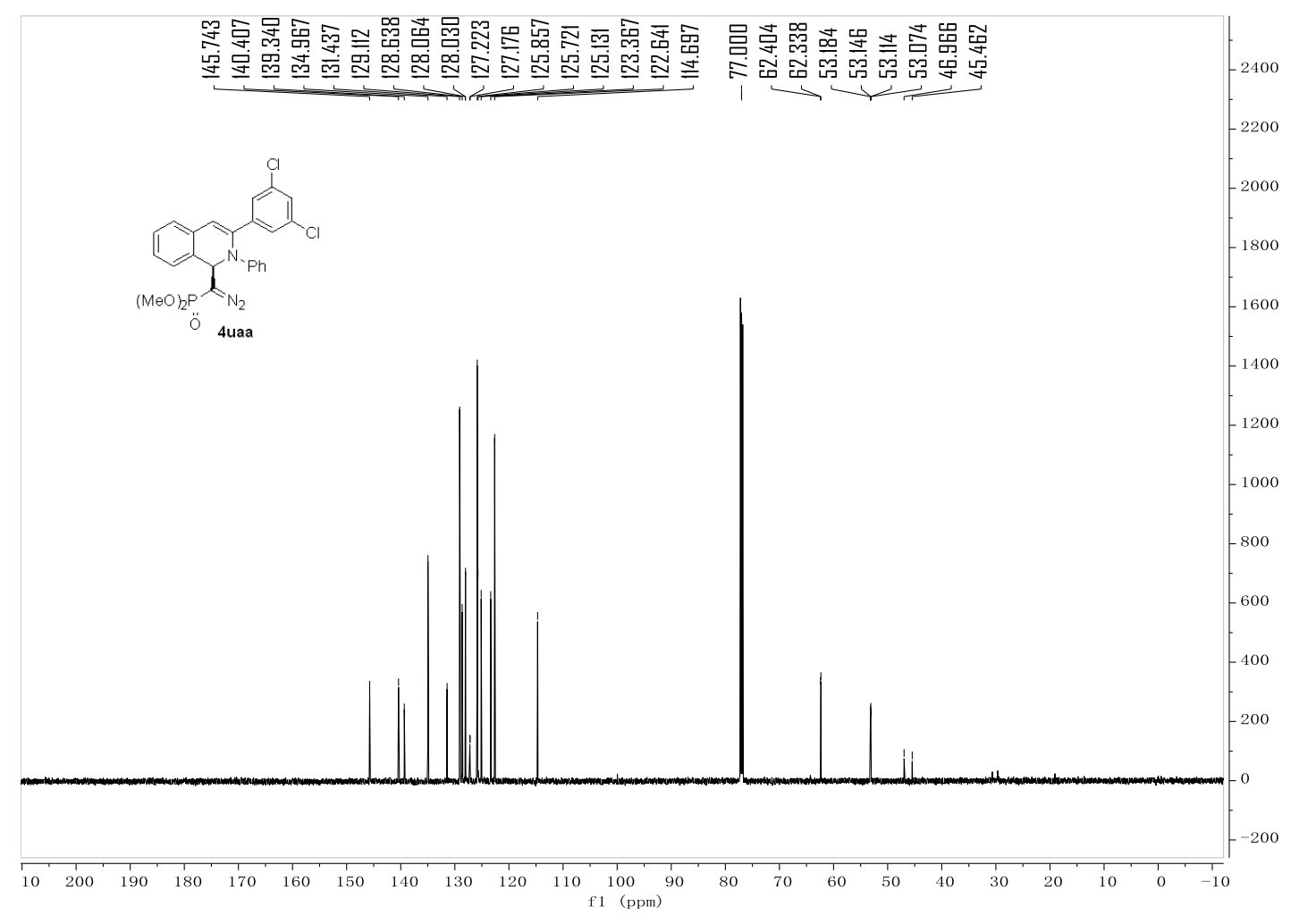




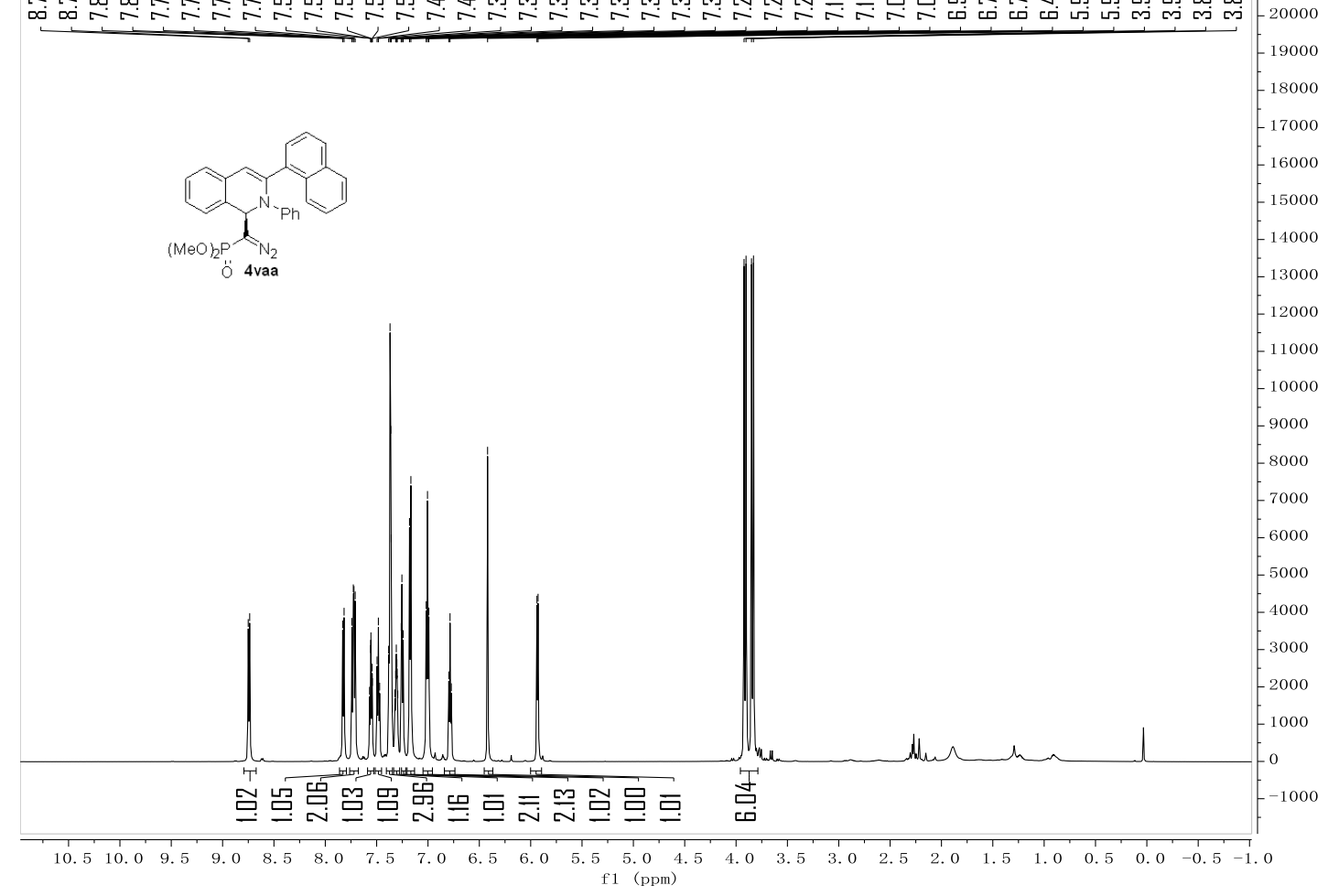

\section{$151 \mathrm{MHz}, \mathrm{CDCl}_{3},{ }^{13} \mathrm{C} \mathrm{NMR}$}

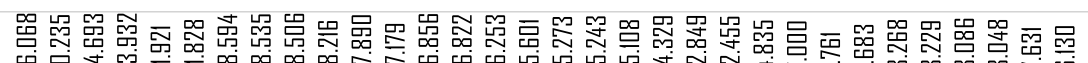

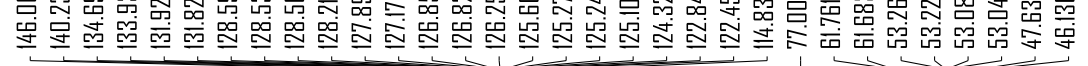
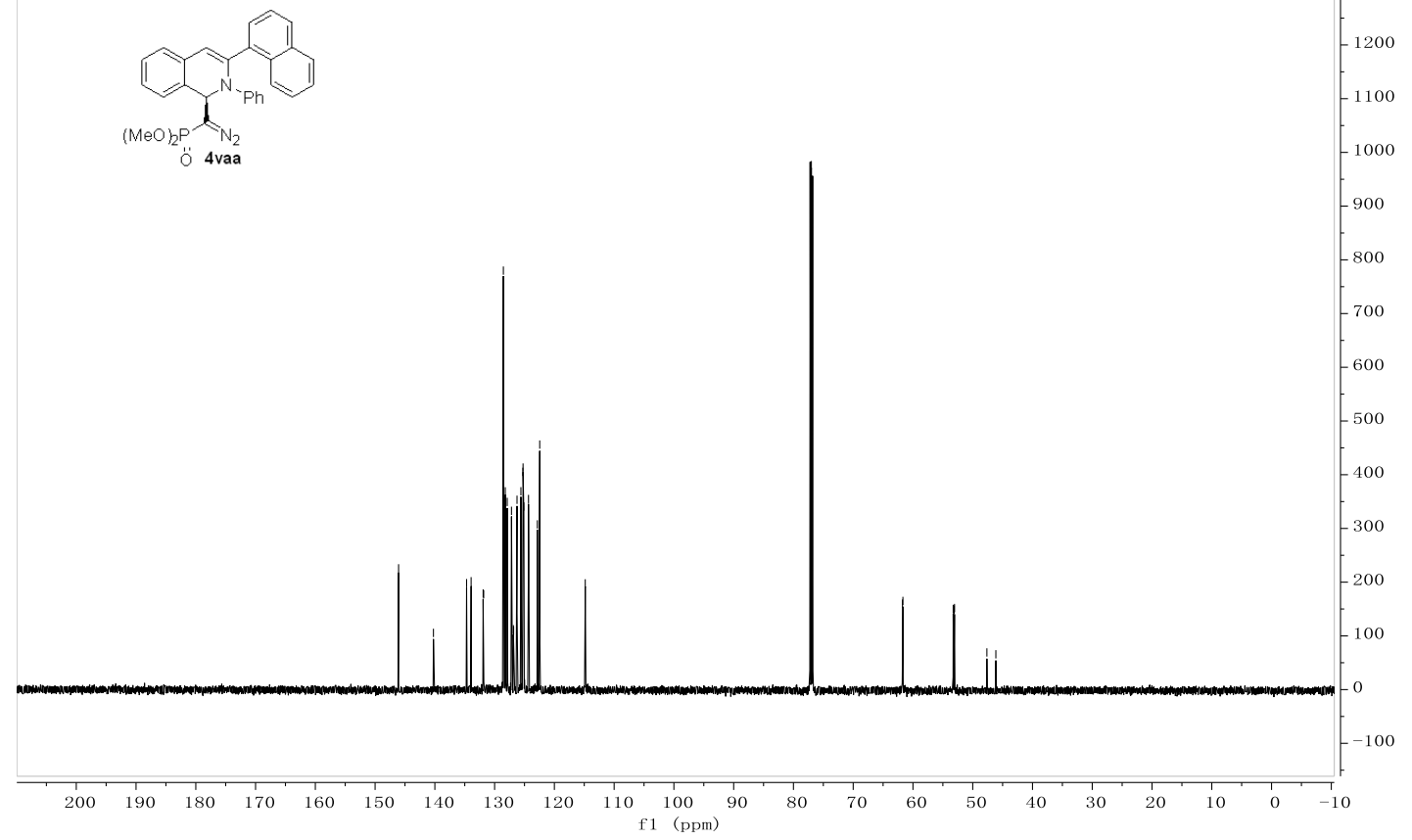
$600 \mathrm{MHz}, \mathrm{CDCl}_{3},{ }^{1} \mathrm{H} \mathrm{NMR}$

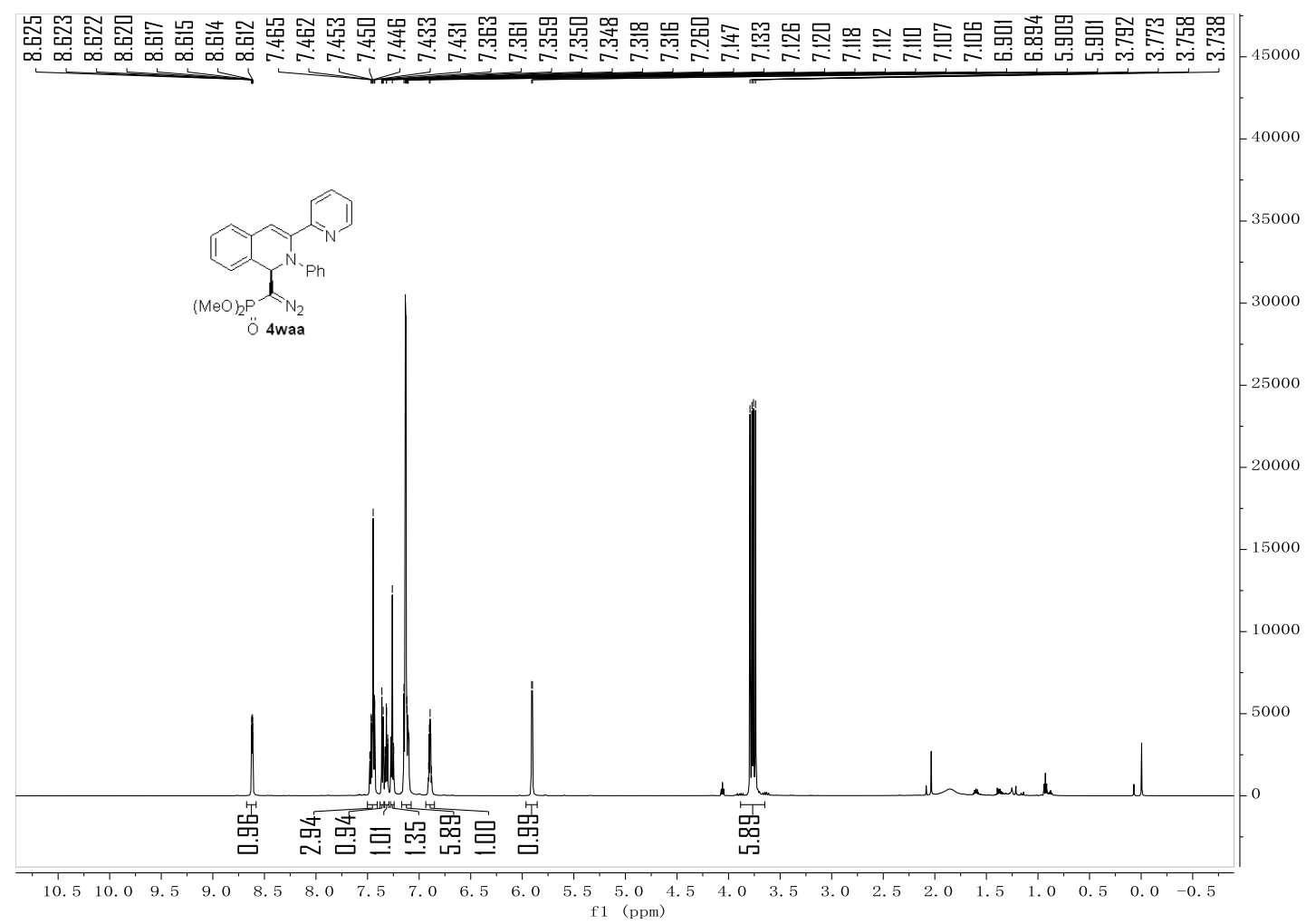

$151 \mathrm{MHz}, \mathrm{CDCl}_{3},{ }^{13} \mathrm{C} \mathrm{NMR}$

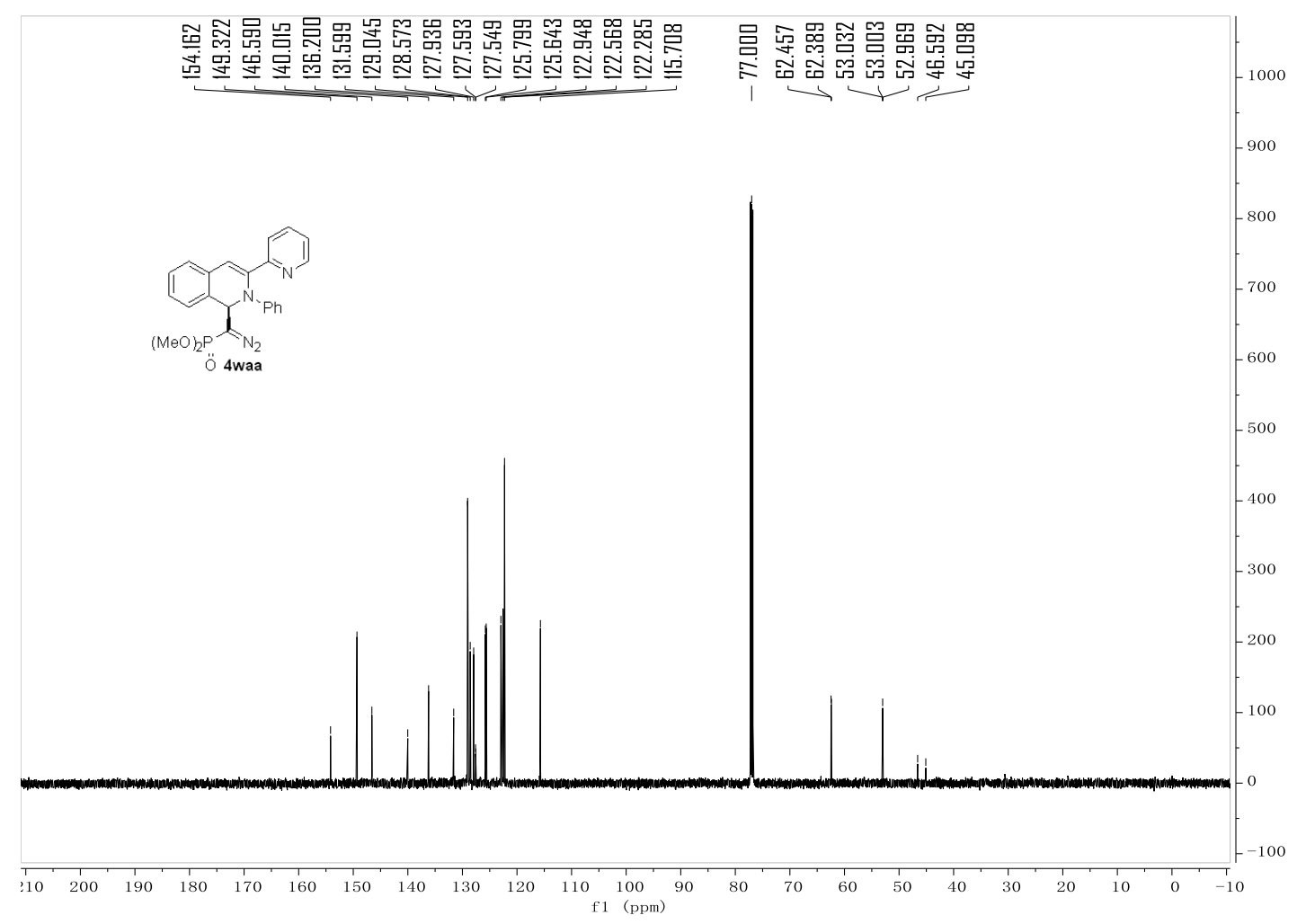




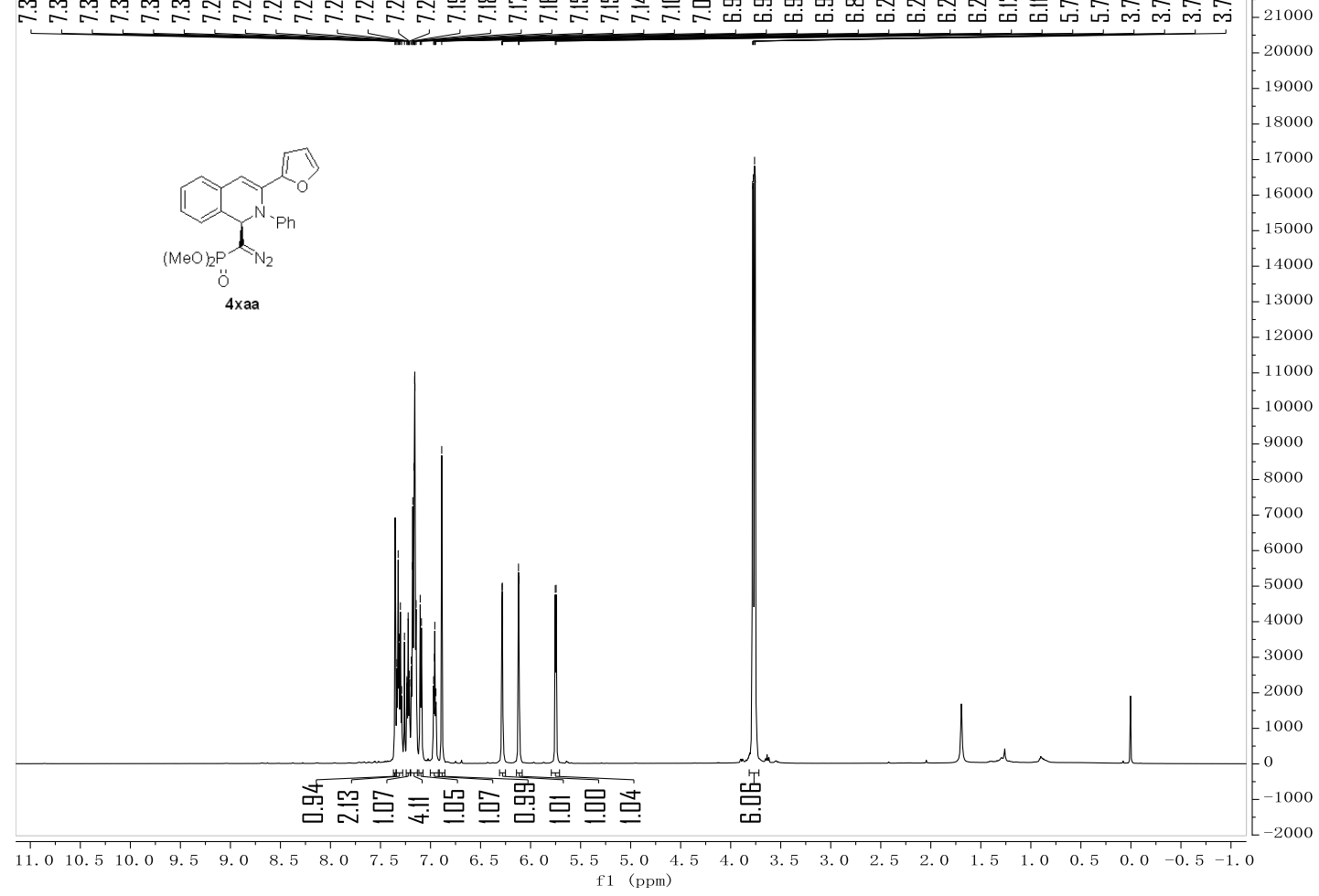

$151 \mathrm{MHz}, \mathrm{CDCl}_{3},{ }^{13} \mathrm{C} \mathrm{NMR}$

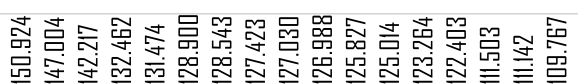

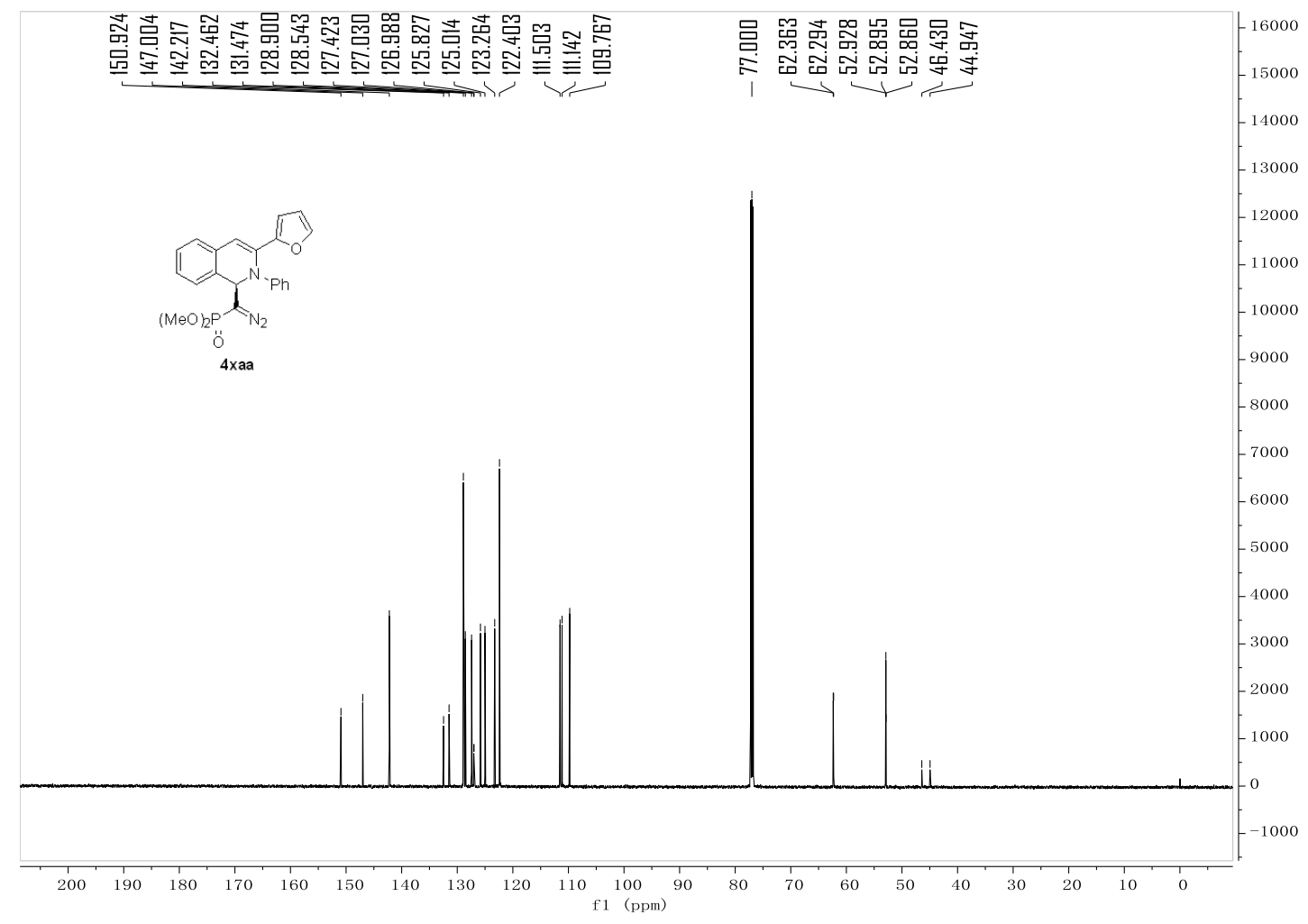


$600 \mathrm{MHz}, \mathrm{CDCl}_{3},{ }^{1} \mathrm{H} \mathrm{NMR}$

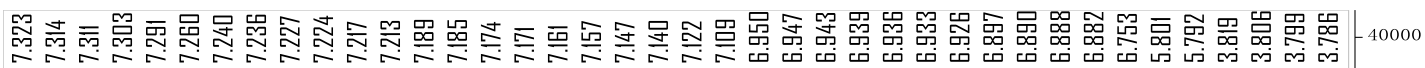

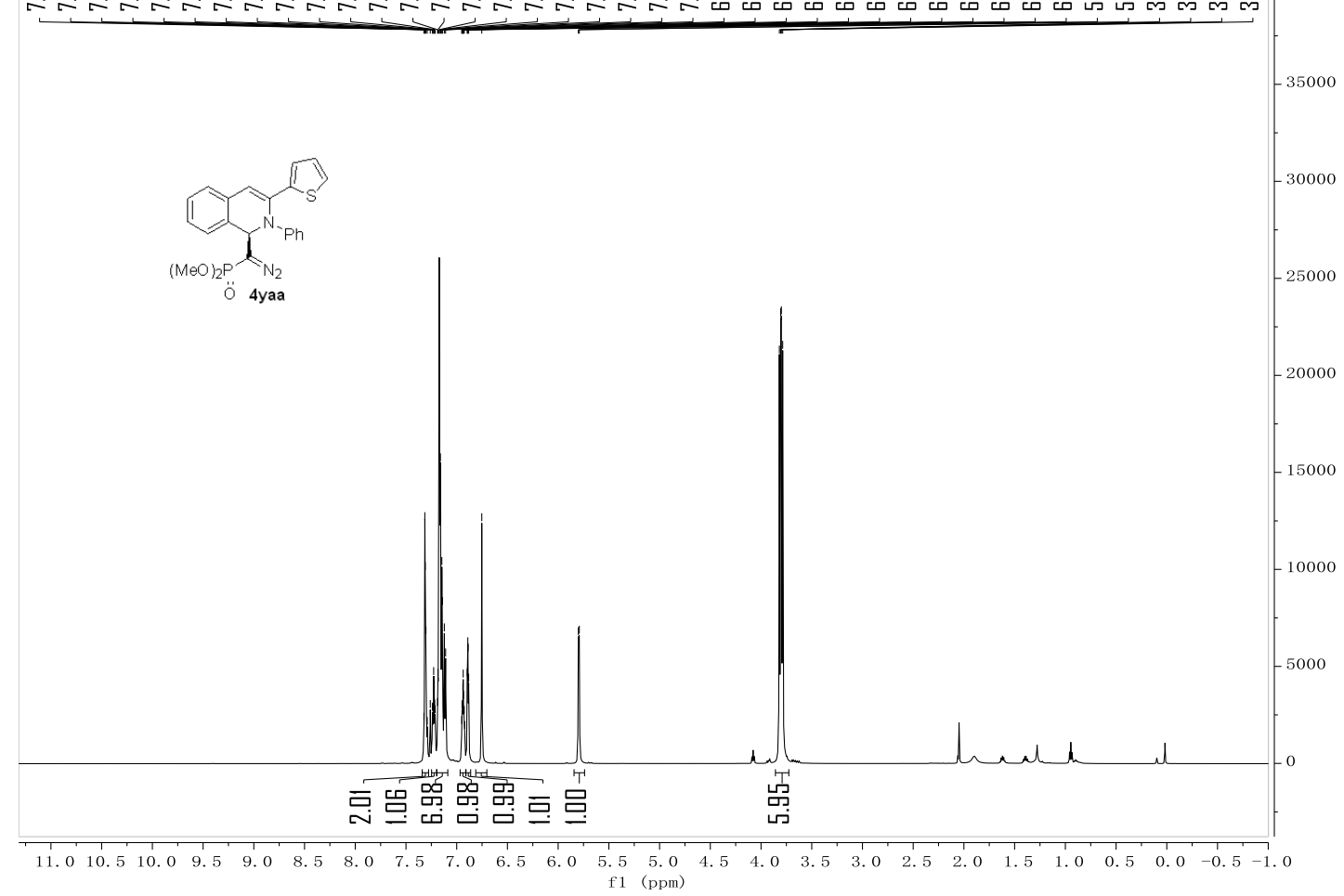

$151 \mathrm{MHz}, \mathrm{CDCl}_{3},{ }^{13} \mathrm{C} \mathrm{NMR}$

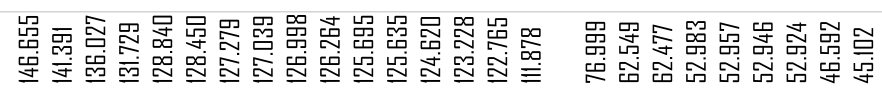

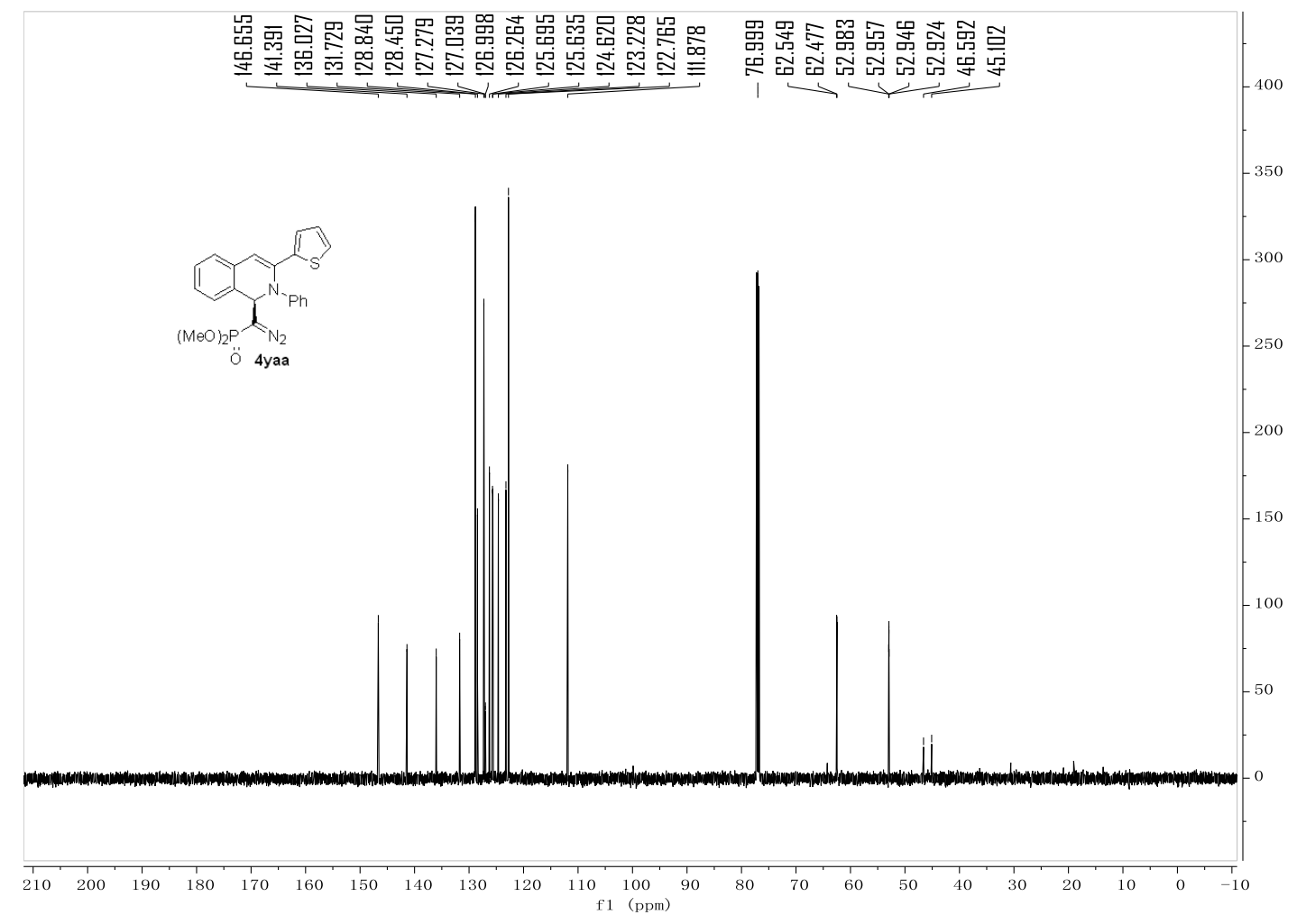




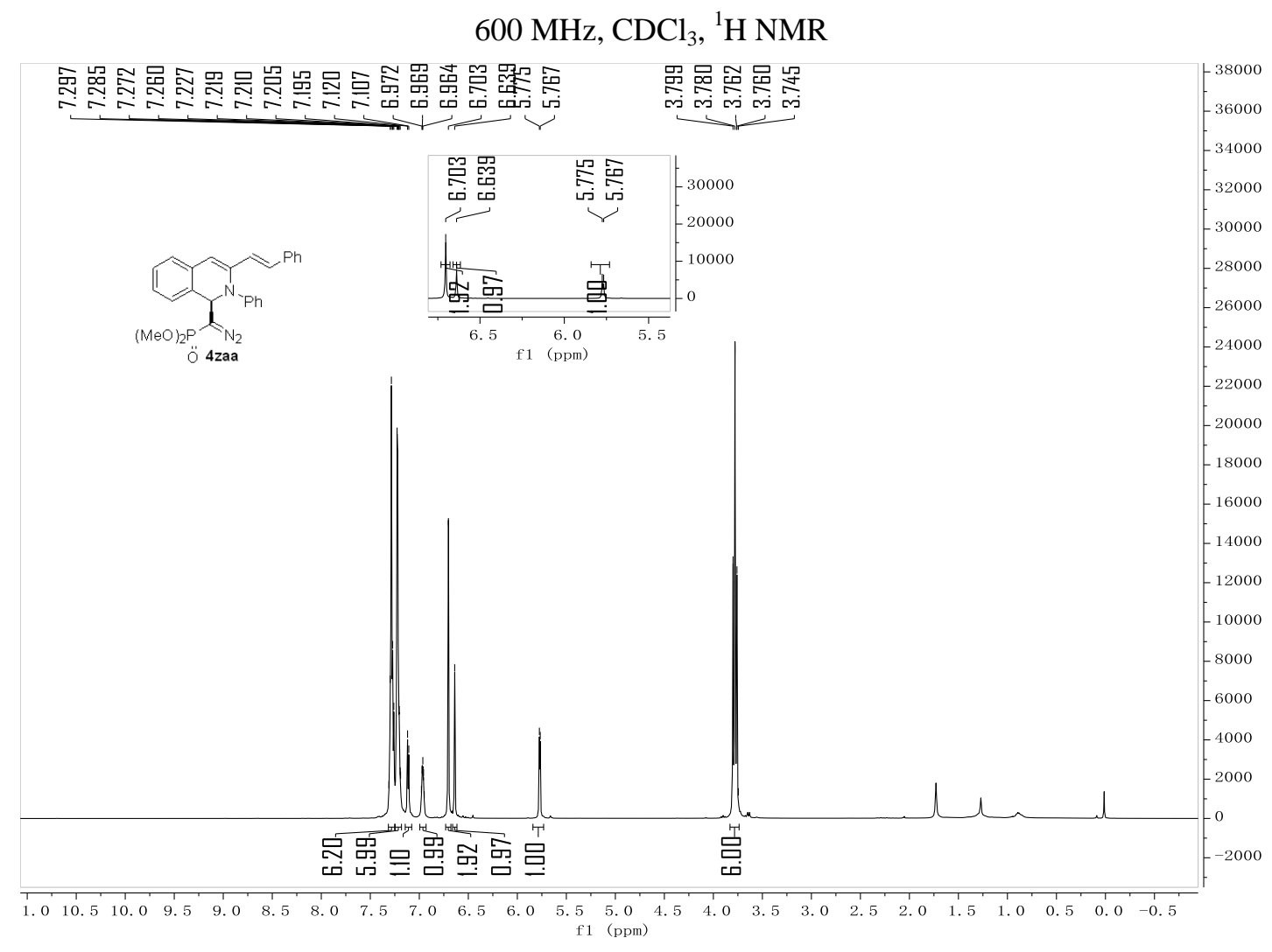

$151 \mathrm{MHz}, \mathrm{CDCl}_{3},{ }^{13} \mathrm{C} \mathrm{NMR}$

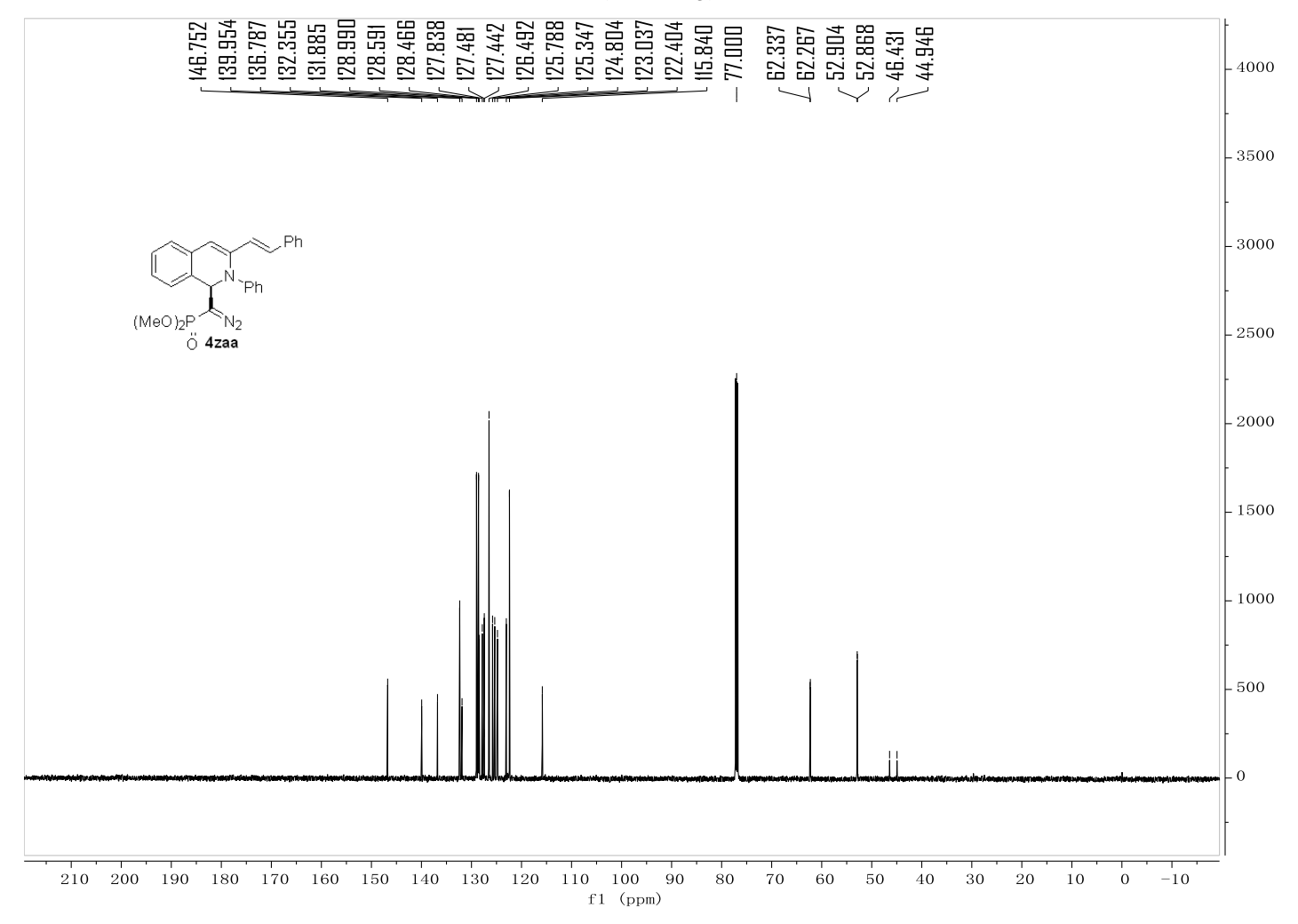




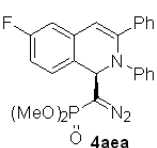

$151 \mathrm{MHz}, \mathrm{CDCl}_{3},{ }^{13} \mathrm{C} \mathrm{NMR}$

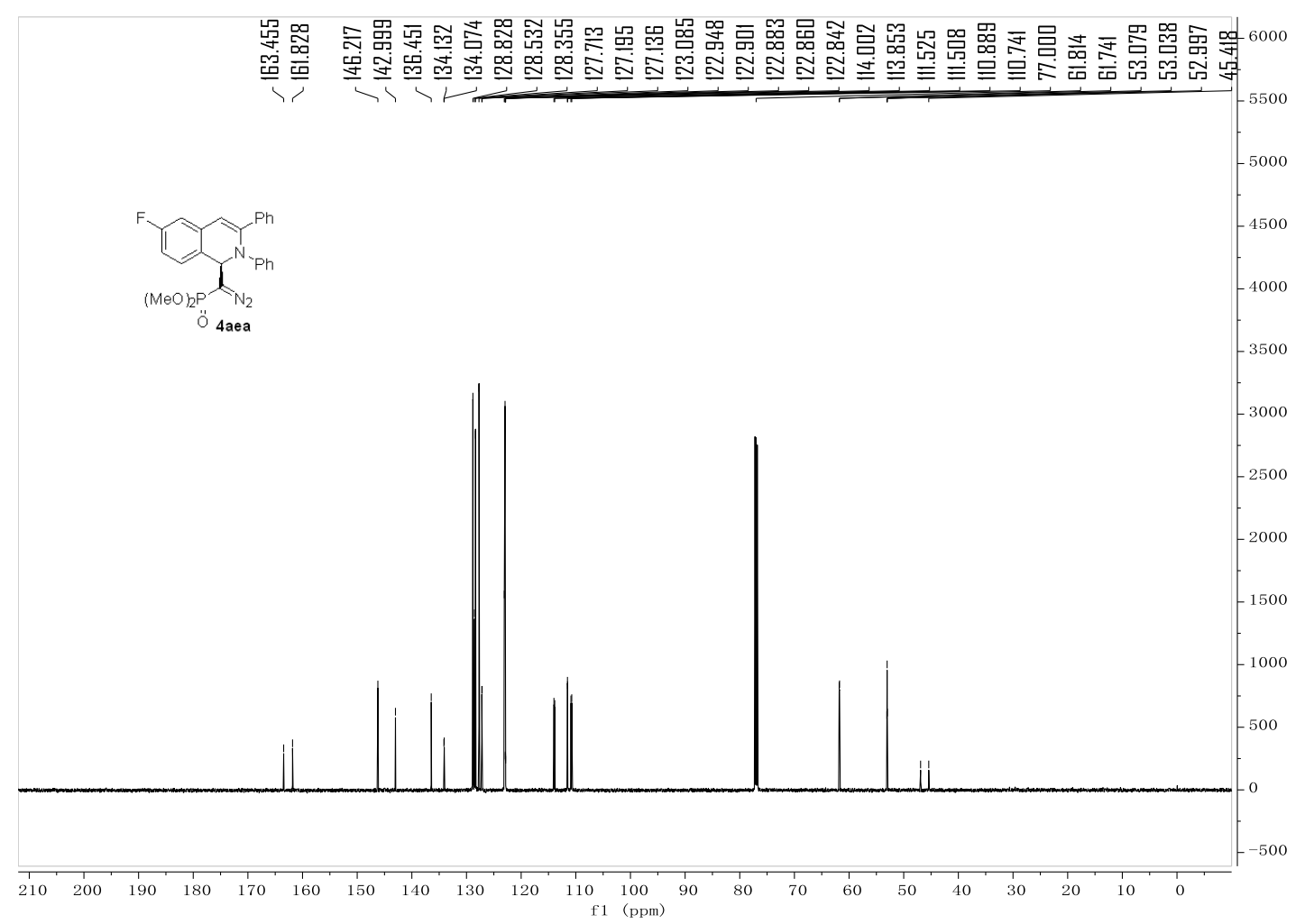




\section{$600 \mathrm{MHz}, \mathrm{CDCl}_{3},{ }^{1} \mathrm{H} \mathrm{NMR}$}

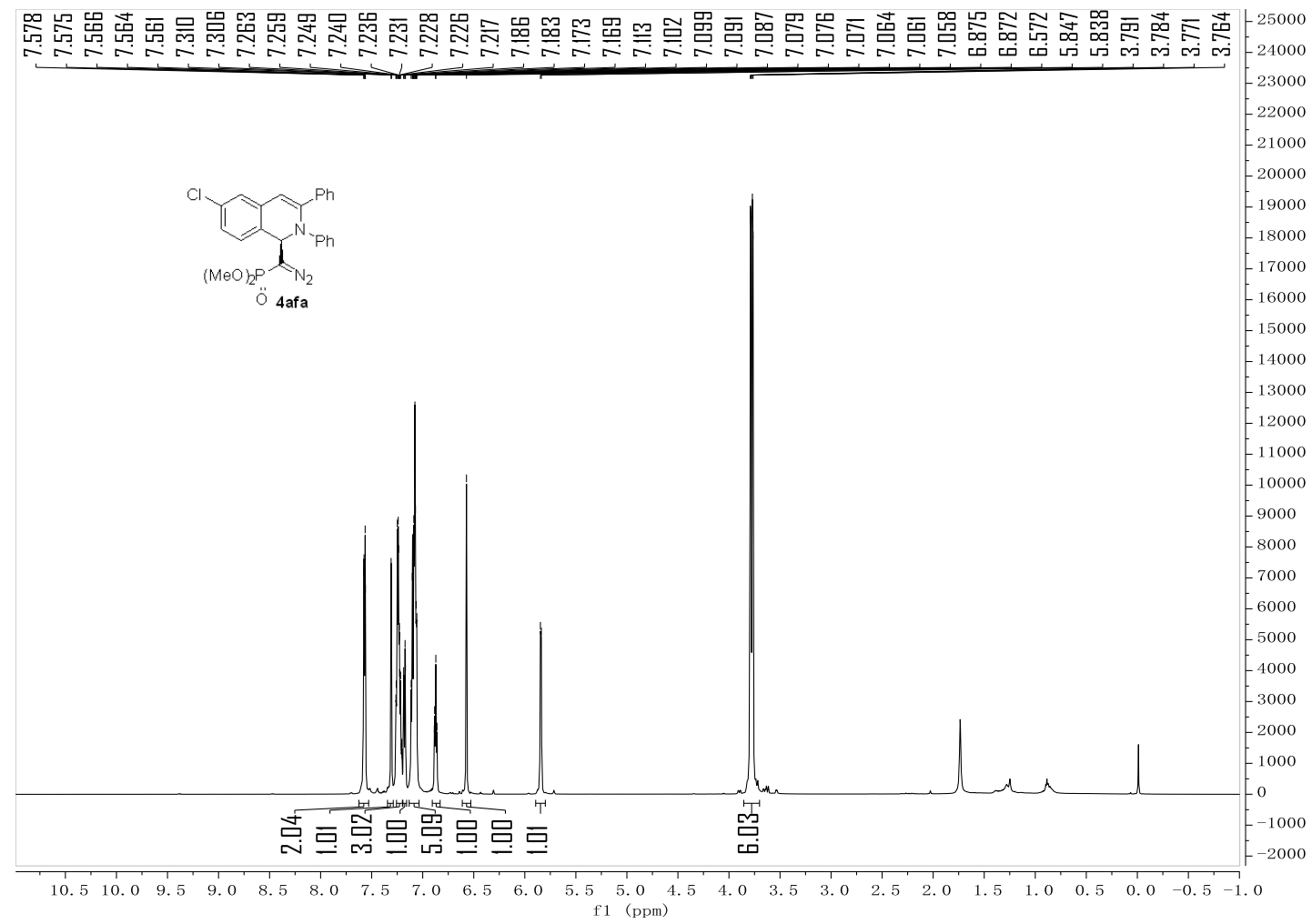

\section{$151 \mathrm{MHz}, \mathrm{CDCl}_{3},{ }^{13} \mathrm{C} \mathrm{NMR}$}

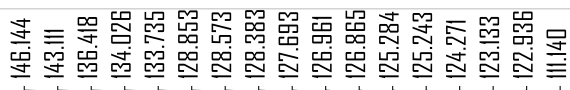

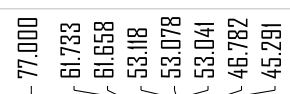

2400

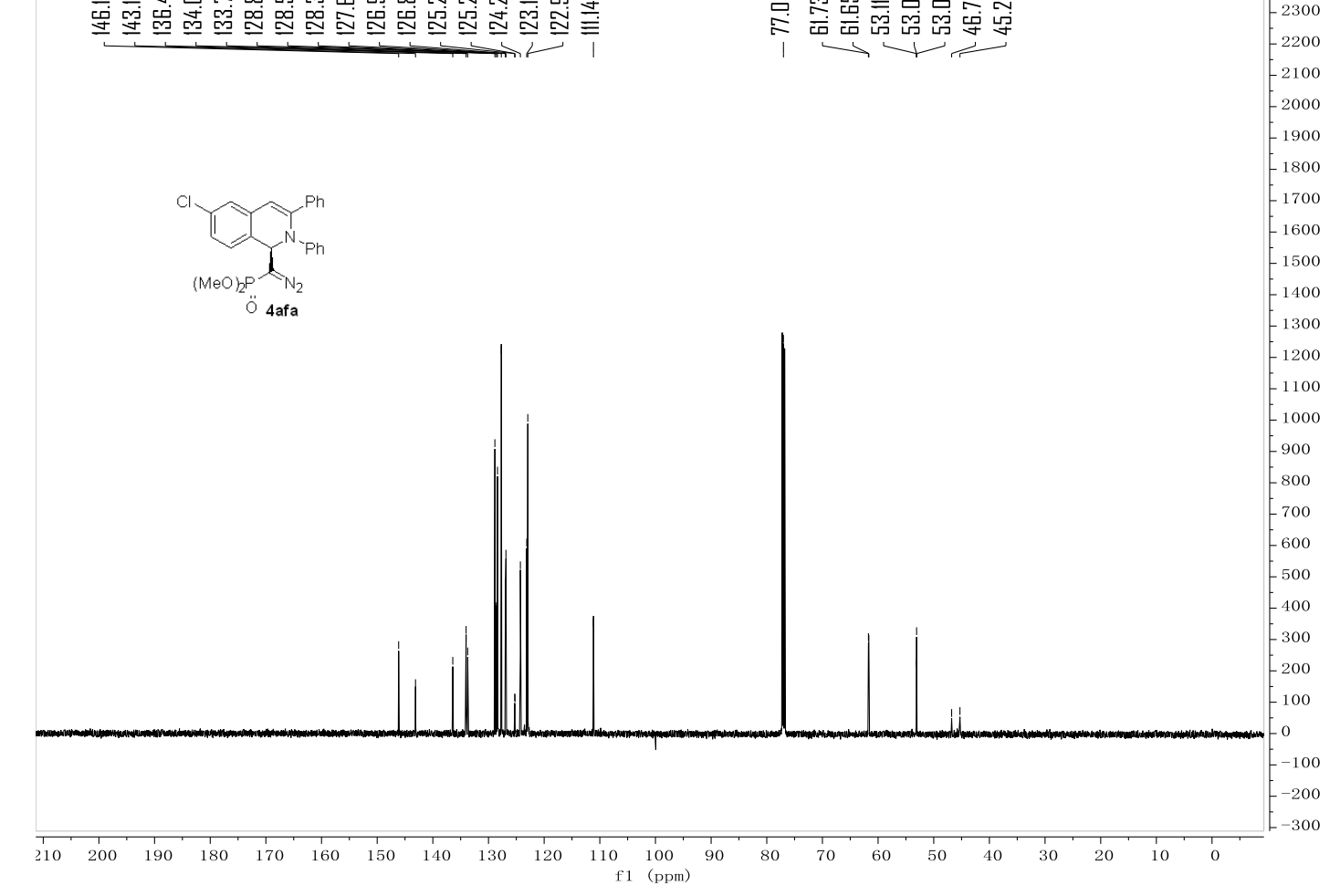




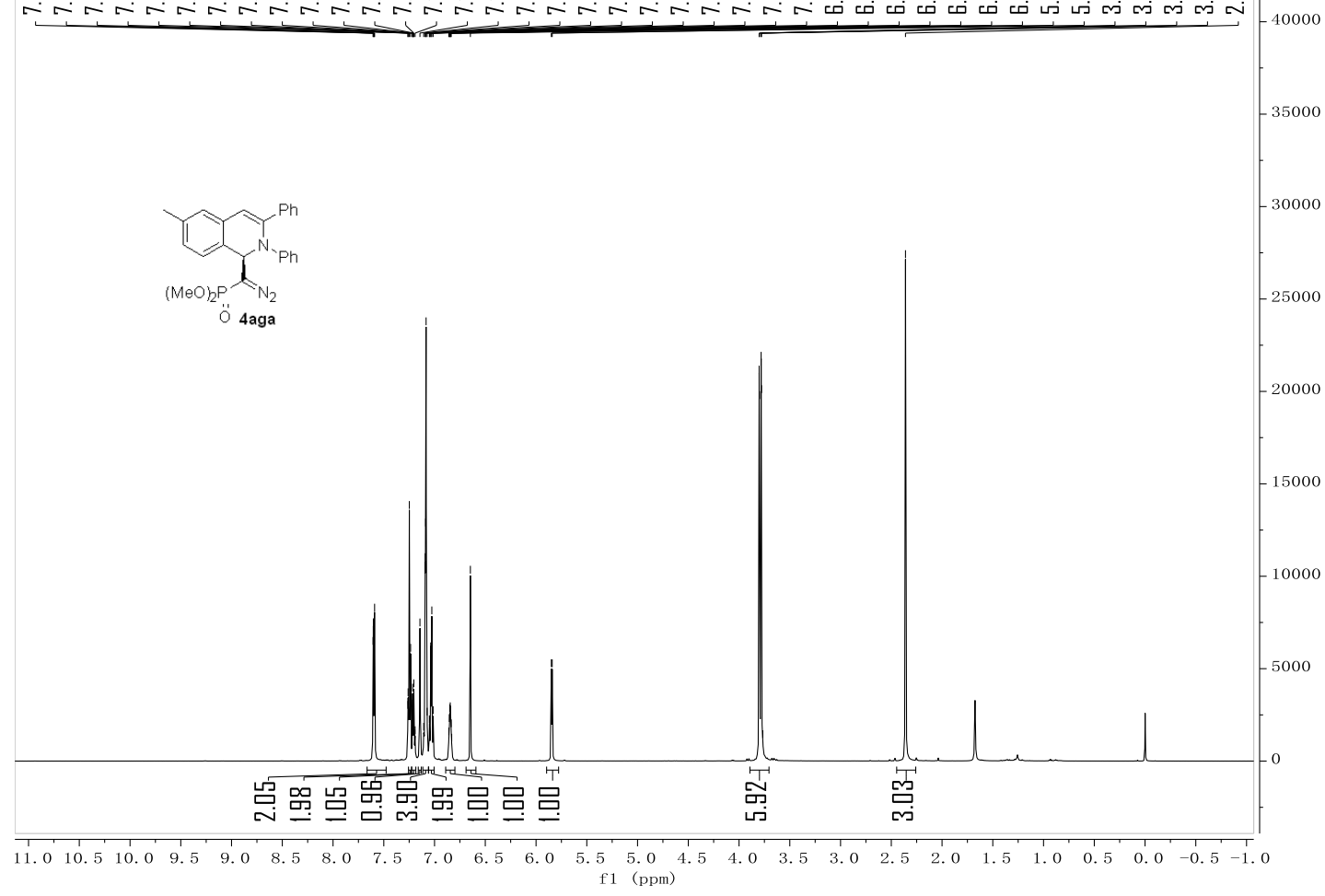

$151 \mathrm{MHz}, \mathrm{CDCl}_{3},{ }^{13} \mathrm{C} \mathrm{NMR}$

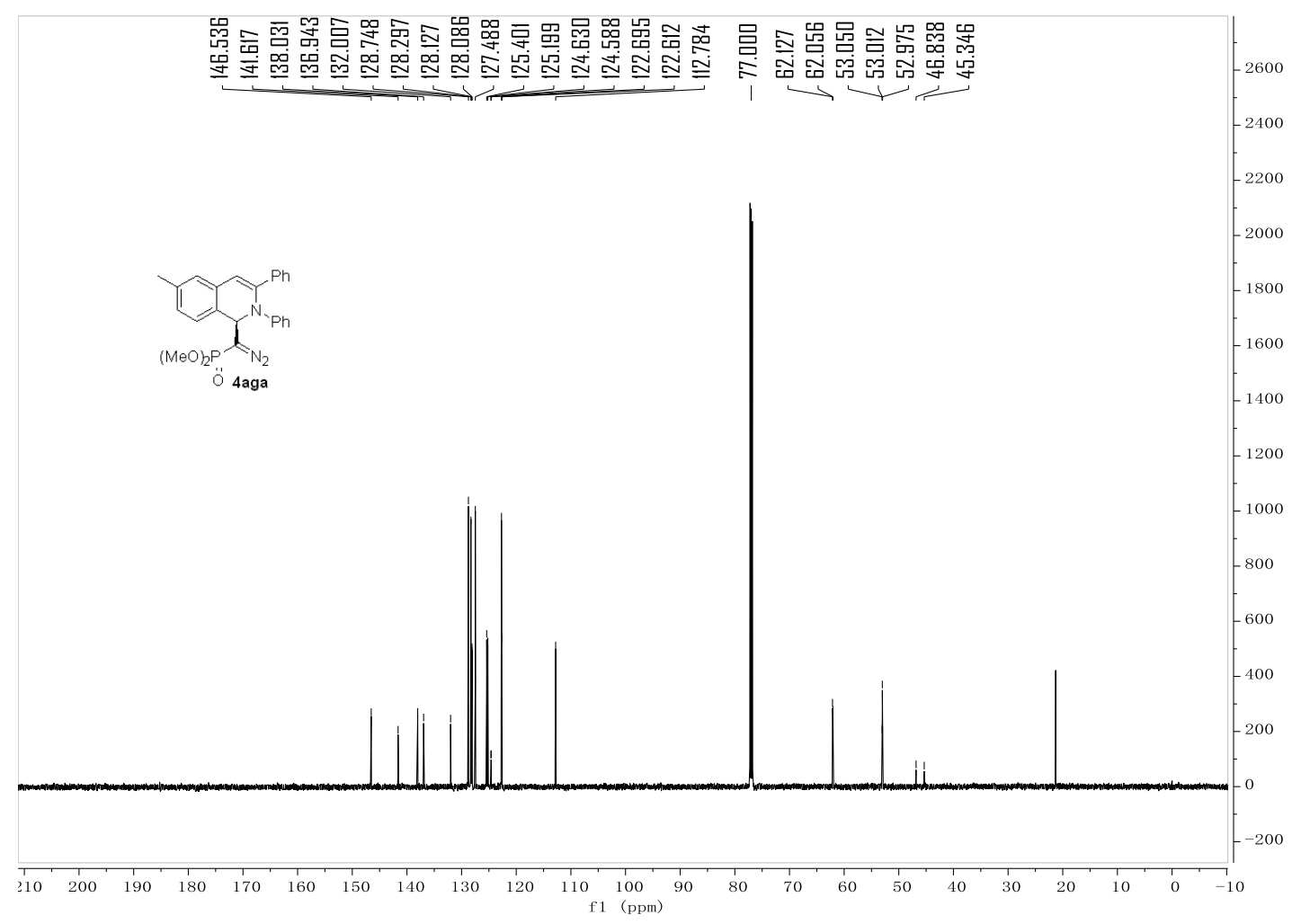


$600 \mathrm{MHz}, \mathrm{CDCl}_{3},{ }^{1} \mathrm{H} \mathrm{NMR}$

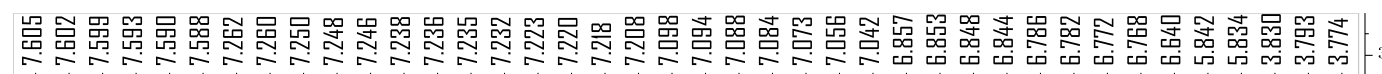

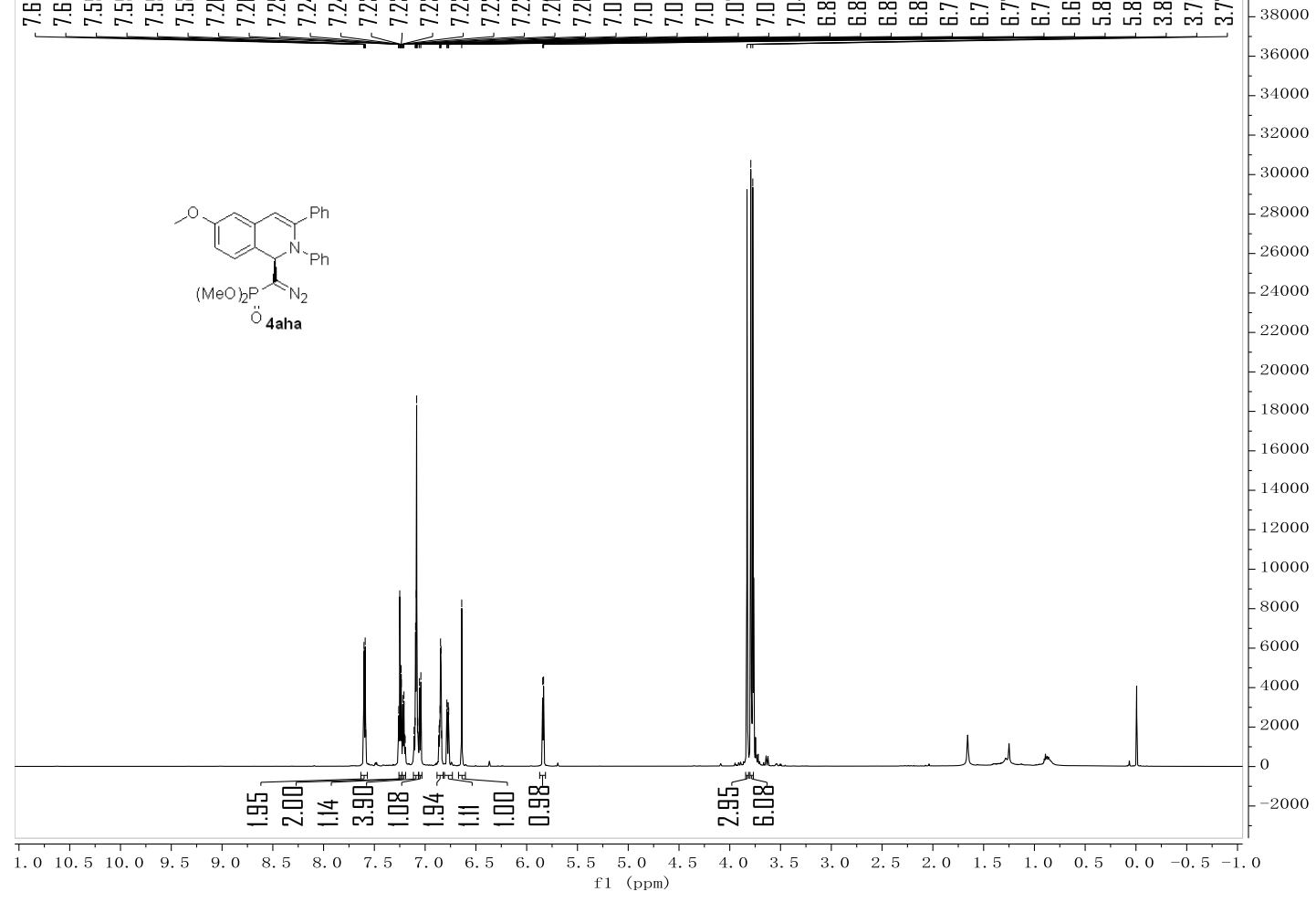

$151 \mathrm{MHz}, \mathrm{CDCl}_{3},{ }^{13} \mathrm{C} \mathrm{NMR}$

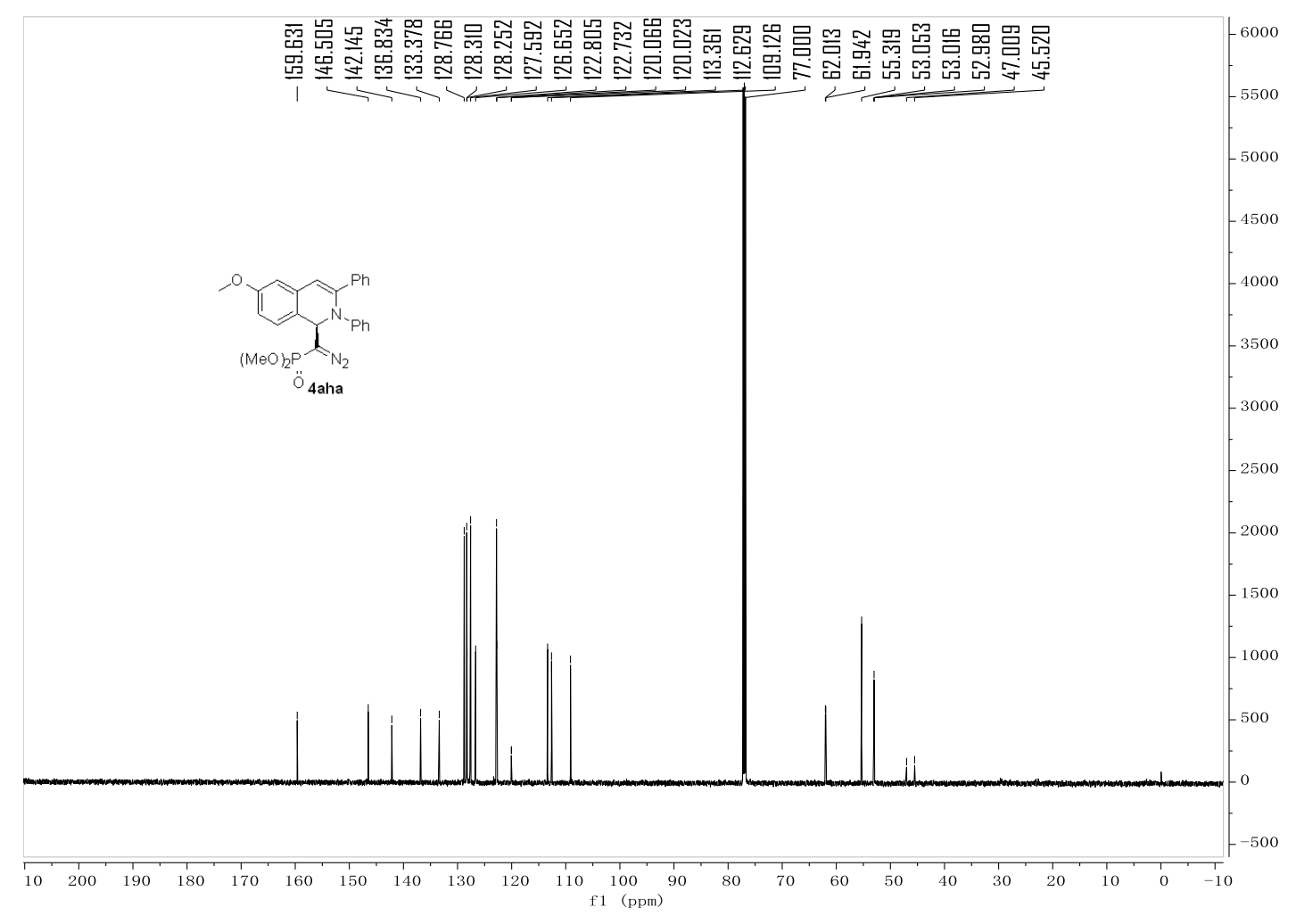


$600 \mathrm{MHz}, \mathrm{CDCl}_{3},{ }^{1} \mathrm{H} \mathrm{NMR}$

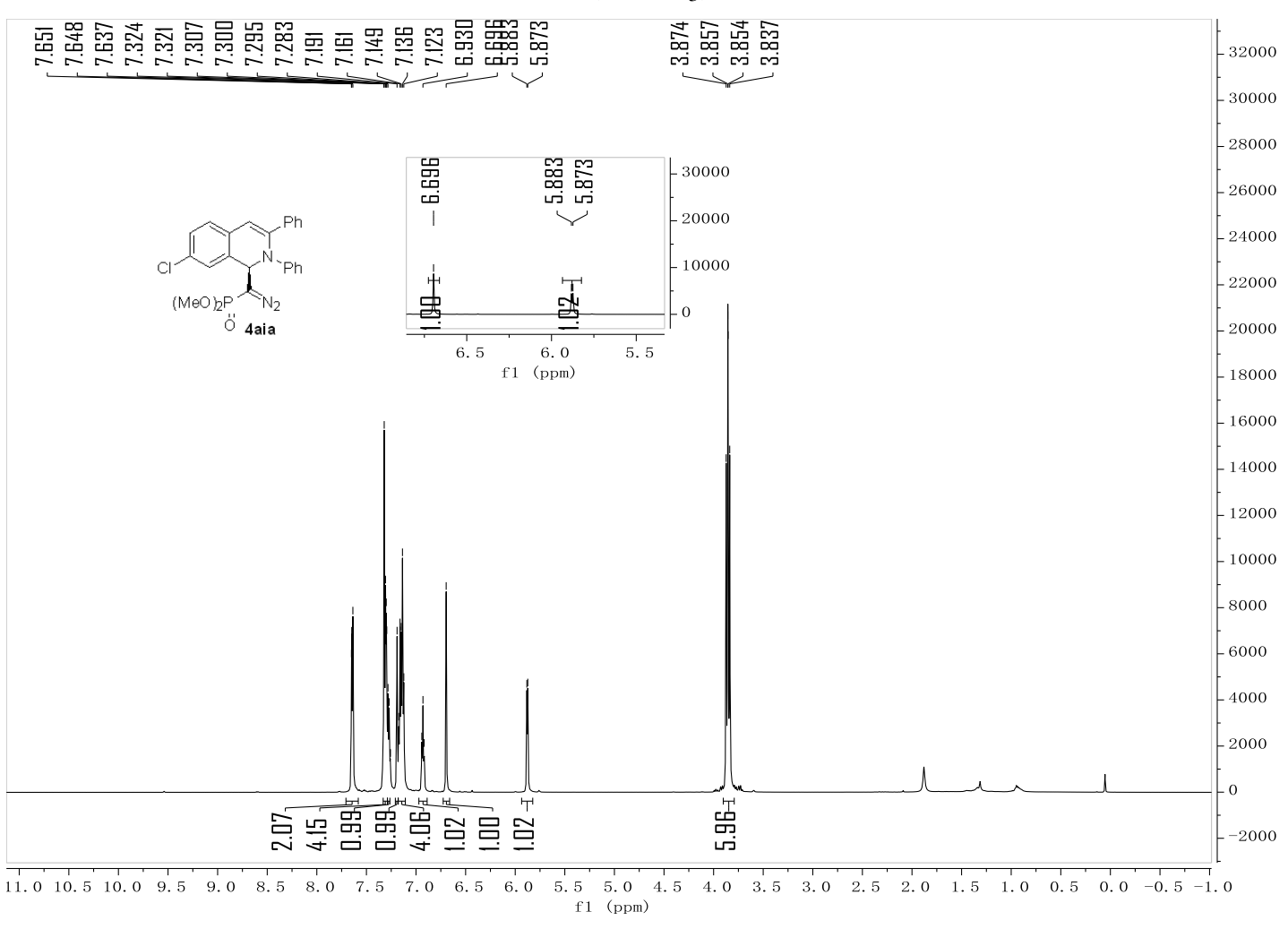

$151 \mathrm{MHz}, \mathrm{CDCl}_{3},{ }^{13} \mathrm{C} \mathrm{NMR}$

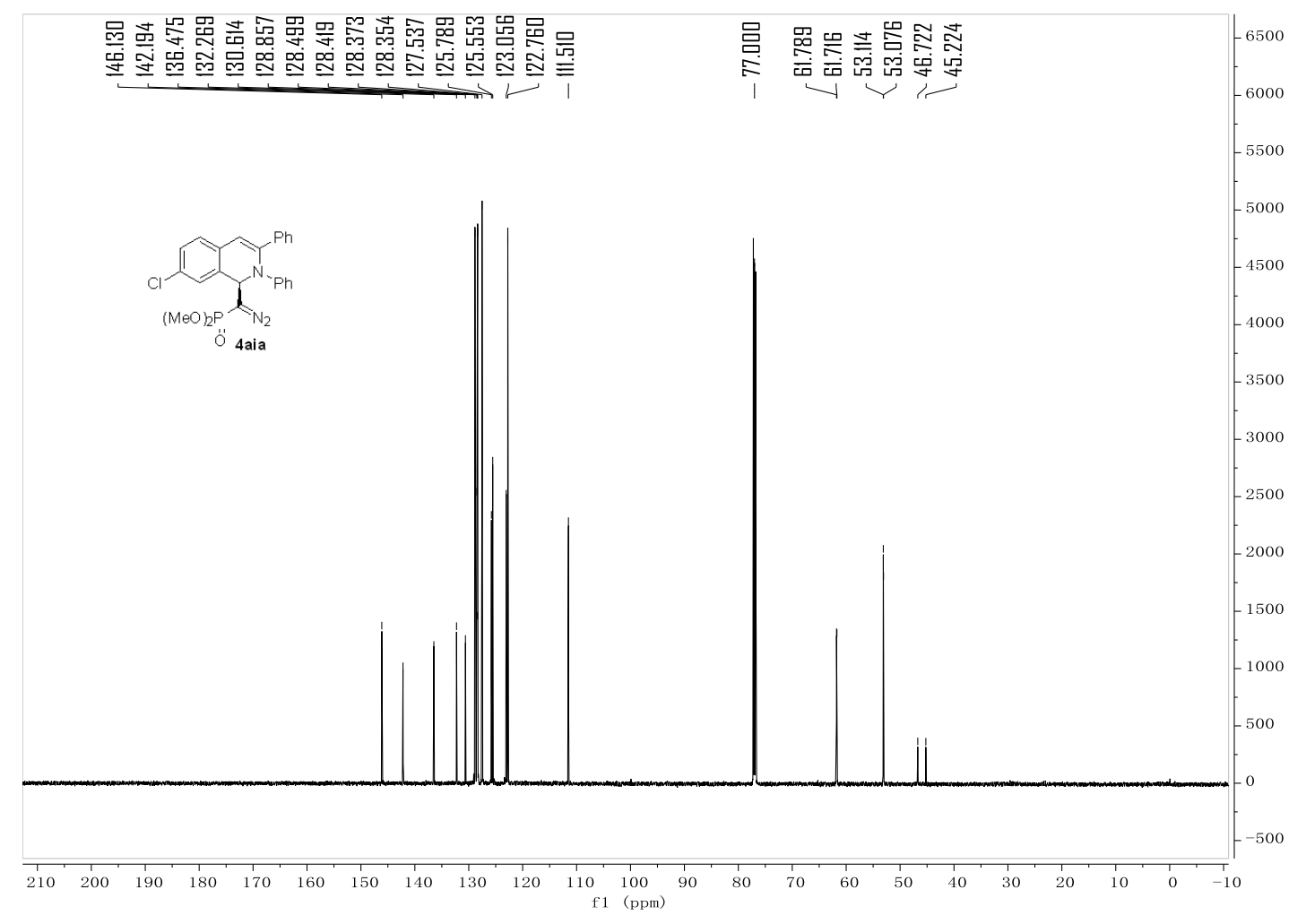




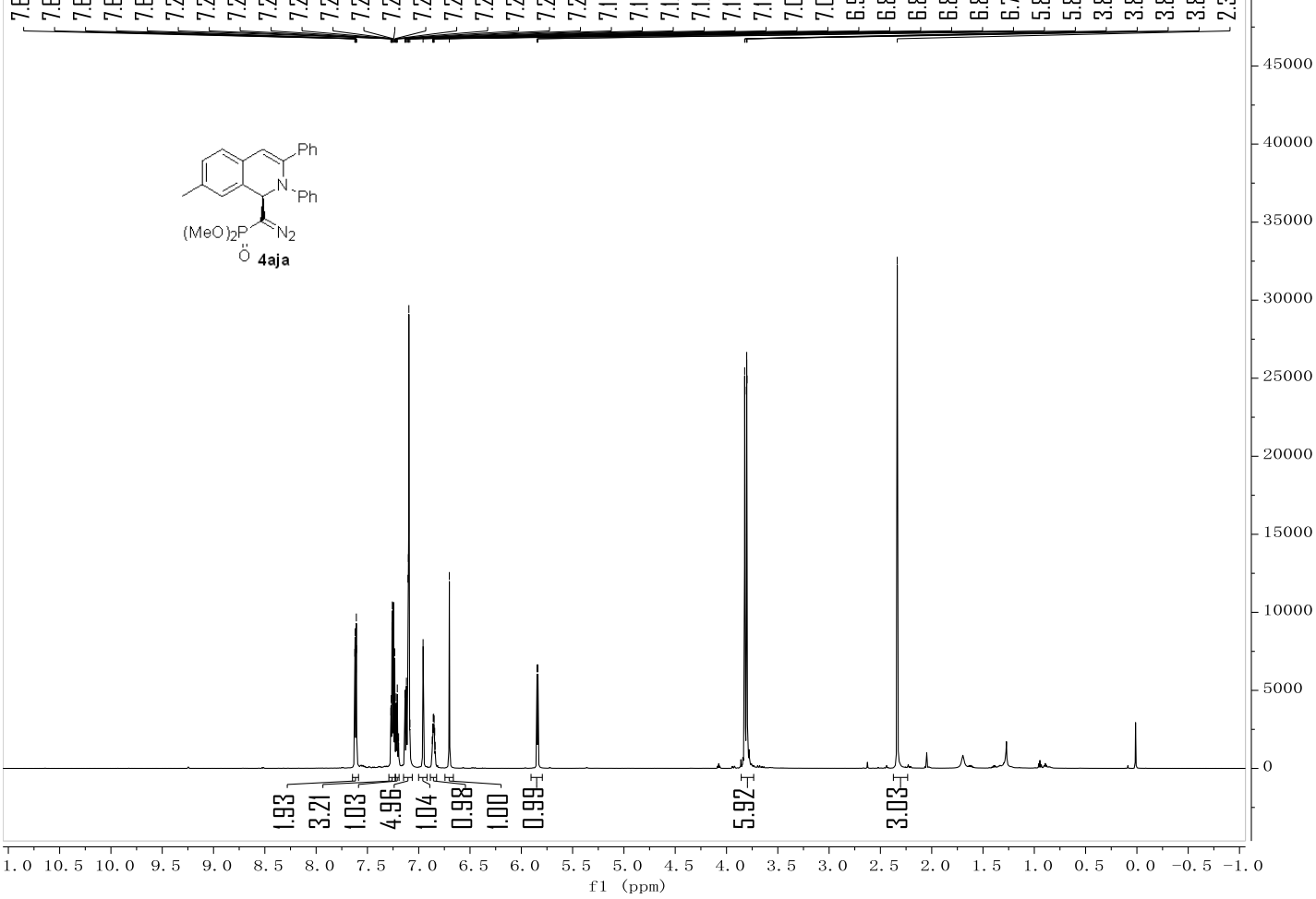

$151 \mathrm{MHz}, \mathrm{CDCl}_{3},{ }^{13} \mathrm{C} \mathrm{NMR}$

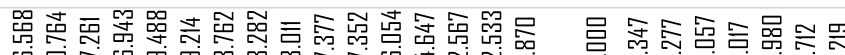

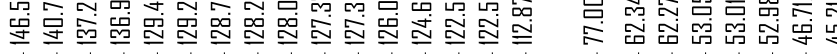

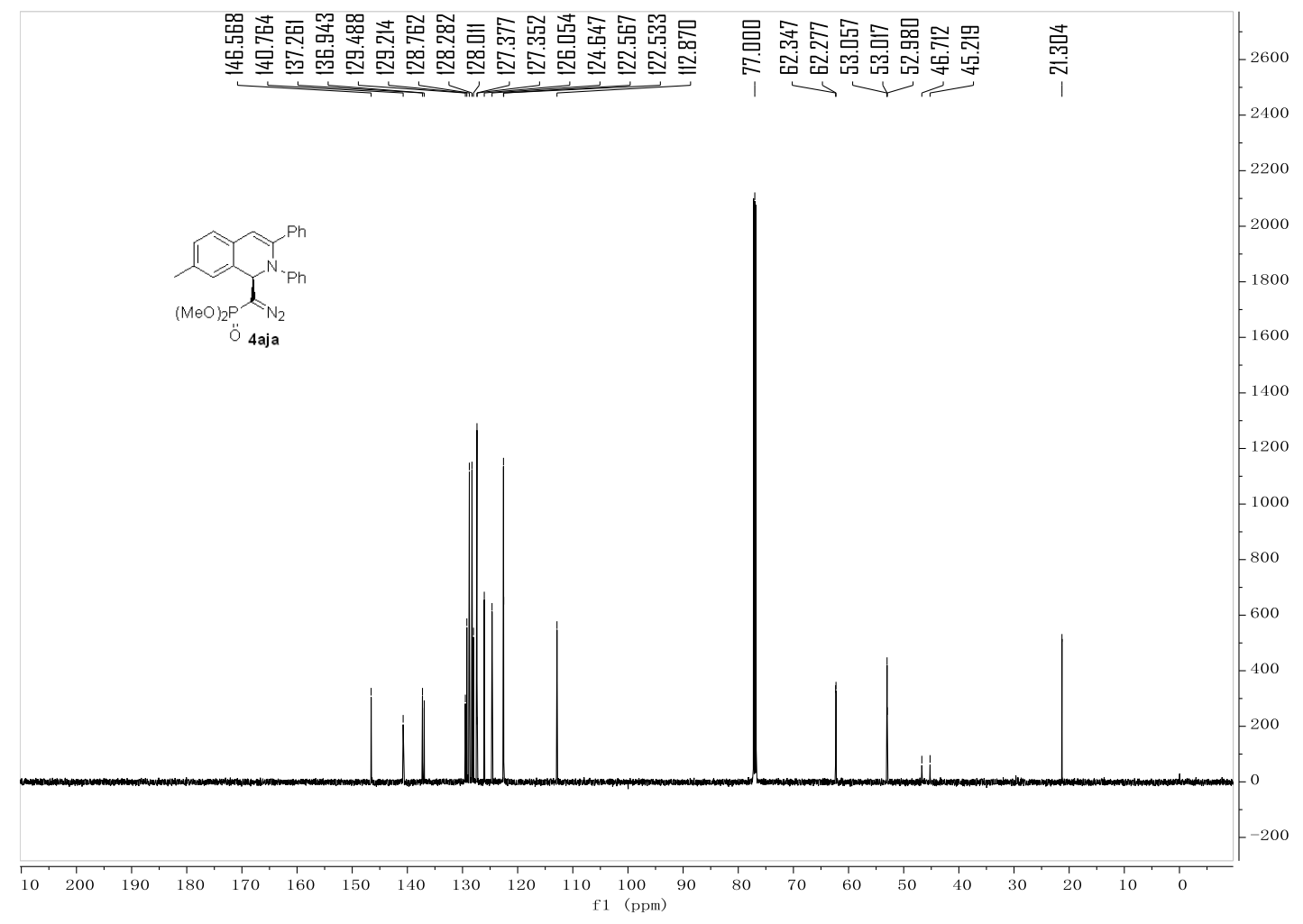




\section{$600 \mathrm{MHz}, \mathrm{CDCl}_{3},{ }^{1} \mathrm{H} \mathrm{NMR}$}

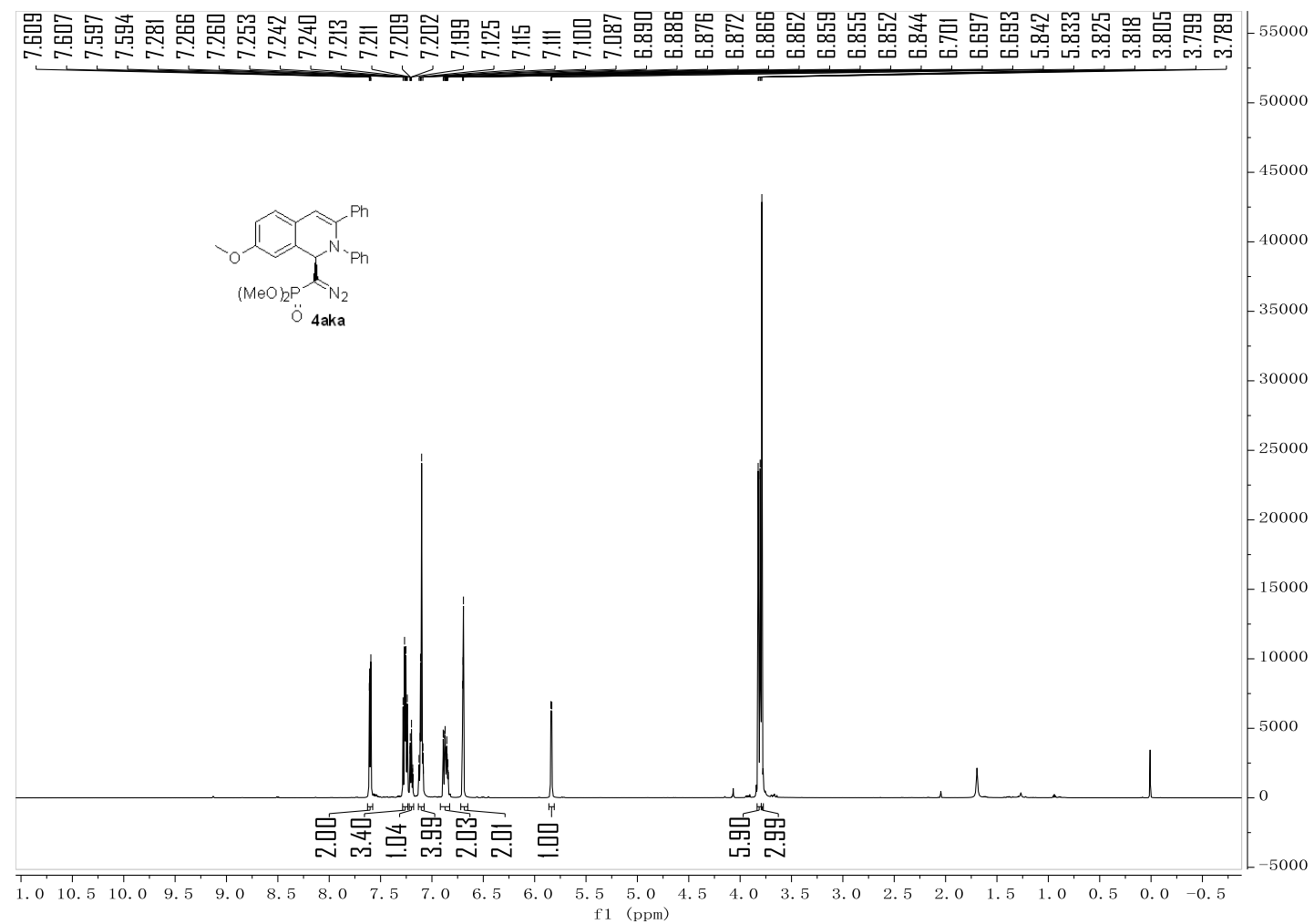

$151 \mathrm{MHz}, \mathrm{CDCl}_{3},{ }^{13} \mathrm{C} \mathrm{NMR}$

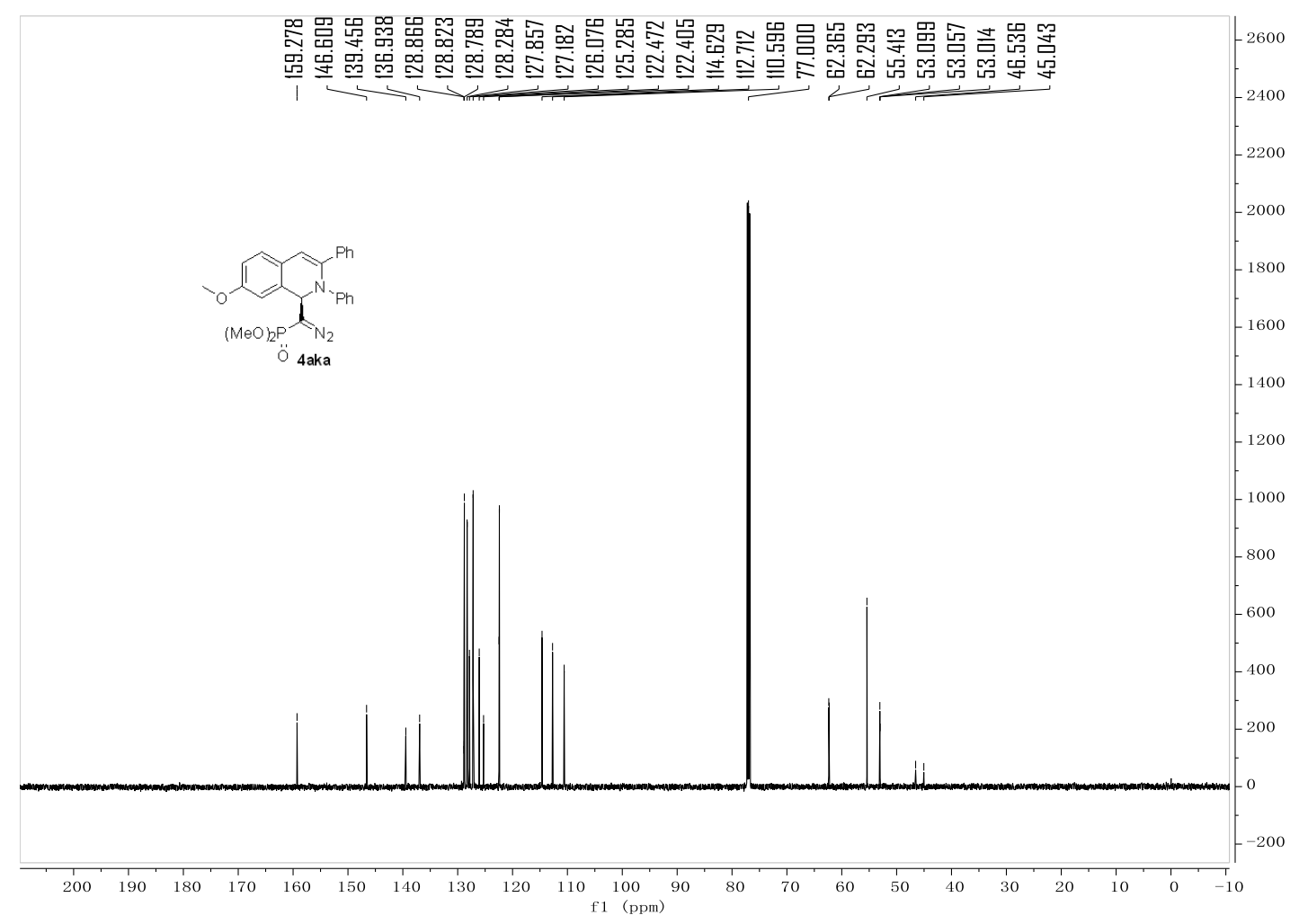




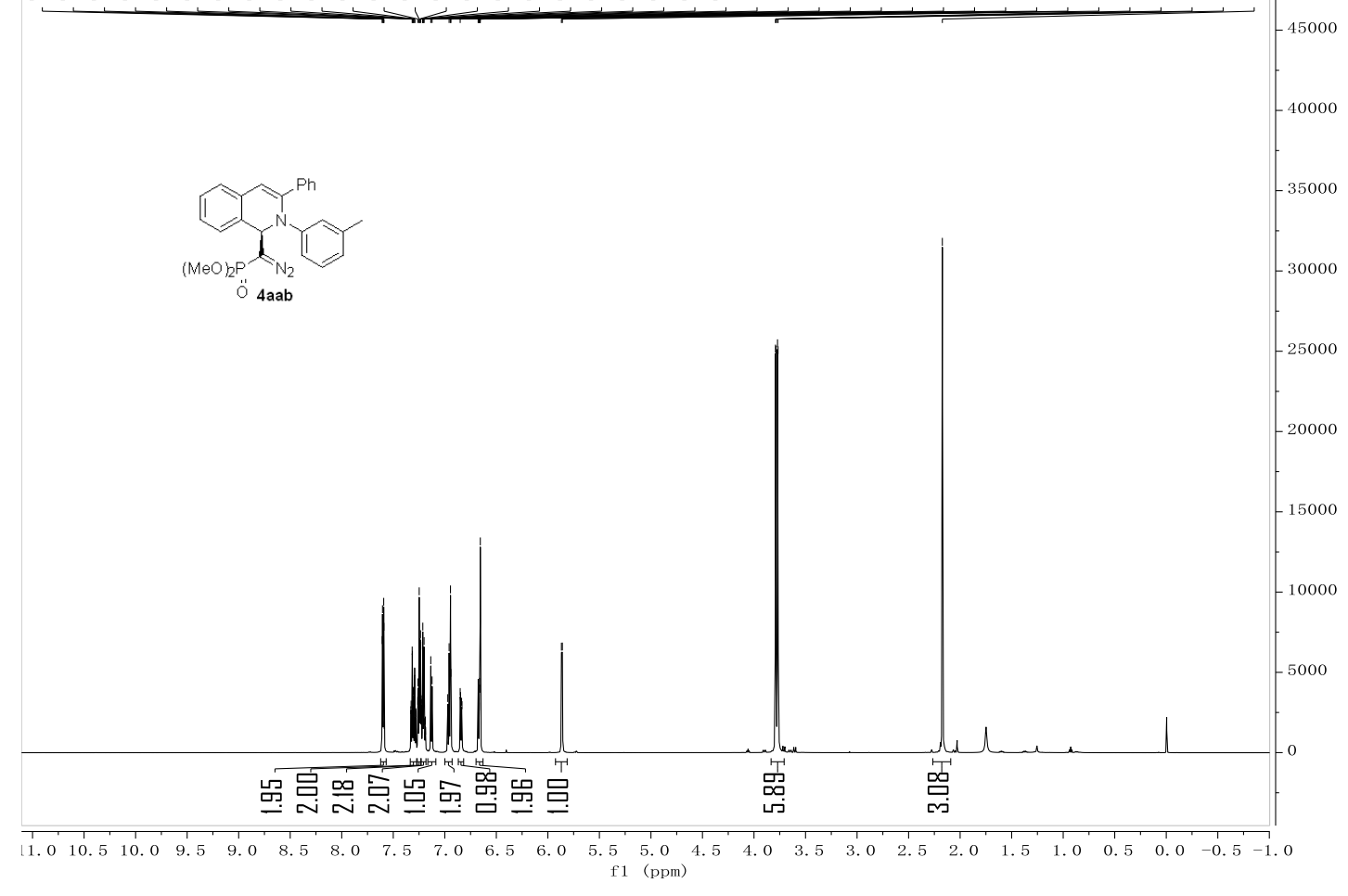

$151 \mathrm{MHz}, \mathrm{CDCl}_{3},{ }^{13} \mathrm{C} \mathrm{NMR}$

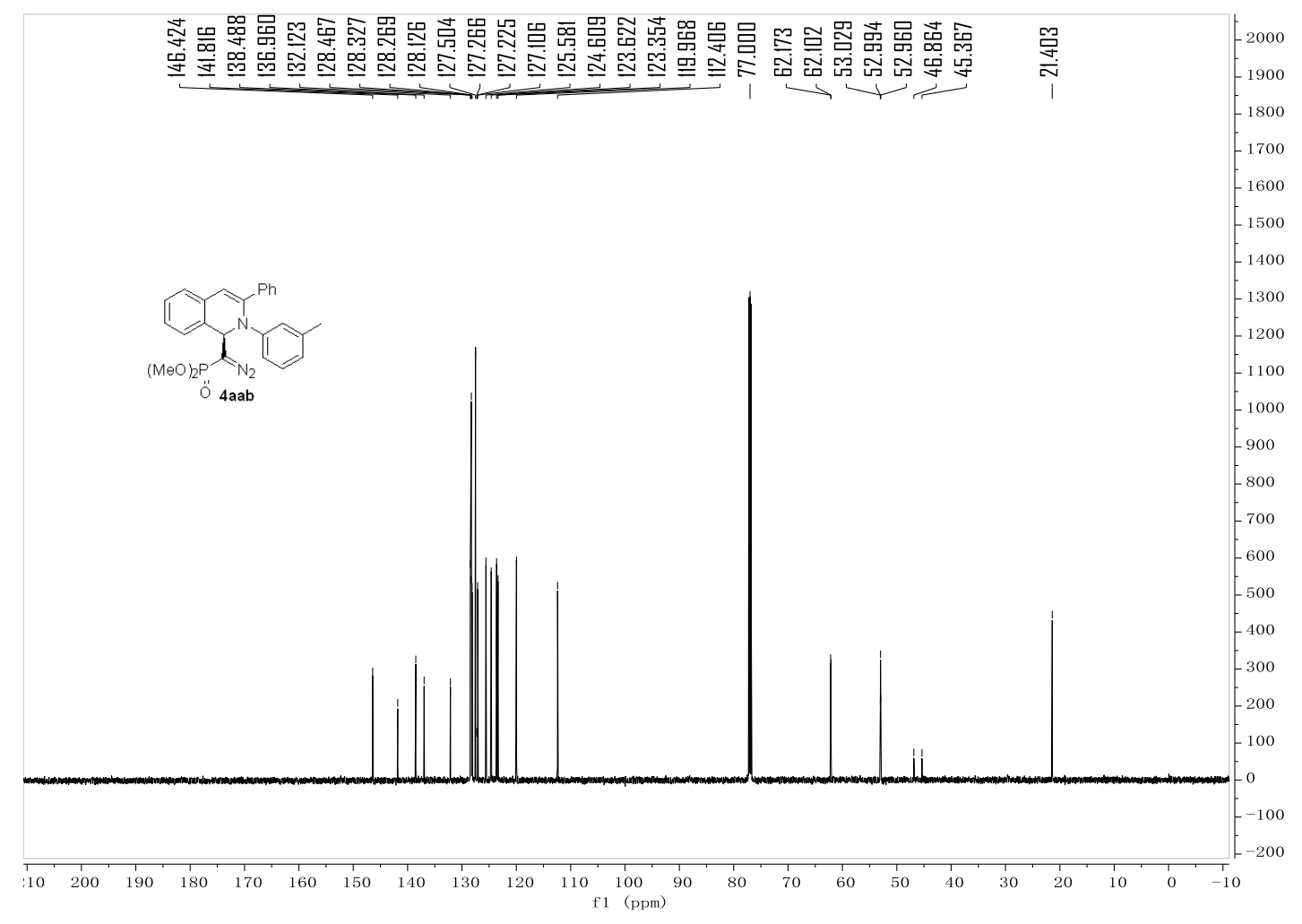




\section{$600 \mathrm{MHz}, \mathrm{CDCl}_{3},{ }^{1} \mathrm{H} \mathrm{NMR}$}

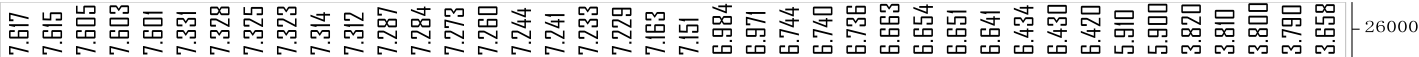

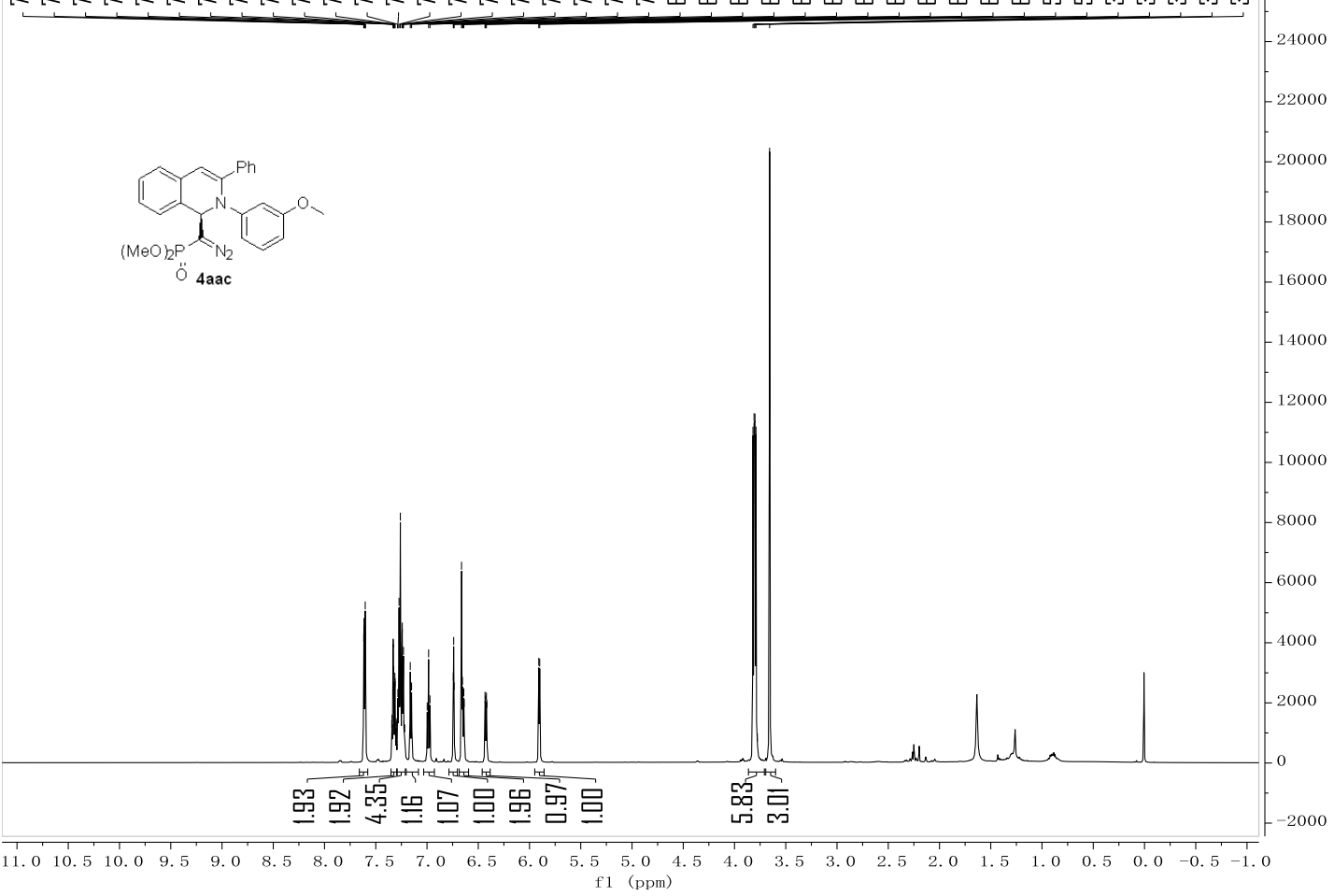

$151 \mathrm{MHz}, \mathrm{CDCl}_{3},{ }^{13} \mathrm{C} \mathrm{NMR}$

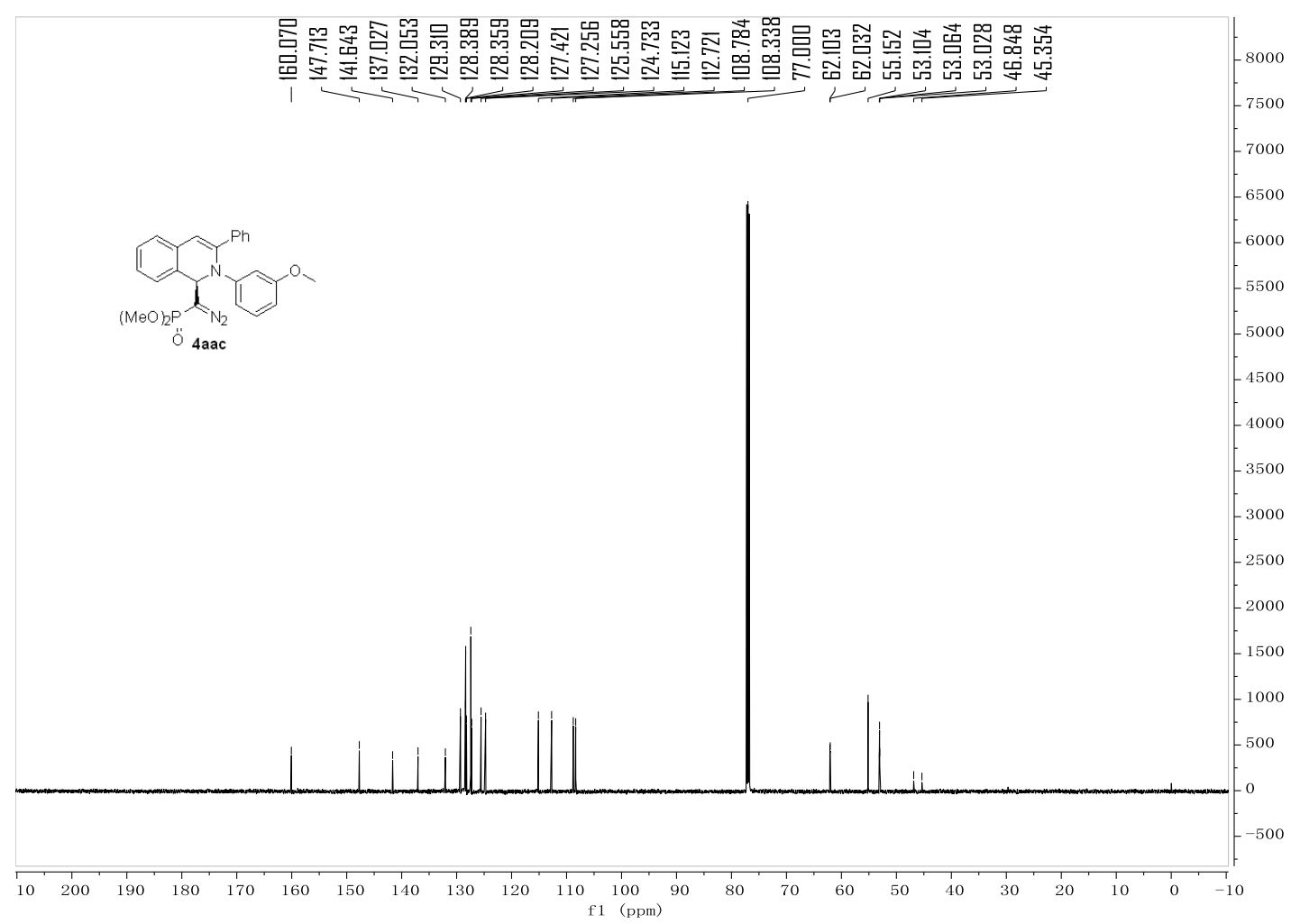




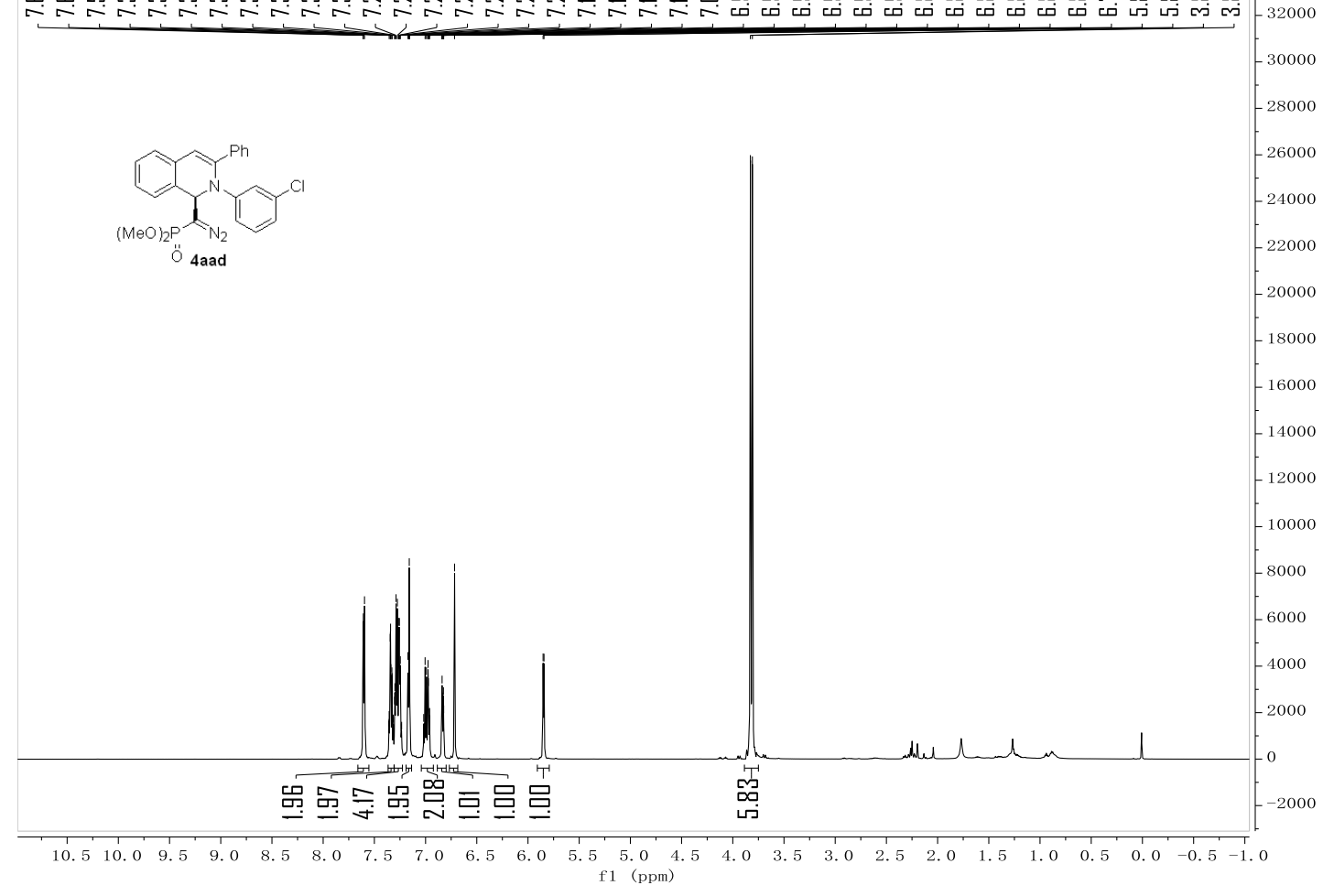

$151 \mathrm{MHz}, \mathrm{CDCl}_{3},{ }^{13} \mathrm{C} \mathrm{NMR}$

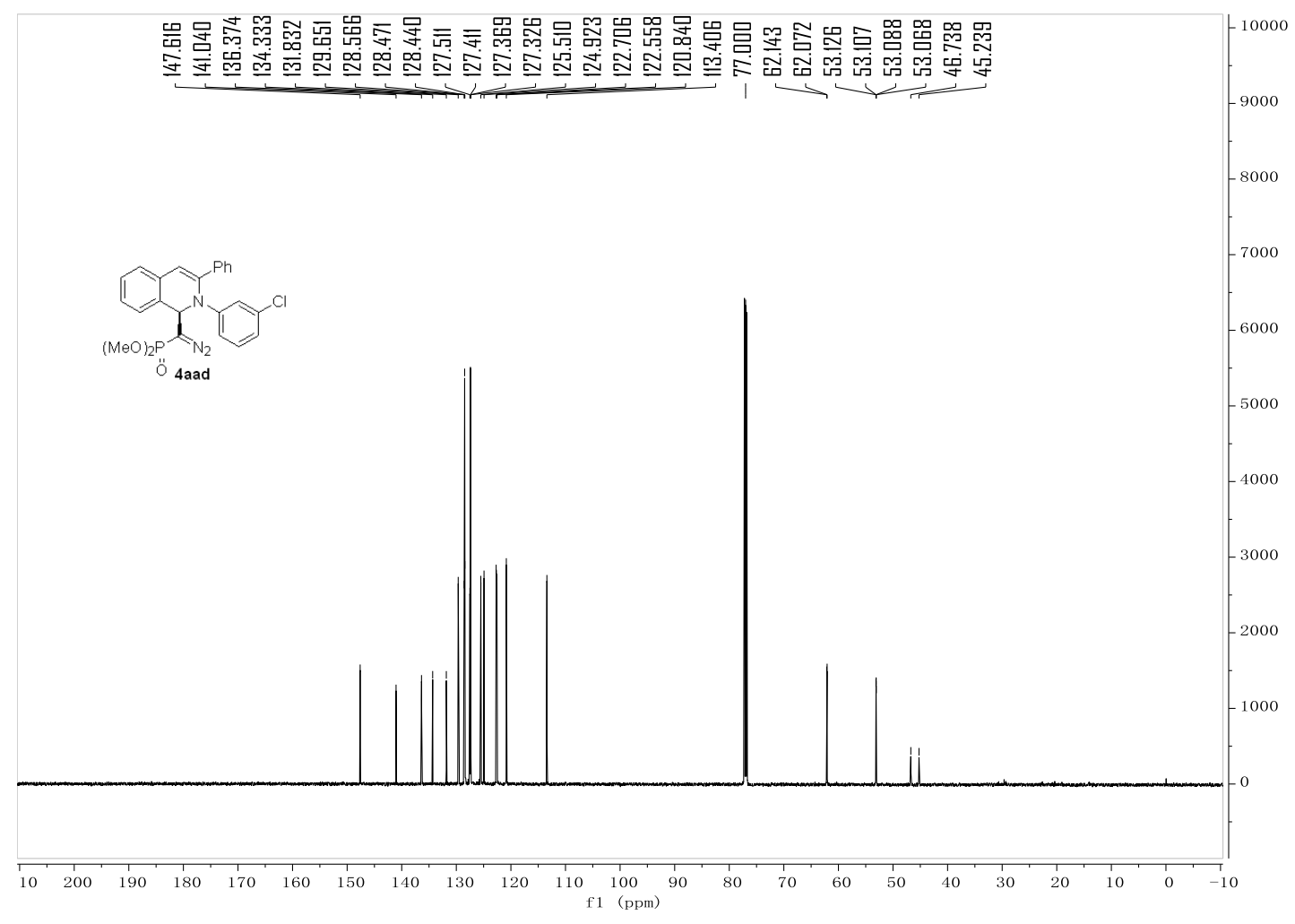


$600 \mathrm{MHz}, \mathrm{CDCl}_{3},{ }^{1} \mathrm{H} \mathrm{NMR}$

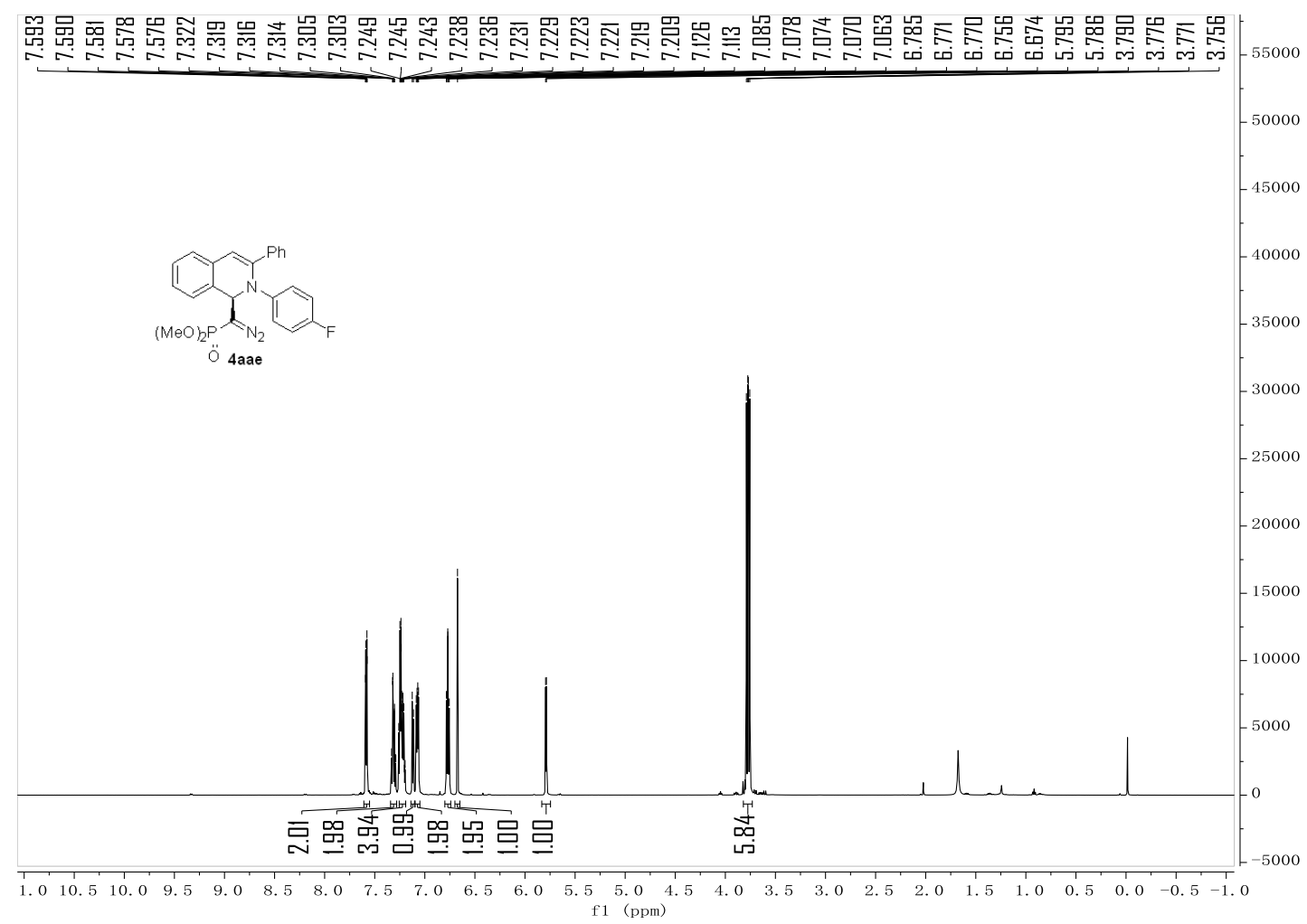

$151 \mathrm{MHz}, \mathrm{CDCl}_{3},{ }^{13} \mathrm{C} \mathrm{NMR}$

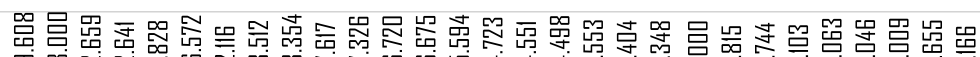

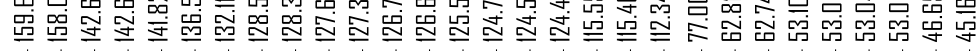

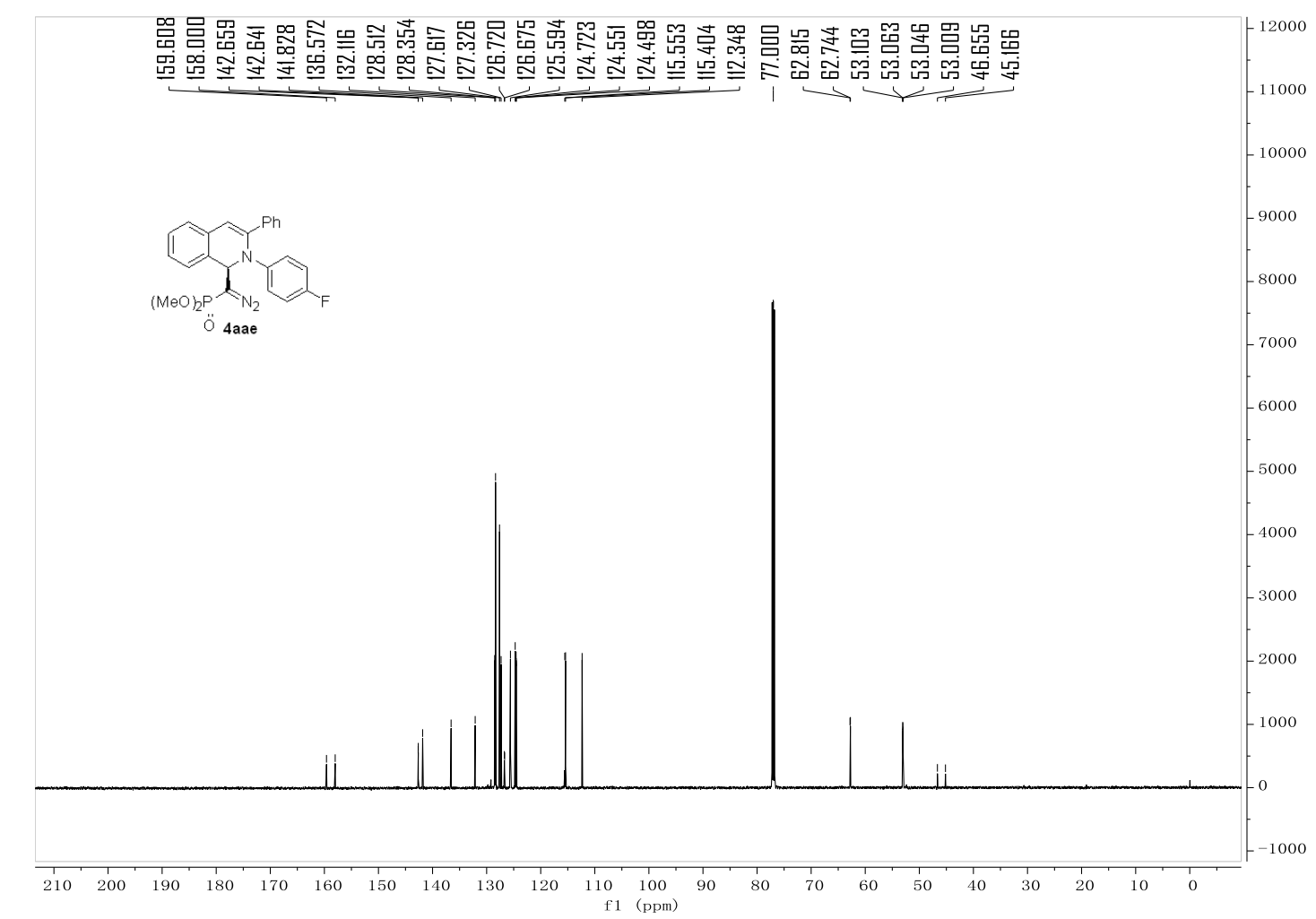



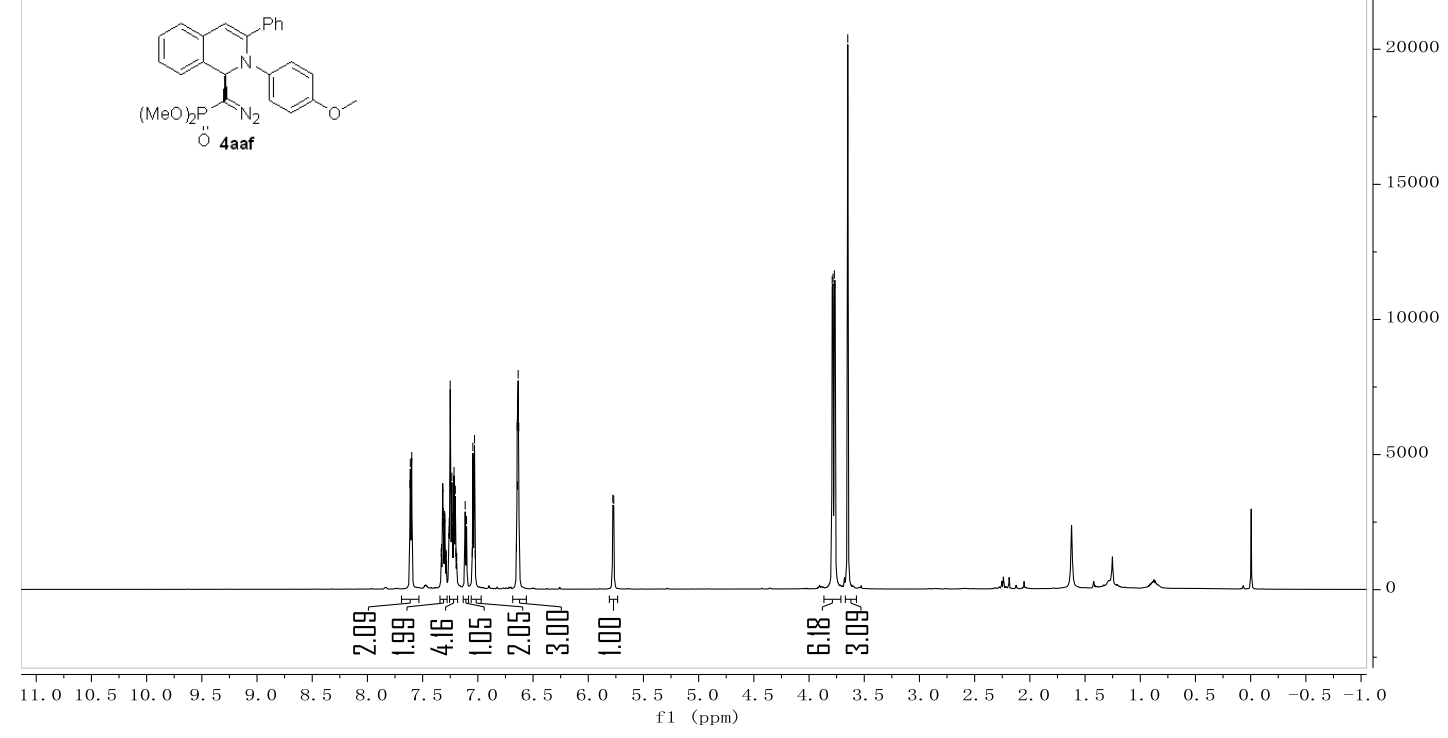

$151 \mathrm{MHz}, \mathrm{CDCl}_{3},{ }^{13} \mathrm{C} \mathrm{NMR}$

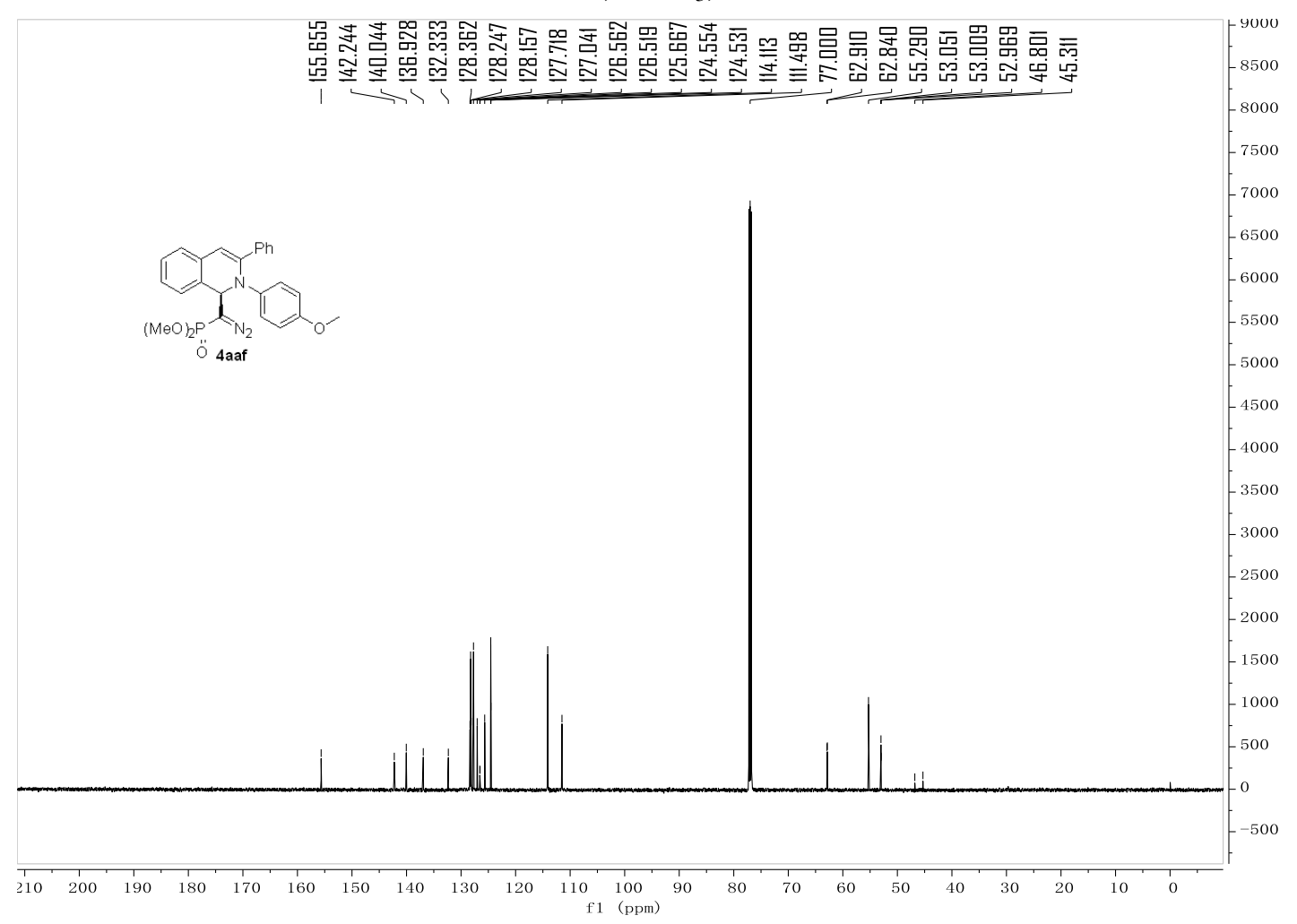




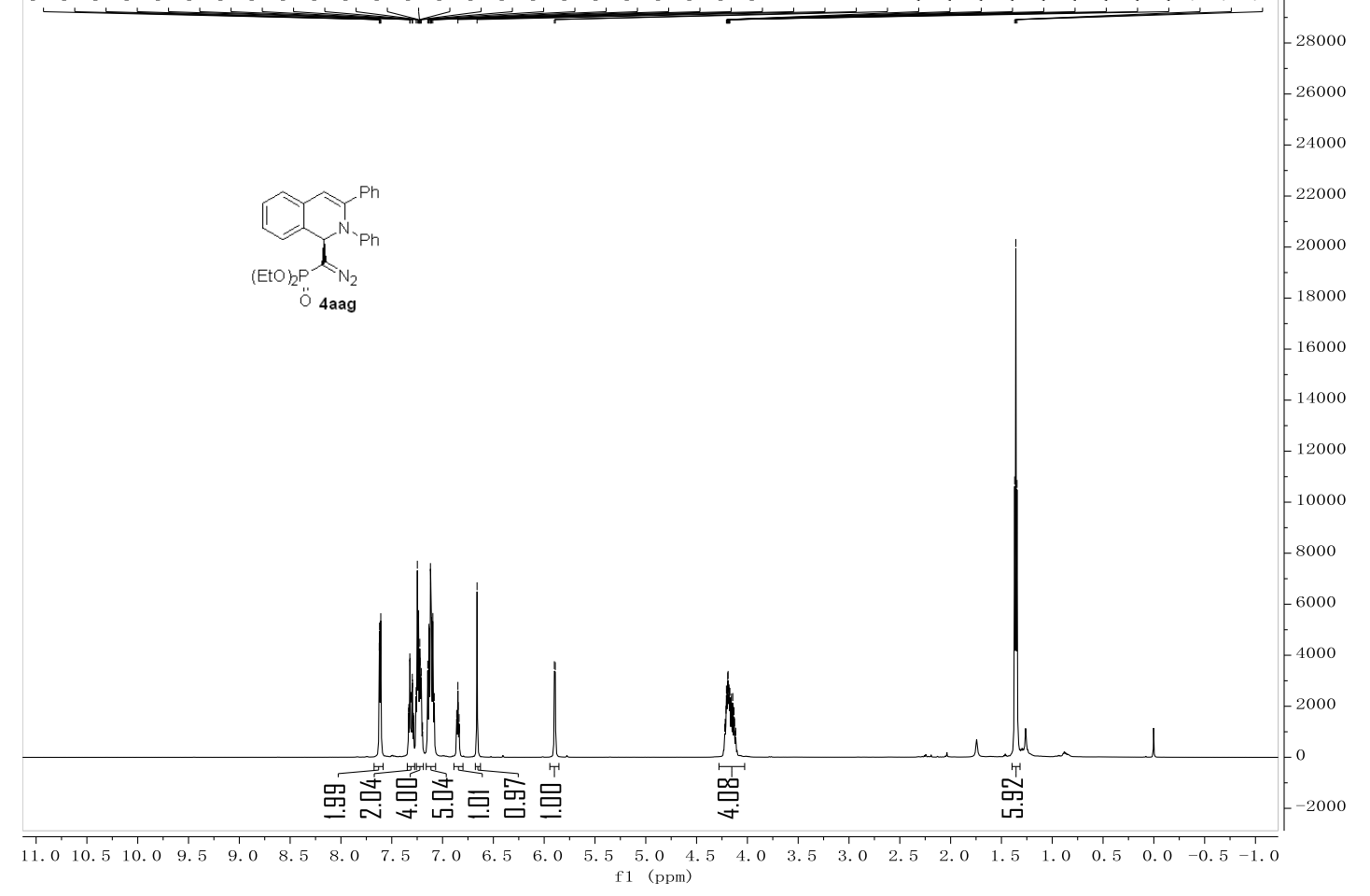

$151 \mathrm{MHz}, \mathrm{CDCl}_{3},{ }^{13} \mathrm{C} \mathrm{NMR}$

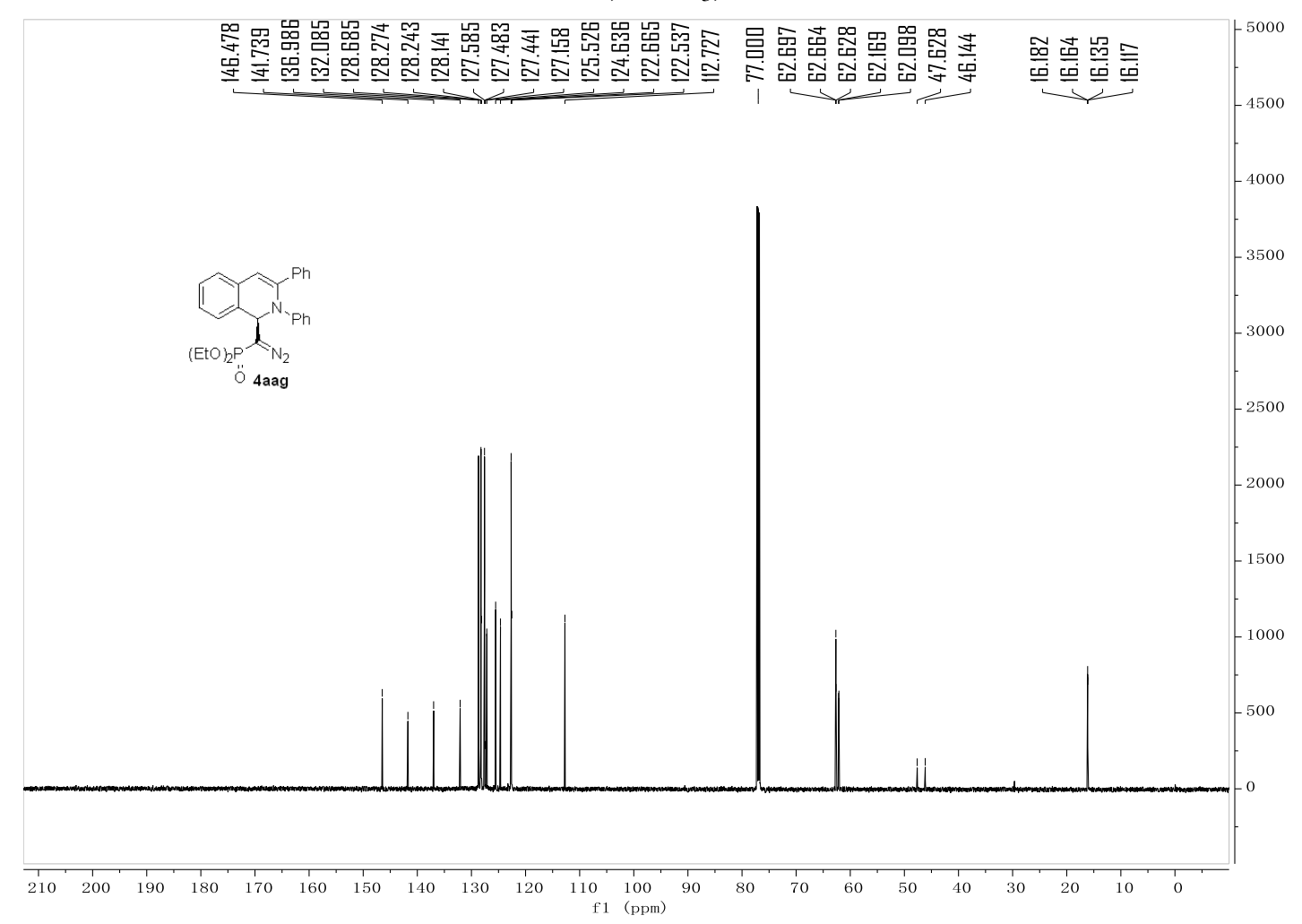




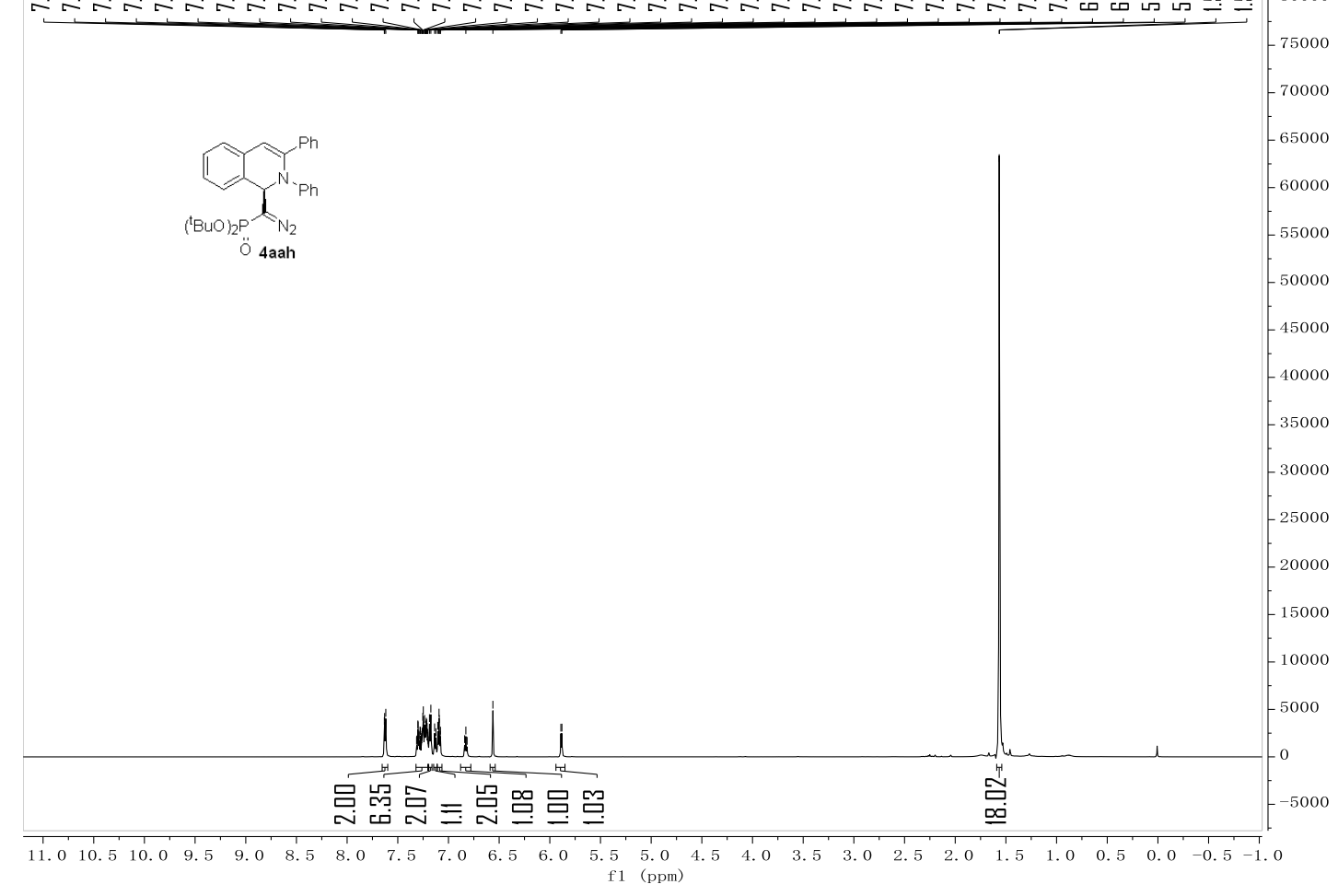

$151 \mathrm{MHz}, \mathrm{CDCl}_{3},{ }^{13} \mathrm{C} \mathrm{NMR}$

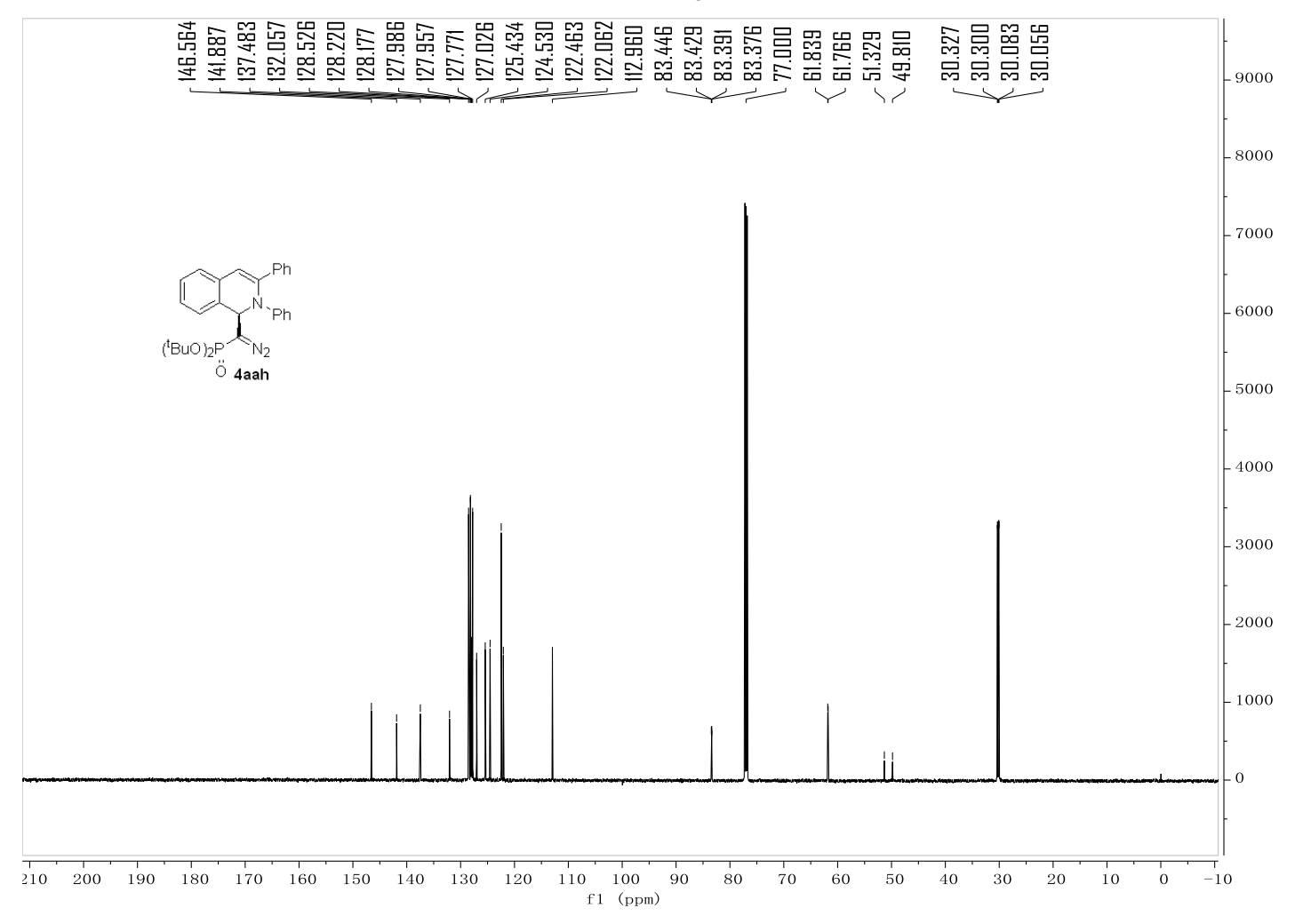




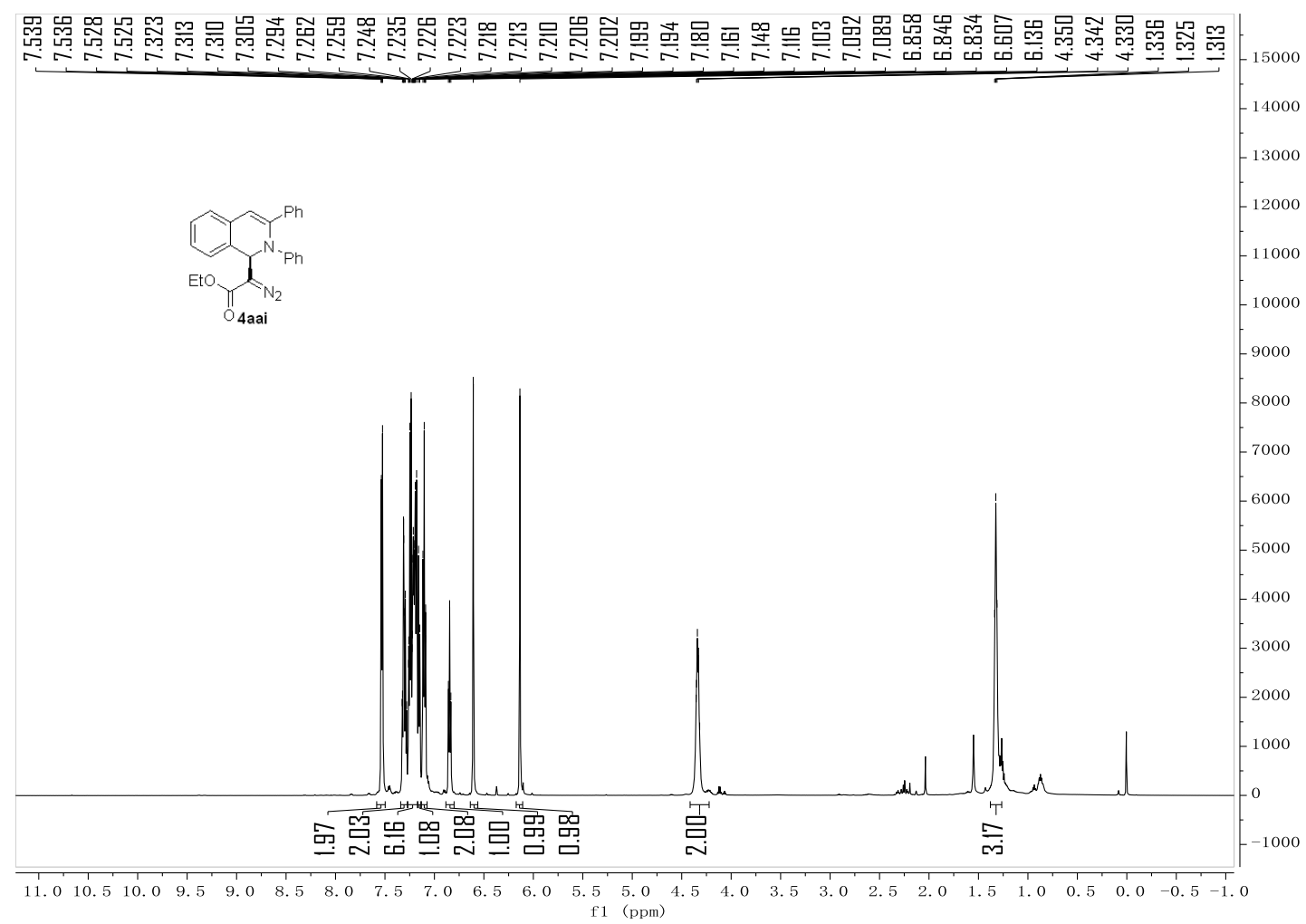

$151 \mathrm{MHz}, \mathrm{CDCl}_{3},{ }^{13} \mathrm{C} \mathrm{NMR}$

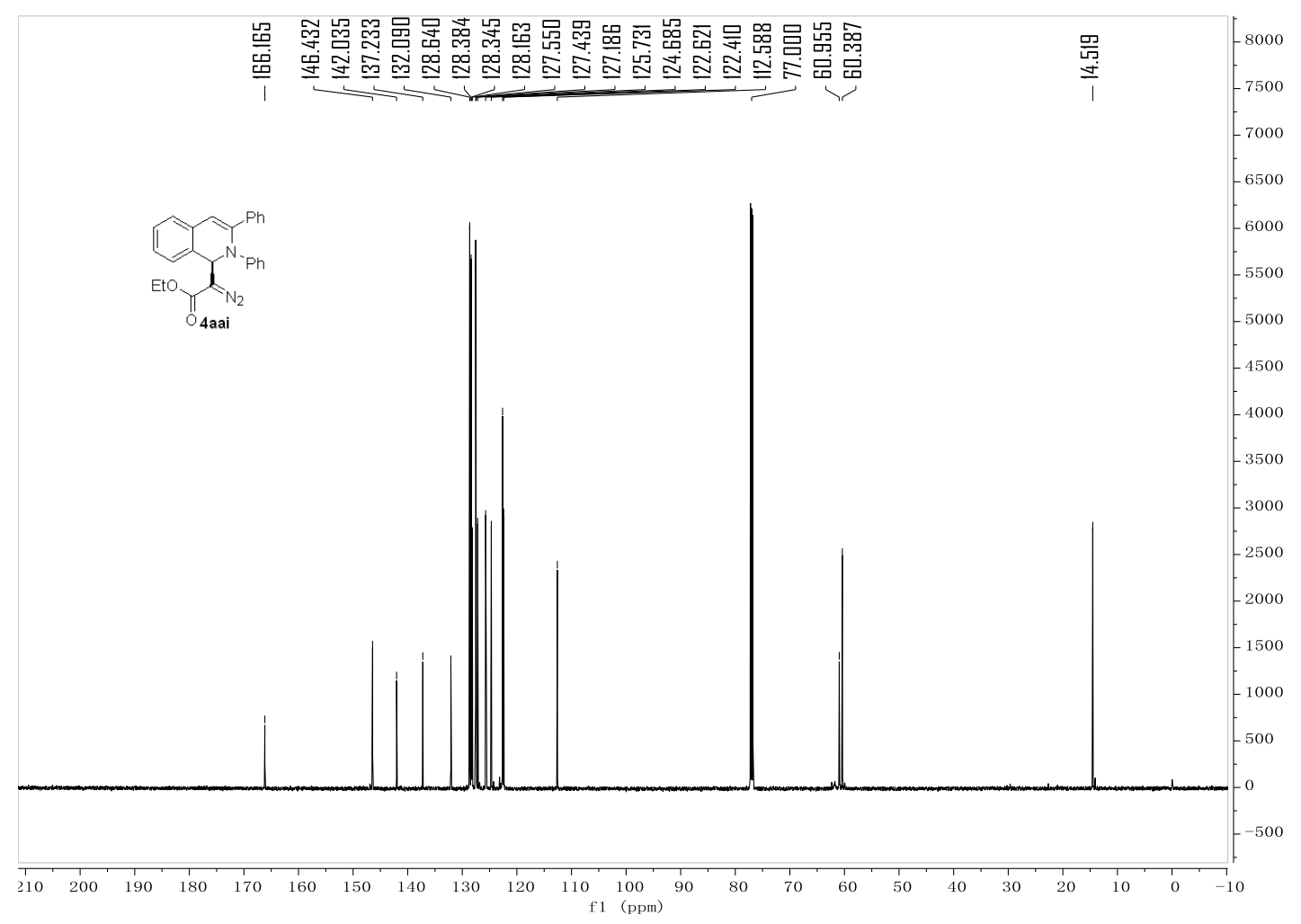




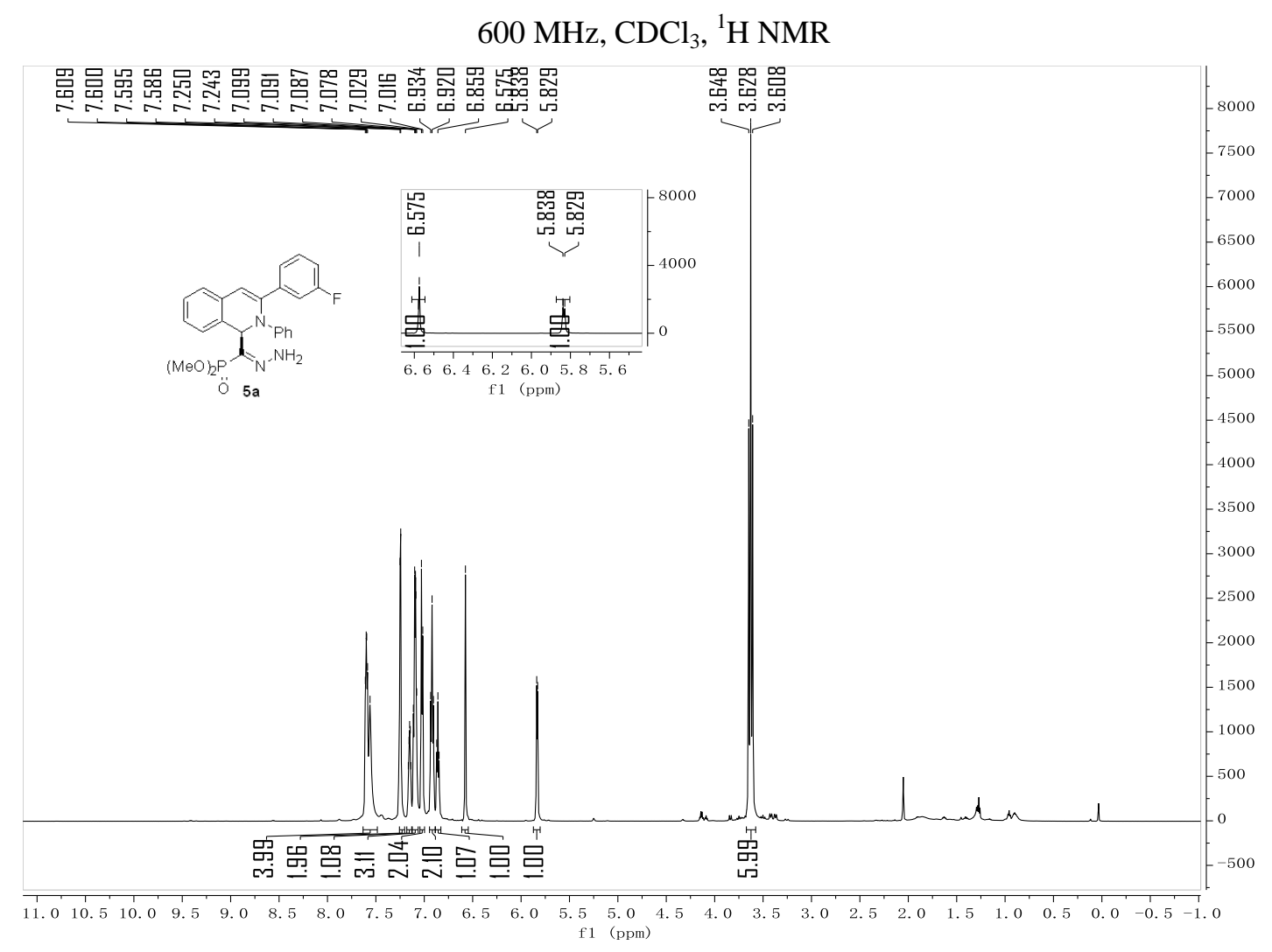

$151 \mathrm{MHz}, \mathrm{CDCl}_{3},{ }^{13} \mathrm{C} \mathrm{NMR}$

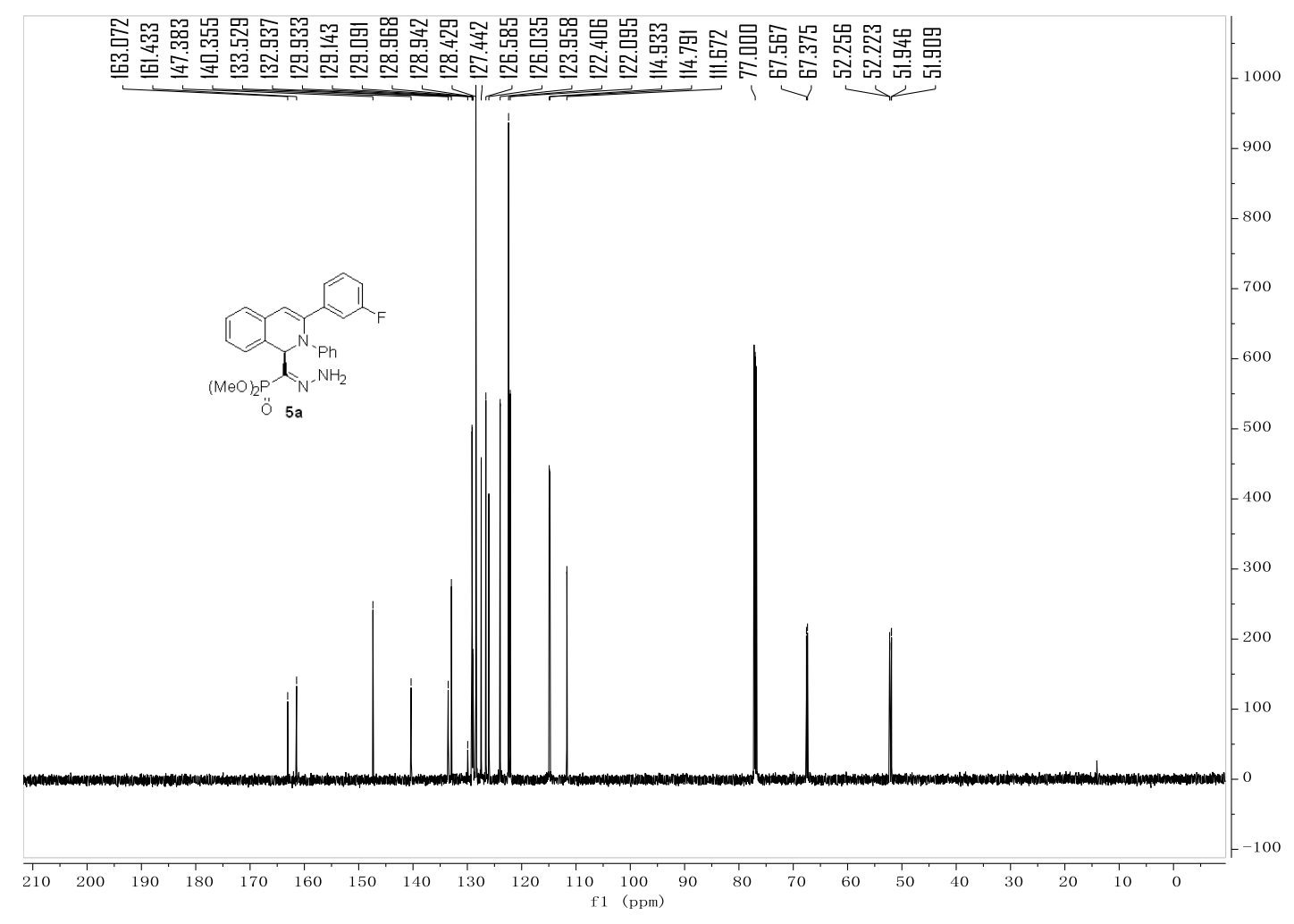




\section{$600 \mathrm{MHz}, \mathrm{CDCl}_{3},{ }^{1} \mathrm{H} \mathrm{NMR}$}

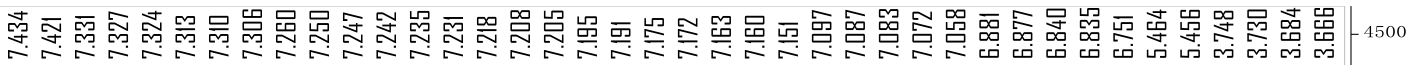

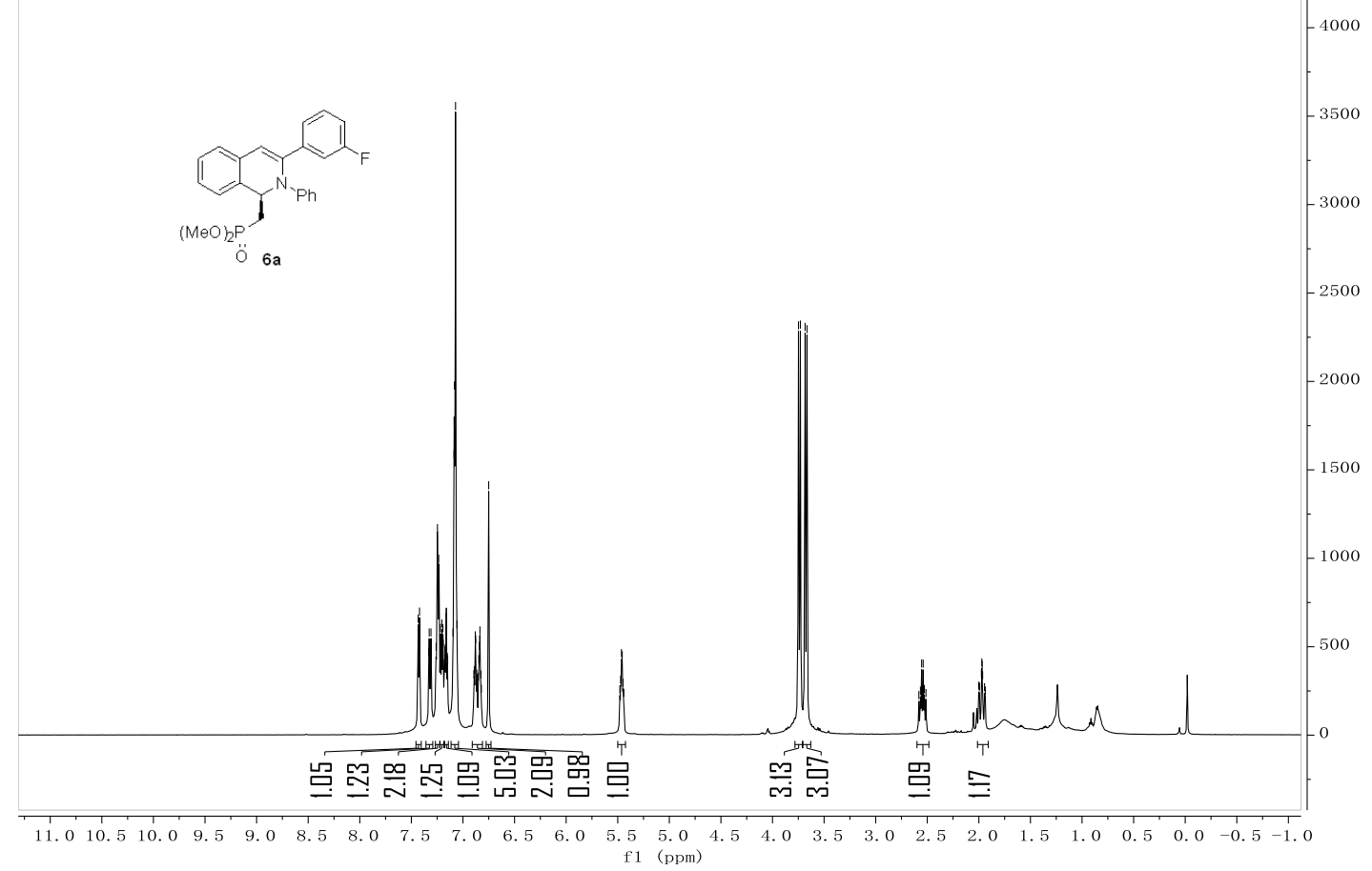

$151 \mathrm{MHz}, \mathrm{CDCl}_{3},{ }^{13} \mathrm{C} \mathrm{NMR}$

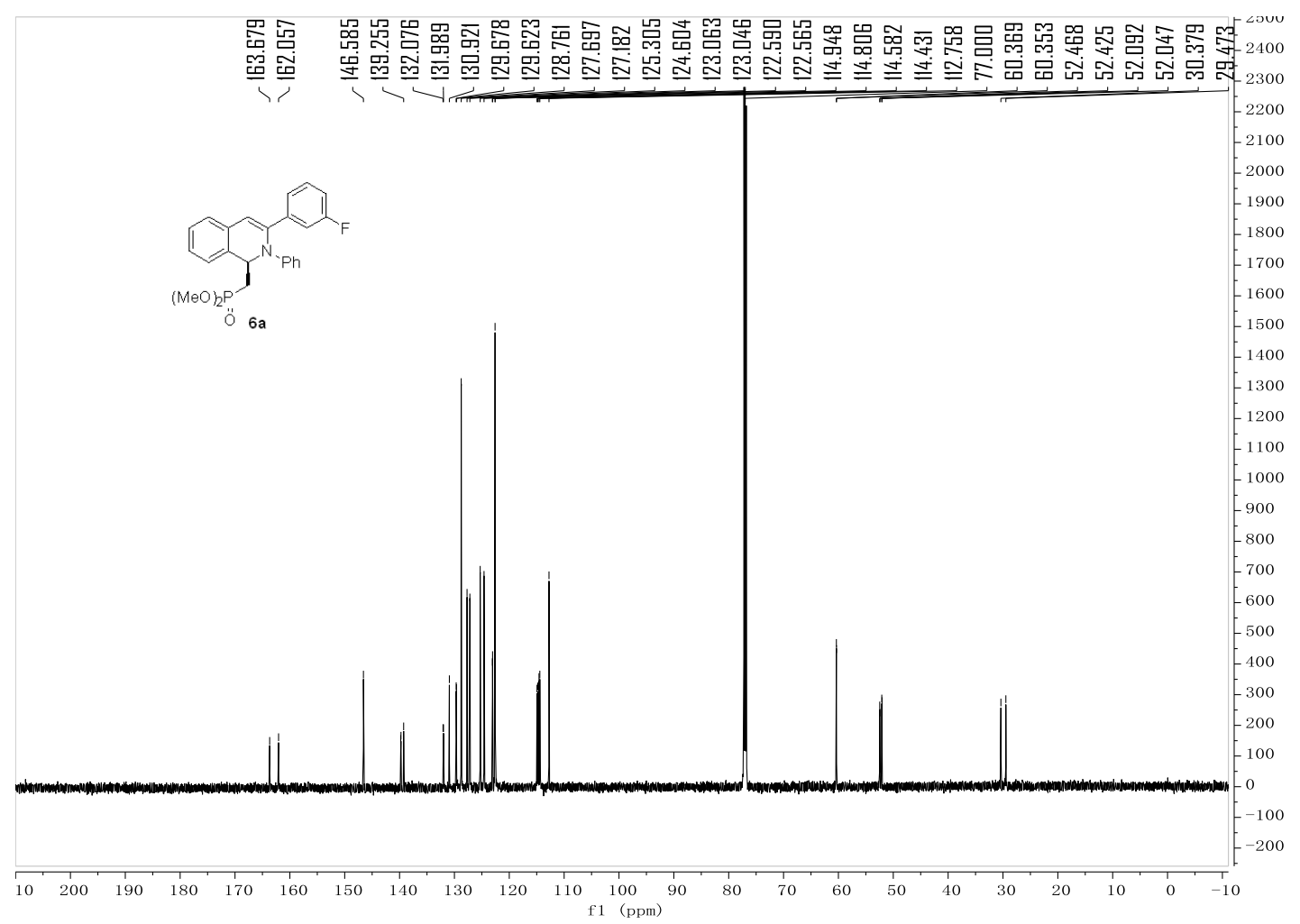


$600 \mathrm{MHz}, \mathrm{CDCl}_{3},{ }^{1} \mathrm{H} \mathrm{NMR}$

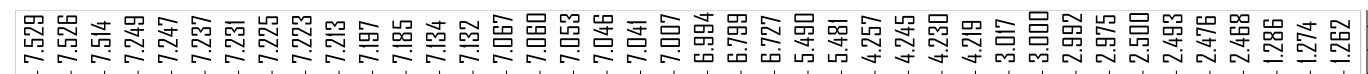

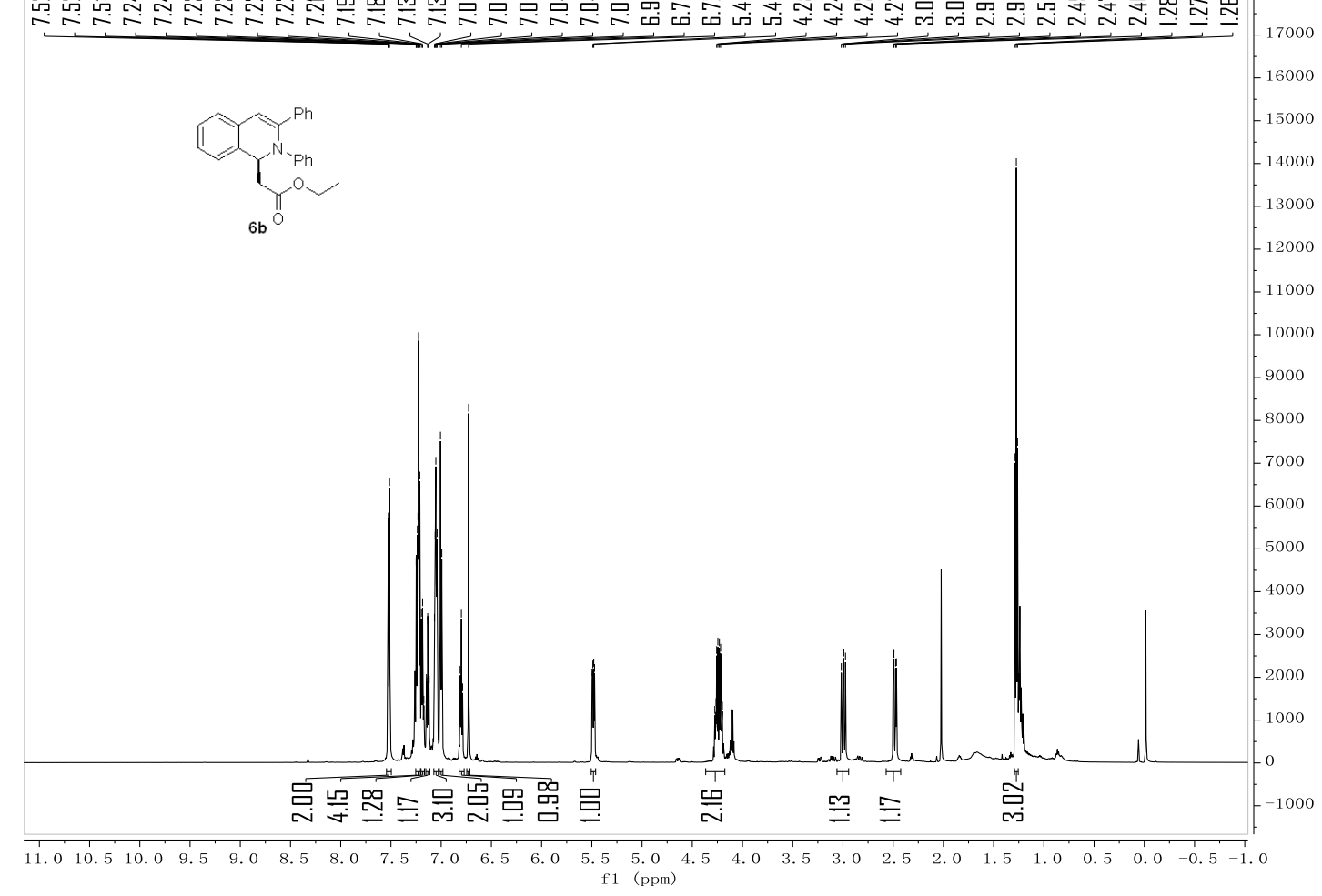

$151 \mathrm{MHz}, \mathrm{CDCl}_{3},{ }^{13} \mathrm{C} \mathrm{NMR}$

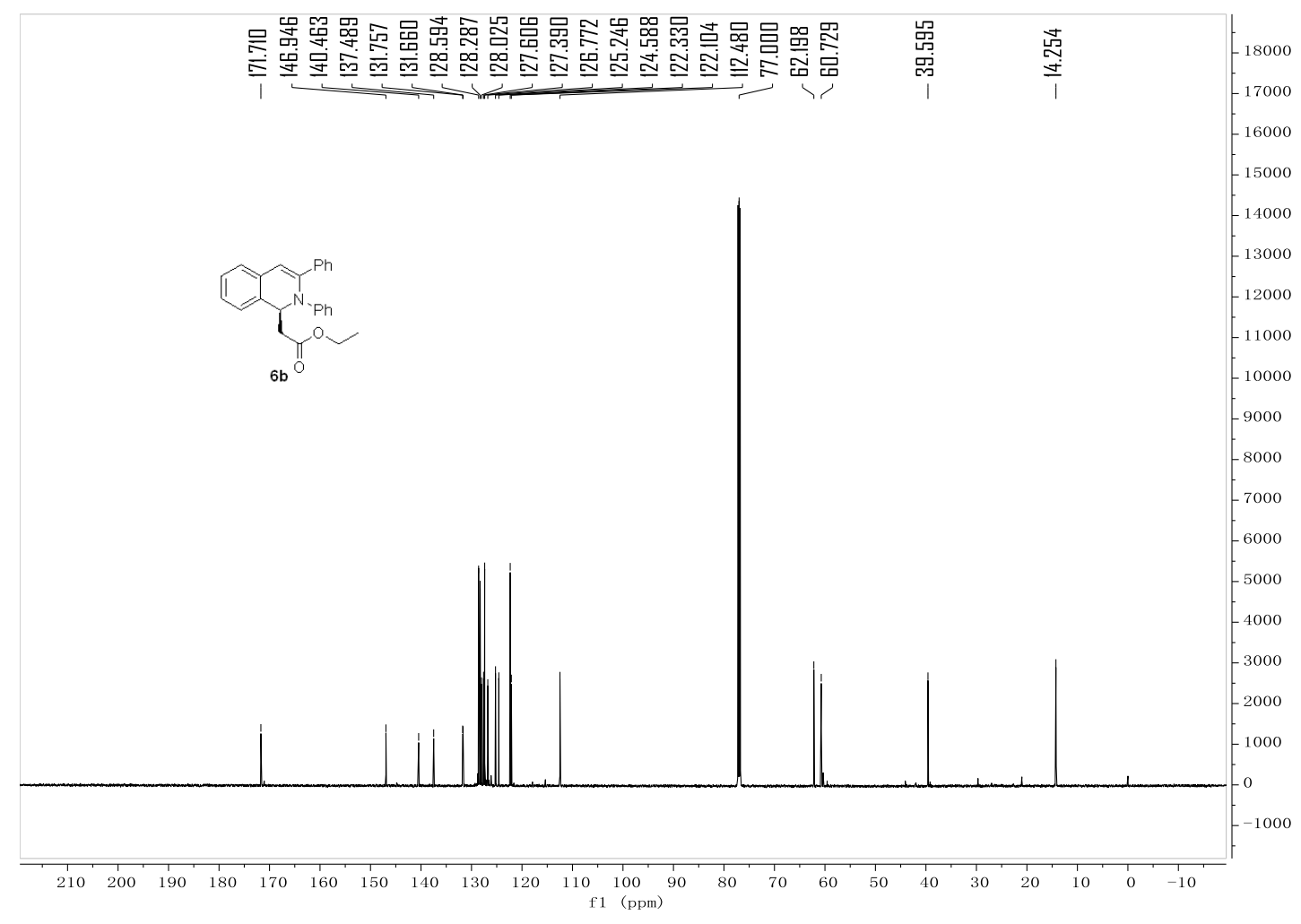


$600 \mathrm{MHz},\left(\mathrm{CD}_{3}\right)_{2} \mathrm{CO},{ }^{1} \mathrm{H} \mathrm{NMR}$

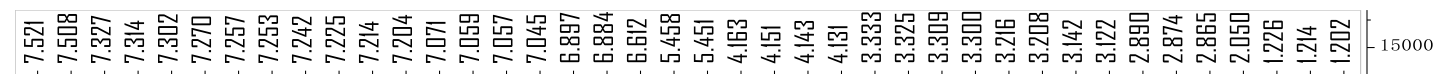

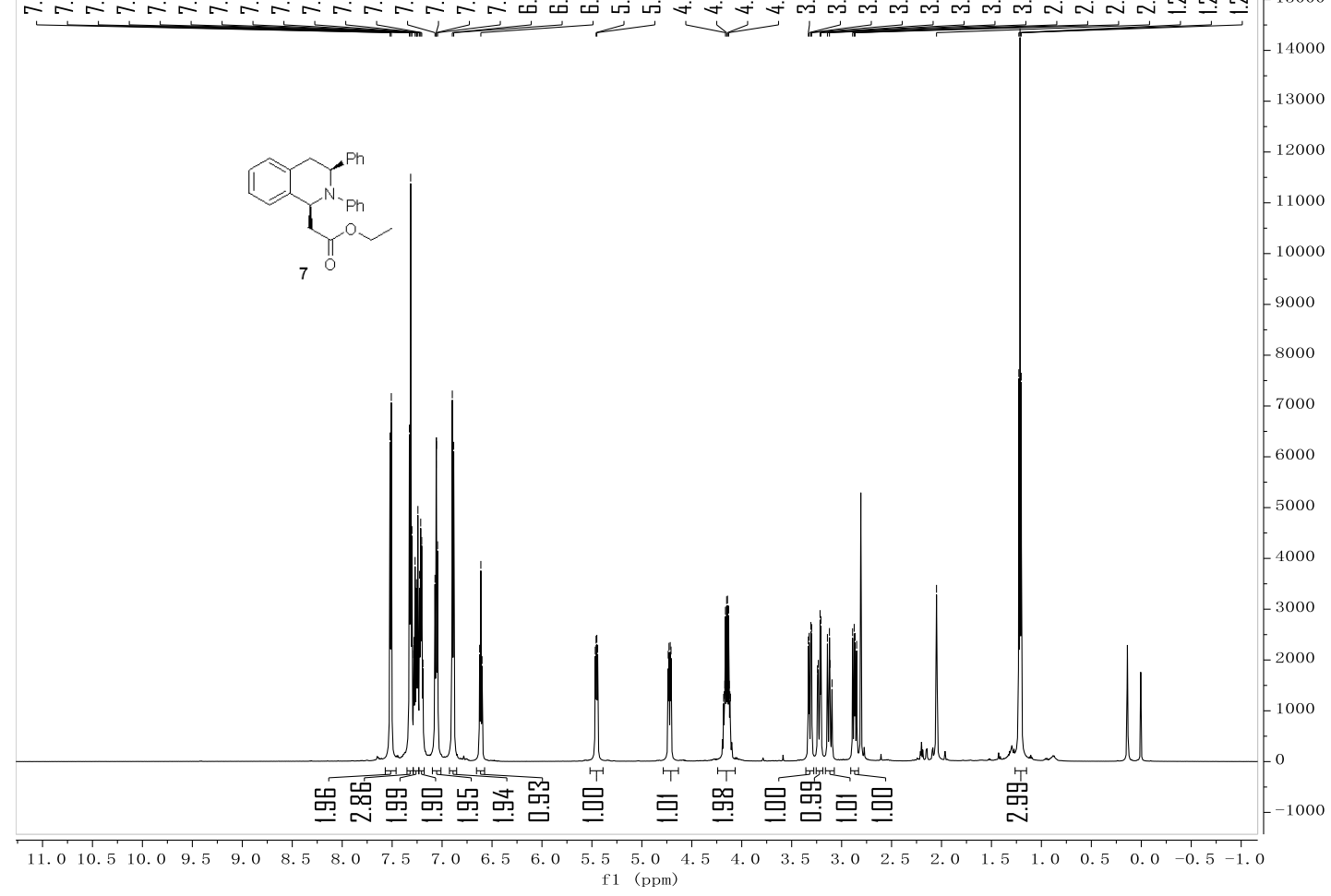

$151 \mathrm{MHz},\left(\mathrm{CD}_{3}\right)_{2} \mathrm{CO},{ }^{13} \mathrm{C}$ NMR

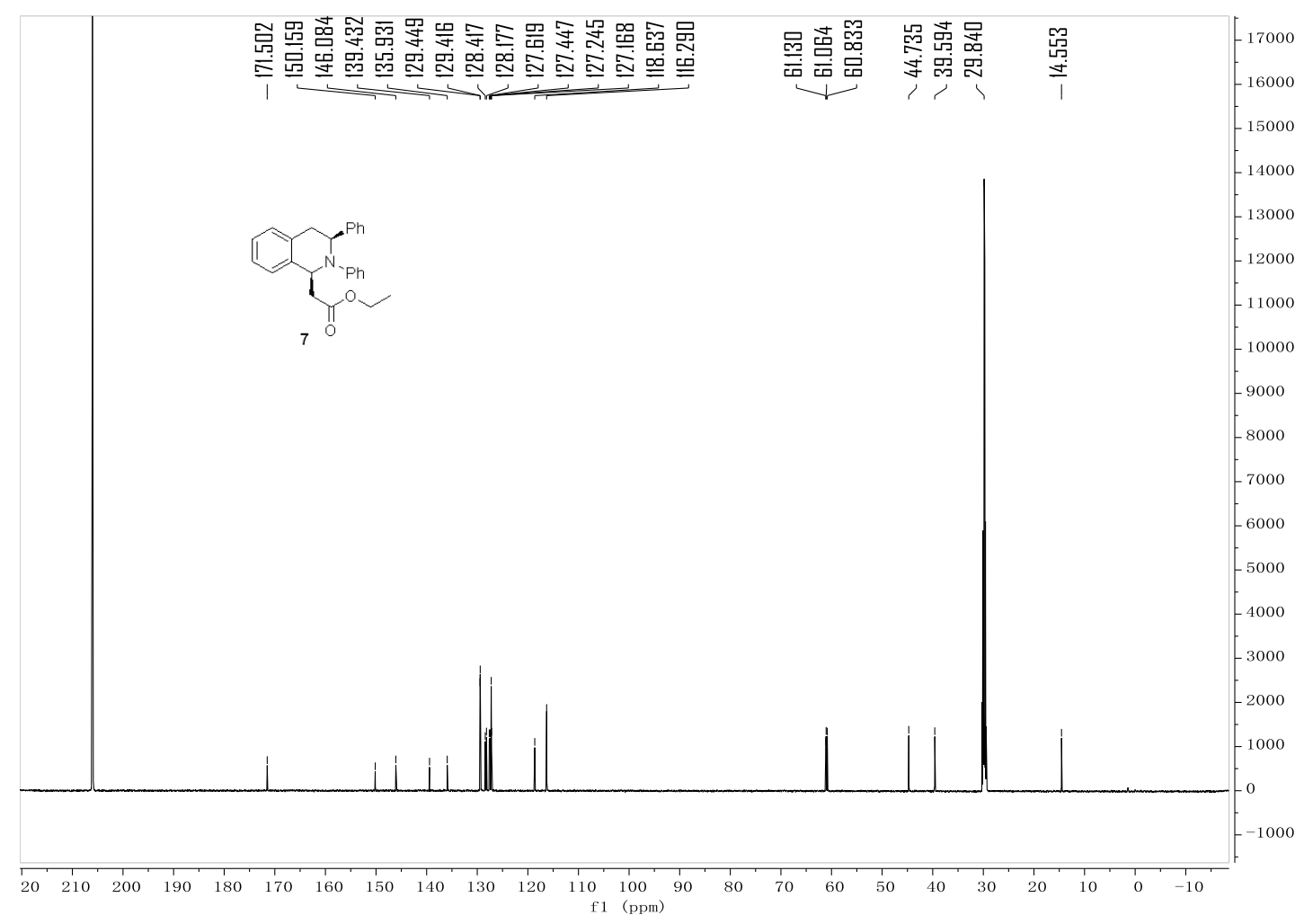


$600 \mathrm{MHz},\left(\mathrm{CD}_{3}\right)_{2} \mathrm{CO},{ }^{1} \mathrm{H} \mathrm{NMR}$

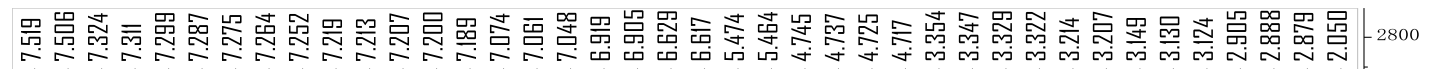

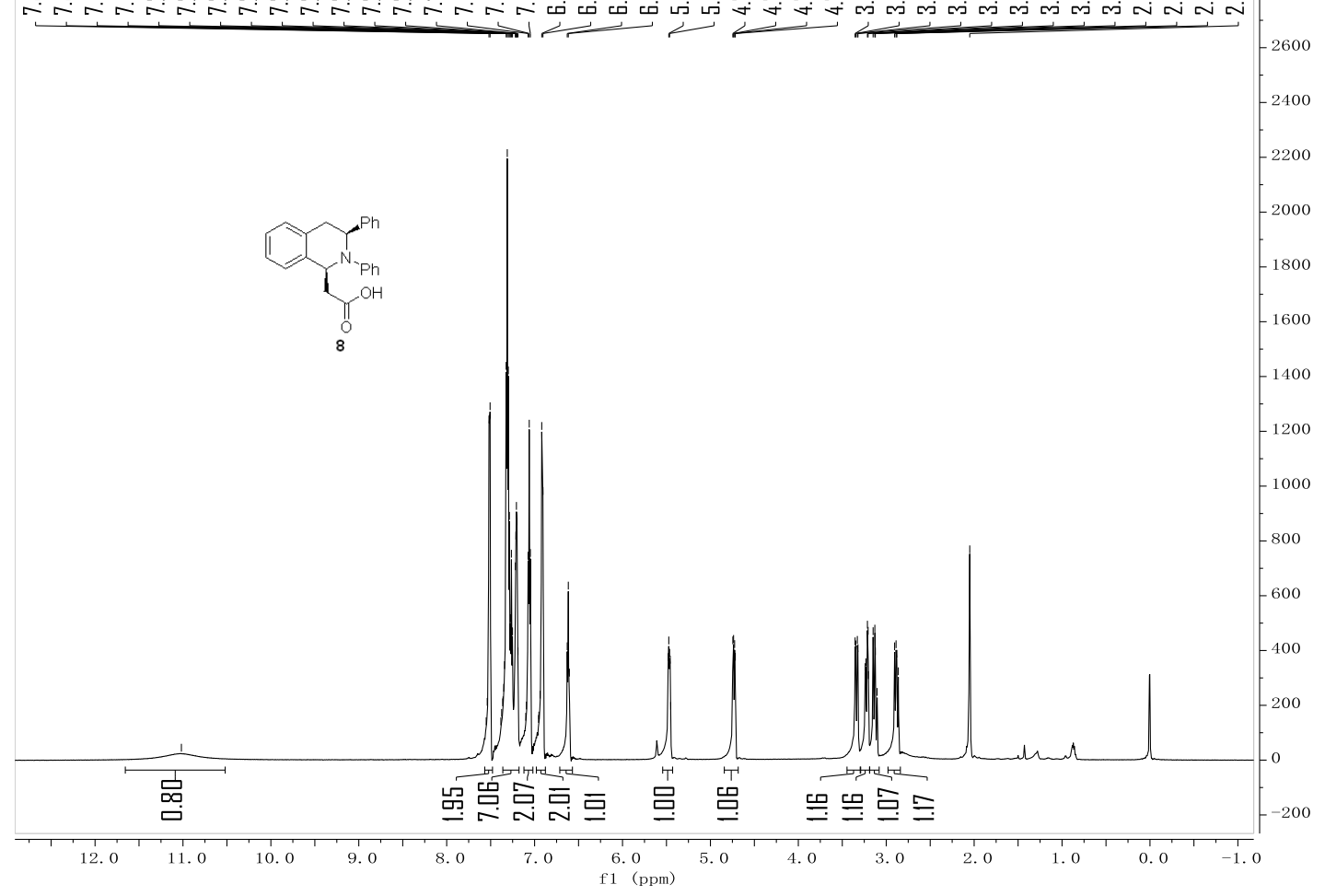

$151 \mathrm{MHz},\left(\mathrm{CD}_{3}\right)_{2} \mathrm{CO},{ }^{13} \mathrm{C} \mathrm{NMR}$

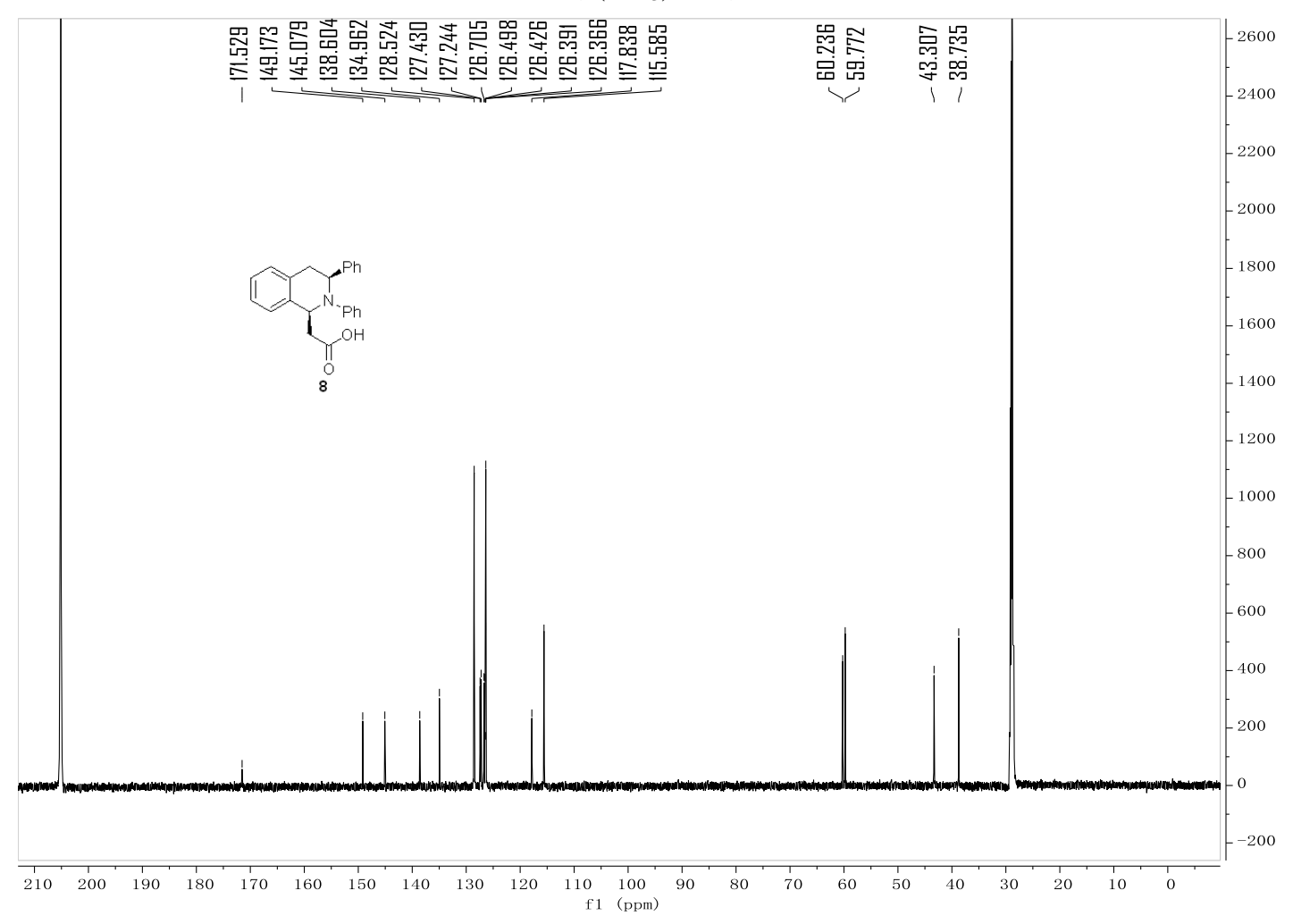




\section{$600 \mathrm{MHz}, \mathrm{CDCl}_{3},{ }^{1} \mathrm{H} \mathrm{NMR}$}

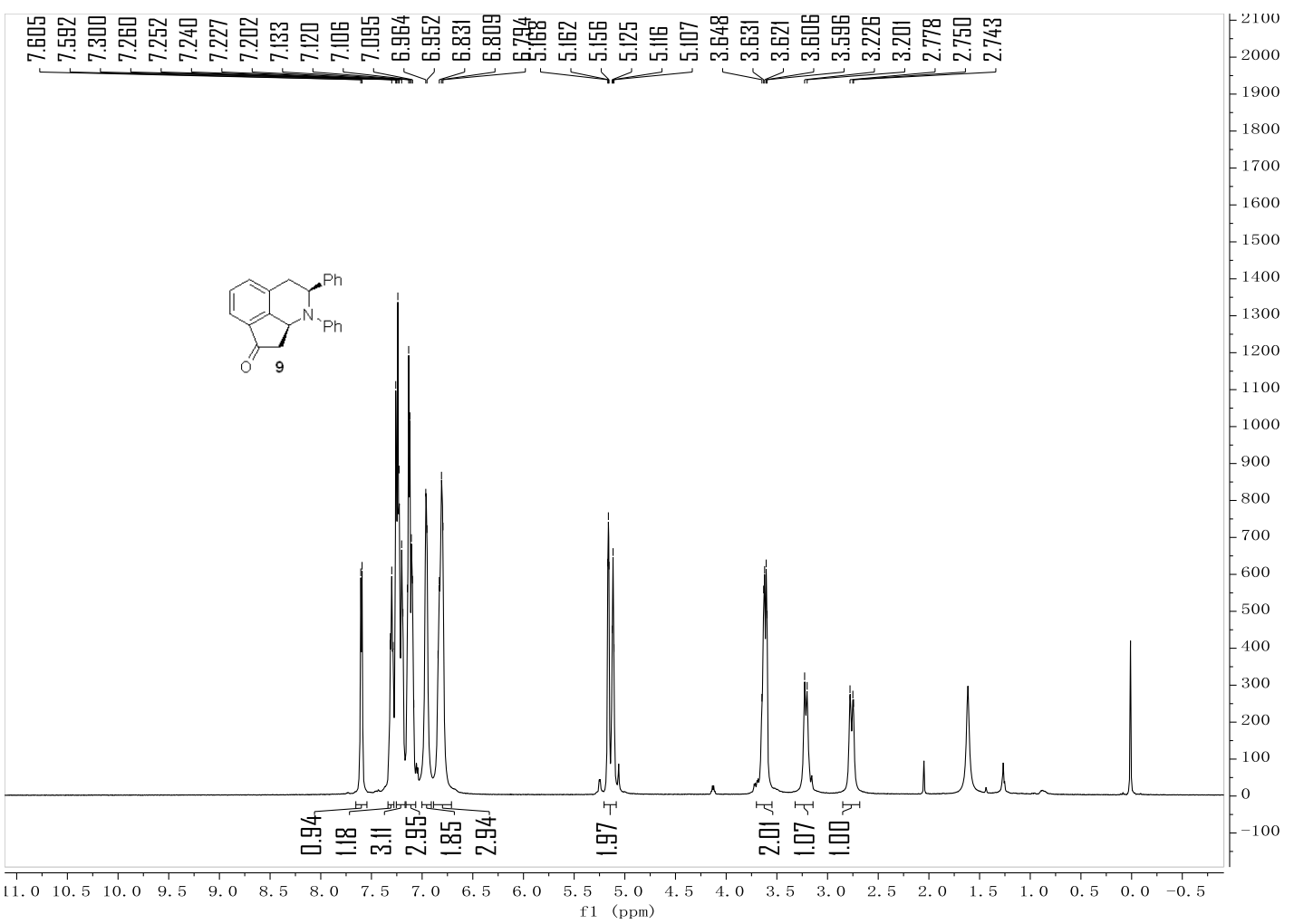

$151 \mathrm{MHz}, \mathrm{CDCl}_{3},{ }^{13} \mathrm{C} \mathrm{NMR}$

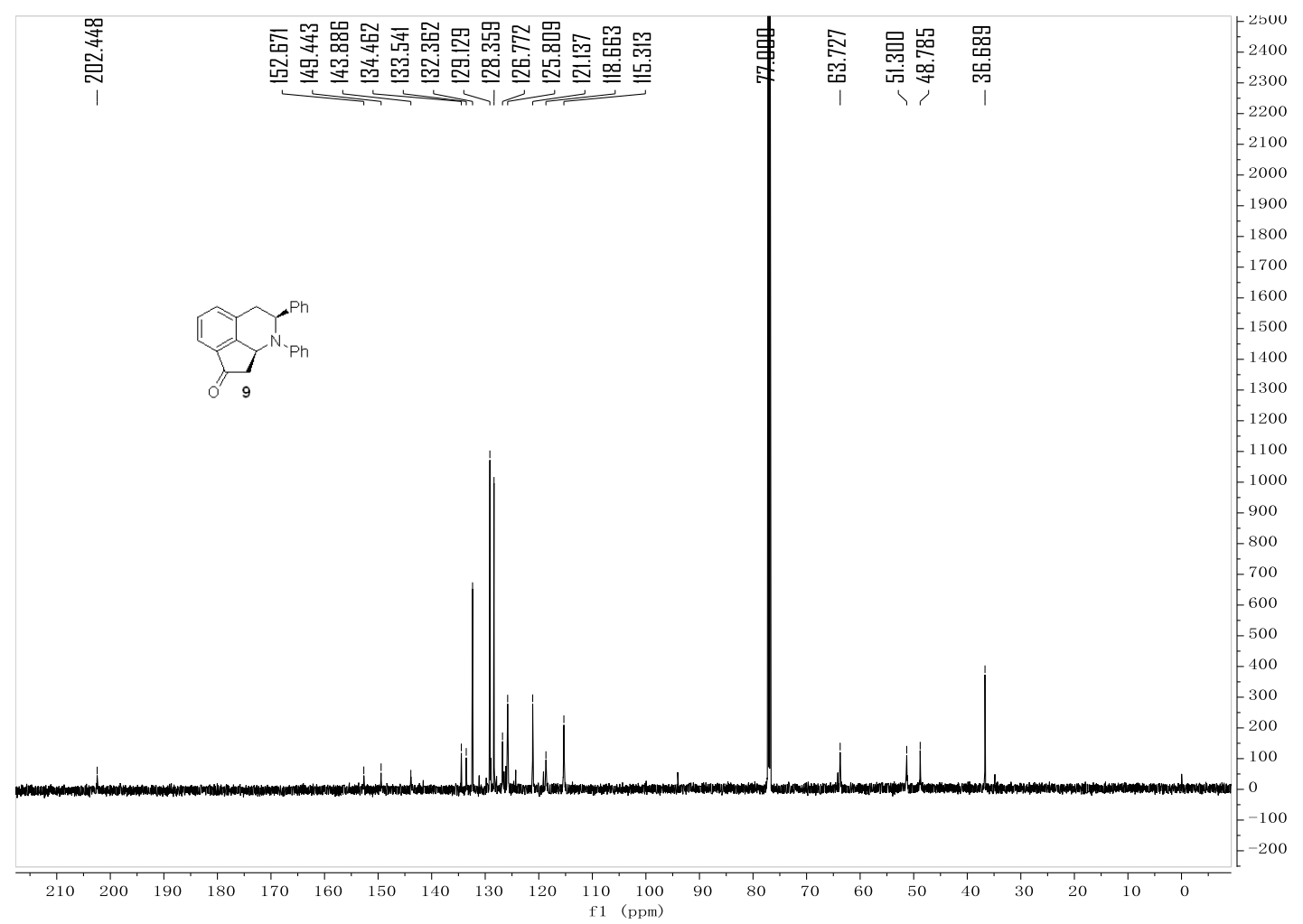




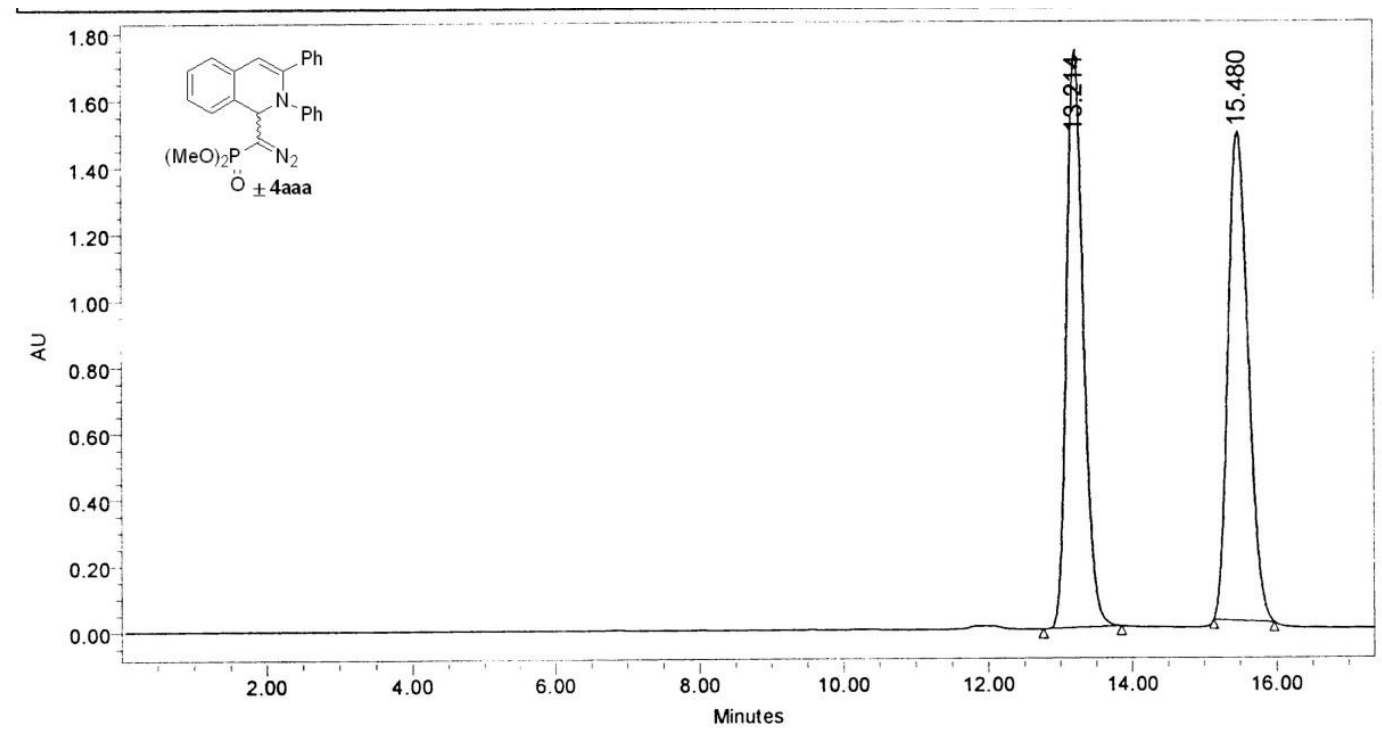

\begin{tabular}{|c|c|c|c|c|c|}
\hline & $\begin{array}{c}\mathrm{RT} \\
(\mathrm{min})\end{array}$ & $\begin{array}{c}\text { Area } \\
\left(\mu \mathrm{V}^{*} \mathrm{sec}\right)\end{array}$ & $\%$ Area & $\begin{array}{c}\text { Height } \\
(\mu \mathrm{V})\end{array}$ & $\begin{array}{c}\% \\
\text { Height }\end{array}$ \\
\hline 1 & 13.214 & 28340902 & 49.92 & 1738228 & 54.08 \\
\hline 2 & 15.480 & 28433923 & 50.08 & 1475672 & 45.92 \\
\hline
\end{tabular}

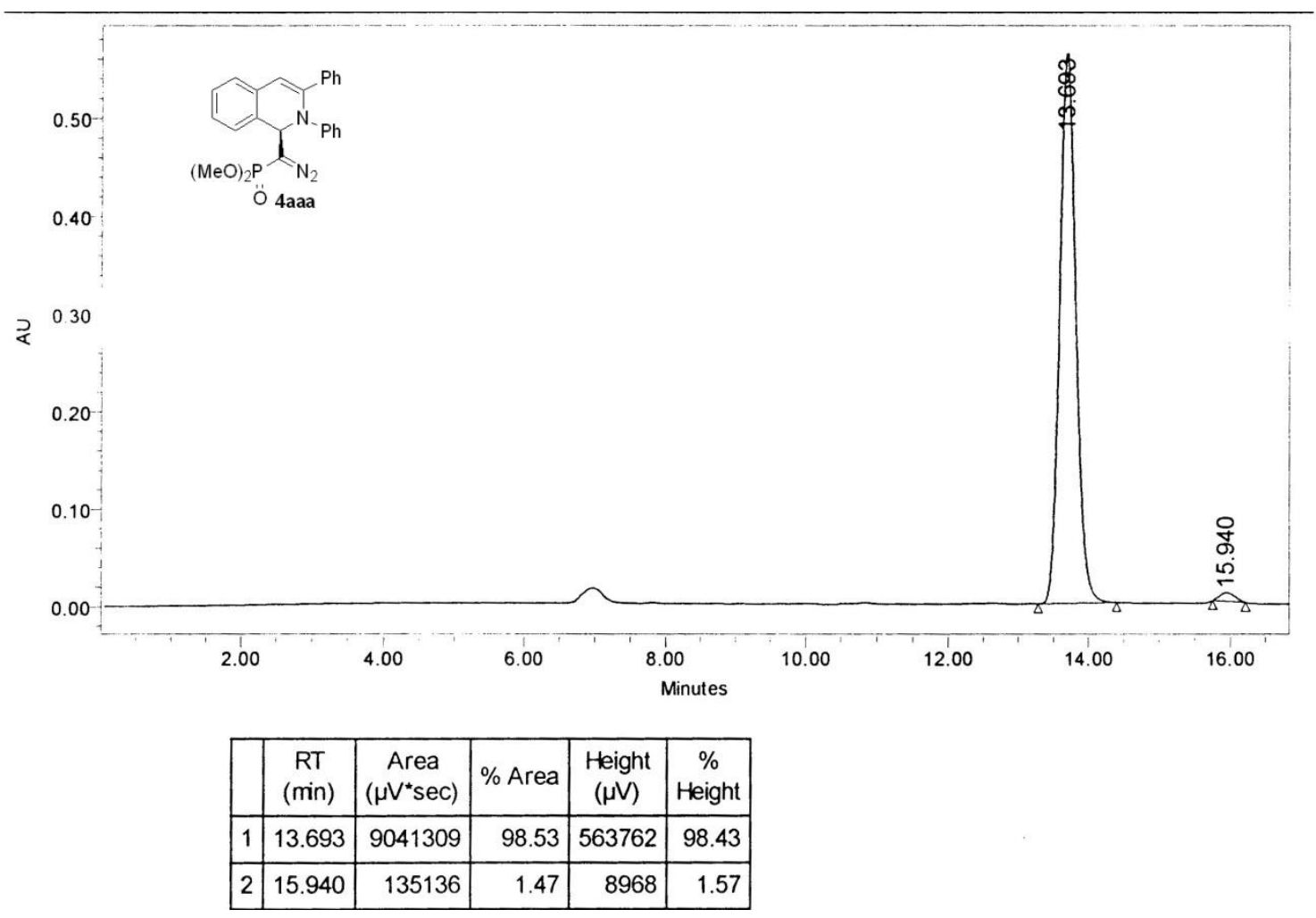



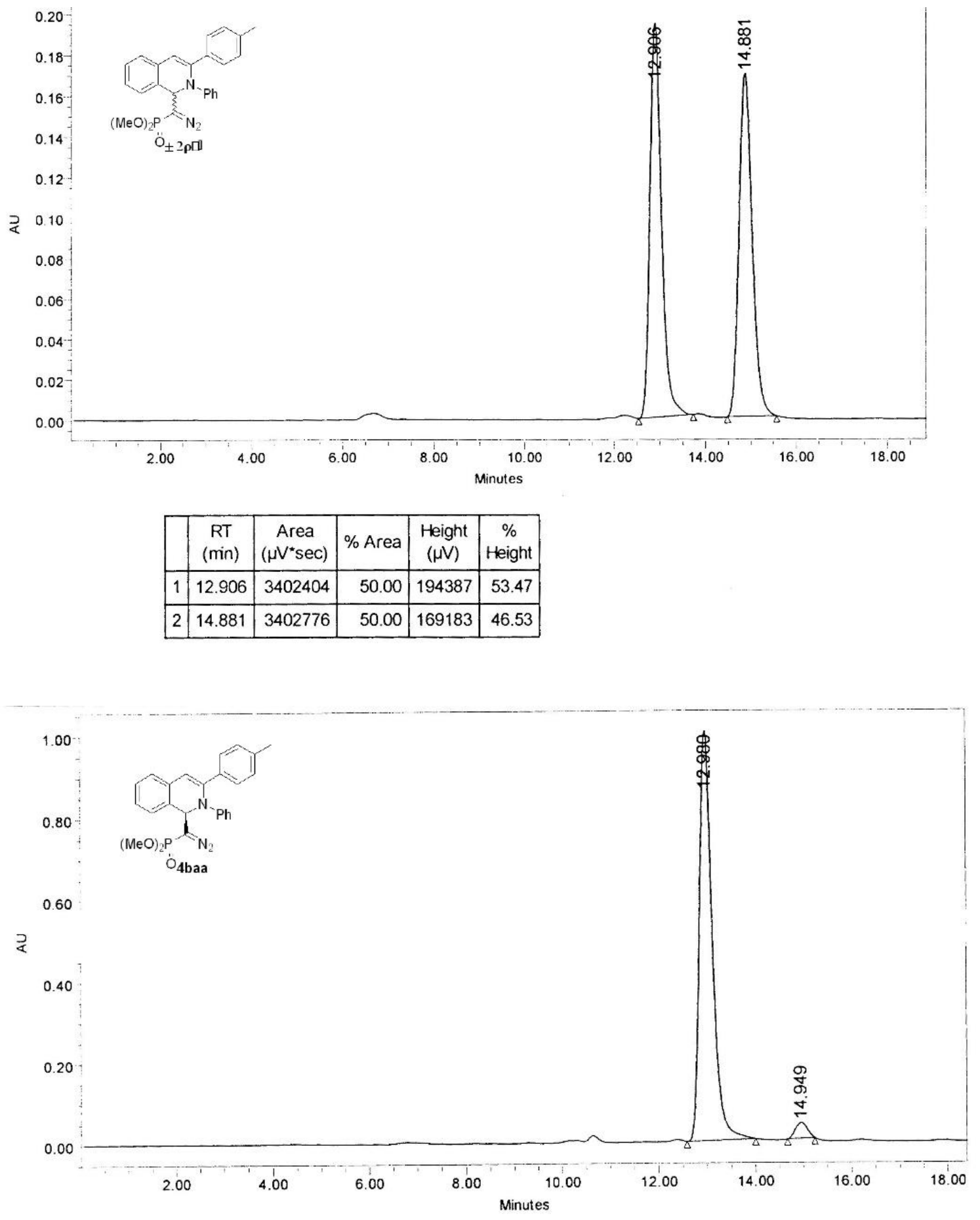

\begin{tabular}{|c|c|c|r|r|r|}
\hline & $\begin{array}{c}\mathrm{RT} \\
(\mathrm{min})\end{array}$ & $\begin{array}{c}\text { Area } \\
(\mu \mathrm{V} * \mathrm{sec})\end{array}$ & $\%$ Area & $\begin{array}{c}\text { Height } \\
(\mu \mathrm{V})\end{array}$ & $\begin{array}{c}\% \\
\text { Height }\end{array}$ \\
\hline 1 & 12.980 & 18365095 & 96.49 & 1003249 & 96.28 \\
\hline 2 & 14.949 & 667347 & 3.51 & 38773 & 3.72 \\
\hline
\end{tabular}



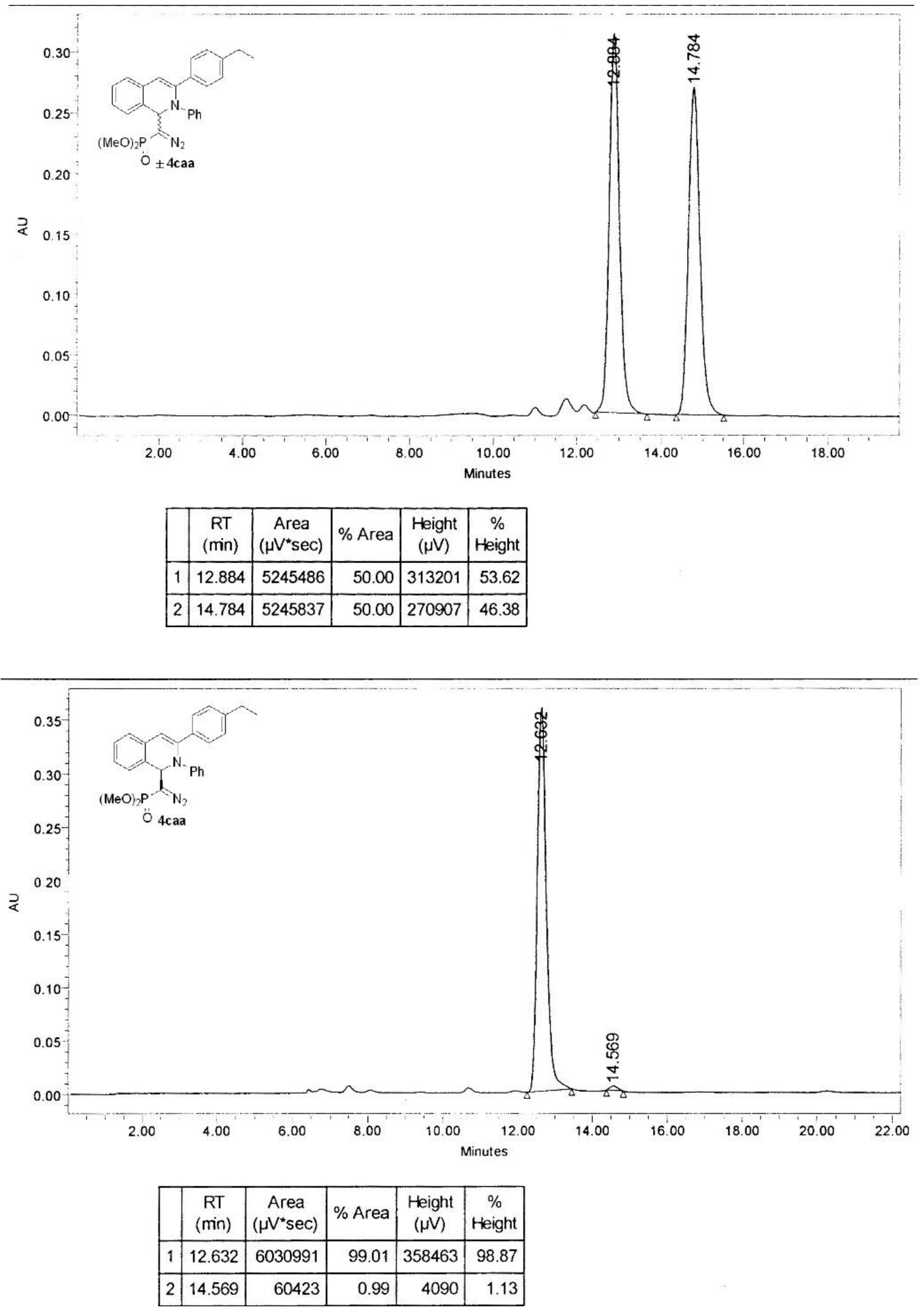


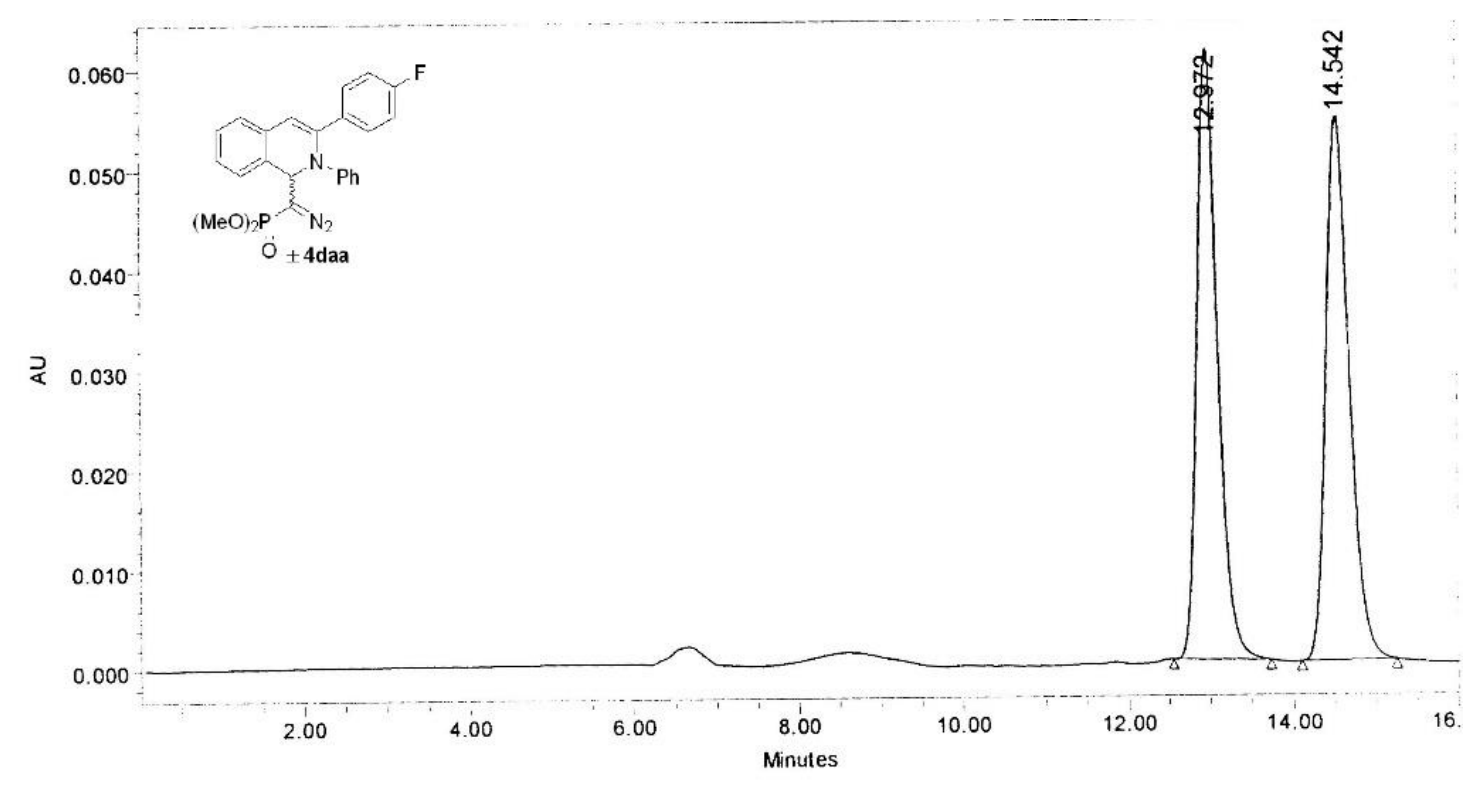

\begin{tabular}{|c|c|c|c|c|c|}
\hline & $\begin{array}{c}\mathrm{RT} \\
(\mathrm{min})\end{array}$ & $\begin{array}{c}\text { Area } \\
(\mu \mathrm{V} * \mathrm{sec})\end{array}$ & $\%$ Area & $\begin{array}{c}\text { Height } \\
(\mu \mathrm{V})\end{array}$ & $\begin{array}{c}\% \\
\text { Height }\end{array}$ \\
\hline 1 & 12.972 & 1064305 & 50.01 & 61083 & 52.84 \\
\hline 2 & 14.542 & 1063692 & 49.99 & 54507 & 47.16 \\
\hline
\end{tabular}

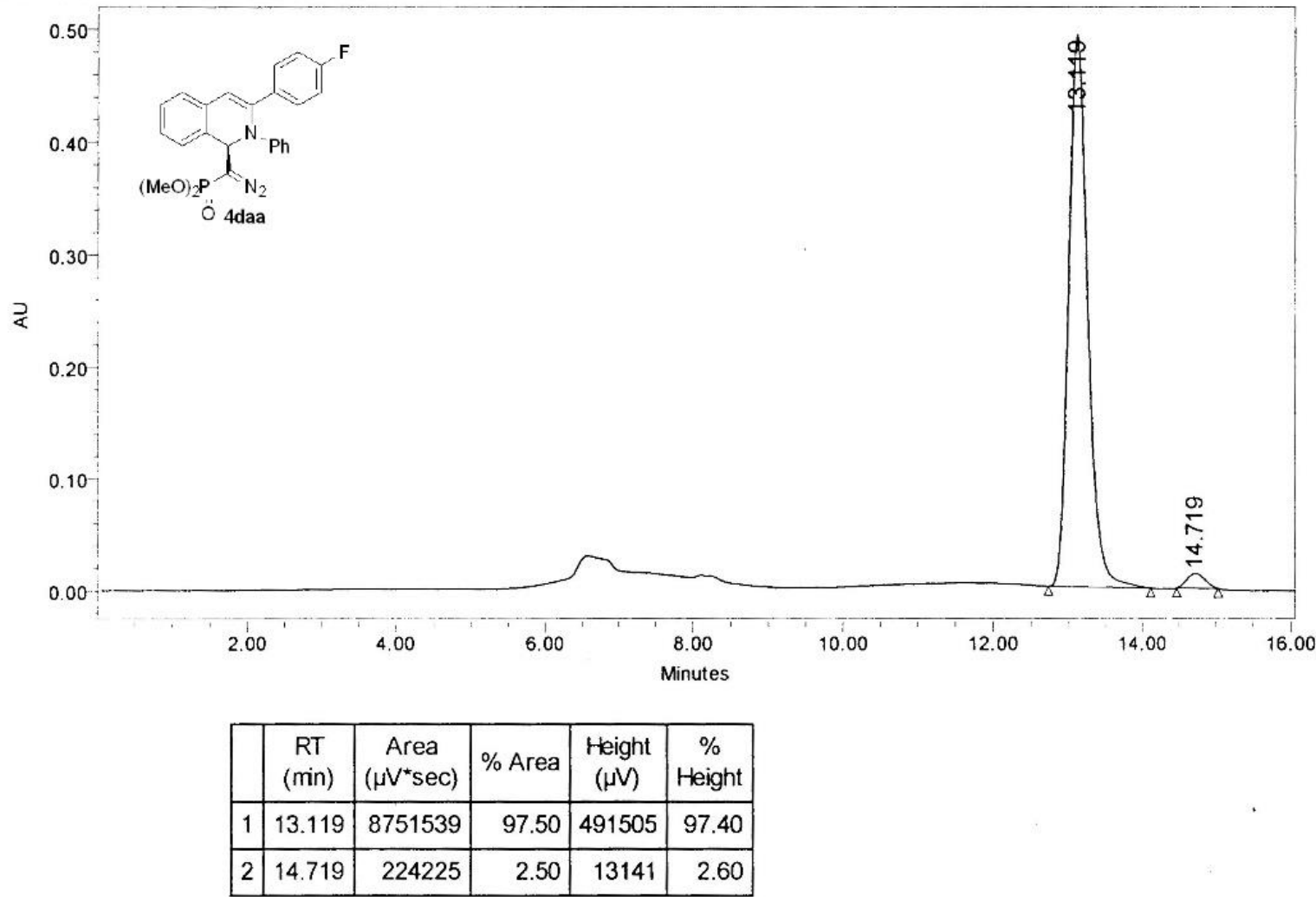



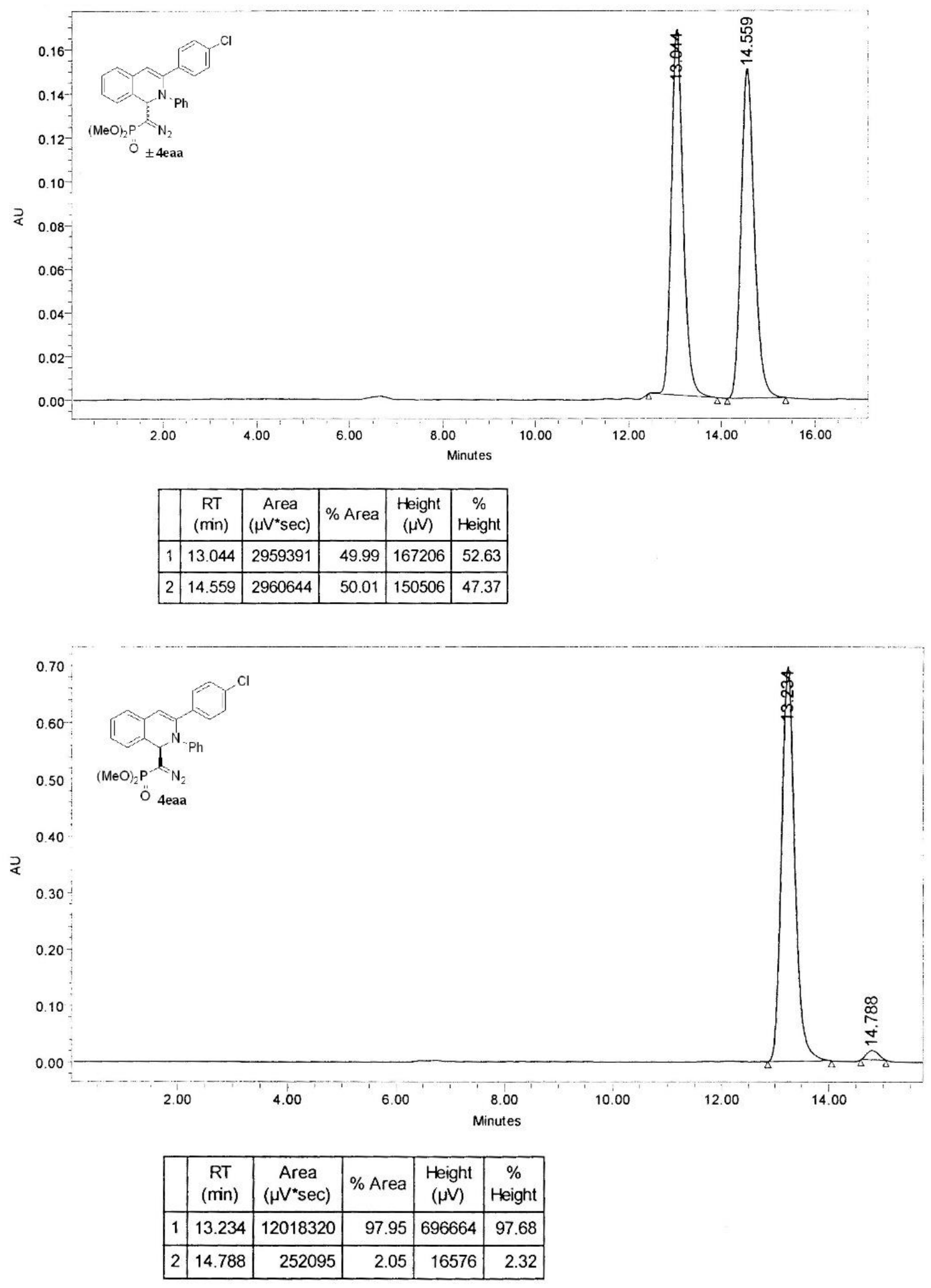

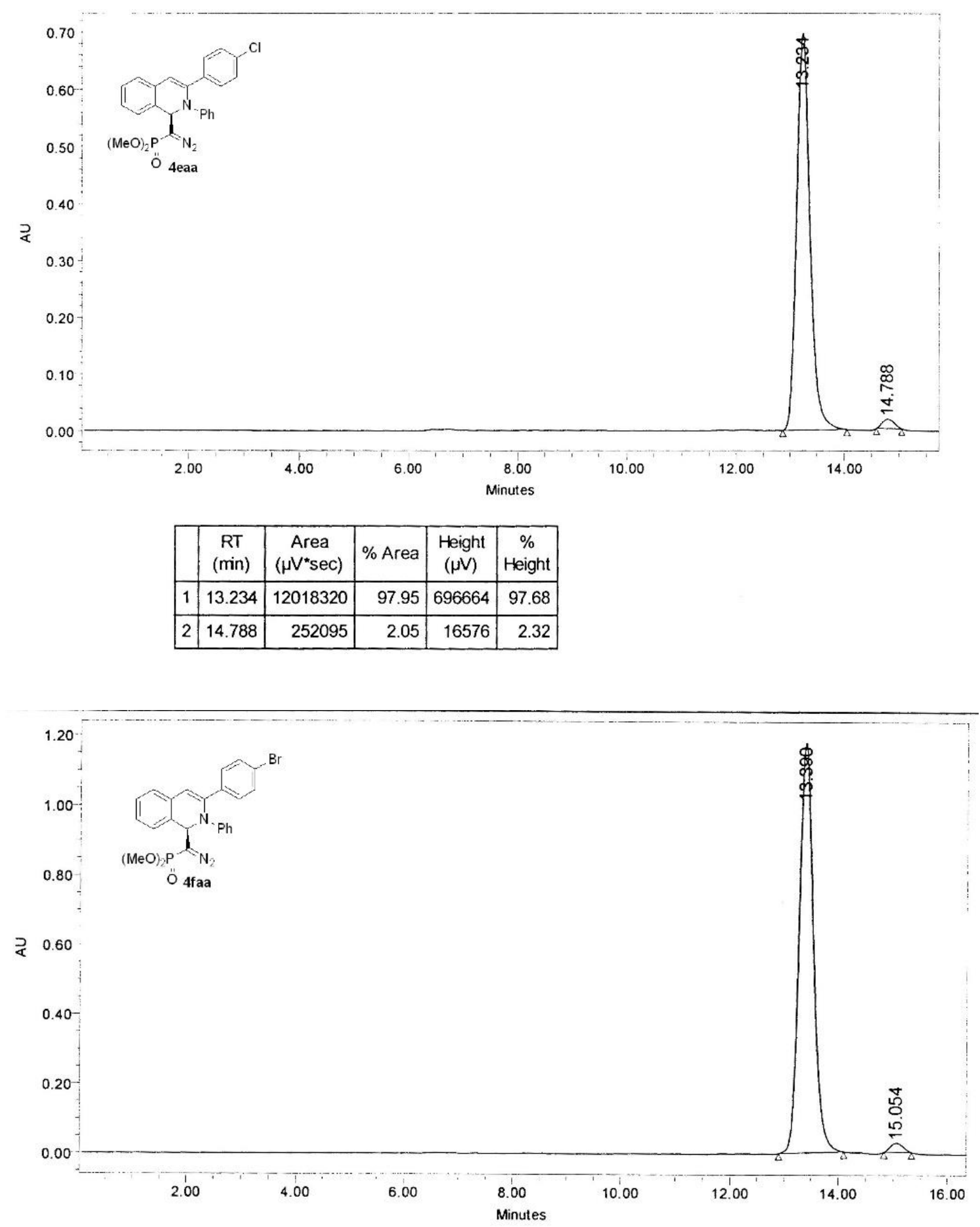

\begin{tabular}{|c|c|r|r|r|r|}
\hline & $\begin{array}{c}\text { RT } \\
(\mathrm{min})\end{array}$ & $\begin{array}{c}\text { Area } \\
\left(\mu \mathrm{V}^{*} \mathrm{sec}\right)\end{array}$ & $\%$ Area & \multicolumn{1}{c|}{$\begin{array}{c}\text { Height } \\
(\mu \mathrm{V})\end{array}$} & $\begin{array}{c}\% \\
\text { Height }\end{array}$ \\
\hline 1 & 13.390 & 21644761 & 98.08 & 1178455 & 97.83 \\
\hline 2 & 15.054 & 423201 & 1.92 & 26196 & 2.17 \\
\hline
\end{tabular}




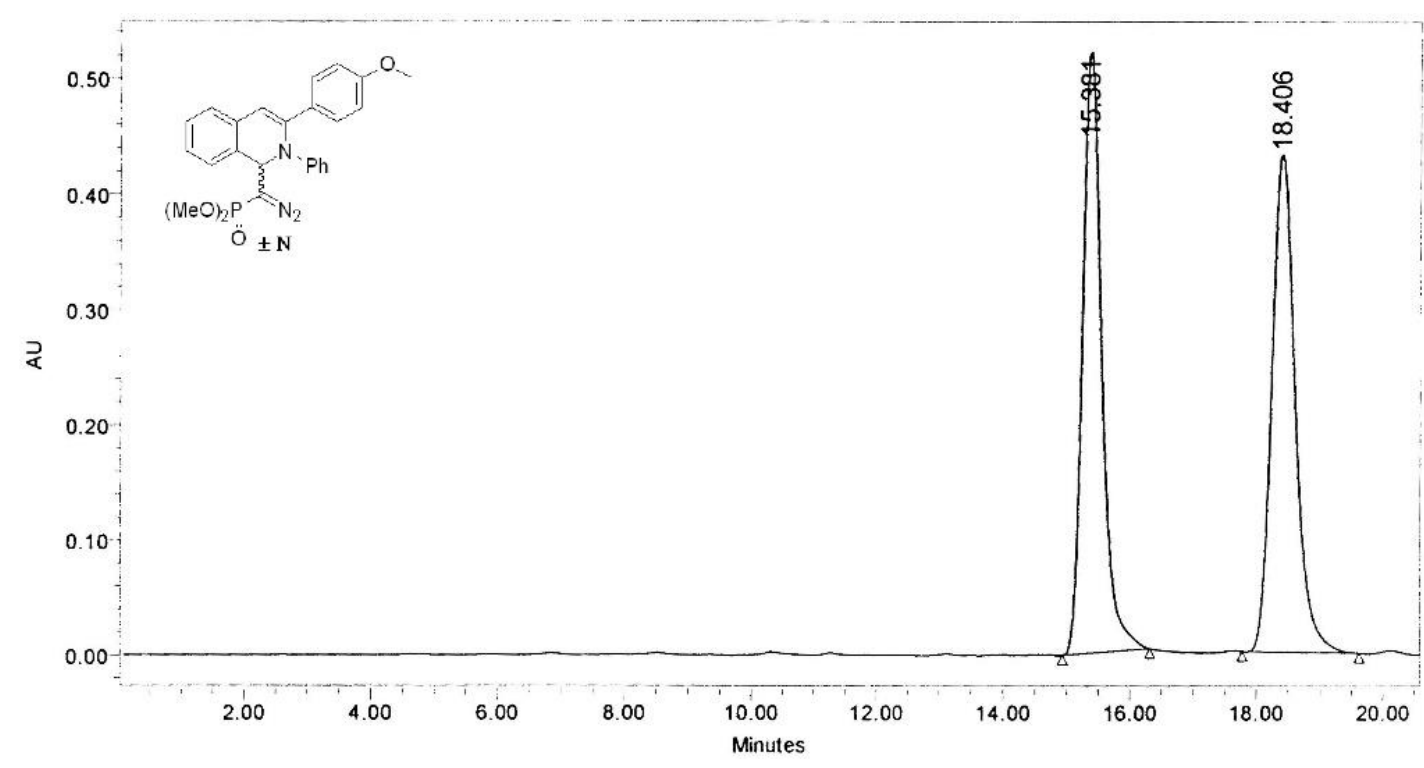

\begin{tabular}{|c|c|c|c|c|c|}
\hline & $\begin{array}{c}\mathrm{RT} \\
(\mathrm{min})\end{array}$ & $\begin{array}{c}\text { Area } \\
(\mu \mathrm{V} * \mathrm{sec})\end{array}$ & $\%$ Area & $\begin{array}{c}\text { Height } \\
(\mu \mathrm{V})\end{array}$ & $\begin{array}{c}\% \\
\text { Height }\end{array}$ \\
\hline 1 & 15.381 & 10975654 & 50.05 & 520563 & 54.66 \\
\hline 2 & 18.406 & 10953431 & 49.95 & 431855 & 45.34 \\
\hline
\end{tabular}

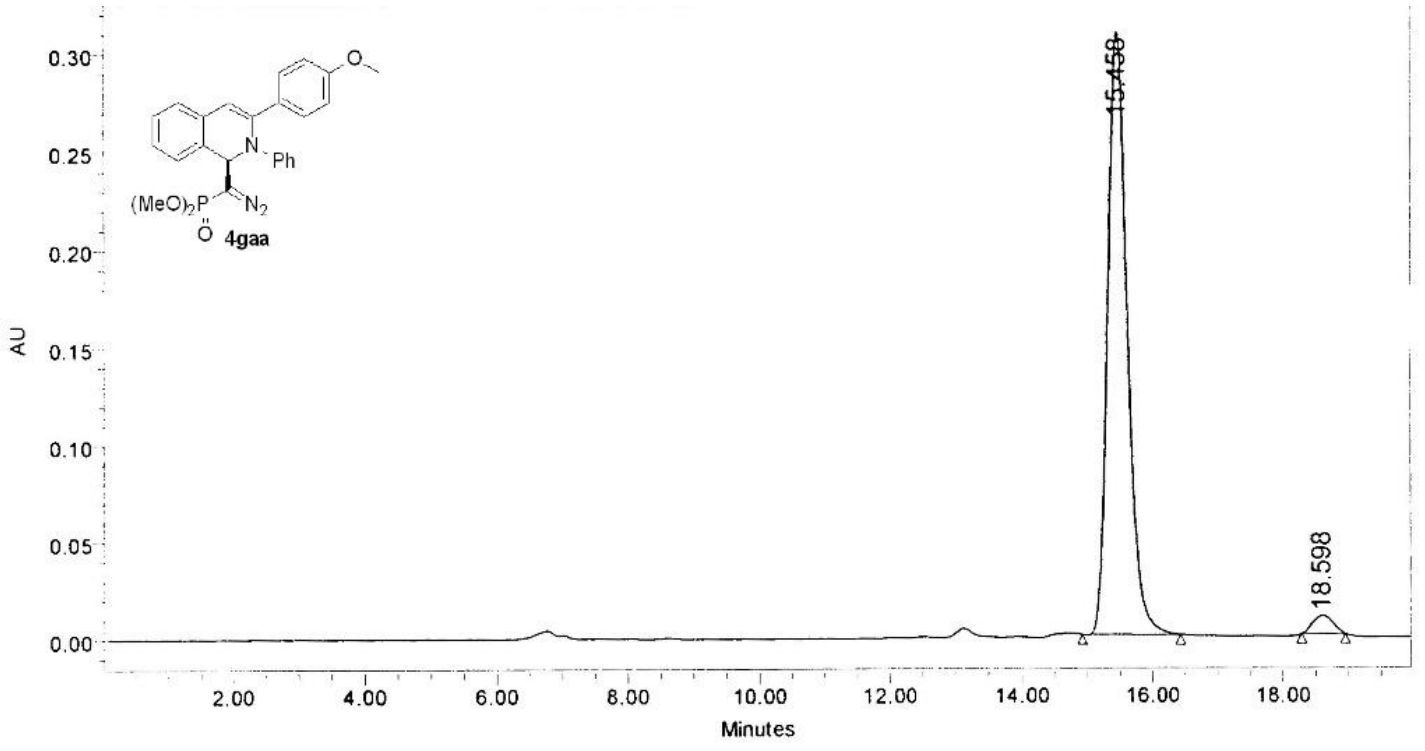

\begin{tabular}{|c|c|c|r|r|r|}
\hline & $\begin{array}{c}\mathrm{RT} \\
(\mathrm{min})\end{array}$ & $\begin{array}{c}\text { Area } \\
\left(\mu \mathrm{V}^{*} \mathrm{sec}\right)\end{array}$ & $\%$ Area & $\begin{array}{c}\text { Height } \\
(\mu \mathrm{V})\end{array}$ & $\begin{array}{c}\% \\
\text { Height }\end{array}$ \\
\hline 1 & 15.458 & 6451425 & 97.06 & 309000 & 97.08 \\
\hline 2 & 18.598 & 195339 & 2.94 & 9293 & 2.92 \\
\hline
\end{tabular}




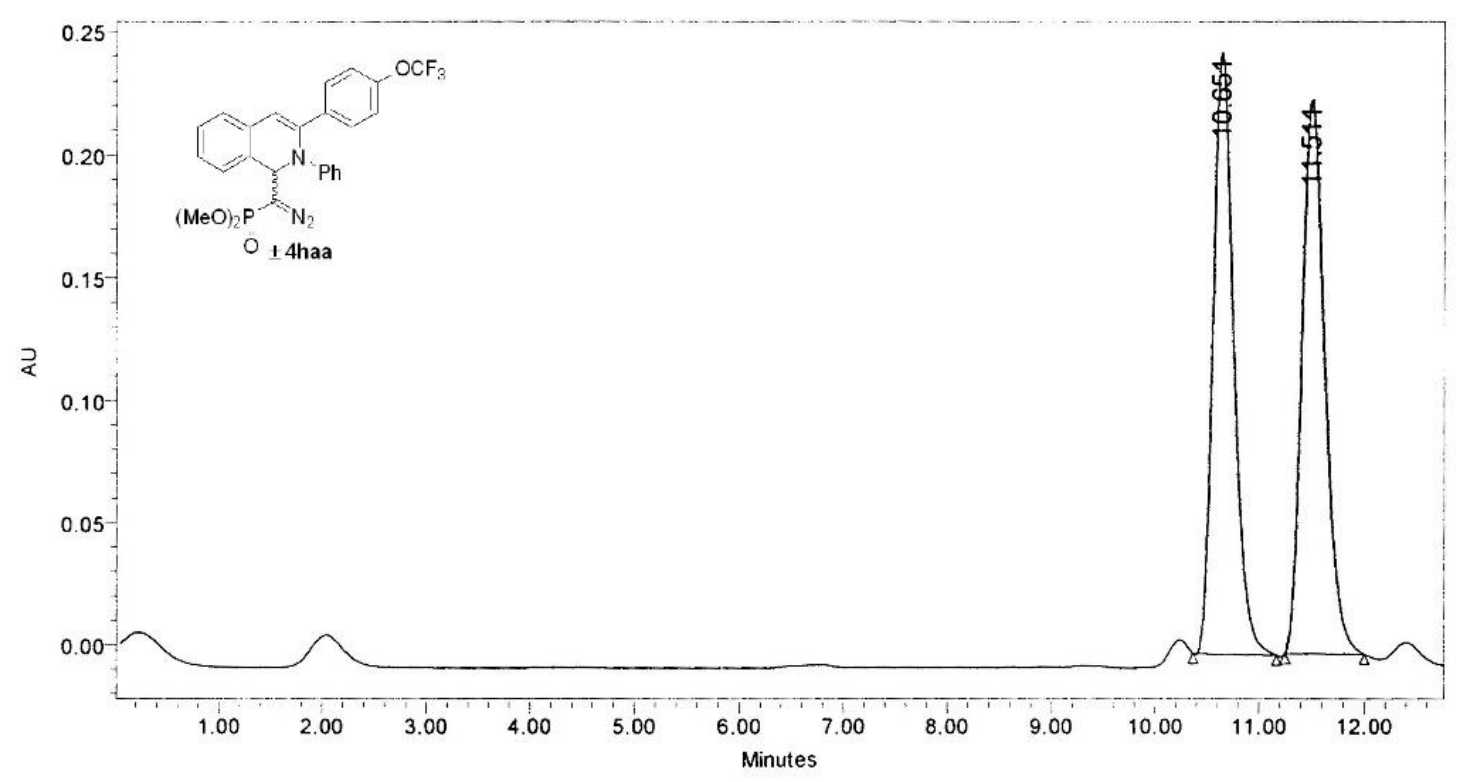

\begin{tabular}{|c|c|c|c|c|c|}
\hline & $\begin{array}{c}\mathrm{RT} \\
(\mathrm{min})\end{array}$ & $\begin{array}{c}\text { Area } \\
\left(\mu \mathrm{V}^{\star} \mathrm{sec}\right)\end{array}$ & $\%$ Area & $\begin{array}{c}\text { Height } \\
(\mu \mathrm{V})\end{array}$ & $\begin{array}{c}\% \\
\text { Height }\end{array}$ \\
\hline 1 & 10.651 & 3372746 & 49.92 & 245247 & 52.00 \\
\hline 2 & 11.511 & 3384071 & 50.08 & 226362 & 48.00 \\
\hline
\end{tabular}

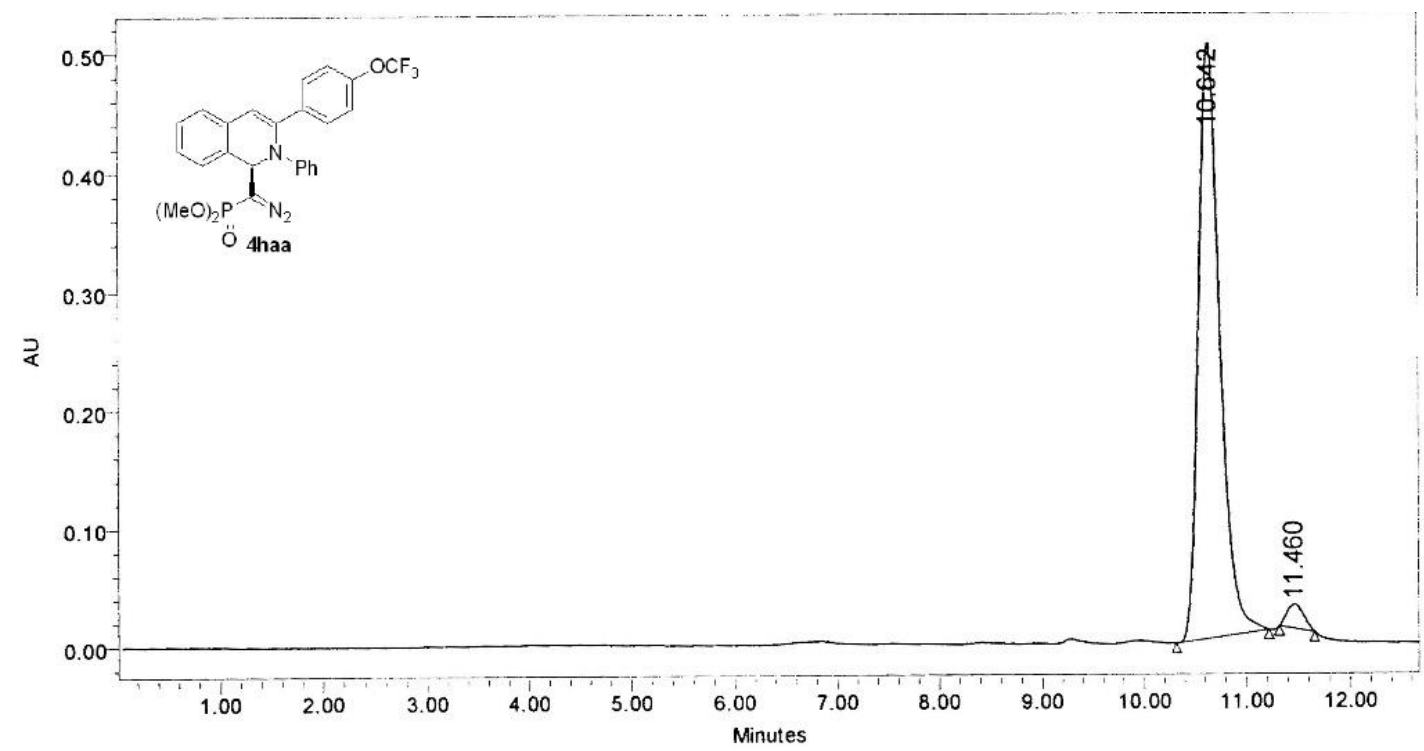

\begin{tabular}{|c|c|c|c|c|c|}
\hline & $\begin{array}{c}\mathrm{RT} \\
(\mathrm{min})\end{array}$ & $\begin{array}{c}\text { Area } \\
\left(\mu \mathrm{V}^{*} \mathrm{sec}\right)\end{array}$ & $\%$ Area & $\begin{array}{c}\text { Height } \\
(\mu \mathrm{V})\end{array}$ & $\begin{array}{c}\% \\
\text { Height }\end{array}$ \\
\hline 1 & 10.642 & 7279758 & 96.98 & 503333 & 96.12 \\
\hline 2 & 11.460 & 226594 & 3.02 & 20306 & 3.88 \\
\hline
\end{tabular}




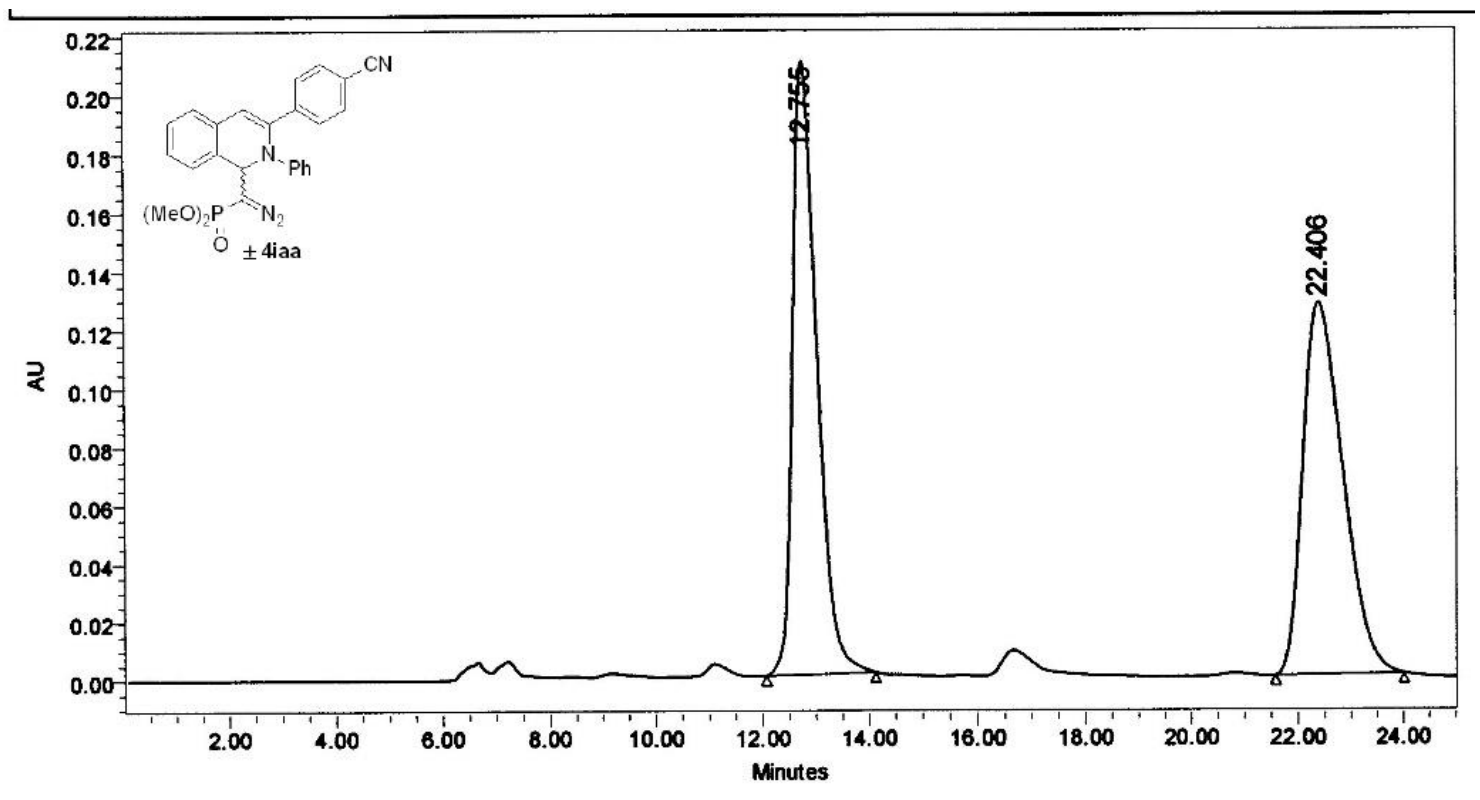

\begin{tabular}{|c|c|c|c|c|c|}
\hline & $\begin{array}{c}\mathrm{RT} \\
(\mathrm{min})\end{array}$ & $\begin{array}{c}\text { Area } \\
(\mu \mathrm{V} * \mathrm{sec})\end{array}$ & $\%$ Area & $\begin{array}{c}\text { Height } \\
(\mu \mathrm{V})\end{array}$ & $\begin{array}{c}\% \\
\text { Height }\end{array}$ \\
\hline 1 & 12.755 & 6604618 & 49.72 & 209671 & 62.18 \\
\hline 2 & 22.406 & 6679352 & 50.28 & 127506 & 37.82 \\
\hline
\end{tabular}

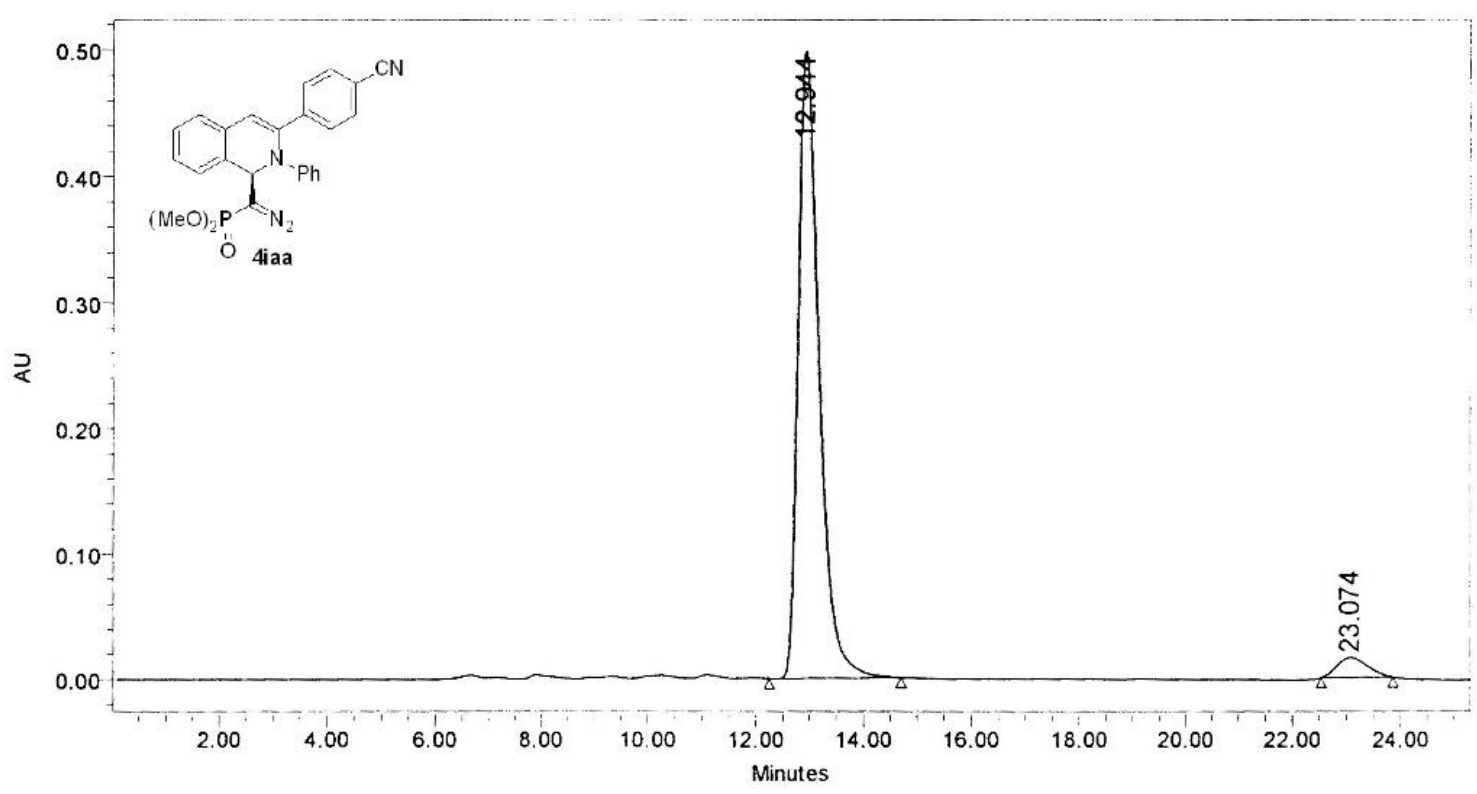

\begin{tabular}{|c|c|c|c|c|c|}
\hline & $\begin{array}{c}\mathrm{RT} \\
(\mathrm{min})\end{array}$ & $\begin{array}{c}\text { Area } \\
\left(\mu \mathrm{V}^{*} \mathrm{sec}\right)\end{array}$ & $\%$ Area & $\begin{array}{c}\text { Height } \\
(\mu \mathrm{V})\end{array}$ & $\begin{array}{c}\% \\
\text { Height }\end{array}$ \\
\hline 1 & 12.944 & 13456622 & 95.55 & 498242 & 96.93 \\
\hline 2 & 23.074 & 627286 & 4.45 & 15790 & 3.07 \\
\hline
\end{tabular}




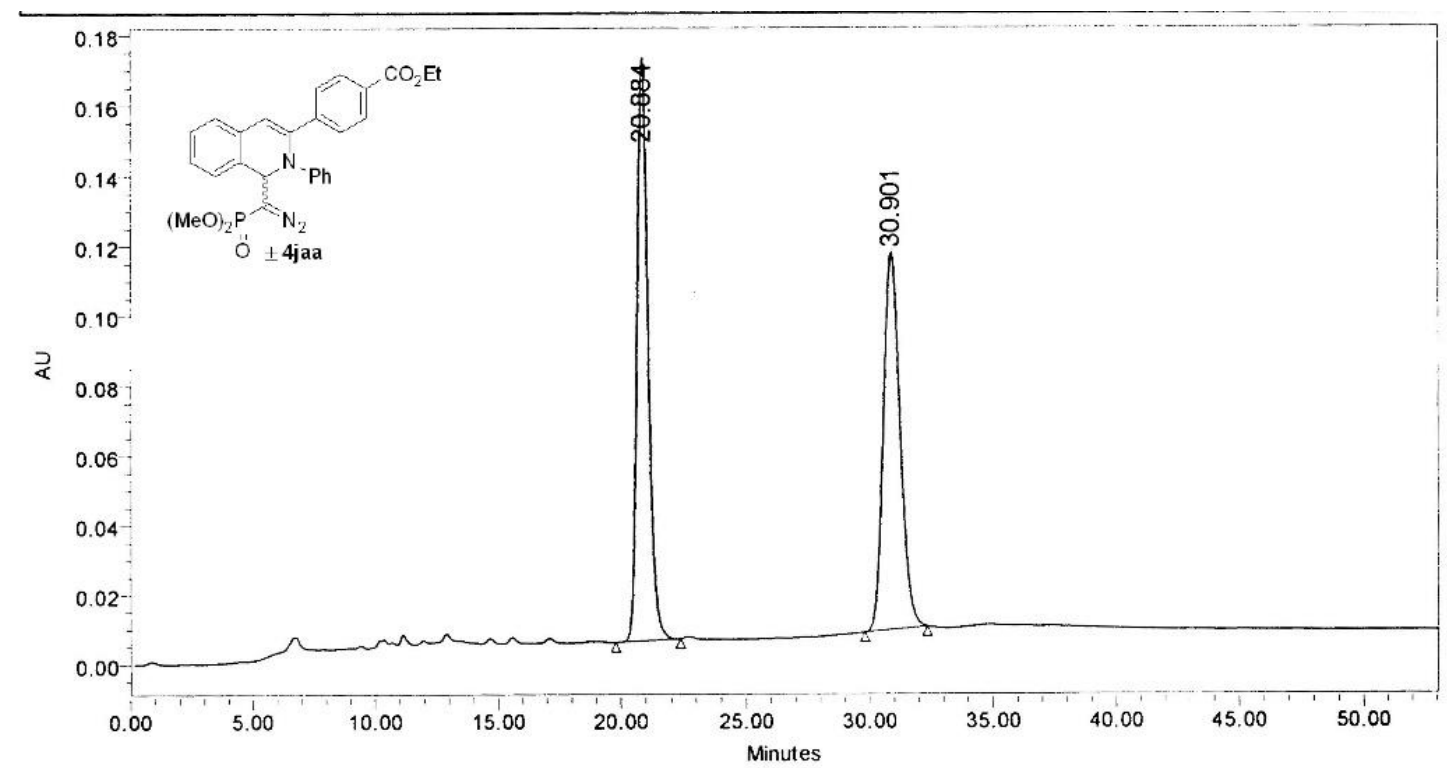

\begin{tabular}{|c|c|c|c|c|c|}
\hline & $\begin{array}{c}\mathrm{RT} \\
(\mathrm{min})\end{array}$ & $\begin{array}{c}\text { Area } \\
\left(\mu \mathrm{V}^{*} \mathrm{sec}\right)\end{array}$ & $\%$ Area & $\begin{array}{c}\text { Height } \\
(\mu \mathrm{V})\end{array}$ & $\begin{array}{c}\% \\
\text { Height }\end{array}$ \\
\hline 1 & 20.884 & 5126785 & 50.02 & 166675 & 60.64 \\
\hline 2 & 30.901 & 5122688 & 49.98 & 108198 & 39.36 \\
\hline
\end{tabular}

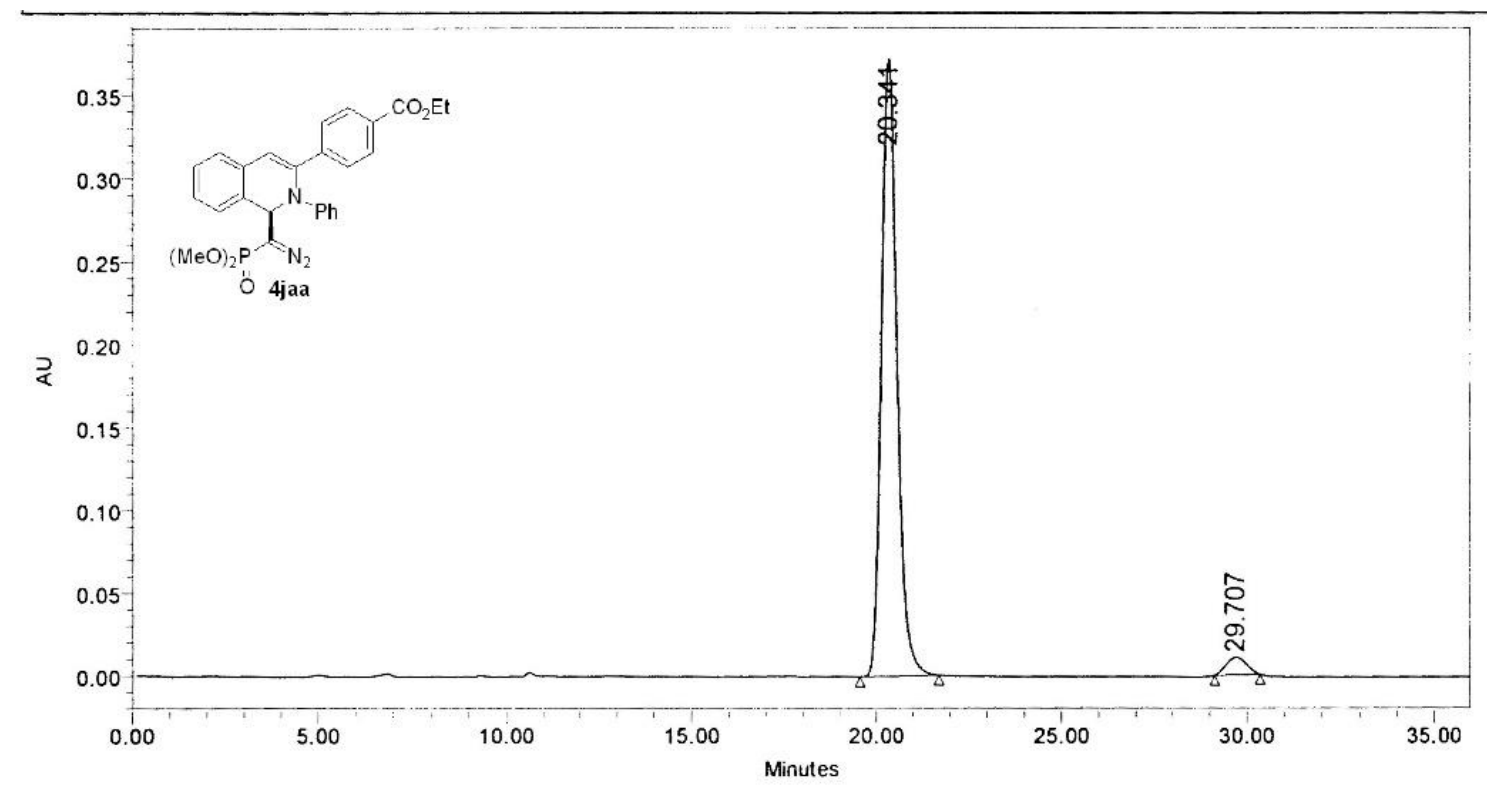

\begin{tabular}{|c|c|r|r|r|r|}
\hline & $\begin{array}{c}\mathrm{RT} \\
(\mathrm{min})\end{array}$ & $\begin{array}{c}\text { Area } \\
\left(\mu \mathrm{V}^{*} \mathrm{sec}\right)\end{array}$ & $\%$ Area & $\begin{array}{c}\text { Height } \\
(\mu \mathrm{V})\end{array}$ & $\begin{array}{c}\% \\
\text { Height }\end{array}$ \\
\hline 1 & 20.341 & 10911742 & 96.49 & 372167 & 97.25 \\
\hline 2 & 29.707 & 397378 & 3.51 & 10540 & 2.75 \\
\hline
\end{tabular}




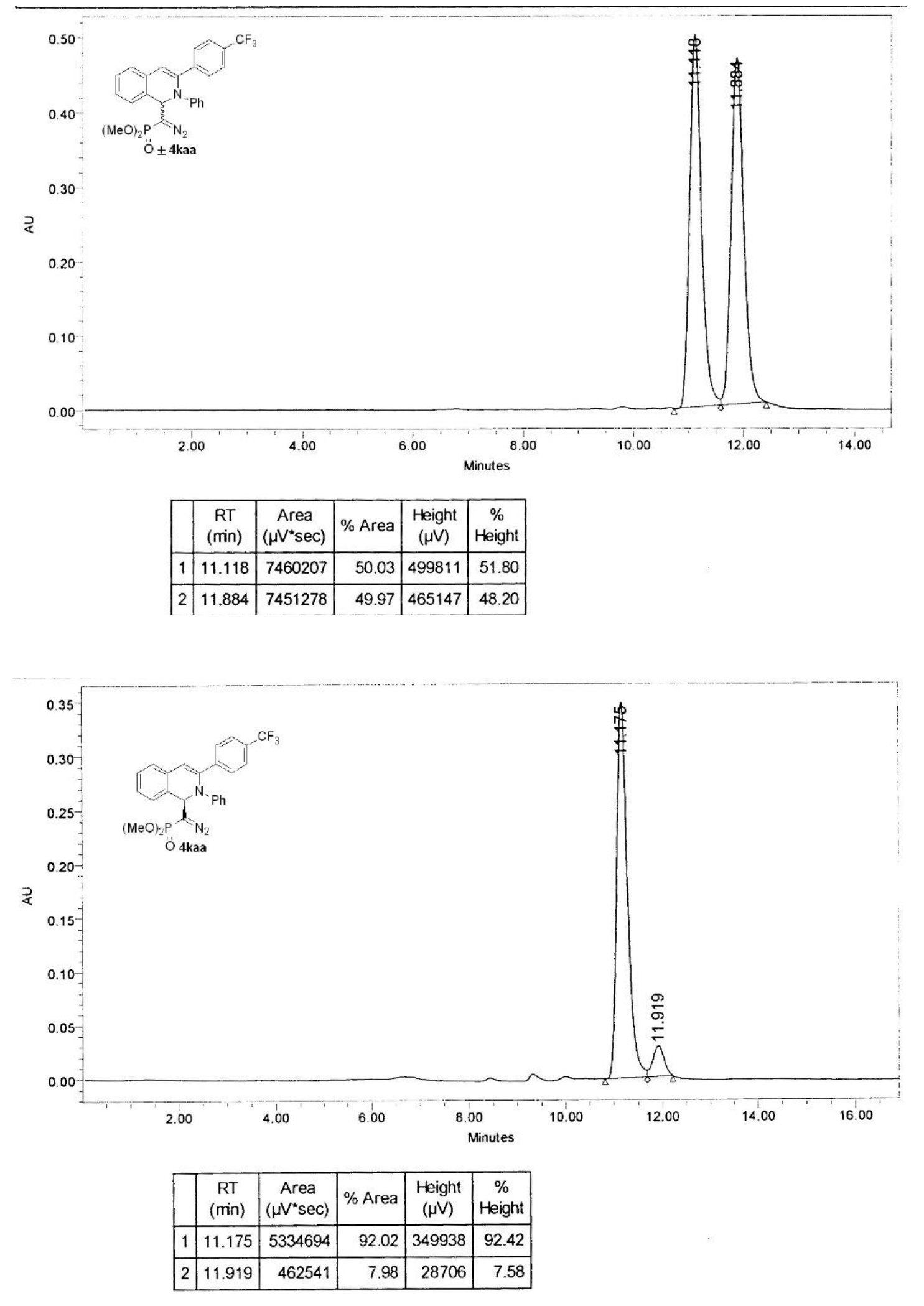




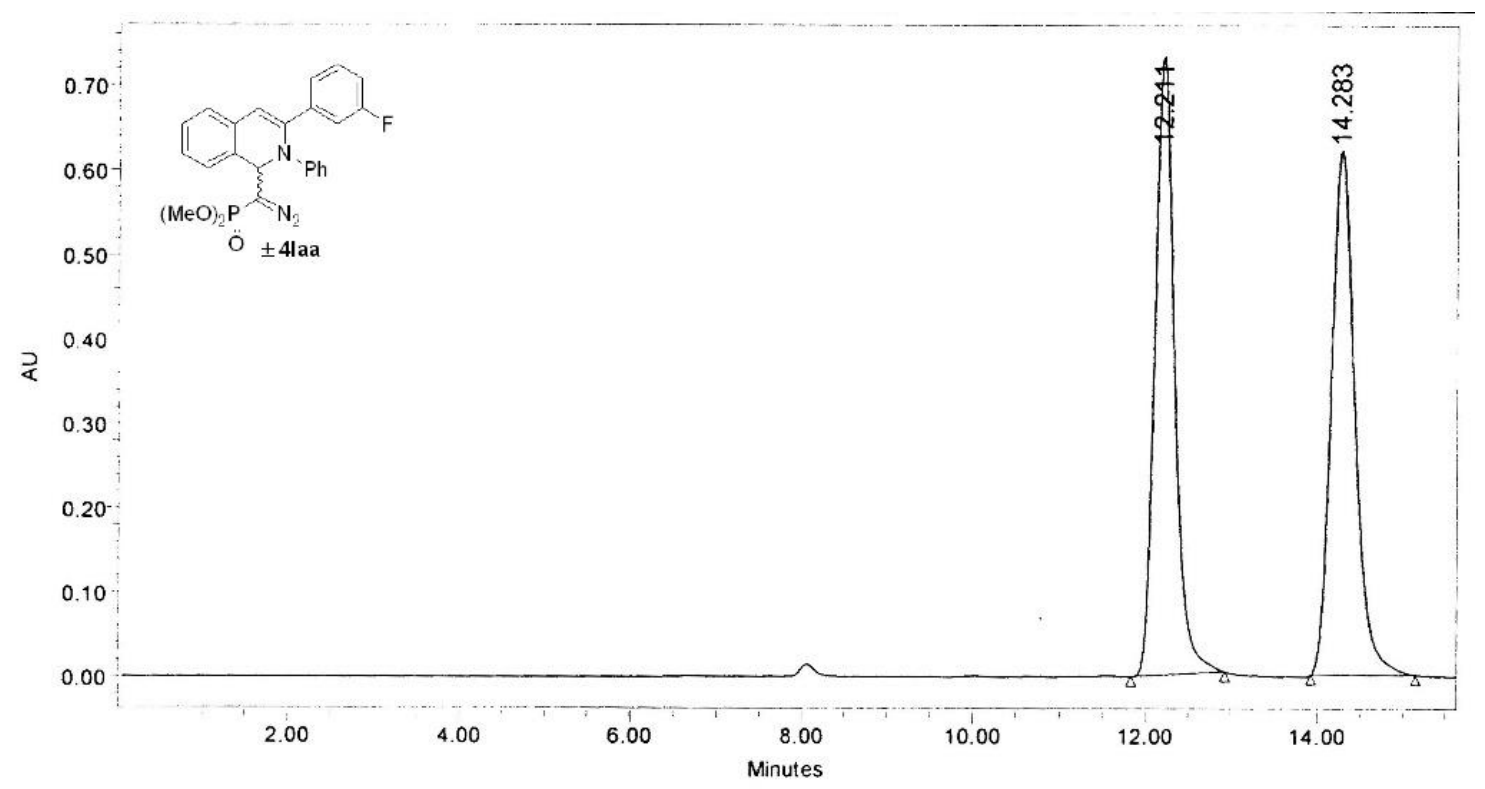

\begin{tabular}{|c|c|c|c|c|c|}
\hline & $\begin{array}{c}\text { RT } \\
(\mathrm{min})\end{array}$ & $\begin{array}{c}\text { Area } \\
\left(\mu \mathrm{V}^{*} \mathrm{sec}\right)\end{array}$ & $\%$ Area & $\begin{array}{c}\text { Height } \\
(\mu \mathrm{V})\end{array}$ & $\begin{array}{c}\% \\
\text { Height }\end{array}$ \\
\hline 1 & 12.211 & 11745055 & 50.00 & 732413 & 54.09 \\
\hline 2 & 14.283 & 11745022 & 50.00 & 621605 & 45.91 \\
\hline
\end{tabular}

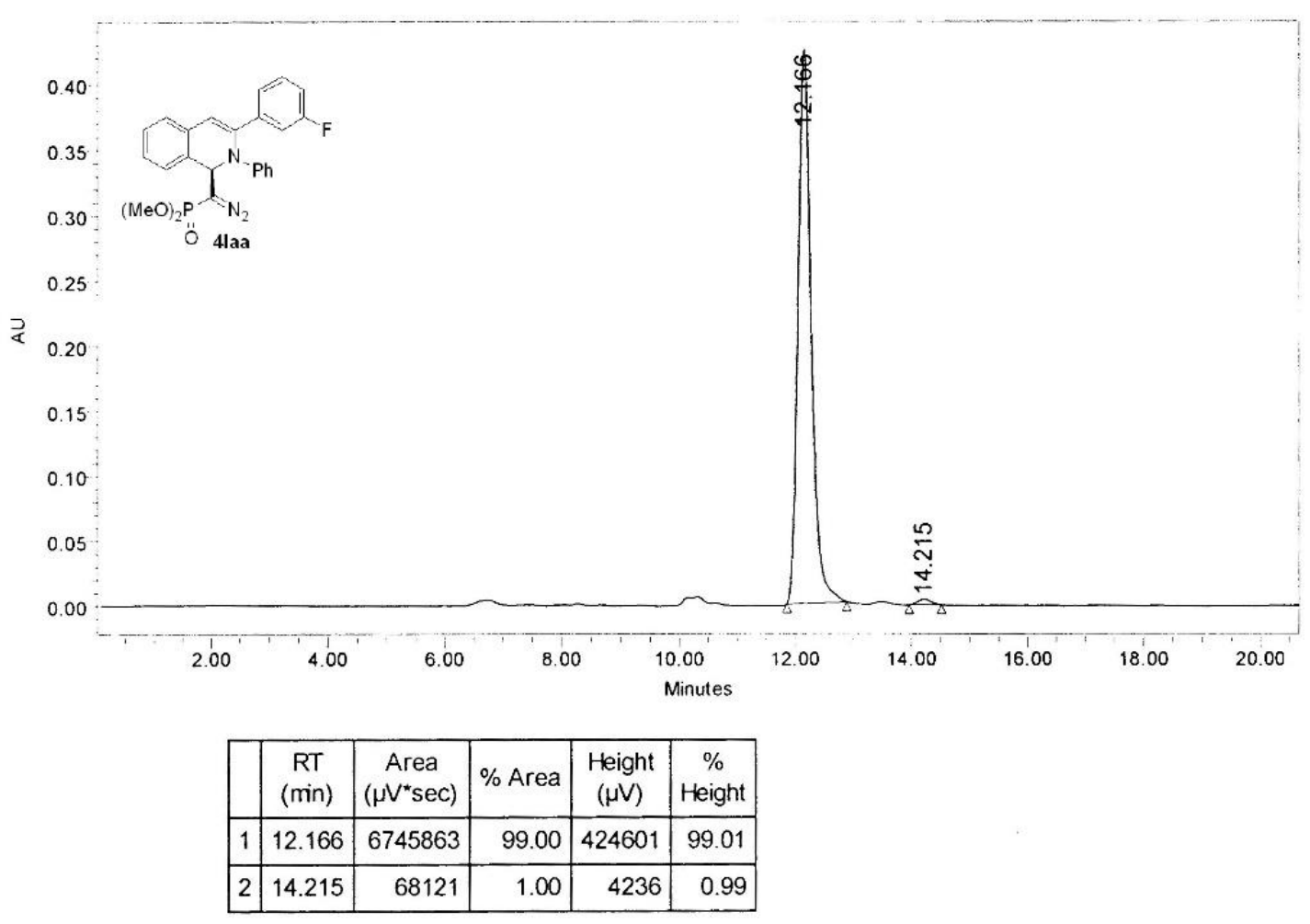




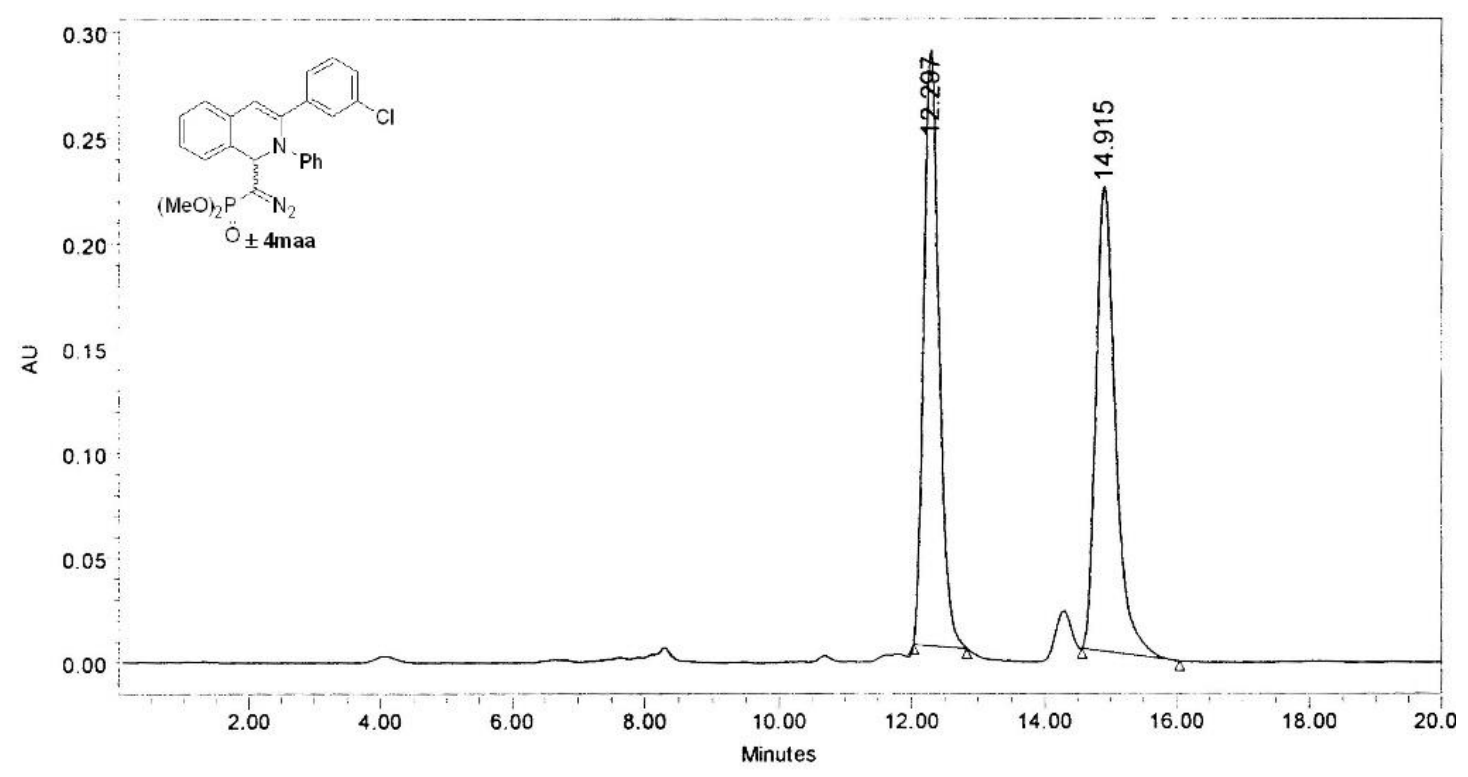

\begin{tabular}{|c|c|c|c|c|c|}
\hline & $\begin{array}{c}\mathrm{RT} \\
(\mathrm{min})\end{array}$ & $\begin{array}{c}\text { Area } \\
\left(\mu \mathrm{V}^{*} \mathrm{sec}\right)\end{array}$ & $\%$ Area & $\begin{array}{c}\text { Height } \\
(\mu \mathrm{V})\end{array}$ & $\begin{array}{c}\% \\
\text { Height }\end{array}$ \\
\hline 1 & 12.297 & 4554280 & 50.08 & 283808 & 56.11 \\
\hline 2 & 14.915 & 4540343 & 49.92 & 221965 & 43.89 \\
\hline
\end{tabular}

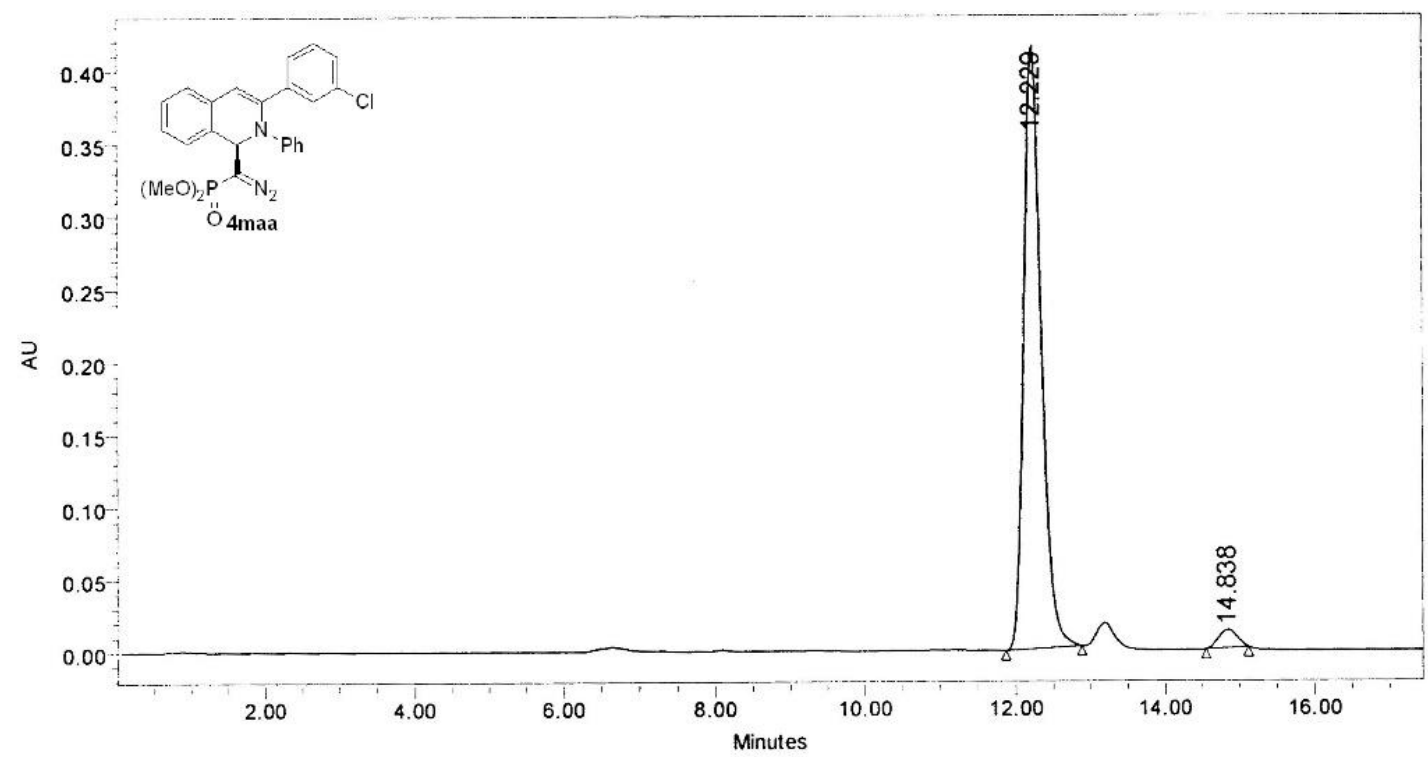

\begin{tabular}{|c|c|c|c|c|c|}
\hline & $\begin{array}{c}\text { RT } \\
(\mathrm{min})\end{array}$ & $\begin{array}{c}\text { Area } \\
\left(\mu \mathrm{V}^{*} \mathrm{sec}\right)\end{array}$ & $\%$ Area & $\begin{array}{c}\text { Height } \\
(\mu \mathrm{V})\end{array}$ & $\begin{array}{c}\% \\
\text { Height }\end{array}$ \\
\hline 1 & 12.229 & 6693186 & 96.92 & 414990 & 97.09 \\
\hline 2 & 14.838 & 212443 & 3.08 & 12439 & 2.91 \\
\hline
\end{tabular}




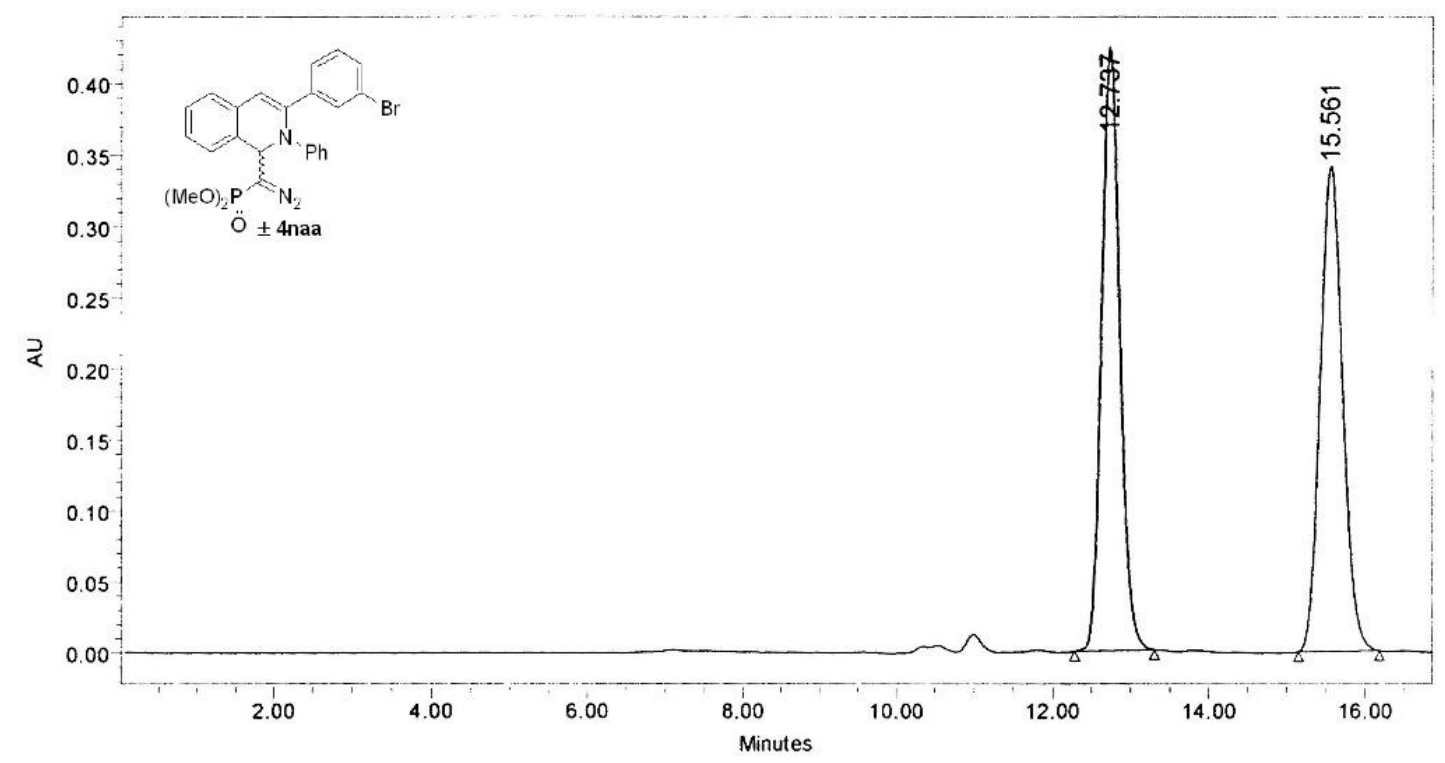

\begin{tabular}{|c|c|c|c|c|c|}
\hline & $\begin{array}{c}\text { RT } \\
(\mathrm{min})\end{array}$ & $\begin{array}{c}\text { Area } \\
\left(\mu \mathrm{V}^{\star} \mathrm{sec}\right)\end{array}$ & $\%$ Area & $\begin{array}{c}\text { Height } \\
(\mu \mathrm{V})\end{array}$ & $\begin{array}{c}\% \\
\text { Height }\end{array}$ \\
\hline 1 & 12.737 & 6818518 & 50.00 & 423953 & 55.40 \\
\hline 2 & 15.561 & 6819204 & 50.00 & 341363 & 44.60 \\
\hline
\end{tabular}

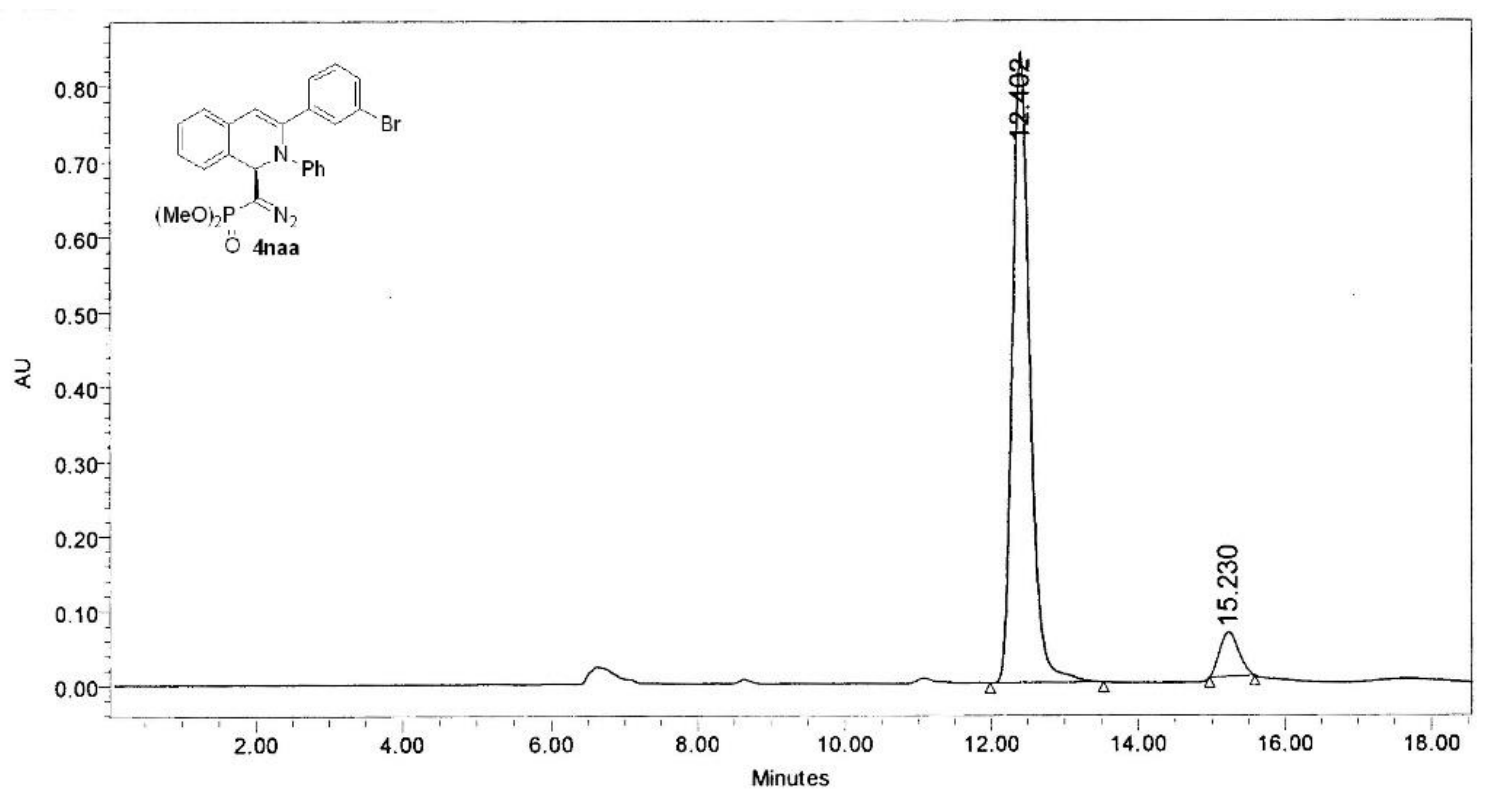

\begin{tabular}{|c|c|c|r|r|r|}
\hline & $\begin{array}{c}\mathrm{RT} \\
(\mathrm{min})\end{array}$ & $\begin{array}{c}\text { Area } \\
\left(\mu \mathrm{V}^{\star} \mathrm{sec}\right)\end{array}$ & $\%$ Area & $\begin{array}{c}\text { Height } \\
(\mu \mathrm{V})\end{array}$ & $\begin{array}{c}\% \\
\text { Height }\end{array}$ \\
\hline 1 & 12.402 & 14056165 & 92.95 & 841875 & 93.40 \\
\hline 2 & 15.230 & 1066051 & 7.05 & 59479 & 6.60 \\
\hline
\end{tabular}




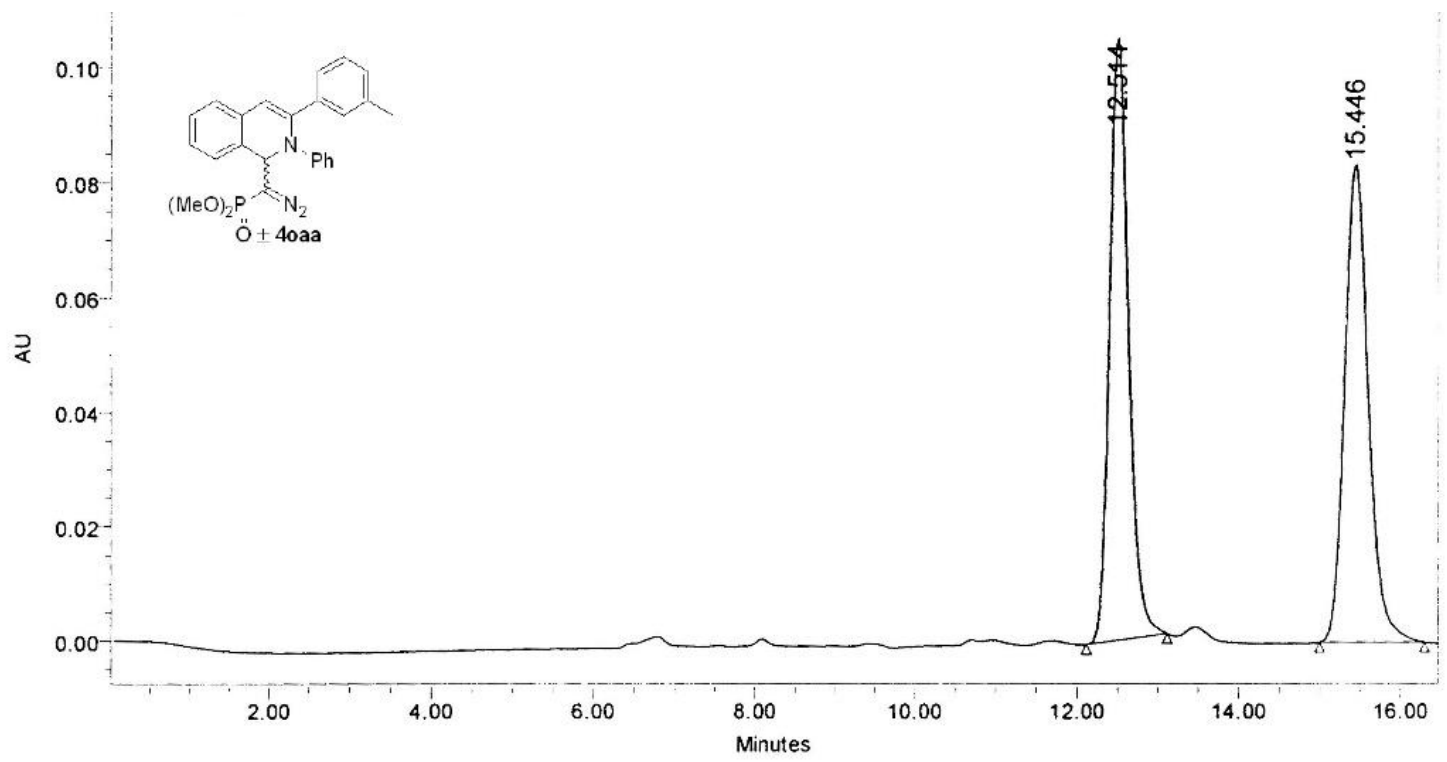

\begin{tabular}{|c|c|c|c|c|c|}
\hline & $\begin{array}{c}\mathrm{RT} \\
(\mathrm{min})\end{array}$ & $\begin{array}{c}\text { Area } \\
(\mu \mathrm{V} * \mathrm{sec})\end{array}$ & $\%$ Area & $\begin{array}{c}\text { Height } \\
(\mu \mathrm{V})\end{array}$ & $\begin{array}{c}\% \\
\text { Height }\end{array}$ \\
\hline 1 & 12.514 & 1701750 & 49.99 & 104892 & 55.69 \\
\hline 2 & 15.446 & 1702592 & 50.01 & 83456 & 44.31 \\
\hline
\end{tabular}

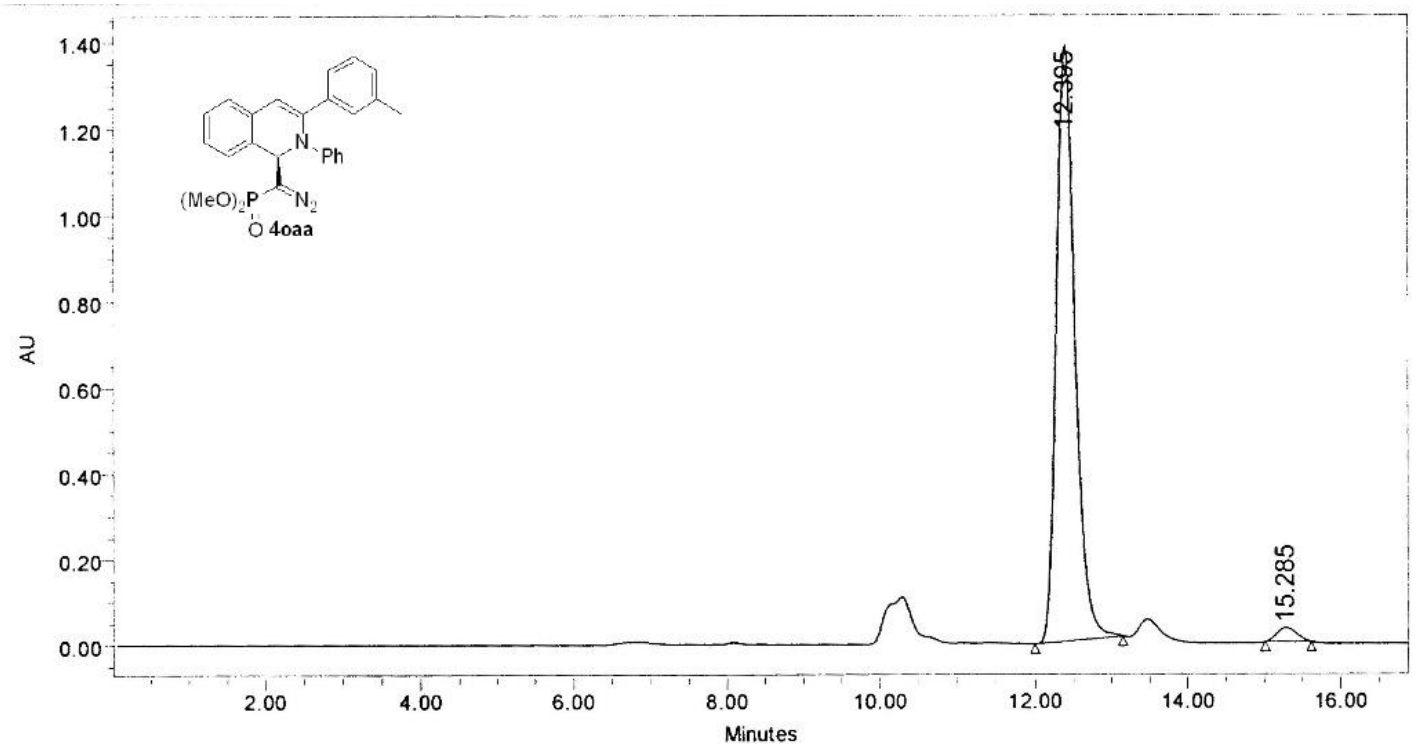

\begin{tabular}{|c|c|r|r|r|r|}
\hline & $\begin{array}{c}\mathrm{RT} \\
(\mathrm{min})\end{array}$ & $\begin{array}{c}\text { Area } \\
\left(\mu \mathrm{V}^{*} \mathrm{sec}\right)\end{array}$ & $\%$ Area & $\begin{array}{c}\text { Height } \\
(\mu \mathrm{V})\end{array}$ & $\begin{array}{c}\% \\
\text { Height }\end{array}$ \\
\hline 1 & 12.395 & 23304466 & 97.55 & 1384830 & 97.70 \\
\hline 2 & 15.285 & 584856 & 2.45 & 32562 & 2.30 \\
\hline
\end{tabular}




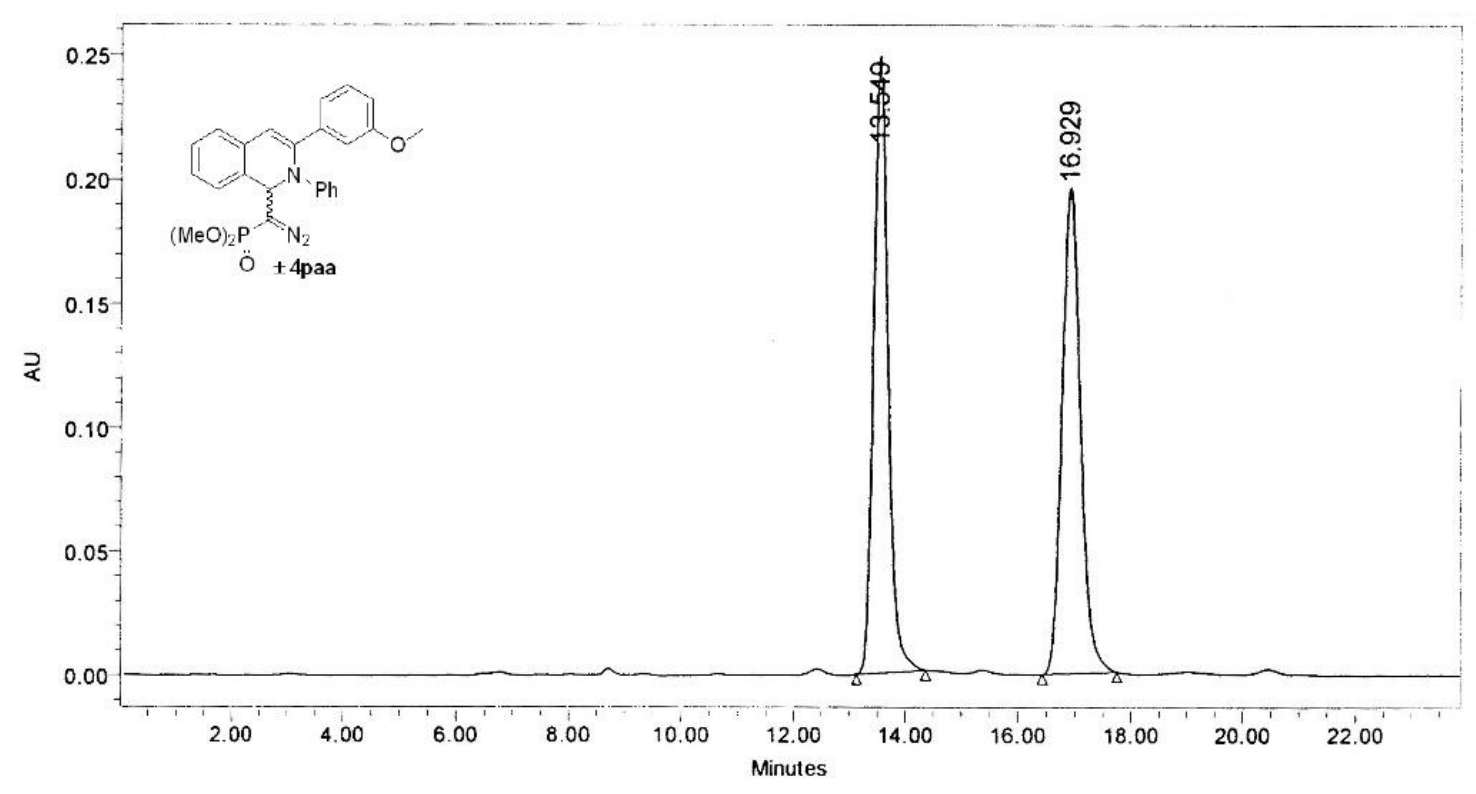

\begin{tabular}{|c|c|c|c|c|c|}
\hline & $\begin{array}{c}\text { RT } \\
(\mathrm{min})\end{array}$ & $\begin{array}{c}\text { Area } \\
\left(\mu \mathrm{V}^{*} \mathrm{sec}\right)\end{array}$ & $\%$ Area & $\begin{array}{c}\text { Height } \\
(\mu \mathrm{V})\end{array}$ & $\begin{array}{c}\% \\
\text { Height }\end{array}$ \\
\hline 1 & 13.549 & 4436771 & 50.01 & 248511 & 55.84 \\
\hline 2 & 16.929 & 4435546 & 49.99 & 196526 & 44.16 \\
\hline
\end{tabular}

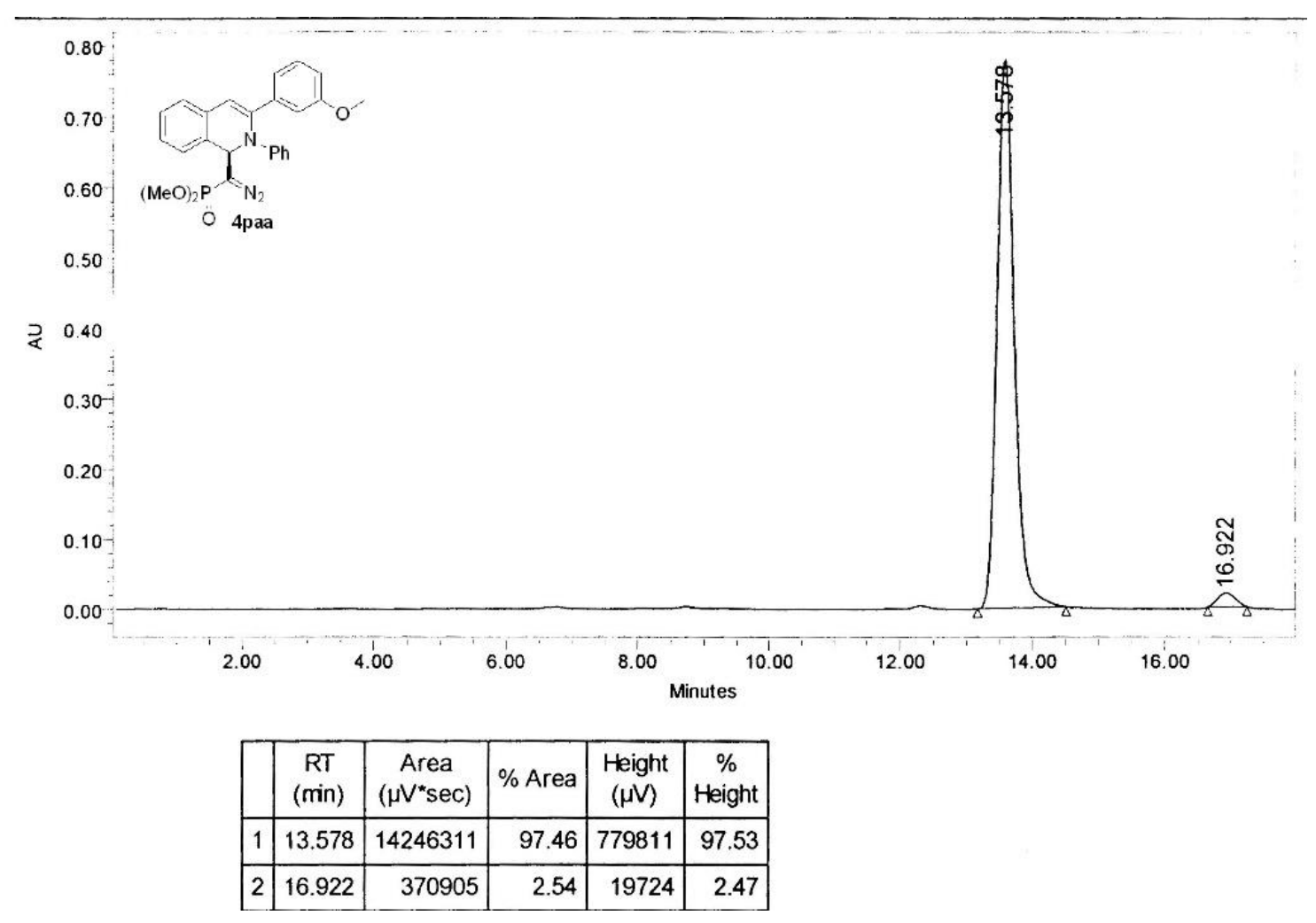




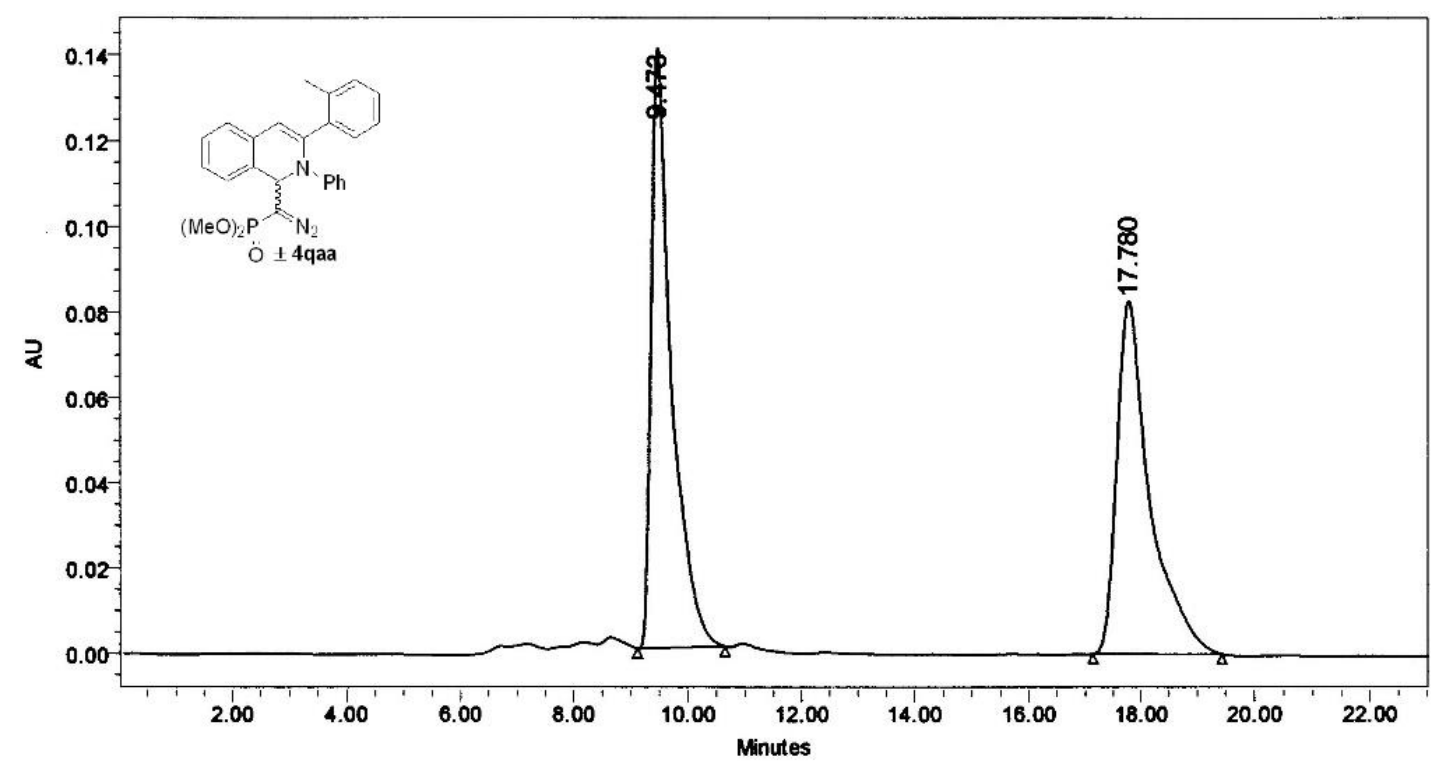

\begin{tabular}{|c|c|c|c|c|c|}
\hline & $\begin{array}{c}\text { RT } \\
(\min )\end{array}$ & $\begin{array}{c}\text { Area } \\
(\mu \mathrm{V} * \mathrm{sec})\end{array}$ & $\%$ Area & $\begin{array}{c}\text { Height } \\
(\mu \mathrm{V})\end{array}$ & $\begin{array}{c}\% \\
\text { Height }\end{array}$ \\
\hline 1 & 9.473 & 3453221 & 50.45 & 140539 & 62.90 \\
\hline 2 & 17.780 & 3391914 & 49.55 & 82910 & 37.10 \\
\hline
\end{tabular}

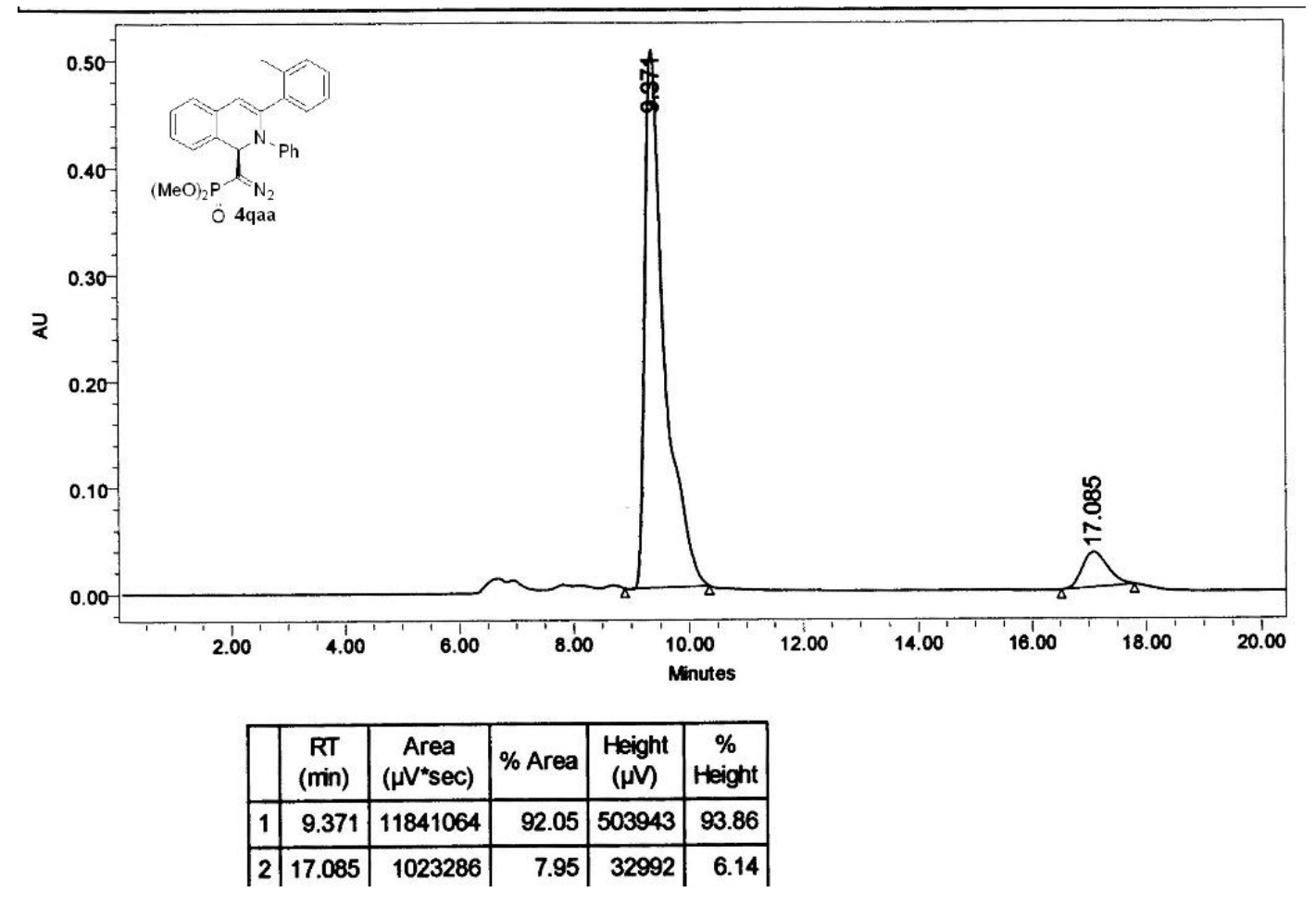




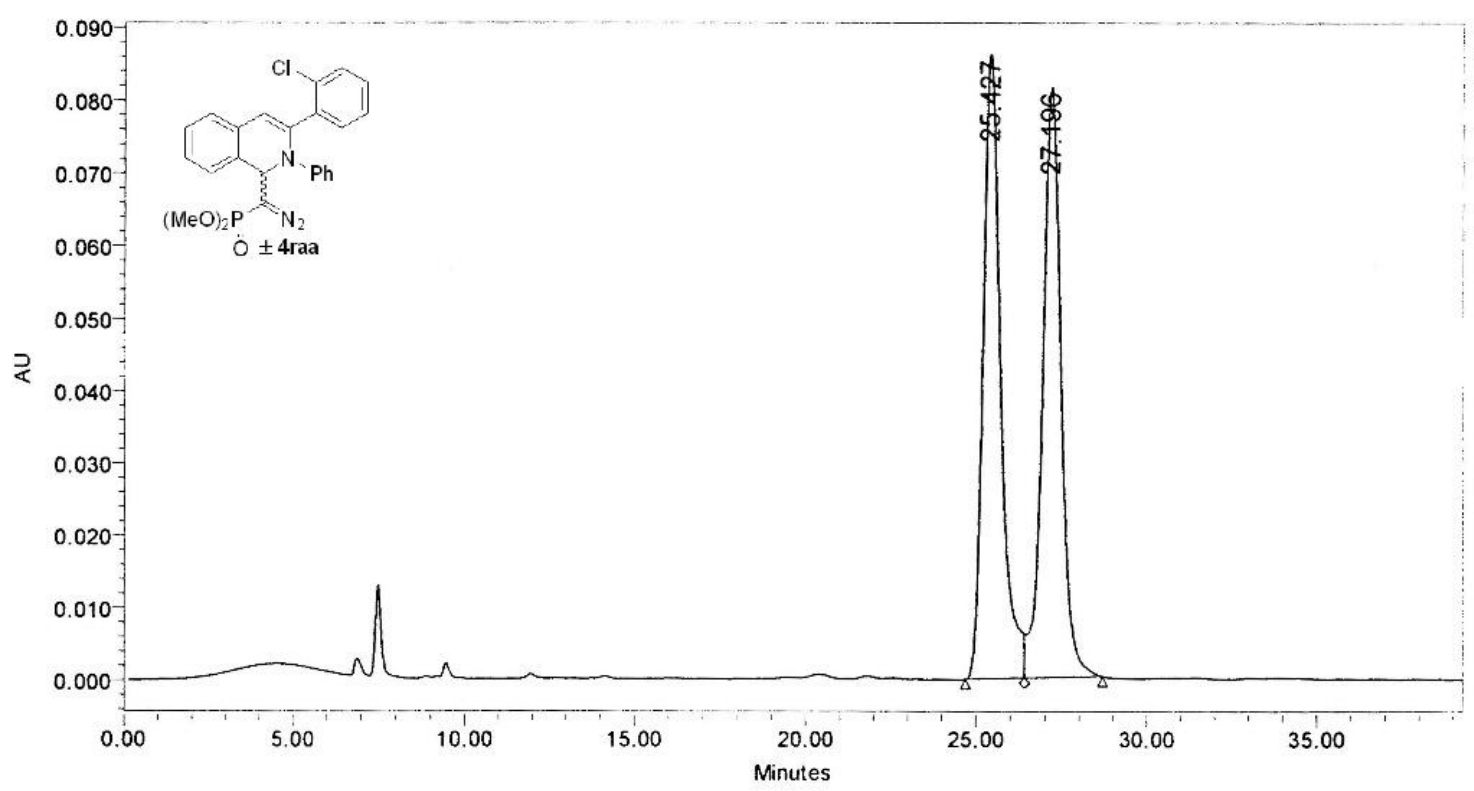

\begin{tabular}{|c|c|c|c|c|c|}
\hline & $\begin{array}{c}\mathrm{RT} \\
(\mathrm{min})\end{array}$ & $\begin{array}{c}\text { Area } \\
\left(\mu \mathrm{V}^{*} \mathrm{sec}\right)\end{array}$ & $\%$ Area & $\begin{array}{c}\text { Height } \\
(\mu \mathrm{V})\end{array}$ & $\begin{array}{c}\% \\
\text { Height }\end{array}$ \\
\hline 1 & 25.427 & 3044110 & 49.90 & 86148 & 51.39 \\
\hline 2 & 27.196 & 3056548 & 50.10 & 81491 & 48.61 \\
\hline
\end{tabular}

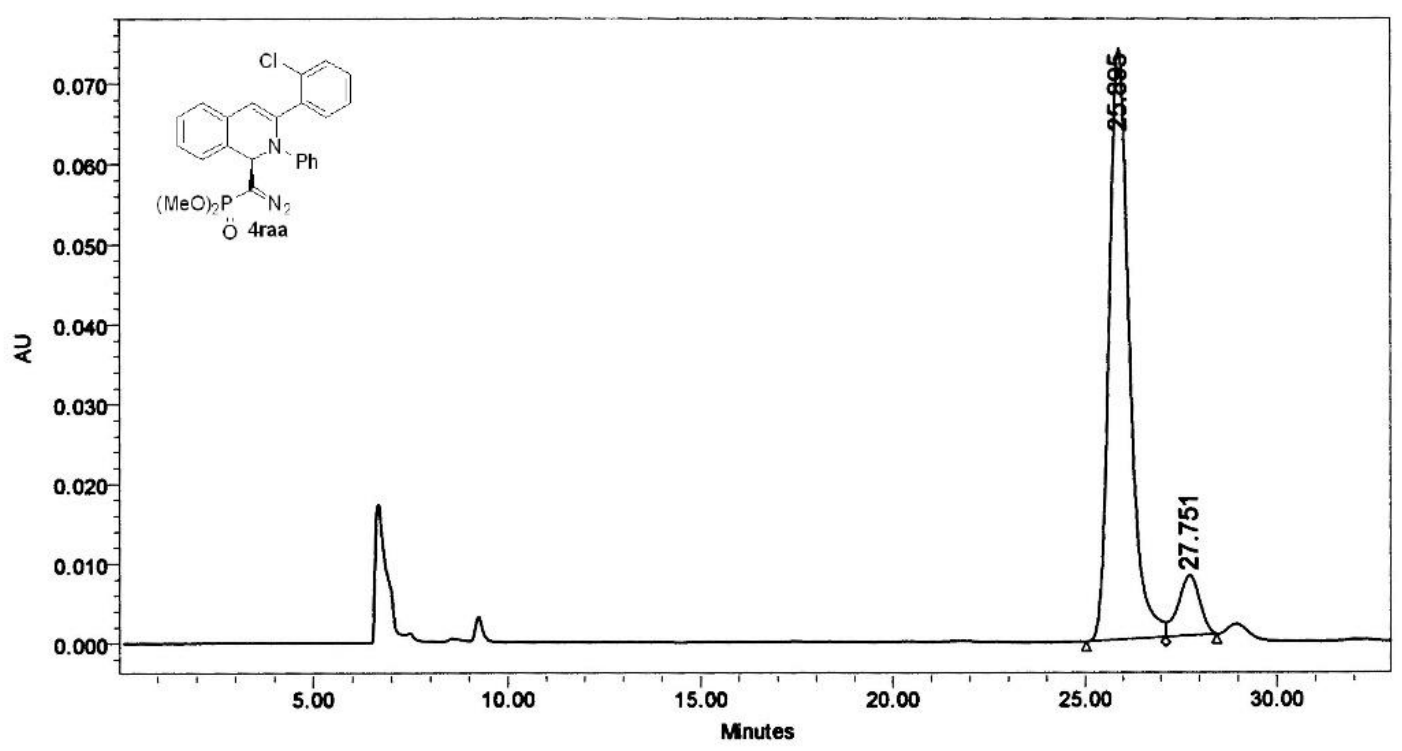

\begin{tabular}{|c|c|c|c|c|c|}
\hline & $\begin{array}{c}\mathrm{RT} \\
(\mathrm{min})\end{array}$ & $\begin{array}{c}\text { Area } \\
\left(\mu \mathrm{V}^{*} \mathrm{sec}\right)\end{array}$ & $\%$ Area & $\begin{array}{c}\text { Height } \\
(\mu \mathrm{V})\end{array}$ & $\begin{array}{c}\% \\
\text { Height }\end{array}$ \\
\hline 1 & 25.895 & 2737581 & 90.19 & 73984 & 90.77 \\
\hline 2 & 27.751 & 297790 & 9.81 & 7520 & 9.23 \\
\hline
\end{tabular}




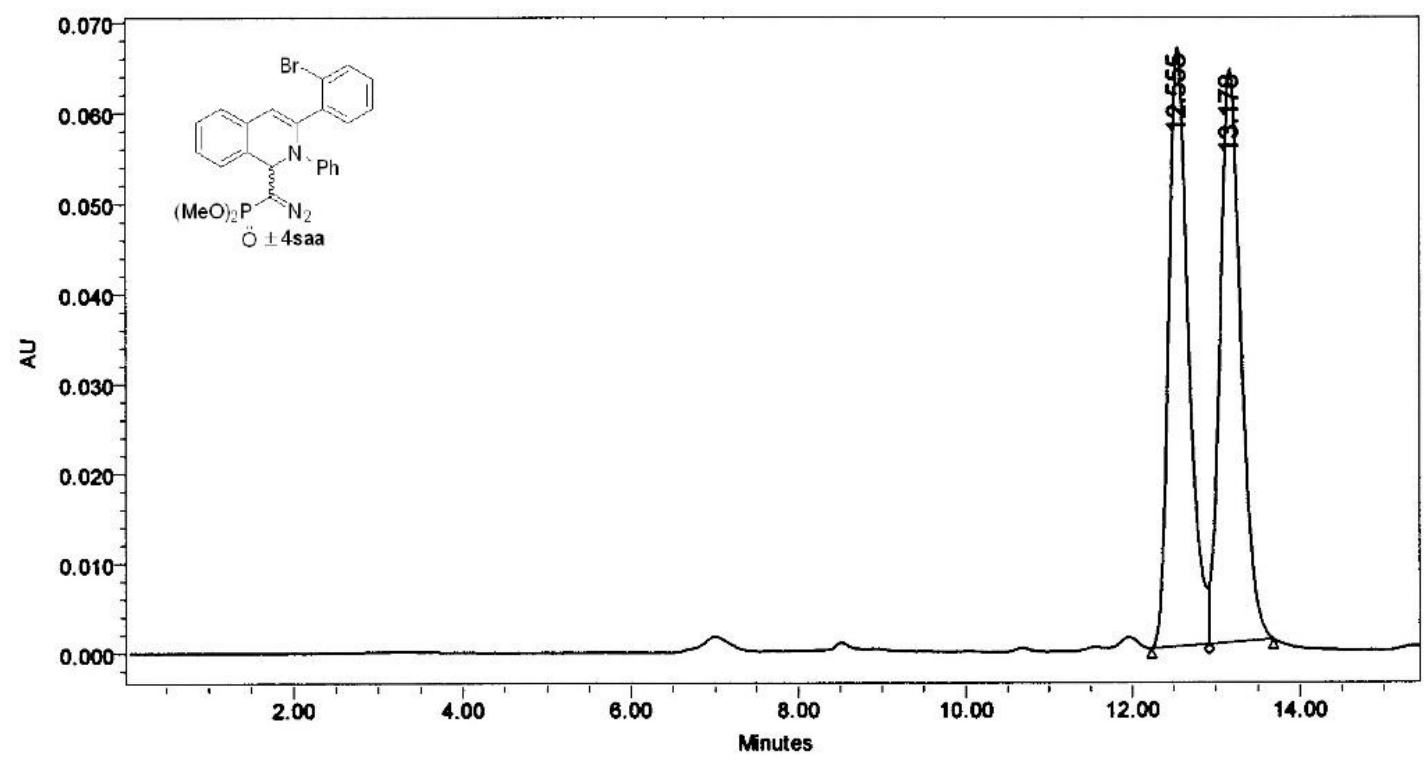

\begin{tabular}{|c|c|c|c|c|c|}
\hline & $\begin{array}{c}\text { RT } \\
(\mathrm{min})\end{array}$ & $\begin{array}{c}\text { Area } \\
\left(\mu \mathrm{N}^{*} \mathrm{sec}\right)\end{array}$ & $\%$ Area & $\begin{array}{c}\text { Height } \\
(\mu \mathrm{V})\end{array}$ & $\begin{array}{c}\% \\
\text { Height }\end{array}$ \\
\hline 1 & 12.555 & 1101077 & 49.87 & 66607 & 51.07 \\
\hline 2 & 13.178 & 1106938 & 50.13 & 63823 & 48.93 \\
\hline
\end{tabular}

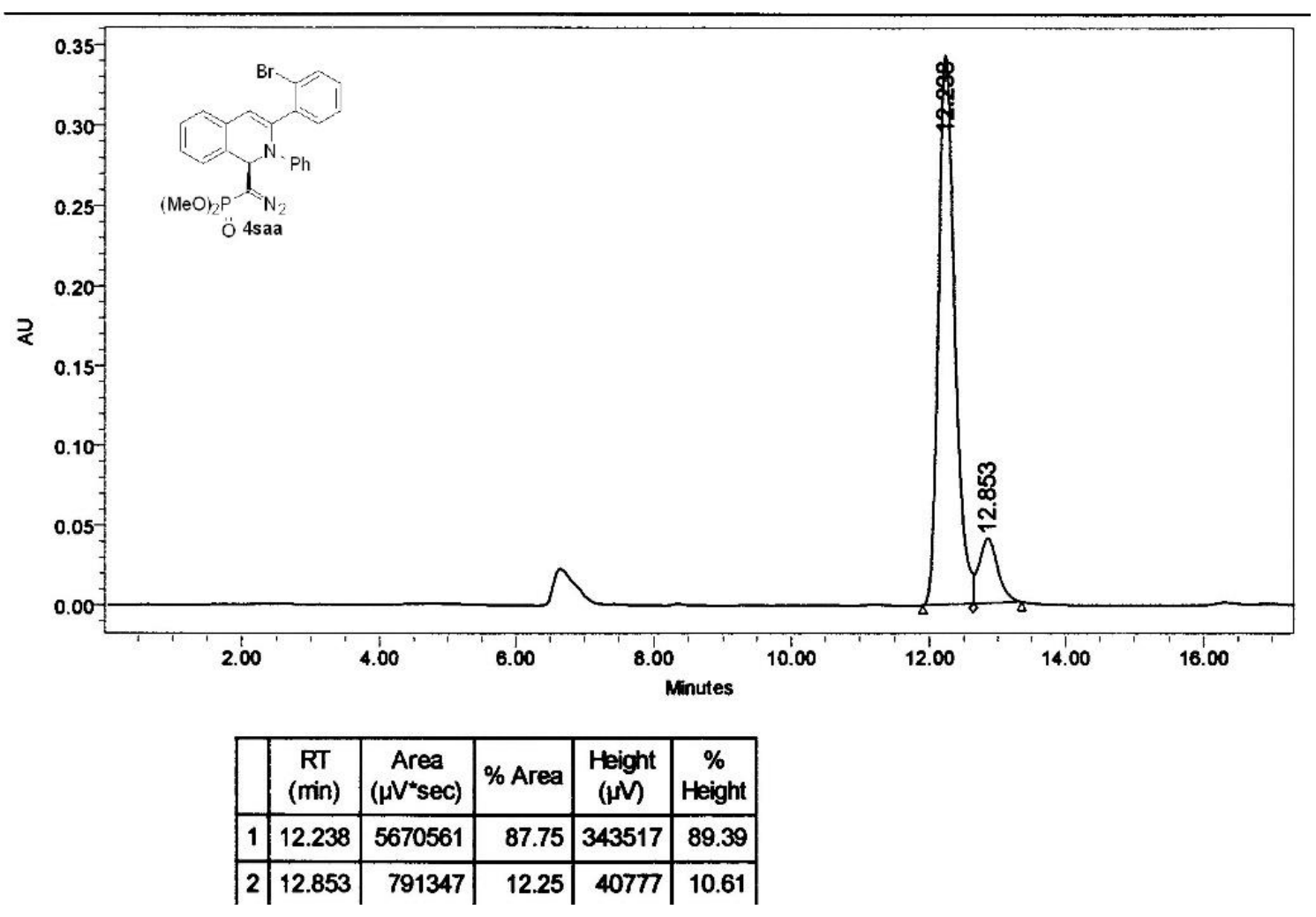




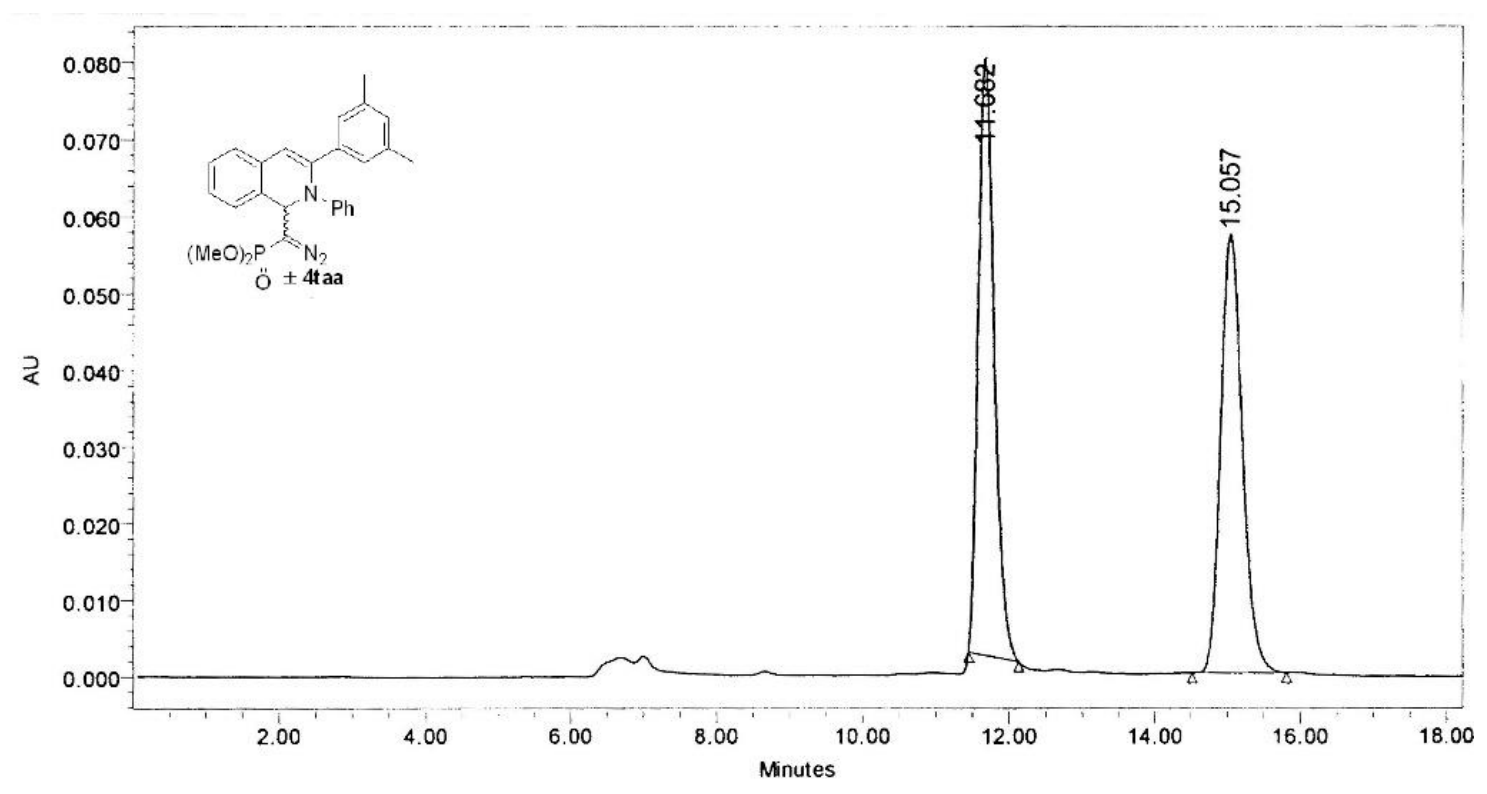

\begin{tabular}{|c|c|c|c|c|c|}
\hline & $\begin{array}{c}\text { RT } \\
(\mathrm{min})\end{array}$ & $\begin{array}{c}\text { Area } \\
\left(\mu \mathrm{V}^{*} \mathrm{sec}\right)\end{array}$ & $\%$ Area & $\begin{array}{c}\text { Height } \\
(\mu \mathrm{V})\end{array}$ & $\begin{array}{c}\% \\
\text { Height }\end{array}$ \\
\hline 1 & 11.682 & 1188379 & 50.06 & 77860 & 57.60 \\
\hline 2 & 15.057 & 1185560 & 49.94 & 57307 & 42.40 \\
\hline
\end{tabular}

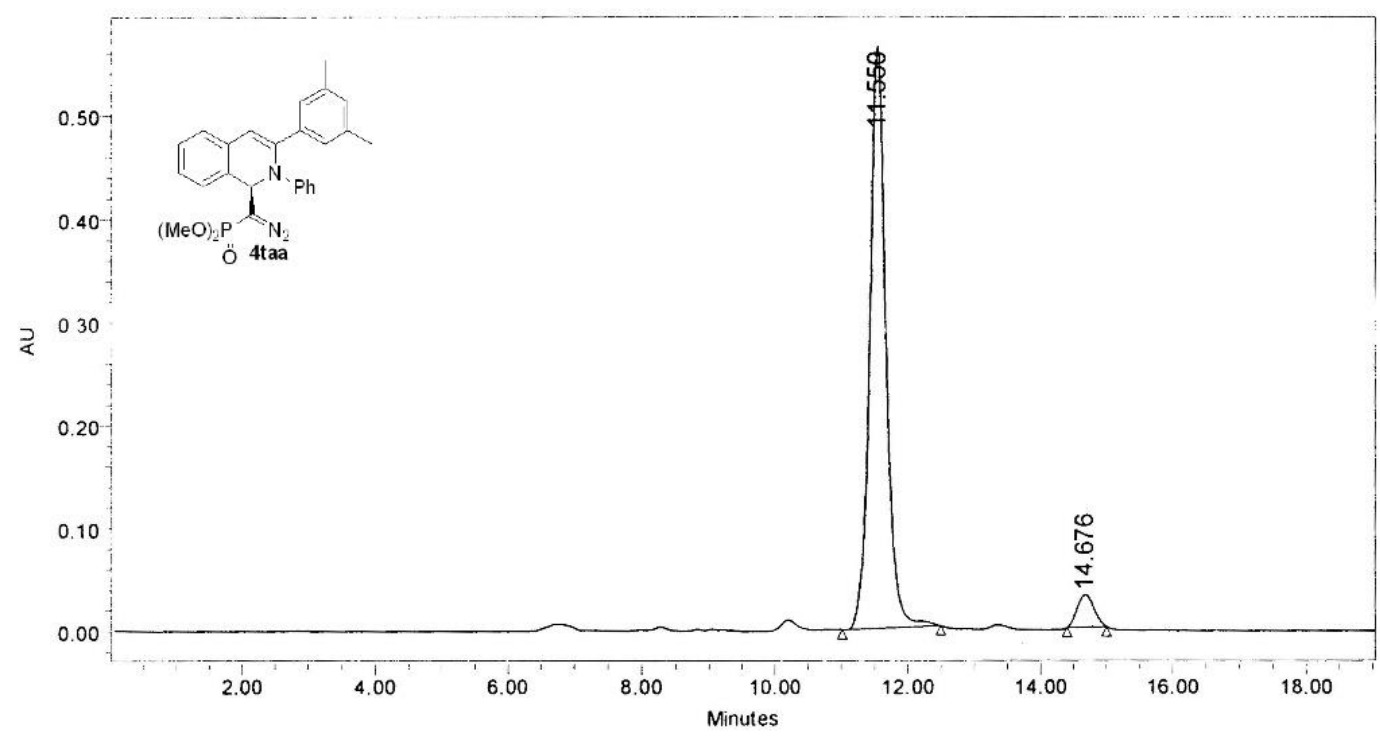

\begin{tabular}{|c|c|c|r|r|r|}
\hline & $\begin{array}{c}\mathrm{RT} \\
(\mathrm{min})\end{array}$ & $\begin{array}{c}\text { Area } \\
\left(\mu \mathrm{V}^{*} \mathrm{sec}\right)\end{array}$ & $\%$ Area & $\begin{array}{c}\text { Height } \\
(\mu \mathrm{V})\end{array}$ & $\begin{array}{c}\% \\
\text { Height }\end{array}$ \\
\hline 1 & 11.550 & 9572800 & 94.48 & 563950 & 94.66 \\
\hline 2 & 14.676 & 559238 & 5.52 & 31795 & 5.34 \\
\hline
\end{tabular}




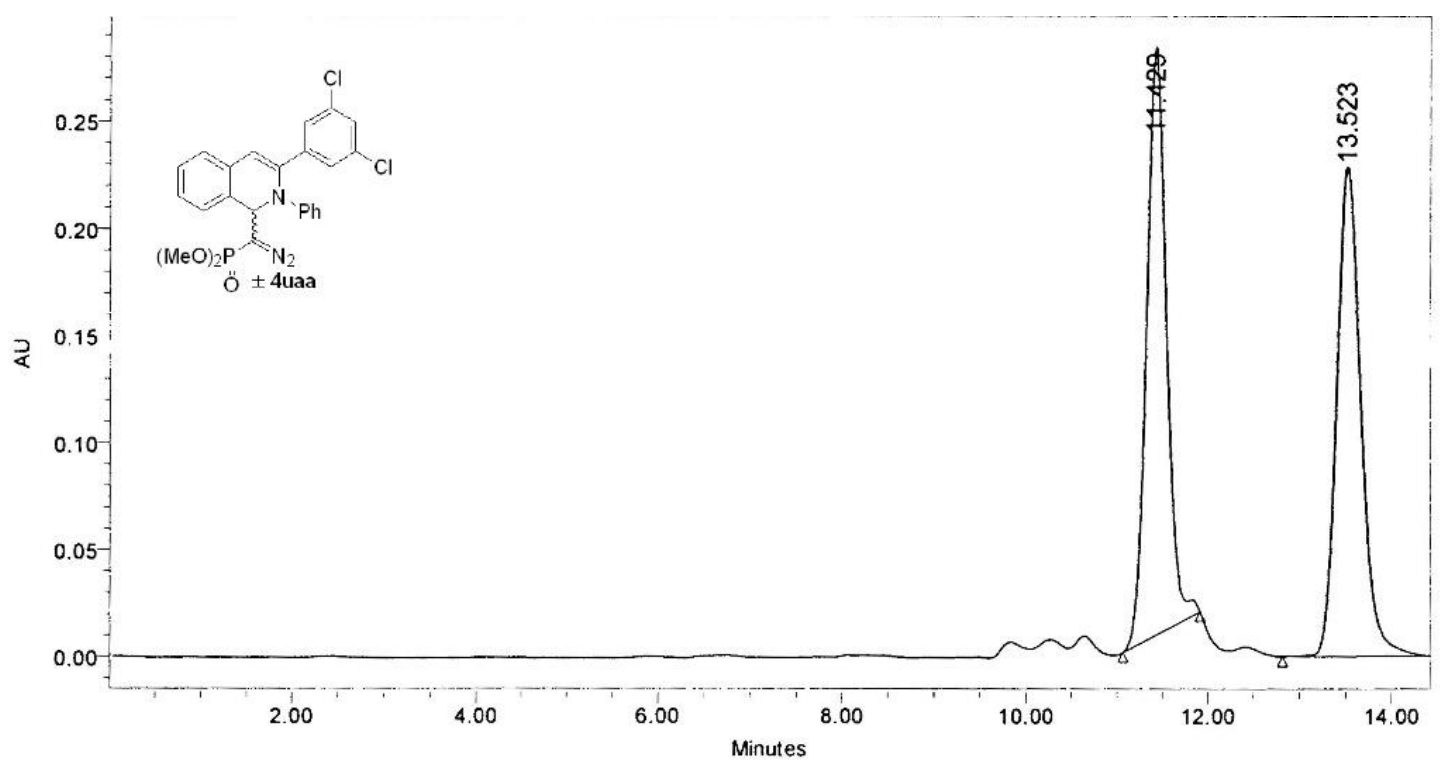

\begin{tabular}{|c|c|c|c|c|c|}
\hline & $\begin{array}{c}\mathrm{RT} \\
(\mathrm{min})\end{array}$ & $\begin{array}{c}\text { Area } \\
\left(\mu \mathrm{V}^{*} \mathrm{sec}\right)\end{array}$ & $\%$ Area & $\begin{array}{c}\text { Height } \\
(\mu \mathrm{V})\end{array}$ & $\begin{array}{c}\% \\
\text { Height }\end{array}$ \\
\hline 1 & 11.429 & 4175677 & 50.04 & 274795 & 54.53 \\
\hline 2 & 13.523 & 4168415 & 49.96 & 229107 & 45.47 \\
\hline
\end{tabular}

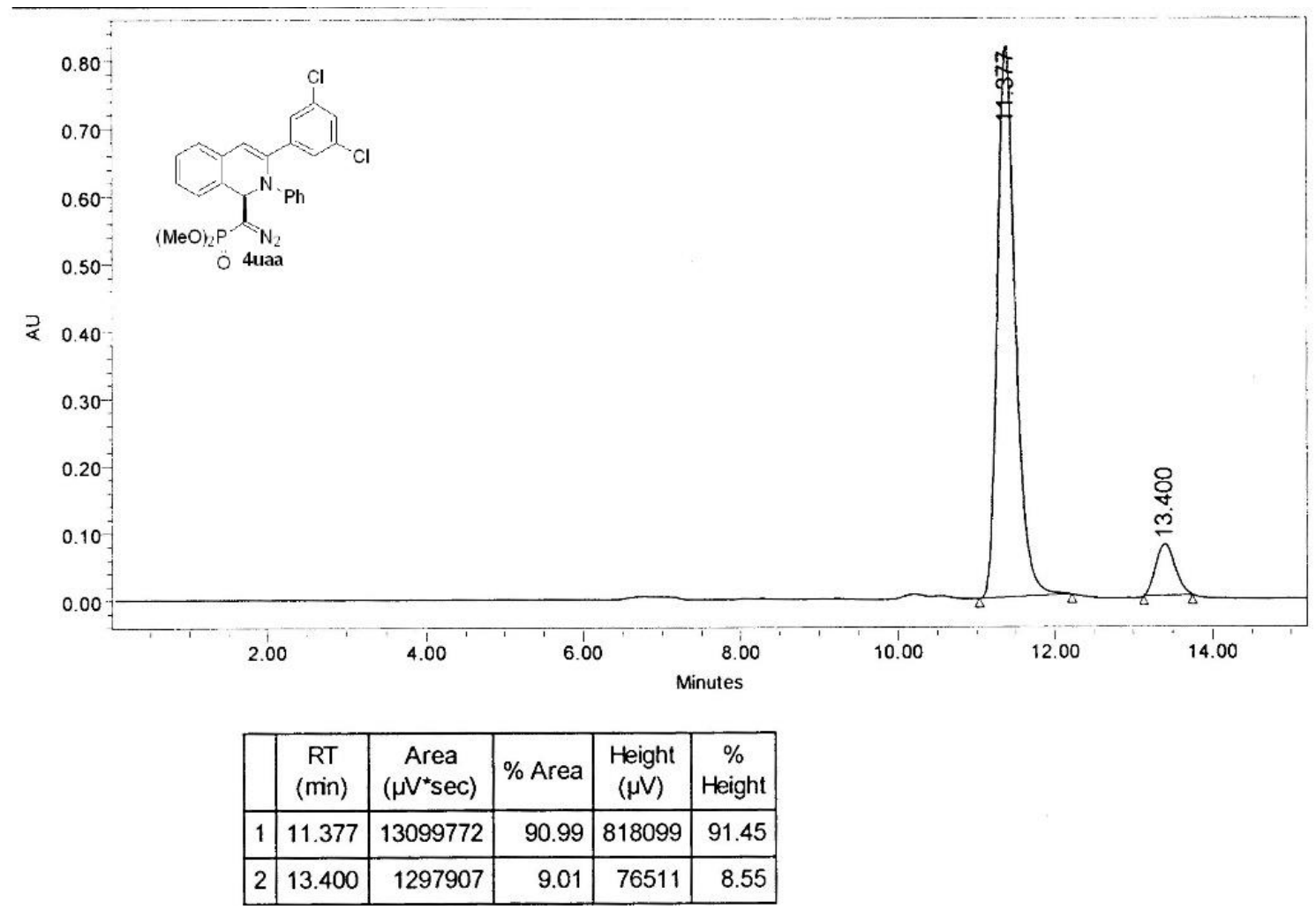




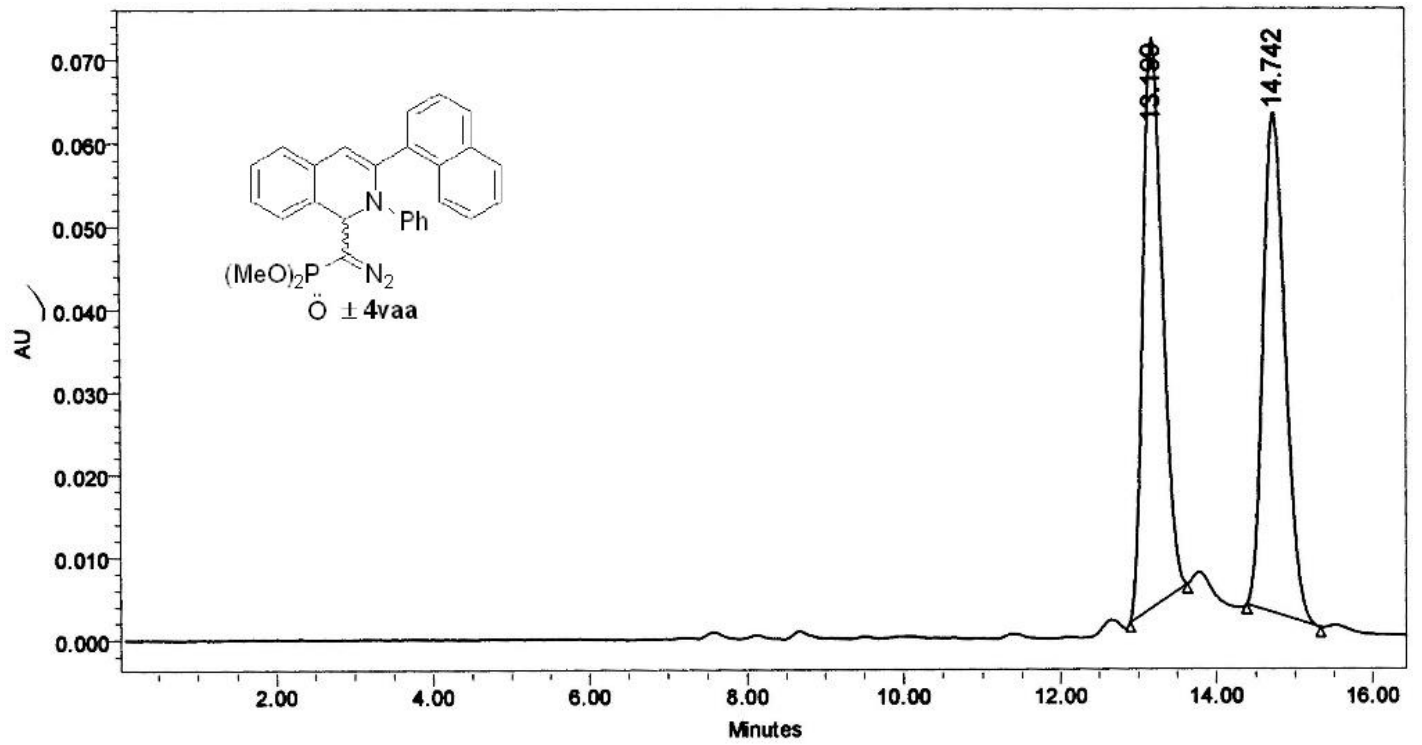

\begin{tabular}{|c|c|c|c|c|c|}
\hline & $\begin{array}{c}\mathrm{RT} \\
(\mathrm{min})\end{array}$ & $\begin{array}{c}\text { Area } \\
\left(\mu \mathrm{V}^{*} \mathrm{sec}\right)\end{array}$ & $\%$ Area & $\begin{array}{c}\text { Height } \\
(\mu \mathrm{V})\end{array}$ & $\begin{array}{c}\% \\
\text { Height }\end{array}$ \\
\hline 1 & 13.199 & 1210349 & 50.49 & 68507 & 53.14 \\
\hline 2 & 14.742 & 1186840 & 49.51 & 60416 & 46.86 \\
\hline
\end{tabular}

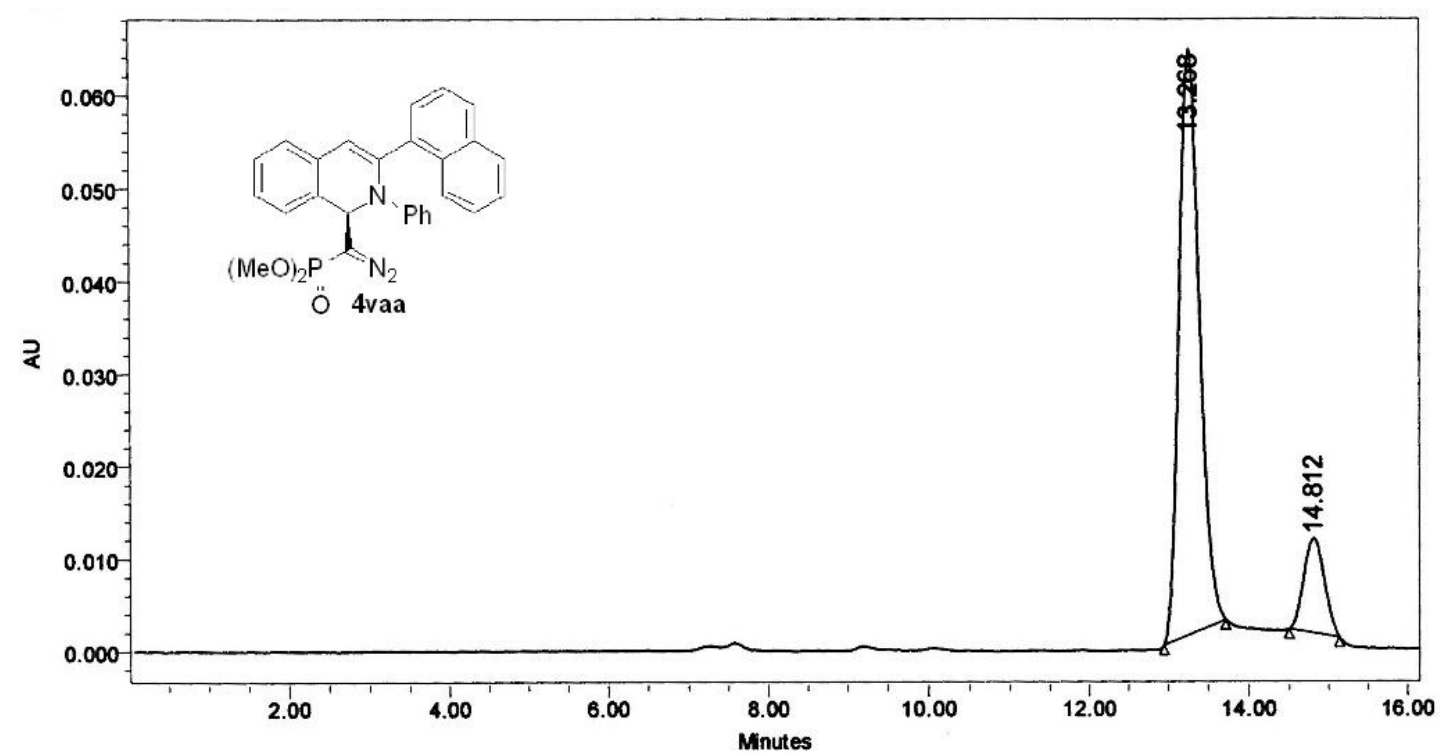

\begin{tabular}{|c|c|c|c|c|c|}
\hline & $\begin{array}{c}\mathrm{RT} \\
(\mathrm{min})\end{array}$ & $\begin{array}{c}\text { Area } \\
(\mu \mathrm{V} * \mathrm{sec})\end{array}$ & $\%$ Area & $\begin{array}{c}\text { Height } \\
(\mu \mathrm{V})\end{array}$ & $\begin{array}{c}\% \\
\text { Height }\end{array}$ \\
\hline 1 & 13.268 & 1125374 & 85.88 & 63150 & 86.07 \\
\hline 2 & 14.812 & 184997 & 14.12 & 10220 & 13.93 \\
\hline
\end{tabular}




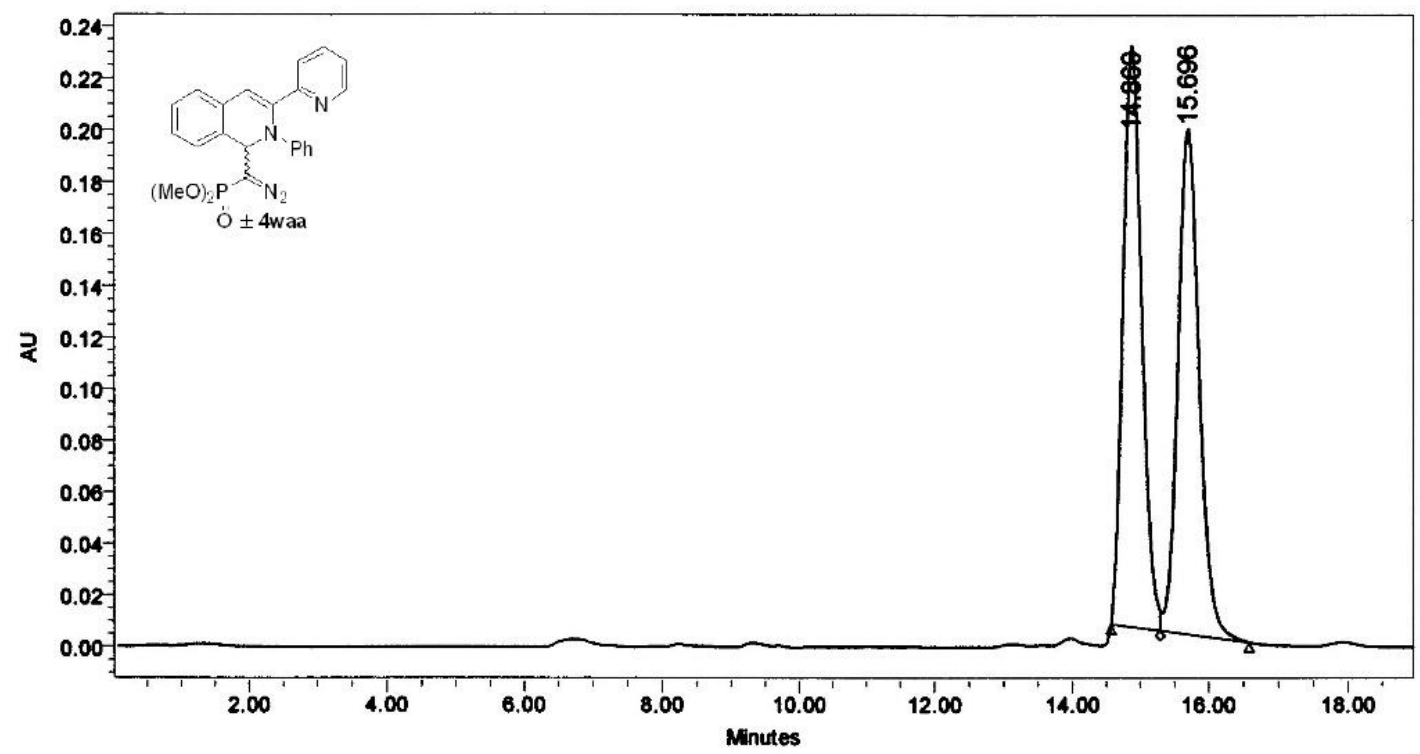

\begin{tabular}{|c|c|c|c|c|c|}
\hline & $\begin{array}{c}\text { RT } \\
(\mathrm{min})\end{array}$ & $\begin{array}{c}\text { Area } \\
(\mu \mathrm{V} \text { "sec })\end{array}$ & $\%$ Area & $\begin{array}{c}\text { Height } \\
(\mu \mathrm{V})\end{array}$ & $\begin{array}{c}\% \\
\text { Height }\end{array}$ \\
\hline 1 & 14.869 & 4346633 & 50.58 & 225274 & 53.45 \\
\hline 2 & 15.696 & 4246186 & 49.42 & 196156 & 46.55 \\
\hline
\end{tabular}

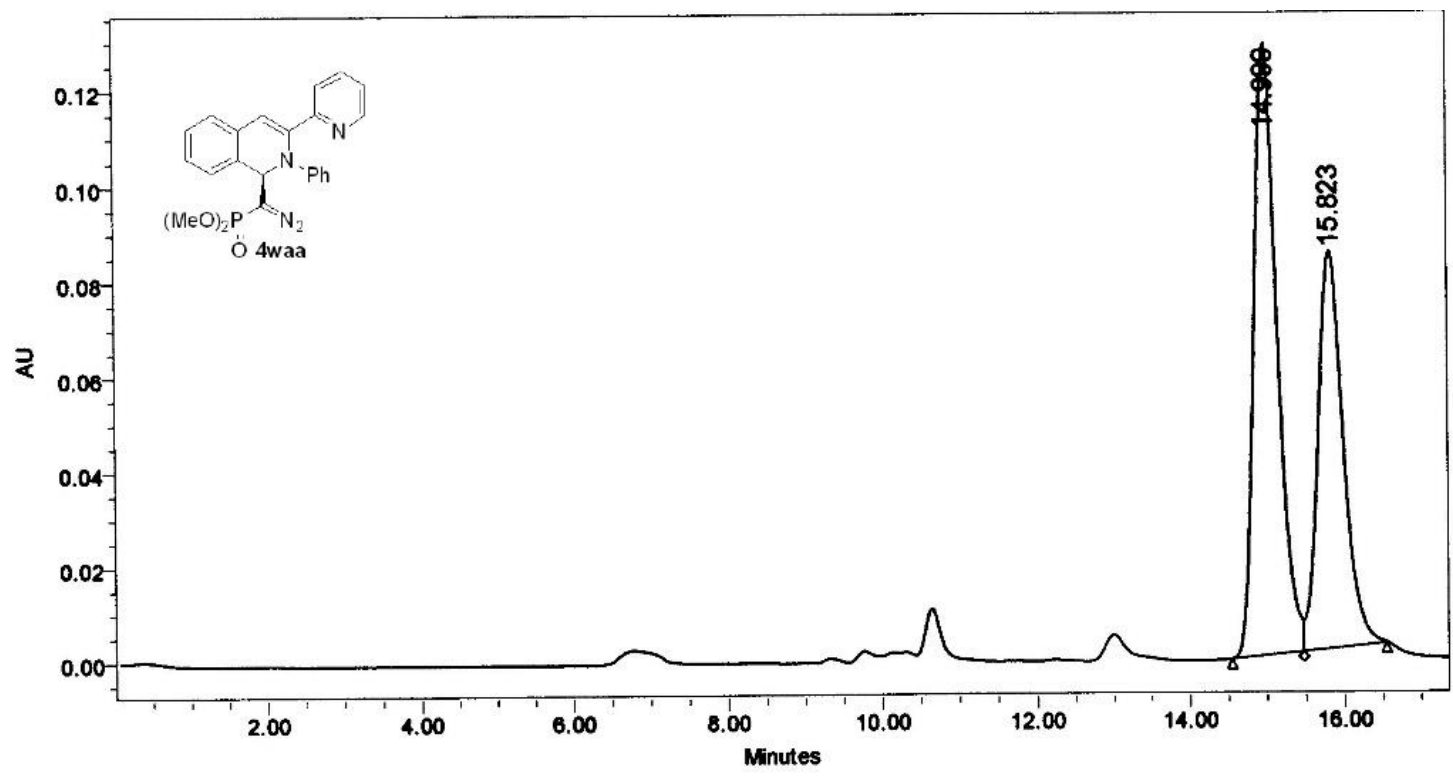

\begin{tabular}{|c|c|c|c|c|c|}
\hline & $\begin{array}{c}\mathrm{RT} \\
(\mathrm{min})\end{array}$ & $\begin{array}{c}\text { Area } \\
(\mu \mathrm{V} * \mathrm{sec})\end{array}$ & $\%$ Area & $\begin{array}{c}\text { Height } \\
(\mu \mathrm{V})\end{array}$ & $\begin{array}{c}\% \\
\text { Height }\end{array}$ \\
\hline 1 & 14.999 & 2662180 & 58.50 & 128657 & 60.59 \\
\hline 2 & 15.823 & 1888533 & 41.50 & 83687 & 39.41 \\
\hline
\end{tabular}




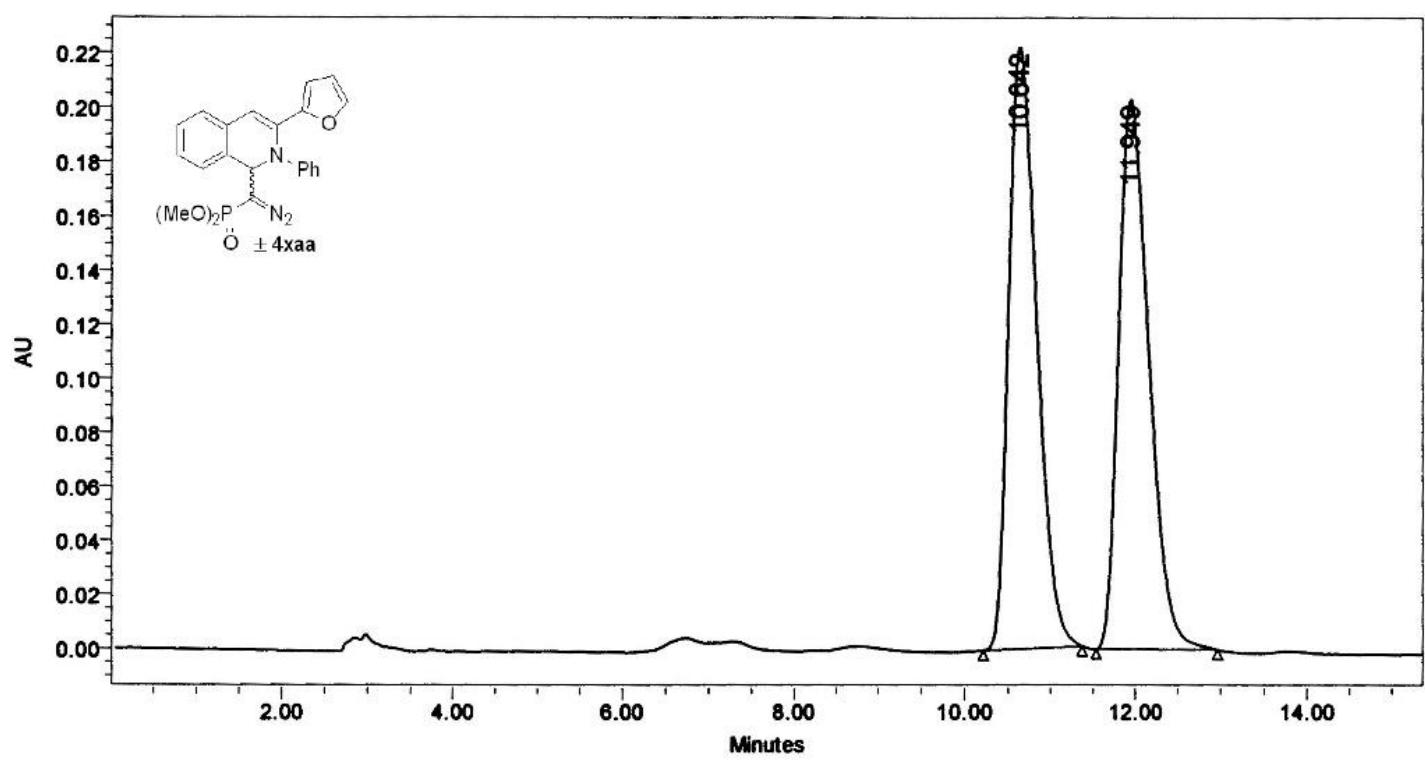

\begin{tabular}{|c|c|c|c|c|c|}
\hline & $\begin{array}{c}\text { RT } \\
(\mathrm{min})\end{array}$ & $\begin{array}{c}\text { Area } \\
\left(\mu \mathrm{V}^{*} \mathrm{sec}\right)\end{array}$ & $\%$ Area & $\begin{array}{c}\text { Height } \\
(\mu \mathrm{V})\end{array}$ & $\begin{array}{c}\% \\
\text { Height }\end{array}$ \\
\hline 1 & 10.642 & 5069284 & 49.82 & 222521 & 52.26 \\
\hline 2 & 11.949 & 5106082 & 50.18 & 203308 & 47.74 \\
\hline
\end{tabular}

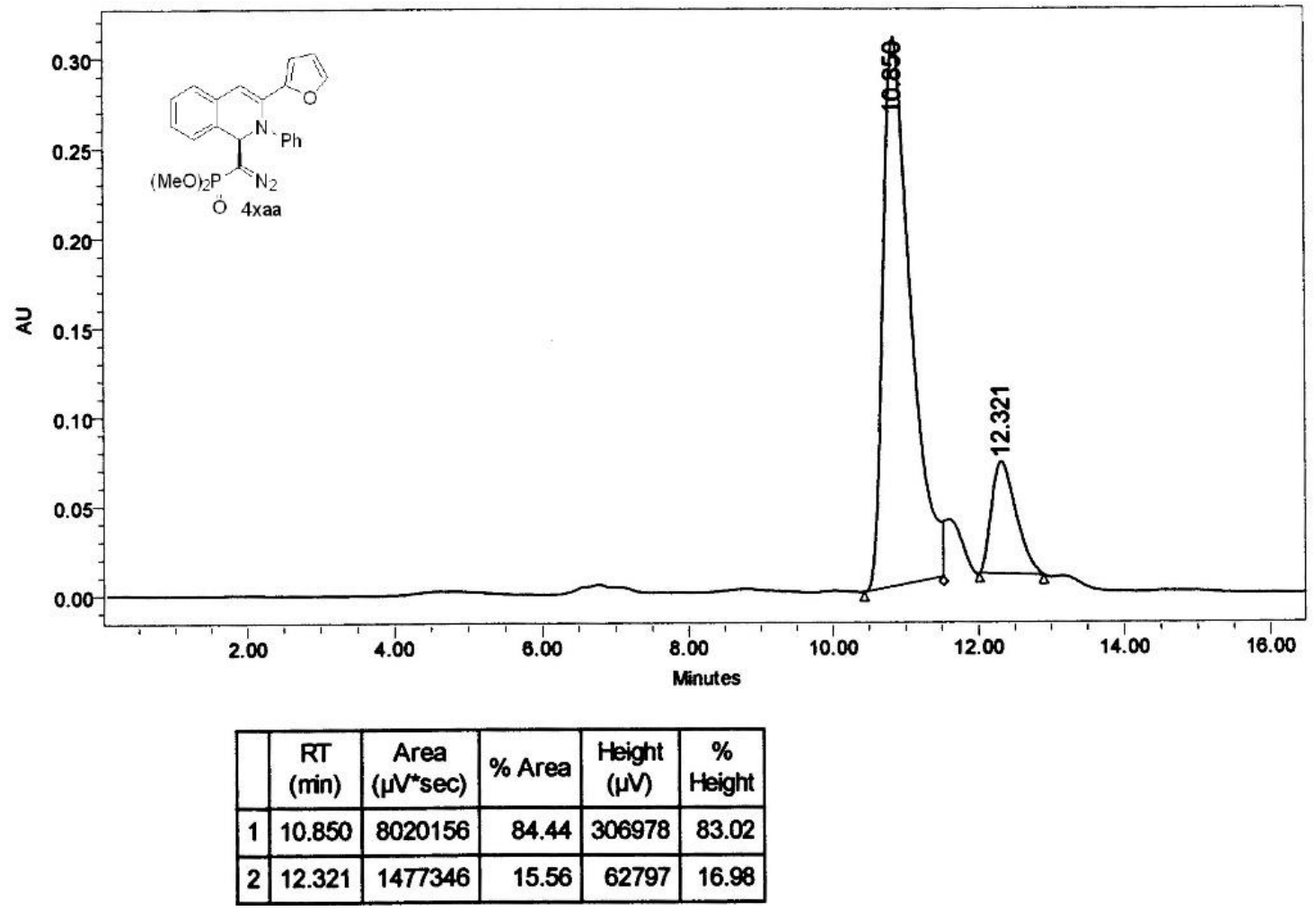




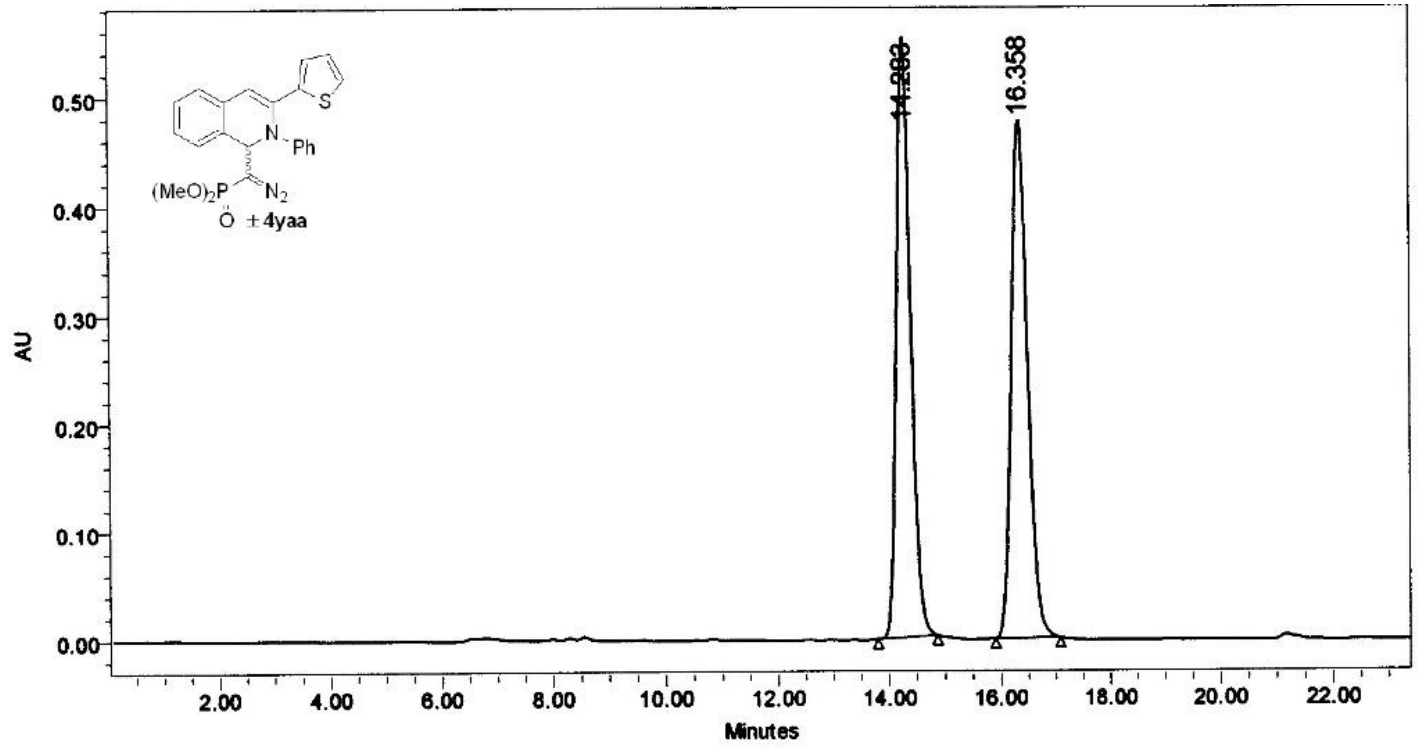

\begin{tabular}{|c|c|c|c|c|c|}
\hline & $\begin{array}{c}R T \\
(\min )\end{array}$ & $\begin{array}{c}\text { Area } \\
\left(\mu N^{*} \mathrm{sec}\right)\end{array}$ & $\%$ Area & $\begin{array}{c}\text { Height } \\
(\mu \mathrm{V})\end{array}$ & $\begin{array}{c}\% \\
\text { Height }\end{array}$ \\
\hline 1 & 14.283 & 9780805 & 49.83 & 552600 & 53.62 \\
\hline 2 & 16.358 & 9845743 & 50.17 & 477980 & 46.38 \\
\hline
\end{tabular}

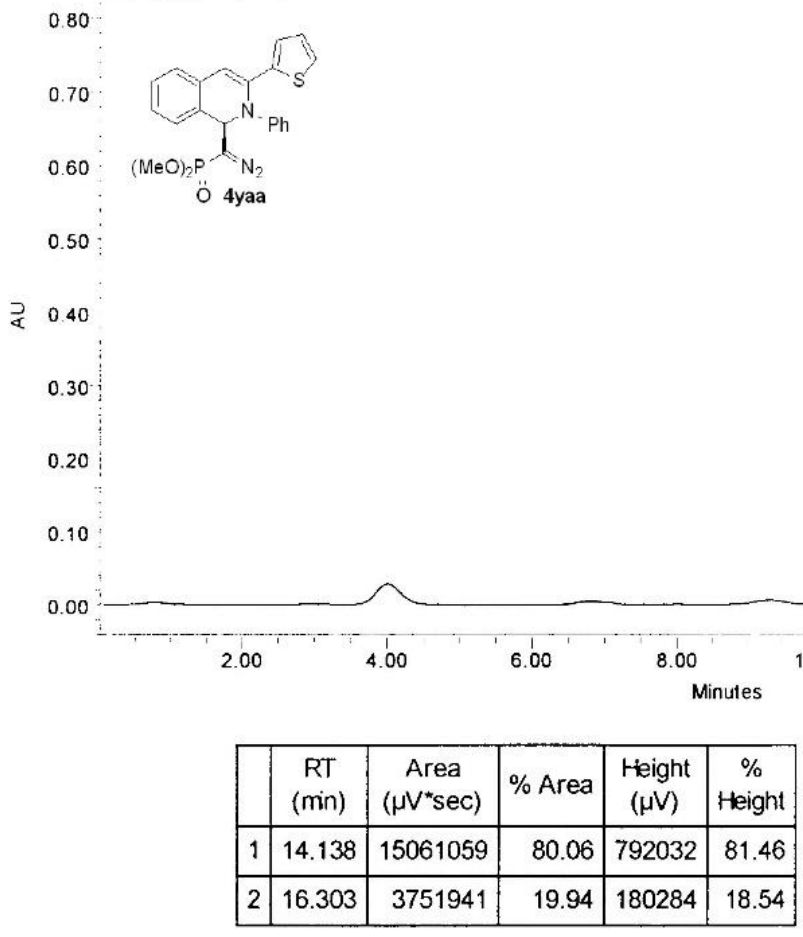




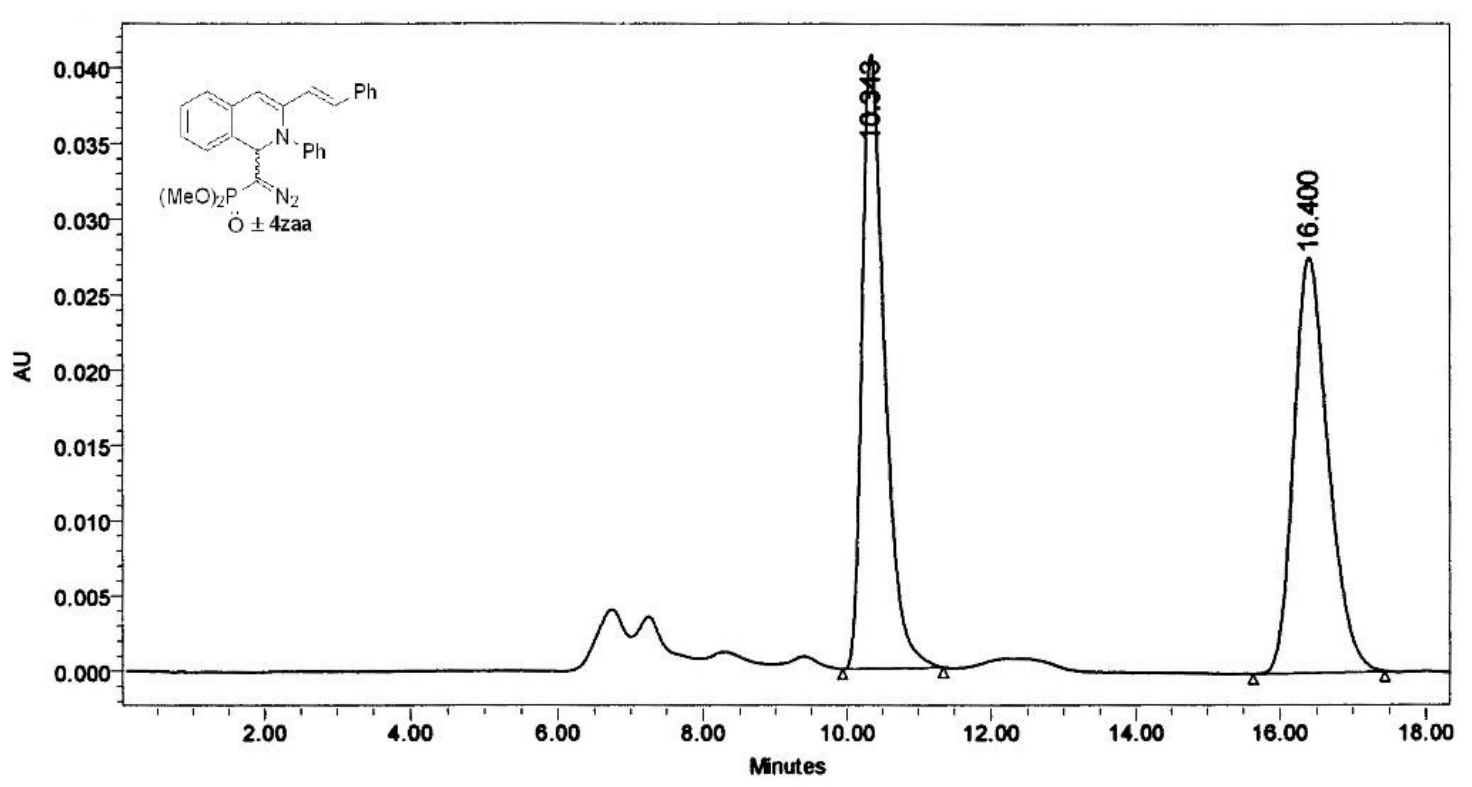

\begin{tabular}{|c|c|c|c|c|c|}
\hline & $\begin{array}{c}\text { RT } \\
(\mathrm{min})\end{array}$ & $\begin{array}{c}\text { Area } \\
(\mu \mathrm{V} \text { *sec })\end{array}$ & \% Area & $\begin{array}{c}\text { Height } \\
(\mu \mathrm{V})\end{array}$ & $\begin{array}{c}\% \\
\text { Height }\end{array}$ \\
\hline 1 & 10.343 & 897666 & 49.92 & 40738 & 59.56 \\
\hline 2 & 16.400 & 900589 & 50.08 & 27661 & 40.44 \\
\hline
\end{tabular}

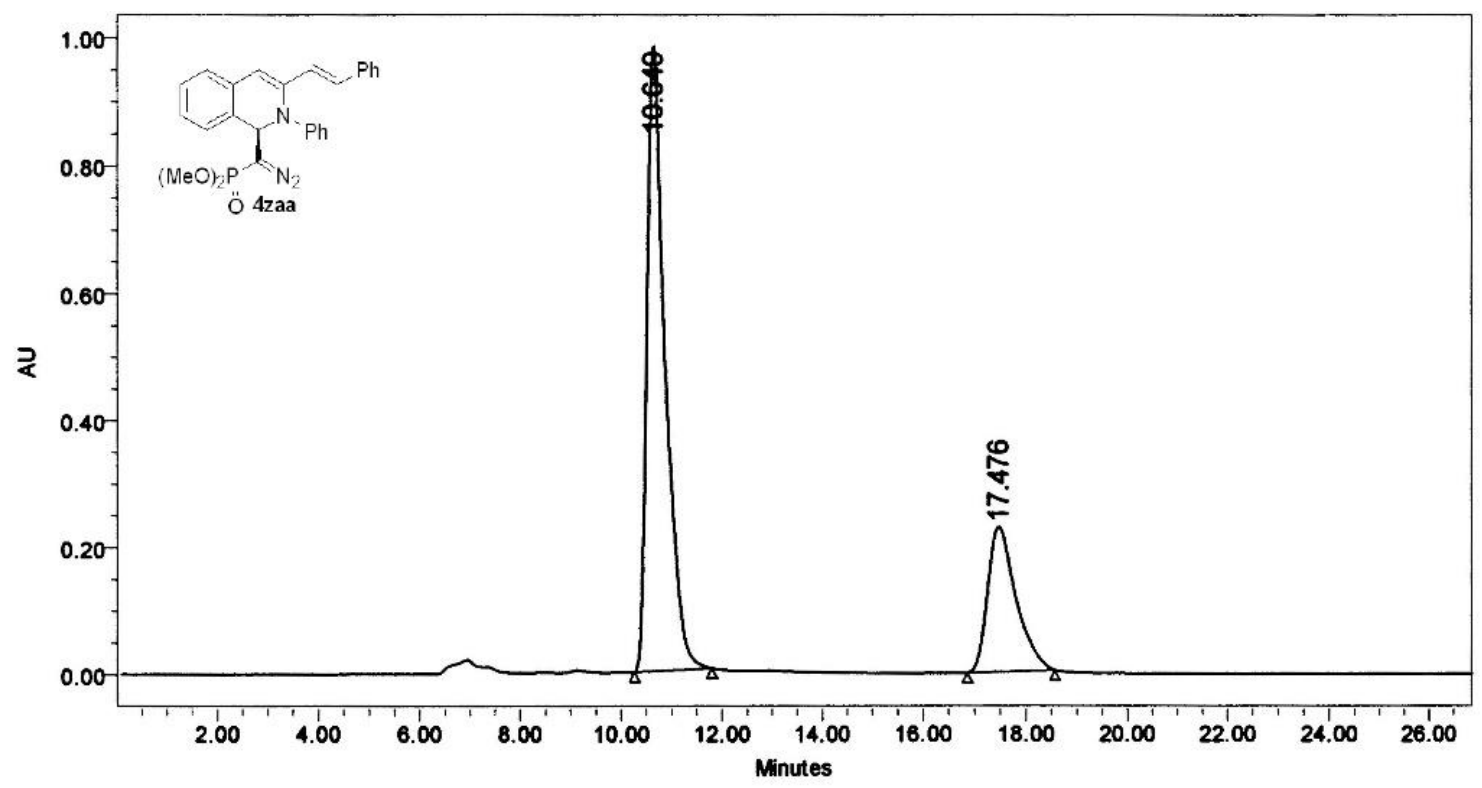

\begin{tabular}{|c|c|c|c|c|c|}
\hline & $\begin{array}{c}\mathrm{RT} \\
(\mathrm{min})\end{array}$ & $\begin{array}{c}\text { Area } \\
\left(\mu \mathrm{V}^{*} \mathrm{sec}\right)\end{array}$ & \% Area & $\begin{array}{c}\text { Height } \\
(\mu \mathrm{V})\end{array}$ & $\begin{array}{c}\% \\
\text { Height }\end{array}$ \\
\hline 1 & 10.649 & 24710447 & 74.07 & 981461 & 81.11 \\
\hline 2 & 17.476 & 8651328 & 25.93 & 228570 & 18.89 \\
\hline
\end{tabular}




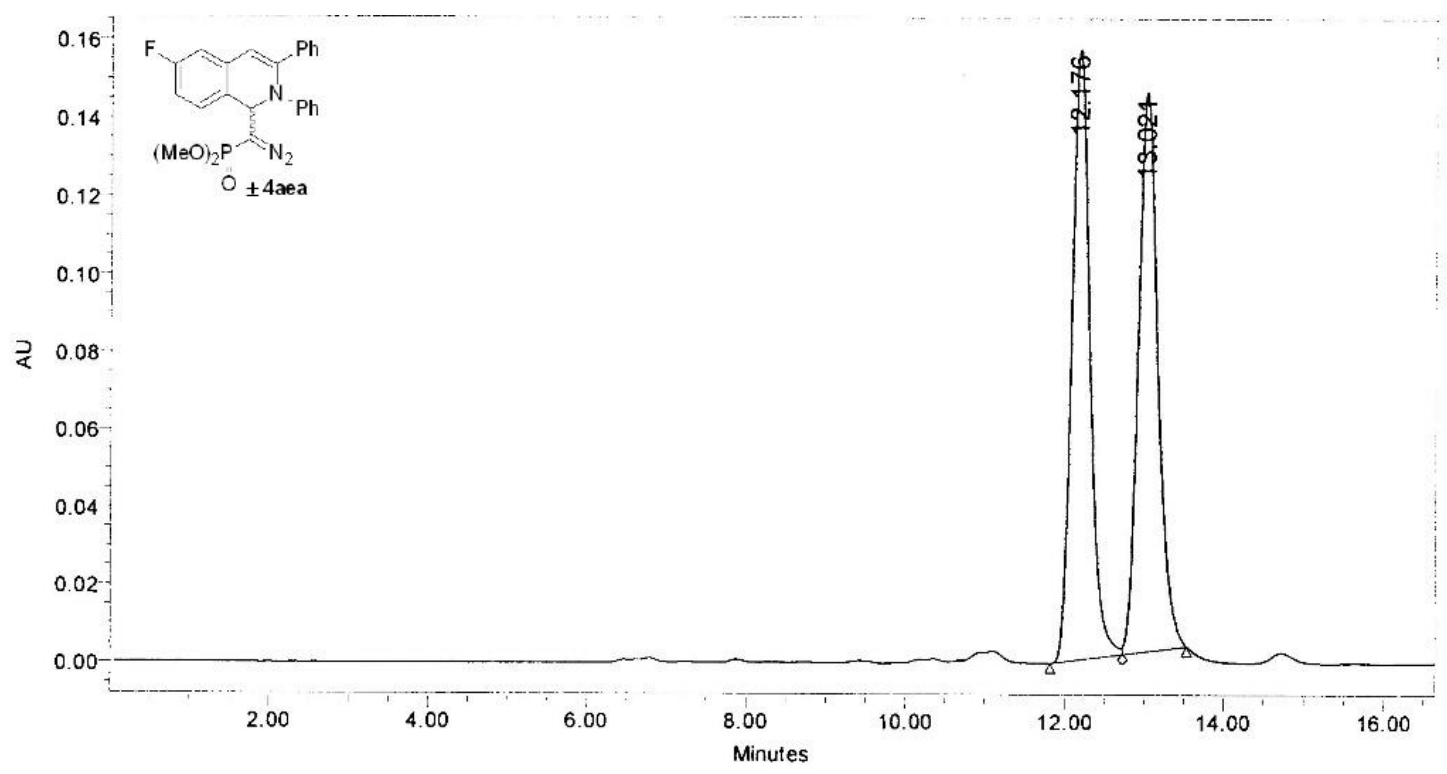

\begin{tabular}{|c|c|c|c|c|c|}
\hline & $\begin{array}{c}\mathrm{RT} \\
(\mathrm{min})\end{array}$ & $\begin{array}{c}\text { Area } \\
\left(\mu \mathrm{V}^{*} \mathrm{sec}\right)\end{array}$ & $\%$ Area & $\begin{array}{c}\text { Height } \\
(\mu \mathrm{V})\end{array}$ & $\begin{array}{c}\% \\
\text { Height }\end{array}$ \\
\hline 1 & 12.176 & 2474152 & 49.88 & 157323 & 52.18 \\
\hline 2 & 13.021 & 2485876 & 50.12 & 144150 & 47.82 \\
\hline
\end{tabular}

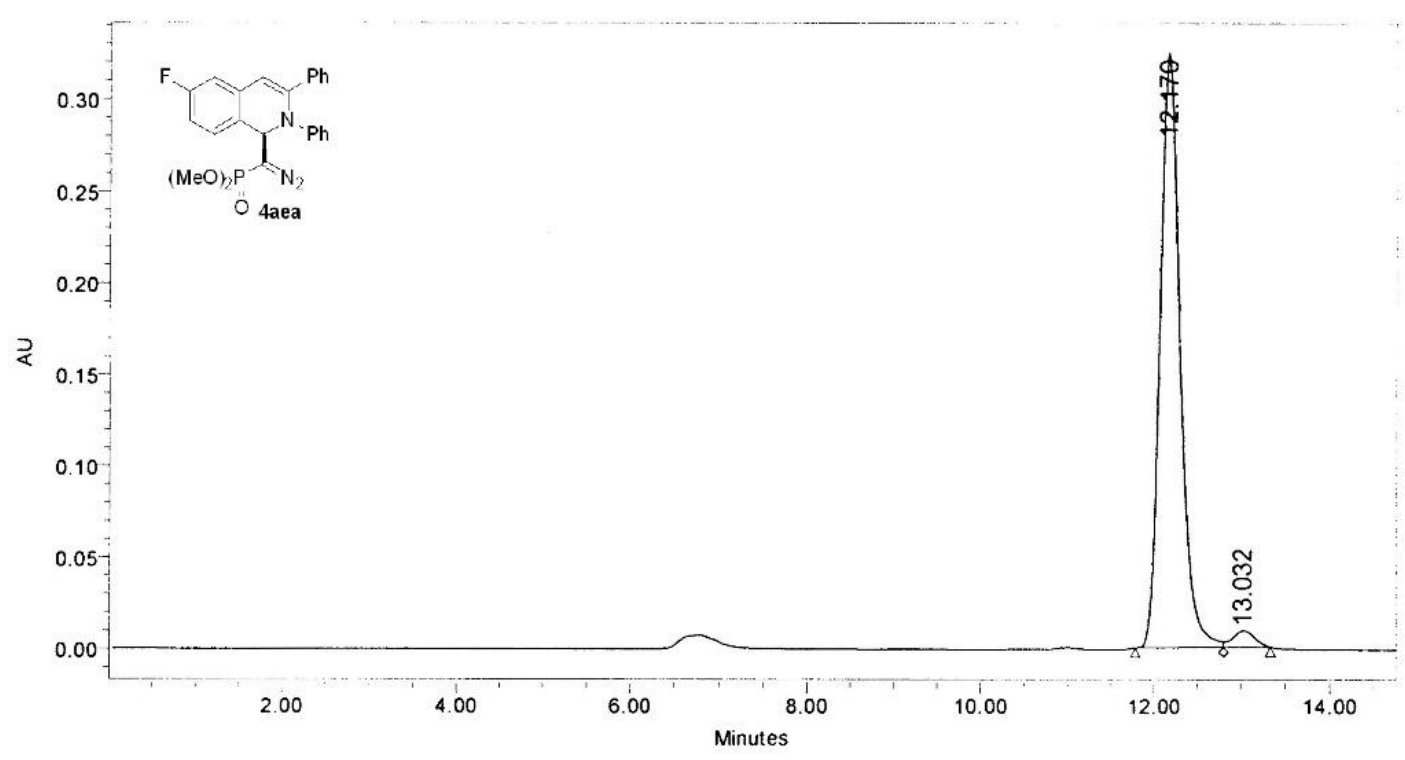

\begin{tabular}{|c|c|c|r|r|r|}
\hline & $\begin{array}{c}\mathrm{RT} \\
(\mathrm{min})\end{array}$ & $\begin{array}{c}\text { Area } \\
\left(\mu \mathrm{V}^{*} \mathrm{sec}\right)\end{array}$ & $\%$ Area & $\begin{array}{c}\text { Height } \\
(\mu \mathrm{V})\end{array}$ & $\begin{array}{c}\% \\
\text { Height }\end{array}$ \\
\hline 1 & 12.170 & 5225938 & 97.05 & 324273 & 97.26 \\
\hline 2 & 13.032 & 158889 & 2.95 & 9152 & 2.74 \\
\hline
\end{tabular}




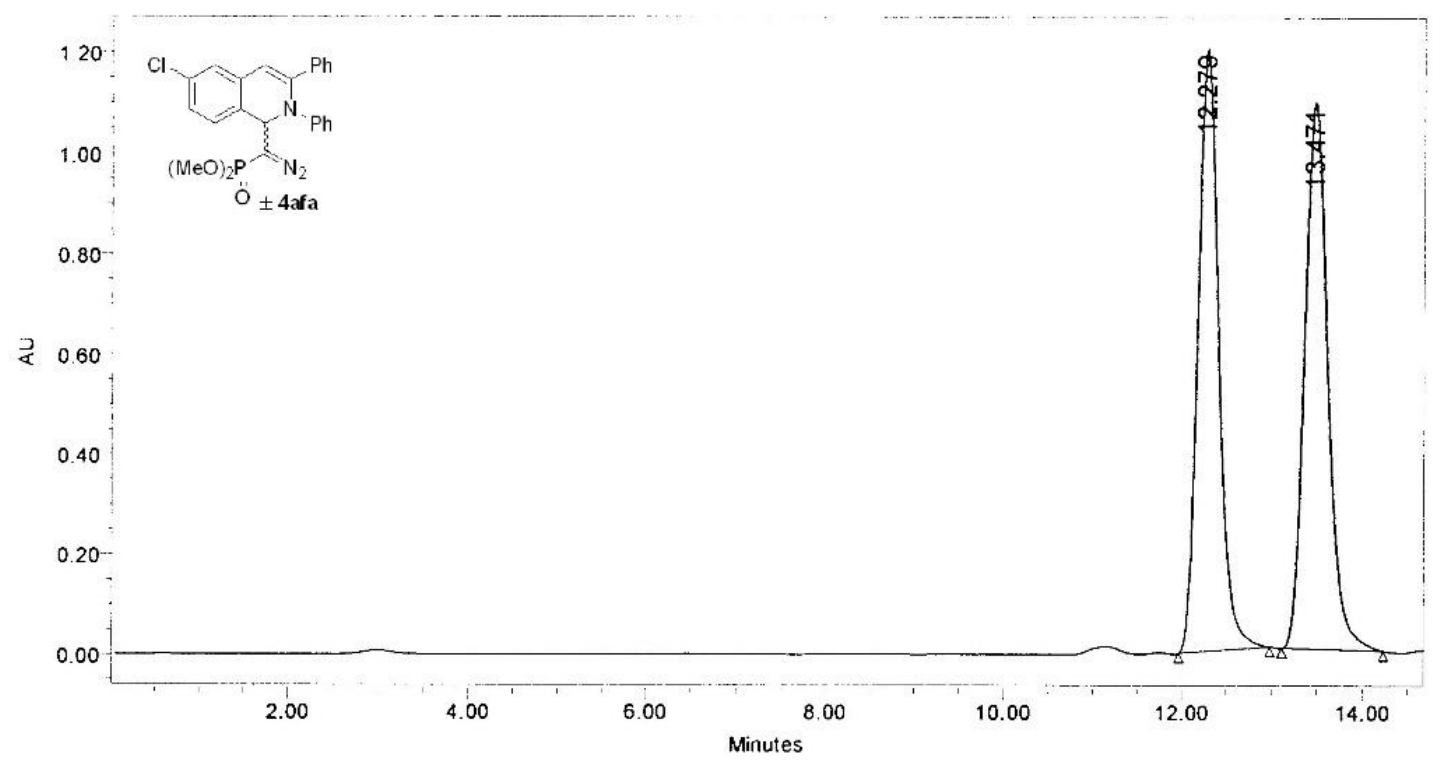

\begin{tabular}{|c|c|c|c|c|c|}
\hline & $\begin{array}{c}\mathrm{RT} \\
(\mathrm{min})\end{array}$ & $\begin{array}{c}\text { Area } \\
(\mu \mathrm{V} * \mathrm{sec})\end{array}$ & $\%$ Area & $\begin{array}{c}\text { Height } \\
(\mu \mathrm{V})\end{array}$ & $\begin{array}{c}\% \\
\text { Height }\end{array}$ \\
\hline 1 & 12.279 & 19217957 & 49.95 & 1202085 & 52.44 \\
\hline 2 & 13.471 & 19257602 & 50.05 & 1090118 & 47.56 \\
\hline
\end{tabular}

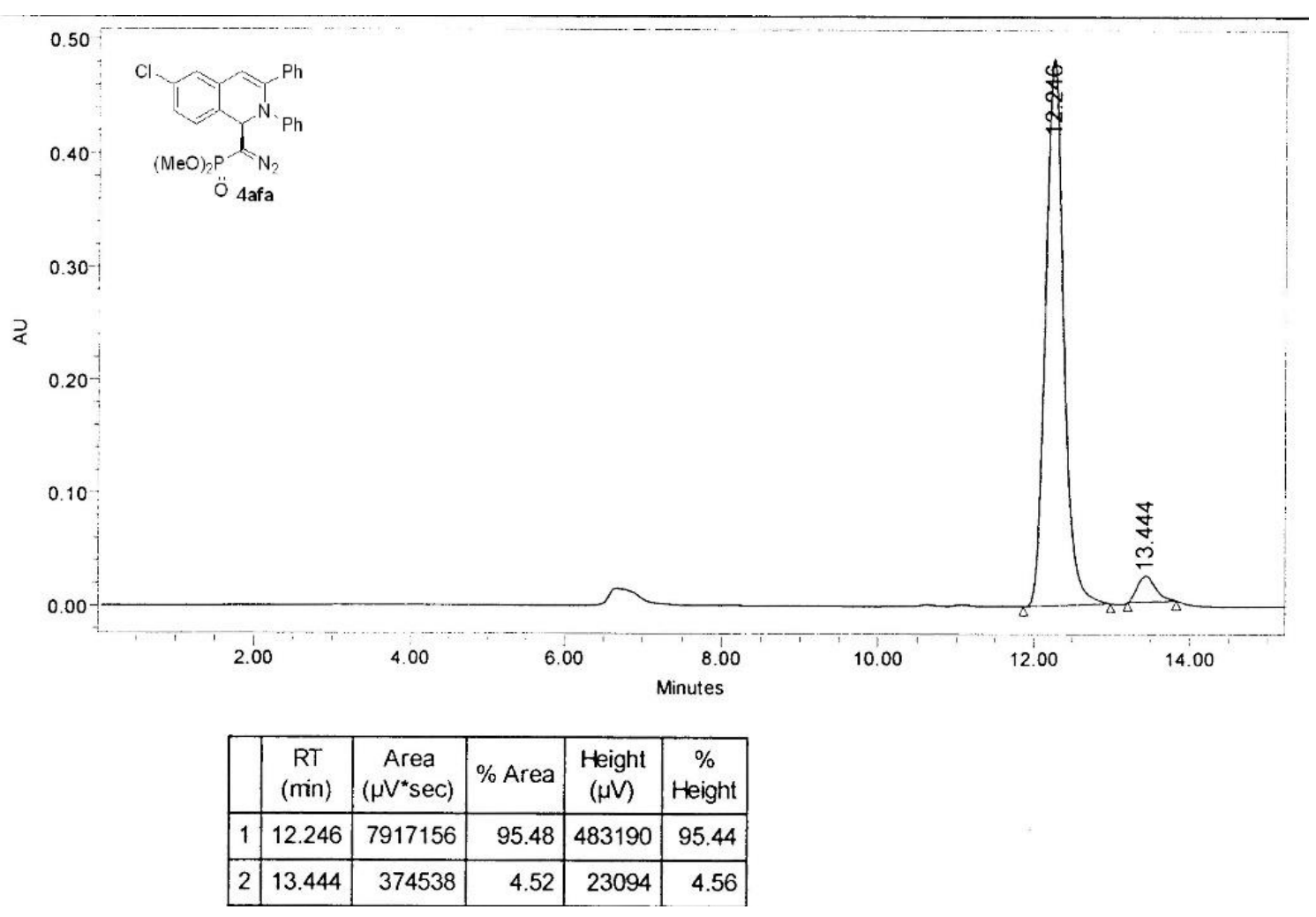



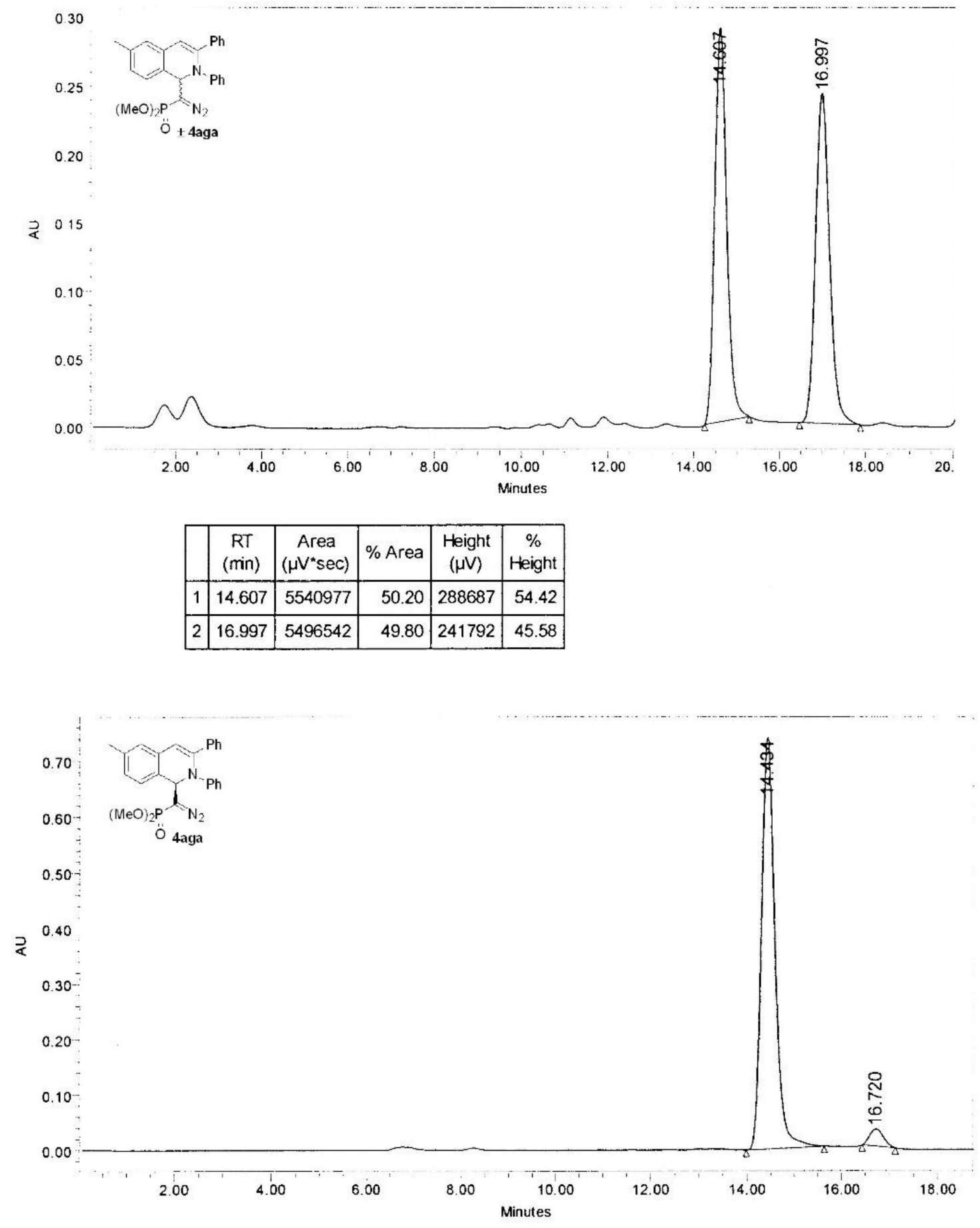

\begin{tabular}{|c|c|c|c|c|c|}
\hline & $\begin{array}{c}\mathrm{RT} \\
(\mathrm{min})\end{array}$ & $\begin{array}{c}\text { Area } \\
\left(\mu \mathrm{V}^{*} \mathrm{sec}\right)\end{array}$ & $\%$ Area & $\begin{array}{c}\text { Height } \\
(\mu \mathrm{V})\end{array}$ & $\begin{array}{c}\% \\
\text { Height }\end{array}$ \\
\hline 1 & 14.434 & 14635072 & 95.97 & 740195 & 95.95 \\
\hline 2 & 16.720 & 614405 & 4.03 & 31263 & 4.05 \\
\hline
\end{tabular}




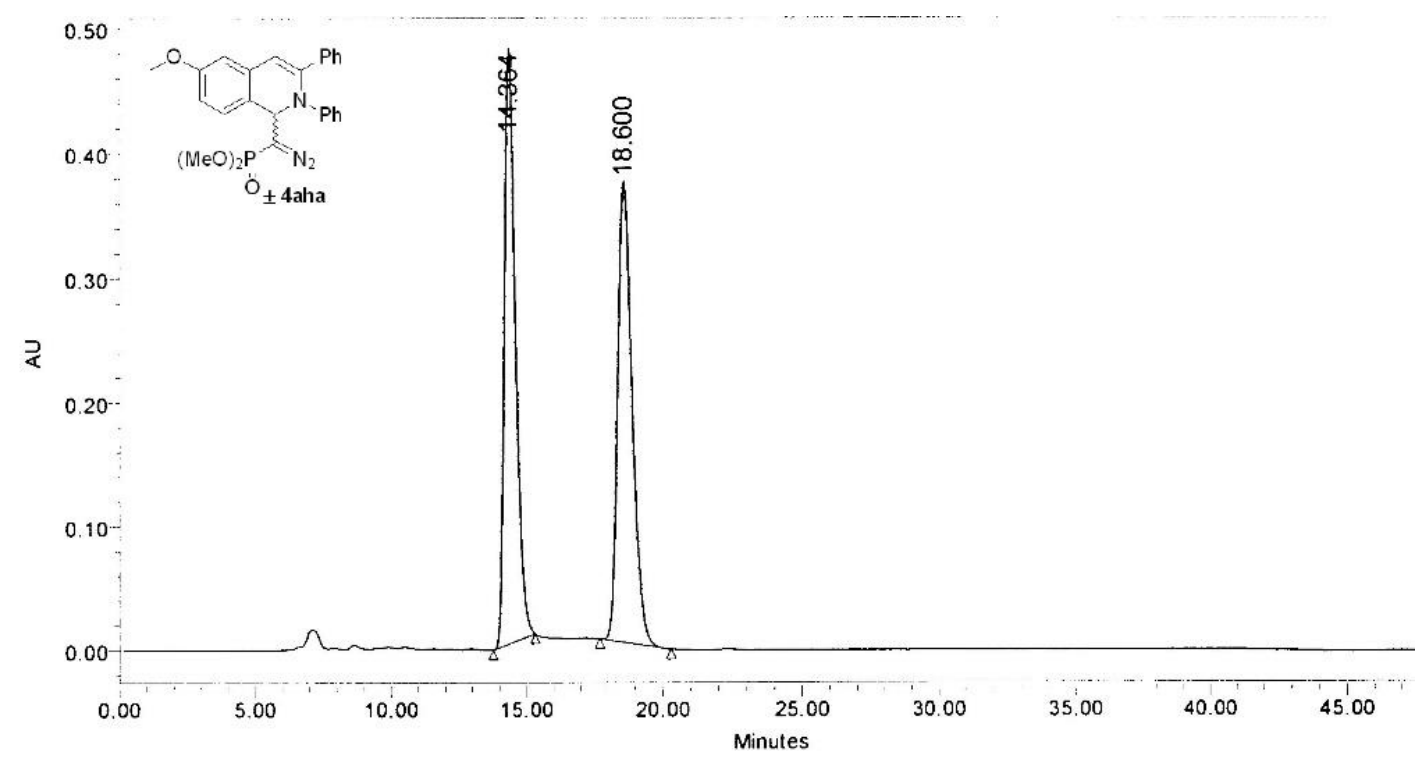

\begin{tabular}{|c|c|c|c|c|c|}
\hline & $\begin{array}{c}\mathrm{RT} \\
(\mathrm{min})\end{array}$ & $\begin{array}{c}\text { Area } \\
\left(\mu \mathrm{V}^{*} \mathrm{sec}\right)\end{array}$ & $\%$ Area & $\begin{array}{c}\text { Height } \\
(\mu \mathrm{V})\end{array}$ & $\begin{array}{c}\% \\
\text { Height }\end{array}$ \\
\hline 1 & 14.364 & 13601503 & 50.05 & 478306 & 56.29 \\
\hline 2 & 18.600 & 13575031 & 49.95 & 371383 & 43.71 \\
\hline
\end{tabular}

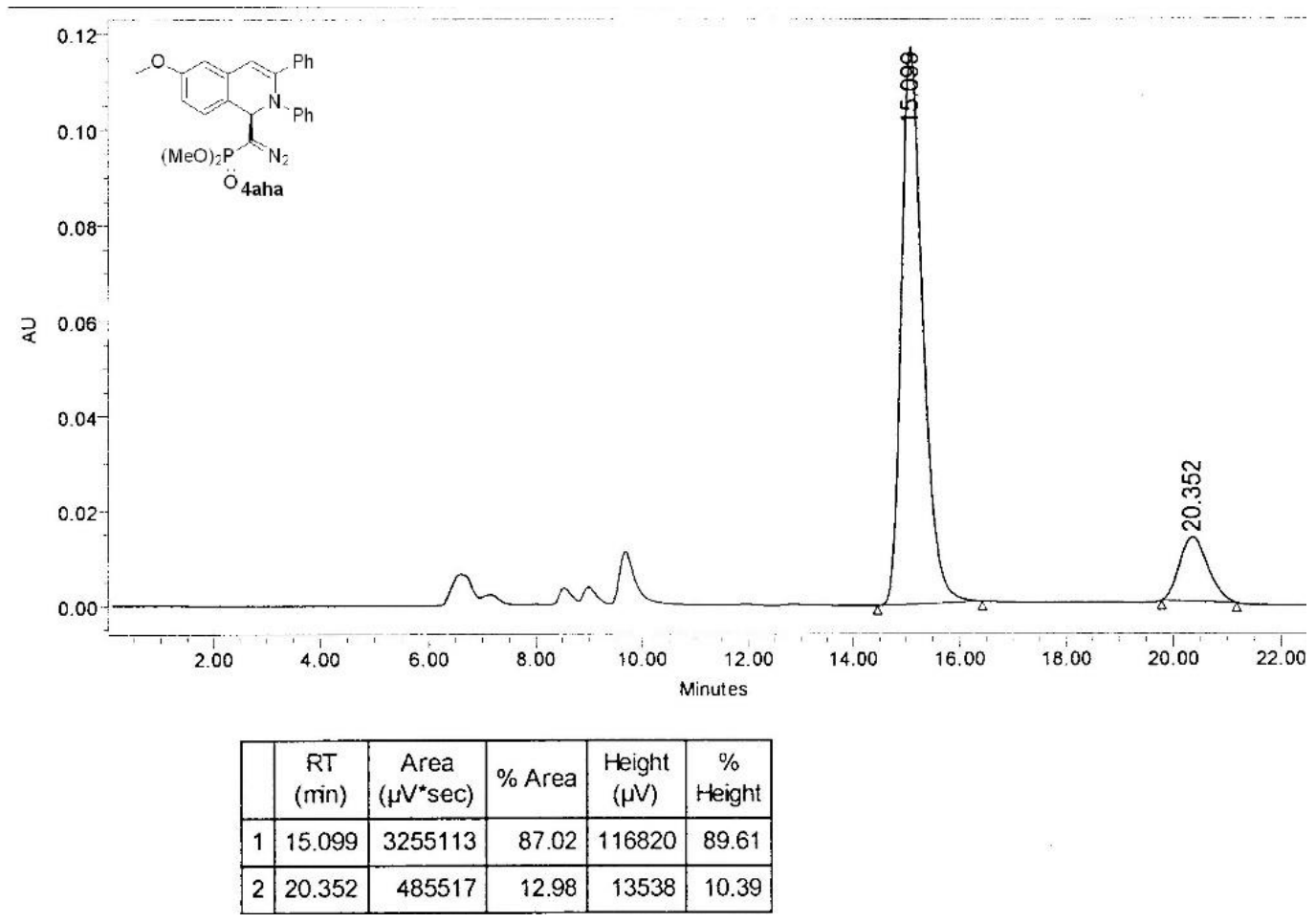




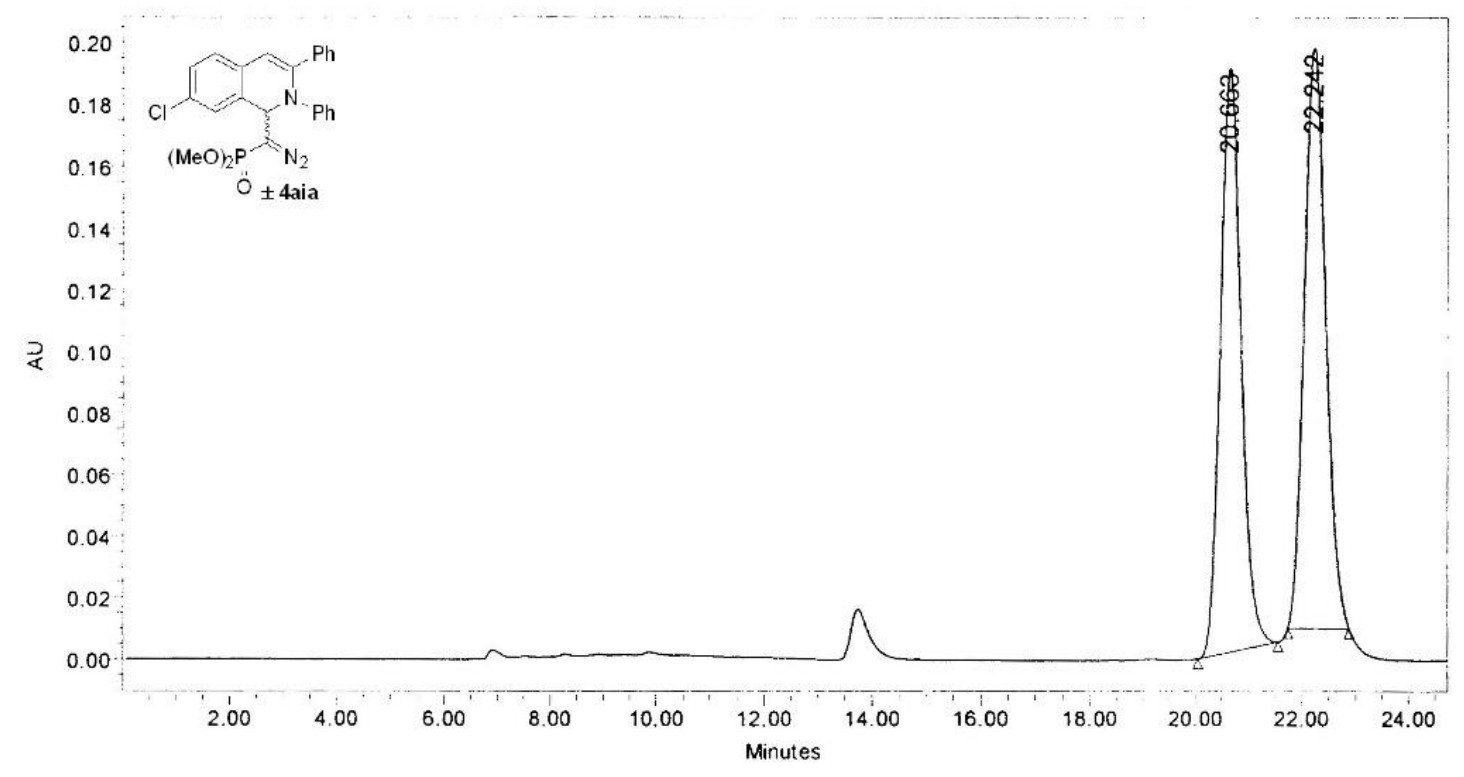

\begin{tabular}{|c|c|c|c|c|c|}
\hline & $\begin{array}{c}\text { RT } \\
(\mathrm{min})\end{array}$ & $\begin{array}{c}\text { Area } \\
\left(\mu \mathrm{V}^{*} \mathrm{sec}\right)\end{array}$ & $\%$ Area & $\begin{array}{c}\text { Height } \\
(\mu \mathrm{V})\end{array}$ & $\begin{array}{c}\% \\
\text { Height }\end{array}$ \\
\hline 1 & 20.663 & 5157557 & 49.74 & 189719 & 50.17 \\
\hline 2 & 22.242 & 5210639 & 50.26 & 188406 & 49.83 \\
\hline
\end{tabular}

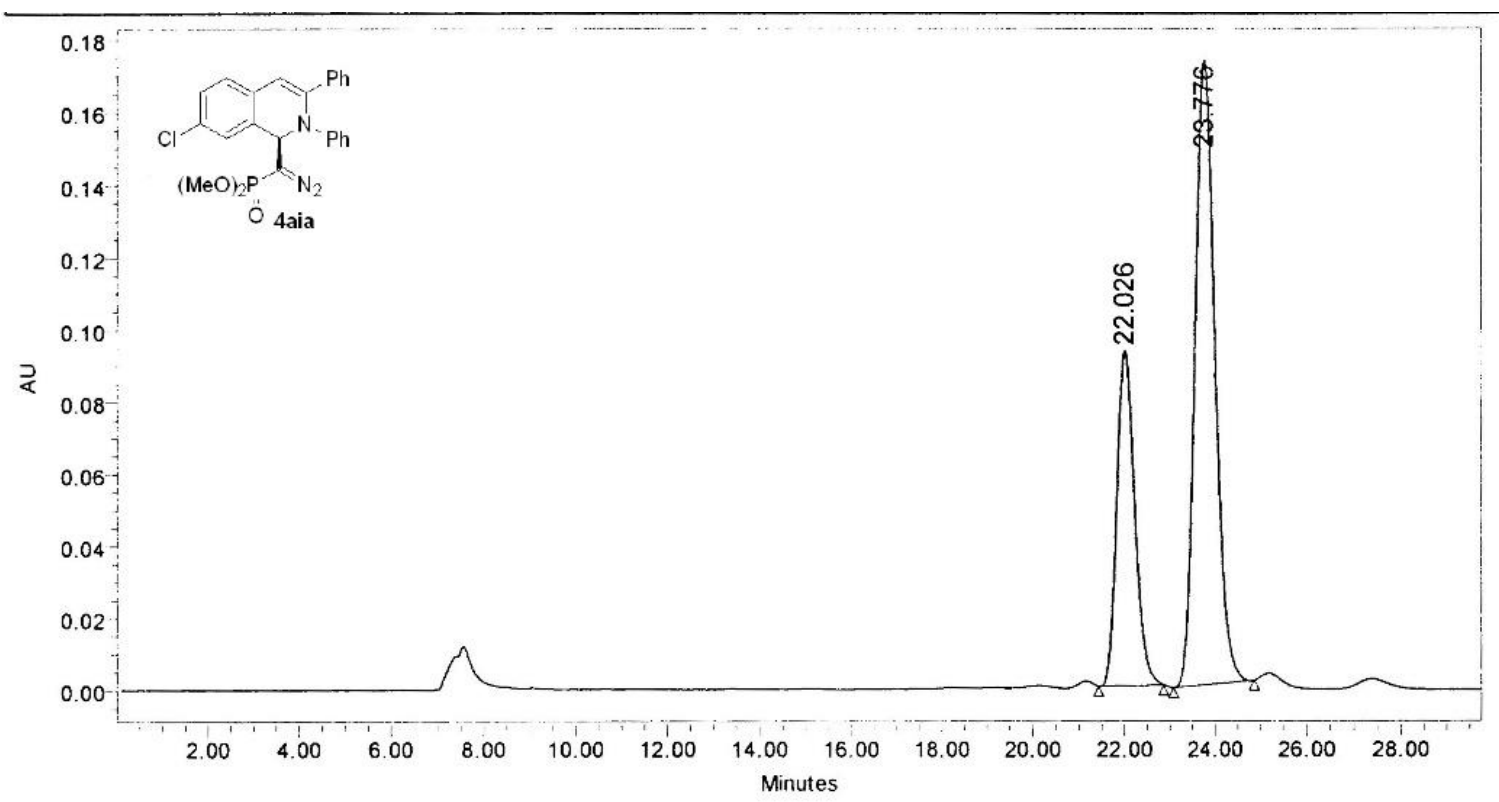

\begin{tabular}{|c|c|c|c|c|c|}
\hline & $\begin{array}{c}\mathrm{RT} \\
(\mathrm{min})\end{array}$ & $\begin{array}{c}\text { Area } \\
\left(\mu \mathrm{V}^{*} \mathrm{sec}\right)\end{array}$ & $\%$ Area & $\begin{array}{c}\text { Height } \\
(\mu \mathrm{V})\end{array}$ & $\begin{array}{c}\% \\
\text { Height }\end{array}$ \\
\hline 1 & 22.026 & 2544050 & 32.48 & 93275 & 35.04 \\
\hline 2 & 23.776 & 5288815 & 67.52 & 172953 & 64.96 \\
\hline
\end{tabular}




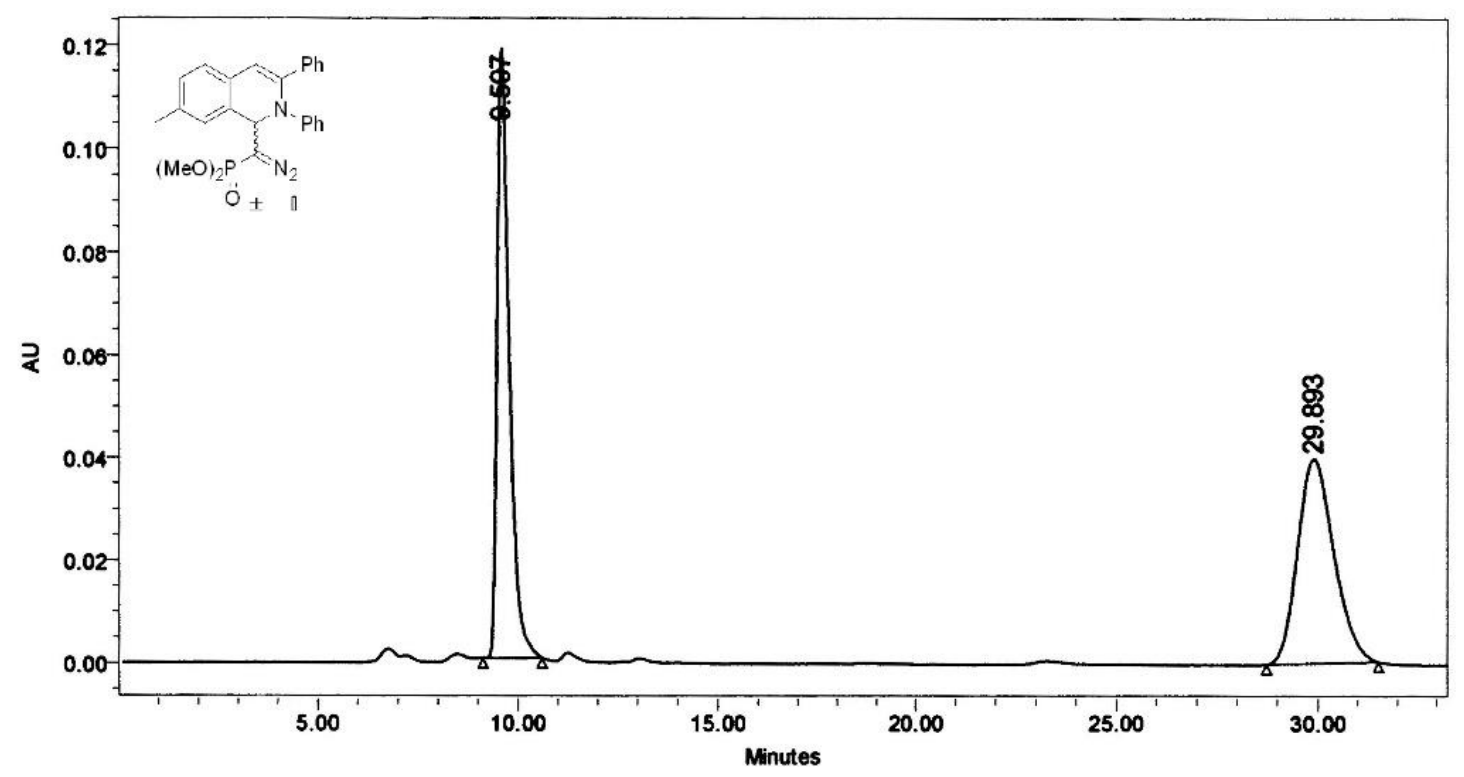

\begin{tabular}{|c|c|c|c|c|c|}
\hline & $\begin{array}{c}\mathrm{RT} \\
(\mathrm{min})\end{array}$ & $\begin{array}{c}\text { Area } \\
\left(\mu \mathrm{N}^{*} \mathrm{sec}\right)\end{array}$ & $\%$ Area & $\begin{array}{c}\text { Height } \\
(\mu \mathrm{V})\end{array}$ & $\begin{array}{c}\% \\
\text { Height }\end{array}$ \\
\hline 1 & 9.597 & 2532921 & 50.31 & 118435 & 74.78 \\
\hline 2 & 29.893 & 2501544 & 49.69 & 39937 & 25.22 \\
\hline
\end{tabular}

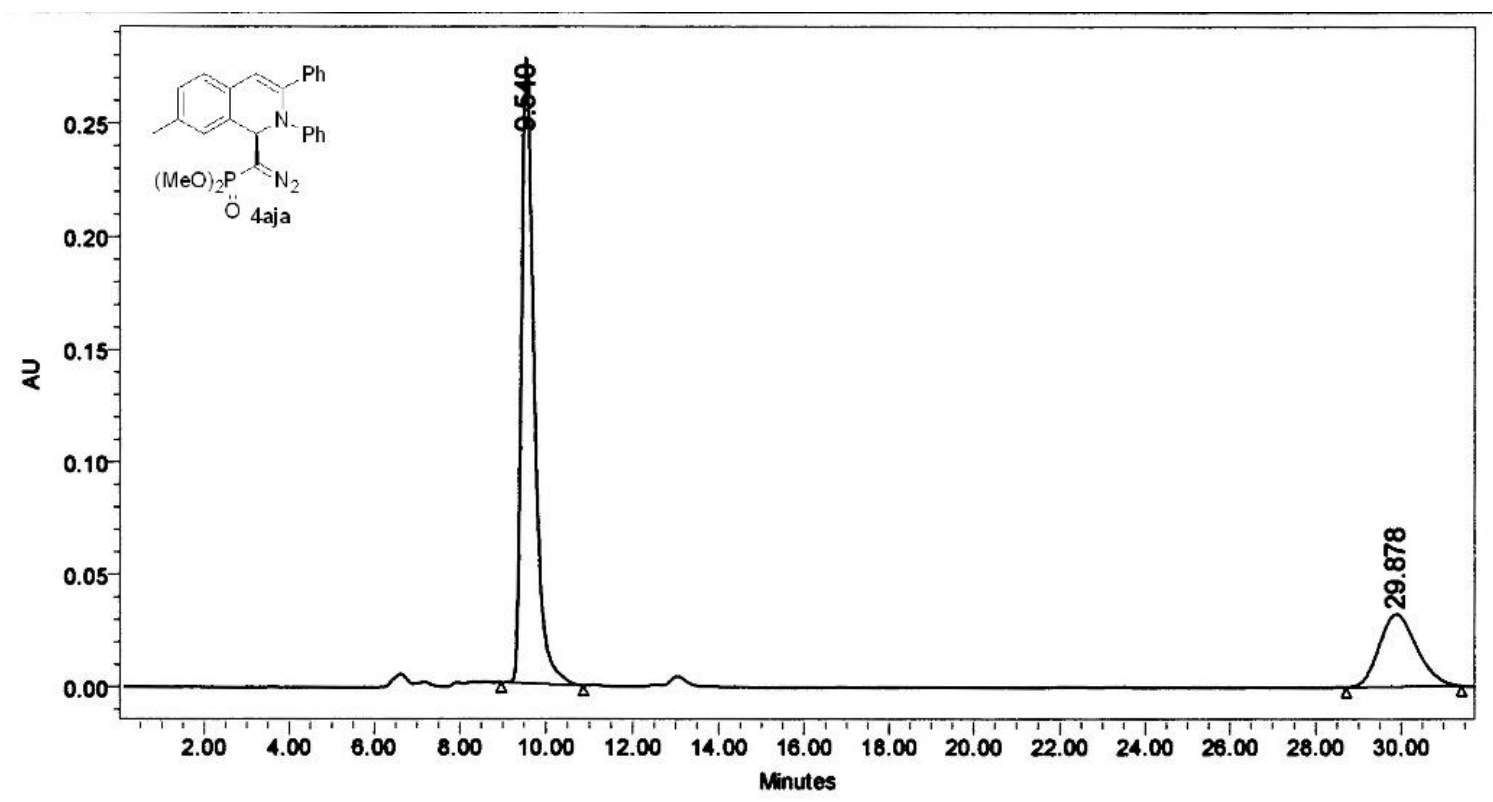

\begin{tabular}{|c|c|c|c|c|c|}
\hline & $\begin{array}{c}\text { RT } \\
(\mathrm{min})\end{array}$ & $\begin{array}{c}\text { Area } \\
(\mu \mathrm{V} \text { "sec })\end{array}$ & \% Area & $\begin{array}{c}\text { Height } \\
(\mu \mathrm{V})\end{array}$ & $\begin{array}{c}\% \\
\text { Height }\end{array}$ \\
\hline 1 & 9.540 & 5740681 & 74.49 & 277777 & 89.62 \\
\hline 2 & 29.878 & 1965501 & 25.51 & 32164 & 10.38 \\
\hline
\end{tabular}



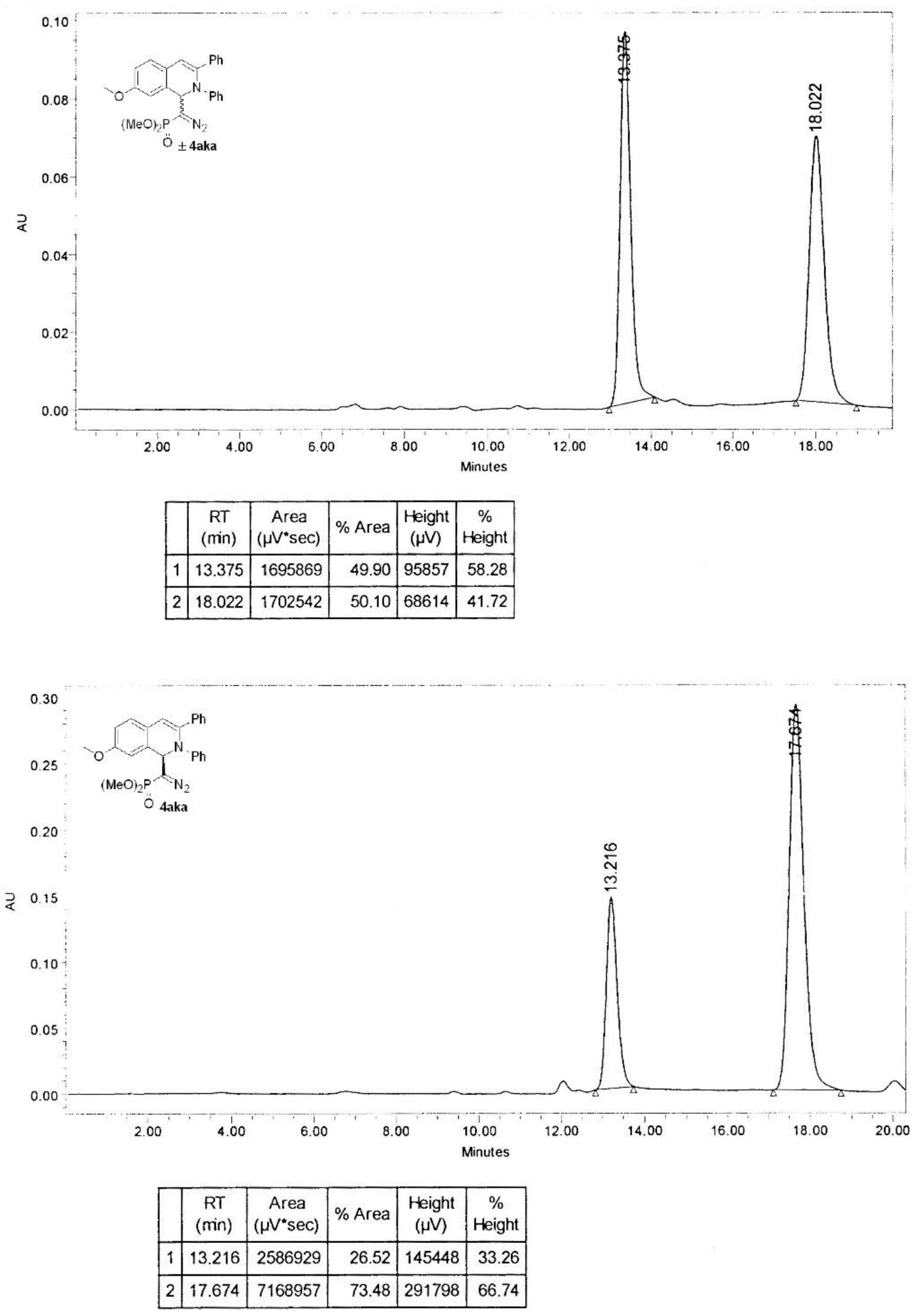


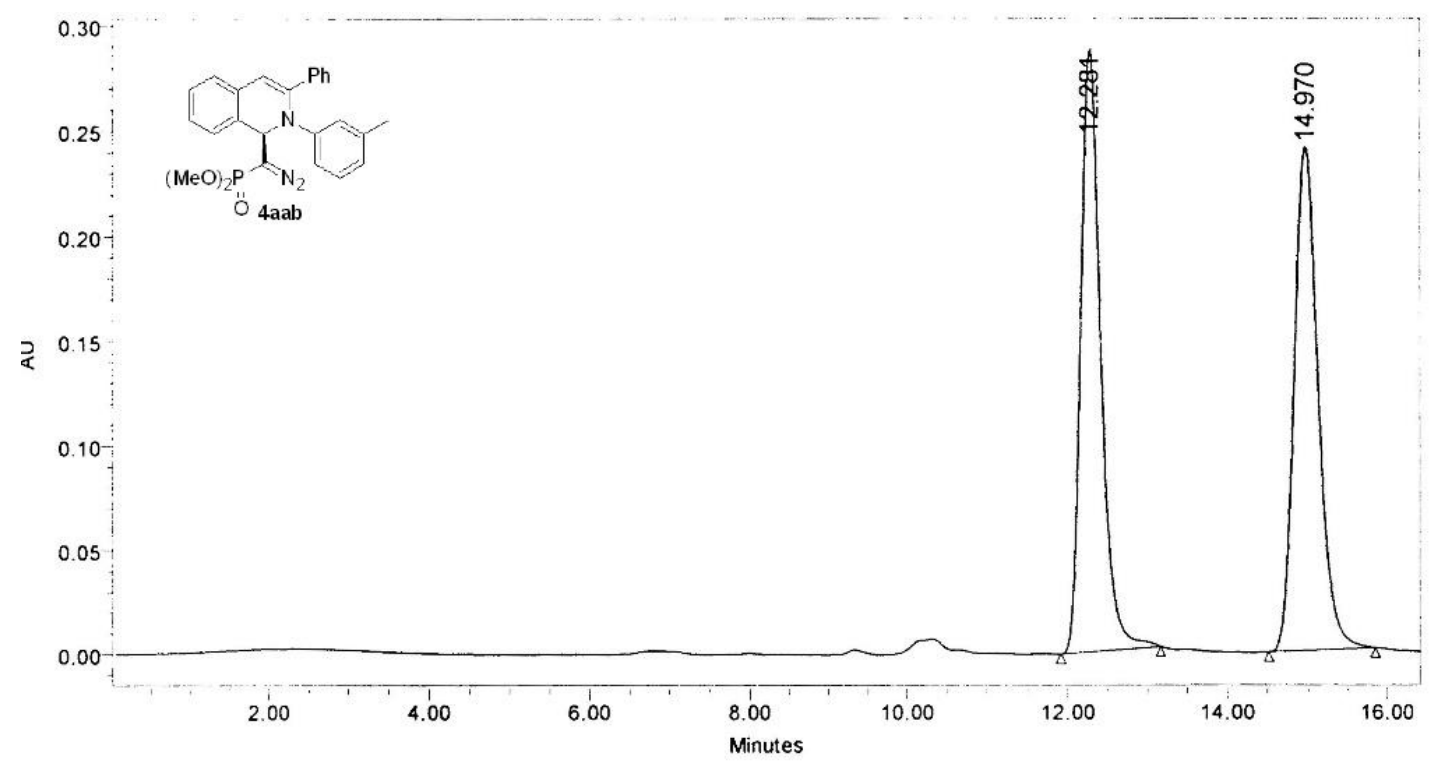

\begin{tabular}{|c|c|c|c|c|c|}
\hline & $\begin{array}{c}\text { RT } \\
(\mathrm{min})\end{array}$ & $\begin{array}{c}\text { Area } \\
\left(\mu \mathrm{V}^{*} \mathrm{sec}\right)\end{array}$ & $\%$ Area & $\begin{array}{c}\text { Height } \\
(\mu \mathrm{V})\end{array}$ & $\begin{array}{c}\% \\
\text { Height }\end{array}$ \\
\hline 1 & 12.281 & 4966201 & 49.93 & 288049 & 54.43 \\
\hline 2 & 14.970 & 4979392 & 50.07 & 241113 & 45.57 \\
\hline
\end{tabular}

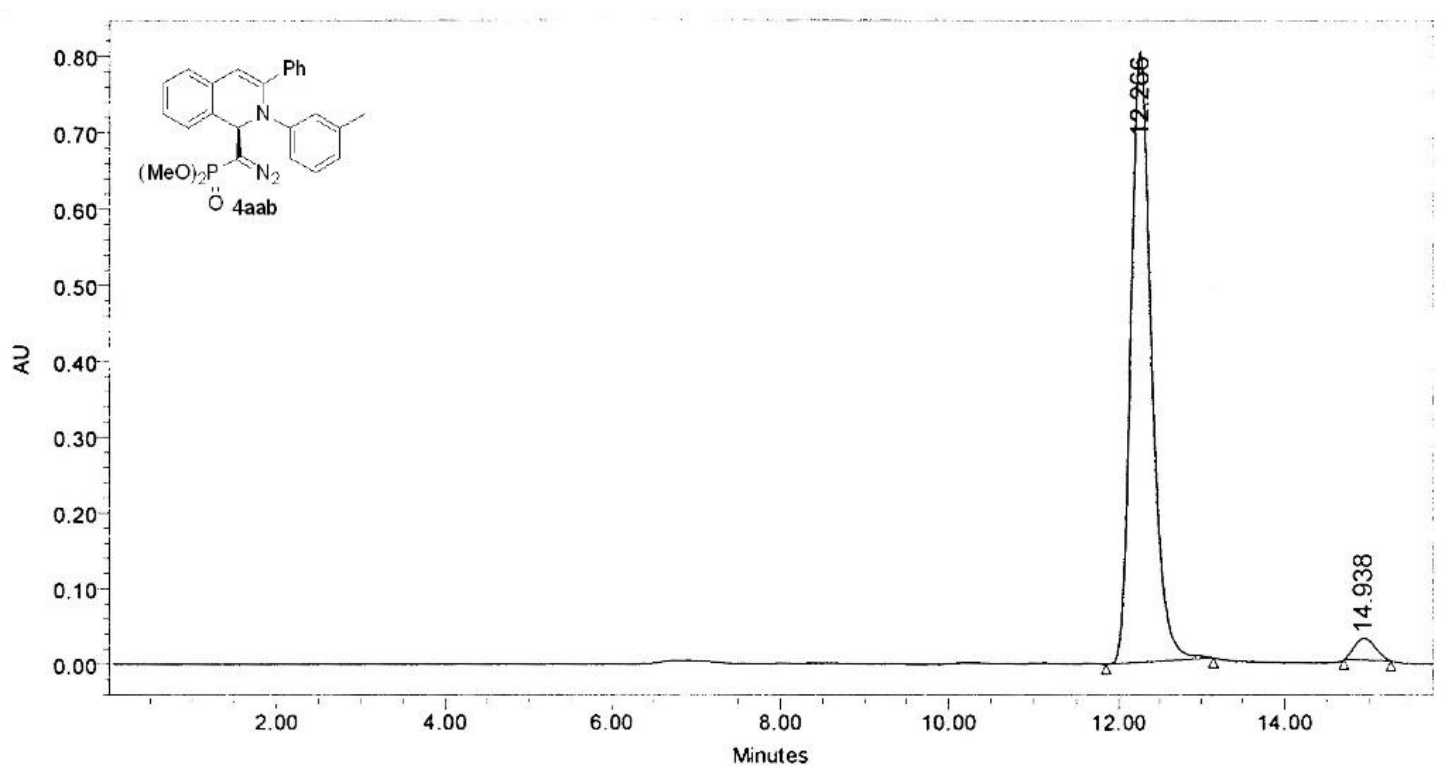

\begin{tabular}{|c|c|c|r|r|r|}
\hline & $\begin{array}{c}\text { RT } \\
(\mathrm{min})\end{array}$ & $\begin{array}{c}\text { Area } \\
\left(\mu \mathrm{V}^{*} \mathrm{sec}\right)\end{array}$ & $\%$ Area & $\begin{array}{c}\text { Height } \\
(\mu \mathrm{V})\end{array}$ & $\begin{array}{c}\% \\
\text { Height }\end{array}$ \\
\hline 1 & 12.266 & 13976457 & 96.52 & 803422 & 96.52 \\
\hline 2 & 14.938 & 504095 & 3.48 & 28951 & 3.48 \\
\hline
\end{tabular}




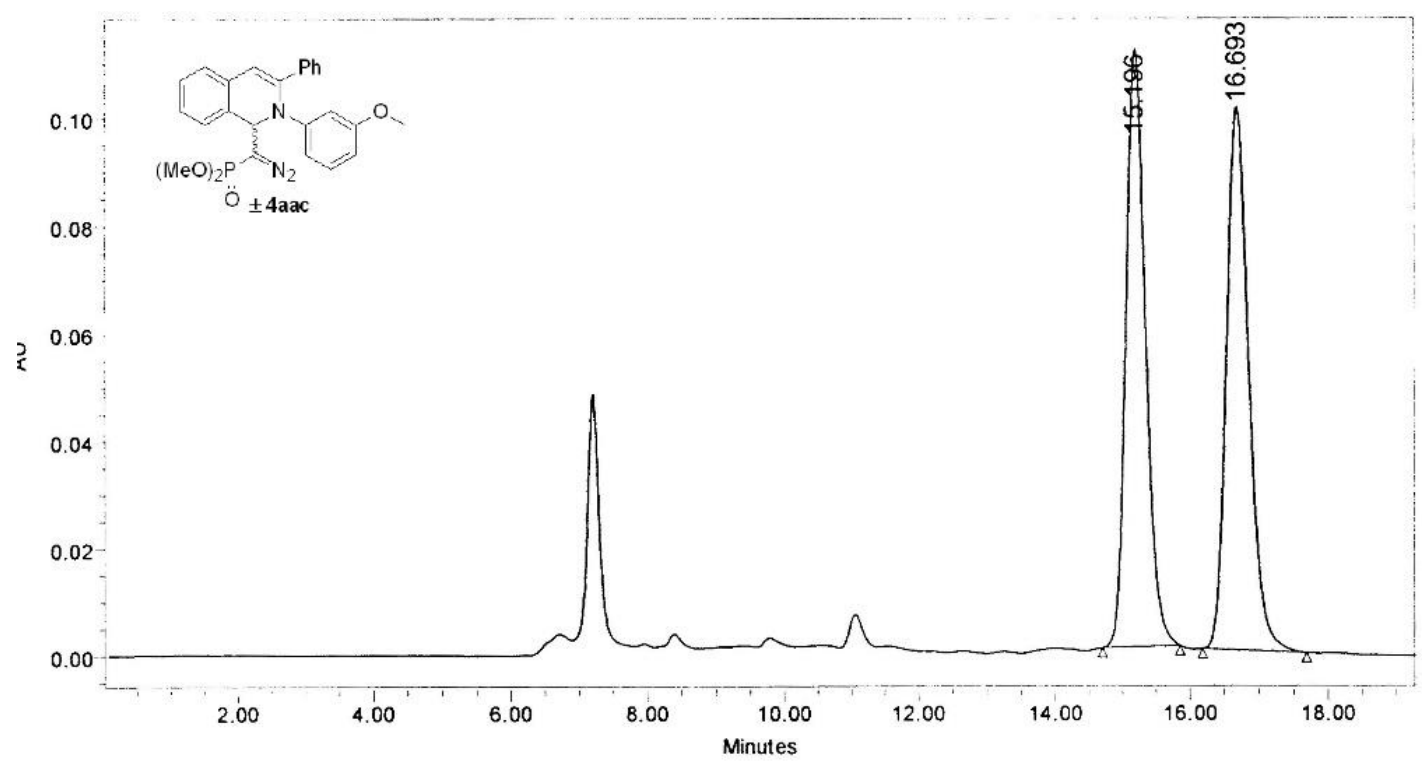

\begin{tabular}{|c|c|c|c|c|c|}
\hline & $\begin{array}{c}\mathrm{RT} \\
(\mathrm{min})\end{array}$ & $\begin{array}{c}\text { Area } \\
\left(\mu \mathrm{V}^{*} \mathrm{sec}\right)\end{array}$ & $\%$ Area & $\begin{array}{c}\text { Height } \\
(\mu \mathrm{V})\end{array}$ & $\begin{array}{c}\% \\
\text { Height }\end{array}$ \\
\hline 1 & 15.196 & 2243701 & 49.90 & 111144 & 52.39 \\
\hline 2 & 16.693 & 2252361 & 50.10 & 100988 & 47.61 \\
\hline
\end{tabular}

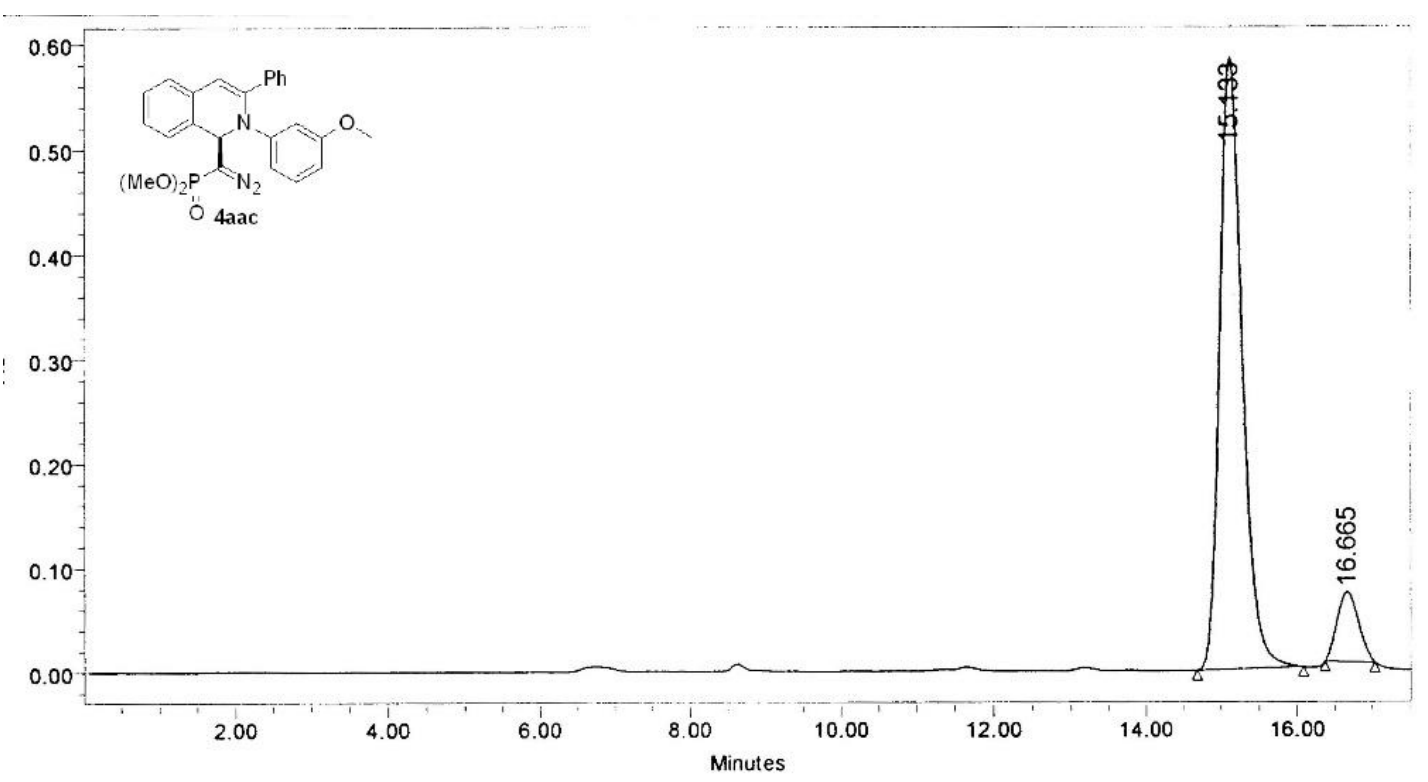

\begin{tabular}{|c|c|c|r|r|c|}
\hline & $\begin{array}{c}\text { RT } \\
(\min )\end{array}$ & $\begin{array}{c}\text { Area } \\
(\mu \mathrm{V} * \mathrm{sec})\end{array}$ & $\%$ Area & $\begin{array}{c}\text { Height } \\
(\mu \mathrm{V})\end{array}$ & $\begin{array}{c}\% \\
\text { Height }\end{array}$ \\
\hline 1 & 15.133 & 12068669 & 90.05 & 584316 & 89.70 \\
\hline 2 & 16.665 & 1333699 & 9.95 & 67121 & 10.30 \\
\hline
\end{tabular}




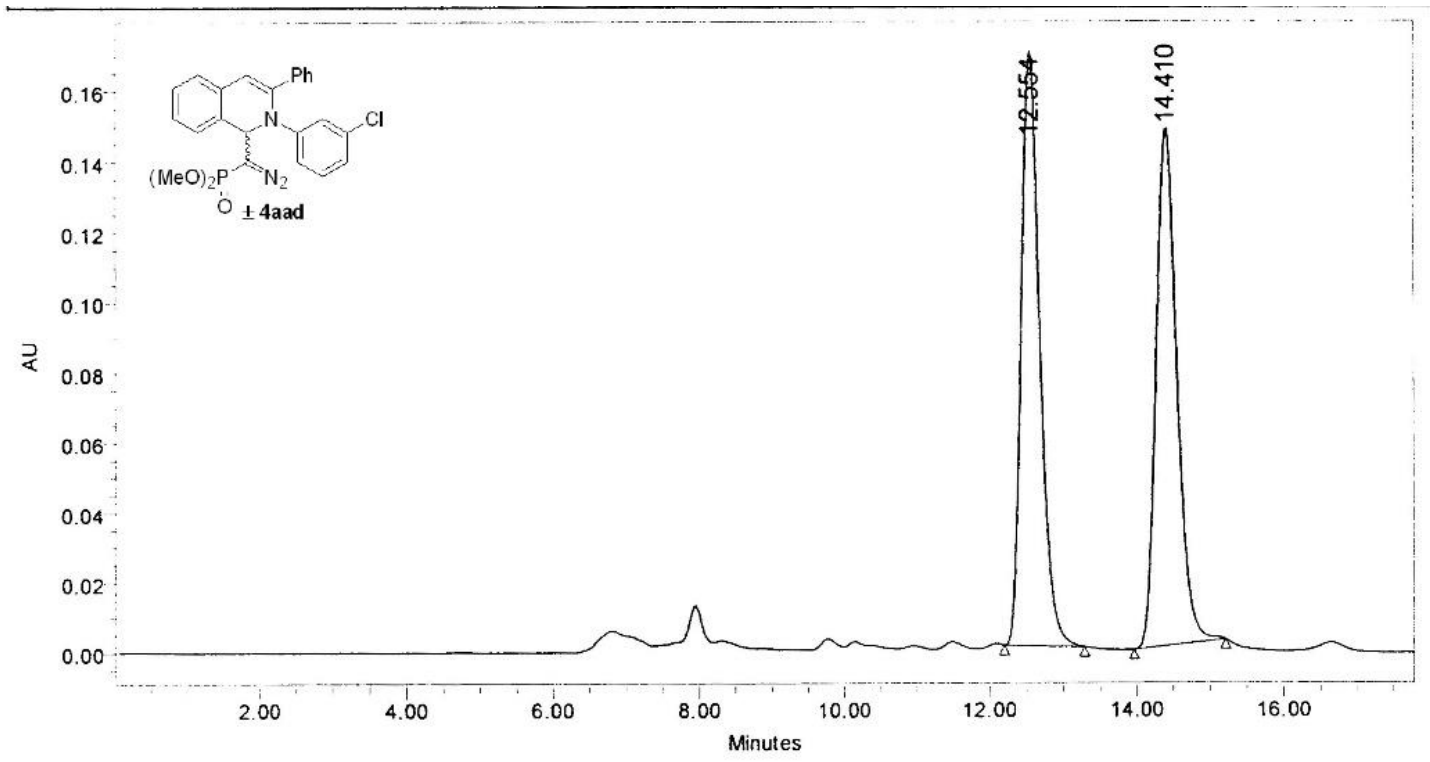

\begin{tabular}{|c|c|c|c|c|c|}
\hline & $\begin{array}{c}\text { RT } \\
(\mathrm{min})\end{array}$ & $\begin{array}{c}\text { Area } \\
(\mu \mathrm{V} * \mathrm{sec})\end{array}$ & $\%$ Area & $\begin{array}{c}\text { Height } \\
(\mu \mathrm{V})\end{array}$ & $\begin{array}{c}\% \\
\text { Height }\end{array}$ \\
\hline 1 & 12.554 & 2928499 & 50.10 & 169385 & 53.42 \\
\hline 2 & 14.410 & 2916424 & 49.90 & 147692 & 46.58 \\
\hline
\end{tabular}

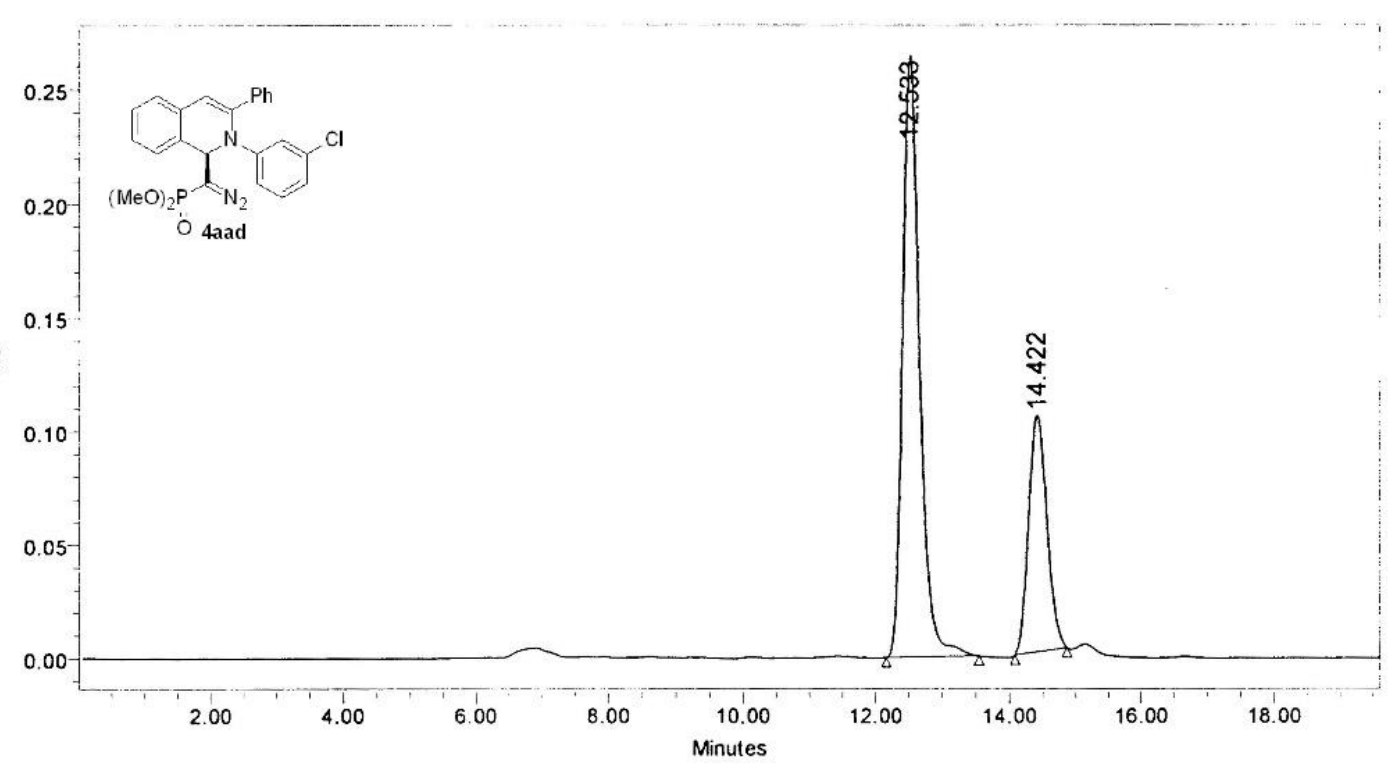

\begin{tabular}{|c|c|c|c|c|c|}
\hline & $\begin{array}{c}\text { RT } \\
(\mathrm{min})\end{array}$ & $\begin{array}{c}\text { Area } \\
\left(\mu \mathrm{V}^{*} \mathrm{sec}\right)\end{array}$ & $\%$ Area & $\begin{array}{c}\text { Height } \\
(\mu \mathrm{V})\end{array}$ & $\begin{array}{c}\% \\
\text { Height }\end{array}$ \\
\hline 1 & 12.533 & 4674472 & 70.09 & 264248 & 71.69 \\
\hline 2 & 14.422 & 1994892 & 29.91 & 104328 & 28.31 \\
\hline
\end{tabular}




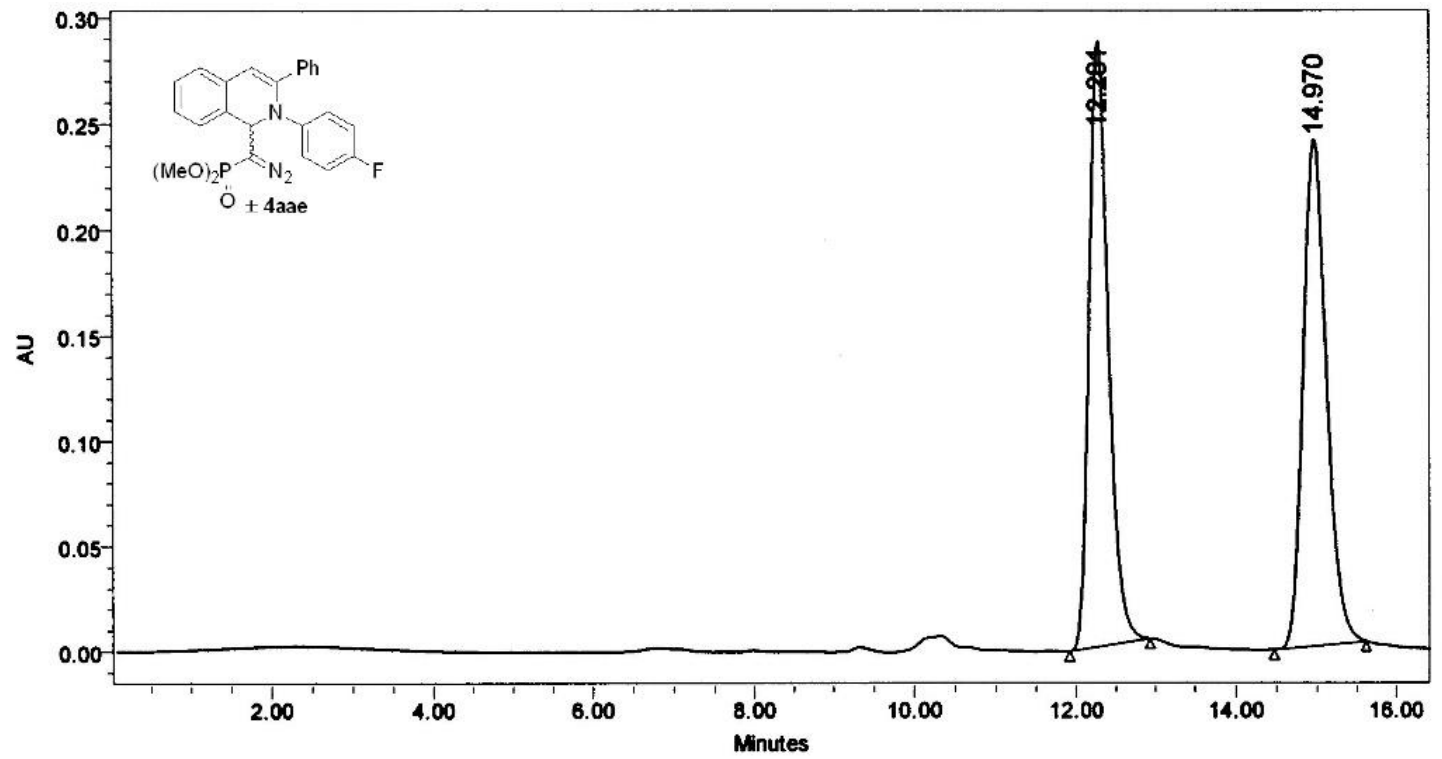

\begin{tabular}{|c|c|c|c|c|c|}
\hline & $\begin{array}{c}\text { RT } \\
(\mathrm{min})\end{array}$ & $\begin{array}{c}\text { Area } \\
\left(\mu \mathrm{N}^{*} \mathrm{sec}\right)\end{array}$ & \% Area & $\begin{array}{c}\text { Height } \\
(\mu \mathrm{V})\end{array}$ & $\begin{array}{c}\% \\
\text { Height }\end{array}$ \\
\hline 1 & 12.281 & 4835484 & 49.69 & 286832 & 54.42 \\
\hline 2 & 14.970 & 4896511 & 50.31 & 240226 & 45.58 \\
\hline
\end{tabular}

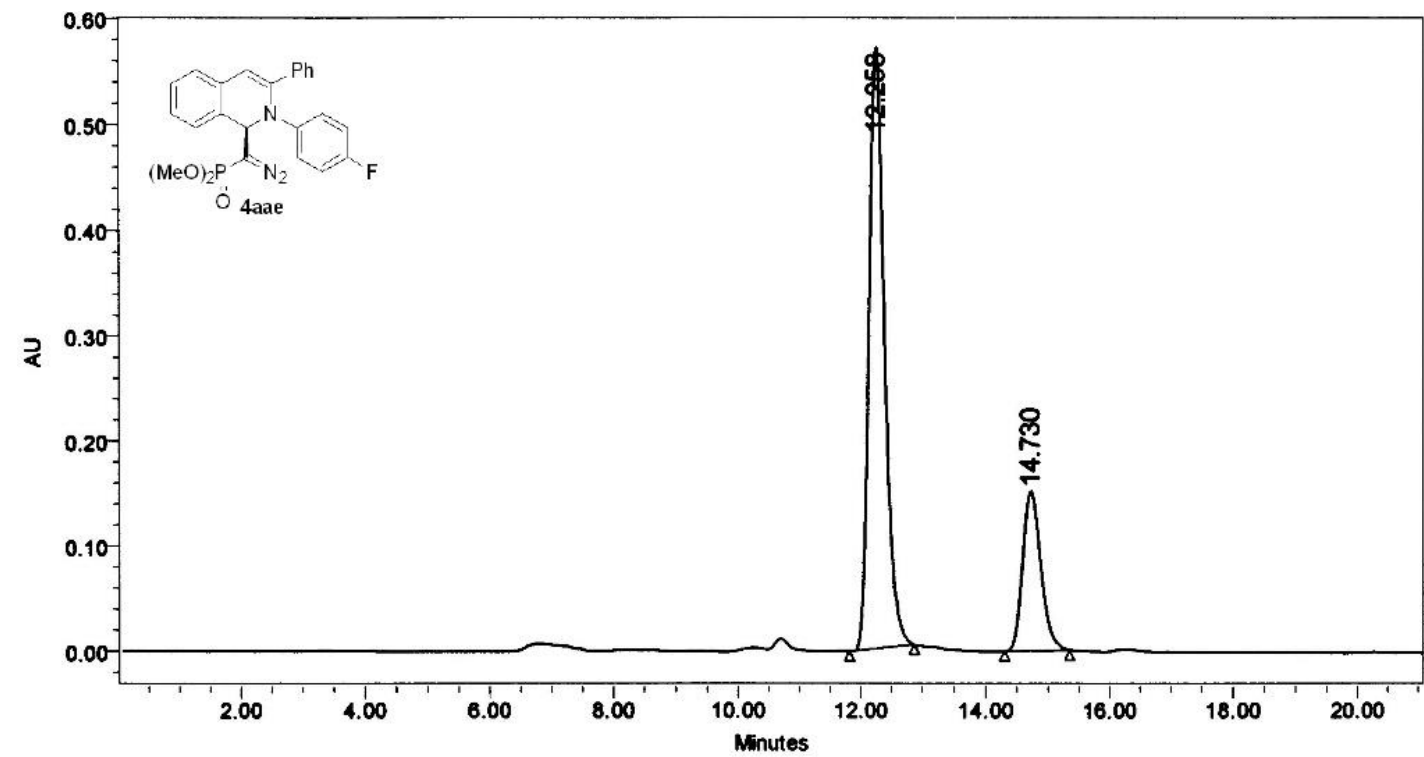

\begin{tabular}{|c|c|c|c|c|c|}
\hline & $\begin{array}{c}\text { RT } \\
(\mathrm{min})\end{array}$ & $\begin{array}{c}\text { Area } \\
(\mu \mathrm{N} * \mathrm{sec})\end{array}$ & \% Area & $\begin{array}{c}\text { Height } \\
(\mu \mathrm{V})\end{array}$ & $\begin{array}{c}\% \\
\text { Height }\end{array}$ \\
\hline 1 & 12.258 & 9986867 & 76.21 & 571676 & 79.00 \\
\hline 2 & 14.730 & 3117513 & 23.79 & 152010 & 21.00 \\
\hline
\end{tabular}




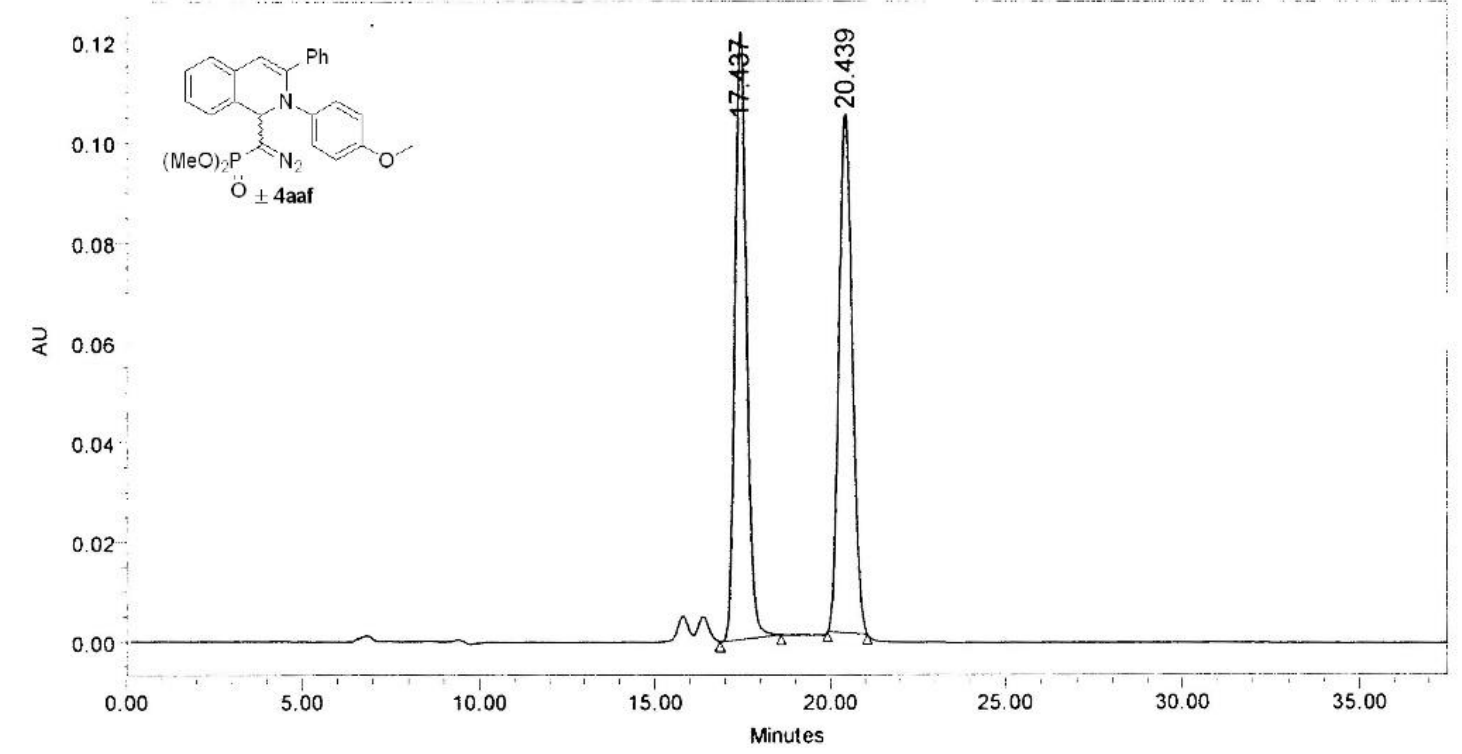

\begin{tabular}{|c|c|c|c|c|c|}
\hline & $\begin{array}{c}\mathrm{RT} \\
(\mathrm{min})\end{array}$ & $\begin{array}{c}\text { Area } \\
\left(\mu \mathrm{V}^{*} \mathrm{sec}\right)\end{array}$ & $\%$ Area & $\begin{array}{c}\text { Height } \\
(\mu \mathrm{V})\end{array}$ & $\begin{array}{c}\% \\
\text { Height }\end{array}$ \\
\hline 1 & 17.437 & 2759520 & 49.79 & 121473 & 53.89 \\
\hline 2 & 20.439 & 2782637 & 50.21 & 103944 & 46.11 \\
\hline
\end{tabular}

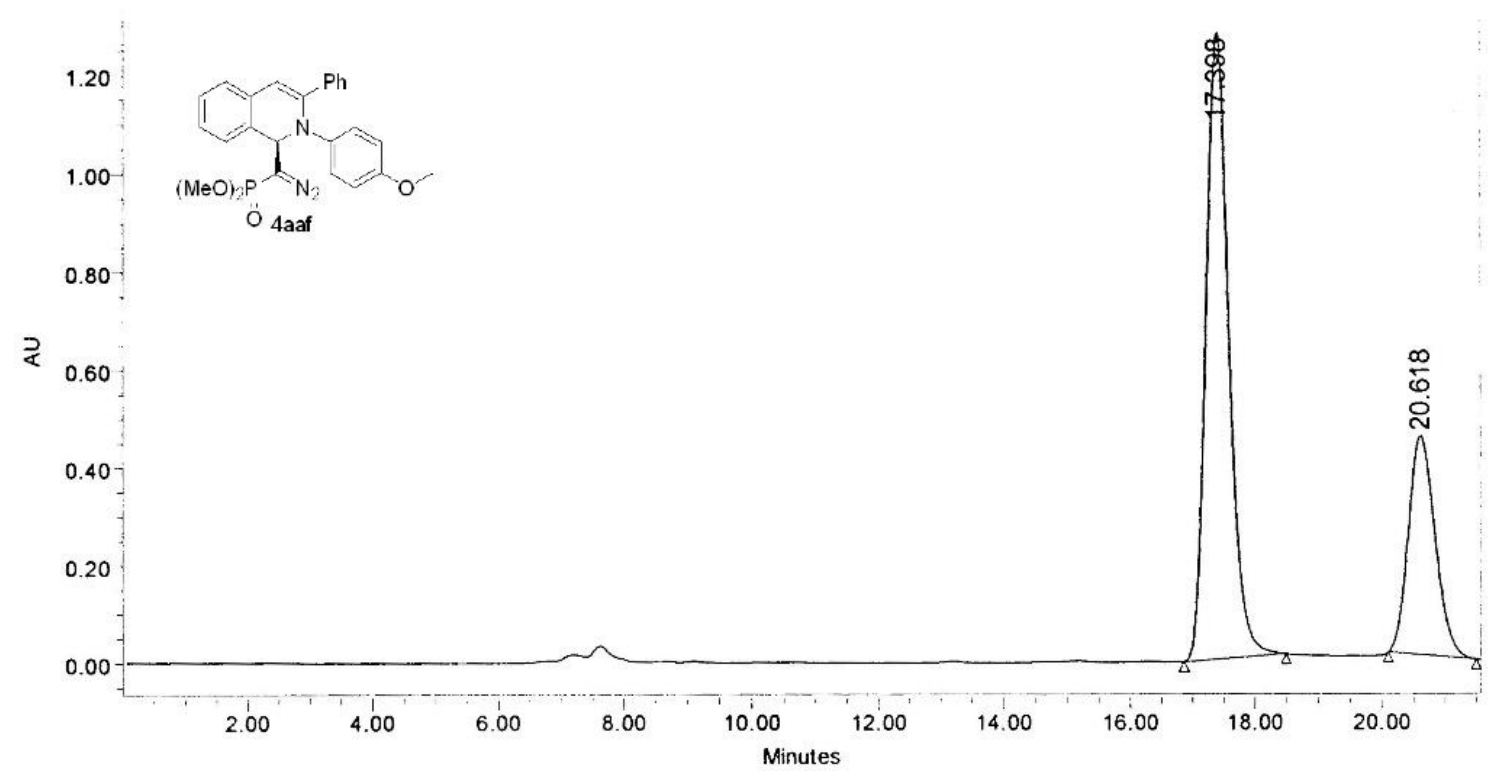

\begin{tabular}{|c|c|c|c|c|c|}
\hline & $\begin{array}{c}\text { RT } \\
(\mathrm{min})\end{array}$ & $\begin{array}{c}\text { Area } \\
(\mu \mathrm{V} \text { sec })\end{array}$ & $\%$ Area & $\begin{array}{c}\text { Height } \\
(\mu \mathrm{V})\end{array}$ & $\begin{array}{c}\% \\
\text { Height }\end{array}$ \\
\hline 1 & 17.398 & 32425083 & 71.00 & 1277885 & 74.04 \\
\hline 2 & 20.618 & 13242035 & 29.00 & 448030 & 25.96 \\
\hline
\end{tabular}




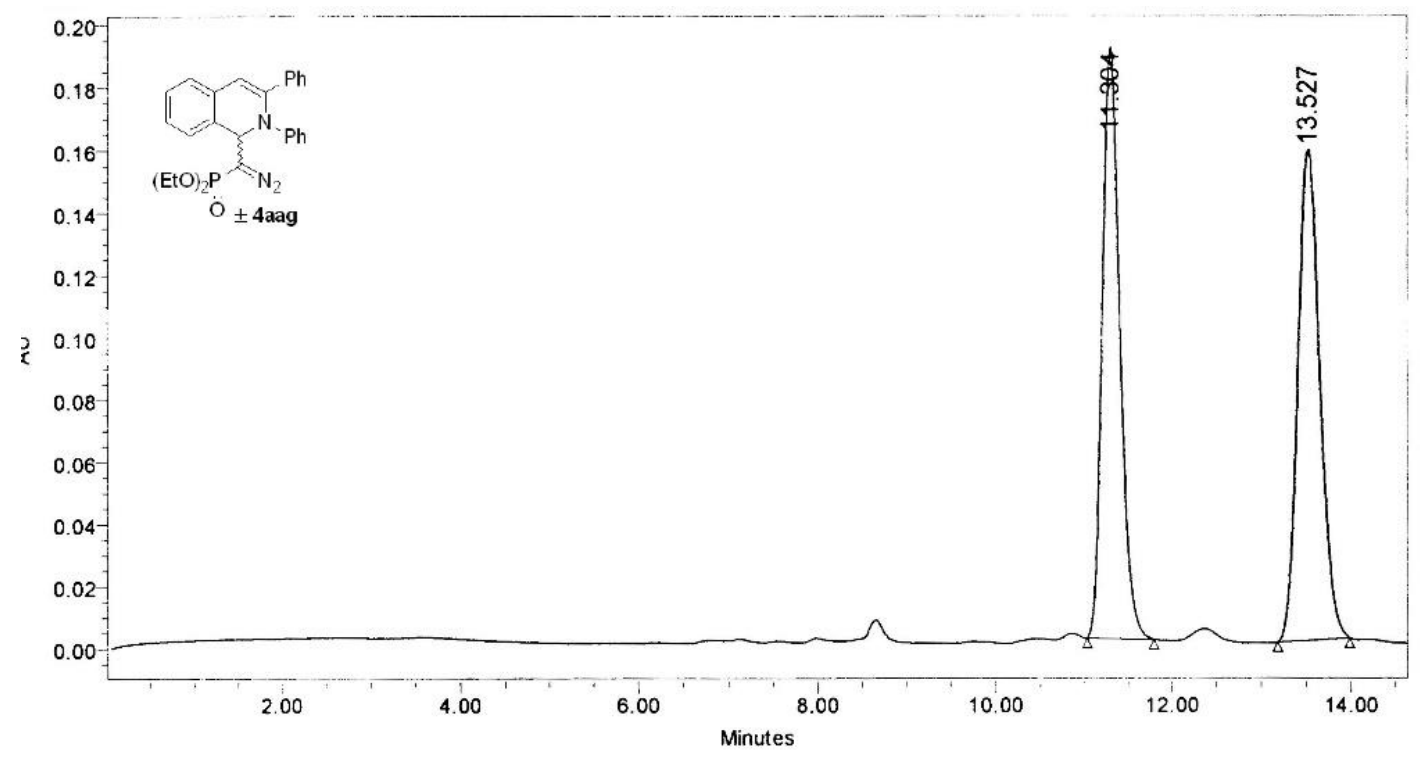

\begin{tabular}{|c|c|c|c|c|c|}
\hline & $\begin{array}{c}\mathrm{RT} \\
(\mathrm{min})\end{array}$ & $\begin{array}{c}\text { Area } \\
(\mu \mathrm{V} * \mathrm{sec})\end{array}$ & $\%$ Area & $\begin{array}{c}\text { Height } \\
(\mu \mathrm{V})\end{array}$ & $\begin{array}{c}\% \\
\text { Height }\end{array}$ \\
\hline 1 & 11.304 & 2647073 & 50.00 & 190009 & 54.63 \\
\hline 2 & 13.527 & 2647338 & 50.00 & 157775 & 45.37 \\
\hline
\end{tabular}

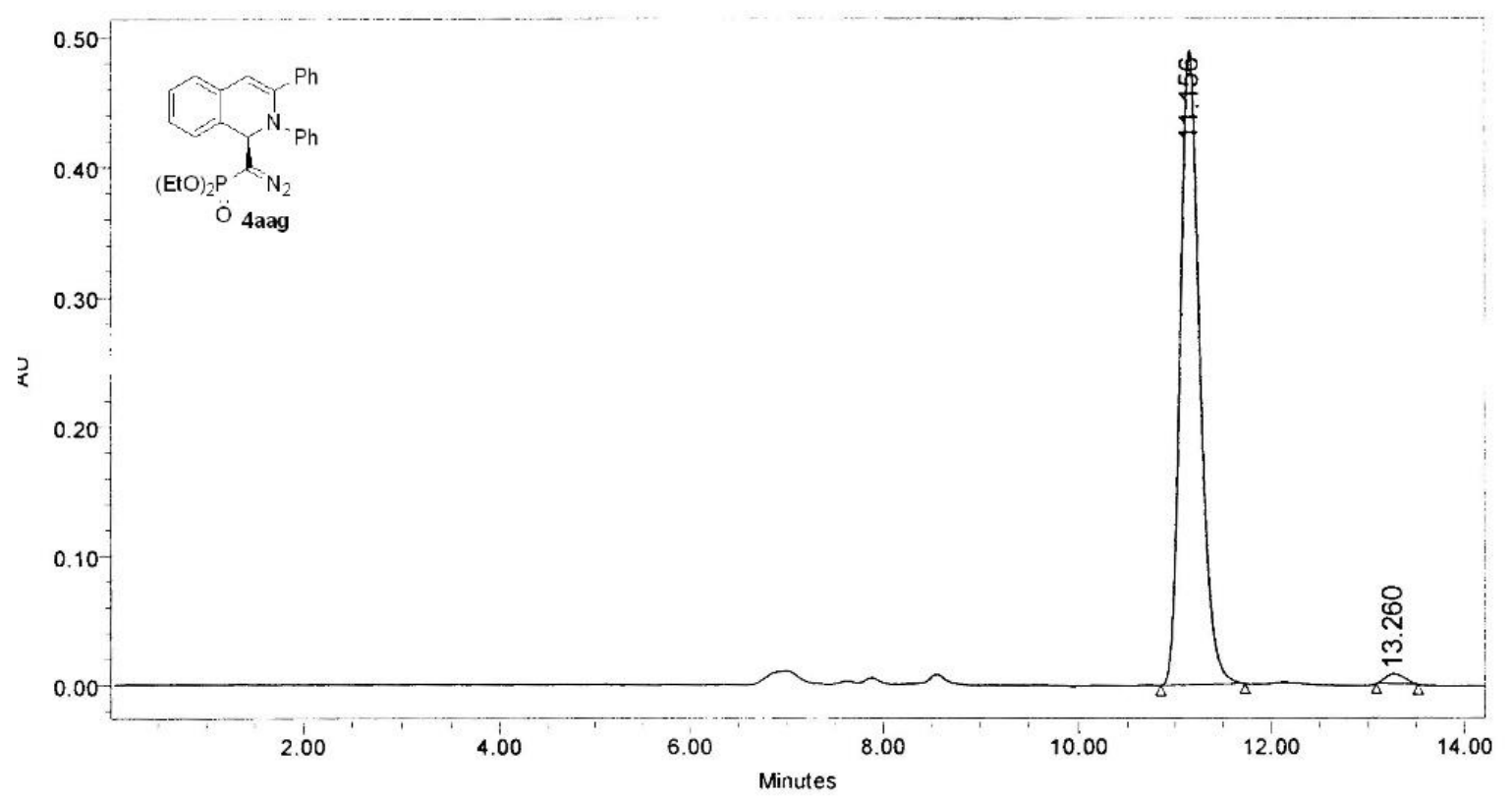

\begin{tabular}{|c|c|r|r|r|r|}
\hline & $\begin{array}{c}\mathrm{RT} \\
(\mathrm{min})\end{array}$ & $\begin{array}{c}\text { Area } \\
\left(\mu \mathrm{V}^{*} \mathrm{sec}\right)\end{array}$ & $\%$ Area & $\begin{array}{c}\text { Height } \\
(\mu \mathrm{V})\end{array}$ & $\begin{array}{c}\% \\
\text { Height }\end{array}$ \\
\hline 1 & 11.156 & 6921284 & 98.52 & 490176 & 98.48 \\
\hline 2 & 13.260 & 103715 & 1.48 & 7553 & 1.52 \\
\hline
\end{tabular}




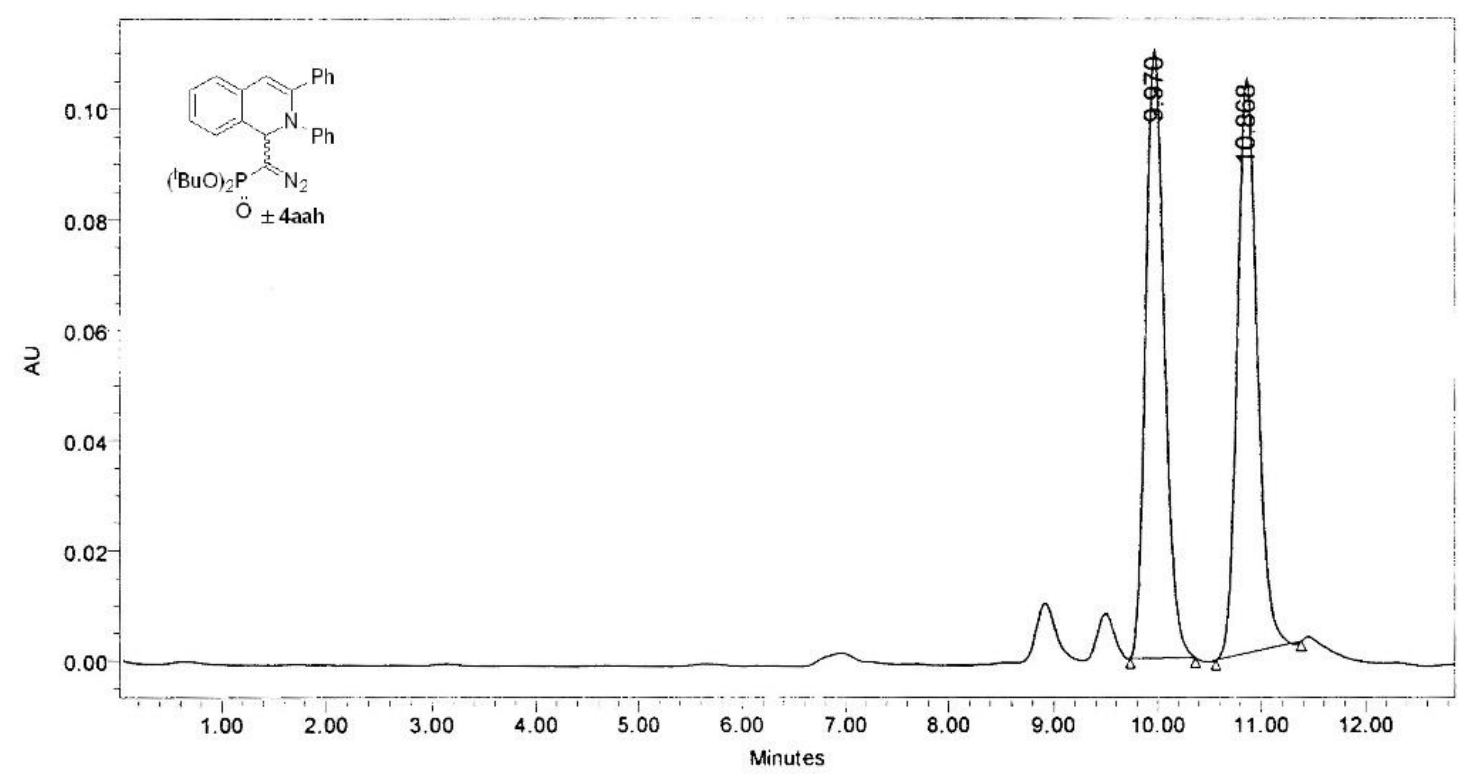

\begin{tabular}{|c|c|c|c|c|c|}
\hline & $\begin{array}{c}\mathrm{RT} \\
(\mathrm{min})\end{array}$ & $\begin{array}{c}\text { Area } \\
\left(\mu \mathrm{V}^{*} \mathrm{sec}\right)\end{array}$ & $\%$ Area & $\begin{array}{c}\text { Height } \\
(\mu \mathrm{V})\end{array}$ & $\begin{array}{c}\% \\
\text { Height }\end{array}$ \\
\hline 1 & 9.970 & 1397483 & 50.08 & 110287 & 51.43 \\
\hline 2 & 10.868 & 1393188 & 49.92 & 104150 & 48.57 \\
\hline
\end{tabular}

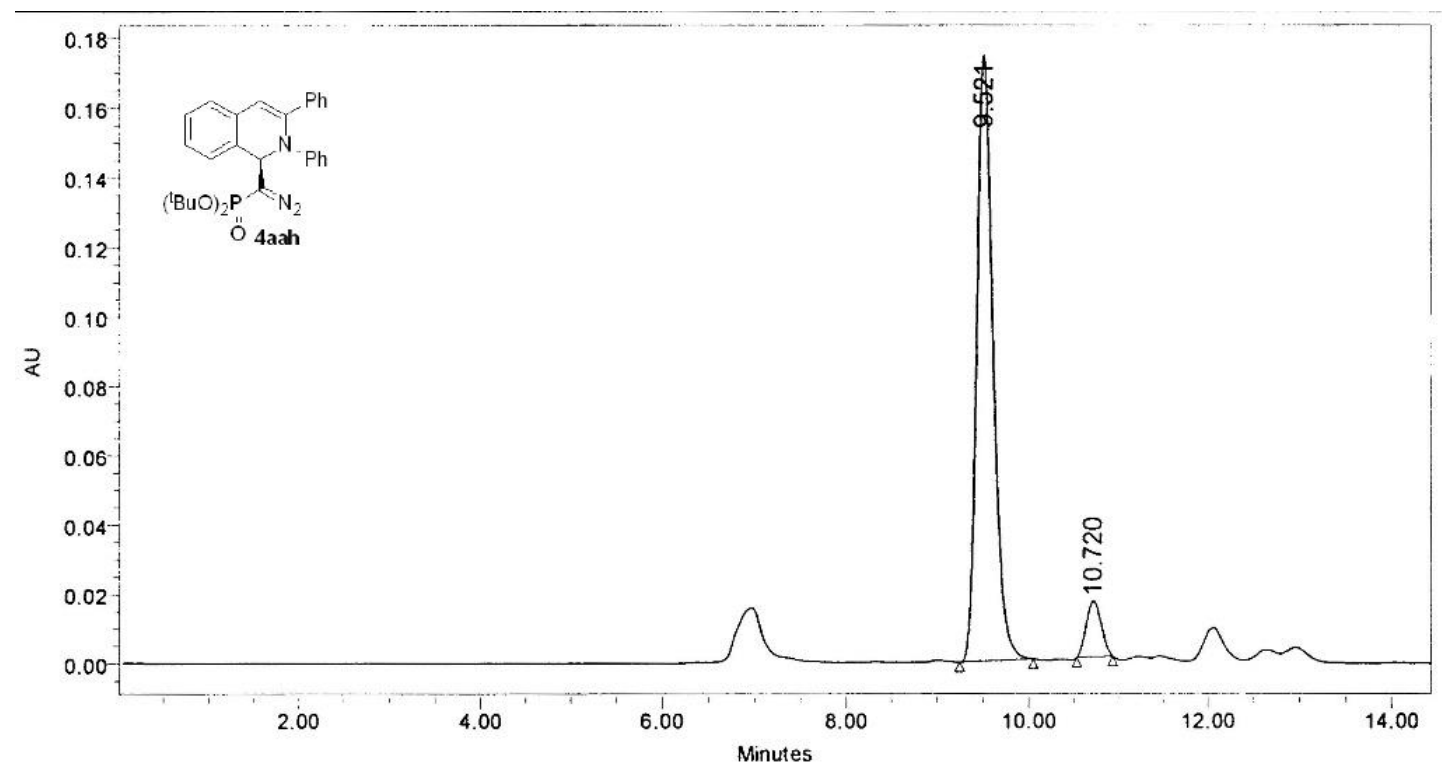

\begin{tabular}{|c|c|c|r|r|r|}
\hline & $\begin{array}{c}\mathrm{RT} \\
(\mathrm{min})\end{array}$ & $\begin{array}{c}\text { Area } \\
\left(\mu \mathrm{V}^{*} \mathrm{sec}\right)\end{array}$ & $\%$ Area & $\begin{array}{c}\text { Height } \\
(\mu \mathrm{V})\end{array}$ & $\begin{array}{c}\% \\
\text { Height }\end{array}$ \\
\hline 1 & 9.521 & 2147239 & 92.02 & 174356 & 91.51 \\
\hline 2 & 10.720 & 186212 & 7.98 & 16184 & 8.49 \\
\hline
\end{tabular}




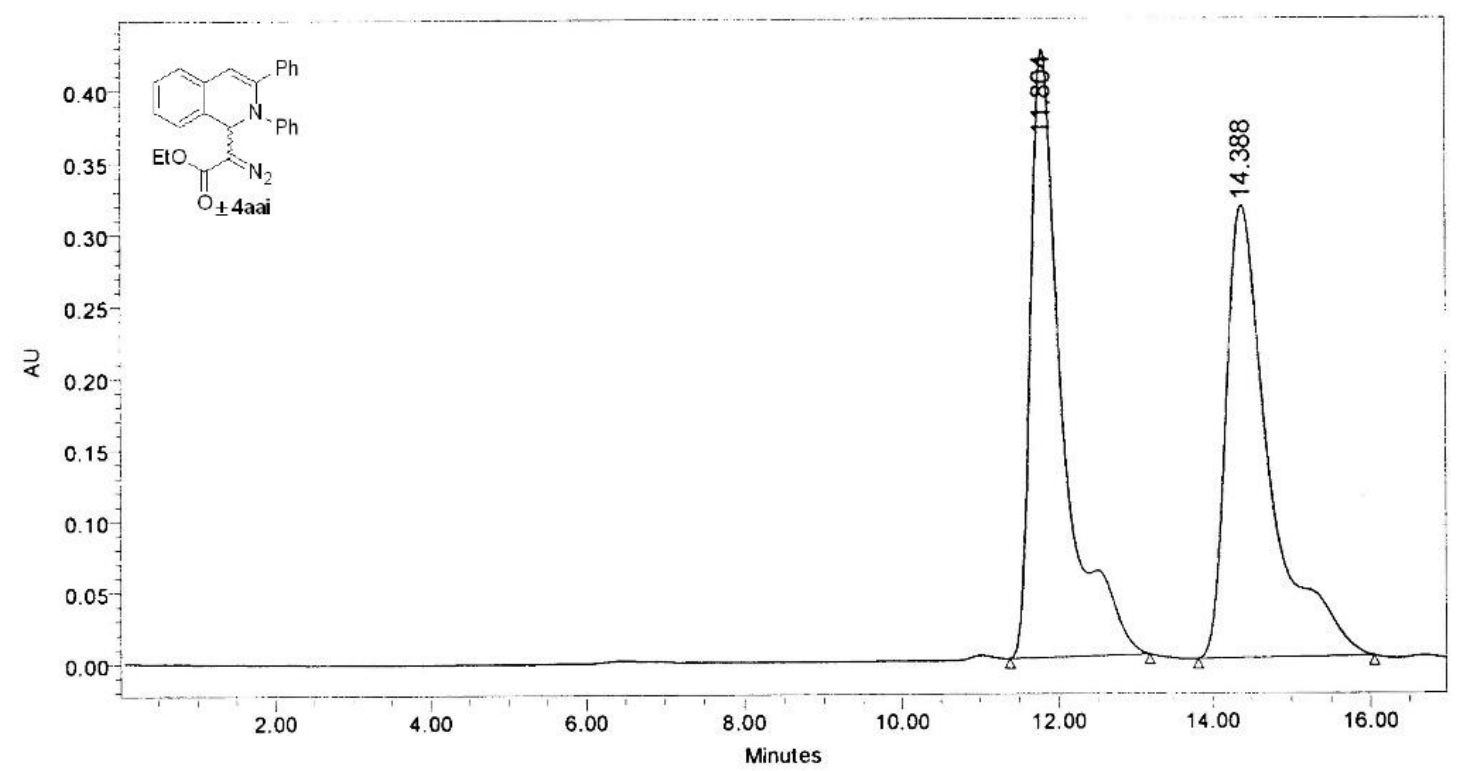

\begin{tabular}{|c|c|c|c|c|c|}
\hline & $\begin{array}{c}\text { RT } \\
(\mathrm{min})\end{array}$ & $\begin{array}{c}\text { Area } \\
(\mu \mathrm{V} * \mathrm{sec})\end{array}$ & $\%$ Area & $\begin{array}{c}\text { Height } \\
(\mu \mathrm{V})\end{array}$ & $\begin{array}{c}\% \\
\text { Height }\end{array}$ \\
\hline 1 & 11.804 & 12047630 & 49.94 & 424483 & 57.29 \\
\hline 2 & 14.388 & 12078734 & 50.06 & 316474 & 42.71 \\
\hline
\end{tabular}

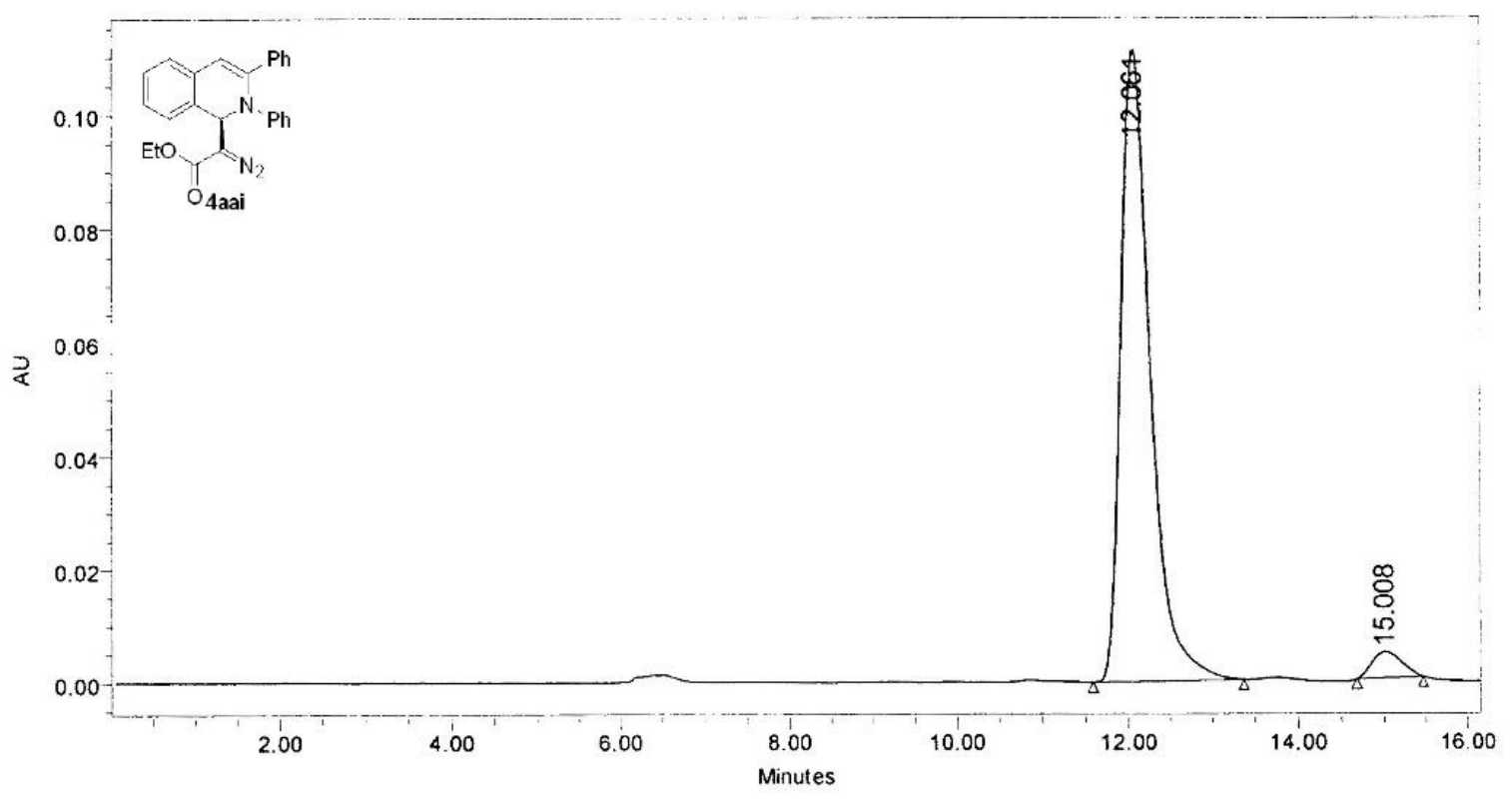

\begin{tabular}{|c|c|c|r|r|r|}
\hline & $\begin{array}{c}\text { RT } \\
(\mathrm{min})\end{array}$ & $\begin{array}{c}\text { Area } \\
\left(\mu \mathrm{V}^{*} \mathrm{sec}\right)\end{array}$ & \% Area & $\begin{array}{c}\text { Height } \\
(\mu \mathrm{V})\end{array}$ & $\begin{array}{c}\% \\
\text { Height }\end{array}$ \\
\hline 1 & 12.061 & 2675829 & 95.98 & 111304 & 96.01 \\
\hline 2 & 15.008 & 112034 & 4.02 & 4624 & 3.99 \\
\hline
\end{tabular}




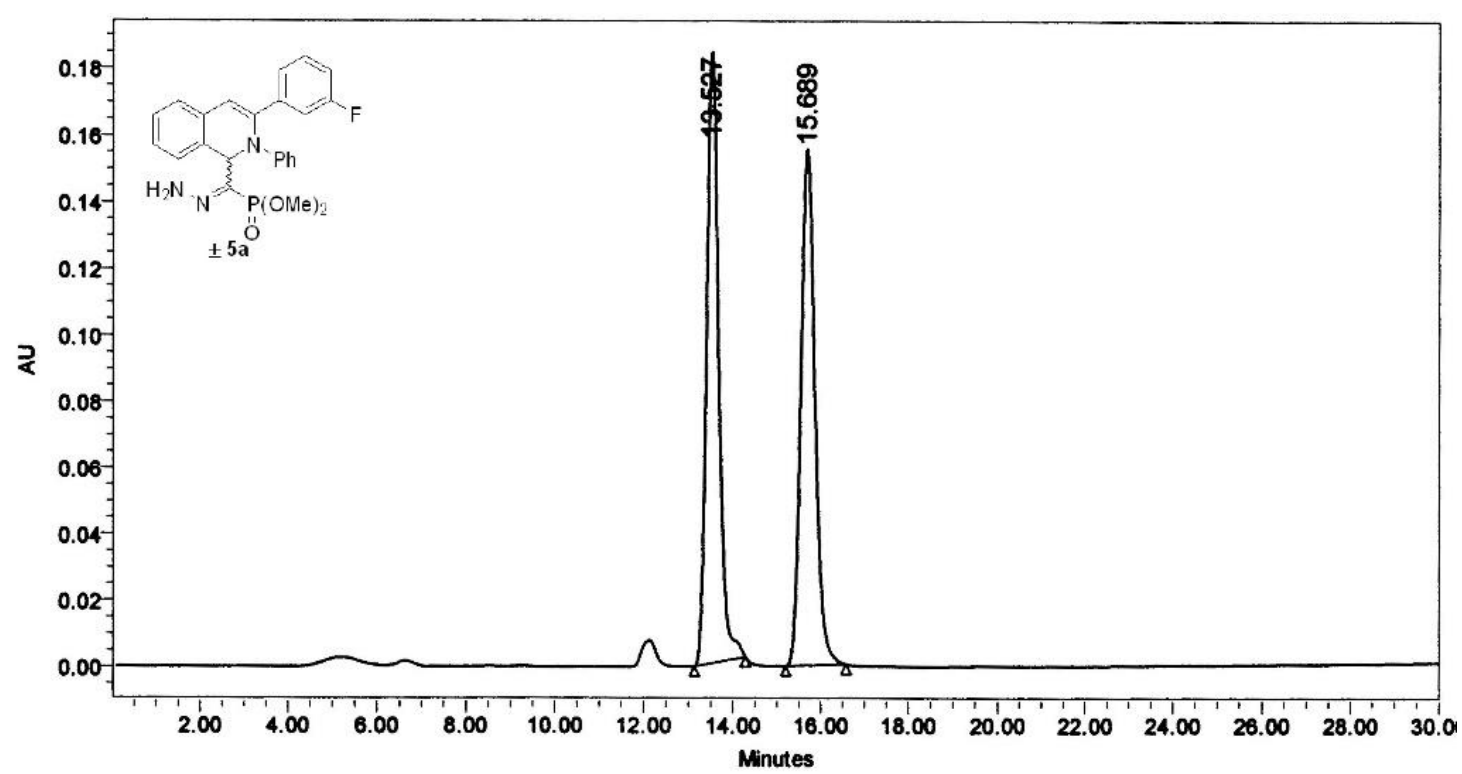

\begin{tabular}{|c|c|c|c|c|c|}
\hline & $\begin{array}{c}\mathrm{RT} \\
(\mathrm{min})\end{array}$ & $\begin{array}{c}\text { Area } \\
(\mu \mathrm{V} \text { *sec })\end{array}$ & $\%$ Area & $\begin{array}{c}\text { Height } \\
(\mu \mathrm{V})\end{array}$ & $\begin{array}{c}\% \\
\text { Height }\end{array}$ \\
\hline 1 & 13.527 & 3492975 & 49.94 & 184170 & 54.19 \\
\hline 2 & 15.689 & 3501733 & 50.06 & 155683 & 45.81 \\
\hline
\end{tabular}

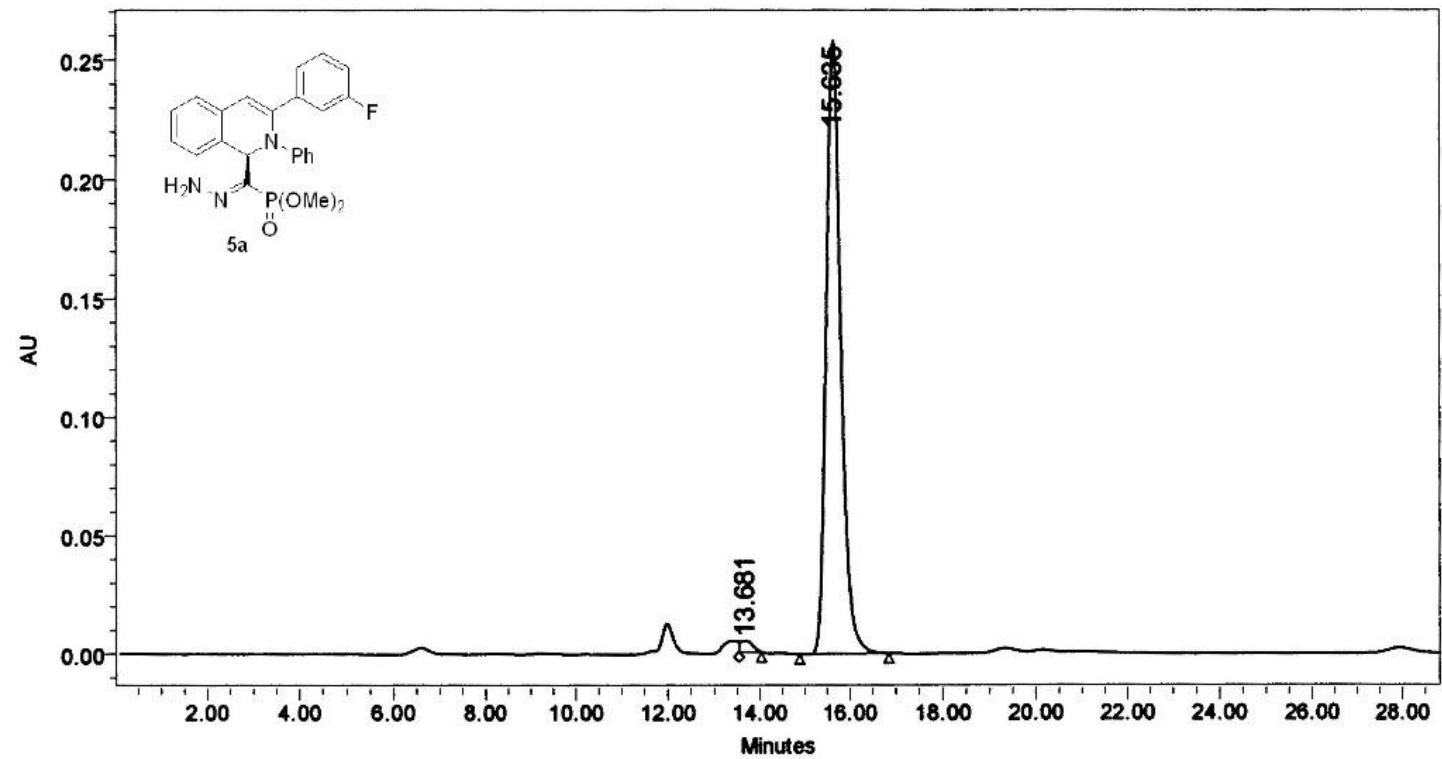

\begin{tabular}{|c|c|r|r|r|r|}
\hline & $\begin{array}{c}\mathrm{RT} \\
(\mathrm{min})\end{array}$ & $\begin{array}{c}\text { Area } \\
(\mu \mathrm{V} * \mathrm{sec})\end{array}$ & \% Area & $\begin{array}{c}\text { Height } \\
(\mu \mathrm{V})\end{array}$ & $\begin{array}{c}\% \\
\text { Height }\end{array}$ \\
\hline 1 & 13.681 & 90856 & 1.54 & 4641 & 1.77 \\
\hline 2 & 15.635 & 5814318 & 98.46 & 258063 & 98.23 \\
\hline
\end{tabular}




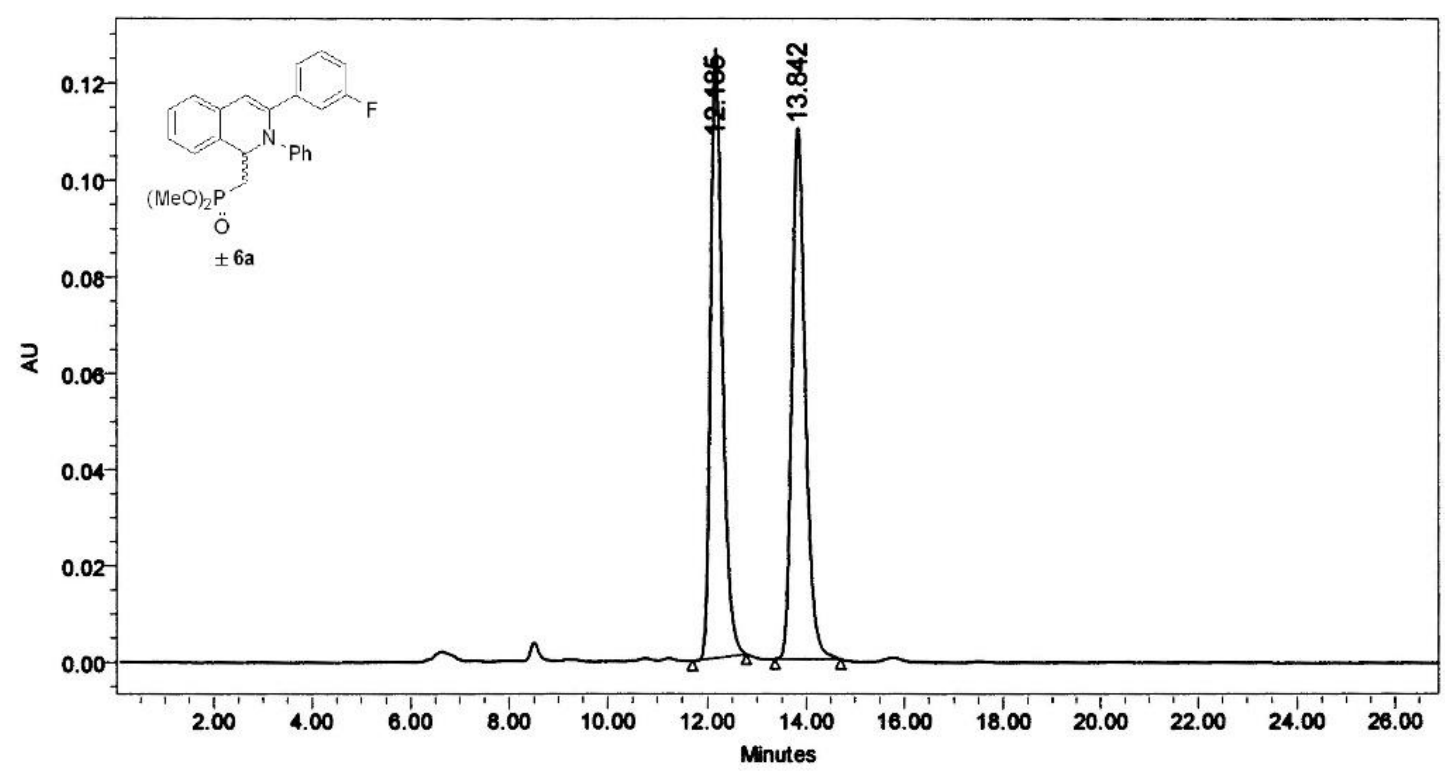

\begin{tabular}{|c|c|c|c|c|c|}
\hline & $\begin{array}{c}\text { RT } \\
(\min )\end{array}$ & $\begin{array}{c}\text { Area } \\
\left(\mu \mathcal{N}^{*} \mathrm{sec}\right)\end{array}$ & \% Area & $\begin{array}{c}\text { Height } \\
(\mu \mathrm{V})\end{array}$ & $\begin{array}{c}\% \\
\text { Height }\end{array}$ \\
\hline 1 & 12.185 & 2066939 & 49.59 & 126066 & 53.34 \\
\hline 2 & 13.842 & 2101237 & 50.41 & 110300 & 46.66 \\
\hline
\end{tabular}

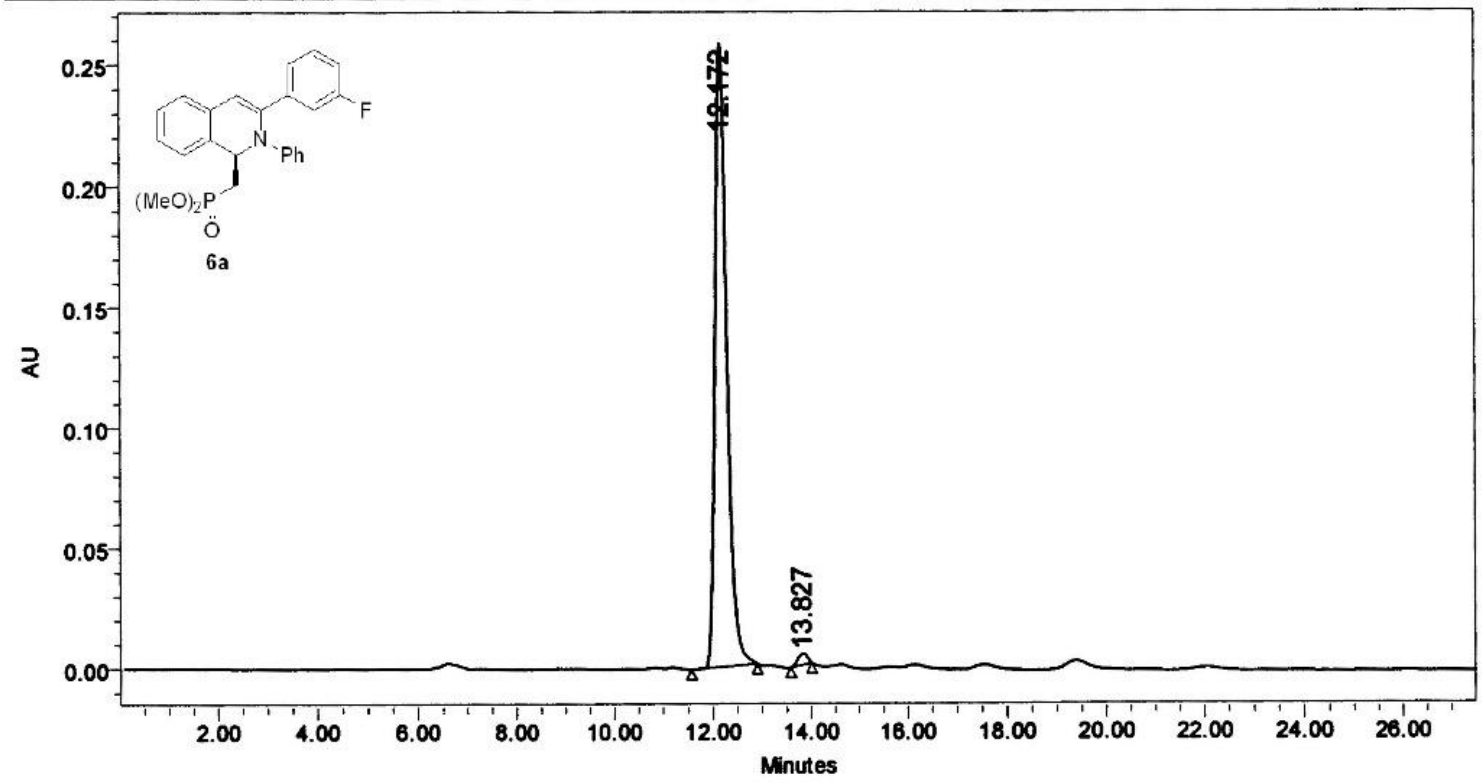

\begin{tabular}{|c|c|c|r|r|r|}
\hline & $\begin{array}{c}\mathrm{RT} \\
(\mathrm{min})\end{array}$ & $\begin{array}{c}\text { Area } \\
\left(\mu \mathrm{V}^{*} \mathrm{sec}\right)\end{array}$ & $\%$ Area & $\begin{array}{c}\text { Height } \\
(\mu \mathrm{V})\end{array}$ & $\begin{array}{c}\% \\
\text { Height }\end{array}$ \\
\hline 1 & 12.172 & 4280661 & 98.52 & 258529 & 98.25 \\
\hline 2 & 13.827 & 64099 & 1.48 & 4610 & 1.75 \\
\hline
\end{tabular}




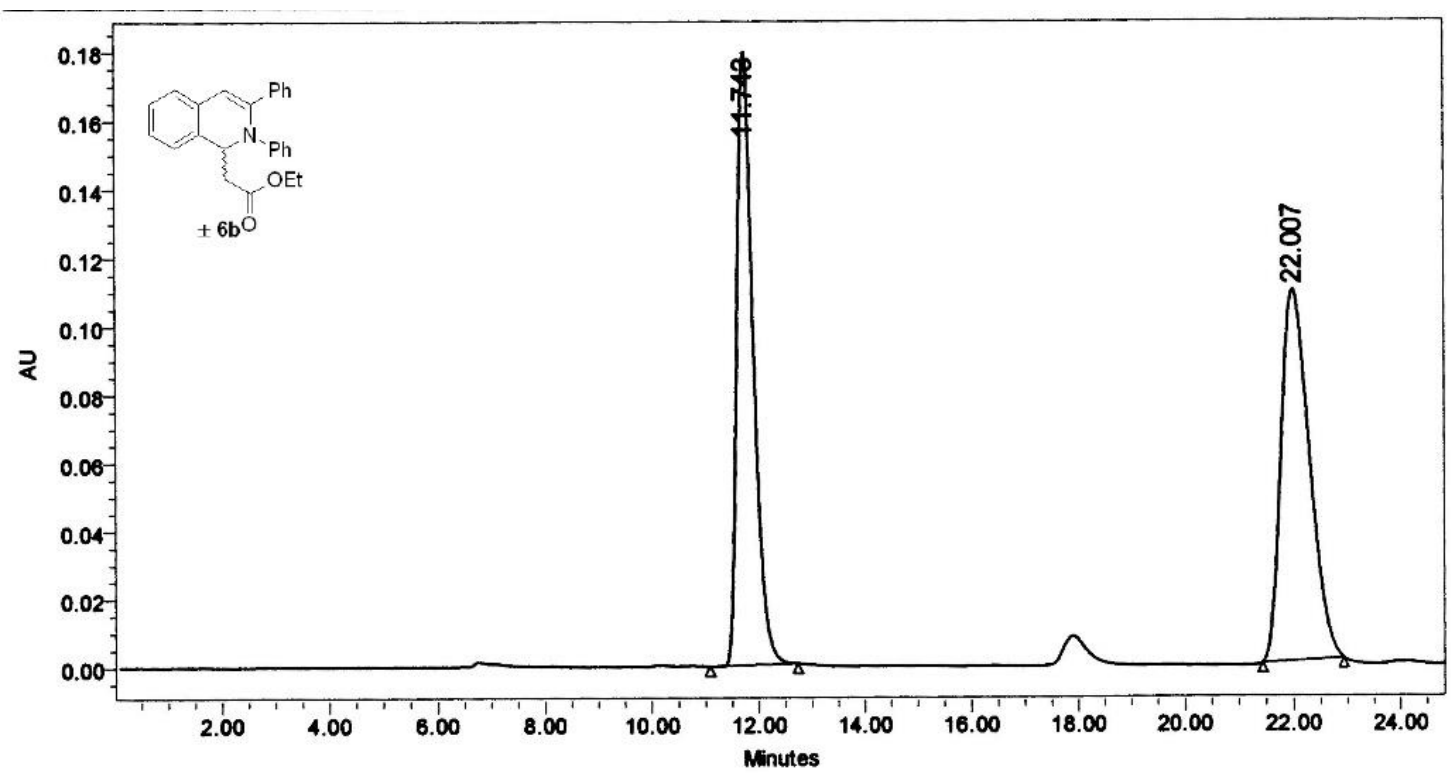

\begin{tabular}{|c|c|c|c|c|c|}
\hline & $\begin{array}{c}\text { RT } \\
(\min )\end{array}$ & $\begin{array}{c}\text { Area } \\
\left(\mu \mathrm{V}^{*} \mathrm{sec}\right)\end{array}$ & $\%$ Area & $\begin{array}{c}\text { Height } \\
(\mu \mathrm{N})\end{array}$ & $\begin{array}{c}\% \\
\text { Height }\end{array}$ \\
\hline 1 & 11.743 & 3836386 & 49.26 & 179504 & 62.17 \\
\hline 2 & 22.007 & 3952195 & 50.74 & 109207 & 37.83 \\
\hline
\end{tabular}

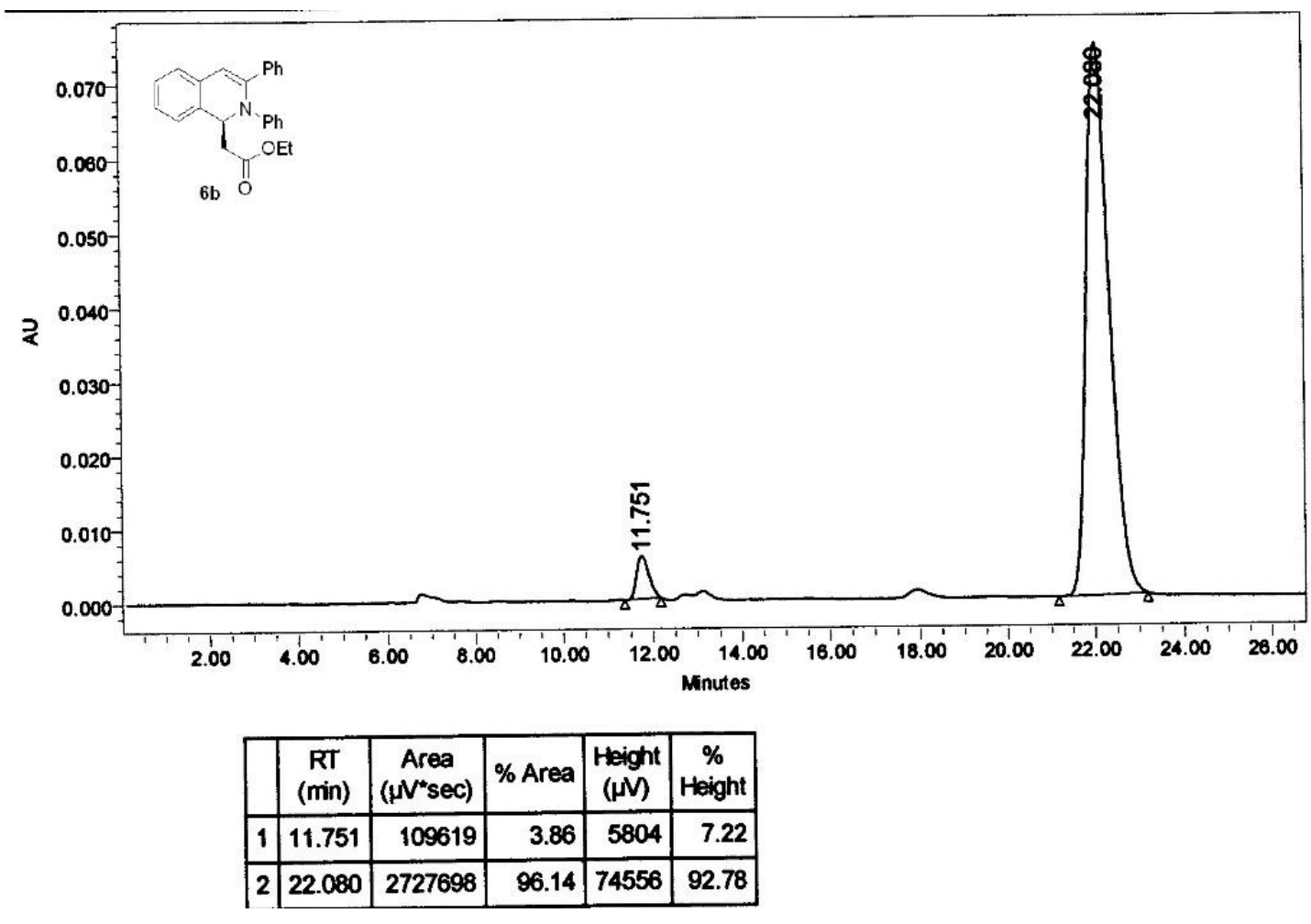




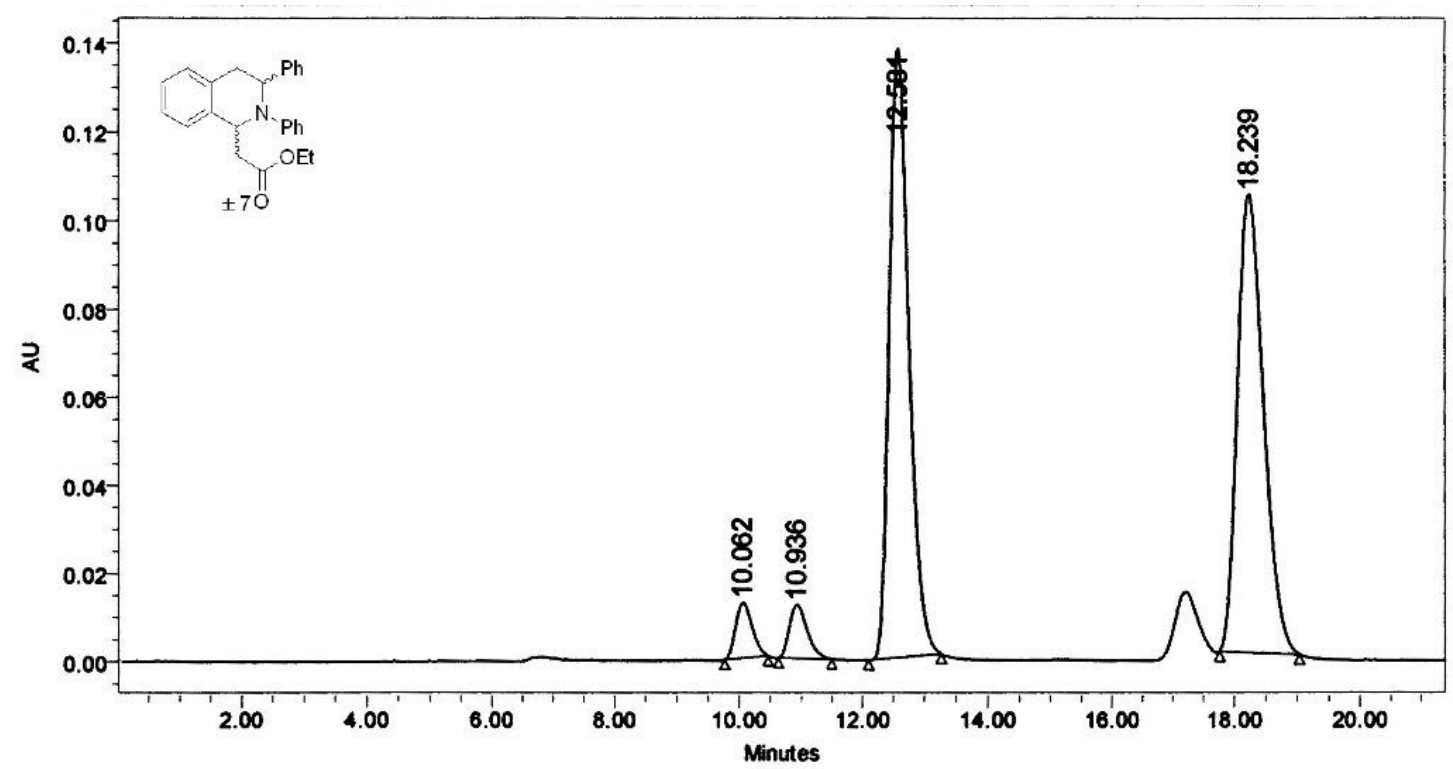

\begin{tabular}{|c|c|r|r|r|r|}
\hline & $\begin{array}{c}\mathrm{RT} \\
(\mathrm{min})\end{array}$ & $\begin{array}{c}\text { Area } \\
(\mu \mathrm{V} * \mathrm{sec})\end{array}$ & $\%$ Area & $\begin{array}{c}\text { Height } \\
(\mu \mathrm{V})\end{array}$ & $\begin{array}{c}\% \\
\text { Height }\end{array}$ \\
\hline 1 & 10.062 & 230699 & 3.52 & 12552 & 4.71 \\
\hline 2 & 10.936 & 240718 & 3.68 & 12155 & 4.56 \\
\hline 3 & 12.581 & 3039925 & 46.44 & 137868 & 51.72 \\
\hline 4 & 18.239 & 3034881 & 46.36 & 103978 & 39.01 \\
\hline
\end{tabular}

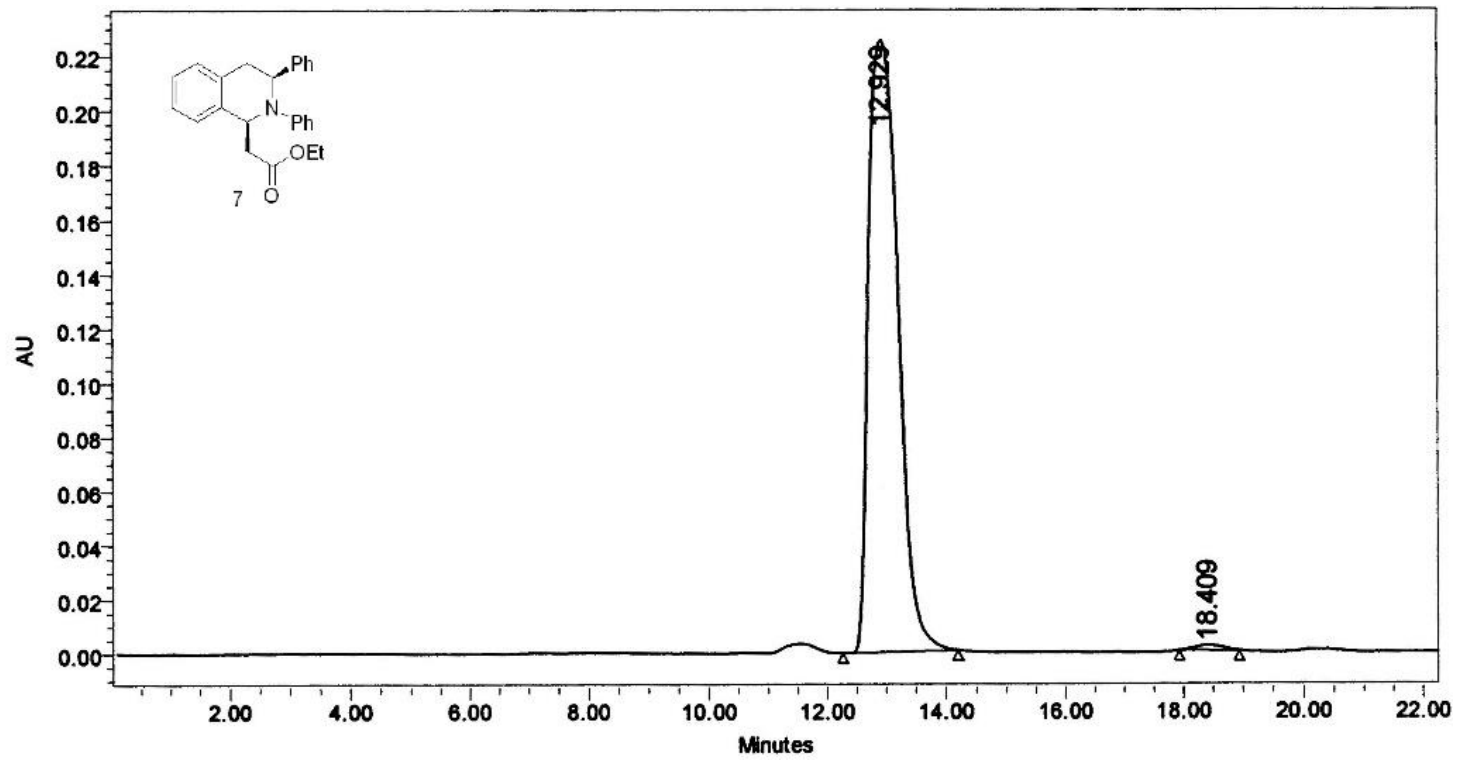

\begin{tabular}{|c|c|c|c|c|c|}
\hline & $\begin{array}{c}\mathrm{RT} \\
(\mathrm{min})\end{array}$ & $\begin{array}{c}\text { Area } \\
\left(\mu \mathrm{V}^{*} \mathrm{sec}\right)\end{array}$ & $\%$ Area & $\begin{array}{c}\text { Height } \\
(\mu \mathrm{V})\end{array}$ & $\begin{array}{c}\% \\
\text { Height }\end{array}$ \\
\hline 1 & 12.929 & 7833570 & 99.21 & 225228 & 99.19 \\
\hline 2 & 18.409 & 62273 & 0.79 & 1828 & 0.81 \\
\hline
\end{tabular}




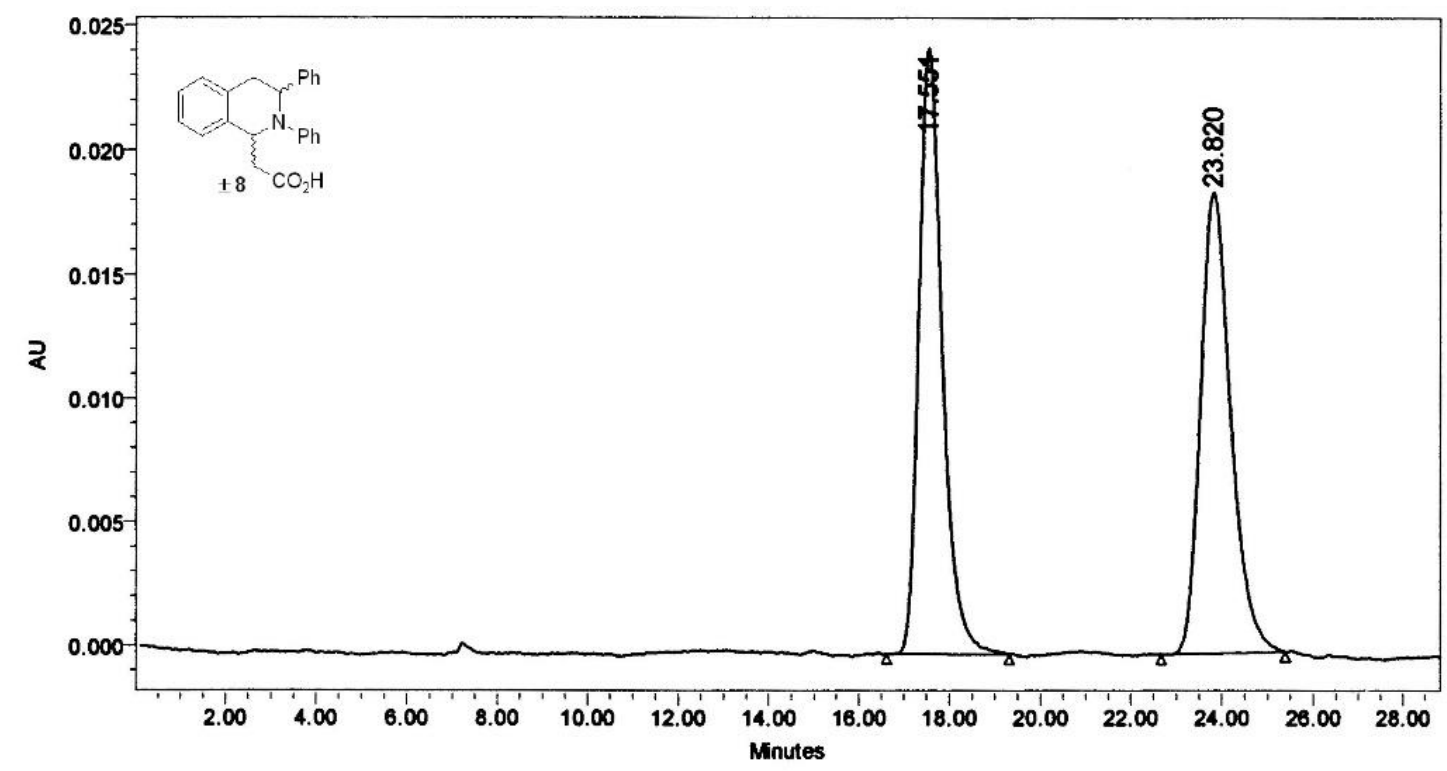

\begin{tabular}{|c|c|c|c|c|c|}
\hline & $\begin{array}{c}\mathrm{R} T \\
(\mathrm{~min})\end{array}$ & $\begin{array}{c}\text { Area } \\
\left(\mu \mathrm{N}^{*} \mathrm{sec}\right)\end{array}$ & $\%$ Area & $\begin{array}{c}\text { Height } \\
(\mu \mathrm{N})\end{array}$ & $\begin{array}{c}\% \\
\text { Height }\end{array}$ \\
\hline 1 & 17.551 & 881531 & 50.60 & 24444 & 56.75 \\
\hline 2 & 23.820 & 860683 & 49.40 & 18629 & 43.25 \\
\hline
\end{tabular}

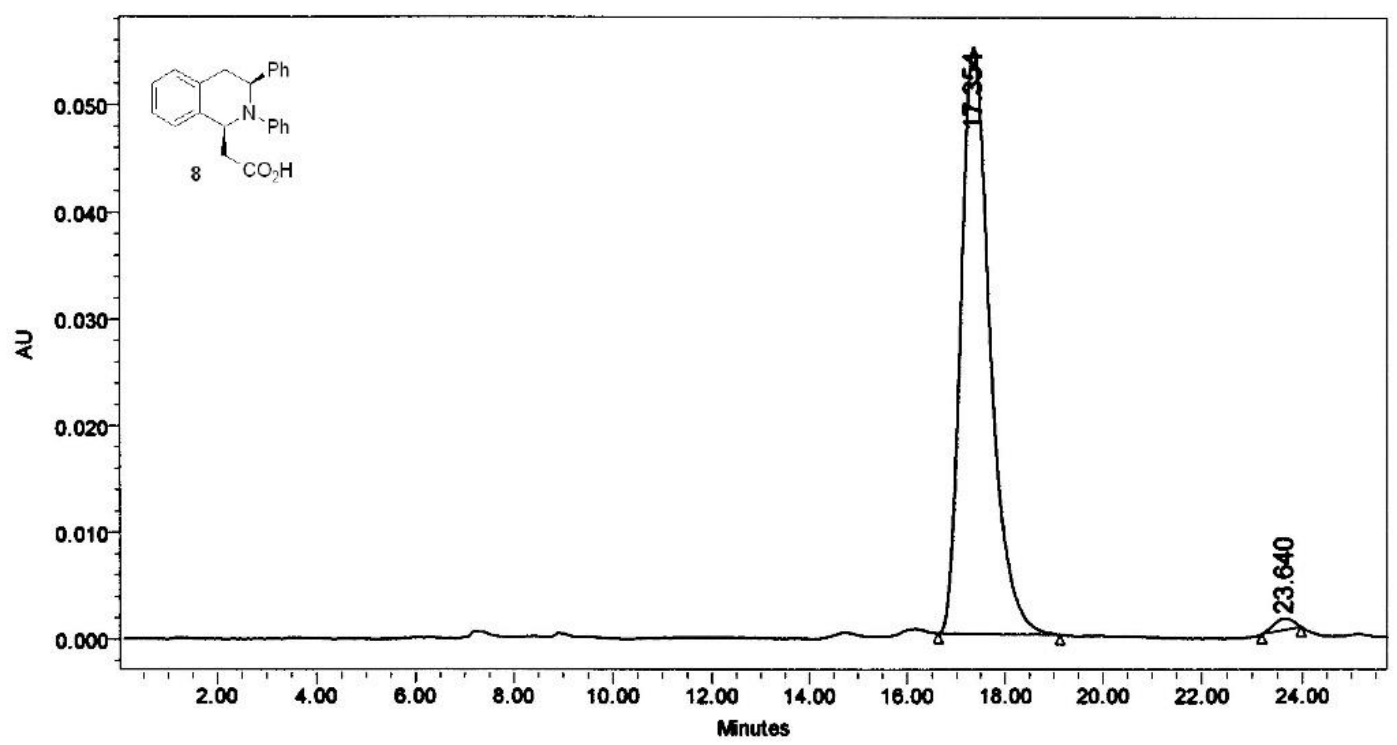

\begin{tabular}{|c|c|c|r|r|r|}
\hline & $\begin{array}{c}\text { RT } \\
(\min )\end{array}$ & $\begin{array}{c}\text { Area } \\
\left(\mu N^{*} \text { sec }\right)\end{array}$ & \% Area & $\begin{array}{c}\text { Height } \\
(\mu \mathrm{N})\end{array}$ & $\begin{array}{c}\% \\
\text { Height }\end{array}$ \\
\hline 1 & 17.354 & 2272750 & 98.80 & 54918 & 98.18 \\
\hline 2 & 23.640 & 27603 & 1.20 & 1016 & 1.82 \\
\hline
\end{tabular}




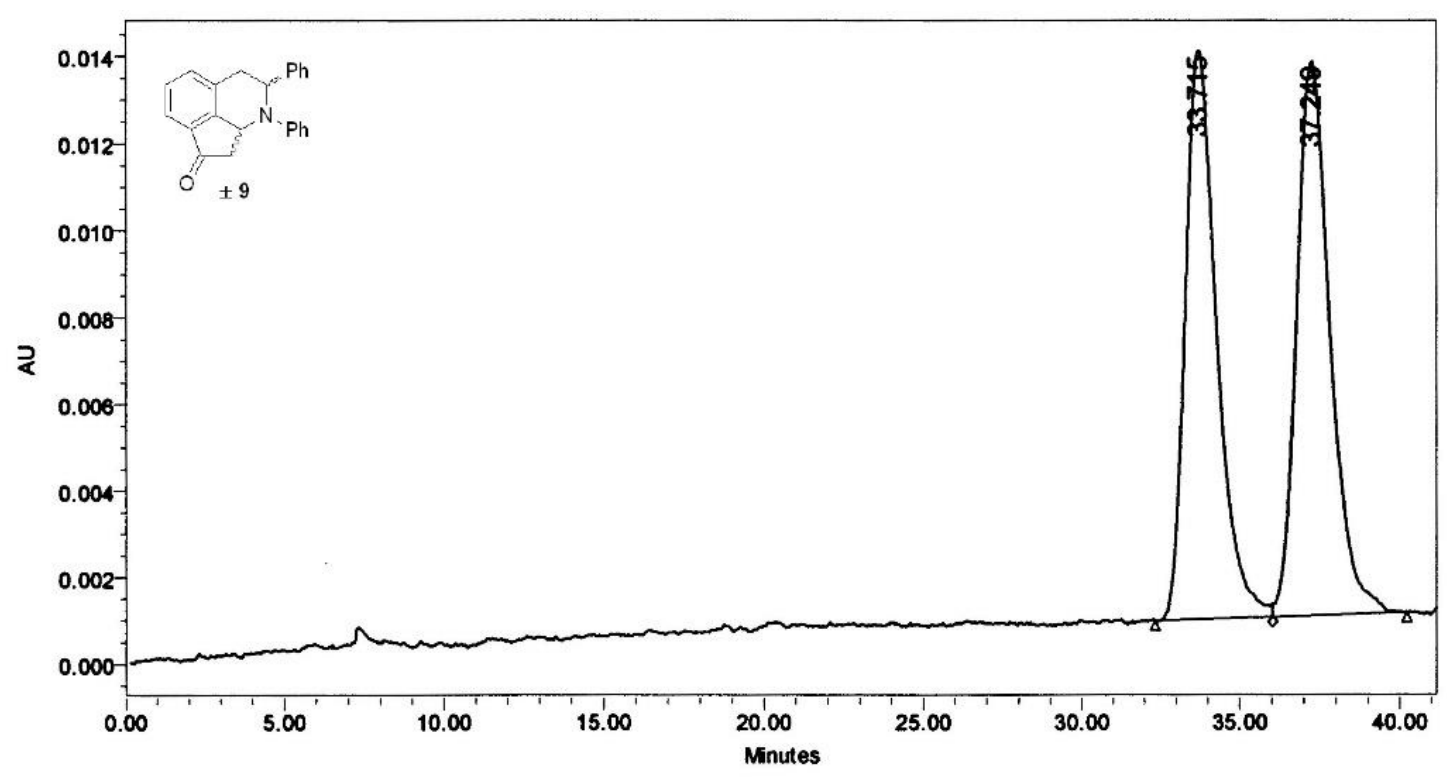

\begin{tabular}{|c|c|c|c|c|c|}
\hline & $\begin{array}{c}\mathrm{RT} \\
(\mathrm{min})\end{array}$ & $\begin{array}{c}\text { Area } \\
(\mu \mathrm{N} \text { *sec })\end{array}$ & $\%$ Area & $\begin{array}{c}\text { Height } \\
(\mu \mathrm{N})\end{array}$ & $\begin{array}{c}\% \\
\text { Height }\end{array}$ \\
\hline 1 & 33.715 & 907577 & 49.41 & 13100 & 50.62 \\
\hline 2 & 37.249 & 929302 & 50.59 & 12780 & 49.38 \\
\hline
\end{tabular}

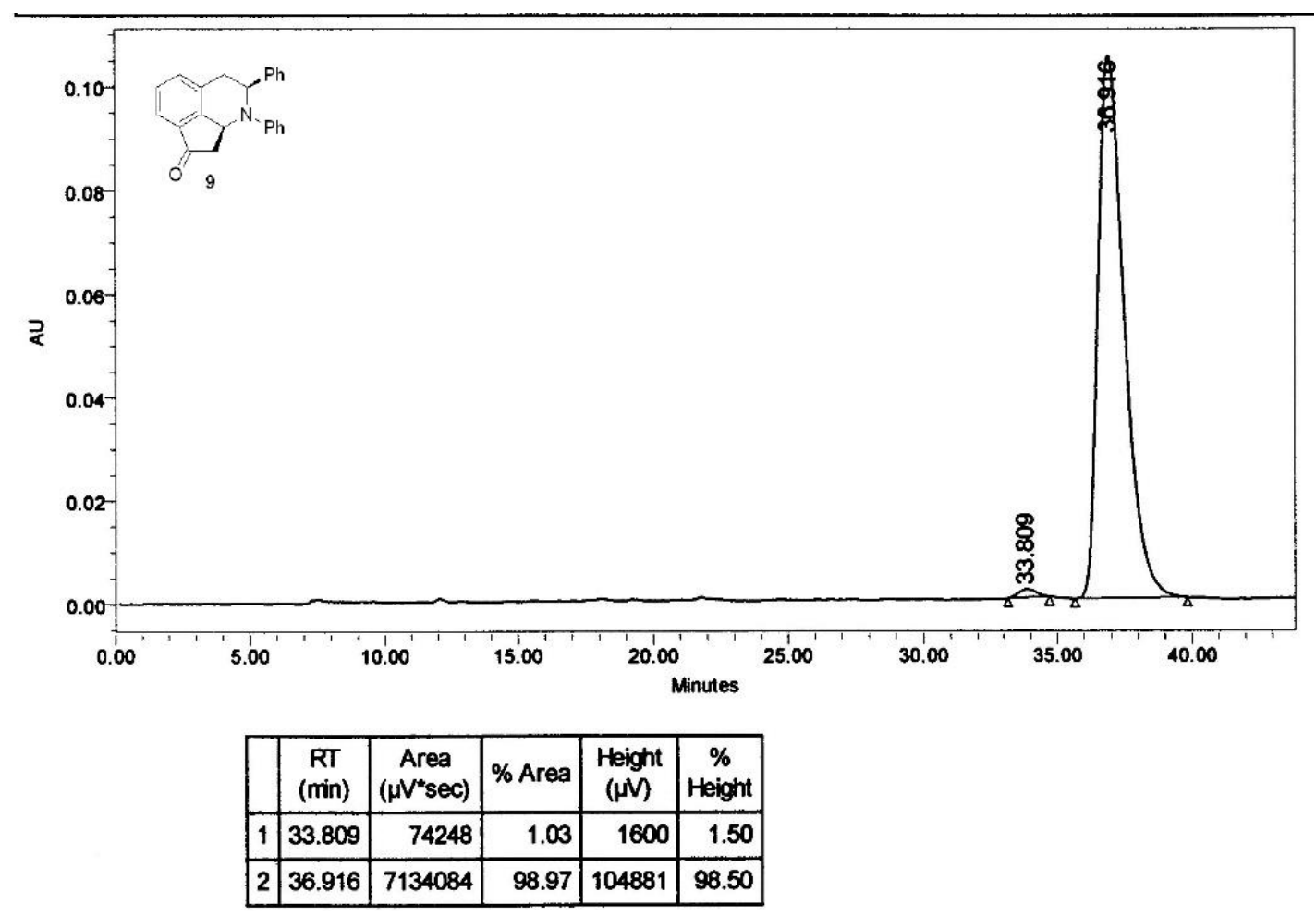

